\title{
Structure and Computational Basis for Backbone Rearrangement in Marine Oxasqualenoids
}

Francisco Cen-Pacheco ${ }^{\dagger, \ddagger}$, Adrián J. Santiago-Benítez ${ }^{\dagger}$, Ka Yi Tsui $^{\S}$, Dean J. Tantillo ${ }^{\S}{ }^{*}$, José J. Fernández $^{+, *}$, Antonio Hernández Daranas ${ }^{\perp, *}$

† Instituto Universitario de Bio-Organica Antonio González (IUBO-AG), Departmento de Química Orgánica, University of La Laguna, Av. Astrofísico Francisco Sánchez 2, 38206 La Laguna, Tenerife, Spain

‡ Facultad de Bioanálisis, Campus-Veracruz, Universidad Veracruzana, 91700, Veracruz, México

$\S$ Department of Chemistry, University of California-Davis, Davis, CA 95616 USA

${ }^{\perp}$ Instituto de Productos Naturales y Agrobiología del CSIC (IPNA-CSIC), Av. Astrofísico Francisco Sánchez s/n, 38206 La Laguna, Tenerife, Spain

\section{Table of Contents}

\begin{tabular}{|c|c|c|}
\hline \multicolumn{3}{|c|}{ A. Isolation and structural elucidation } \\
\hline Scheme S1 & Isolation procedure for compounds 1-6. & S7 \\
\hline Table S1 & $\begin{array}{l}{ }^{13} \mathrm{C} \text { and }{ }^{1} \mathrm{H} \text { NMR data of laurokanol } \mathrm{A}(1) \text { in } \mathrm{CDCl}_{3}(\mathrm{~A}) \text { and benzene- } d_{6} \\
\text { (B) at } 298 \mathrm{~K}, 600 \mathrm{MHz} \text {. }\end{array}$ & S8 \\
\hline Figure S1 & ${ }^{1} \mathrm{H}-\mathrm{NMR}$ spectrum of laurokanol A (1) in $\mathrm{CDCl}_{3}$ at $298 \mathrm{~K}, 600 \mathrm{MHz}$. & S9 \\
\hline Figure S2 & ${ }^{1} \mathrm{H}-\mathrm{NMR}$ spectrum of laurokanol A (1) in benzene-d 6 at $298 \mathrm{~K}, 600 \mathrm{MHz}$. & $\mathbf{S 1 0}$ \\
\hline Figure S3 & ${ }^{13} \mathrm{C}\left\{{ }^{1} \mathrm{H}\right\}$ NMR spectrum of laurokanol A (1) in $\mathrm{CDCl}_{3}$ at $298 \mathrm{~K}, 150 \mathrm{MHz}$. & S11 \\
\hline Figure S4 & $\begin{array}{l}{ }^{13} \mathrm{C}\left\{{ }^{1} \mathrm{H}\right\} \text { NMR spectrum of laurokanol A (1) in benzene- } d_{6} \text { at } 298 \mathrm{~K}, 150 \\
\mathrm{MHz} .\end{array}$ & $\mathbf{S 1 2}$ \\
\hline Figure S5 & COSY spectrum of laurokanol A (1) in $\mathrm{CDCl}_{3}$ at $298 \mathrm{~K}, 600 \mathrm{MHz}$. & $\mathbf{S 1 3}$ \\
\hline Figure S6 & TOCSY-1D spectra of laurokanol A (1) in $\mathrm{CDCl}_{3}$ at $298 \mathrm{~K}, 600 \mathrm{MHz}$. & S14 \\
\hline Figure S7 & TOCSY-1D spectra of laurokanol A (1) in benzene-d ${ }_{6}$ at $298 \mathrm{~K}, 600 \mathrm{MHz}$. & S15 \\
\hline Figure S8 & $\mathrm{HSQC}$ spectrum of laurokanol A (1) in $\mathrm{CDCl}_{3}$ at $298 \mathrm{~K}, 600 \mathrm{MHz}$. & S16 \\
\hline Figure S9 & HMBC spectrum of laurokanol A (1) in $\mathrm{CDCl}_{3}$ at $298 \mathrm{~K}, 600 \mathrm{MHz}$. & S17 \\
\hline Figure S10 & NOESY spectrum of laurokanol A (1) in $\mathrm{CDCl}_{3}$ at $298 \mathrm{~K}, 600 \mathrm{MHz}$. & S18 \\
\hline Figure S11 & Mass spectrum of laurokanol A (1). & $\mathbf{S 1 9}$ \\
\hline Table S2 & ${ }^{13} \mathrm{C}$ and ${ }^{1} \mathrm{H}$ NMR data of laurokanol B (2) in $\mathrm{CDCl}_{3}$ at $298 \mathrm{~K}, 600 \mathrm{MHz}$ & $\mathbf{S 2 0}$ \\
\hline Figure S12 & ${ }^{1} \mathrm{H}-\mathrm{NMR}$ spectrum of laurokanol B (2) in $\mathrm{CDCl}_{3}$ at $298 \mathrm{~K}, 600 \mathrm{MHz}$. & $\mathbf{S 2 1}$ \\
\hline Figure S13 & ${ }^{13} \mathrm{C}\left\{{ }^{1} \mathrm{H}\right\}$ NMR spectrum of laurokanol B (2) in $\mathrm{CDCl}_{3}$ at $298 \mathrm{~K}, 150 \mathrm{MHz}$. & $\mathbf{S 2 2}$ \\
\hline Figure S14 & COSY spectrum of laurokanol B (2) in $\mathrm{CDCl}_{3}$ at $298 \mathrm{~K}, 600 \mathrm{MHz}$. & $\mathbf{S 2 3}$ \\
\hline Figure S15 & TOCSY-1D spectra of laurokanol B (2) in $\mathrm{CDCl}_{3}$ at $298 \mathrm{~K}, 600 \mathrm{MHz}$. & $\mathbf{S 2 4}$ \\
\hline Figure S16 & HSQC spectrum of laurokanol B (2) in $\mathrm{CDCl}_{3}$ at $298 \mathrm{~K}, 600 \mathrm{MHz}$. & $\mathbf{S 2 5}$ \\
\hline Figure S17 & $\mathrm{HMBC}$ spectrum of laurokanol B (2) in $\mathrm{CDCl}_{3}$ at $298 \mathrm{~K}, 600 \mathrm{MHz}$. & S26 \\
\hline
\end{tabular}




\begin{tabular}{|c|c|c|}
\hline Figure S18 & NOESY spectrum of laurokanol B (2) in $\mathrm{CDCl}_{3}$ at $298 \mathrm{~K}, 600 \mathrm{MHz}$. & $\mathbf{S 2 7}$ \\
\hline Figure S19 & Mass spectrum of laurokanol B (2). & $\mathbf{S 2 8}$ \\
\hline Table S3 & ${ }^{13} \mathrm{C}$ and ${ }^{1} \mathrm{H}$ NMR data of laurokanol C (3) in $\mathrm{CDCl}_{3}$ at $298 \mathrm{~K}, 600 \mathrm{MHz}$ & $\mathbf{S 2 9}$ \\
\hline Table S4 & ${ }^{13} \mathrm{C}$ and ${ }^{1} \mathrm{H}$ NMR data of laurokanol D (4) in $\mathrm{CDCl}_{3}$ at $298 \mathrm{~K}, 600 \mathrm{MHz}$ & S30 \\
\hline Figure S20 & $\begin{array}{l}{ }^{1} \mathrm{H}-\mathrm{NMR} \text { spectrum of laurokanol C (3) and laurokanol D (4) mixture in } \\
\mathrm{CDCl}_{3} \text { at } 298 \mathrm{~K}, 600 \mathrm{MHz} \text {. }\end{array}$ & S31 \\
\hline Figure S21 & $\begin{array}{l}{ }^{13} \mathrm{C}\left\{{ }^{1} \mathrm{H}\right\} \text { NMR spectrum of laurokanol C (3) and laurokanol D (4) mixture } \\
\text { in } \mathrm{CDCl}_{3} \text { at } 298 \mathrm{~K}, 150 \mathrm{MHz} \text {. }\end{array}$ & S32 \\
\hline Figure S22 & $\begin{array}{l}\text { COSY spectrum of laurokanol C (3) and laurokanol D (4) mixture in } \\
\mathrm{CDCl}_{3} \text { at } 298 \mathrm{~K}, 600 \mathrm{MHz} \text {. }\end{array}$ & S33 \\
\hline Figure S23 & $\begin{array}{l}\text { HSQCed spectrum of laurokanol C (3) and laurokanol D (4) mixture in } \\
\mathrm{CDCl}_{3} \text { at } 298 \mathrm{~K}, 600 \mathrm{MHz} \text {. }\end{array}$ & S34 \\
\hline Figure S24 & $\begin{array}{l}\mathrm{HMBC} \text { spectrum of laurokanol C (3) and laurokanol D (4) mixture in } \\
\mathrm{CDCl}_{3} \text { at } 298 \mathrm{~K}, 600 \mathrm{MHz} \text {. }\end{array}$ & S35 \\
\hline Figure S25 & $\begin{array}{l}\text { NOESY spectrum of laurokanol C (3) and laurokanol D (4) mixture in } \\
\mathrm{CDCl}_{3} \text { at } 298 \mathrm{~K}, 600 \mathrm{MHz} \text {. }\end{array}$ & S36 \\
\hline Figure S26 & Mass spectrum of laurokanol C (3) and laurokanol D (4) mixture. & S37 \\
\hline Table S5 & ${ }^{13} \mathrm{C}$ and ${ }^{1} \mathrm{H}$ NMR data of laurokanol $\mathrm{E}(5)$ in $\mathrm{CDCl}_{3}$ at $298 \mathrm{~K}, 600 \mathrm{MHz}$. & S38 \\
\hline Figure S27 & ${ }^{1} \mathrm{H}-\mathrm{NMR}$ spectrum of laurokanol E (5) in $\mathrm{CDCl}_{3}$ at $298 \mathrm{~K}, 600 \mathrm{MHz}$. & $\mathbf{S 3 9}$ \\
\hline Figure S28 & ${ }^{13} \mathrm{C}\left\{{ }^{1} \mathrm{H}\right\}$ NMR spectrum of laurokanol E (5) in $\mathrm{CDCl}_{3}$ at $298 \mathrm{~K}, 150 \mathrm{MHz}$. & S40 \\
\hline Figure S29 & COSY spectrum of laurokanol E (5) in $\mathrm{CDCl}_{3}$ at $298 \mathrm{~K}, 600 \mathrm{MHz}$. & S41 \\
\hline Figure S30 & HSQCed spectrum of laurokanol E (5) in $\mathrm{CDCl}_{3}$ at $298 \mathrm{~K}, 600 \mathrm{MHz}$. & S42 \\
\hline Figure S31 & HMBC spectrum of laurokanol E (5) in $\mathrm{CDCl}_{3}$ at $298 \mathrm{~K}, 600 \mathrm{MHz}$. & S43 \\
\hline Figure S32 & NOESY spectrum of laurokanol E (5) in $\mathrm{CDCl}_{3}$ at $298 \mathrm{~K}, 600 \mathrm{MHz}$. & S44 \\
\hline Figure S33 & Mass spectrum of laurokanol E (5). & S45 \\
\hline Table S6 & ${ }^{13} \mathrm{C}$ and ${ }^{1} \mathrm{H}$ NMR data of yucatecone $(6)$ in $\mathrm{CDCl}_{3}$ at $298 \mathrm{~K}, 600 \mathrm{MHz}$. & S46 \\
\hline Figure S34 & ${ }^{1} \mathrm{H}-\mathrm{NMR}$ spectrum of yucatecone $(6)$ in $\mathrm{CDCl}_{3}$ at $298 \mathrm{~K}, 600 \mathrm{MHz}$. & S47 \\
\hline Figure S35 & $\begin{array}{l}\text { Section of the }{ }^{1} \mathrm{H}-\mathrm{NMR} \text { decoupling spectrum of yucatecone }(6) \text { in } \mathrm{CDCl}_{3} \\
\text { at } 298 \mathrm{~K}, 600 \mathrm{MHz} \text {. }\end{array}$ & S48 \\
\hline Figure S36 & ${ }^{13} \mathrm{C}\left\{{ }^{1} \mathrm{H}\right\}$ NMR spectrum of yucatecone (6) in $\mathrm{CDCl}_{3}$ at $298 \mathrm{~K}, 150 \mathrm{MHz}$. & $\mathbf{S 4 9}$ \\
\hline Figure S37 & COSY spectrum of yucatecone (6) in $\mathrm{CDCl}_{3}$ at $298 \mathrm{~K}, 600 \mathrm{MHz}$. & S50 \\
\hline Figure S38 & TOCSY spectrum of yucatecone $(6)$ in $\mathrm{CDCl}_{3}$ at $298 \mathrm{~K}, 600 \mathrm{MHz}$. & S51 \\
\hline Figure S39 & HSQC spectrum of yucatecone (6) in $\mathrm{CDCl}_{3}$ at $298 \mathrm{~K}, 600 \mathrm{MHz}$. & S52 \\
\hline Figure S40 & HMBC spectrum of yucatecone (6) in $\mathrm{CDCl}_{3}$ at $298 \mathrm{~K}, 600 \mathrm{MHz}$. & S53 \\
\hline Figure S41 & NOESY spectrum of yucatecone (6) in $\mathrm{CDCl}_{3}$ at $298 \mathrm{~K}, 600 \mathrm{MHz}$. & S54 \\
\hline Figure S42 & $\begin{array}{l}\text { HSQC-HECADE spectrum of yucatecone (6) in } \mathrm{CDCl}_{3} \text { at } 298 \mathrm{~K}, 600 \\
\mathrm{MHz} \text {. }\end{array}$ & S55 \\
\hline Figure S43 & J-HMBC spectrum of yucatecone (6) in $\mathrm{CDCl}_{3}$ at $298 \mathrm{~K}, 600 \mathrm{MHz}$. & S56 \\
\hline Figure S44 & MS spectrum of yucatecone (6). & S57 \\
\hline \multicolumn{3}{|c|}{ B. Computational study } \\
\hline Table S7 & $\begin{array}{l}\text { Scaled }{ }^{13} \mathrm{C} \text { average chemical } \\
\mathrm{CHCl}_{3} .\end{array}$ & S58 \\
\hline
\end{tabular}




\begin{tabular}{|c|c|c|}
\hline Table S8 & $\begin{array}{l}\text { Scaled }{ }^{1} \mathrm{H} \text { average chemical shifts for } 1 \mathrm{a}-1 \mathrm{~d} \text { on B3LYP-D3 in PCM- } \\
\mathrm{CHCl}_{3} \text {. }\end{array}$ & S58 \\
\hline Figure S45 & Correlations graphs for ${ }^{13} \mathrm{C}$ calculated $v s{ }^{13} \mathrm{C}$ experimental data. & S59 \\
\hline Figure S46 & Correlations graphs for ${ }^{1} \mathrm{H}$ calculated $v s{ }^{1} \mathrm{H}$ experimental data. & S61 \\
\hline Table S9 & 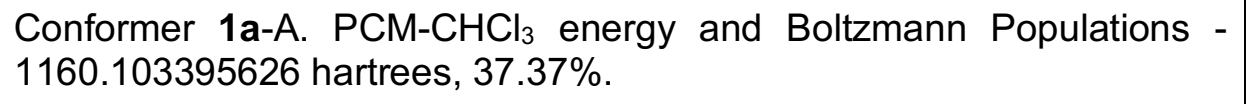 & S63 \\
\hline Table S10 & $\begin{array}{l}\text { Conformer 1a-B. PCM-CHCl }{ }_{3} \text { energy and Boltzmann Populations - } \\
\text { 1160.10365751868 hartrees, } 25.86 \% \text {. }\end{array}$ & S65 \\
\hline Table S11 & $\begin{array}{l}\text { Conformer 1a-C. PCM-CHCl } \mathrm{PH}_{3} \text { energy and Boltzmann Populations - } \\
1160.10379625597 \text { hartrees, } 22.62 \% \text {. }\end{array}$ & S67 \\
\hline Table S12 & $\begin{array}{l}\text { Conformer 1a-D. PCM- } \mathrm{CHCl}_{3} \text { energy and Boltzmann Populations - } \\
\text { 1160.10382129745 hartrees, } 12.94 \% \text {. }\end{array}$ & S69 \\
\hline Table S13 & $\begin{array}{l}\text { Conformer 1b-A. PCM- } \mathrm{CHCl}_{3} \text { energy and Boltzmann Populations - } \\
1160.10215148922 \text { hartrees, } 36.68 \% \text {. }\end{array}$ & S71 \\
\hline Table S14 & $\begin{array}{l}\text { Conformer 1b-B. PCM- } \mathrm{CHCl}_{3} \text { energy and Boltzmann Populations - } \\
\text { 1160.10218973569 hartrees, } 38.57 \% \text {. }\end{array}$ & S73 \\
\hline Table S15 & $\begin{array}{l}\text { Conformer 1b-C. PCM- } \mathrm{CHCl}_{3} \text { energy and Boltzmann Populations - } \\
1160.0972655957 \text { hartrees, } 23.94 \% \text {. }\end{array}$ & S75 \\
\hline Table S16 & 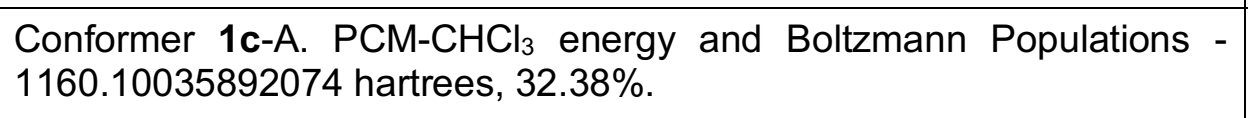 & S77 \\
\hline Table S17 & 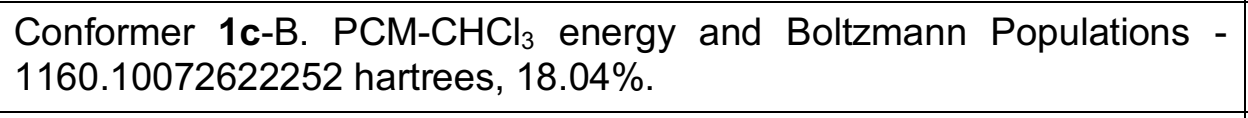 & S80 \\
\hline Table S18 & 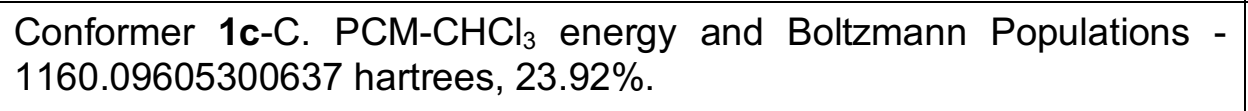 & S81 \\
\hline Table S19 & $\begin{array}{l}\text { Conformer 1c-D. PCM- } \mathrm{CHCl}_{3} \text { energy and Boltzmann Populations - } \\
1160.09564920643 \text { hartrees, } 24.33 \% \text {. }\end{array}$ & S83 \\
\hline Table S20 & 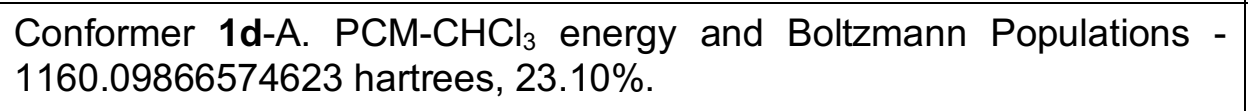 & S85 \\
\hline Table S21 & $\begin{array}{l}\text { Conformer 1d-B. PCM- } \mathrm{CHCl}_{3} \text { energy and Boltzmann Populations - } \\
\text { 1160.09895700992 hartrees, } 17.37 \% \text {. }\end{array}$ & S87 \\
\hline Table S22 & 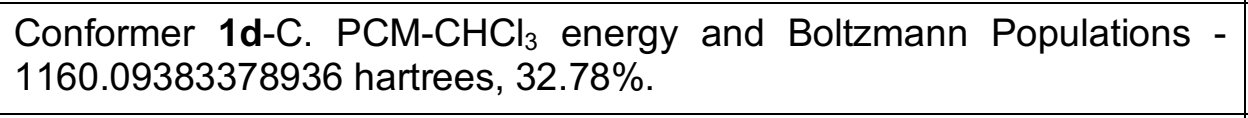 & S89 \\
\hline Table S23 & $\begin{array}{l}\text { Conformer } 1 \mathrm{~d}-\mathrm{D} \text {. } \mathrm{PCM}-\mathrm{CHCl}_{3} \text { energy and Boltzmann Populations - } \\
\text { 1160.09408876724 hartrees, } 24.33 \% \text {. }\end{array}$ & s91 \\
\hline Table S24 & J-DP4 results for 1a-1d (Isomers 1-4). & S93 \\
\hline Table S25 & J-DP4 results for $\mathbf{5 a - 5 d}$ (Isomers 1-4). & S95 \\
\hline Table S26 & $\begin{array}{l}\text { Scaled }{ }^{13} \mathrm{C} \text { average chemical shifts for } 2 a-2 d / 3 a-3 d \text { on B3LYP-D3 in } \\
\text { PCM-CHCl }\end{array}$ & S97 \\
\hline Table S27 & $\begin{array}{l}\text { Scaled }{ }^{1} \mathrm{H} \text { average chemical shifts for } \mathbf{2 a - 2} \mathbf{d} / \mathbf{3 a}-\mathbf{3 d} \text { on B3LYP-D3 in } \\
\text { PCM-CHCl } 3 \text {. }\end{array}$ & S97 \\
\hline Table S28 & $\begin{array}{l}\text { Conformer } 2 \mathrm{a}-\mathrm{A} / 3 \mathrm{a}-\mathrm{A} \text {. } \mathrm{PCM}-\mathrm{CHCl}_{3} \text { energy and Boltzmann Populations - } \\
1694.8125931 \text { hartrees, } 35.55 \% \text {. }\end{array}$ & S98 \\
\hline Table S29 & $\begin{array}{l}\text { Conformer } 2 \mathrm{a}-\mathrm{B} / 3 \mathrm{a}-\mathrm{B} \text {. PCM-CHCl} \\
\text { 1694.8119361 energy and Boltzmann Populations - } \\
\text { hartrees, } 17.73 \% \text {. }\end{array}$ & S100 \\
\hline
\end{tabular}




\begin{tabular}{|c|c|c|}
\hline Table S30 & $\begin{array}{l}\text { Conformer } 2 \mathrm{a}-\mathrm{C} / 3 \mathrm{a}-\mathrm{C} \text {. } \mathrm{PCM}-\mathrm{CHCl}_{3} \text { energy and Boltzmann Populations - } \\
1694.81162066 \text { hartrees, } 12.69 \% \text {. }\end{array}$ & S102 \\
\hline Table S31 & $\begin{array}{l}\text { Conformer 2a-D/3a-D. PCM-CHCl }{ }_{3} \text { energy and Boltzmann Populations - } \\
1694.8121798 \text { hartrees, } 22.95 \% \text {. }\end{array}$ & S104 \\
\hline Table S32 & $\begin{array}{l}\text { Conformer } 2 \text { b-A/3b-A. PCM-CHCl }{ }_{3} \text { energy and Boltzmann Populations - } \\
1694.81068 \text { hartrees, } 9.30 \% \text {. }\end{array}$ & S106 \\
\hline Table S33 & $\begin{array}{l}\text { Conformer } 2 \text { b-B/3b-B. PCM-CHCl }{ }_{3} \text { energy and Boltzmann Populations - } \\
1694.81128 \text { hartrees, } 17.07 \% \text {. }\end{array}$ & S108 \\
\hline Table S34 & $\begin{array}{l}\text { Conformer } 2 \text { b-C/3b-C. } \mathrm{PCM}-\mathrm{CHCl}_{3} \text { energy and Boltzmann Populations } \\
-1694.81034 \text { hartrees, } 6.49 \% \text {. }\end{array}$ & S110 \\
\hline Table S35 & $\begin{array}{l}\text { Conformer } \mathbf{2 b} \text {-D/3b-D. PCM- } \mathrm{CHCl}_{3} \text { energy and Boltzmann Populations } \\
-1694.81083 \text { hartrees, } 10.91 \% \text {. }\end{array}$ & S112 \\
\hline Table S36 & $\begin{array}{l}\text { Conformer } 2 \mathrm{~b}-\mathrm{E} / \mathbf{3 b} \text {-E. PCM-CHCl }{ }_{3} \text { energy and Boltzmann Populations - } \\
1694.81036 \text { hartrees, } 6.63 \% \text {. }\end{array}$ & S114 \\
\hline Table S37 & $\begin{array}{l}\text { Conformer } 2 \mathrm{~b}-\mathrm{F} / 3 \mathrm{~b}-\mathrm{F} . \mathrm{PCM}-\mathrm{CHCl}_{3} \text { energy and Boltzmann Populations - } \\
1694.81146 \text { hartrees, } 21.44 \% \text {. }\end{array}$ & S116 \\
\hline Table S38 & $\begin{array}{l}\text { Conformer } 2 \mathrm{~b}-\mathrm{G} / 3 \mathrm{~b}-\mathrm{G} . \mathrm{PCM}-\mathrm{CHCl}_{3} \text { energy and Boltzmann Populations } \\
-1694.81003 \text { hartrees, } 4.69 \% \text {. }\end{array}$ & S118 \\
\hline Table S39 & $\begin{array}{l}\text { Conformer } \mathbf{2 b}-\mathrm{H} / \mathbf{3} \mathbf{b}-\mathrm{H} \text {. } \mathrm{PCM}-\mathrm{CHCl}_{3} \text { energy and Boltzmann Populations } \\
-1694.81103 \text { hartrees, } 13.57 \% \text {. }\end{array}$ & S120 \\
\hline Table S40 & $\begin{array}{l}\text { Conformer 2c-A/3c-A. PCM- } \mathrm{CHCl}_{3} \text { energy and Boltzmann Populations - } \\
1694.808387 \text { hartrees, } 28.31 \% \text {. }\end{array}$ & S122 \\
\hline Table S41 & $\begin{array}{l}\text { Conformer 2c-B/3c-B. PCM- } \mathrm{CHCl}_{3} \text { energy and Boltzmann Populations - } \\
1694.807499 \text { hartrees, } 11.06 \% \text {. }\end{array}$ & S124 \\
\hline Table S42 & $\begin{array}{l}\text { Conformer } 2 \mathrm{c}-\mathrm{C} / 3 \mathrm{c}-\mathrm{C} . \mathrm{PCM}-\mathrm{CHCl}_{3} \text { energy and Boltzmann Populations - } \\
1694.807950 \text { hartrees, } 17.82 \% \text {. }\end{array}$ & S126 \\
\hline Table S43 & $\begin{array}{l}\text { Conformer } 2 \mathrm{c}-\mathrm{D} / 3 \mathrm{c}-\mathrm{D} . \mathrm{PCM}-\mathrm{CHCl}_{3} \text { energy and Boltzmann Populations - } \\
1694.807078 \text { hartrees, } 7.08 \% \text {. }\end{array}$ & S128 \\
\hline Table S44 & $\begin{array}{l}\text { Conformer 2c-E/3c-E. PCM-CHCl }{ }_{3} \text { energy and Boltzmann Populations - } \\
1694.806944 \text { hartrees, } 6.14 \% \text {. }\end{array}$ & $\mathbf{S} 130$ \\
\hline Table S45 & $\begin{array}{l}\text { Conformer } 2 \mathrm{c}-\mathrm{F} / 3 \mathrm{c}-\mathrm{F} . \mathrm{PCM}-\mathrm{CHCl}_{3} \text { energy and Boltzmann Populations - } \\
1694.806519 \text { hartrees, } 3.92 \% \text {. }\end{array}$ & S132 \\
\hline Table S46 & $\begin{array}{l}\text { Conformer 2c-G/3c-G. PCM-CHCl }{ }_{3} \text { energy and Boltzmann Populations } \\
-1694.806626 \text { hartrees, } 4.38 \% \text {. }\end{array}$ & S134 \\
\hline Table S47 & $\begin{array}{l}\text { Conformer } 2 \mathrm{c}-\mathrm{H} / 3 \mathrm{c}-\mathrm{H} . \mathrm{PCM}-\mathrm{CHCl}_{3} \text { energy and Boltzmann Populations - } \\
1694.806744 \text { hartrees, } 4.97 \% \text {. }\end{array}$ & $\mathbf{S 1 3 6}$ \\
\hline Table S48 & $\begin{array}{l}\text { Conformer } 2 \mathrm{~d}-\mathrm{A} / 3 \mathrm{~d}-\mathrm{A} . \mathrm{PCM}-\mathrm{CHCl}_{3} \text { energy and Boltzmann Populations - } \\
1694.806402 \text { hartrees, } 12.46 \% \text {. }\end{array}$ & S138 \\
\hline Table S49 & $\begin{array}{l}\text { Conformer } 2 \mathrm{~d}-\mathrm{B} / 3 \mathrm{~d}-\mathrm{B} . \mathrm{PCM}-\mathrm{CHCl}_{3} \text { energy and Boltzmann Populations - } \\
1694.805943 \text { hartrees, } 7.66 \% \text {. }\end{array}$ & S140 \\
\hline Table S50 & $\begin{array}{l}\text { Conformer } 2 \mathrm{~d}-\mathrm{C} / \mathbf{3} \mathrm{d}-\mathrm{C} . \mathrm{PCM}-\mathrm{CHCl}_{3} \text { energy and Boltzmann Populations } \\
-1694.806929 \text { hartrees, } 21.78 \% \text {. }\end{array}$ & S142 \\
\hline Table S51 & $\begin{array}{l}\text { Conformer } 2 \mathrm{~d} \text {-D/3d-D. } \mathrm{PCM}-\mathrm{CHCl}_{3} \text { energy and Boltzmann Populations } \\
-1694.806502 \text { hartrees, } 13.86 \% \text {. }\end{array}$ & S144 \\
\hline
\end{tabular}




\begin{tabular}{|c|c|c|}
\hline Table S52 & $\begin{array}{l}\text { Conformer } 2 \mathrm{~d}-\mathrm{E} / 3 \mathrm{~d}-\mathrm{E} . \mathrm{PCM}-\mathrm{CHCl}_{3} \text { energy and Boltzmann Populations - } \\
1694.806597 \text { hartrees, } 15.33 \% \text {. }\end{array}$ & S146 \\
\hline Table S53 & $\begin{array}{l}\text { Conformer } 2 \mathrm{~d}-\mathrm{F} / 3 \mathrm{~d}-\mathrm{F} . \mathrm{PCM}-\mathrm{CHCl}_{3} \text { energy and Boltzmann Populations - } \\
1694.806092 \text { hartrees, } 8.98 \% \text {. }\end{array}$ & S148 \\
\hline Table S54 & J-DP4 results for $\mathbf{2 a - 2 d}$ (Isomers $1-4$ ). & S150 \\
\hline Table S55 & DP4 results for $\mathbf{3 a - 3 d}$ (Isomers $1-4)$ & S152 \\
\hline Table S56 & $\begin{array}{l}\text { Scaled }{ }^{13} \mathrm{C} \text { average chemical shifts for } 4 a-4 d \text { on B3LYP-D3 in PCM- } \\
\mathrm{CHCl}_{3 .}\end{array}$ & S153 \\
\hline Table S57 & $\begin{array}{l}\text { Scaled }{ }^{1} \mathrm{H} \text { average chemical shifts for } 4 a-4 d \text { on B3LYP-D3 in PCM- } \\
\mathrm{CHCl}_{3} \text {. }\end{array}$ & S153 \\
\hline Table S58 & $\begin{array}{l}\text { Conformer 4a-A. PCM- } \mathrm{CHCl}_{3} \text { energy and Boltzmann Populations - } \\
\text { 3806.322917 hartrees, } 16.17 \% \text {. }\end{array}$ & S154 \\
\hline Table S59 & $\begin{array}{l}\text { Conformer 4a-B. } \mathrm{PCM}^{-\mathrm{CHCl}_{3}} \text { energy and Boltzmann Populations - } \\
\text { 3806.323604 hartrees, } 33.49 \% \text {. }\end{array}$ & S156 \\
\hline Table S60 & $\begin{array}{l}\text { Conformer } 4 \mathrm{a}-\mathrm{C} \text {. } \mathrm{PCM}-\mathrm{CHCl}_{3} \text { energy and Boltzmann Populations - } \\
3806.322622 \text { hartrees, } 11.84 \% \text {. }\end{array}$ & S158 \\
\hline Table S61 & 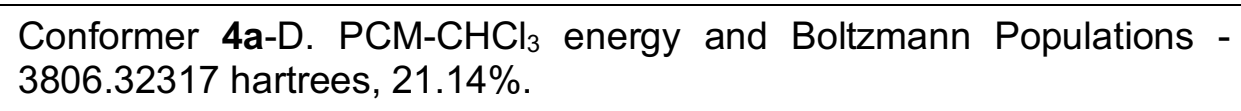 & $\mathbf{S 1 6 0}$ \\
\hline Table S62. & $\begin{array}{l}\text { Conformer 4b-A. } \mathrm{PCM}-\mathrm{CHCl}_{3} \text { energy and Boltzmann Populations - } \\
3806.322662 \text { hartrees, } 15.35 \% \text {. }\end{array}$ & S162 \\
\hline Table S63 & $\begin{array}{l}\text { Conformer 4b-B. PCM- } \mathrm{CHCl}_{3} \text { energy and Boltzmann Populations - } \\
3806.323268 \text { hartrees, } 29.17 \% \text {. }\end{array}$ & S164 \\
\hline Table S64 & $\begin{array}{l}\text { Conformer 4b-C. PCM- } \mathrm{CHCl}_{3} \text { energy and Boltzmann Populations - } \\
\text { 3806.322286 hartrees, } 10.31 \% \text {. }\end{array}$ & S166 \\
\hline Table S65. & $\begin{array}{l}\text { Conformer 4b-D. PCM- } \mathrm{CHCl}_{3} \text { energy and Boltzmann Populations - } \\
3806.322782 \text { hartrees, } 17.43 \% \text {. }\end{array}$ & S168 \\
\hline Table S66 & $\begin{array}{l}\text { Conformer } 4 \text { b-E. PCM- } \mathrm{CHCl}_{3} \text { energy and Boltzmann Populations - } \\
\text { 3806.322446 hartrees, } 12.20 \% \text {. }\end{array}$ & $\mathbf{S 1 7 0}$ \\
\hline Table S67 & $\begin{array}{l}\text { Conformer 4b-F. PCM- } \mathrm{CHCl}_{3} \text { energy and Boltzmann Populations - } \\
3806.321970 \text { hartrees, } 7.37 \% \text {. }\end{array}$ & S172 \\
\hline Table S68. & $\begin{array}{l}\text { Conformer 4c-A. PCM- } \mathrm{CHCl}_{3} \text { energy and Boltzmann Populations - } \\
3806.321079 \text { hartrees, } 36.14 \% \text {. }\end{array}$ & S174 \\
\hline Table S69 & $\begin{array}{l}\text { Conformer 4c-B. PCM- } \mathrm{CHCl}_{3} \text { energy and Boltzmann Populations - } \\
3806.320139 \text { hartrees, } 13.36 \% \text {. }\end{array}$ & S176 \\
\hline Table S70 & $\begin{array}{l}\text { Conformer } 4 \mathrm{c}-\mathrm{C} \text {. PCM- } \mathrm{CHCl}_{3} \text { energy and Boltzmann Populations - } \\
3806.320567 \text { hartrees, } 21.02 \% \text {. }\end{array}$ & S178 \\
\hline Table S71 & $\begin{array}{l}\text { Conformer 4c-D. PCM- } \mathrm{CHCl}_{3} \text { energy and Boltzmann Populations - } \\
3806.319669 \text { hartrees, } 8.12 \% \text {. }\end{array}$ & $\mathbf{S 1 8 0}$ \\
\hline Table S72 & $\begin{array}{l}\text { Conformer 4c-E. PCM- } \mathrm{CHCl}_{3} \text { energy and Boltzmann Populations - } \\
3806.319029 \text { hartrees, } 4.12 \% \text {. }\end{array}$ & $\mathbf{S 1 8 2}$ \\
\hline Table S73 & $\begin{array}{l}\text { Conformer 4d-A. PCM- } \mathrm{CHCl}_{3} \text { energy and Boltzmann Populations - } \\
3806.319568 \text { hartrees, } 5.27 \% \text {. }\end{array}$ & $\mathbf{S 1 8 4}$ \\
\hline Table S74 & $\begin{array}{l}\text { Conformer 4d-B. PCM- } \mathrm{CHCl}_{3} \text { energy and Boltzmann Populations - } \\
3806.320124 \text { hartrees, } 9.50 \% \text {. }\end{array}$ & S186 \\
\hline
\end{tabular}




\begin{tabular}{|c|c|c|}
\hline Table S75 & $\begin{array}{l}\text { Conformer 4d-C. PCM- } \mathrm{CHCl}_{3} \text { energy and Boltzmann Populations - } \\
3806.319635 \text { hartrees, } 5.66 \% \text {. }\end{array}$ & S188 \\
\hline Table S76 & $\begin{array}{l}\text { Conformer 4d-D. PCM- } \mathrm{CHCl}_{3} \text { energy and Boltzmann Populations - } \\
3806.320655 \text { hartrees, } 16.66 \% \text {. }\end{array}$ & $\mathbf{S 1 9 0}$ \\
\hline Table S77 & $\begin{array}{l}\text { Conformer 4d-E. PCM- } \mathrm{CHCl}_{3} \text { energy and Boltzmann Populations - } \\
3806.320082 \text { hartrees, } 9.09 \% \text {. }\end{array}$ & S192 \\
\hline Table S78 & $\begin{array}{l}\text { Conformer 4d-F. PCM- } \mathrm{CHCl}_{3} \text { energy and Boltzmann Populations - } \\
3806.321204 \text { hartrees, } 29.81 \% \text {. }\end{array}$ & $\mathbf{S 1 9 4}$ \\
\hline Table S79 & $\begin{array}{l}\text { Conformer 4d-G. PCM- } \mathrm{CHCl}_{3} \text { energy and Boltzmann Populations - } \\
3806.320636 \text { hartrees, } 16.33 \% \text {. }\end{array}$ & S196 \\
\hline \multirow[t]{2}{*}{ Table S80 } & DP4 results for $\mathbf{4 a - 4 d}$ (Isomers $1-4)$. & S198 \\
\hline & Computational Details and Full Gaussian reference & S199 \\
\hline Table S81 & Cartesian coordinates structures in Path A. & $\mathbf{S 2 0 0}$ \\
\hline Table S82 & Coordinates and energies of structures in Path B. & $\mathbf{S 2 1 8}$ \\
\hline Table S83 & $\begin{array}{l}\text { Cartesian coordinates of structures in proposed mechanism in forming } \\
\text { yucatecone. }\end{array}$ & $\mathbf{S 2 3 6}$ \\
\hline
\end{tabular}




\section{A. Isolation and structural elucidation}

Scheme S1. Isolation procedure for compounds 1-6.

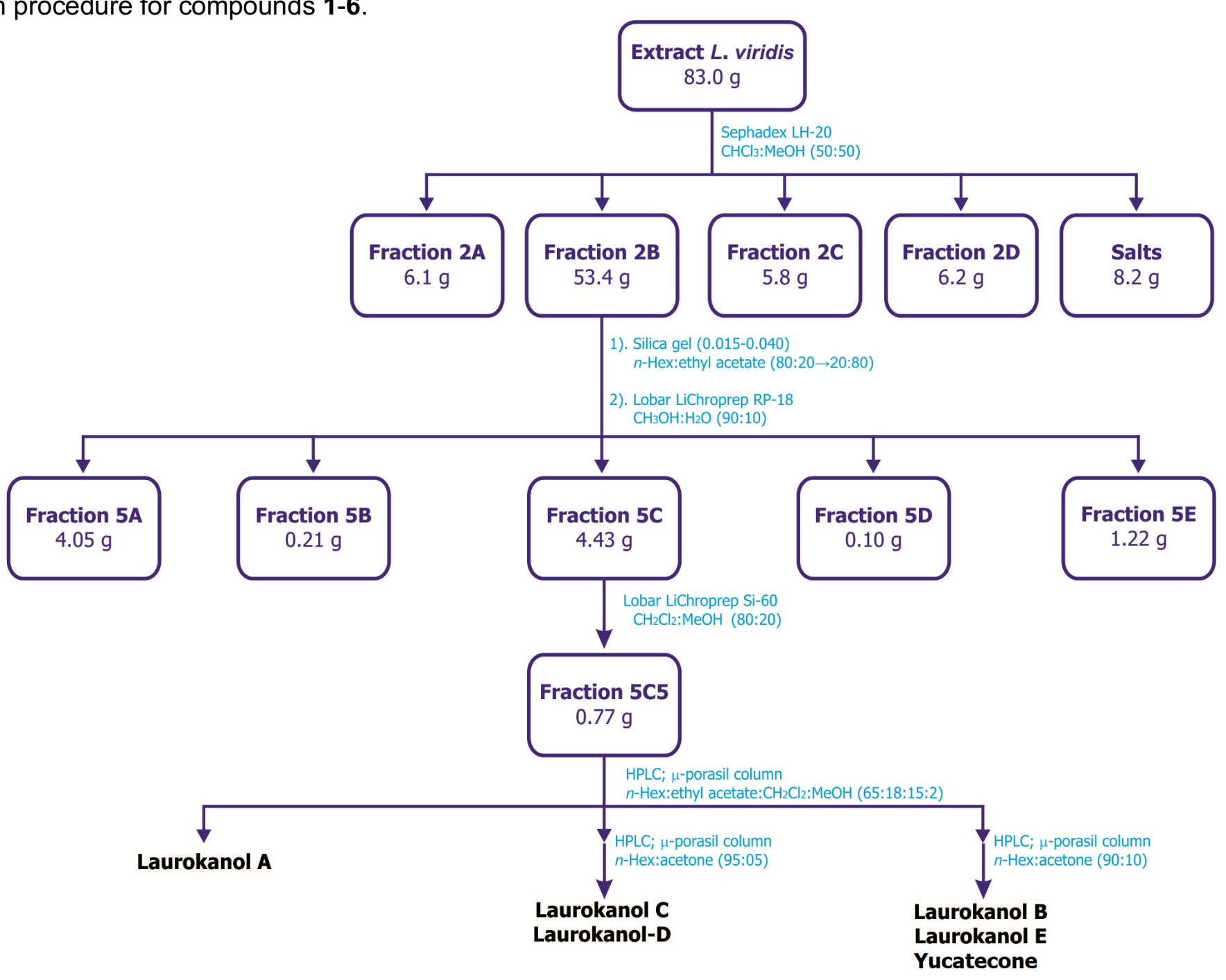


Table S1. ${ }^{13} \mathrm{C}$ and ${ }^{1} \mathrm{H}$ NMR data of laurokanol $\mathrm{A}(1)$ in $\mathrm{CDCl}_{3}(\mathrm{~A})$ and benzene-d $(\mathrm{B})$ at $298 \mathrm{~K}, 600 \mathrm{MHz}$.

\begin{tabular}{|c|c|c|c|c|c|c|c|}
\hline C & Mult. & $\delta_{c}(A)$ & $\delta_{c}(B)$ & $\delta_{H}(A)$ & $\delta_{\mathrm{H}}(\mathrm{B})$ & $J$ in $\mathrm{Hz}(\mathrm{A})$ & $J$ in $\mathrm{Hz}(\mathrm{B})$ \\
\hline 1 & $\mathrm{CH}_{3}$ & 31.2 & 31.3 & 1.25 & 1.32 & s & s \\
\hline 2 & C & 75.0 & 75.2 & & & & \\
\hline 3 & $\mathrm{CH}$ & 59.4 & 59.3 & 3.85 & 3.81 & $\mathrm{dd}(4.1,12.3)$ & $\mathrm{dd}(4.0,12.5)$ \\
\hline 4 & $\mathrm{CH}_{2}$ & 28.4 & 28.6 & $\begin{array}{l}2.07 \\
2.21\end{array}$ & $\begin{array}{l}1.91 \\
2.09\end{array}$ & $\begin{array}{l}\text { dddd }(3.6,4.1,4.3,13.2) \\
\text { dddd }(3.9,12.3,13.2,13.8)\end{array}$ & $\begin{array}{l}\text { dddd }(4.0,4.0,4.0,13.4) \\
\text { dddd }(2.0,12.5,13.4,13.4)\end{array}$ \\
\hline 5 & $\mathrm{CH}_{2}$ & 37.2 & 37.5 & $\begin{array}{l}1.48 \\
1.77\end{array}$ & $\begin{array}{l}1.52 \\
1.73\end{array}$ & $\begin{array}{l}\text { ddd }(4.3,13.8,13.8) \\
\text { ddd }(3.6,3.9,13.8)\end{array}$ & $\begin{array}{l}\text { ddd }(4.0,13.4,13.4) \\
\text { ddd }(2.0,4.0,13.4)\end{array}$ \\
\hline 6 & C & 74.9 & 74.8 & & & & \\
\hline 7 & $\mathrm{CH}$ & 86.0 & 86.4 & 3.06 & 3.28 & $\mathrm{dd}(2.4,11.4)$ & $\mathrm{dd}(2.6,11.8)$ \\
\hline 8 & $\mathrm{CH}_{2}$ & 23.3 & 23.7 & $\begin{array}{l}1.43 \\
1.73\end{array}$ & $\begin{array}{l}1.48 \\
1.82\end{array}$ & $\begin{array}{l}\text { dddd }(2.6,11.4,13.5,13.5) \\
\text { dddd }(2.4,3.0,4.3,13.5)\end{array}$ & $\begin{array}{l}\text { dddd }(2.9,11.8,13.5,13.5) \\
\text { dddd }(2.6,2.9,4.5,13.5)\end{array}$ \\
\hline 9 & $\mathrm{CH}_{2}$ & 38.7 & 39.2 & $\begin{array}{l}1.59 \\
1.78\end{array}$ & $\begin{array}{l}1.80 \\
1.90\end{array}$ & $\begin{array}{l}\text { ddd }(4.3,12.2,13.5) \\
\text { ddd }(2.6,3.0,12.2)\end{array}$ & $\begin{array}{l}\text { ddd }(4.5,12.7,13.5) \\
\text { ddd }(2.9,2.9,12.7)\end{array}$ \\
\hline 10 & C & 72.0 & 72.3 & & & & \\
\hline 11 & $\mathrm{CH}$ & 75.6 & 75.9 & 3.96 & 4.22 & $\mathrm{dd}(8.1,9.8)$ & $\mathrm{dd}(8.0,10.0)$ \\
\hline 12 & $\mathrm{CH}_{2}$ & 20.3 & 20.7 & $\begin{array}{l}1.35 \\
1.87\end{array}$ & $\begin{array}{l}1.50 \\
2.07\end{array}$ & $\begin{array}{l}\text { dddd }(1.0,9.8,11.2,13.5) \\
\text { dddd }(8.0,8.1,10.5,13.5)\end{array}$ & $\begin{array}{l}\text { dddd }(1.3,10.0,11.1,13.0) \\
\text { dddd }(7.7,8.0,10.5,13.0)\end{array}$ \\
\hline 13 & $\mathrm{CH}_{2}$ & 27.9 & 28.2 & $\begin{array}{l}1.55 \\
2.17\end{array}$ & $\begin{array}{l}1.48 \\
2.03\end{array}$ & $\begin{array}{l}\text { ddd }(8.0,11.2,14.2) \\
\text { ddd }(1.0,10.5,14.2)\end{array}$ & $\begin{array}{l}\text { ddd }(1.3,10.5,14.1) \\
\text { ddd }(7.7,11.1,14.1)\end{array}$ \\
\hline 14 & C & 99.1 & 99.4 & & & & \\
\hline 15 & $\mathrm{CH}$ & 38.8 & 39.1 & 1.35 & 1.25 & $\mathrm{~m}$ & $\operatorname{ddt}(3.8,6.7,6.7,6.7,12.5)$ \\
\hline 16 & $\mathrm{CH}_{2}$ & 27.4 & 27.7 & $\begin{array}{l}1.38 \\
1.56\end{array}$ & $\begin{array}{l}1.33 \\
1.70\end{array}$ & $\begin{array}{l}\mathrm{m} \\
\text { dddd }(4.1,13.0,13.0,13.0)\end{array}$ & $\begin{array}{l}\text { dddd }(2.9,3.8,4.4,13.0) \\
\text { dddd }(4.2,12.5,13.0,13.0)\end{array}$ \\
\hline 17 & $\mathrm{CH}_{2}$ & 27.1 & 27.4 & $\begin{array}{l}1.18 \\
1.53\end{array}$ & $\begin{array}{l}1.12 \\
1.44\end{array}$ & $\begin{array}{l}\text { dddd }(4.1,12.5,12.5,13.0) \\
m\end{array}$ & $\begin{array}{l}\text { dddd }(4.4,12.5,12.5,13.0) \\
\text { dddd }(2.4,2.9,4.2,12.5)\end{array}$ \\
\hline 18 & $\mathrm{CH}$ & 74.6 & 75.0 & 3.77 & 3.92 & $\mathrm{dd}(2.3,12.5)$ & $d d(2.4,12.5)$ \\
\hline 19 & C & 84.8 & 84.7 & & & & \\
\hline 20 & $\mathrm{CH}_{2}$ & 32.9 & 33.2 & $\begin{array}{l}1.52 \\
2.13\end{array}$ & $\begin{array}{l}1.39 \\
2.12\end{array}$ & $\begin{array}{l}\text { ddd }(2.8,7.8,11.8) \\
\text { ddd }(8.7,11.7,11.8)\end{array}$ & $\begin{array}{l}\text { ddd }(2.5,7.6,11.9) \\
\text { ddd }(8.5,11.5,11.9)\end{array}$ \\
\hline 21 & $\mathrm{CH}_{2}$ & 26.3 & 26.9 & 1.79 & $\begin{array}{l}1.60 \\
1.78\end{array}$ & & $\begin{array}{l}\text { dddd }(2.5,5.9,8.5,12.5) \\
\text { dddd }(7.6,9.7,11.5,12.5)\end{array}$ \\
\hline 22 & $\mathrm{CH}$ & 87.1 & 87.6 & 3.74 & 3.78 & $\mathrm{dd}(6.1,9.3)$ & $\mathrm{dd}(5.9,9.7)$ \\
\hline 23 & C & 70.2 & 70.2 & & & & \\
\hline 24 & $\mathrm{CH}_{3}$ & 24.6 & 24.9 & 1.12 & 1.12 & s & s \\
\hline 25 & $\mathrm{CH}_{3}$ & 23.7 & 23.9 & 1.39 & 1.38 & $s$ & $\mathrm{~s}$ \\
\hline 26 & $\mathrm{CH}_{3}$ & 20.1 & 20.1 & 1.19 & 1.17 & $S$ & $\mathrm{~s}$ \\
\hline 27 & $\mathrm{CH}_{3}$ & 19.7 & 19.9 & 1.12 & 1.20 & $\mathrm{~s}$ & $\mathrm{~s}$ \\
\hline 28 & $\mathrm{CH}_{3}$ & 17.1 & 17.3 & 0.84 & 0.87 & $d(6.5)$ & $d(6.7)$ \\
\hline 29 & $\mathrm{CH}_{3}$ & 24.5 & 24.3 & 1.12 & 1.04 & $s$ & $s$ \\
\hline 30 & $\mathrm{CH}_{3}$ & 28.7 & 28.5 & 1.26 & 1.32 & $s$ & $\mathrm{~s}$ \\
\hline
\end{tabular}


Figure S1. ${ }^{1} \mathrm{H}-\mathrm{NMR}$ spectrum of laurokanol A (1) in $\mathrm{CDCl}_{3}$ at $298 \mathrm{~K}, 600 \mathrm{MHz}$.

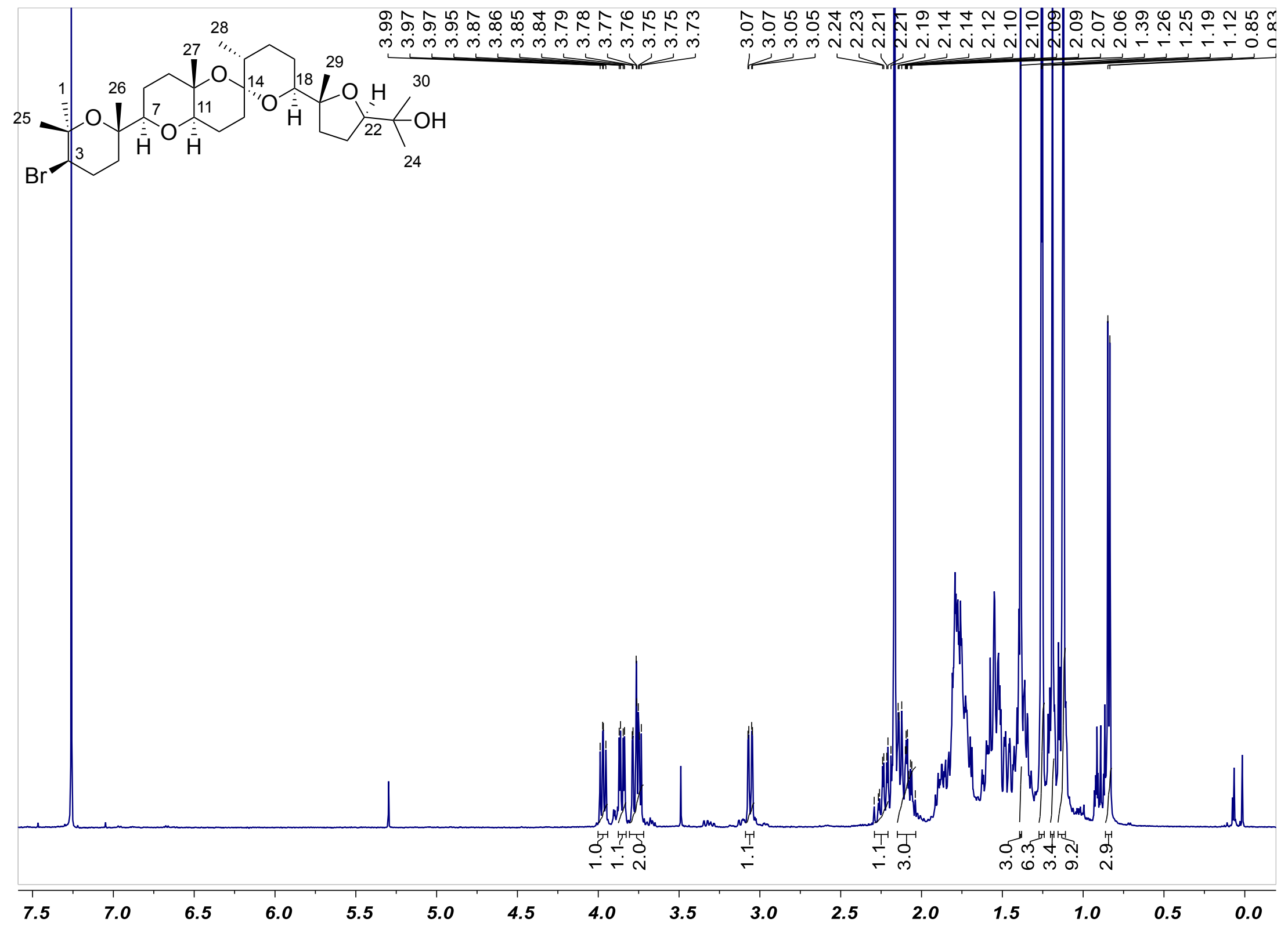


Figure S2. ${ }^{1} \mathrm{H}-\mathrm{NMR}$ spectrum of laurokanol A (1) in benzene- $d_{6}$ at $298 \mathrm{~K}, 600 \mathrm{MHz}$.

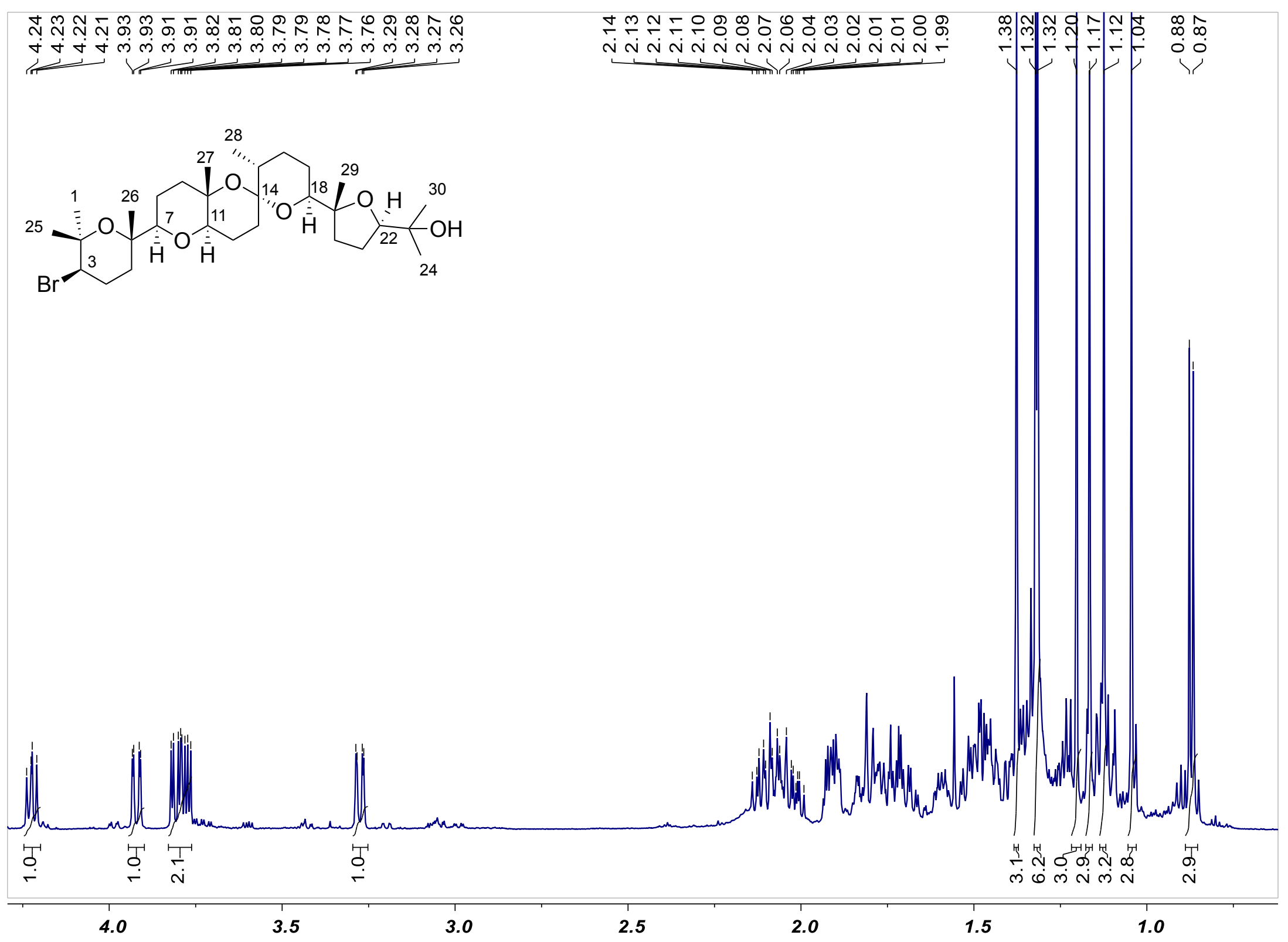


Figure S3. ${ }^{13} \mathrm{C}\left\{{ }^{1} \mathrm{H}\right\}$ NMR spectrum of laurokanol $\mathrm{A}(\mathbf{1})$ in $\mathrm{CDCl}_{3}$ at $298 \mathrm{~K}, 150 \mathrm{MHz}$.

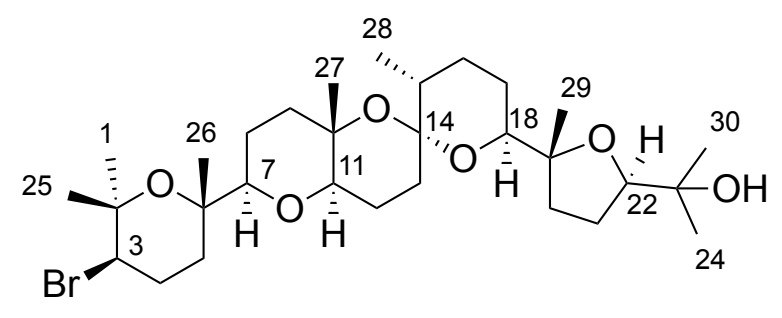

r.

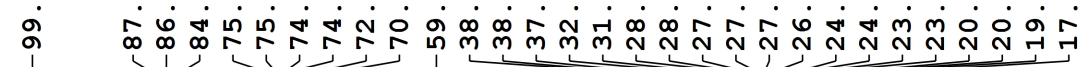

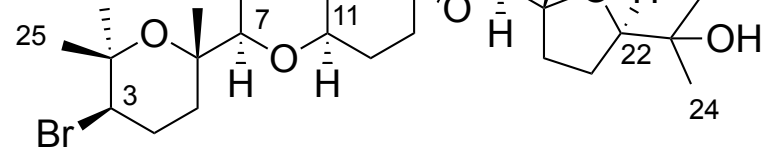

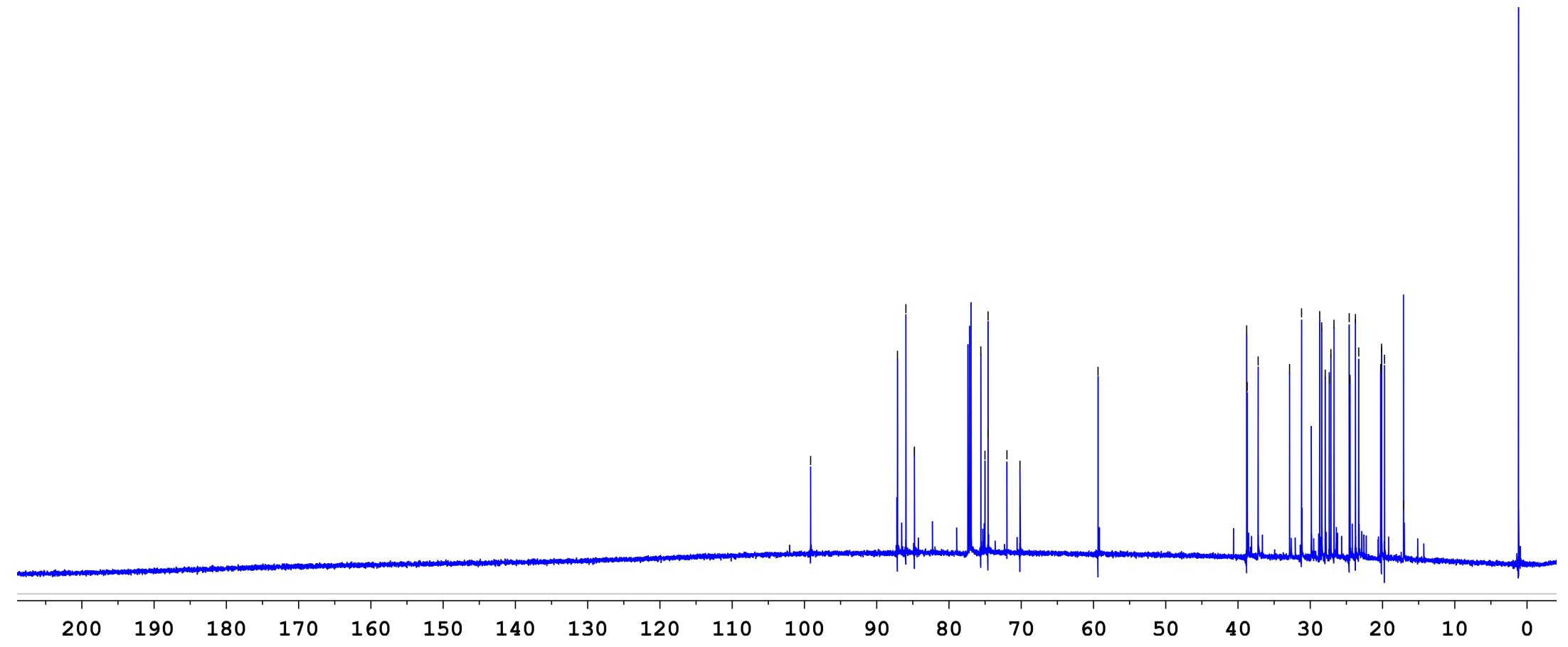


Figure S4. ${ }^{13} \mathrm{C}\left\{{ }^{1} \mathrm{H}\right\}$ NMR spectrum of laurokanol A (1) in benzene- $d_{6}$ at $298 \mathrm{~K}, 150 \mathrm{MHz}$.

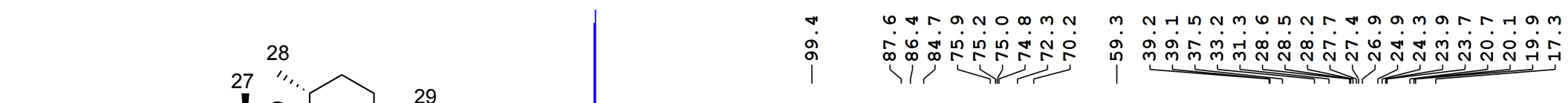

S 12 
Figure S5. COSY spectrum of laurokanol A (1) in $\mathrm{CDCl}_{3}$ at $298 \mathrm{~K}, 600 \mathrm{MHz}$.

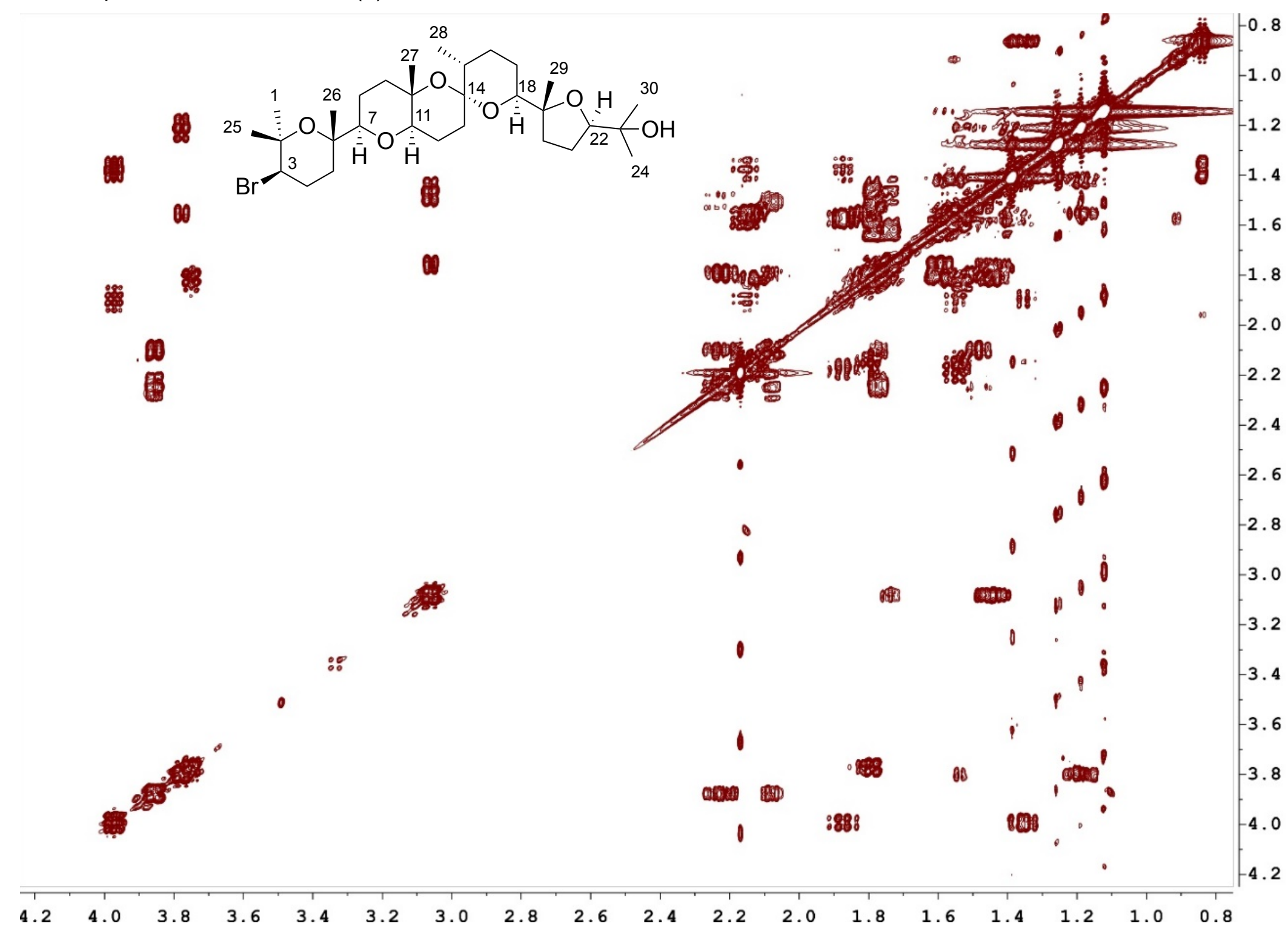


Figure S6. TOCSY-1D spectra of laurokanol A (1) in $\mathrm{CDCl}_{3}$ at $298 \mathrm{~K}, 600 \mathrm{MHz}$.
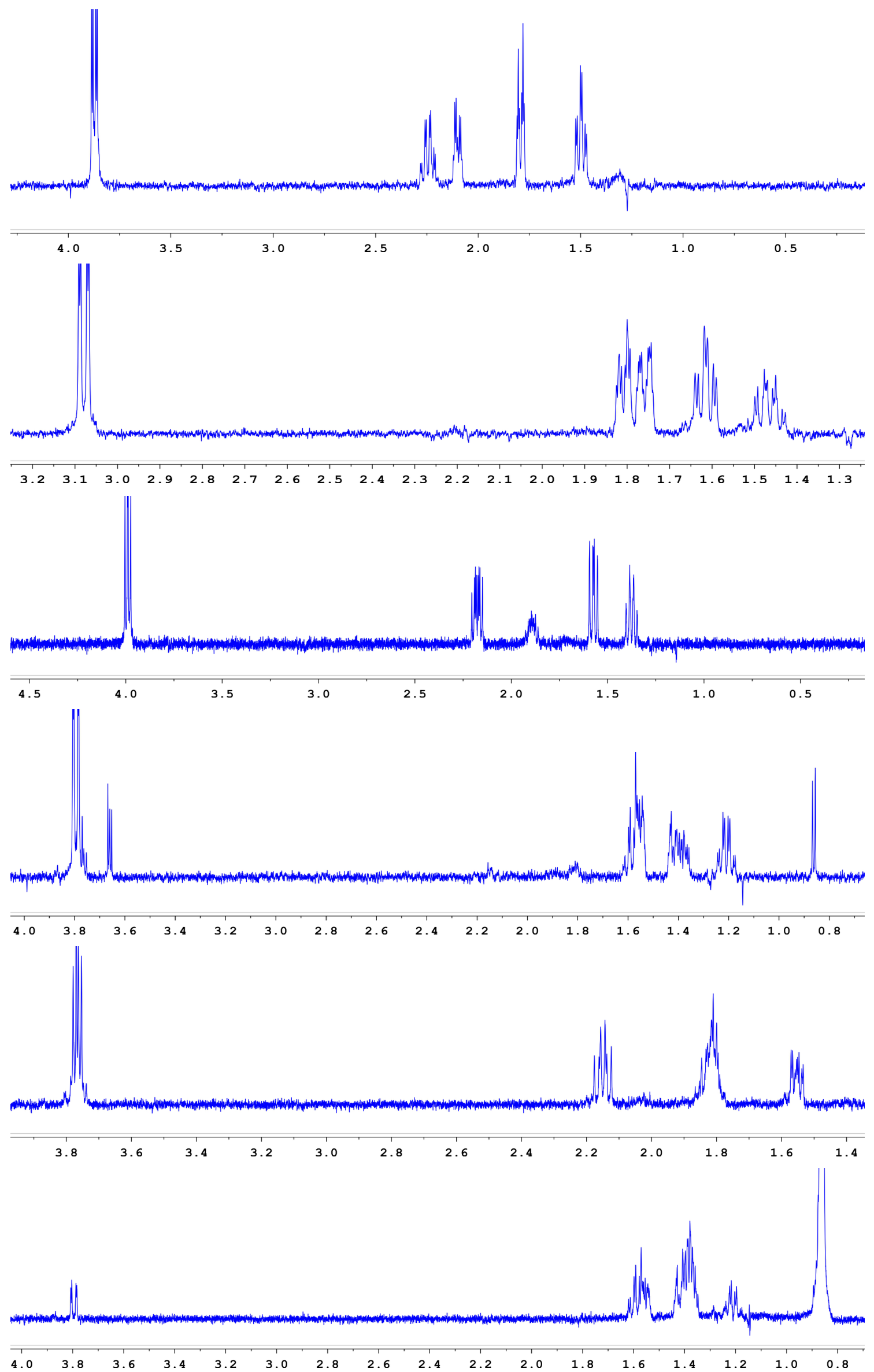
Figure S7. TOCSY-1D spectra of laurokanol A (1) in benzene- $d_{6}$ at $298 \mathrm{~K}, 600 \mathrm{MHz}$.
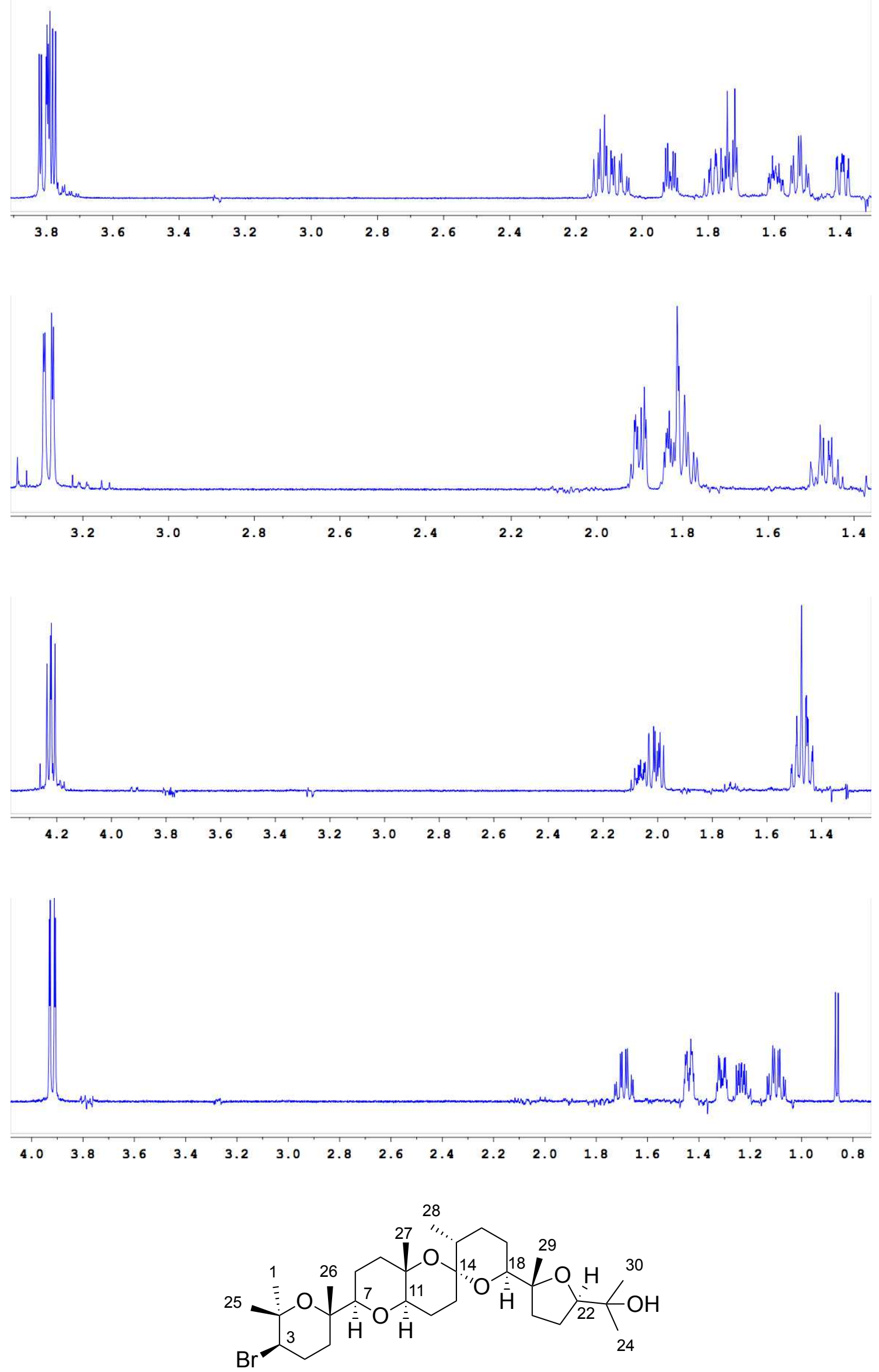
Figure S8. HSQC spectrum of laurokanol A (1) in $\mathrm{CDCl}_{3}$ at $298 \mathrm{~K}, 600 \mathrm{MHz}$.

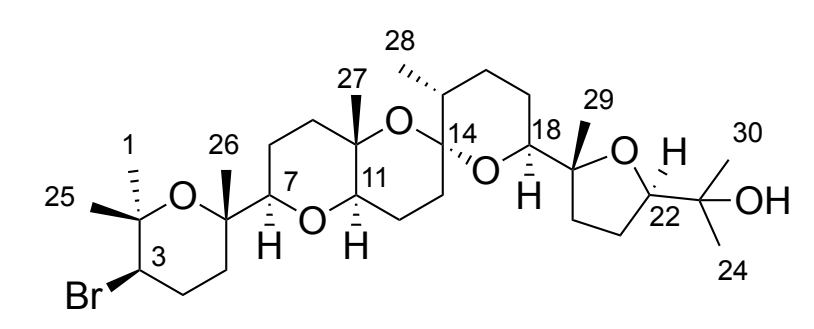

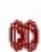

6

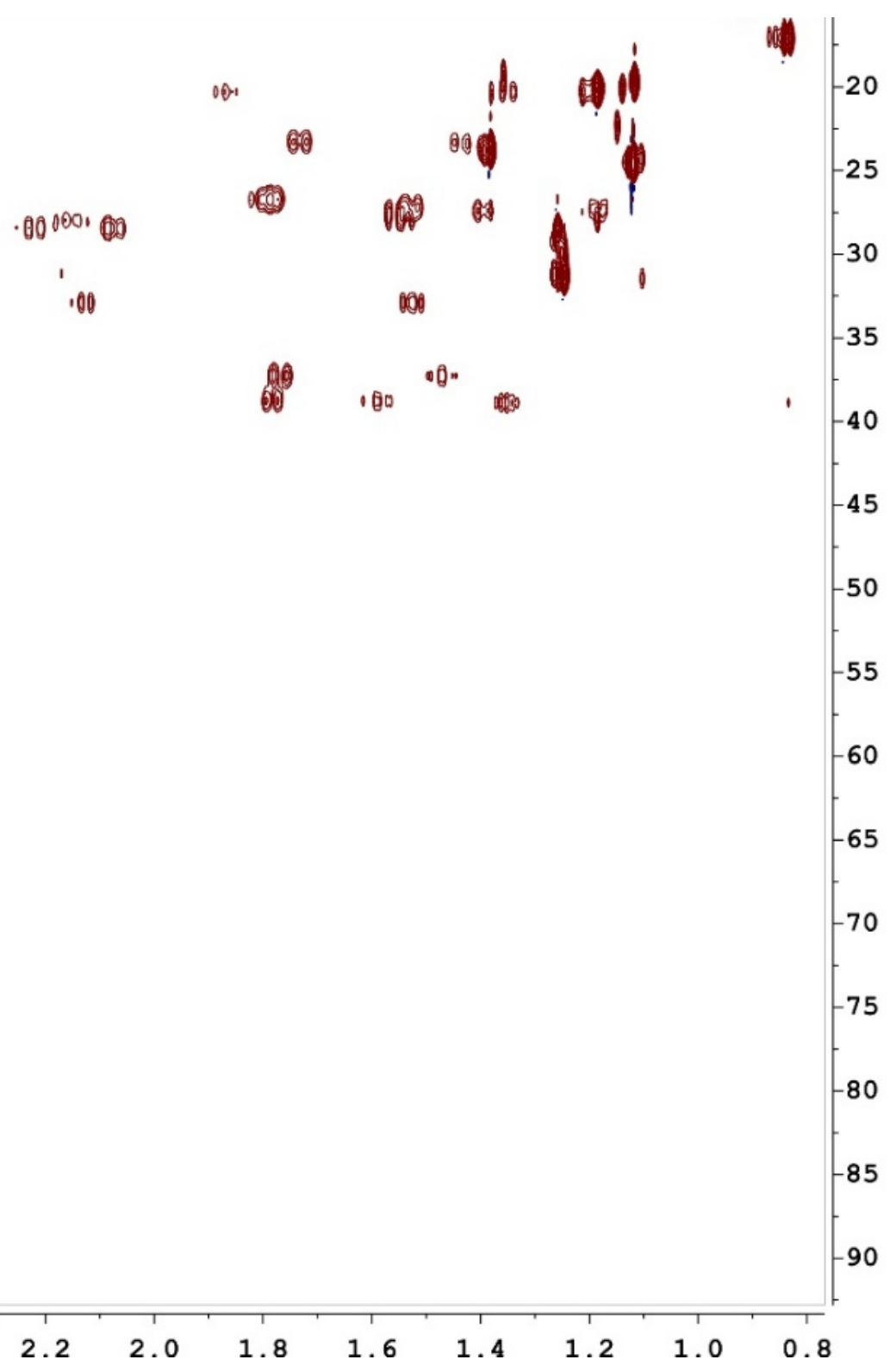


Figure S9. $\mathrm{HMBC}$ spectrum of laurokanol $\mathrm{A}(1)$ in $\mathrm{CDCl}_{3}$ at $298 \mathrm{~K}, 600 \mathrm{MHz}$.

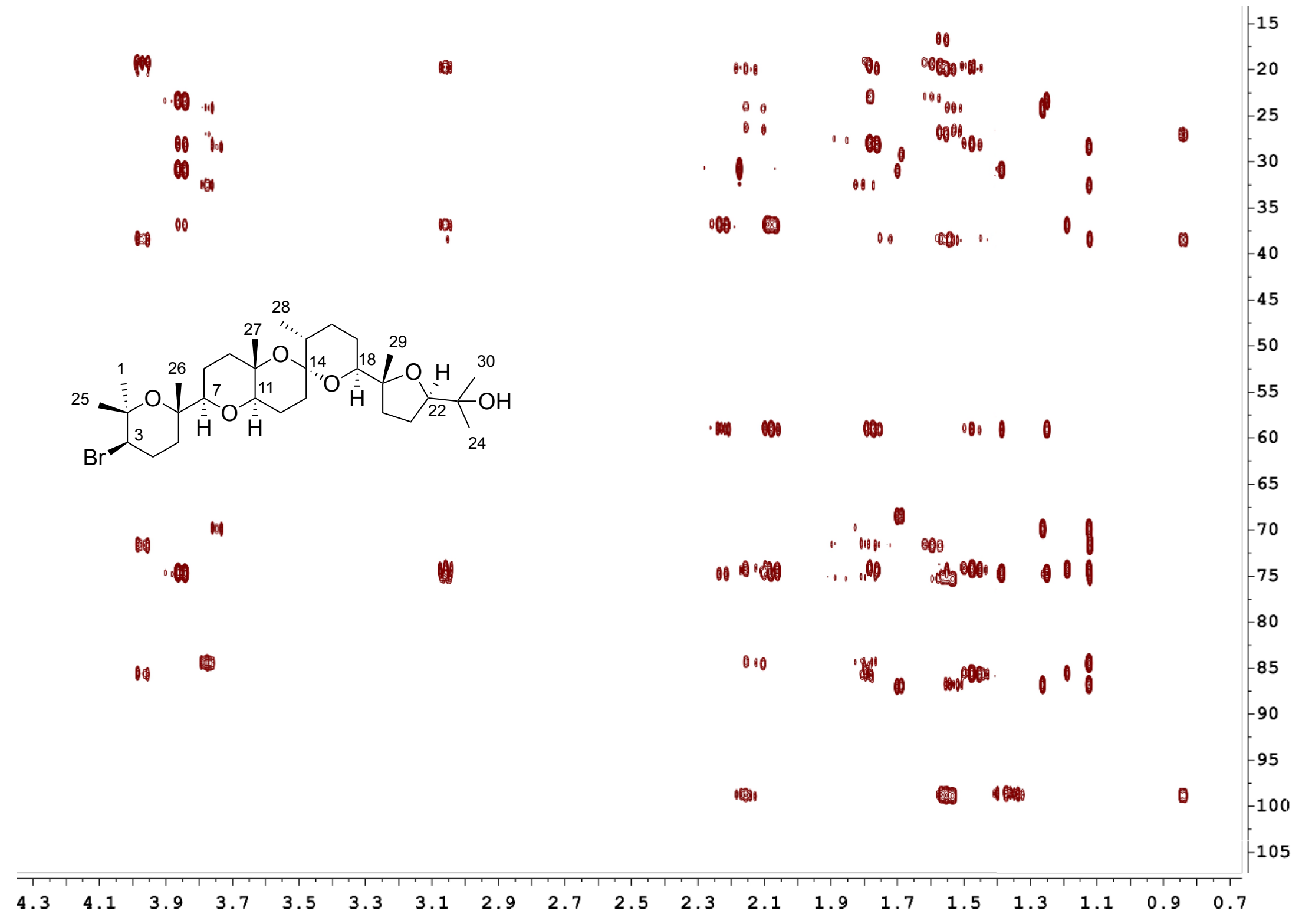


Figure S10. NOESY spectrum of laurokanol A (1) in $\mathrm{CDCl}_{3}$ at $298 \mathrm{~K}, 600 \mathrm{MHz}$.

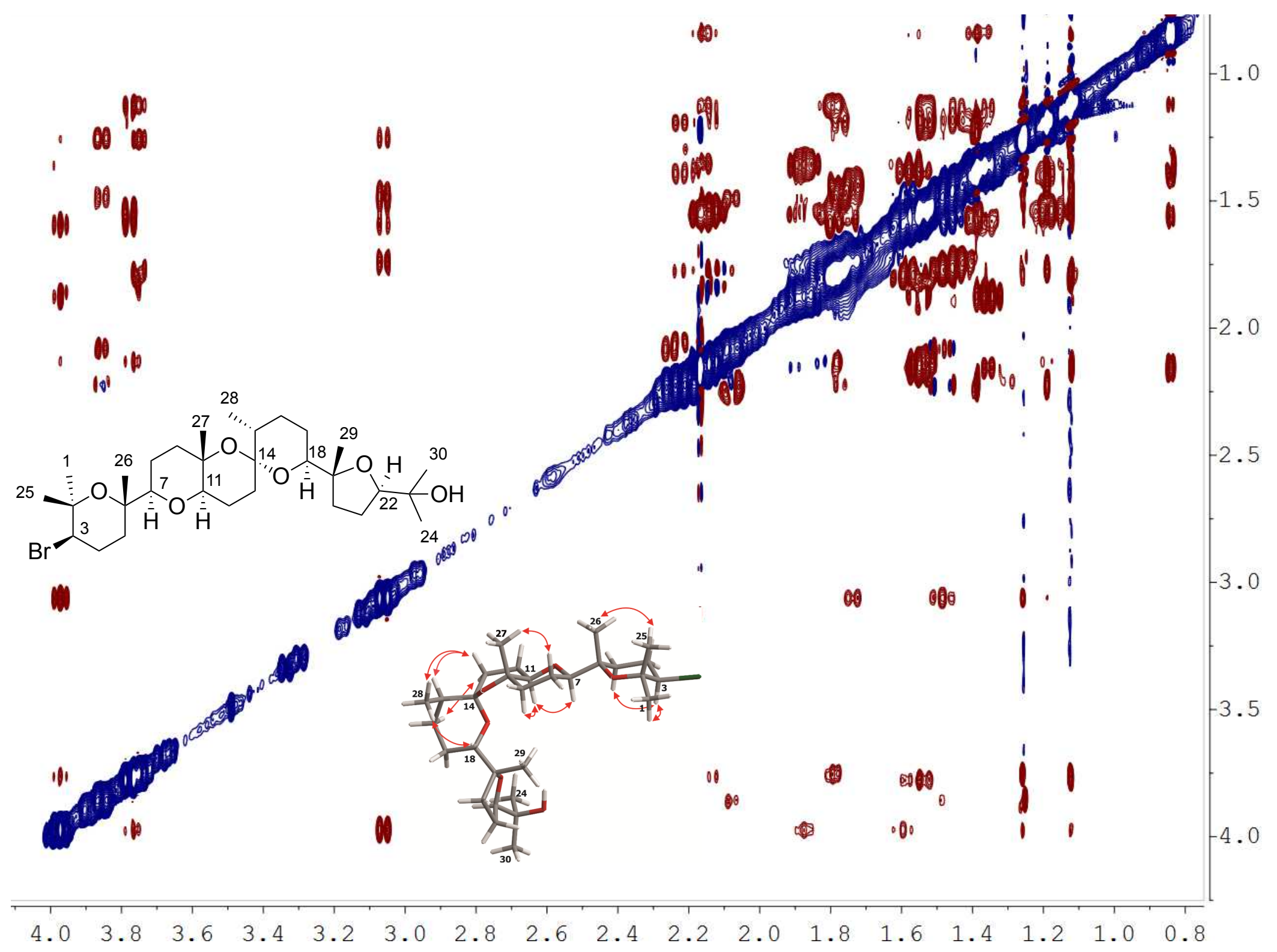


Figure S11. Mass spectrum of laurokanol A (1).

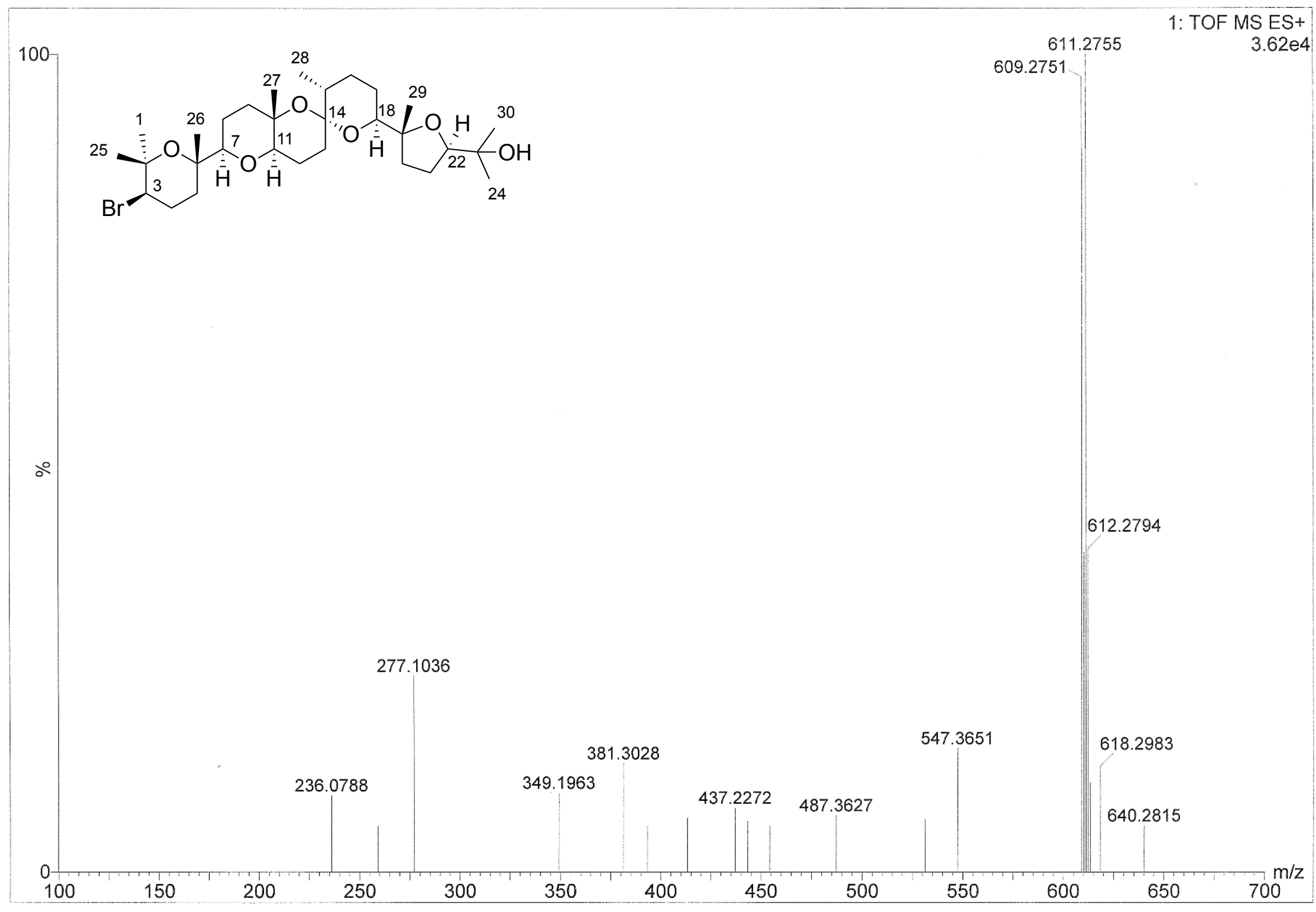


Table S2. ${ }^{13} \mathrm{C}$ and ${ }^{1} \mathrm{H}$ NMR data of laurokanol B (2) in $\mathrm{CDCl}_{3}$ at $298 \mathrm{~K}, 600 \mathrm{MHz}$.

\begin{tabular}{|c|c|c|c|c|}
\hline $\mathrm{C}$ & Mult. & $\delta_{\mathrm{C}}$ & $\delta_{\mathrm{H}}$ & $J$ in $\mathrm{Hz}$ \\
\hline 1 & $\mathrm{CH}_{3}$ & 31.1 & 1.25 & $\mathrm{~s}$ \\
\hline 2 & C & 75.0 & & \\
\hline 3 & $\mathrm{CH}$ & 59.0 & 3.83 & $\mathrm{dd}(4.1,12.5)$ \\
\hline 4 & $\mathrm{CH}_{2}$ & 28.2 & $\begin{array}{l}2.08 \\
2.23\end{array}$ & $\begin{array}{l}\text { dddd }(3.7,4.1,4.0,13.3) \\
\text { dddd }(3.7,13.1,13.2,13.3)\end{array}$ \\
\hline 5 & $\mathrm{CH}_{2}$ & 37.0 & $\begin{array}{l}1.46 \\
1.76\end{array}$ & $\begin{array}{l}\text { ddd }(4.0,13.1,13.9) \\
\mathrm{dt}(3.7,3.7,13.9)\end{array}$ \\
\hline 6 & $\mathrm{C}$ & 74.4 & & \\
\hline 7 & $\mathrm{CH}$ & 85.8 & 3.05 & $\mathrm{dd}(2.5,11.6)$ \\
\hline 8 & $\mathrm{CH}_{2}$ & 23.1 & $\begin{array}{l}1.45 \\
1.74\end{array}$ & $\begin{array}{l}\text { dddd }(4.5,11.6,12.3,13) \\
\text { dddd }(2.5,2.7,4.3,13)\end{array}$ \\
\hline 9 & $\mathrm{CH}_{2}$ & 38.2 & $\begin{array}{l}1.61 \\
1.78\end{array}$ & $\begin{array}{l}\operatorname{ddd}(4.3,12.3,12.3) \\
\operatorname{ddd}(4.5,2.7,12.3)\end{array}$ \\
\hline 10 & $\mathrm{C}$ & 73.5 & & \\
\hline 11 & $\mathrm{CH}$ & 75.0 & 3.91 & $\mathrm{dd}(8.1,9.8)$ \\
\hline 12 & $\mathrm{CH}_{2}$ & 19.7 & $\begin{array}{l}1.40 \\
1.88\end{array}$ & $\begin{array}{l}\text { dddd }(1.3,9.8,11.1,13.0) \\
\text { dddd }(7.7,8.1,10.5,13.0)\end{array}$ \\
\hline 13 & $\mathrm{CH}_{2}$ & 23.6 & $\begin{array}{l}1.95 \\
2.04\end{array}$ & $\begin{array}{l}\text { ddd }(1.3,10.5,14.1) \\
\text { ddd }(7.7,11.1,14.1)\end{array}$ \\
\hline 14 & $\mathrm{C}$ & 100.0 & & \\
\hline 15 & C & 71.5 & & \\
\hline 16 & $\mathrm{CH}_{2}$ & 28.0 & $\begin{array}{l}1.85 \\
1.91\end{array}$ & $\begin{array}{l}\text { ddd }(4.3,13.1,13.4) \\
\text { ddd }(5.5,5.5,13.4)\end{array}$ \\
\hline 17 & $\mathrm{CH}_{2}$ & 21.8 & $\begin{array}{l}1.47 \\
1.50\end{array}$ & $\begin{array}{l}\text { dddd }(3.7,5.5,13.1,13.4) \\
\text { dddd }(5.5,4.3,11.1,13.4)\end{array}$ \\
\hline 18 & $\mathrm{CH}$ & 74.2 & 3.83 & $\mathrm{dd}(3.7,11.1)$ \\
\hline 19 & $\mathrm{C}$ & 84.3 & & \\
\hline 20 & $\mathrm{CH}_{2}$ & 32.3 & $\begin{array}{l}1.59 \\
2.14\end{array}$ & $\begin{array}{l}\text { ddd }(2.5,8.2,11.8) \\
\text { ddd }(8.2,8.3,11.8)\end{array}$ \\
\hline 21 & $\mathrm{CH}_{2}$ & 26.5 & $\begin{array}{l}1.80 \\
1.84\end{array}$ & \\
\hline 22 & $\mathrm{CH}$ & 87.3 & 3.74 & $\mathrm{dd}(5.2,9.8)$ \\
\hline 23 & C & 69.9 & & \\
\hline 24 & $\mathrm{CH}_{3}$ & 24.5 & 1.12 & $s$ \\
\hline 25 & $\mathrm{CH}_{3}$ & 23.6 & 1.38 & $s$ \\
\hline 26 & $\mathrm{CH}_{3}$ & 19.9 & 1.18 & s \\
\hline 27 & $\mathrm{CH}_{3}$ & 19.7 & 1.14 & $s$ \\
\hline 28 & $\mathrm{CH}_{2}$ & 51.7 & $\begin{array}{l}3.64 \\
3.75\end{array}$ & $d(11.4)$ \\
\hline 29 & $\mathrm{CH}_{3}$ & 24.6 & 1.16 & $\mathrm{~s}$ \\
\hline 30 & $\mathrm{CH}_{3}$ & 28.6 & 1.26 & $\mathrm{~s}$ \\
\hline
\end{tabular}


Figure S12. ${ }^{1} \mathrm{H}-\mathrm{NMR}$ spectrum of laurokanol B (2) in $\mathrm{CDCl}_{3}$ at $298 \mathrm{~K}, 600 \mathrm{MHz}$.

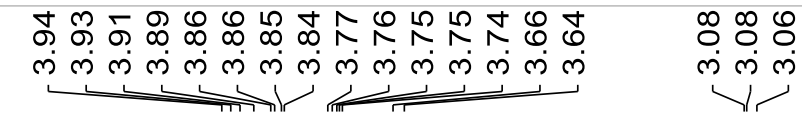
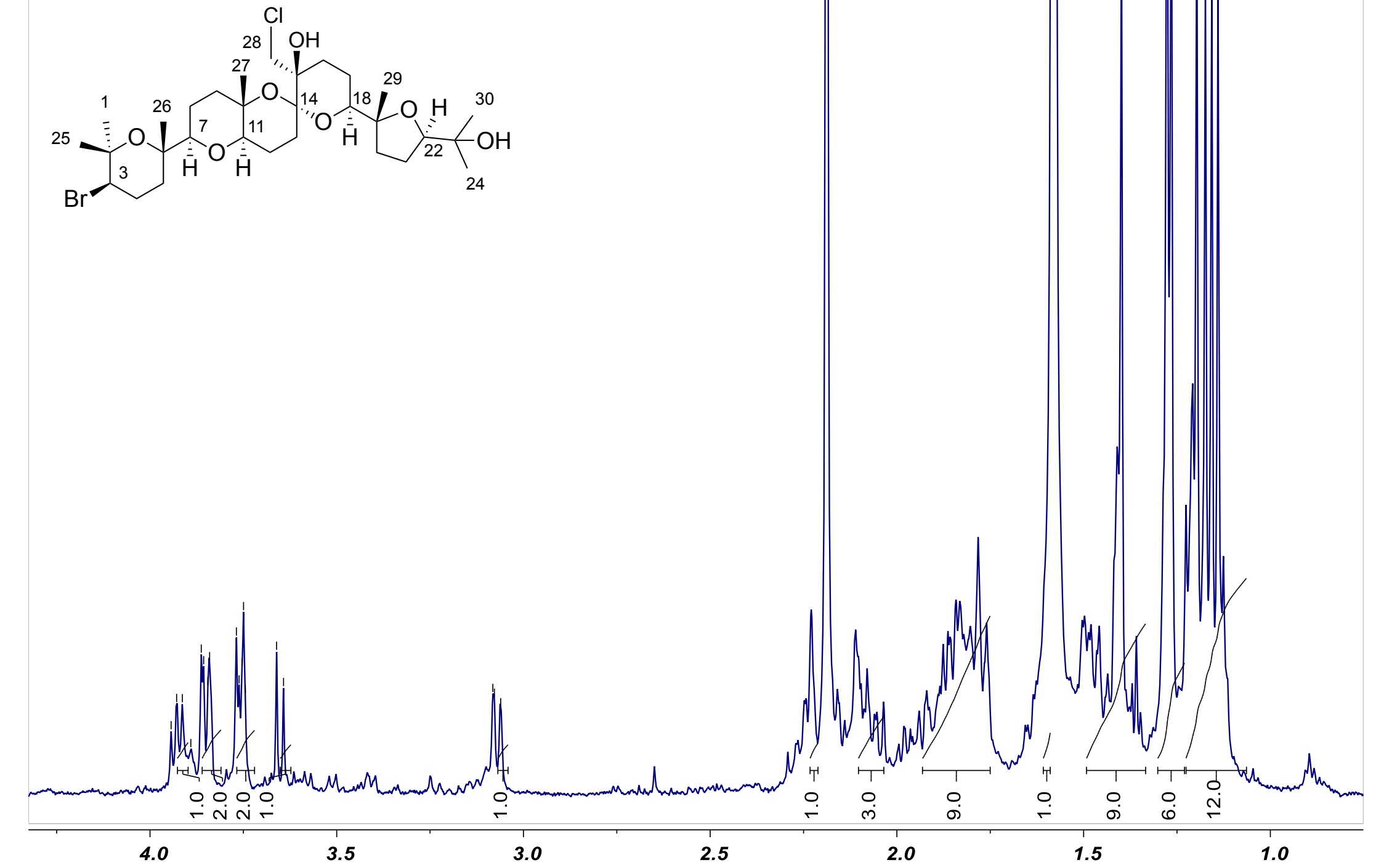
Figure S13. ${ }^{13} \mathrm{C}\left\{{ }^{1} \mathrm{H}\right\} \mathrm{NMR}$ spectrum of laurokanol B (2) in $\mathrm{CDCl}_{3}$ at $298 \mathrm{~K}, 150 \mathrm{MHz}$.

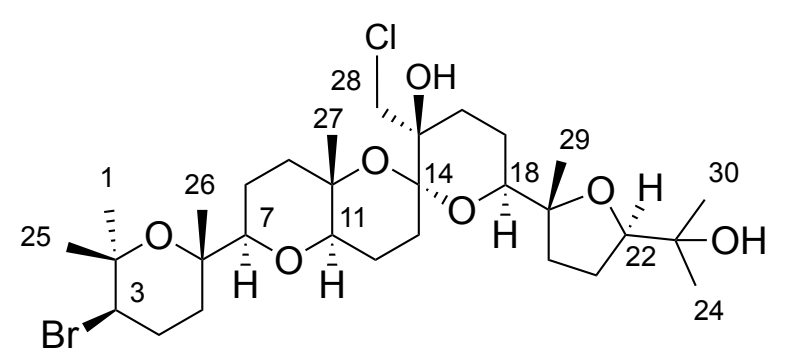

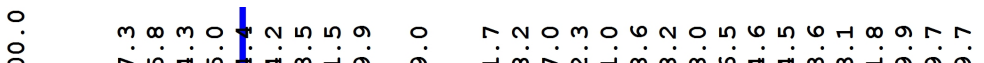

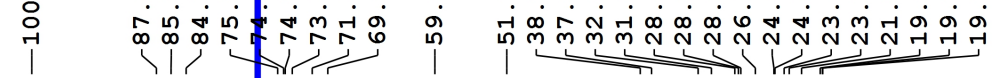

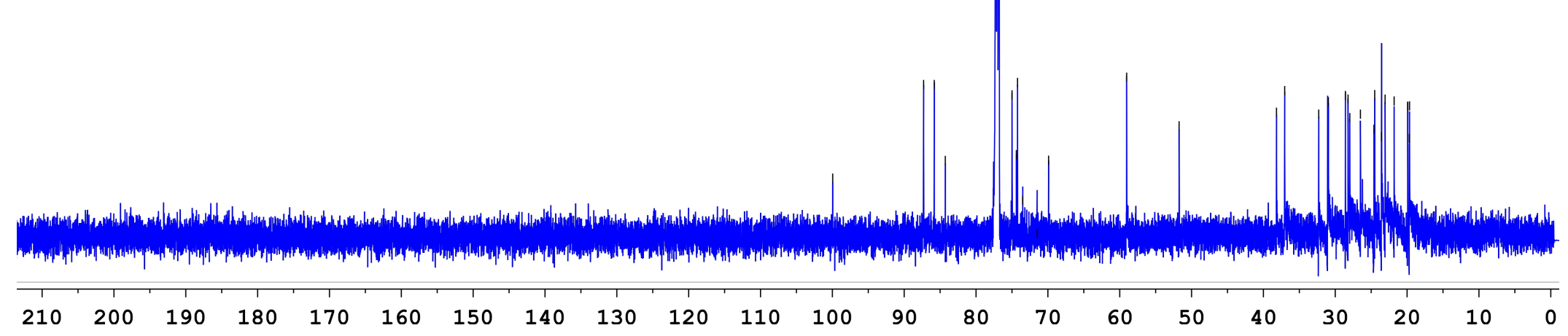


Figure S14. COSY spectrum of laurokanol B (2) in $\mathrm{CDCl}_{3}$ at $298 \mathrm{~K}, 600 \mathrm{MHz}$.

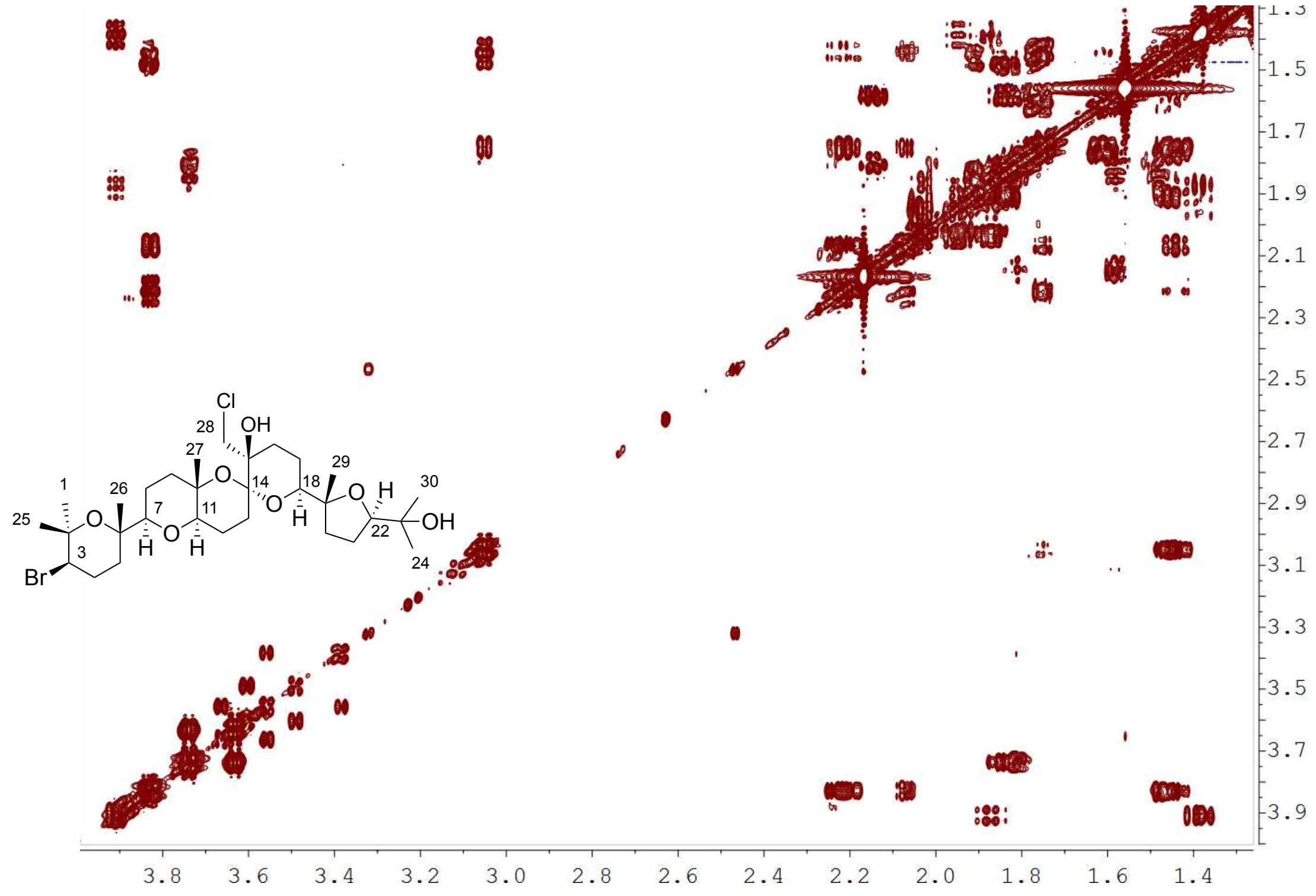


Figure S15. TOCSY-1D spectra of laurokanol B (2) in $\mathrm{CDCl}_{3}$ at $298 \mathrm{~K}, 600 \mathrm{MHz}$.
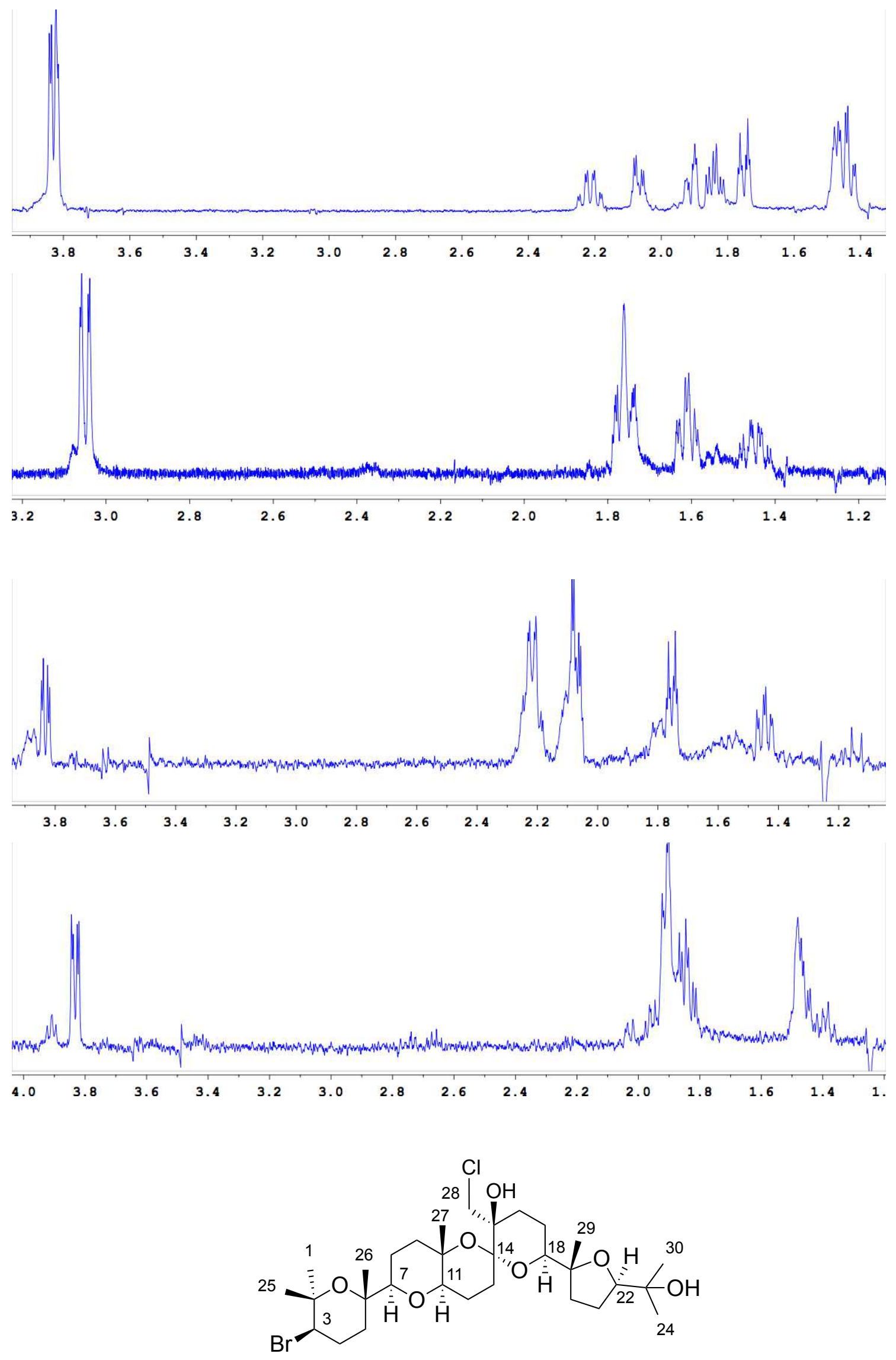
Figure S16. HSQC spectrum of laurokanol B (2) in $\mathrm{CDCl}_{3}$ at $298 \mathrm{~K}, 600 \mathrm{MHz}$.
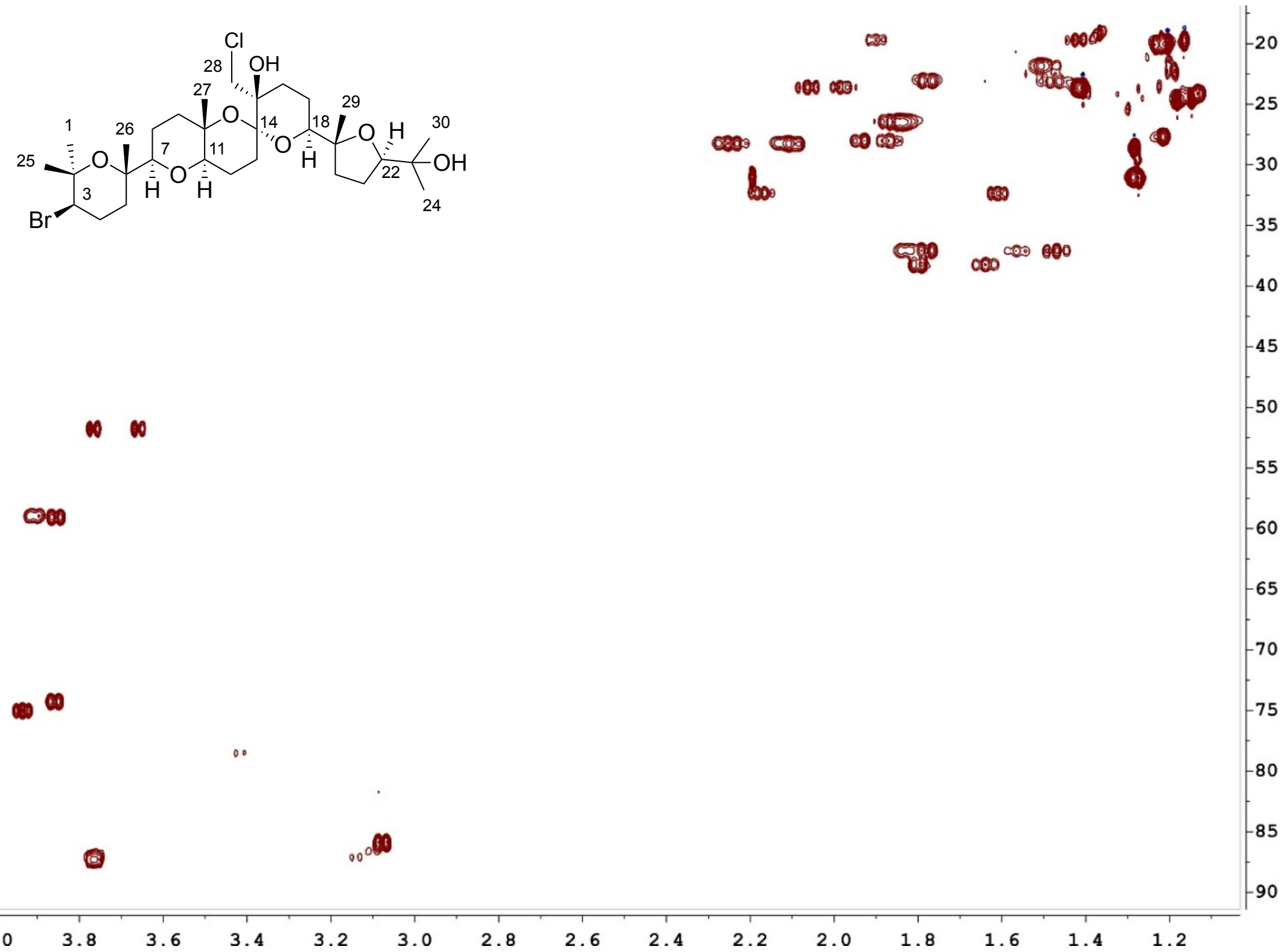
Figure 17. $\mathrm{HMBC}$ spectrum of laurokanol $\mathrm{B}(2)$ in $\mathrm{CDCl}_{3}$ at $298 \mathrm{~K}, 600 \mathrm{MHz}$.

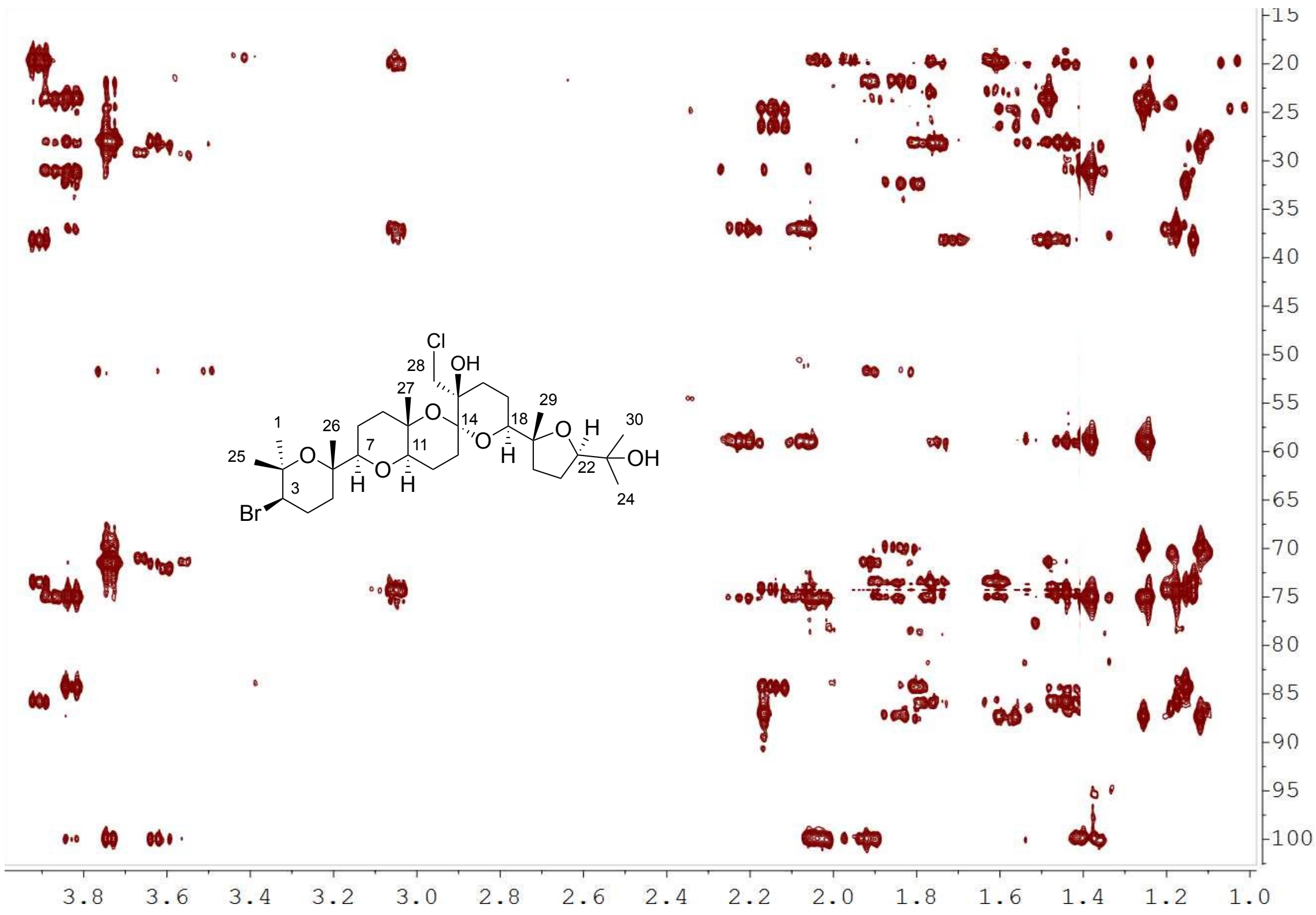


Figure S18. NOESY spectrum of laurokanol B (2) in $\mathrm{CDCl}_{3}$ at $298 \mathrm{~K}, 600 \mathrm{MHz}$.

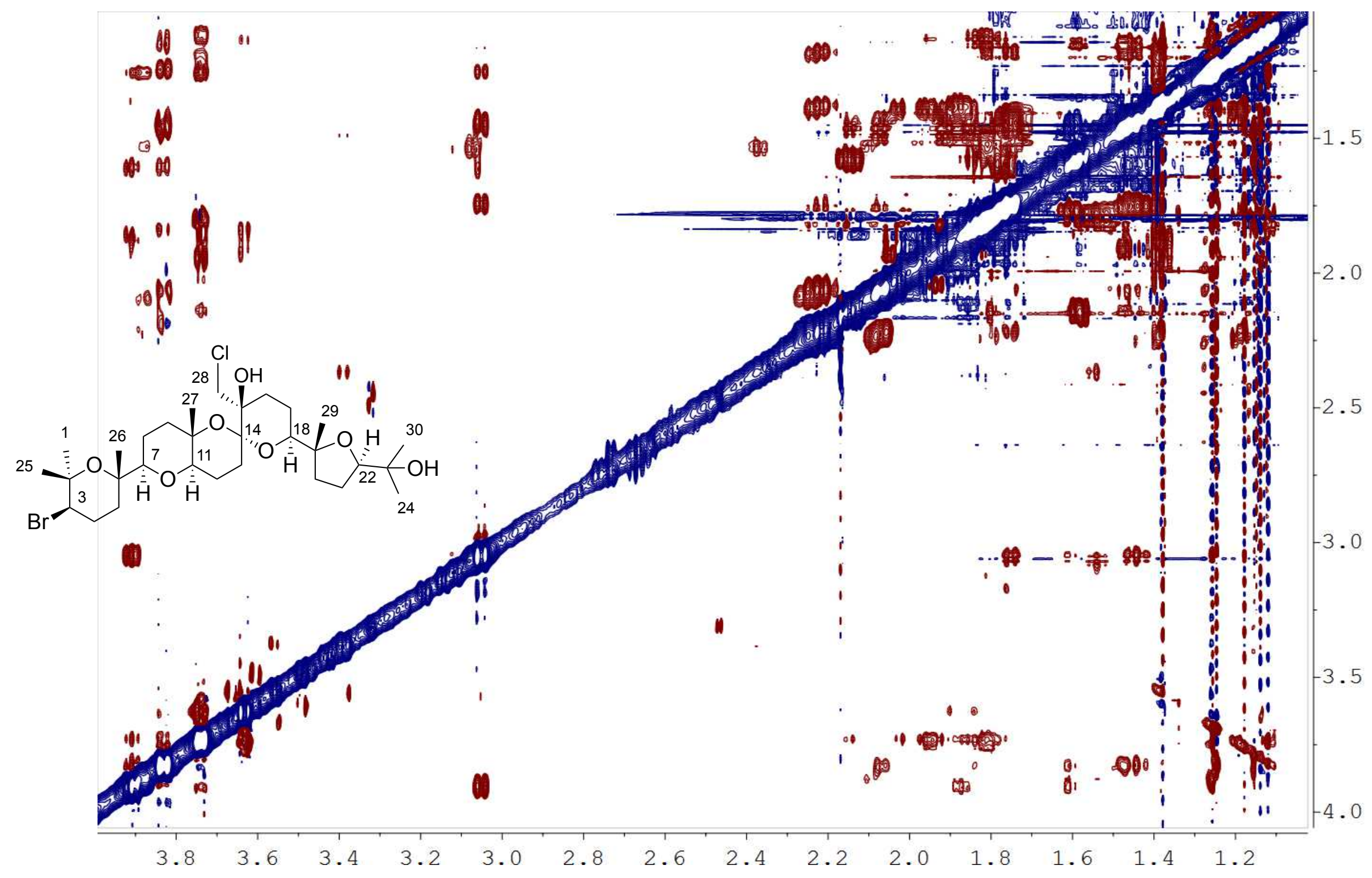


Figure S19. Mass spectrum of laurokanol B (2).
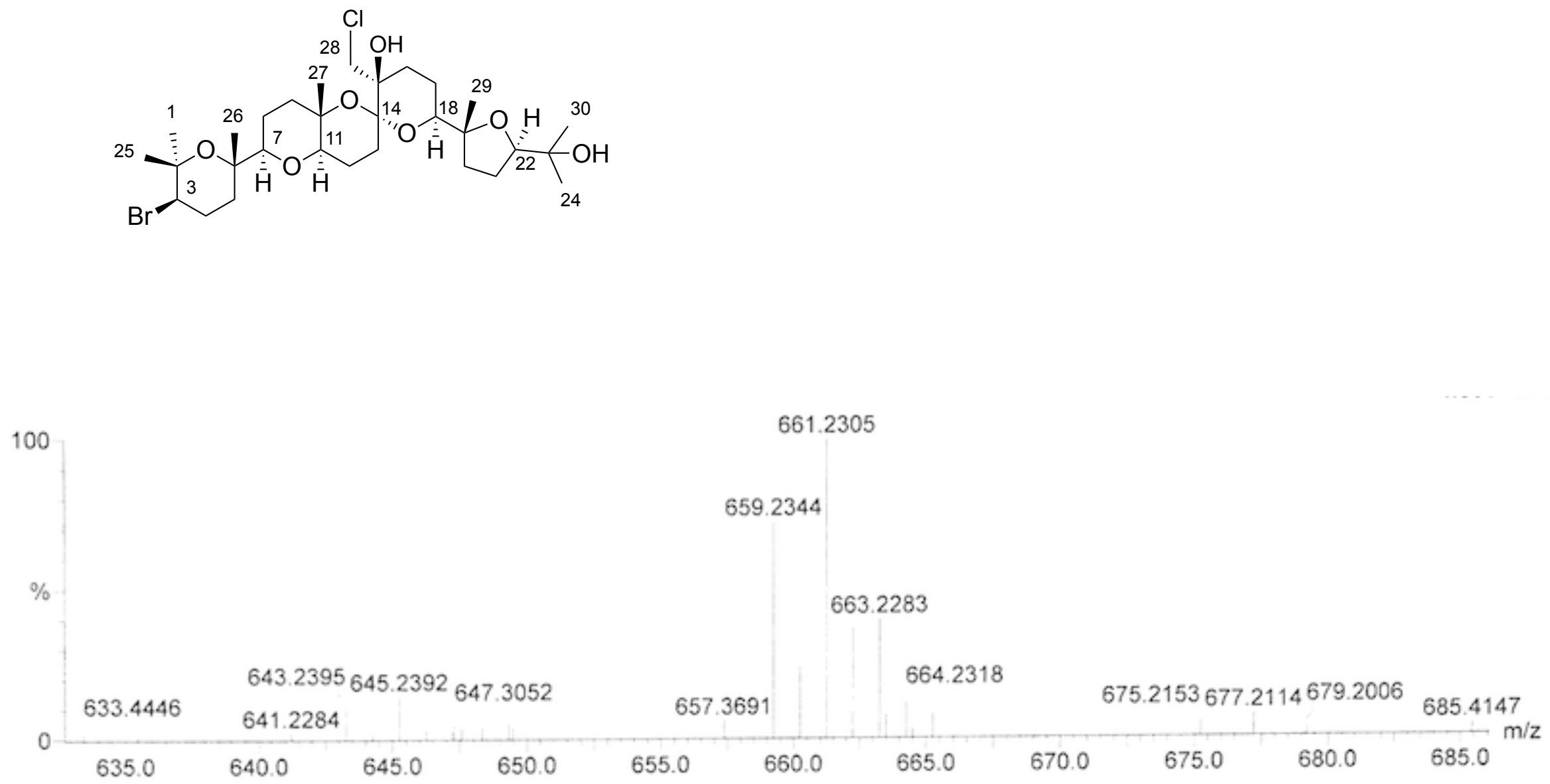
Table S3. ${ }^{13} \mathrm{C}$ and ${ }^{1} \mathrm{H}$ NMR data of laurokanol C (3) in $\mathrm{CDCl}_{3}$ at $298 \mathrm{~K}, 600 \mathrm{MHz}$.

\begin{tabular}{|c|c|c|c|c|}
\hline C & Multiplicity & $\delta_{\mathrm{C}}$ & $\delta_{\mathrm{H}}$ & $J$ in $\mathrm{Hz}$ \\
\hline $\begin{array}{l}1 \\
2\end{array}$ & $\begin{array}{l}\mathrm{CH}_{3} \\
\mathrm{C}\end{array}$ & $\begin{array}{l}31.2 \\
75.1\end{array}$ & 1.25 & $s$ \\
\hline 3 & $\mathrm{CH}$ & 59.1 & 3.85 & $\mathrm{dd}(4.1,12.4)$ \\
\hline 4 & $\mathrm{CH}_{2}$ & 28.4 & $\begin{array}{l}2.08 \\
2.22\end{array}$ & \\
\hline 5 & $\mathrm{CH}_{2}$ & 37.2 & $\begin{array}{l}1.44 \\
1.76\end{array}$ & \\
\hline 6 & $C$ & 74.5 & & \\
\hline 7 & $\mathrm{CH}$ & 86.1 & 3.05 & $\mathrm{dd}(2.5,11.6)$ \\
\hline 8 & $\mathrm{CH}_{2}$ & 23.4 & $\begin{array}{l}1.46 \\
1.78\end{array}$ & \\
\hline 9 & $\mathrm{CH}_{2}$ & 38.5 & $\begin{array}{l}1.65 \\
1.84\end{array}$ & \\
\hline 10 & C & 74.0 & & \\
\hline 11 & $\mathrm{CH}$ & 75.0 & 3.87 & dd $(7.8,10.4)$ \\
\hline 12 & $\mathrm{CH}_{2}$ & 19.4 & $\begin{array}{l}1.43 \\
1.83\end{array}$ & \\
\hline 13 & $\mathrm{CH}_{2}$ & 23.2 & $\begin{array}{l}1.62 \\
2.19\end{array}$ & \\
\hline $\begin{array}{l}14 \\
15\end{array}$ & $\begin{array}{l}C \\
C\end{array}$ & $\begin{array}{l}99.9 \\
72.3\end{array}$ & & \\
\hline 16 & $\mathrm{CH}_{2}$ & 28.4 & $\begin{array}{l}1.80 \\
2.17\end{array}$ & \\
\hline 17 & $\mathrm{CH}_{2}$ & 24.8 & $\begin{array}{l}1.24 \\
1.56\end{array}$ & \\
\hline 18 & $\mathrm{CH}$ & 74.9 & 3.85 & $\mathrm{dd}(2.5,12,2)$ \\
\hline 19 & C & 84.1 & & \\
\hline 20 & $\mathrm{CH}_{2}$ & 32.7 & $\begin{array}{l}1.59 \\
2.03\end{array}$ & \\
\hline 21 & $\mathrm{CH}_{2}$ & 26.7 & $\begin{array}{l}1.79 \\
1.84\end{array}$ & \\
\hline 22 & $\mathrm{CH}$ & 87.4 & 3.71 & $\mathrm{dd}(5.3,10.3)$ \\
\hline 23 & C & 70.1 & & \\
\hline 24 & $\mathrm{CH}_{3}$ & 24.7 & 1.12 & s \\
\hline 25 & $\mathrm{CH}_{3}$ & 23.7 & 1.39 & s \\
\hline 26 & $\mathrm{CH}_{3}$ & 20.1 & 1.18 & $s$ \\
\hline 27 & $\mathrm{CH}_{3}$ & 19.7 & 1.20 & $\mathrm{~s}$ \\
\hline 28 & $\mathrm{CH}_{2}$ & 47.4 & $\begin{array}{l}3.78 \\
3.65\end{array}$ & $d(11.7)$ \\
\hline 29 & $\mathrm{CH}_{3}$ & 24.7 & 1.14 & s \\
\hline 30 & $\mathrm{CH}_{3}$ & 28.6 & 1.26 & $\mathrm{~s}$ \\
\hline
\end{tabular}


Table S4. ${ }^{13} \mathrm{C}$ and ${ }^{1} \mathrm{H}$ NMR data of laurokanol D (4) in $\mathrm{CDCl}_{3}$ at $298 \mathrm{~K}, 600 \mathrm{MHz}$.

\begin{tabular}{|c|c|c|c|c|}
\hline C & Multiplicity & $\delta_{\mathrm{c}}$ & $\delta \mathrm{H}$ & $J$ in $\mathrm{Hz}$ \\
\hline $\begin{array}{l}1 \\
2\end{array}$ & $\begin{array}{l}\mathrm{CH}_{3} \\
\mathrm{C}\end{array}$ & $\begin{array}{l}31.2 \\
75.1\end{array}$ & 1.25 & s \\
\hline 3 & $\mathrm{CH}$ & 59.1 & 3.85 & $\mathrm{dd}(4.1,12.4)$ \\
\hline 4 & $\mathrm{CH}_{2}$ & 28.4 & $\begin{array}{l}2.08 \\
2.22\end{array}$ & \\
\hline 5 & $\mathrm{CH}_{2}$ & 37.2 & $\begin{array}{l}1.44 \\
1.76\end{array}$ & \\
\hline 6 & C & 74.5 & & \\
\hline 7 & $\mathrm{CH}$ & 86.1 & 3.05 & $\mathrm{dd}(2.5,11.6)$ \\
\hline 8 & $\mathrm{CH}_{2}$ & 23.4 & $\begin{array}{l}1.46 \\
1.78\end{array}$ & \\
\hline 9 & $\mathrm{CH}_{2}$ & 38.5 & $\begin{array}{l}1.65 \\
1.84\end{array}$ & \\
\hline 10 & C & 74.0 & & \\
\hline 11 & $\mathrm{CH}$ & 75.0 & 3.87 & $\mathrm{dd}(7.8,10.4)$ \\
\hline 12 & $\mathrm{CH}_{2}$ & 19.4 & $\begin{array}{l}1.43 \\
1.83\end{array}$ & \\
\hline 13 & $\mathrm{CH}_{2}$ & 23.2 & $\begin{array}{l}1.68 \\
2.19\end{array}$ & \\
\hline $\begin{array}{l}14 \\
15\end{array}$ & $\begin{array}{l}\mathrm{C} \\
\mathrm{C}\end{array}$ & $\begin{array}{l}99.9 \\
71.6\end{array}$ & & \\
\hline 16 & $\mathrm{CH}_{2}$ & 29.9 & $\begin{array}{l}1.84 \\
2.17\end{array}$ & \\
\hline 17 & $\mathrm{CH}_{2}$ & 24.8 & $\begin{array}{l}1.24 \\
1.56\end{array}$ & \\
\hline 18 & $\mathrm{CH}$ & 74.9 & 3.85 & $\mathrm{dd}(2.5,12.5)$ \\
\hline 19 & C & 84.1 & & \\
\hline 20 & $\mathrm{CH}_{2}$ & 32.7 & $\begin{array}{l}1.59 \\
2.03\end{array}$ & \\
\hline 21 & $\mathrm{CH}_{2}$ & 26.7 & $\begin{array}{l}1.79 \\
1.84\end{array}$ & \\
\hline 22 & $\mathrm{CH}$ & 87.4 & 3.71 & dd $(5.3,10.3)$ \\
\hline 23 & C & 70.1 & & \\
\hline 24 & $\mathrm{CH}_{3}$ & 24.7 & 1.12 & s \\
\hline 25 & $\mathrm{CH}_{3}$ & 23.7 & 1.39 & s \\
\hline 26 & $\mathrm{CH}_{3}$ & 20.1 & 1.18 & s \\
\hline 27 & $\mathrm{CH}_{3}$ & 19.7 & 1.20 & $\mathrm{~s}$ \\
\hline 28 & $\mathrm{CH}_{2}$ & 37.4 & $\begin{array}{l}3.66 \\
3.57\end{array}$ & $d(10.9)$ \\
\hline 29 & $\mathrm{CH}_{3}$ & 24.7 & 1.14 & s \\
\hline 30 & $\mathrm{CH}_{3}$ & 28.6 & 1.26 & s \\
\hline
\end{tabular}


Figure S20. ${ }^{1} \mathrm{H}-\mathrm{NMR}$ spectrum of laurokanol C (3) and laurokanol D (4) mixture in $\mathrm{CDCl}_{3}$ at $298 \mathrm{~K}, 600 \mathrm{MHz}$.

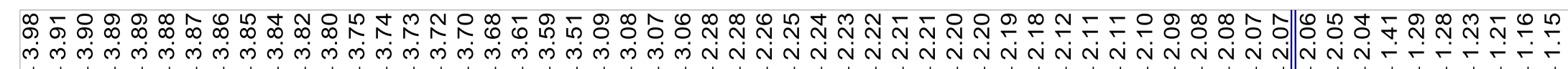

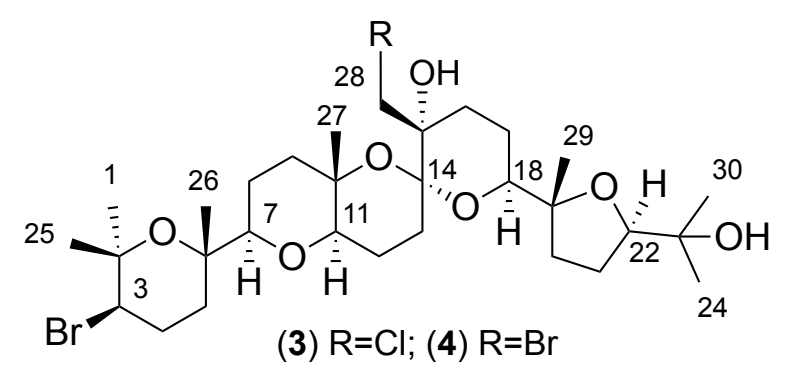

(3) $\mathrm{R}=\mathrm{Cl}$; (4) $\mathrm{R}=\mathrm{Br}$

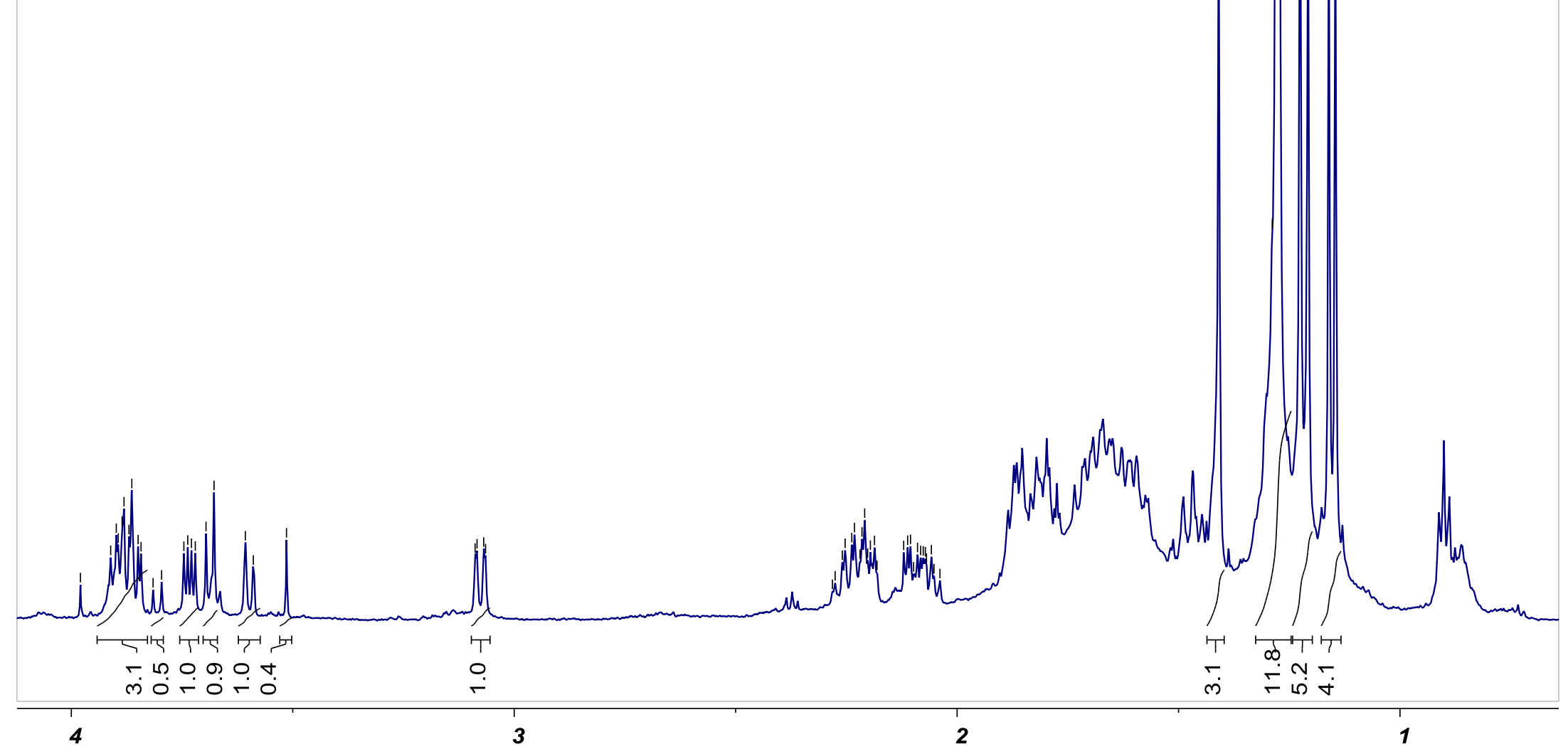


Figure S21. ${ }^{13} \mathrm{C}\left\{{ }^{1} \mathrm{H}\right\}$ NMR spectrum of laurokanol C (3) and laurokanol D (4) mixture in $\mathrm{CDCl}_{3}$ at $298 \mathrm{~K}, 150 \mathrm{MHz}$.

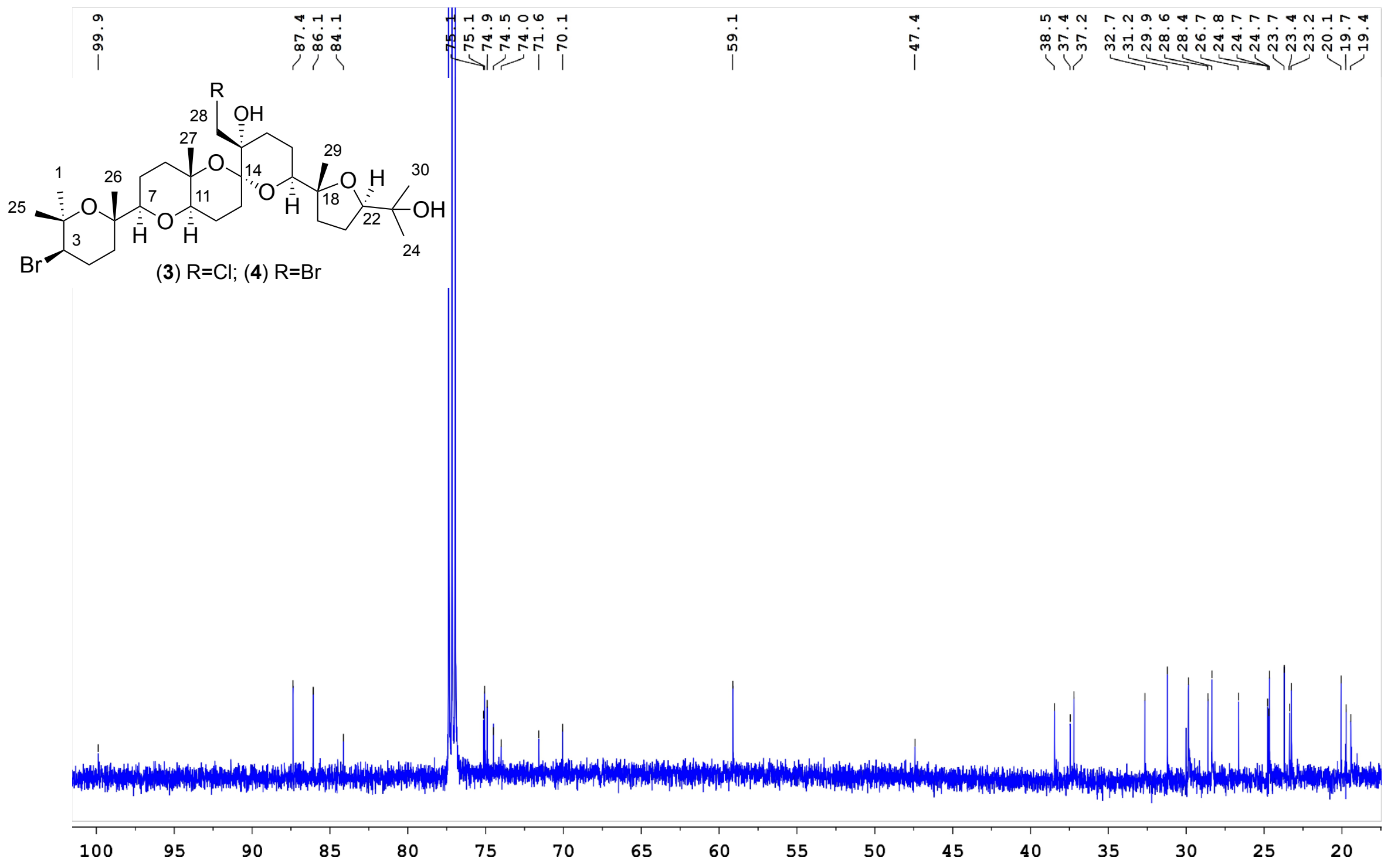


Figure S22. COSY spectrum of laurokanol C (3) and laurokanol D (4) mixture in $\mathrm{CDCl}_{3}$ at $298 \mathrm{~K}, 600 \mathrm{MHz}$.

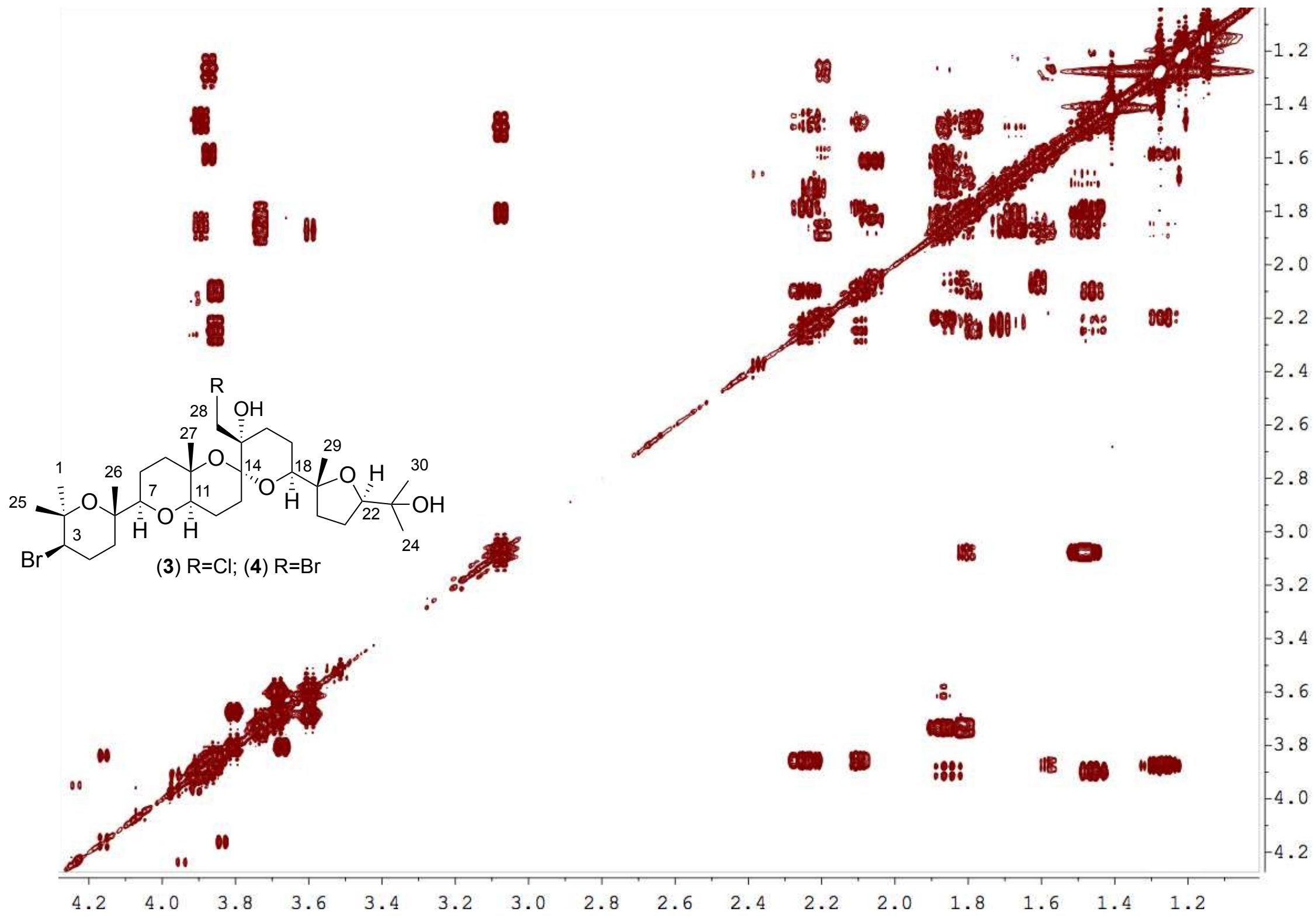


Figure S23. HSQC edited spectrum of laurokanol C (3) and laurokanol D (4) mixture in $\mathrm{CDCl}_{3}$ at $298 \mathrm{~K}, 600 \mathrm{MHz}$.
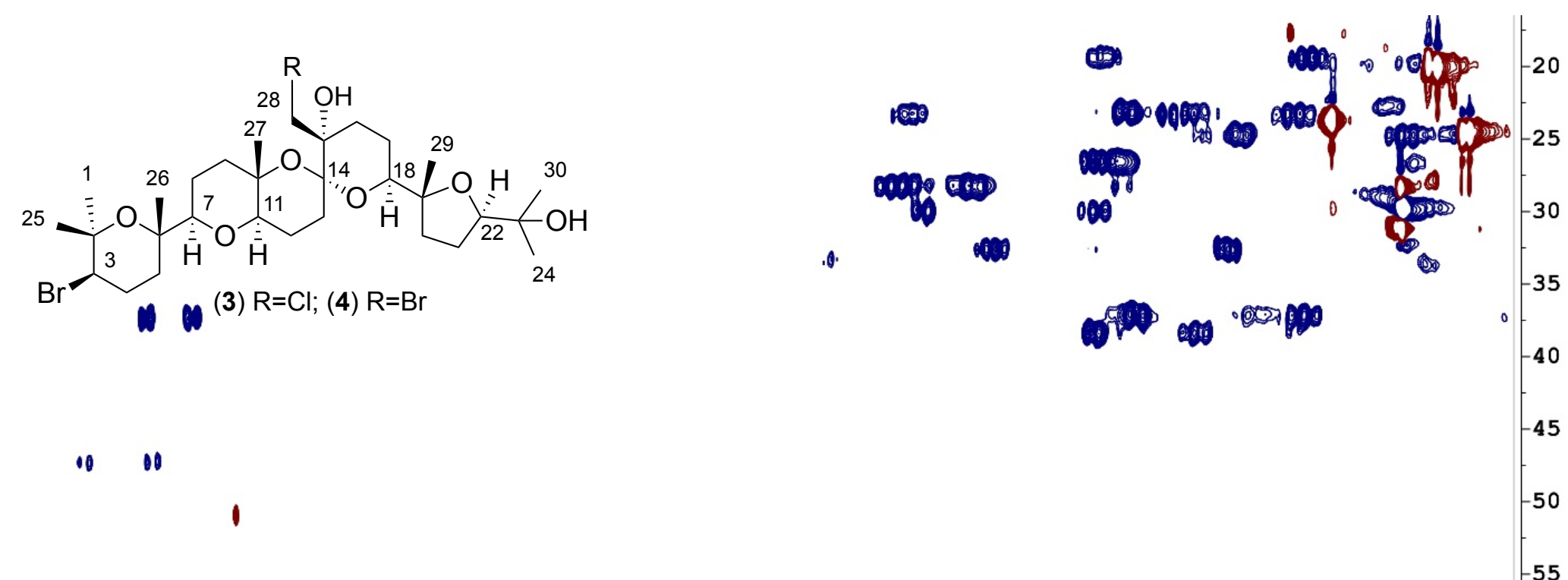

on

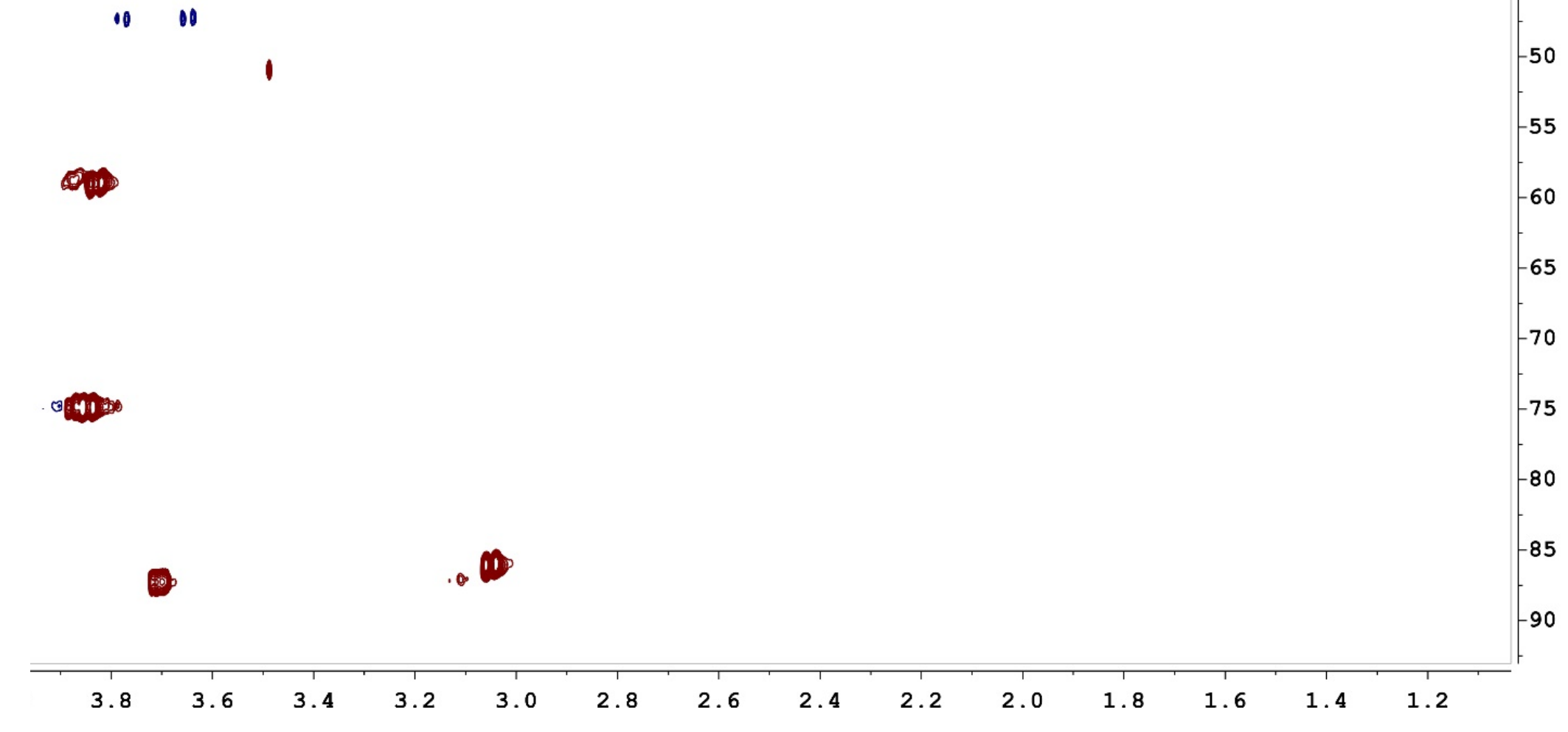


Figure S24. HMBC spectrum of laurokanol C (3) and laurokanol D (4) mixture in $\mathrm{CDCl}_{3}$ at $298 \mathrm{~K}, 600 \mathrm{MHz}$.

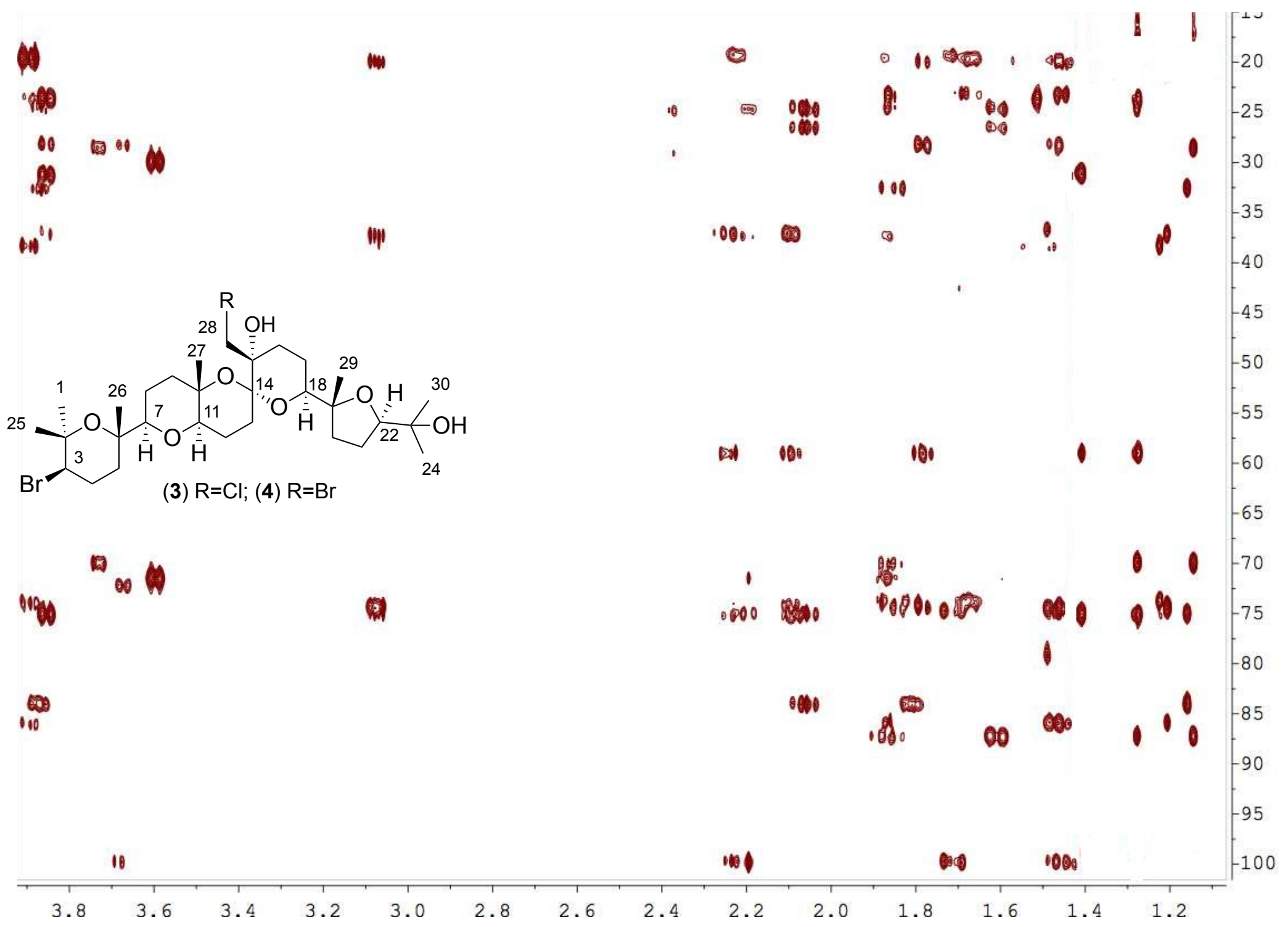


Figure S25. NOESY spectrum of laurokanol C (3) and laurokanol D (4) mixture in $\mathrm{CDCl}_{3}$ at $298 \mathrm{~K}, 600 \mathrm{MHz}$.

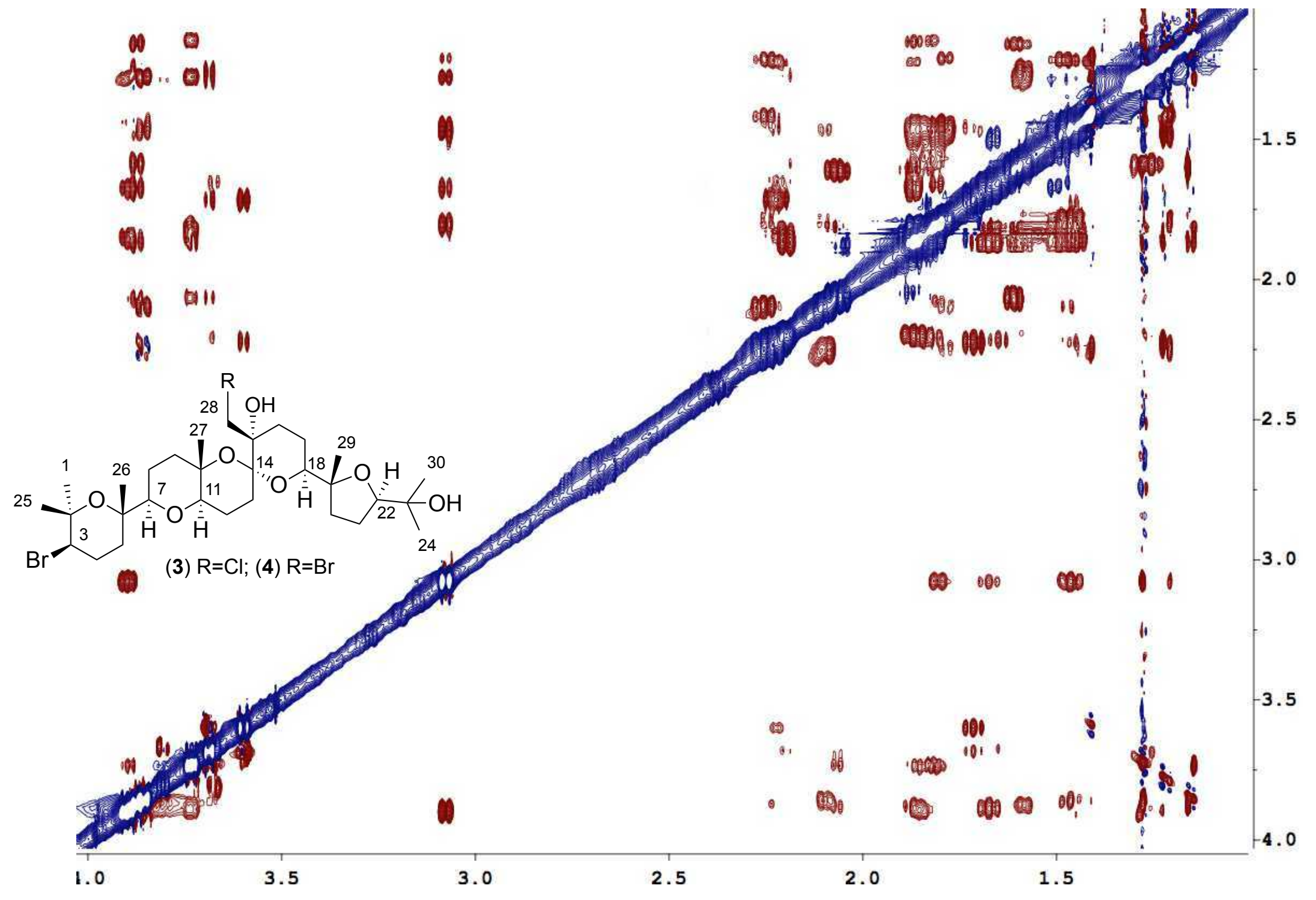


Figure S26. Mass spectra of laurokanol C (3) and laurokanol D (4).

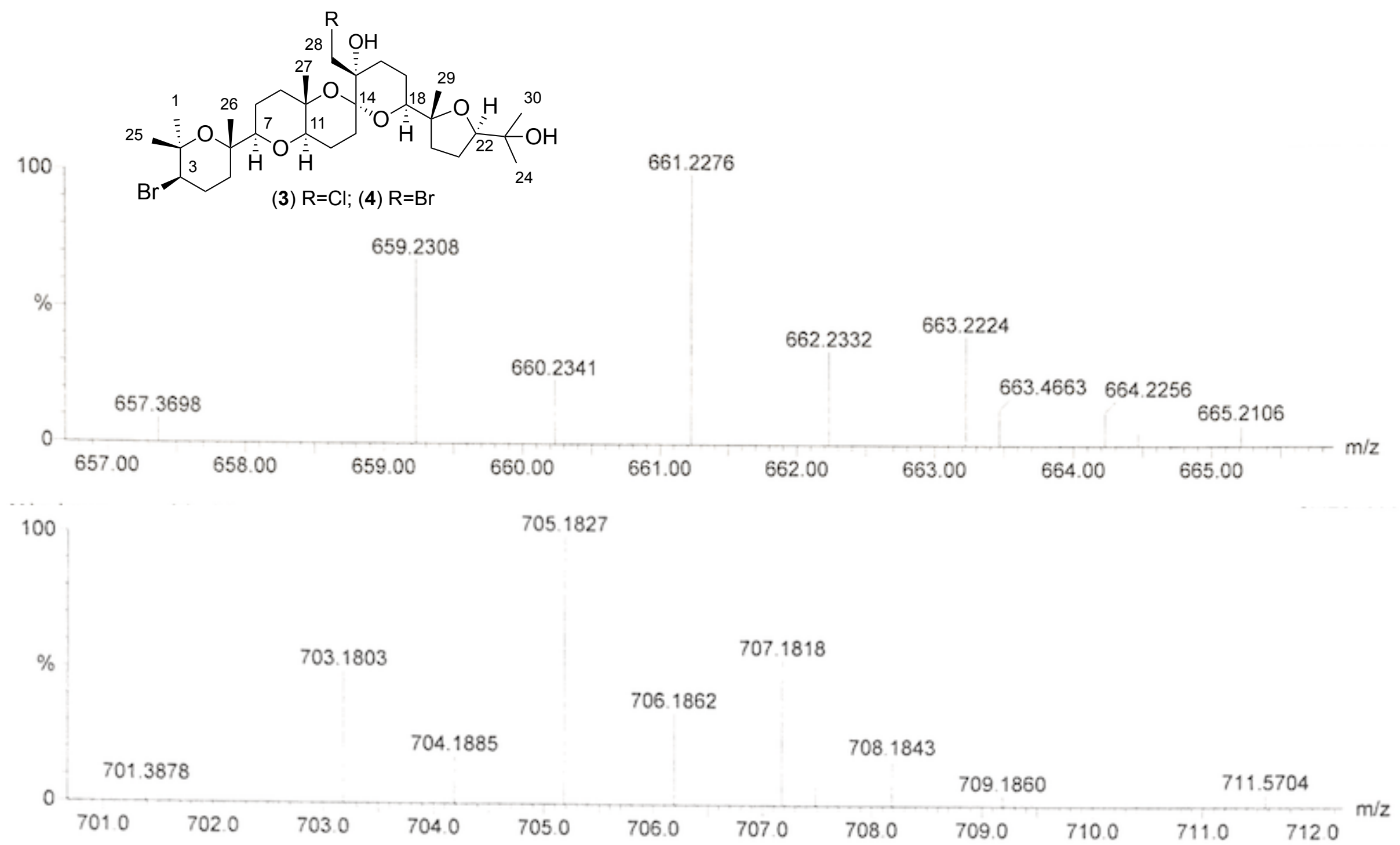


Table S5. ${ }^{13} \mathrm{C}$ and ${ }^{1} \mathrm{H}$ NMR data of laurokanol E (5) in $\mathrm{CDCl}_{3}$ at $298 \mathrm{~K}, 600 \mathrm{MHz}$.

\begin{tabular}{|c|c|c|c|c|}
\hline $\mathrm{C}$ & Multiplicity & $\delta_{\mathrm{C}}$ & $\delta_{\mathrm{H}}$ & $J$ in $\mathrm{Hz}$ \\
\hline 1 & $\mathrm{CH}_{3}$ & 24.0 & 1.12 & s \\
\hline 2 & C & 70.5 & & \\
\hline 3 & $\mathrm{CH}$ & 86.4 & 3.71 & $\mathrm{t}(7.6)$ \\
\hline 4 & $\mathrm{CH}_{2}$ & 26.3 & 1.78 & \\
\hline 5 & $\mathrm{CH}_{2}$ & 35.5 & $\begin{array}{l}1.67 \\
2.01\end{array}$ & ddd $(8.4,11.5,12.1)$ \\
\hline 6 & C & 83.9 & & \\
\hline 7 & $\mathrm{CH}$ & 83.2 & 3.30 & $\mathrm{dd}(2.6,11.8)$ \\
\hline 8 & $\mathrm{CH}_{2}$ & 24.5 & $\begin{array}{l}1.51 \\
1.69\end{array}$ & $\begin{array}{l}\text { dddd }(2.9,11.8,13.5,13.5) \\
\text { dddd }(2.6,2.9,4.5,13.5)\end{array}$ \\
\hline 9 & $\mathrm{CH}_{2}$ & 38.7 & $\begin{array}{l}1.65 \\
1.83\end{array}$ & $\begin{array}{l}\text { ddd }(4.5,12.7,13.5) \\
\text { ddd }(2.9,2.9,12.7)\end{array}$ \\
\hline 10 & C & 71.8 & & \\
\hline 11 & $\mathrm{CH}$ & 75.5 & 4.03 & $\mathrm{dd}(8.1,10.0)$ \\
\hline 12 & $\mathrm{CH}_{2}$ & 20.2 & $\begin{array}{l}1.42 \\
1.93\end{array}$ & $\begin{array}{l}\text { dddd }(1.3,10.0,11.1,13.0) \\
\text { dddd }(7.5,8.1,10.4,13.0)\end{array}$ \\
\hline 13 & $\mathrm{CH}_{2}$ & 27.8 & $\begin{array}{l}1.58 \\
2.19\end{array}$ & $\begin{array}{l}\text { ddd }(1.3,10.4,14.1) \\
\text { ddd }(7.5,11.1,14.1)\end{array}$ \\
\hline 14 & C & 99.0 & & \\
\hline 15 & $\mathrm{CH}$ & 38.6 & 1.38 & $\operatorname{ddt}(3.8,6.7,6.7,6.7,12.5)$ \\
\hline 16 & $\mathrm{CH}_{2}$ & 27.2 & $\begin{array}{l}1.42 \\
1.57\end{array}$ & $\begin{array}{l}\text { dddd }(2.9,3.8,4.4,13.0) \\
\text { dddd }(4.2,12.5,13.0,13.0)\end{array}$ \\
\hline 17 & $\mathrm{CH}_{2}$ & 27.0 & $\begin{array}{l}1.20 \\
1.55\end{array}$ & $\begin{array}{l}\text { dddd }(4.4,12.5,12.5,13.0) \\
\text { dddd }(2.3,2.9,4.2,12.5)\end{array}$ \\
\hline 18 & $\mathrm{CH}$ & 74.5 & 3.80 & $d d(2.3,12.5)$ \\
\hline 19 & $\mathrm{C}$ & 84.7 & & \\
\hline 20 & $\mathrm{CH}_{2}$ & 32.6 & $\begin{array}{l}1.55 \\
2.16\end{array}$ & $\begin{array}{l}\text { ddd }(2.5,7.6,11.9) \\
\text { ddd }(8.5,11.5,11.9)\end{array}$ \\
\hline 21 & $\mathrm{CH}_{2}$ & 26.6 & 1.80 & \\
\hline 22 & $\mathrm{CH}$ & 87.0 & 3.78 & $\mathrm{dd}(5.7,9.9)$ \\
\hline 23 & C & 70.0 & & \\
\hline 24 & $\mathrm{CH}_{3}$ & 24.4 & 1.13 & s \\
\hline 25 & $\mathrm{CH}_{3}$ & 27.5 & 1.20 & s \\
\hline 26 & $\mathrm{CH}_{3}$ & 22.5 & 1.14 & $s$ \\
\hline 27 & $\mathrm{CH}_{3}$ & 19.5 & 1.17 & $\mathrm{~s}$ \\
\hline 28 & $\mathrm{CH}_{3}$ & 16.9 & 0.87 & $d(6.6)$ \\
\hline 29 & $\mathrm{CH}_{3}$ & 24.4 & 1.15 & s \\
\hline 30 & $\mathrm{CH}_{3}$ & 28.4 & 1.28 & $s$ \\
\hline
\end{tabular}


Figure S27. ${ }^{1} \mathrm{H}-\mathrm{NMR}$ spectrum of laurokanol $\mathrm{E}(5)$ in $\mathrm{CDCl}_{3}$ at $298 \mathrm{~K}, 600 \mathrm{MHz}$.

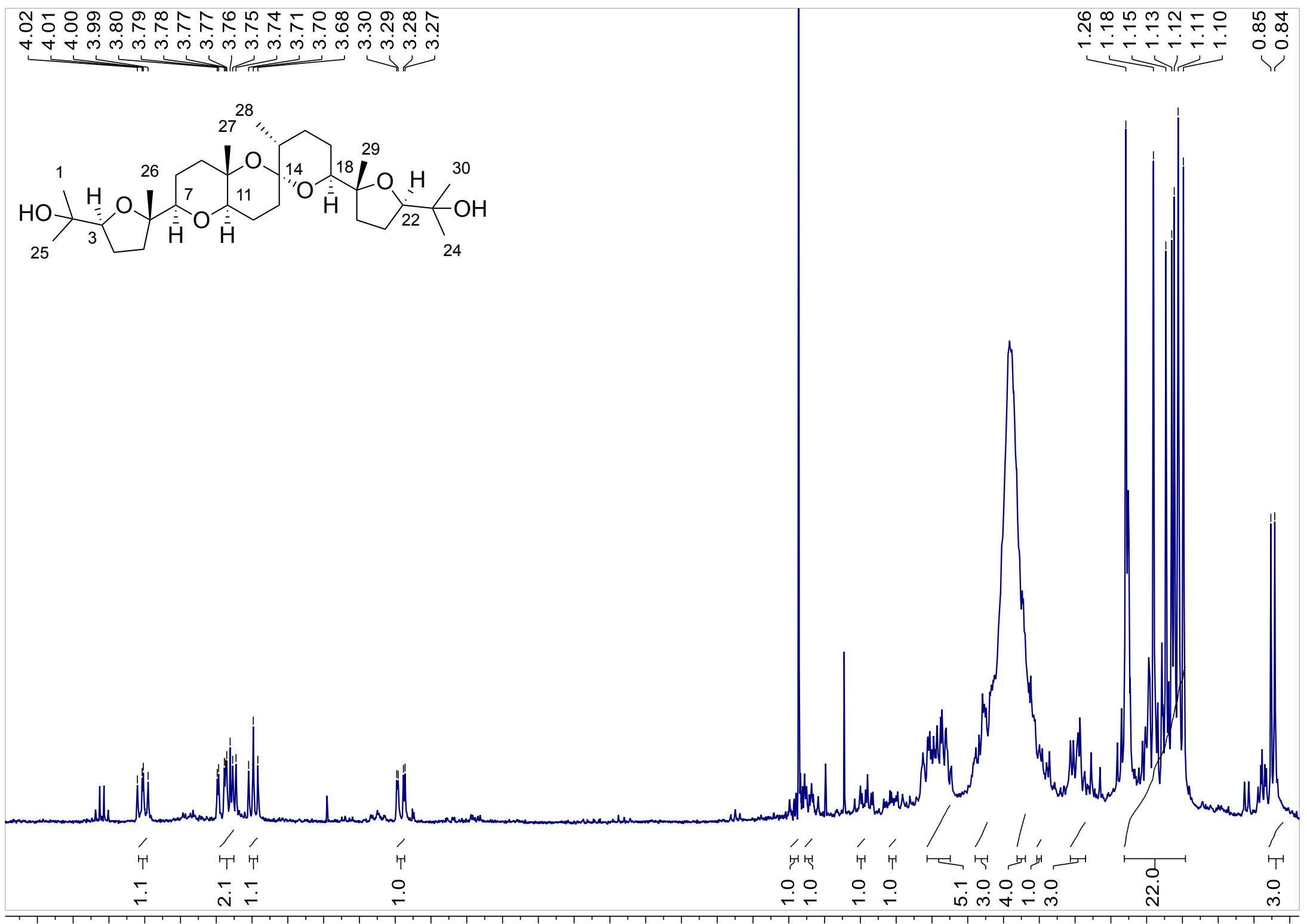

4.34 .24 .14 .03 .93 .83 .73 .63 .53 .43 .33 .23 .13 .02 .92 .82 .72 .62 .52 .42 .32 .22 .12 .01 .91 .81 .71 .61 .51 .41 .31 .21 .11 .00 .90 .1 
Figure S28. ${ }^{13} \mathrm{C}\left\{{ }^{1} \mathrm{H}\right\} \mathrm{NMR}$ spectrum of laurokanol $\mathrm{E}(\mathbf{5})$ in $\mathrm{CDCl}_{3}$ at $298 \mathrm{~K}, 150 \mathrm{MHz}$.

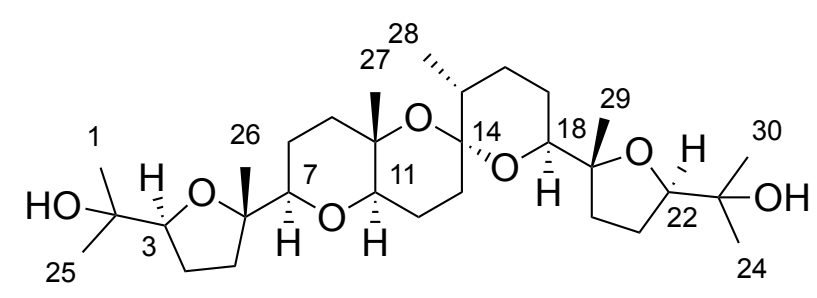

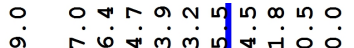

م่

更

の

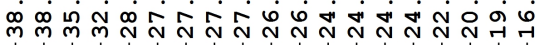

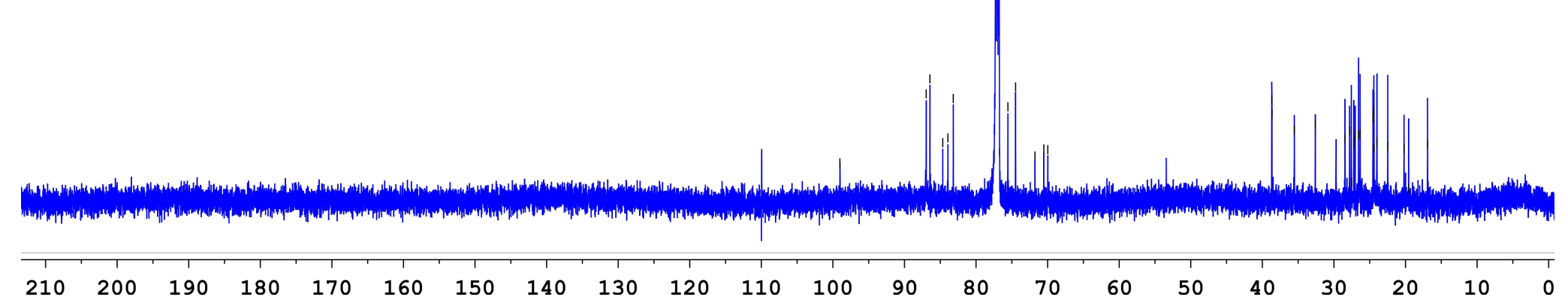


Figure S29. COSY spectrum of laurokanol E (5) in $\mathrm{CDCl}_{3}$ at $298 \mathrm{~K}, 600 \mathrm{MHz}$.

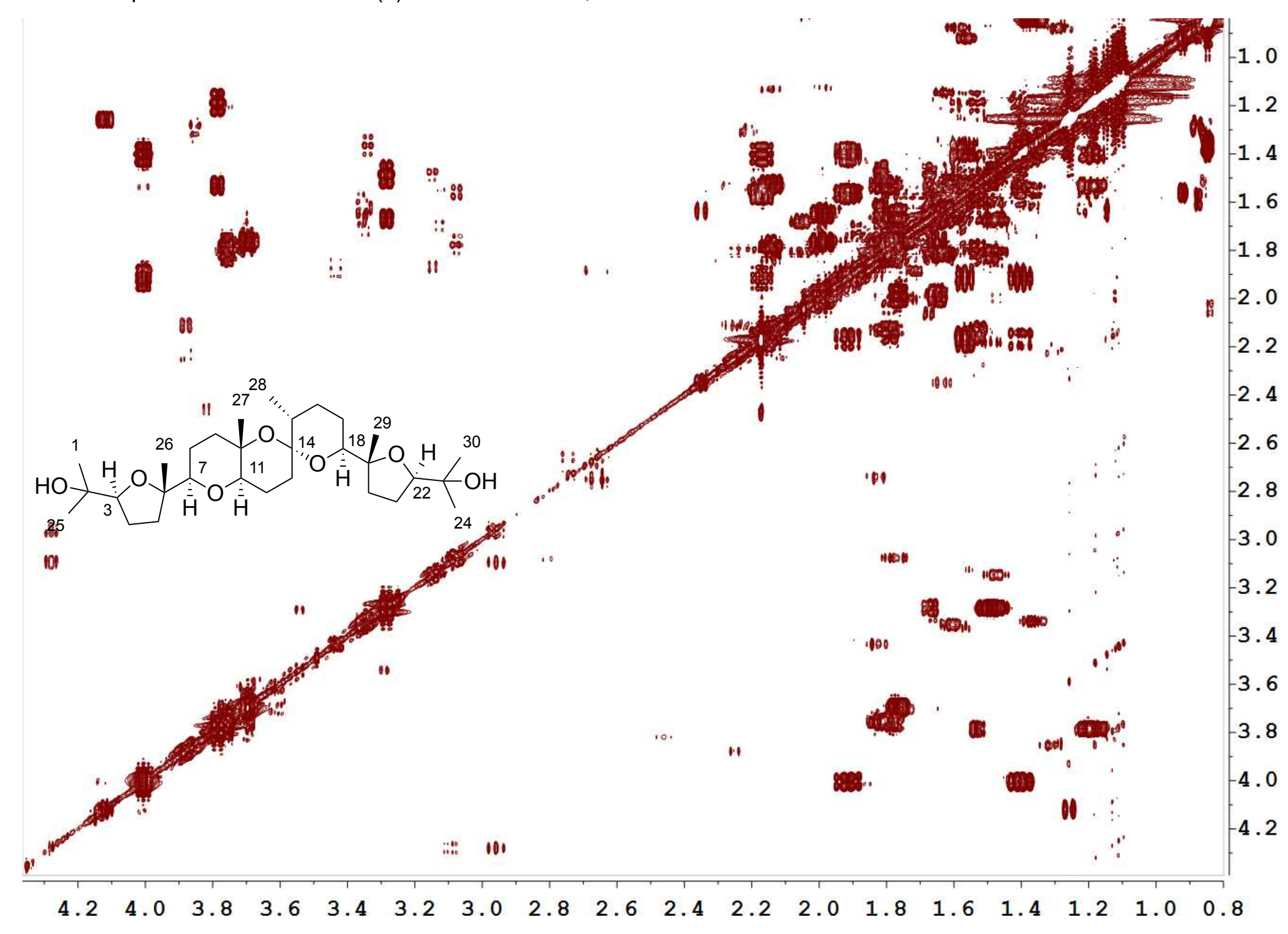


Figure S30. HSQCed spectrum of laurokanol E (5) in $\mathrm{CDCl}_{3}$ at $298 \mathrm{~K}, 600 \mathrm{MHz}$.
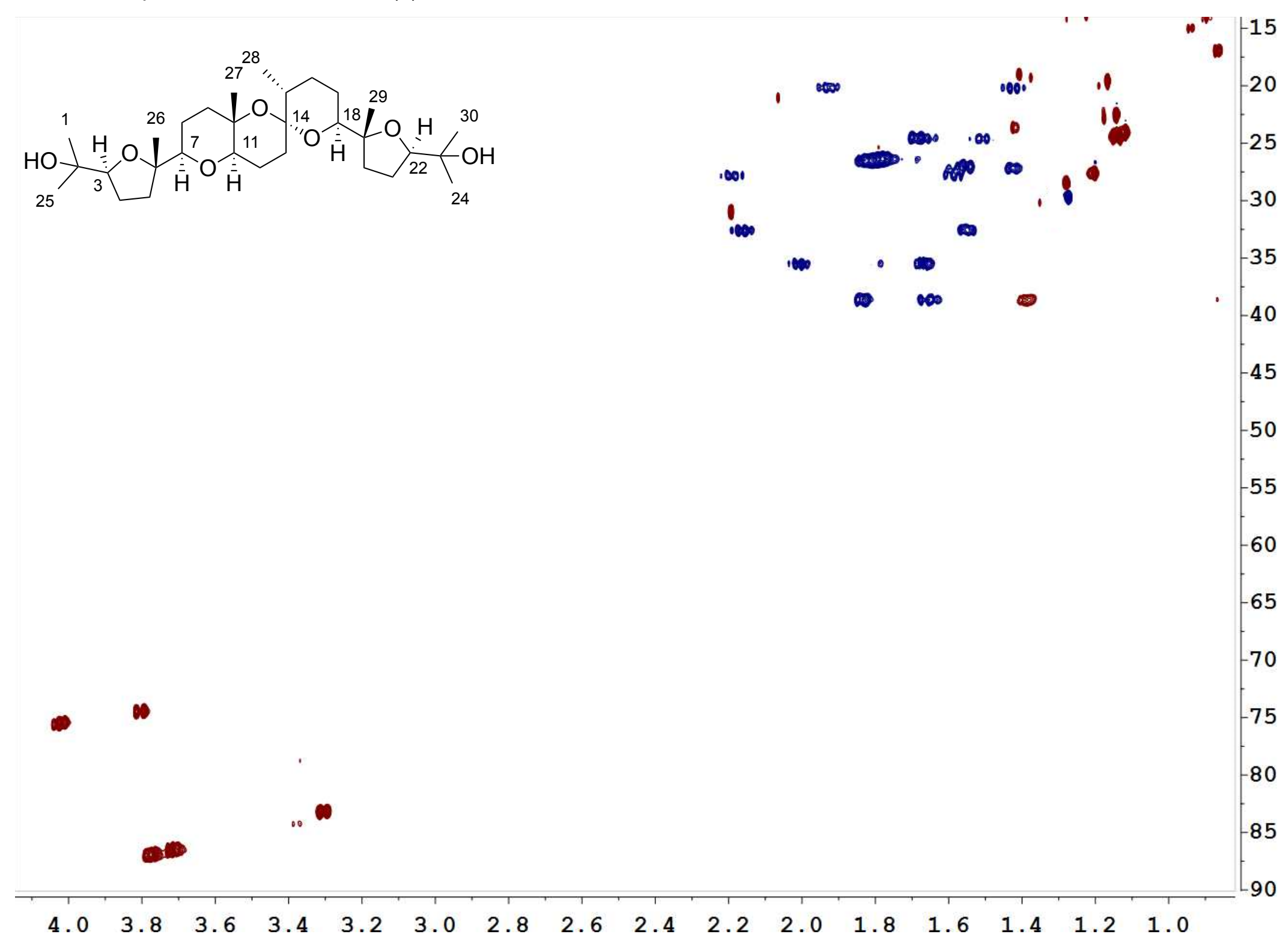
Figure S31. HMBC spectrum of laurokanol E (5) in $\mathrm{CDCl}_{3}$ at $298 \mathrm{~K}, 600 \mathrm{MHz}$.

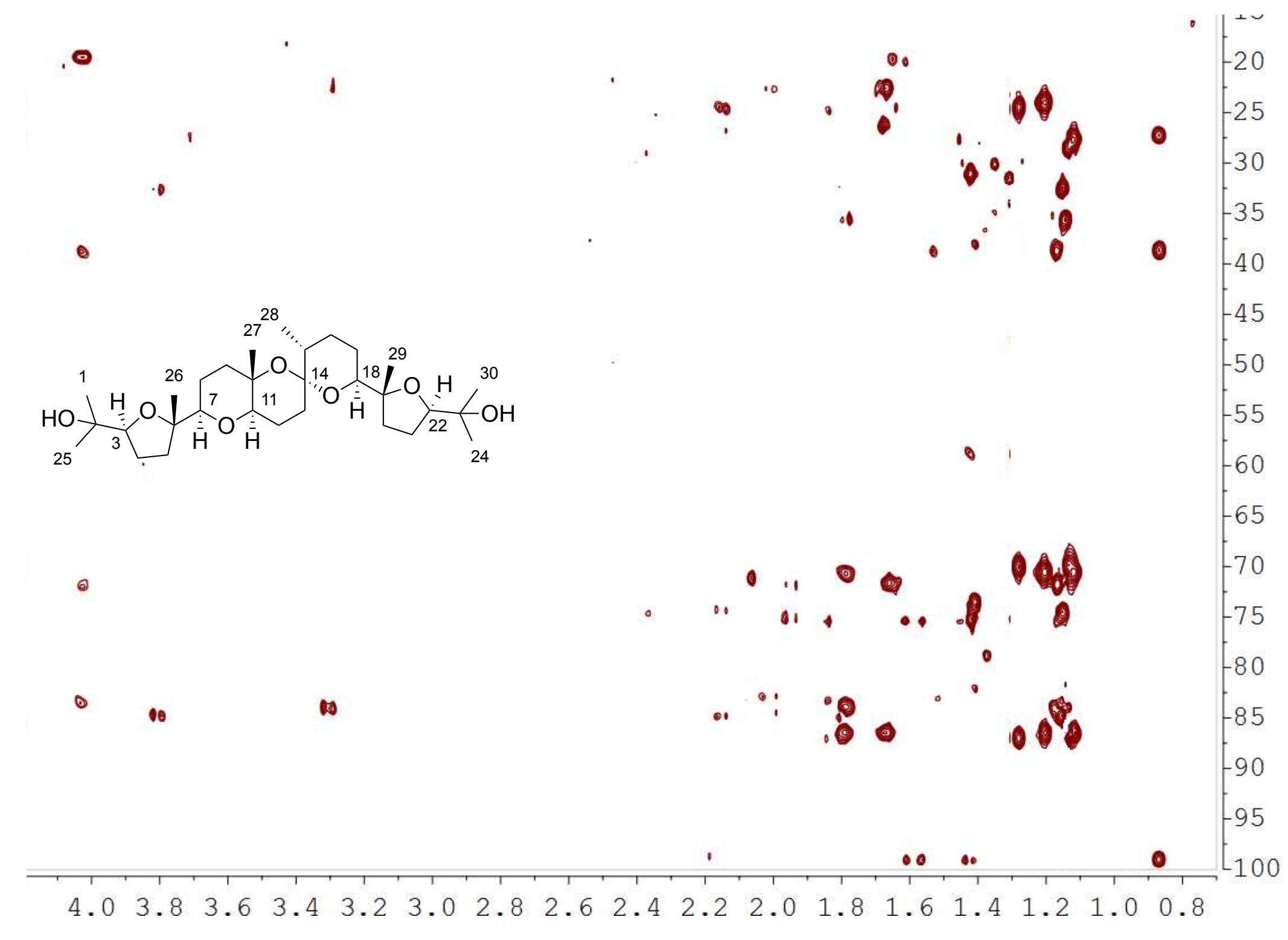


Figure S32. NOESY spectrum of laurokanol E (5) in $\mathrm{CDCl}_{3}$ at $298 \mathrm{~K}, 600 \mathrm{MHz}$.

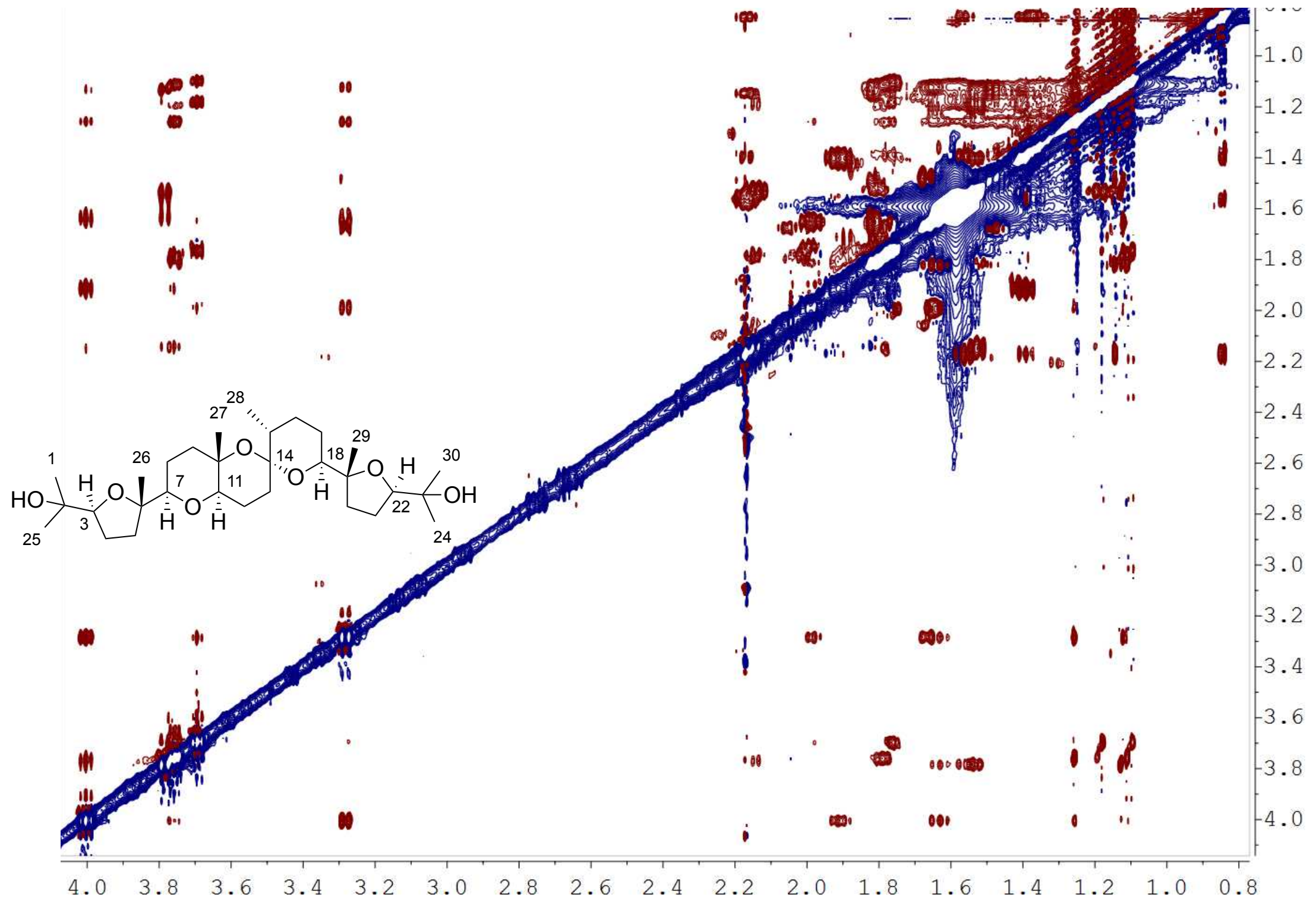


Figure S33. Mass spectrum of laurokanol E (5).

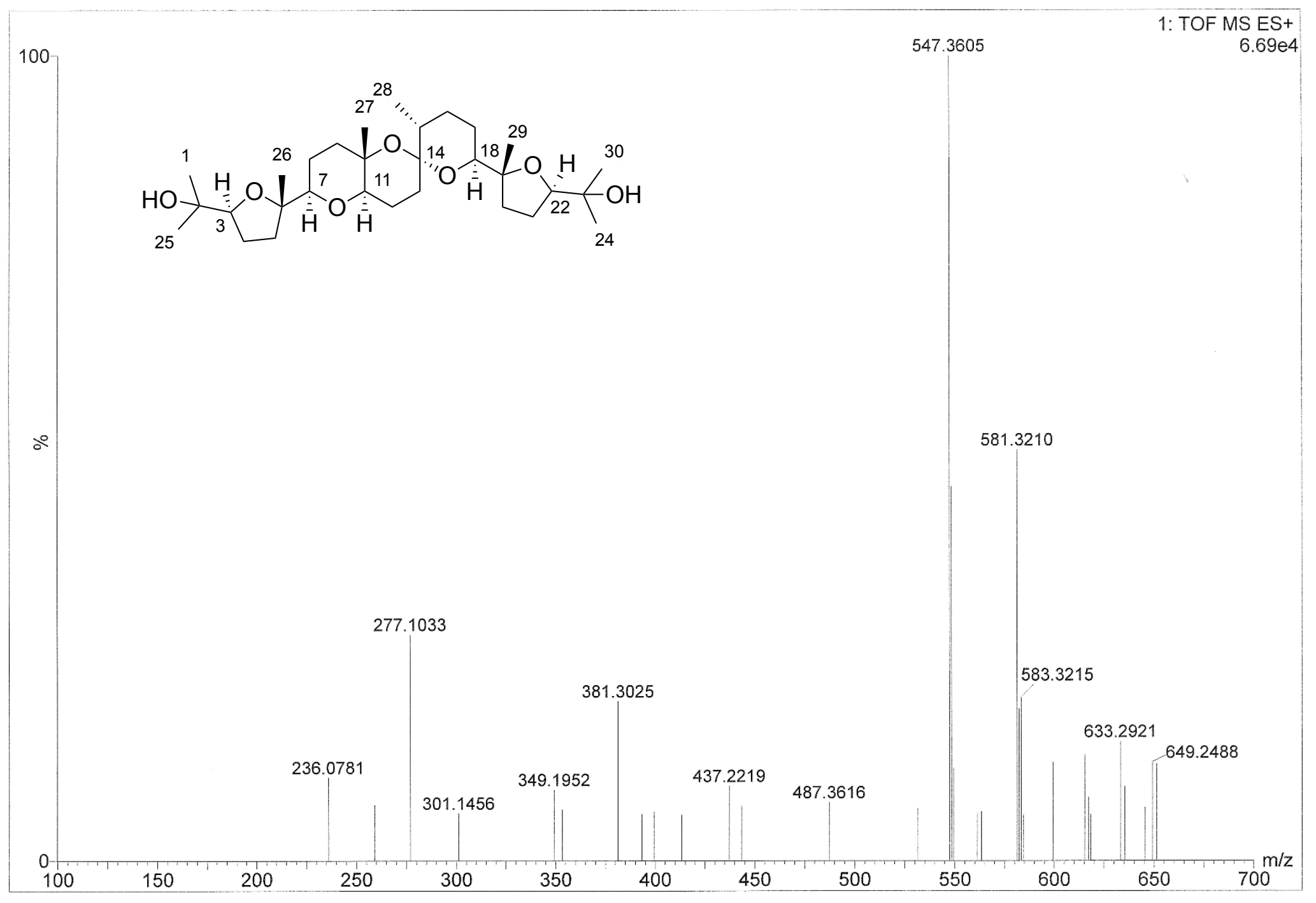


Table S6. ${ }^{13} \mathrm{C}$ and ${ }^{1} \mathrm{H}$ NMR data of yucatecone (6) in $\mathrm{CDCl}_{3}$ at $298 \mathrm{~K}, 600 \mathrm{MHz}$.

\begin{tabular}{|c|c|c|c|c|c|}
\hline C & Multiplicity & $\delta_{c}$ & $\delta_{H}$ & Multiplicity & $J$ in $\mathrm{Hz}$ \\
\hline $\begin{array}{l}1 \\
2 \\
2\end{array}$ & $\begin{array}{l}\mathrm{CH}_{3} \\
\mathrm{C}\end{array}$ & $\begin{array}{l}31.0 \\
74.9 \\
\end{array}$ & 1.27 & s & $d d(10123)$ \\
\hline 3 & & 59.1 & 3.90 & & $\mathrm{dd}(4.0,12.3)$ \\
\hline 4 & $\mathrm{CH}_{2}$ & 27.7 & $\begin{array}{l}2.09 \\
2.25\end{array}$ & & $\begin{array}{l}\text { dddd }(3.5,4.0,4.3,13.3) \\
\text { dddd }(3.9,12.0,13.3,13.5)\end{array}$ \\
\hline 5 & $\mathrm{CH}_{2}$ & 37.2 & $\begin{array}{l}1.55 \\
1.80\end{array}$ & & $\begin{array}{l}\text { ddd }(4.3,13.5,13.6) \\
\text { ddd }(3.5,3.9,13.6)\end{array}$ \\
\hline $\begin{array}{l}6 \\
7\end{array}$ & $\stackrel{\mathrm{C}}{\mathrm{CH}}$ & $\begin{array}{l}74.4 \\
86.8\end{array}$ & 3.09 & & dd $(2.4,11.2$ \\
\hline 8 & $\mathrm{CH}_{2}$ & 22.9 & $\begin{array}{l}1.53 \\
1.76\end{array}$ & & $\begin{array}{l}\mathrm{m} \\
\mathrm{m}\end{array}$ \\
\hline 9 & $\mathrm{CH}_{2}$ & 37.7 & $\begin{array}{l}1.50 \\
1.70\end{array}$ & & $\begin{array}{l}\mathrm{m} \\
\mathrm{m}\end{array}$ \\
\hline $\begin{array}{l}10 \\
11\end{array}$ & $\begin{array}{l}\mathrm{C} \\
\mathrm{CH}\end{array}$ & $\begin{array}{l}72.1 \\
81.2\end{array}$ & 3.00 & & dd $(3.8,11.8)$ \\
\hline 12 & $\mathrm{CH}_{2}$ & 24.8 & $\begin{array}{l}1.51 \\
1.64\end{array}$ & & $\begin{array}{l}\text { dddd }(3.2,3.8,4.0,12.2) \\
\text { dddd }(2.9,11.8,12.0,12.2)\end{array}$ \\
\hline 13 & $\mathrm{CH}_{2}$ & 28.2 & $\begin{array}{l}1.37 \\
1.65\end{array}$ & & $\begin{array}{l}\text { dddd }(4.0,11.7,12.0,12.0) \\
\text { dddd }(2.1,2.9,3.2,12.0)\end{array}$ \\
\hline $\begin{array}{l}14 \\
15\end{array}$ & $\begin{array}{l}\mathrm{CH} \\
\mathrm{CH}\end{array}$ & $\begin{array}{l}72.6 \\
37.1\end{array}$ & $\begin{array}{l}3.47 \\
1.44\end{array}$ & & $\begin{array}{l}\text { ddd }(2.1 ; 4.6 ; 11.7) \\
\mathrm{m}\end{array}$ \\
\hline 16 & $\mathrm{CH}_{2}$ & 26.2 & $\begin{array}{l}1.34 \\
1.73\end{array}$ & & $\begin{array}{l}\mathrm{m} \\
\mathrm{m}\end{array}$ \\
\hline 17 & $\mathrm{CH}_{2}$ & 34.5 & $\begin{array}{l}2.56 \\
2.71\end{array}$ & & $\begin{array}{l}\text { ddd }(5.8,9.7,17.7) \\
\text { ddd }(5.3,9.9,17.6)\end{array}$ \\
\hline $\begin{array}{l}18 \\
19\end{array}$ & $\begin{array}{l}\mathrm{C} \\
\mathrm{C}\end{array}$ & $\begin{array}{l}215.6 \\
88.8\end{array}$ & & & \\
\hline 20 & $\mathrm{CH}_{2}$ & 35.0 & $\begin{array}{l}1.77 \\
2.16\end{array}$ & & $\begin{array}{l}\mathrm{m} \\
\mathrm{m}\end{array}$ \\
\hline $\begin{array}{l}21 \\
22 \\
23\end{array}$ & $\begin{array}{l}\mathrm{CH}_{2} \\
\mathrm{CH} \\
\mathrm{C}\end{array}$ & $\begin{array}{l}26.0 \\
87.3 \\
70.7\end{array}$ & $\begin{array}{l}1.83 \\
3.77\end{array}$ & & $\begin{array}{l}\mathrm{m} \\
\mathrm{dd}(6.2,8.7)\end{array}$ \\
\hline 24 & $\mathrm{CH}_{3}$ & 23.7 & 1.15 & & $\mathrm{~s}$ \\
\hline 25 & $\mathrm{CH}_{3}$ & 24.2 & 1.40 & & s \\
\hline 26 & $\mathrm{CH}_{3}$ & 20.1 & 1.21 & & s \\
\hline 27 & $\mathrm{CH}_{3}$ & 14.7 & 1.14 & & $\mathrm{~S}$ \\
\hline 28 & $\mathrm{CH}_{3}$ & 15.4 & 0.88 & & $\mathrm{~d}(6.8)$ \\
\hline 29 & $\mathrm{CH}_{3}$ & 24.4 & 1.33 & & $\mathrm{~s}$ \\
\hline 30 & $\mathrm{CH}_{3}$ & 27.3 & 1.26 & & $\mathrm{~s}$ \\
\hline
\end{tabular}


Figure S34. ${ }^{1} \mathrm{H}-\mathrm{NMR}$ spectrum of yucatecone (6) in $\mathrm{CDCl}_{3}$ at $298 \mathrm{~K}, 600 \mathrm{MHz}$.

б

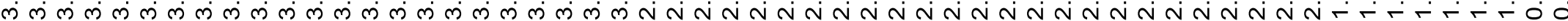
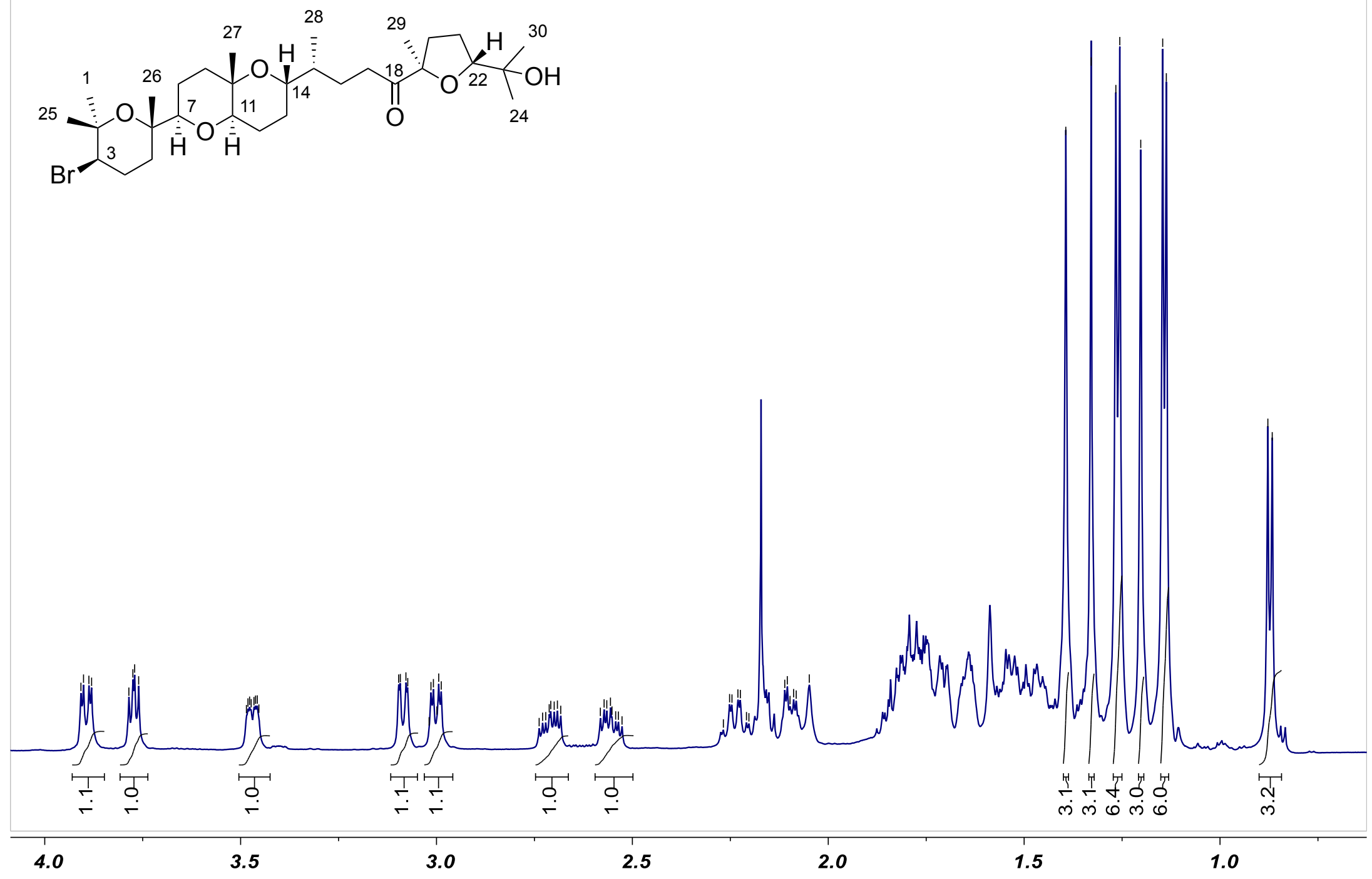
Figure S35. Section of the ${ }^{1} \mathrm{H}-\mathrm{NMR}$ decoupling spectrum of yucatecone (6) in $\mathrm{CDCl}_{3}$ at $298 \mathrm{~K}, 600 \mathrm{MHz}$.

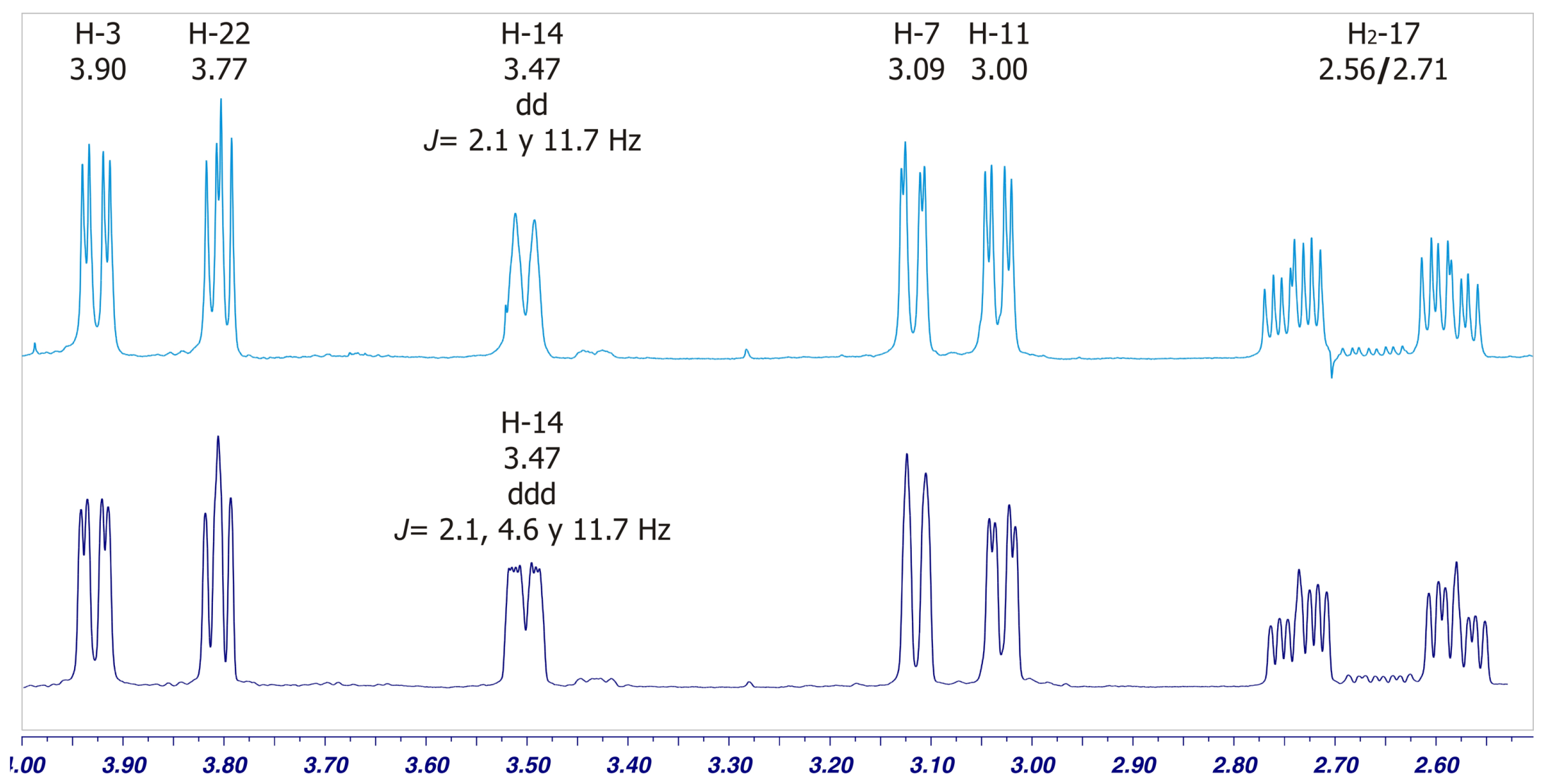


Figure S36. ${ }^{13} \mathrm{C}\{\mathrm{H}\}$ NMR spectrum of yucatecone (6) in $\mathrm{CDCl}_{3}$ at $298 \mathrm{~K}, 150 \mathrm{MHz}$.

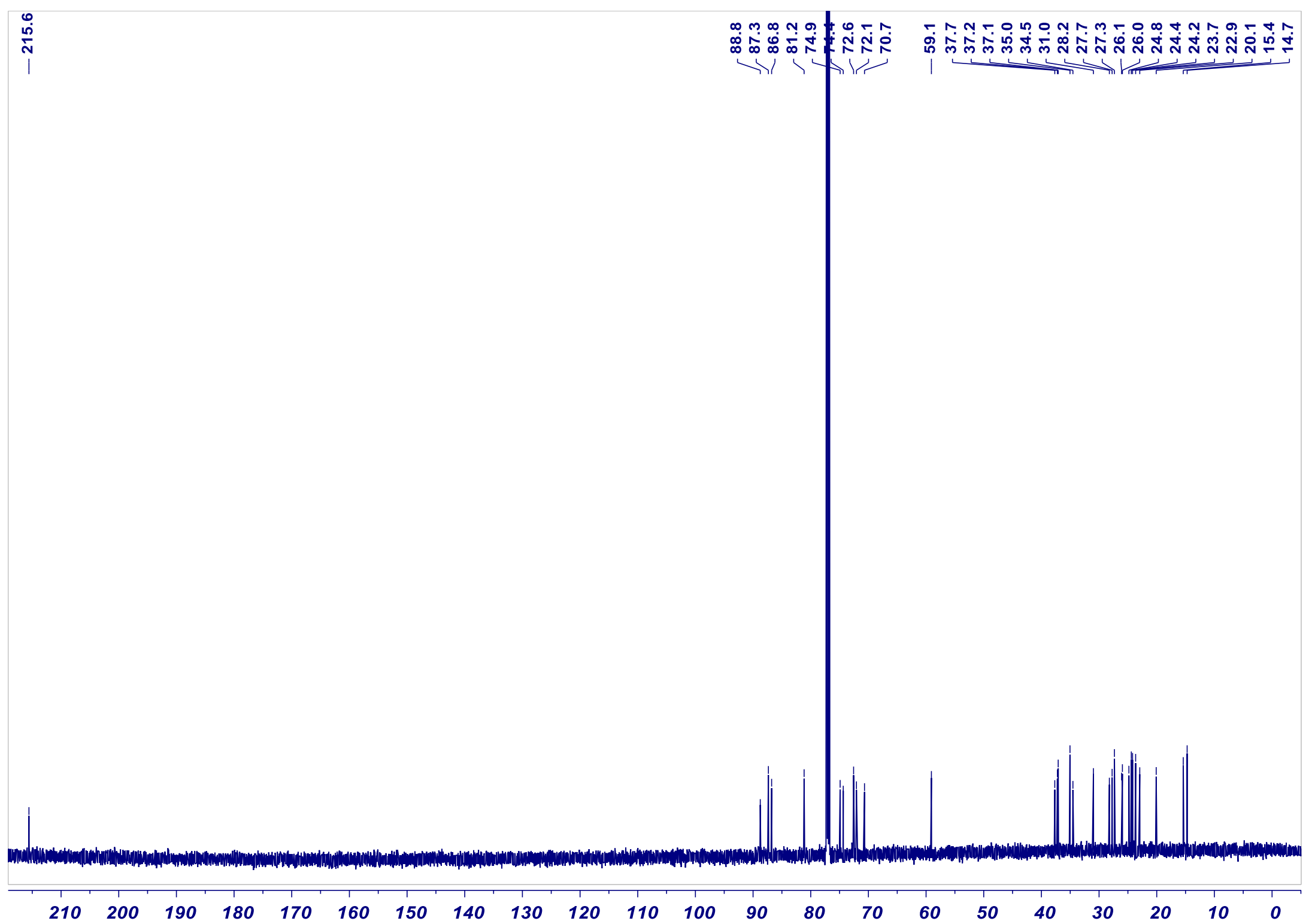


Figure S37. COSY spectrum of yucatecone (6) in $\mathrm{CDCl}_{3}$ at $298 \mathrm{~K}, 600 \mathrm{MHz}$.

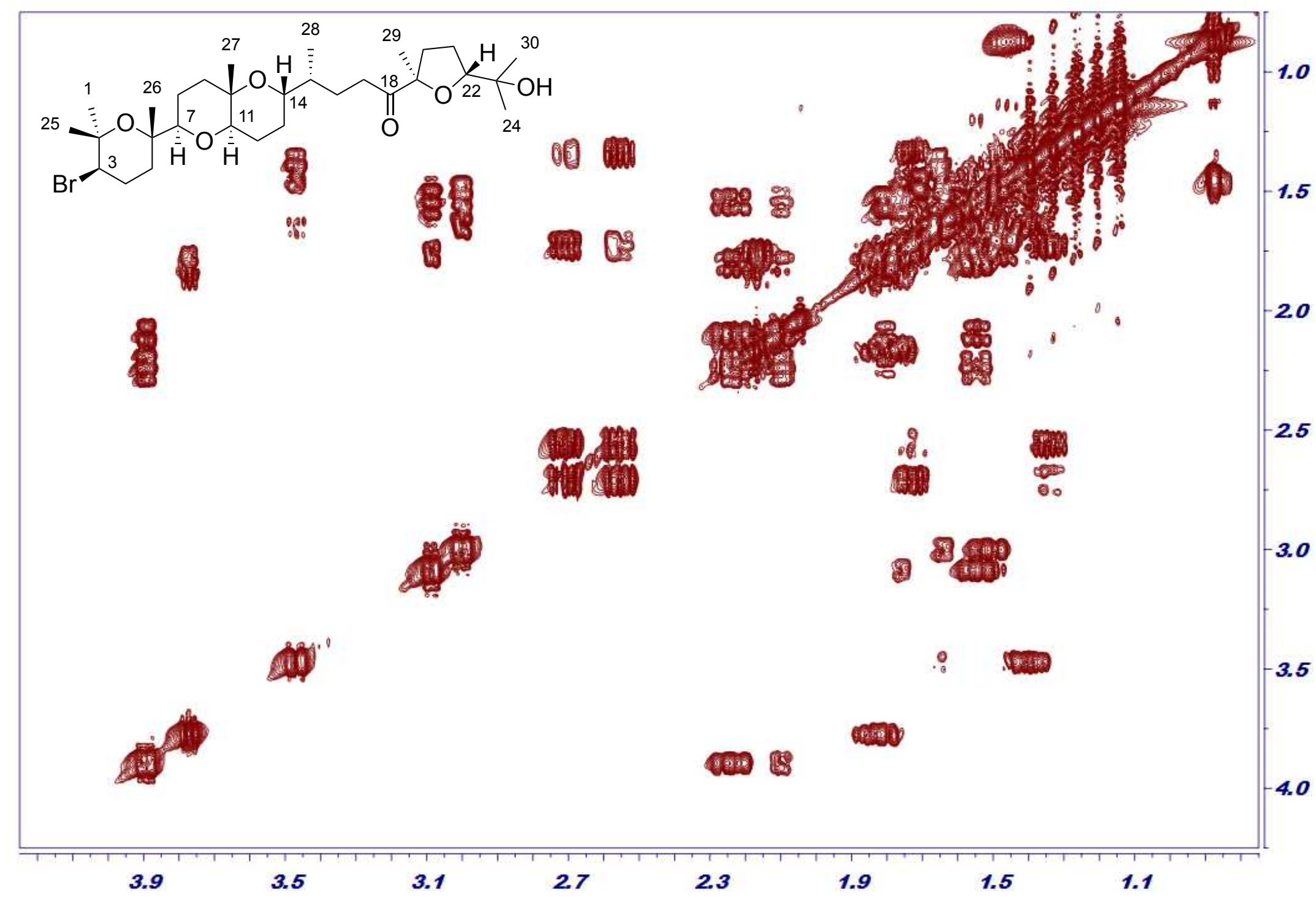


Figure S38. TOCSY spectrum of yucatecone (6) in $\mathrm{CDCl}_{3}$ at $298 \mathrm{~K}, 600 \mathrm{MHz}$.

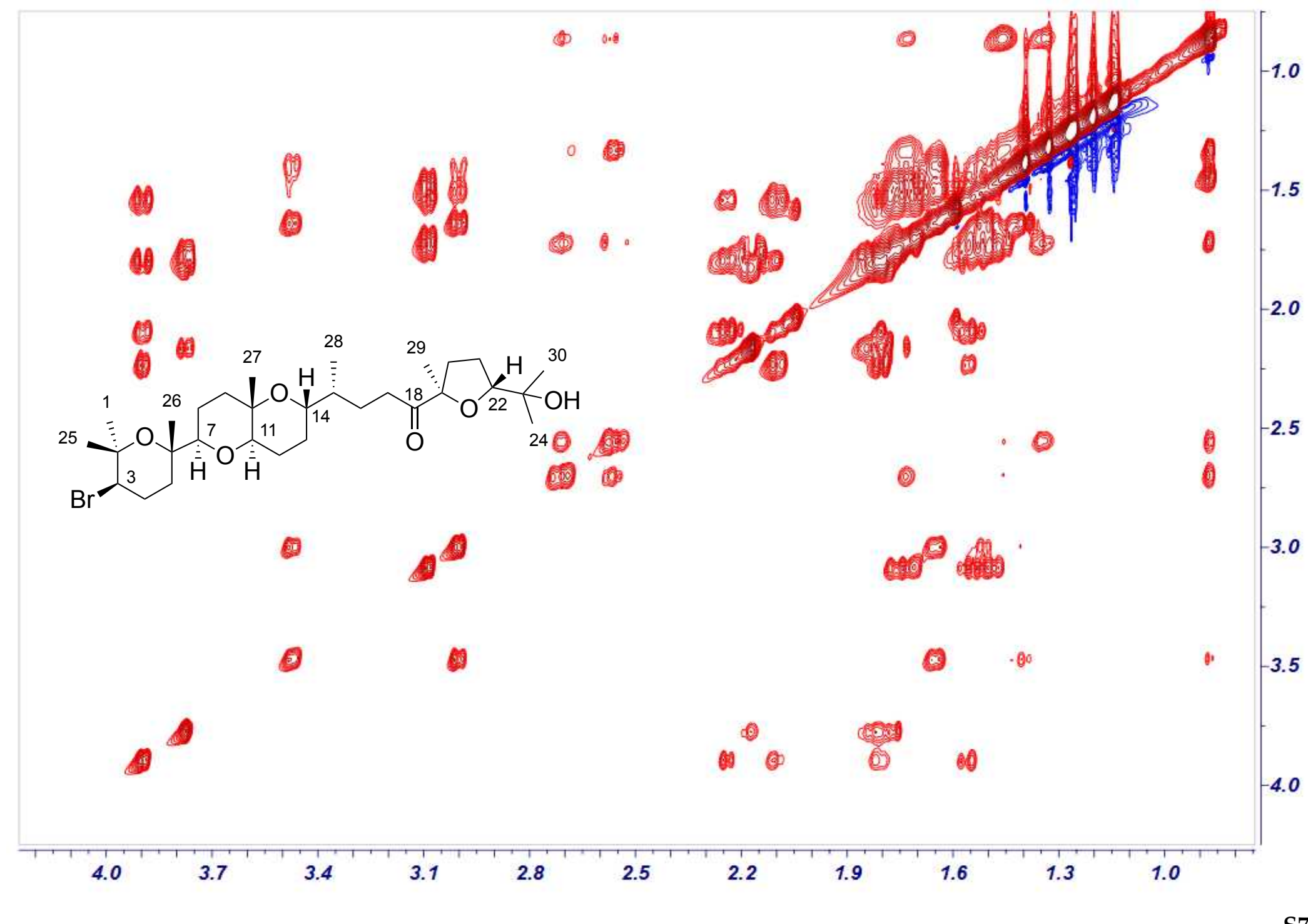


Figure S39. HSQC spectrum of yucatecone (6) in $\mathrm{CDCl}_{3}$ at $298 \mathrm{~K}, 600 \mathrm{MHz}$.

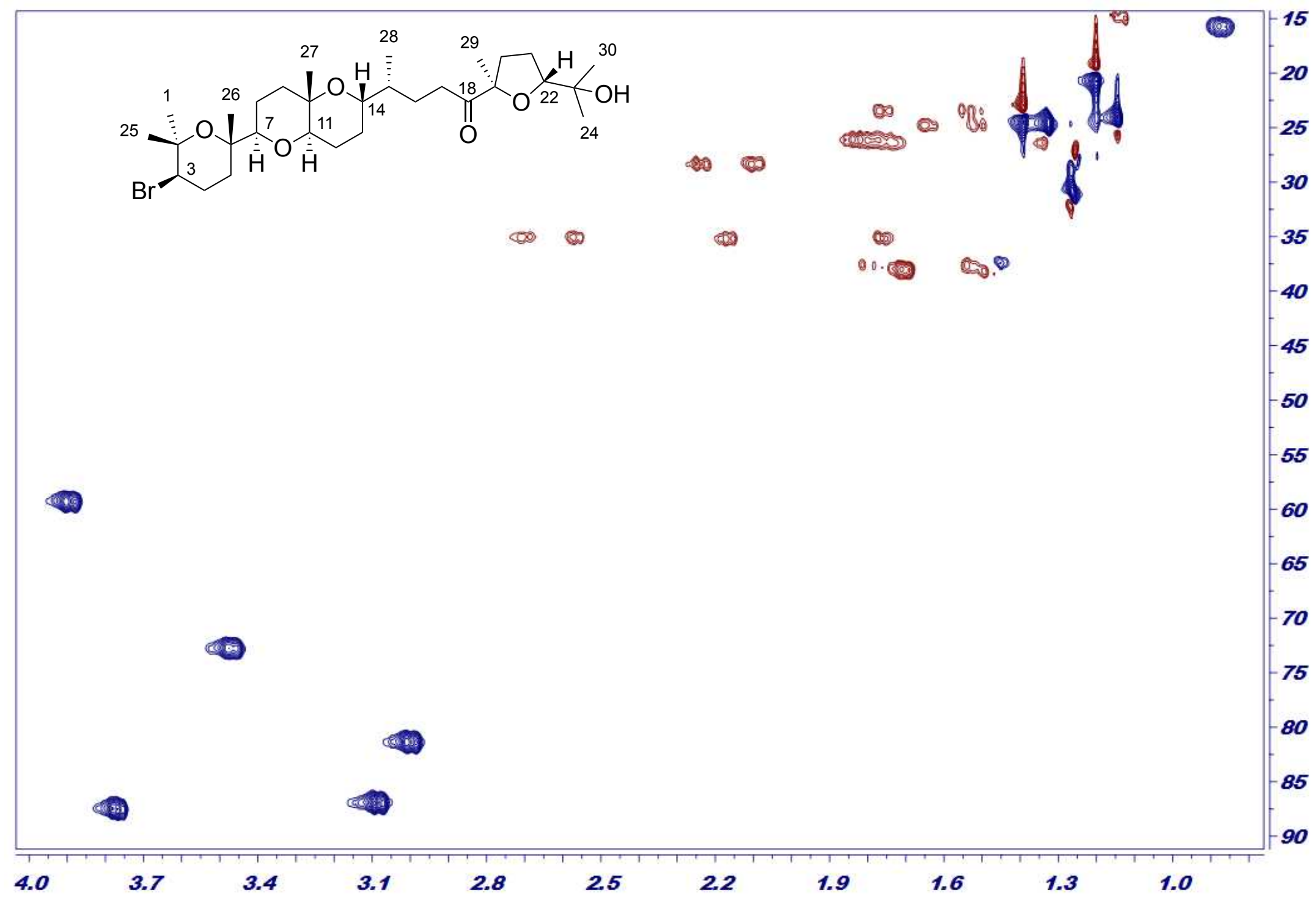


Figure S40. HMBC spectrum of yucatecone (2) in $\mathrm{CDCl}_{3}$ at $298 \mathrm{~K}, 600 \mathrm{MHz}$.

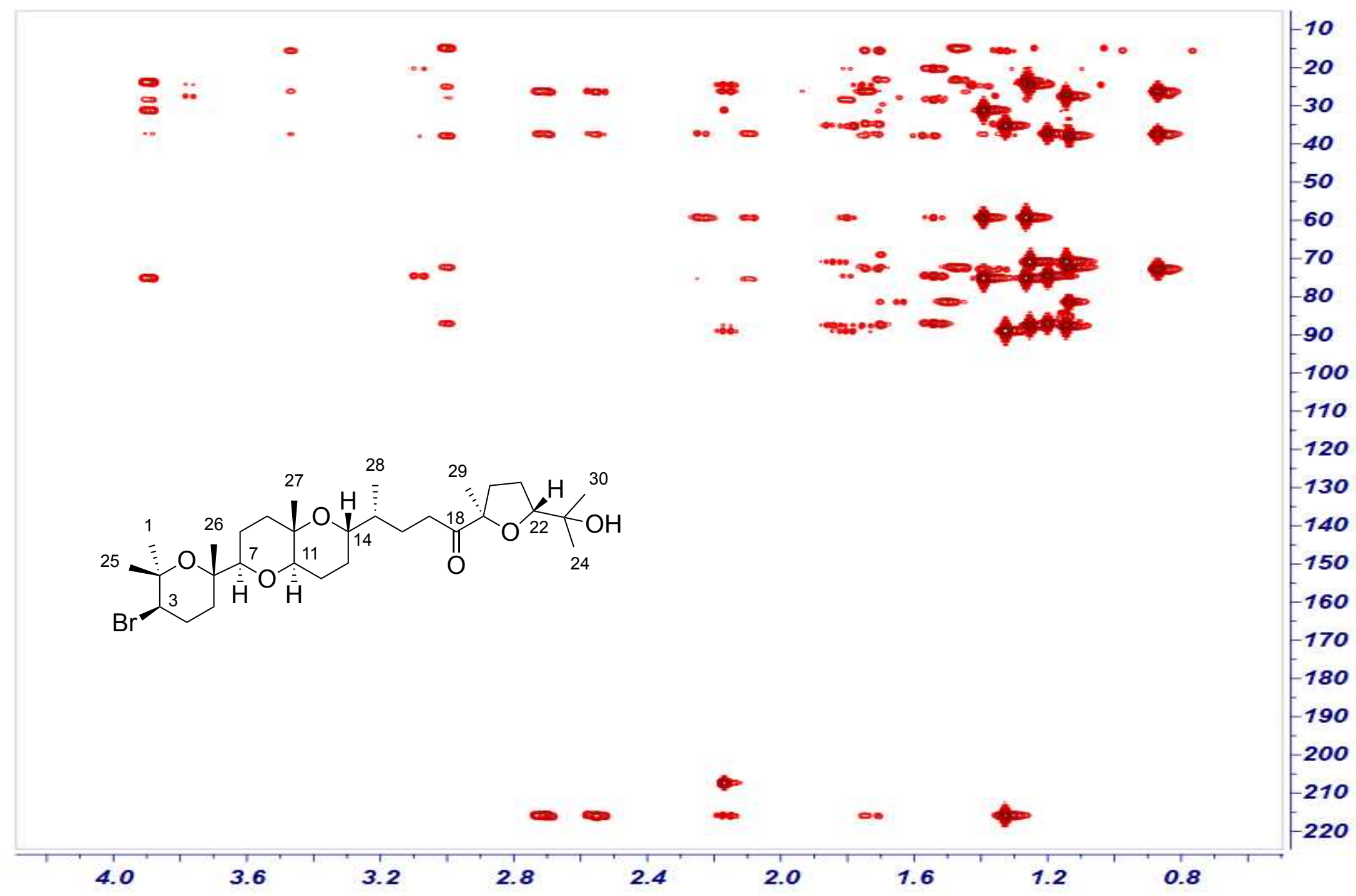


Figure S41. NOESY spectrum of yucatecone (2) in $\mathrm{CDCl}_{3}$ at $298 \mathrm{~K}, 600 \mathrm{MHz}$.

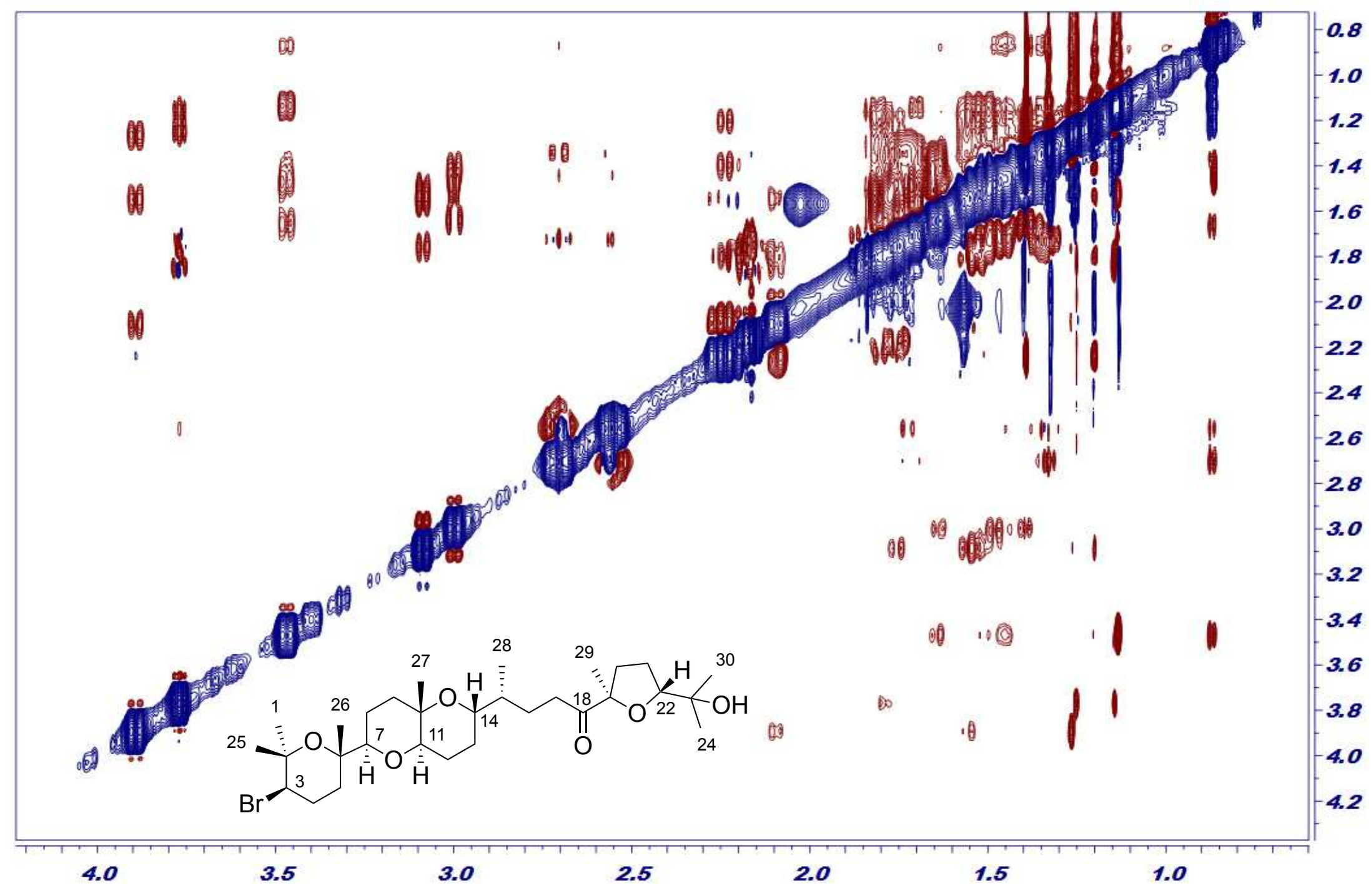


Figure S42. HSQC-HECADE spectrum of yucatecone (2) in $\mathrm{CDCl}_{3}$ at $298 \mathrm{~K}, 600 \mathrm{MHz}$.

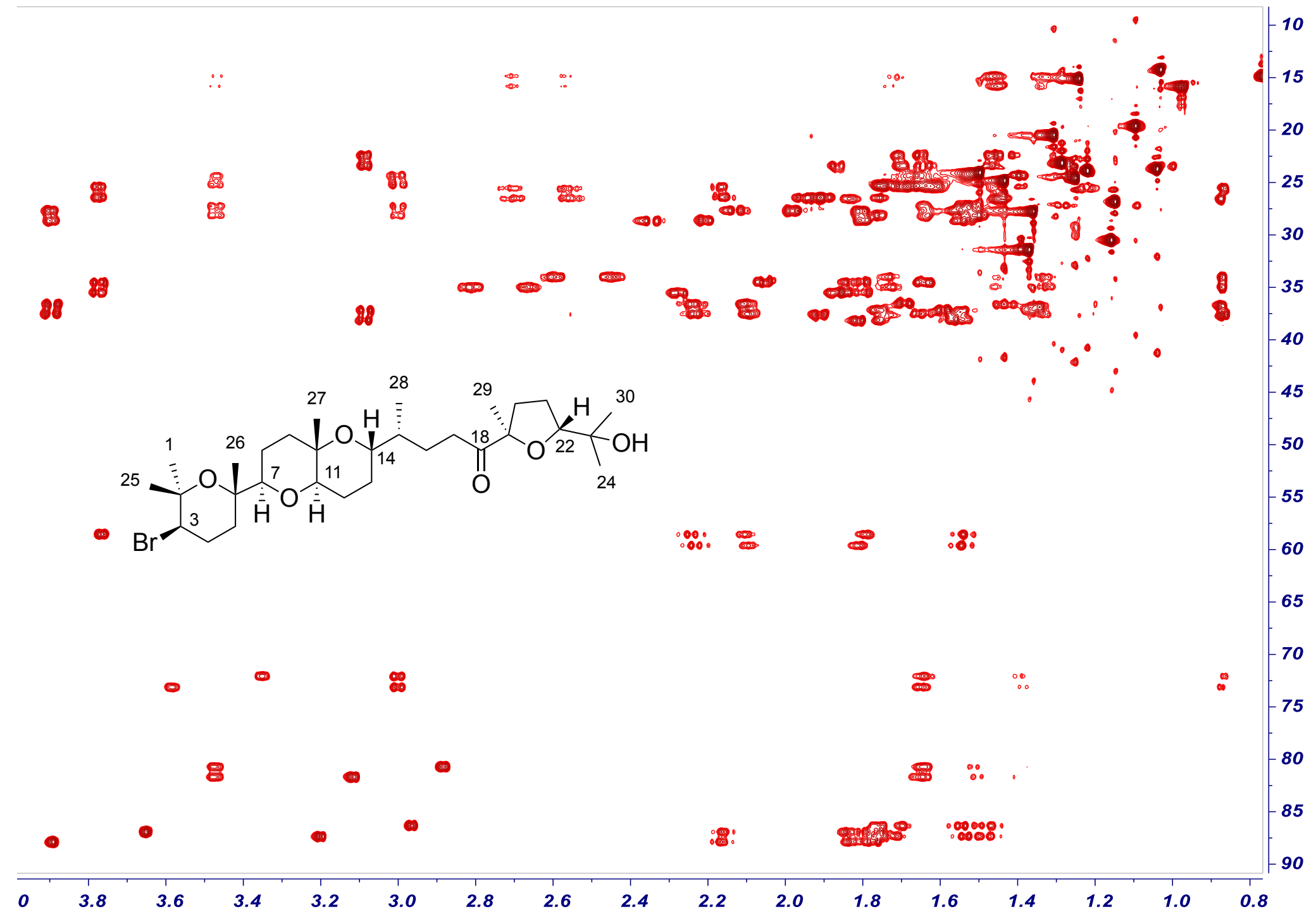


Figure S43. J-HMBC spectrum of yucatecone (2) in $\mathrm{CDCl}_{3}$ at $298 \mathrm{~K}, 600 \mathrm{MHz}$.

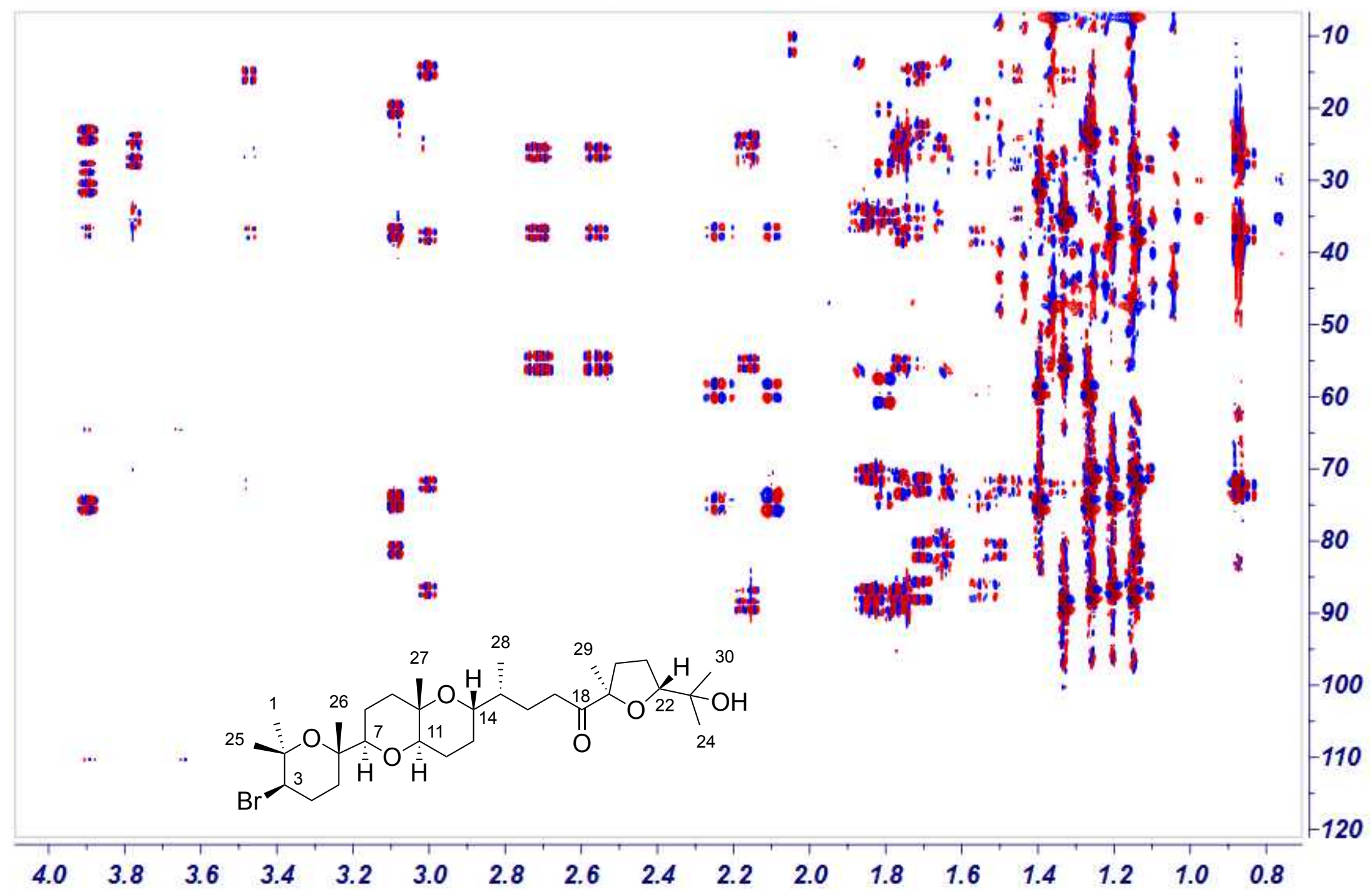


Figure S44. Mass spectrum of yucatecone (2).

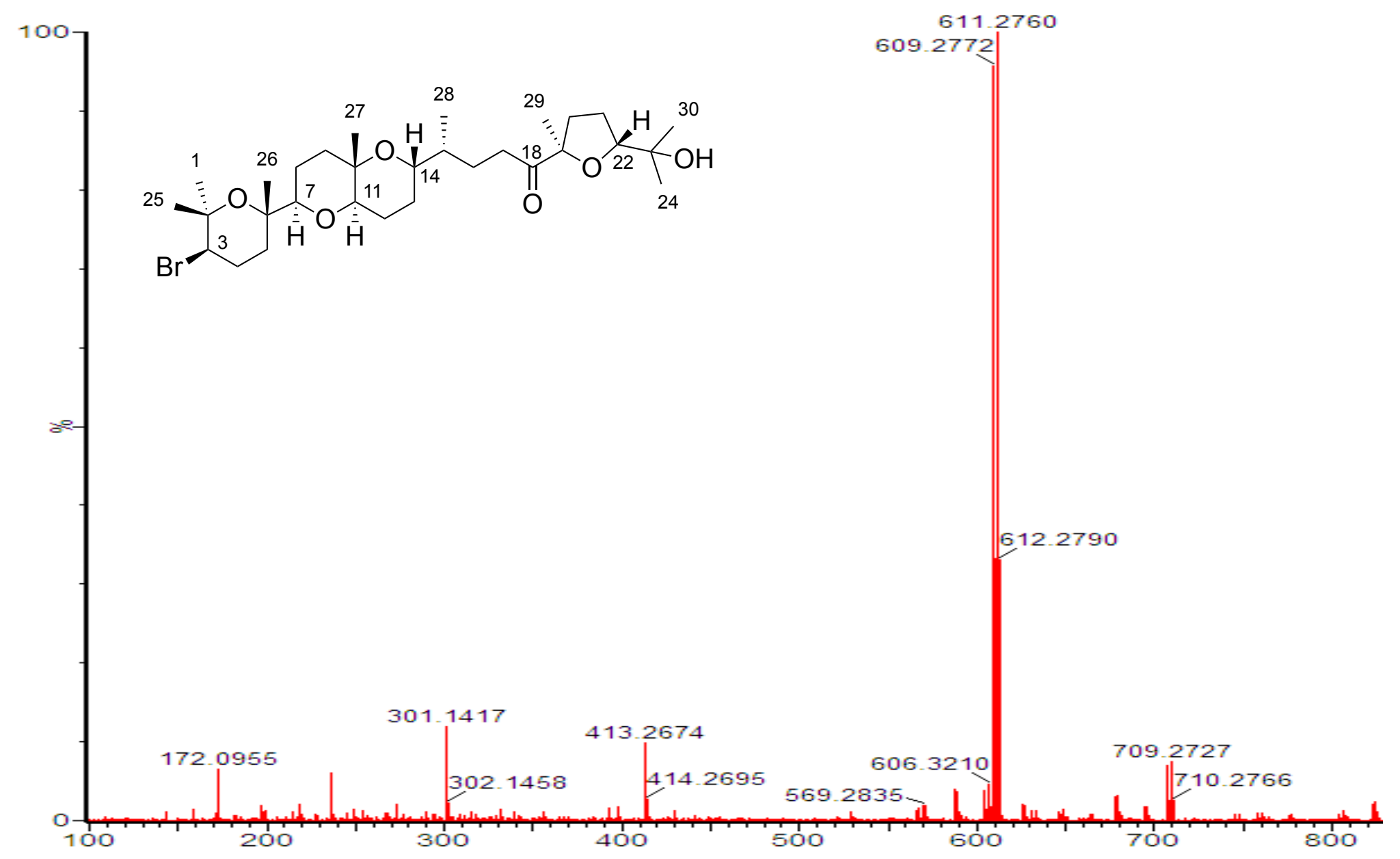




\section{B. Computational study}

Table S7. Scaled ${ }^{13} \mathrm{C}$ average chemical shifts for $\mathbf{1 a}-\mathbf{1} \mathbf{d} / \mathbf{5 a}-\mathbf{5 d}$ on B3LYP-D3 in PCM-CHCl 3.

\begin{tabular}{ccccc}
\hline $\mathbf{C}$ & $\mathbf{1 a / 5 a}(\mathbf{1 4 R}-\mathbf{1 5 R})$ & $\mathbf{1 b} / \mathbf{5 b}(\mathbf{1 4 R}-\mathbf{1 5 S})$ & $\mathbf{1 c / 5 \mathbf { c } ( 1 4 S - 1 5 R )}$ & $\mathbf{1 d} / \mathbf{5 d}(\mathbf{1 4 S}-\mathbf{1 5 S})$ \\
\hline $\mathbf{7}$ & 83.0769077 & 83.1682184 & 81.5374287 & 82.2358117 \\
$\mathbf{8}$ & 24.7582483 & 25.8124601 & 24.9456819 & 25.2108145 \\
$\mathbf{9}$ & 38.2676413 & 38.9423061 & 37.2705321 & 37.6851904 \\
$\mathbf{1 0}$ & 72.4522878 & 72.6026279 & 71.6671695 & 72.0409000 \\
$\mathbf{1 1}$ & 74.9881668 & 74.9675765 & 77.8034757 & 78.3236006 \\
$\mathbf{1 2}$ & 20.6139939 & 21.7708348 & 21.7669635 & 22.6536548 \\
$\mathbf{1 3}$ & 28.2515904 & 30.0490180 & 24.0652581 & 31.2389444 \\
$\mathbf{1 4}$ & 100.9216562 & 100.3146894 & 99.8265230 & 98.7456358 \\
$\mathbf{1 5}$ & 39.6675239 & 39.3748304 & 40.6134364 & 39.1297103 \\
$\mathbf{1 6}$ & 27.5199919 & 27.3348573 & 28.1420643 & 27.4501484 \\
$\mathbf{1 7}$ & 26.0107094 & 21.5491132 & 25.9970188 & 21.4981774 \\
$\mathbf{1 8}$ & 75.4927497 & 76.2312836 & 77.3654102 & 77.0577022 \\
$\mathbf{2 7}$ & 18.9236008 & 19.9299242 & 19.5782466 & 19.8323241 \\
$\mathbf{2 8}$ & 16.6549319 & 15.5522599 & 17.0207912 & 14.4973854 \\
\hline
\end{tabular}

Table S8. Scaled ${ }^{1} \mathrm{H}$ average chemical shifts for $\mathbf{1 a}-\mathbf{1 d} / \mathbf{5 a}-\mathbf{5 d}$ on B3LYP-D3 in PCM-CHCl 3 .

\begin{tabular}{ccccc}
\hline $\mathbf{H}$ & $\mathbf{1 a / 5 a}(14 R-15 R)$ & $\mathbf{1 b} / \mathbf{5 b}(14 R-15 S)$ & $\mathbf{1 c / 5 c}(14 S-15 R)$ & $\mathbf{1 d} / \mathbf{5 d}(14 S-15 S)$ \\
\hline $\mathbf{7}$ & 3.1891618 & 3.2205891 & 3.7126515 & 3.6596601 \\
$\mathbf{8 a}$ & 1.4159544 & 1.3819927 & 1.3869516 & 1.2782809 \\
$\mathbf{8 b}$ & 1.7213176 & 1.6843958 & 1.9134656 & 1.8177093 \\
$\mathbf{9 a}$ & 1.6726301 & 1.6468117 & 1.3961552 & 1.2925017 \\
$\mathbf{9 b}$ & 1.7193144 & 1.6724584 & 1.6638348 & 1.6252363 \\
$\mathbf{1 1}$ & 3.9453926 & 3.9440070 & 3.5329679 & 3.5319236 \\
$\mathbf{1 2 a}$ & 1.9183220 & 1.8507123 & 1.3931828 & 1.2940673 \\
$\mathbf{1 2 b}$ & 1.4201071 & 1.3968056 & 1.9246920 & 1.8599805 \\
$\mathbf{1 3 a}$ & 1.4970335 & 1.6995817 & 1.4585916 & 1.1134853 \\
$\mathbf{1 3 b}$ & 2.1489023 & 1.7898263 & 2.2622235 & 2.6908119 \\
$\mathbf{1 5}$ & 1.3504132 & 1.3987670 & 1.4385698 & 1.3559436 \\
$\mathbf{1 6 a}$ & 1.5933026 & 2.0971242 & 1.1446154 & 1.6306860 \\
$\mathbf{1 6 b}$ & 1.2108464 & 1.1100290 & 1.4102429 & 1.2778329 \\
$\mathbf{1 7 a}$ & 1.4025804 & 1.0908854 & 1.3061379 & 0.8942591 \\
$\mathbf{1 7 b}$ & 1.3530013 & 1.4850921 & 1.4427617 & 1.7334728 \\
$\mathbf{1 8}$ & 3.7140386 & 3.8232329 & 3.9024755 & 3.9511131 \\
$\mathbf{2 7}$ & 1.1567226 & 1.0974119 & 1.3163088 & 1.3194211 \\
$\mathbf{2 8}$ & 0.7909591 & 0.8302768 & 0.6141715 & 0.8936143 \\
\hline
\end{tabular}


Figure S45. Correlations graphs for ${ }^{13} \mathrm{C}$ calculated $v s{ }^{13} \mathrm{C}$ experimental data.
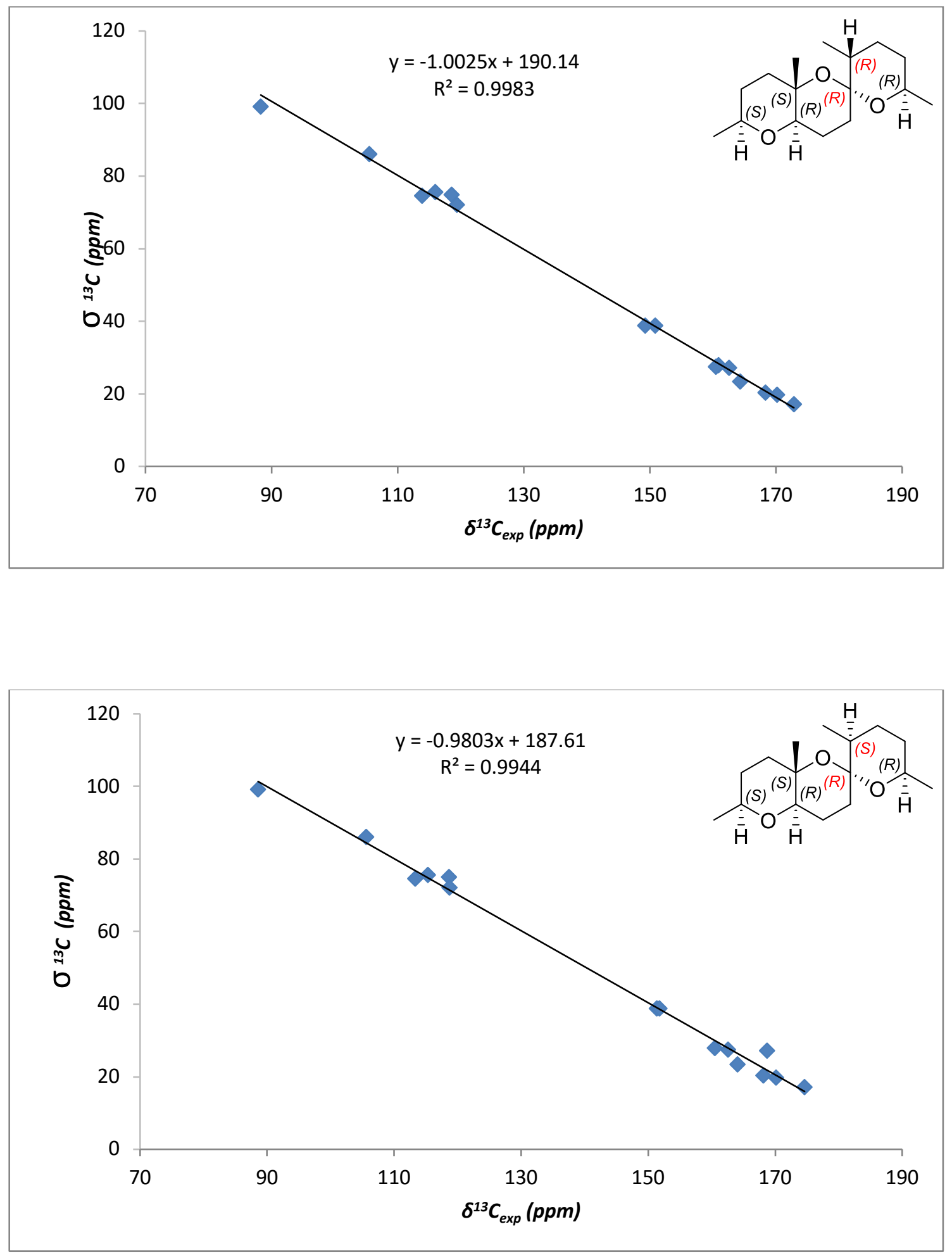

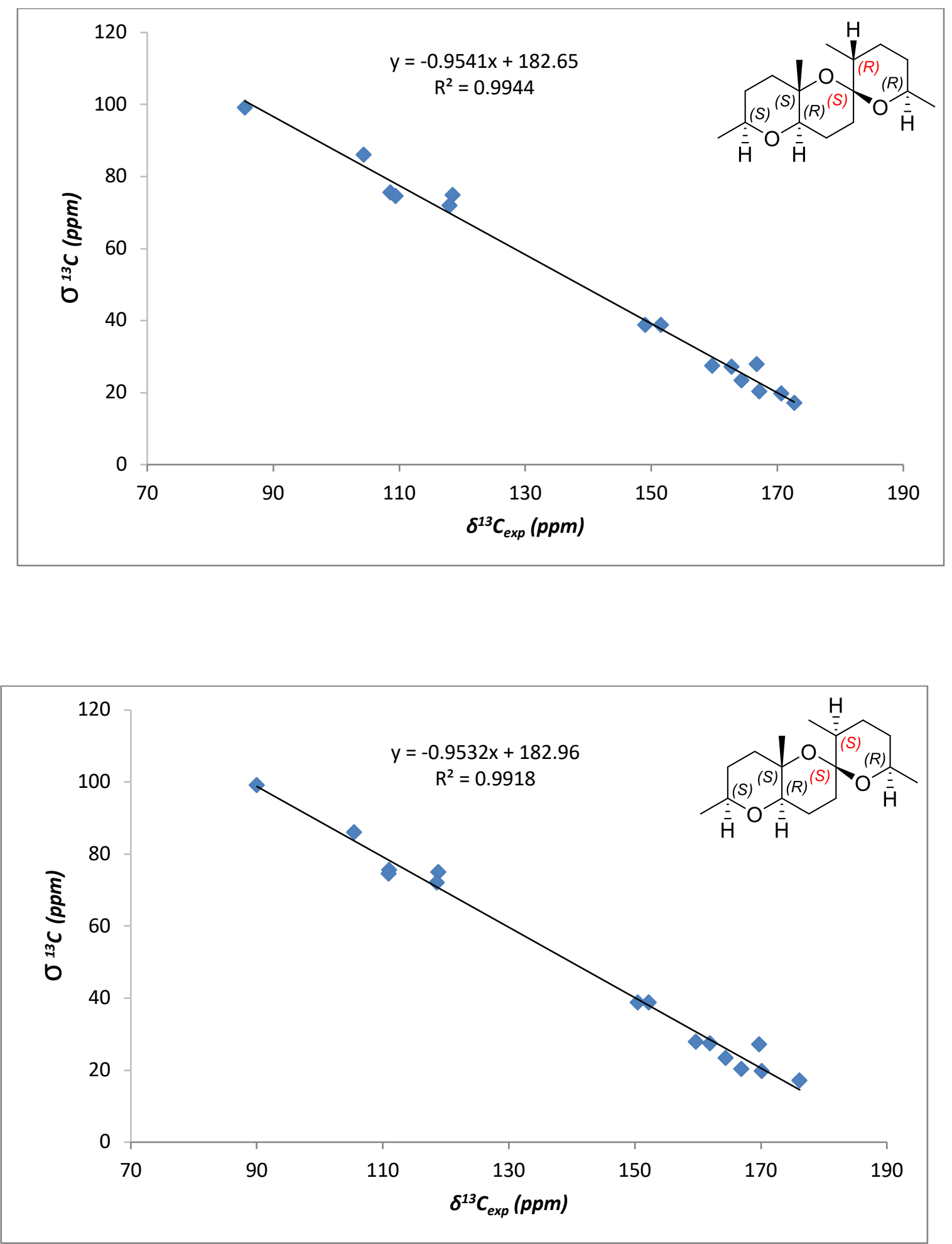
Figure S46. Correlations graphs for ${ }^{1} \mathrm{H}$ calculated $v s^{1} \mathrm{H}$ experimental data.
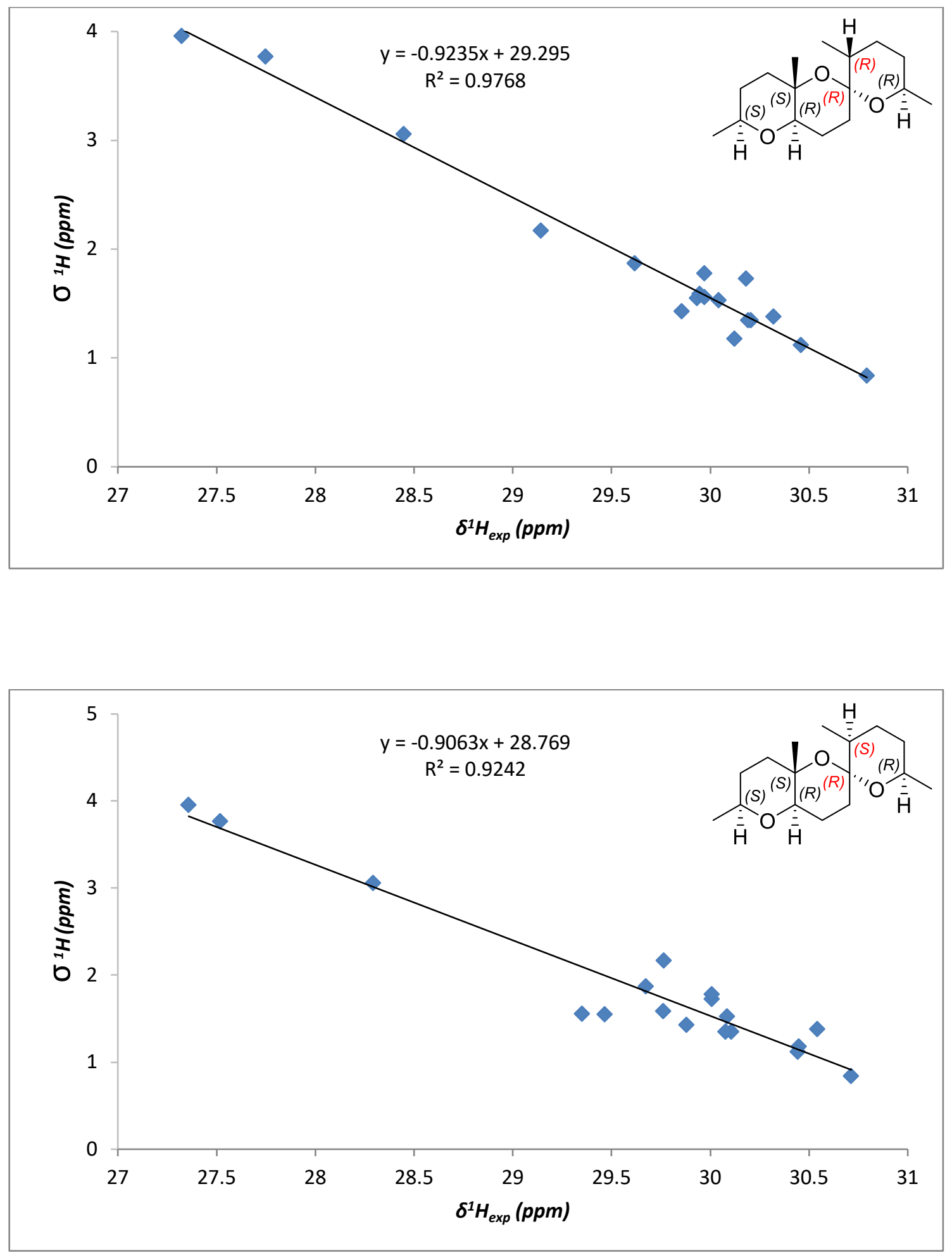

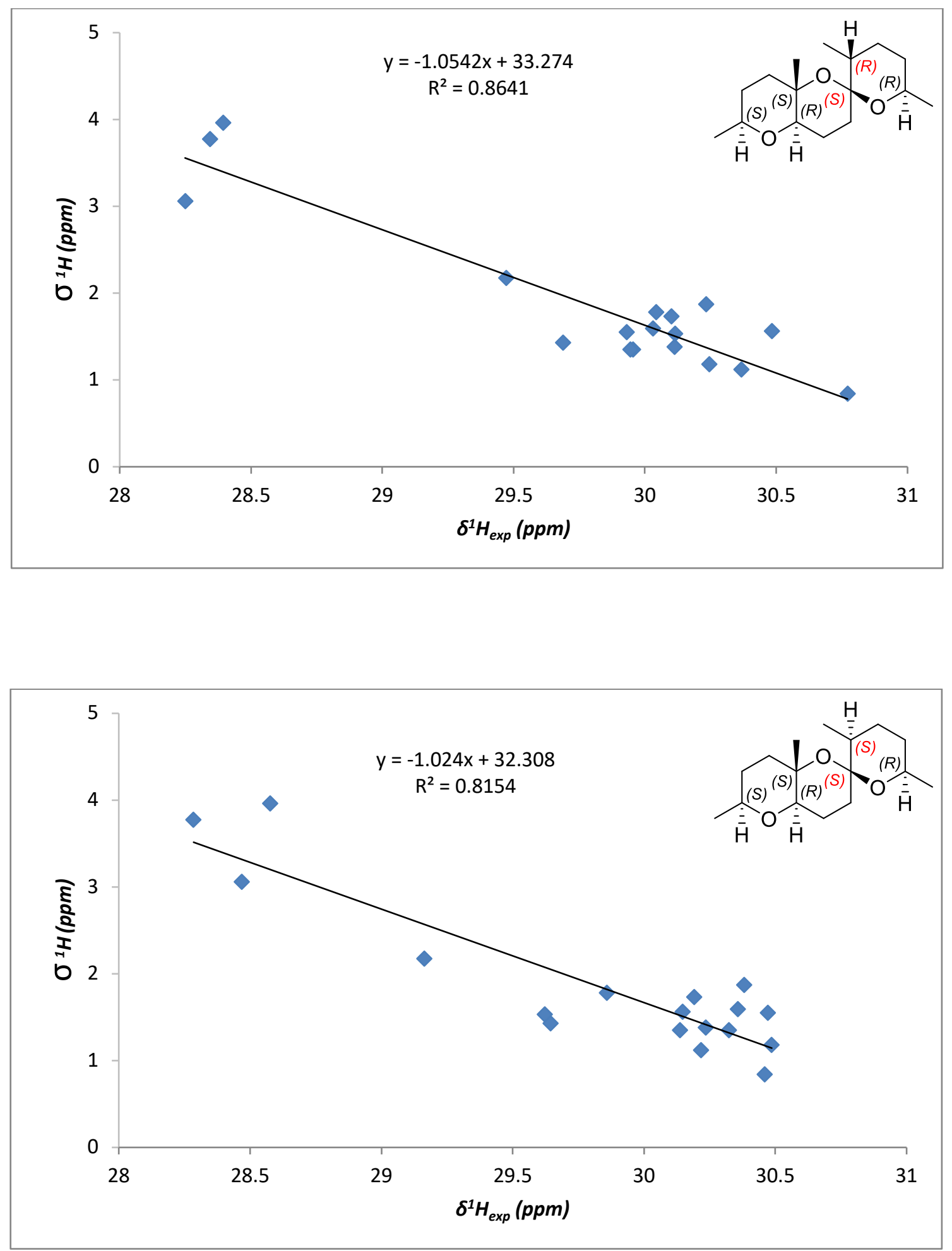


\section{Energies and Coordinates}

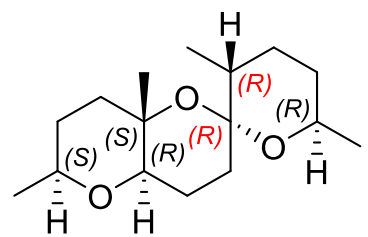

Table S9. Conformer 1a-A/5a-A. PCM-CHCl 3 energy and Boltzmann Populations 1160.103395626 hartrees, $37.37 \%$.

\begin{tabular}{rrrrr}
\hline Atom number & \multicolumn{3}{r}{ Coordinates } & \\
\hline & & $\mathbf{X}$ & $\mathbf{Y}$ & $\mathbf{Z}$ \\
$\mathbf{1}$ & $\mathrm{O} 1$ & -0.529252 & 1.335185 & -1.061805 \\
$\mathbf{2}$ & $\mathrm{C} 2$ & -0.263564 & 2.529078 & -1.803221 \\
$\mathbf{3}$ & $\mathrm{C} 3$ & -1.310797 & 3.616436 & -1.503623 \\
$\mathbf{4}$ & $\mathrm{C} 4$ & -2.681687 & 3.041897 & -1.906654 \\
$\mathbf{5}$ & $\mathrm{C} 5$ & -2.941172 & 1.670069 & -1.278339 \\
$\mathbf{6}$ & $\mathrm{C} 6$ & -1.759810 & 0.704307 & -1.490078 \\
$\mathbf{7}$ & $\mathrm{C} 7$ & 1.147638 & 3.024710 & -1.513421 \\
$\mathbf{8}$ & $\mathrm{C} 8$ & 1.315240 & 4.508134 & -1.857842 \\
$\mathbf{9}$ & $\mathrm{C} 9$ & 0.206333 & 5.045341 & -2.789853 \\
$\mathbf{1 0}$ & $\mathrm{O} 10$ & -1.105814 & 4.819232 & -2.258075 \\
$\mathbf{1 1}$ & $\mathrm{O} 11$ & 0.369493 & 4.350470 & -4.039571 \\
$\mathbf{1 2}$ & $\mathrm{C} 12$ & -0.583337 & 4.720751 & -5.056469 \\
$\mathbf{1 3}$ & $\mathrm{C} 13$ & -0.416356 & 6.207411 & -5.385649 \\
$\mathbf{1 4}$ & $\mathrm{C} 14$ & -0.564931 & 7.057072 & -4.131479 \\
$\mathbf{1 5}$ & $\mathrm{C} 15$ & 0.388160 & 6.569452 & -3.031645 \\
$\mathbf{1 6}$ & $\mathrm{C} 16$ & -1.894992 & -0.627052 & -0.694160 \\
$\mathbf{1 7}$ & $\mathrm{C} 17$ & -0.709542 & -1.558331 & -0.983748 \\
$\mathbf{1 8}$ & $\mathrm{O} 18$ & -1.864550 & -0.332376 & 0.711253 \\
$\mathbf{1 9}$ & $\mathrm{C} 19$ & -3.204616 & -1.360498 & -0.986697 \\
$\mathbf{2 0}$ & $\mathrm{C} 20$ & -1.353484 & 4.015831 & -0.015222 \\
$\mathbf{2 1}$ & $\mathrm{C} 21$ & 0.178797 & 7.425114 & -1.777350 \\
$\mathbf{2 2}$ & $\mathrm{C} 22$ & -0.336561 & 3.791674 & -6.283673 \\
$\mathbf{2 3}$ & $\mathrm{C} 23$ & -0.509069 & 2.315706 & -5.900591 \\
$\mathbf{2 4}$ & $\mathrm{C} 24$ & -1.253334 & 4.119883 & -7.463852 \\
$\mathbf{2 5}$ & $\mathrm{O} 25$ & 1.019480 & 3.950519 & -6.729619 \\
$\mathbf{2 6}$ & $\mathrm{H} 26$ & -1.673030 & 0.468127 & -2.559431 \\
$\mathbf{2 7}$ & $\mathrm{H} 27$ & -0.297205 & 2.269055 & -2.868128 \\
$\mathbf{2 8}$ & $\mathrm{H} 28$ & -1.598128 & 4.535978 & -4.682277 \\
$\mathbf{2 9}$ & $\mathrm{H} 29$ & -2.726865 & 2.949421 & -3.000290 \\
$\mathbf{3 0}$ & $\mathrm{H} 30$ & -3.487888 & 3.737873 & -1.643123 \\
$\mathbf{3 1}$ & $\mathrm{H} 31$ & -3.857296 & 1.252802 & -1.710361 \\
$\mathbf{3 2}$ & $\mathrm{H} 32$ & -3.127715 & 1.804729 & -0.206764 \\
$\mathbf{3 3}$ & $\mathrm{H} 33$ & 1.859214 & 2.423002 & -2.093197 \\
$\mathbf{3 4}$ & $\mathrm{H} 34$ & 1.418365 & 2.852458 & -0.464162 \\
$\mathbf{3 5}$ & $\mathrm{H} 35$ & 2.302134 & 4.650945 & -2.315502 \\
$\mathbf{3 6}$ & $\mathrm{H} 36$ & 1.324689 & 5.063797 & -0.915207 \\
$\mathbf{3 7}$ & $\mathrm{H} 37$ & -1.156845 & 6.532936 & -6.123177 \\
$\mathbf{3 8}$ & $\mathrm{H} 38$ & 0.578153 & 6.389942 & -5.810826 \\
$\mathbf{3 9}$ & $\mathrm{H} 39$ & -1.604388 & 7.014256 & -3.782220 \\
$\mathbf{4 0}$ & $\mathrm{H} 40$ & -0.355927 & 8.105345 & -4.376585 \\
$\mathbf{4 1}$ & $\mathrm{H} 41$ & 1.411309 & 6.740253 & -3.394913 \\
$\mathbf{4 2}$ & $\mathrm{H} 42$ & -0.658940 & -1.823661 & -2.044719 \\
$\mathbf{4 3}$ & $\mathrm{H} 43$ & -1.098887 & -0.693714 \\
$\mathbf{4 4}$ & $\mathrm{H} 44$ & -2.482041 & -0.398821
\end{tabular}




\begin{tabular}{llrrr}
$\mathbf{4 5}$ & $\mathrm{H} 45$ & -1.107752 & 0.276481 & 0.826814 \\
$\mathbf{4 6}$ & $\mathrm{H} 46$ & -3.242042 & -2.316700 & -0.451606 \\
$\mathbf{4 7}$ & $\mathrm{H} 47$ & -4.071095 & -0.790909 & -0.634695 \\
$\mathbf{4 8}$ & $\mathrm{H} 48$ & -3.322767 & -1.560122 & -2.056480 \\
$\mathbf{4 9}$ & $\mathrm{H} 49$ & -1.471473 & 3.154883 & 0.649162 \\
$\mathbf{5 0}$ & $\mathrm{H} 50$ & -0.451487 & 4.551166 & 0.292870 \\
$\mathbf{5 1}$ & $\mathrm{H} 51$ & -2.183362 & 4.708067 & 0.170615 \\
$\mathbf{5 2}$ & $\mathrm{H} 52$ & 0.274731 & 8.489746 & -2.017942 \\
$\mathbf{5 3}$ & $\mathrm{H} 53$ & -0.814485 & 7.270456 & -1.343480 \\
$\mathbf{5 4}$ & $\mathrm{H} 54$ & 0.925199 & 7.203842 & -1.009668 \\
$\mathbf{5 5}$ & $\mathrm{H} 55$ & -0.352432 & 1.667076 & -6.770432 \\
$\mathbf{5 6}$ & $\mathrm{H} 56$ & 0.235990 & 2.005084 & -5.160538 \\
$\mathbf{5 7}$ & $\mathrm{H} 57$ & -1.508213 & 2.119041 & -5.498659 \\
$\mathbf{5 8}$ & $\mathrm{H} 58$ & -1.097498 & 3.411269 & -8.285893 \\
$\mathbf{5 9}$ & $\mathrm{H} 59$ & -2.308492 & 4.083991 & -7.174659 \\
$\mathbf{6 0}$ & $\mathrm{H} 60$ & -1.033158 & 5.107585 & -7.881989 \\
$\mathbf{6 1}$ & $\mathrm{H} 61$ & 1.563427 & 3.915806 & -5.918265 \\
\hline
\end{tabular}


Table S10. Conformer 1a-B/5a-B. PCM-CHCl 3 energy and Boltzmann Populations 1160.10365751868 hartrees, $25.86 \%$.

\begin{tabular}{|c|c|c|c|c|}
\hline \multicolumn{2}{|c|}{ Atom number } & \multicolumn{3}{|c|}{ Coordinates } \\
\hline & & $\mathbf{X}$ & $\mathbf{Y}$ & $\mathbf{Z}$ \\
\hline 1 & 01 & -0.618024 & 1.436171 & -0.933677 \\
\hline 2 & $\mathrm{C} 2$ & -0.331208 & 2.607700 & -1.703618 \\
\hline 3 & C3 & -1.378801 & 3.710695 & -1.464527 \\
\hline 4 & C4 & -2.746324 & 3.134191 & -1.880117 \\
\hline 5 & C5 & -3.035453 & 1.790773 & -1.205075 \\
\hline 6 & C6 & -1.857308 & 0.815780 & -1.353527 \\
\hline 7 & C7 & 1.076075 & 3.105487 & -1.398025 \\
\hline 8 & C8 & 1.260907 & 4.575167 & -1.789933 \\
\hline 9 & C9 & 0.176514 & 5.085202 & -2.764933 \\
\hline 10 & 010 & -1.148232 & 4.886668 & -2.253387 \\
\hline 11 & 011 & 0.362138 & 4.344691 & -3.984874 \\
\hline 12 & C12 & -0.567033 & 4.682077 & -5.034360 \\
\hline 13 & C13 & -0.383282 & 6.154600 & -5.414256 \\
\hline 14 & C14 & -0.552404 & 7.050836 & -4.195423 \\
\hline 15 & C15 & 0.373561 & 6.598326 & -3.057952 \\
\hline 16 & C16 & -2.007438 & -0.510580 & -0.555465 \\
\hline 17 & C17 & -3.209449 & -1.331071 & -1.023417 \\
\hline 18 & 018 & -0.831909 & -1.301069 & -0.819279 \\
\hline 19 & C19 & -2.064795 & -0.303905 & 0.961893 \\
\hline 20 & C20 & -1.451102 & 4.159742 & 0.008537 \\
\hline 21 & C21 & 0.142843 & 7.500361 & -1.840487 \\
\hline 22 & $\mathrm{C} 22$ & -0.301771 & 3.706453 & -6.221025 \\
\hline 23 & $\mathrm{C} 23$ & -0.487633 & 2.246415 & -5.786233 \\
\hline 24 & C24 & -1.195638 & 3.993832 & -7.429101 \\
\hline 25 & $\mathrm{O} 25$ & 1.062691 & 3.843557 & -6.647974 \\
\hline 26 & $\mathrm{H} 26$ & -1.735145 & 0.555617 & -2.414513 \\
\hline 27 & $\mathrm{H} 27$ & -0.342123 & 2.314622 & -2.760271 \\
\hline 28 & $\mathrm{H} 28$ & -1.590509 & 4.517343 & -4.674760 \\
\hline 29 & $\mathrm{H} 29$ & -2.765542 & 2.999950 & -2.970115 \\
\hline 30 & $\mathrm{H} 30$ & -3.550967 & 3.847955 & -1.663583 \\
\hline 31 & H31 & -3.945240 & 1.360511 & -1.637356 \\
\hline 32 & H32 & -3.244445 & 1.977333 & -0.146792 \\
\hline 33 & H33 & 1.797384 & 2.479941 & -1.939255 \\
\hline 34 & H34 & 1.320706 & 2.968678 & -0.337193 \\
\hline 35 & H35 & 2.258676 & 4.696326 & -2.229864 \\
\hline 36 & H36 & 1.252588 & 5.163525 & -0.867340 \\
\hline 37 & $\mathrm{H} 37$ & -1.105790 & 6.457149 & -6.178885 \\
\hline 38 & H38 & 0.621170 & 6.315474 & -5.824498 \\
\hline 39 & H39 & -1.599329 & 7.027487 & -3.867285 \\
\hline 40 & $\mathrm{H} 40$ & -0.330911 & 8.088035 & -4.474319 \\
\hline 41 & $\mathrm{H} 41$ & 1.405408 & 6.749825 & -3.404826 \\
\hline 42 & $\mathrm{H} 42$ & -3.163186 & -1.506271 & -2.104212 \\
\hline 43 & $\mathrm{H} 43$ & -3.211955 & -2.320576 & -0.551539 \\
\hline 44 & $\mathrm{H} 44$ & -4.158943 & -0.839855 & -0.790330 \\
\hline 45 & $\mathrm{H} 45$ & -0.081132 & -0.682886 & -0.710104 \\
\hline 46 & $\mathrm{H} 46$ & -2.970567 & 0.225251 & 1.271840 \\
\hline 47 & $\mathrm{H} 47$ & -2.041735 & -1.268636 & 1.482460 \\
\hline 48 & $\mathrm{H} 48$ & -1.194104 & 0.255360 & 1.321346 \\
\hline 49 & $\mathrm{H} 49$ & -1.584679 & 3.321634 & 0.698685 \\
\hline 50 & $\mathrm{H} 50$ & -0.554271 & 4.703471 & 0.317090 \\
\hline 51 & H51 & -2.282849 & 4.859302 & 0.154095 \\
\hline
\end{tabular}




\begin{tabular}{llrll}
$\mathbf{5 2}$ & H52 & -0.860510 & 7.367227 & -1.422947 \\
$\mathbf{5 3}$ & H53 & 0.871162 & 7.302902 & -1.049295 \\
$\mathbf{5 4}$ & H54 & 0.250763 & 8.554947 & -2.117492 \\
$\mathbf{5 5}$ & H55 & -0.320162 & 1.565242 & -6.628733 \\
$\mathbf{5 6}$ & H56 & 0.243987 & 1.960351 & -5.023230 \\
$\mathbf{5 7}$ & H57 & -1.494089 & 2.069066 & -5.393692 \\
$\mathbf{5 8}$ & H58 & -1.027532 & 3.254953 & -8.221547 \\
$\mathbf{5 9}$ & H59 & -2.256024 & 3.971840 & -7.158272 \\
$\mathbf{6 0}$ & H60 & -0.964248 & 4.964770 & -7.879148 \\
$\mathbf{6 1}$ & H61 & 1.592152 & 3.833088 & -5.826497 \\
\hline
\end{tabular}


Table S11. Conformer 1a-C/5a-C. PCM- $\mathrm{CHCl}_{3}$ energy and Boltzmann Populations 1160.10379625597 hartrees, $22.62 \%$.

\begin{tabular}{|c|c|c|c|c|}
\hline \multicolumn{2}{|c|}{ Atom number } & \multicolumn{3}{|c|}{ Coordinates } \\
\hline & & $X$ & $\mathbf{X}$ & $\mathbf{Z}$ \\
\hline 1 & O1 & -0.477623 & 1.388822 & -1.105509 \\
\hline 2 & $\mathrm{C} 2$ & -0.213447 & 2.597099 & -1.823463 \\
\hline 3 & C3 & -1.287748 & 3.662100 & -1.540377 \\
\hline 4 & $\mathrm{C} 4$ & -2.637325 & 3.069561 & -1.986290 \\
\hline 5 & C5 & -2.888207 & 1.684532 & -1.383871 \\
\hline 6 & C6 & -1.684620 & 0.743375 & -1.579562 \\
\hline 7 & $\mathrm{C} 7$ & 1.180033 & 3.115976 & -1.489608 \\
\hline 8 & C8 & 1.331254 & 4.604521 & -1.822477 \\
\hline 9 & C9 & 0.236303 & 5.126474 & -2.779130 \\
\hline 10 & 010 & -1.082364 & 4.878145 & -2.273269 \\
\hline 11 & 011 & 0.439189 & 4.436126 & -4.026816 \\
\hline 12 & C12 & -0.490759 & 4.806089 & -5.065758 \\
\hline 13 & C13 & -0.359164 & 6.295118 & -5.384945 \\
\hline 14 & C14 & -0.553833 & 7.129706 & -4.126587 \\
\hline 15 & C15 & 0.393062 & 6.655270 & -3.013565 \\
\hline 16 & C16 & -1.816631 & -0.604140 & -0.811243 \\
\hline 17 & C17 & -0.609398 & -1.510261 & -1.090624 \\
\hline 18 & 018 & -1.822869 & -0.334906 & 0.599722 \\
\hline 19 & C19 & -3.106834 & -1.353278 & -1.146868 \\
\hline 20 & $\mathrm{C} 20$ & -1.376283 & 4.042469 & -0.048981 \\
\hline 21 & $\mathrm{C} 21$ & 0.148400 & 7.502167 & -1.759700 \\
\hline 22 & $\mathrm{C} 22$ & -0.233750 & 3.870747 & -6.283999 \\
\hline 23 & $\mathrm{C} 23$ & -1.287302 & 4.050121 & -7.377969 \\
\hline 24 & $\mathrm{C} 24$ & 1.172488 & 4.014371 & -6.877112 \\
\hline 25 & $\mathrm{O} 25$ & -0.347079 & 2.512308 & -5.817073 \\
\hline 26 & $\mathrm{H} 26$ & -1.564369 & 0.527748 & -2.650005 \\
\hline 27 & $\mathrm{H} 27$ & -0.212793 & 2.348462 & -2.891595 \\
\hline 28 & $\mathrm{H} 28$ & -1.501712 & 4.593388 & -4.694993 \\
\hline 29 & $\mathrm{H} 29$ & -2.652747 & 2.991604 & -3.081860 \\
\hline 30 & $\mathrm{H} 30$ & -3.462285 & 3.747578 & -1.734281 \\
\hline 31 & H31 & -3.785600 & 1.257815 & -1.844922 \\
\hline 32 & H32 & -3.103514 & 1.800155 & -0.315472 \\
\hline 33 & $\mathrm{H} 33$ & 1.919036 & 2.529856 & -2.050701 \\
\hline 34 & H34 & 1.422429 & 2.943012 & -0.433579 \\
\hline 35 & H35 & 2.326414 & 4.766639 & -2.254968 \\
\hline 36 & H36 & 1.306967 & 5.155198 & -0.877131 \\
\hline 37 & $\mathrm{H} 37$ & -1.088914 & 6.602208 & -6.140776 \\
\hline 38 & H38 & 0.639713 & 6.513076 & -5.779771 \\
\hline 39 & H39 & -1.597946 & 7.057723 & -3.796745 \\
\hline 40 & $\mathrm{H} 40$ & -0.366653 & 8.184973 & -4.358882 \\
\hline 41 & $\mathrm{H} 41$ & 1.418334 & 6.846481 & -3.360245 \\
\hline 42 & $\mathrm{H} 42$ & -0.686445 & -2.445664 & -0.524219 \\
\hline 43 & $\mathrm{H} 43$ & -0.530661 & -1.755453 & -2.154714 \\
\hline 44 & $\mathrm{H} 44$ & 0.327368 & -1.040502 & -0.771005 \\
\hline 45 & $\mathrm{H} 45$ & -1.082358 & 0.288176 & 0.742125 \\
\hline 46 & $\mathrm{H} 46$ & -3.139952 & -2.320033 & -0.630788 \\
\hline 47 & $\mathrm{H} 47$ & -3.990323 & -0.804795 & -0.803656 \\
\hline 48 & $\mathrm{H} 48$ & -3.197984 & -1.534292 & -2.222597 \\
\hline 49 & $\mathrm{H} 49$ & -2.222754 & 4.717934 & 0.123230 \\
\hline 50 & H50 & -1.496249 & 3.171563 & 0.601944 \\
\hline 51 & H51 & -0.491926 & 4.589679 & 0.288416 \\
\hline
\end{tabular}




\begin{tabular}{llrll}
$\mathbf{5 2}$ & $\mathrm{H} 52$ & -0.847873 & 7.326442 & -1.341005 \\
$\mathbf{5 3}$ & $\mathrm{H} 53$ & 0.887881 & 7.292860 & -0.981996 \\
$\mathbf{5 4}$ & $\mathrm{H} 54$ & 0.226867 & 8.569359 & -1.995280 \\
$\mathbf{5 5}$ & $\mathrm{H} 55$ & -1.173068 & 3.286360 & -8.156111 \\
$\mathbf{5 6}$ & $\mathrm{H} 56$ & -2.296510 & 3.923796 & -6.969893 \\
$\mathbf{5 7}$ & $\mathrm{H} 57$ & -1.224598 & 5.033010 & -7.854294 \\
$\mathbf{5 8}$ & $\mathrm{H} 58$ & 1.349936 & 3.244664 & -7.637576 \\
$\mathbf{5 9}$ & $\mathrm{H} 59$ & 1.324516 & 4.991752 & -7.344403 \\
$\mathbf{6 0}$ & $\mathrm{H} 60$ & 1.943430 & 3.869719 & -6.112326 \\
$\mathbf{6 1}$ & $\mathrm{H} 61$ & 0.254814 & 2.461613 & -5.049885 \\
\hline
\end{tabular}


Table S12. Conformer 1a-D/5a-D. PCM- $\mathrm{CHCl}_{3}$ energy and Boltzmann Populations 1160.10382129745 hartrees, $12.94 \%$.

\begin{tabular}{|c|c|c|c|c|}
\hline \multicolumn{2}{|c|}{ Atom number } & \multicolumn{3}{|c|}{ Coordinates } \\
\hline & & $X$ & $\mathbf{Y}$ & $\mathbf{Z}$ \\
\hline 1 & 01 & -0.573355 & 1.480281 & -0.978867 \\
\hline 2 & $\mathrm{C} 2$ & -0.286826 & 2.663825 & -1.730256 \\
\hline 3 & C3 & -1.360725 & 3.745230 & -1.511255 \\
\hline 4 & C4 & -2.704342 & 3.148875 & -1.973527 \\
\hline 5 & C5 & -2.988579 & 1.793039 & -1.321632 \\
\hline 6 & C6 & -1.788276 & 0.841044 & -1.441521 \\
\hline 7 & $\mathrm{C} 7$ & 1.102104 & 3.184715 & -1.381096 \\
\hline 8 & C8 & 1.271916 & 4.660374 & -1.758088 \\
\hline 9 & C9 & 0.204905 & 5.158390 & -2.757990 \\
\hline 10 & 010 & -1.127745 & 4.935126 & -2.278036 \\
\hline 11 & 011 & 0.433038 & 4.427278 & -3.977607 \\
\hline 12 & C12 & -0.465715 & 4.772495 & -5.051939 \\
\hline 13 & C13 & -0.312730 & 6.249858 & -5.413222 \\
\hline 14 & C14 & -0.534338 & 7.124348 & -4.187051 \\
\hline 15 & C15 & 0.379485 & 6.678129 & -3.035633 \\
\hline 16 & C16 & -1.938262 & -0.493993 & -0.657694 \\
\hline 17 & C17 & -3.105813 & -1.335695 & -1.172770 \\
\hline 18 & 018 & -0.737743 & -1.258314 & -0.883983 \\
\hline 19 & C19 & -2.053013 & -0.299441 & 0.858072 \\
\hline 20 & $\mathrm{C} 20$ & -1.483877 & 4.175942 & -0.036055 \\
\hline 21 & $\mathrm{C} 21$ & 0.109170 & 7.565659 & -1.815550 \\
\hline 22 & $\mathrm{C} 22$ & -0.185117 & 3.798721 & -6.234342 \\
\hline 23 & $\mathrm{C} 23$ & -1.201721 & 3.960418 & -7.365516 \\
\hline 24 & $\mathrm{C} 24$ & 1.240638 & 3.905899 & -6.787179 \\
\hline 25 & $\mathrm{O} 25$ & -0.333111 & 2.456215 & -5.733281 \\
\hline 26 & $\mathrm{H} 26$ & -1.628759 & 0.591510 & -2.500035 \\
\hline 27 & $\mathrm{H} 27$ & -0.262344 & 2.379145 & -2.789153 \\
\hline 28 & $\mathrm{H} 28$ & -1.487992 & 4.579435 & -4.701945 \\
\hline 29 & $\mathrm{H} 29$ & -2.688499 & 3.026218 & -3.064970 \\
\hline 30 & $\mathrm{H} 30$ & -3.528054 & 3.845587 & -1.773791 \\
\hline 31 & H31 & -3.876109 & 1.350232 & -1.786326 \\
\hline 32 & H32 & -3.234325 & 1.965122 & -0.268807 \\
\hline 33 & H33 & 1.849820 & 2.575885 & -1.905269 \\
\hline 34 & H34 & 1.318645 & 3.044797 & -0.314589 \\
\hline 35 & H35 & 2.278624 & 4.802129 & -2.170566 \\
\hline 36 & H36 & 1.228281 & 5.241398 & -0.831797 \\
\hline 37 & H37 & -1.019323 & 6.539232 & -6.197490 \\
\hline 38 & H38 & 0.698208 & 6.447660 & -5.787427 \\
\hline 39 & H39 & -1.587250 & 7.069628 & -3.882862 \\
\hline 40 & $\mathrm{H} 40$ & -0.333144 & 8.170615 & -4.446760 \\
\hline 41 & H41 & 1.414842 & 6.851262 & -3.361216 \\
\hline 42 & $\mathrm{H} 42$ & -3.104957 & -2.327931 & -0.706695 \\
\hline 43 & $\mathrm{H} 43$ & -4.072822 & -0.865149 & -0.971203 \\
\hline 44 & $\mathrm{H} 44$ & -3.017463 & -1.503271 & -2.252139 \\
\hline 45 & $\mathrm{H} 45$ & -0.004987 & -0.623448 & -0.750883 \\
\hline 46 & $\mathrm{H} 46$ & -2.025002 & -1.267044 & 1.373058 \\
\hline 47 & $\mathrm{H} 47$ & -1.208817 & 0.277660 & 1.250951 \\
\hline 48 & $\mathrm{H} 48$ & -2.981326 & 0.205877 & 1.139842 \\
\hline 49 & $\mathrm{H} 49$ & -1.623297 & 3.328012 & 0.640805 \\
\hline 50 & H50 & -0.605486 & 4.730941 & 0.304196 \\
\hline 51 & H51 & -2.331250 & 4.859897 & 0.092579 \\
\hline
\end{tabular}




\begin{tabular}{llrll}
$\mathbf{5 2}$ & $\mathrm{H} 52$ & 0.201895 & 8.624374 & -2.082196 \\
$\mathbf{5 3}$ & $\mathrm{H} 53$ & -0.898855 & 7.410007 & -1.417570 \\
$\mathbf{5 4}$ & $\mathrm{H} 54$ & 0.826796 & 7.375913 & -1.012764 \\
$\mathbf{5 5}$ & $\mathrm{H} 55$ & -1.076330 & 3.171202 & -8.116069 \\
$\mathbf{5 6}$ & $\mathrm{H} 56$ & -2.224842 & 3.862468 & -6.985241 \\
$\mathbf{5 7}$ & $\mathrm{H} 57$ & -1.108347 & 4.926888 & -7.869572 \\
$\mathbf{5 8}$ & $\mathrm{H} 58$ & 1.429338 & 3.113579 & -7.521236 \\
$\mathbf{5 9}$ & $\mathrm{H} 59$ & 1.421911 & 4.868095 & -7.275054 \\
$\mathbf{6 0}$ & $\mathrm{H} 60$ & 1.985612 & 3.770420 & -5.995467 \\
$\mathbf{6 1}$ & $\mathrm{H} 61$ & 0.242965 & 2.417331 & -4.945933 \\
\hline
\end{tabular}




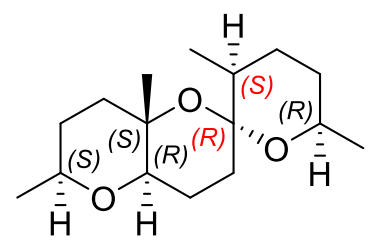

Table S13. Conformer 1b-A/5b-A. PCM- $\mathrm{CHCl}_{3}$ energy and Boltzmann Populations 1160.10215148922 hartrees, $36.68 \%$.

\begin{tabular}{rrrrr}
\hline Atom number & \multicolumn{3}{r}{ Coordinates } & \\
\hline & & $\mathbf{X}$ & $\mathbf{Y}$ & $\mathbf{Z}$ \\
$\mathbf{1}$ & $\mathrm{O} 1$ & 0.378821 & 0.197000 & 7.984911 \\
$\mathbf{2}$ & $\mathrm{C} 2$ & 0.825744 & 0.245440 & 6.627481 \\
$\mathbf{3}$ & $\mathrm{C} 3$ & 0.238880 & -0.911772 & 5.803201 \\
$\mathbf{4}$ & $\mathrm{C} 4$ & 0.704021 & -2.221196 & 6.466454 \\
$\mathbf{5}$ & $\mathrm{C} 5$ & 0.406209 & -2.246207 & 7.967943 \\
$\mathbf{6}$ & $\mathrm{C} 6$ & 0.896197 & -0.967564 & 8.673077 \\
$\mathbf{7}$ & $\mathrm{C} 7$ & 0.470387 & 1.587912 & 6.004518 \\
$\mathbf{8}$ & $\mathrm{C} 8$ & 0.514926 & 1.538442 & 4.473588 \\
$\mathbf{9}$ & $\mathrm{C} 9$ & 1.247518 & 0.297798 & 3.916578 \\
$\mathbf{1 0}$ & $\mathrm{O} 10$ & 0.696677 & -0.921277 & 4.444713 \\
$\mathbf{1 1}$ & $\mathrm{O} 11$ & 2.632018 & 0.429542 & 4.277655 \\
$\mathbf{1 2}$ & $\mathrm{C} 12$ & 3.458256 & -0.677937 & 3.857618 \\
$\mathbf{1 3}$ & $\mathrm{C} 13$ & 3.416429 & -0.848452 & 2.336612 \\
$\mathbf{1 4}$ & $\mathrm{C} 14$ & 1.978347 & -0.949274 & 1.839782 \\
$\mathbf{1 5}$ & $\mathrm{C} 15$ & 1.118647 & 0.211062 & 2.369676 \\
$\mathbf{1 6}$ & $\mathrm{C} 16$ & 0.441901 & -0.862443 & 10.158307 \\
$\mathbf{1 7}$ & $\mathrm{C} 17$ & 1.005955 & 0.407311 & 10.811011 \\
$\mathbf{1 8}$ & $\mathrm{O} 18$ & -0.990003 & -0.755898 & 10.198933 \\
$\mathbf{1 9}$ & $\mathrm{C} 19$ & 0.840411 & -2.083936 & 10.987515 \\
$\mathbf{2 0}$ & $\mathrm{C} 20$ & -1.302143 & -0.893120 & 5.757163 \\
$\mathbf{2 1}$ & $\mathrm{C} 21$ & 1.489696 & 1.512925 & 1.645449 \\
$\mathbf{2 2}$ & $\mathrm{C} 22$ & 4.885534 & -0.439620 & 4.432434 \\
$\mathbf{2 3}$ & $\mathrm{C} 23$ & 5.805592 & -1.639473 & 4.204315 \\
$\mathbf{2 4}$ & $\mathrm{C} 24$ & 5.546988 & 0.840418 & 3.909085 \\
$\mathbf{2 5}$ & $\mathrm{O} 25$ & 4.753517 & -0.280873 & 5.858799 \\
$\mathbf{2 6}$ & $\mathrm{H} 26$ & 1.993565 & -0.932196 & 8.637035 \\
$\mathbf{2 7}$ & $\mathrm{H} 27$ & 1.920604 & 0.176581 & 6.639009 \\
$\mathbf{2 8}$ & $\mathrm{H} 28$ & 3.061077 & -1.581960 & 4.336402 \\
$\mathbf{2 9}$ & $\mathrm{H} 29$ & 1.785397 & -2.338817 & 6.315050 \\
$\mathbf{3 0}$ & $\mathrm{H} 30$ & 0.248317 & -3.089797 & 5.974943 \\
$\mathbf{3 1}$ & $\mathrm{H} 31$ & 0.881613 & -3.130455 & 8.406143 \\
$\mathbf{3 2}$ & $\mathrm{H} 32$ & -0.674366 & -2.361930 & 8.109940 \\
$\mathbf{3 3}$ & $\mathrm{H} 33$ & 1.171884 & 2.345986 & 6.375895 \\
$\mathbf{3 4}$ & $\mathrm{H} 34$ & -0.517375 & 1.929426 & 6.338601 \\
$\mathbf{3 5}$ & $\mathrm{H} 35$ & 0.992587 & 2.463172 & 4.129072 \\
$\mathbf{3 6}$ & $\mathrm{H} 36$ & -0.515159 & 1.551678 & 4.097514 \\
$\mathbf{3 7}$ & $\mathrm{H} 37$ & 3.967130 & -1.744519 & 2.031760 \\
$\mathbf{3 8}$ & $\mathrm{H} 38$ & 3.891228 & 0.005040 & 1.841705 \\
$\mathbf{3 9}$ & $\mathrm{H} 39$ & 1.550807 & -1.903245 & 2.173473 \\
$\mathbf{4 0}$ & $\mathrm{H} 40$ & 1.963378 & -0.973638 & 0.743766 \\
$\mathbf{4 1}$ & $\mathrm{H} 41$ & 0.075320 & -0.021440 & 2.116125 \\
$\mathbf{4 2}$ & $\mathrm{H} 42$ & 0.659967 & 1.310558 & 10.296432 \\
$\mathbf{4 3}$ & $\mathrm{H} 43$ & 0.659142 & 0.498580 & 11.846915 \\
$\mathbf{4 4}$ & $\mathrm{H} 44$ & 0.404594 & 10.811494
\end{tabular}




\begin{tabular}{lrrrr}
$\mathbf{4 5}$ & $\mathrm{H} 45$ & -1.213258 & -0.083103 & 9.524837 \\
$\mathbf{4 6}$ & $\mathrm{H} 46$ & 0.318343 & -2.985603 & 10.650430 \\
$\mathbf{4 7}$ & $\mathrm{H} 47$ & 1.919525 & -2.262973 & 10.946012 \\
$\mathbf{4 8}$ & $\mathrm{H} 48$ & 0.553368 & -1.951034 & 12.037277 \\
$\mathbf{4 9}$ & $\mathrm{H} 49$ & -1.753022 & -0.839324 & 6.752378 \\
$\mathbf{5 0}$ & $\mathrm{H} 50$ & -1.683735 & -0.051322 & 5.172489 \\
$\mathbf{5 1}$ & $\mathrm{H} 51$ & -1.680602 & -1.793096 & 5.257944 \\
$\mathbf{5 2}$ & $\mathrm{H} 52$ & 2.467974 & 1.892508 & 1.956482 \\
$\mathbf{5 3}$ & $\mathrm{H} 53$ & 1.513587 & 1.362237 & 0.560727 \\
$\mathbf{5 4}$ & $\mathrm{H} 54$ & 0.747642 & 2.293806 & 1.840005 \\
$\mathbf{5 5}$ & $\mathrm{H} 55$ & 6.753282 & -1.509656 & 4.739949 \\
$\mathbf{5 6}$ & $\mathrm{H} 56$ & 5.353232 & -2.556453 & 4.598631 \\
$\mathbf{5 7}$ & $\mathrm{H} 57$ & 6.031460 & -1.790168 & 3.144460 \\
$\mathbf{5 8}$ & $\mathrm{H} 58$ & 4.911238 & 1.715907 & 4.080907 \\
$\mathbf{5 9}$ & $\mathrm{H} 59$ & 6.484209 & 1.035446 & 4.443690 \\
$\mathbf{6 0}$ & $\mathrm{H} 60$ & 5.774464 & 0.779911 & 2.840831 \\
$\mathbf{6 1}$ & $\mathrm{H} 61$ & 4.068052 & 0.405842 & 5.970866 \\
\hline
\end{tabular}


Table S14. Conformer 1b-B/5b-B. PCM- $\mathrm{CHCl}_{3}$ energy and Boltzmann Populations 1160.10218973569 hartrees, $38.57 \%$.

\begin{tabular}{|c|c|c|c|c|}
\hline \multicolumn{2}{|c|}{ Atom number } & \multicolumn{3}{|c|}{ Coordinates } \\
\hline & & $x$ & $\mathbf{Y}$ & $\mathbf{Z}$ \\
\hline 1 & 01 & 0.225316 & 0.103476 & 7.931677 \\
\hline 2 & $\mathrm{C} 2$ & 0.713186 & 0.172855 & 6.588481 \\
\hline 3 & C3 & 0.188509 & -0.994547 & 5.735792 \\
\hline 4 & $\mathrm{C} 4$ & 0.673405 & -2.297325 & 6.400521 \\
\hline 5 & C5 & 0.317340 & -2.352734 & 7.888457 \\
\hline 6 & $\mathrm{C} 6$ & 0.731884 & -1.066352 & 8.619427 \\
\hline 7 & $\mathrm{C} 7$ & 0.340192 & 1.509965 & 5.964474 \\
\hline 8 & $\mathrm{C} 8$ & 0.434753 & 1.475332 & 4.435687 \\
\hline 9 & $\mathrm{C} 9$ & 1.219333 & 0.261123 & 3.891509 \\
\hline 10 & 010 & 0.687853 & -0.977835 & 4.392113 \\
\hline 11 & 011 & 2.587949 & 0.427881 & 4.295654 \\
\hline 12 & C12 & 3.458137 & -0.650873 & 3.889563 \\
\hline 13 & C13 & 3.465611 & -0.807678 & 2.366488 \\
\hline 14 & C14 & 2.046420 & -0.944629 & 1.826367 \\
\hline 15 & C15 & 1.139018 & 0.185800 & 2.340598 \\
\hline 16 & C16 & 0.256744 & -0.978355 & 10.097843 \\
\hline 17 & C17 & 0.861906 & -2.083450 & 10.963582 \\
\hline 18 & 018 & 0.732742 & 0.275611 & 10.624665 \\
\hline 19 & C19 & -1.268461 & -0.963847 & 10.246391 \\
\hline 20 & $\mathrm{C} 20$ & -1.350237 & -1.021005 & 5.640905 \\
\hline 21 & $\mathrm{C} 21$ & 1.494335 & 1.504541 & 1.639257 \\
\hline 22 & $\mathrm{C} 22$ & 4.861232 & -0.377790 & 4.507123 \\
\hline 23 & $\mathrm{C} 23$ & 5.825113 & -1.544062 & 4.285143 \\
\hline 24 & $\mathrm{C} 24$ & 5.495473 & 0.931072 & 4.022821 \\
\hline 25 & $\mathrm{O} 25$ & 4.686707 & -0.247970 & 5.931535 \\
\hline 26 & $\mathrm{H} 26$ & 1.827941 & -0.984188 & 8.614487 \\
\hline 27 & $\mathrm{H} 27$ & 1.808710 & 0.135158 & 6.634934 \\
\hline 28 & $\mathrm{H} 28$ & 3.073825 & -1.570744 & 4.348277 \\
\hline 29 & $\mathrm{H} 29$ & 1.763237 & -2.375859 & 6.290112 \\
\hline 30 & $\mathrm{H} 30$ & 0.267081 & -3.173746 & 5.880392 \\
\hline 31 & H31 & 0.802408 & -3.223995 & 8.341558 \\
\hline 32 & H32 & -0.762633 & -2.509320 & 7.975784 \\
\hline 33 & $\mathrm{H} 33$ & 1.007403 & 2.284403 & 6.364216 \\
\hline 34 & H34 & -0.667109 & 1.820538 & 6.269134 \\
\hline 35 & H35 & 0.896337 & 2.416325 & 4.114031 \\
\hline 36 & H36 & -0.582995 & 1.462875 & 4.027312 \\
\hline 37 & H37 & 4.050140 & -1.684959 & 2.070027 \\
\hline 38 & $\mathrm{H} 38$ & 3.930470 & 0.063519 & 1.893457 \\
\hline 39 & H39 & 1.636657 & -1.913474 & 2.138863 \\
\hline 40 & $\mathrm{H} 40$ & 2.064459 & -0.958913 & 0.730214 \\
\hline 41 & $\mathrm{H} 41$ & 0.110685 & -0.073406 & 2.053821 \\
\hline 42 & $\mathrm{H} 42$ & 0.492082 & -3.074483 & 10.683850 \\
\hline 43 & $\mathrm{H} 43$ & 1.954952 & -2.083879 & 10.885009 \\
\hline 44 & $\mathrm{H} 44$ & 0.630049 & -1.918642 & 12.022288 \\
\hline 45 & $\mathrm{H} 45$ & 0.513775 & 0.937793 & 9.938161 \\
\hline 46 & $\mathrm{H} 46$ & -1.718834 & -0.158476 & 9.655953 \\
\hline 47 & $\mathrm{H} 47$ & -1.721264 & -1.912462 & 9.943748 \\
\hline 48 & $\mathrm{H} 48$ & -1.551481 & -0.771200 & 11.288048 \\
\hline 49 & $\mathrm{H} 49$ & -1.83402 & -0.987127 & 6.621424 \\
\hline 50 & $\mathrm{H} 50$ & -1.737679 & -0.186646 & 5.049390 \\
\hline 51 & H51 & -1.685900 & -1.928236 & 5.124534 \\
\hline
\end{tabular}




\begin{tabular}{llrrr}
$\mathbf{5 2}$ & $\mathrm{H} 52$ & 2.451460 & 1.908714 & 1.983303 \\
$\mathbf{5 3}$ & $\mathrm{H} 53$ & 1.555346 & 1.365007 & 0.554487 \\
$\mathbf{5 4}$ & $\mathrm{H} 54$ & 0.724695 & 2.262200 & 1.817829 \\
$\mathbf{5 5}$ & $\mathrm{H} 55$ & 6.752677 & -1.394327 & 4.850033 \\
$\mathbf{5 6}$ & $\mathrm{H} 56$ & 5.391106 & -2.481668 & 4.650490 \\
$\mathbf{5 7}$ & $\mathrm{H} 57$ & 6.085757 & -1.668506 & 3.229861 \\
$\mathbf{5 8}$ & $\mathrm{H} 58$ & 5.750801 & 0.897002 & 2.959727 \\
$\mathbf{5 9}$ & $\mathrm{H} 59$ & 4.829141 & 1.783637 & 4.194029 \\
$\mathbf{6 0}$ & $\mathrm{H} 60$ & 6.412850 & 1.144721 & 4.584104 \\
$\mathbf{6 1}$ & $\mathrm{H} 61$ & 3.977085 & 0.414549 & 6.038287 \\
\hline
\end{tabular}


Table S15 . Conformer 1b-C/5b-C. PCM-CHCl 3 energy and Boltzmann Populations 1160.0972655957 hartrees, $23.94 \%$.

\begin{tabular}{|c|c|c|c|c|}
\hline \multicolumn{2}{|c|}{ Atom number } & \multicolumn{3}{|c|}{ Coordinates } \\
\hline & & $X$ & $\mathbf{Y}$ & $\mathbf{Z}$ \\
\hline 1 & O1 & 0.174028 & 0.328379 & 7.877859 \\
\hline 2 & $\mathrm{C} 2$ & 0.705502 & 0.325328 & 6.550116 \\
\hline 3 & C3 & 0.130730 & -0.831240 & 5.714532 \\
\hline 4 & $\mathrm{C} 4$ & 0.502898 & -2.142064 & 6.434251 \\
\hline 5 & C5 & 0.095900 & -2.122051 & 7.909981 \\
\hline 6 & C6 & 0.582504 & -0.840373 & 8.613732 \\
\hline 7 & $\mathrm{C7}$ & 0.440303 & 1.663988 & 5.876852 \\
\hline 8 & C8 & 0.575394 & 1.575872 & 4.353551 \\
\hline 9 & C9 & 1.294434 & 0.297745 & 3.868037 \\
\hline 10 & 010 & 0.676131 & -0.891060 & 4.389710 \\
\hline 11 & 011 & 2.659722 & 0.392081 & 4.302656 \\
\hline 12 & C12 & 3.467155 & -0.758485 & 3.974355 \\
\hline 13 & C13 & 3.511479 & -0.950113 & 2.452712 \\
\hline 14 & C14 & 2.106145 & -1.012692 & 1.864780 \\
\hline 15 & C15 & 1.252564 & 0.182175 & 2.318633 \\
\hline 16 & C16 & 0.105729 & -0.724107 & 10.091233 \\
\hline 17 & C17 & -1.418648 & -0.612701 & 10.217349 \\
\hline 18 & 018 & 0.531745 & -1.891603 & 10.808101 \\
\hline 19 & C19 & 0.756887 & 0.495629 & 10.759703 \\
\hline 20 & $\mathrm{C} 20$ & -1.402322 & -0.758182 & 5.565678 \\
\hline 21 & $\mathrm{C} 21$ & 1.706428 & 1.455707 & 1.590873 \\
\hline 22 & $\mathrm{C} 22$ & 4.874335 & -0.526448 & 4.603199 \\
\hline 23 & $\mathrm{C} 23$ & 4.774958 & -0.363113 & 6.126007 \\
\hline 24 & $\mathrm{C} 24$ & 5.859796 & -1.649729 & 4.275271 \\
\hline 25 & $\mathrm{O} 25$ & 5.422626 & 0.696968 & 4.087413 \\
\hline 26 & $\mathrm{H} 26$ & 1.681870 & -0.870149 & 8.624780 \\
\hline 27 & $\mathrm{H} 27$ & 1.794356 & 0.217829 & 6.630918 \\
\hline 28 & $\mathrm{H} 28$ & 3.026776 & -1.648610 & 4.440354 \\
\hline 29 & $\mathrm{H} 29$ & 1.587539 & -2.298859 & 6.363361 \\
\hline 30 & $\mathrm{H} 30$ & 0.052915 & -3.005202 & 5.928067 \\
\hline 31 & H31 & 0.499038 & -3.009686 & 8.410254 \\
\hline 32 & H32 & -0.994957 & -2.195499 & 7.962952 \\
\hline 33 & $\mathrm{H} 33$ & 1.146133 & 2.404820 & 6.274014 \\
\hline 34 & H34 & -0.552278 & 2.049433 & 6.141764 \\
\hline 35 & H35 & 1.106084 & 2.475084 & 4.019100 \\
\hline 36 & H36 & -0.428908 & 1.616799 & 3.914973 \\
\hline 37 & $\mathrm{H} 37$ & 4.042978 & -1.872109 & 2.194199 \\
\hline 38 & H38 & 4.054756 & -0.122193 & 1.982789 \\
\hline 39 & H39 & 1.626637 & -1.946350 & 2.185313 \\
\hline 40 & $\mathrm{H} 40$ & 2.161356 & -1.057130 & 0.770674 \\
\hline 41 & $\mathrm{H} 41$ & 0.219178 & -0.024498 & 2.008413 \\
\hline 42 & $\mathrm{H} 42$ & -1.916165 & -1.528487 & 9.882697 \\
\hline 43 & $\mathrm{H} 43$ & -1.711352 & -0.483135 & 11.266124 \\
\hline 44 & $\mathrm{H} 44$ & -1.815783 & 0.233040 & 9.647434 \\
\hline 45 & $\mathrm{H} 45$ & 0.232591 & -1.796914 & 11.730041 \\
\hline 46 & $\mathrm{H} 46$ & 1.849648 & 0.420922 & 10.721560 \\
\hline 47 & $\mathrm{H} 47$ & 0.455440 & 1.433717 & 10.282850 \\
\hline 48 & $\mathrm{H} 48$ & 0.490234 & 0.549093 & 11.821612 \\
\hline 49 & $\mathrm{H} 49$ & -1.915041 & -0.657638 & 6.526746 \\
\hline 50 & H50 & -1.712995 & 0.078479 & 4.933642 \\
\hline 51 & H51 & -1.781336 & -1.658656 & 5.067757 \\
\hline
\end{tabular}




\begin{tabular}{llrrr}
$\mathbf{5 2}$ & $\mathrm{H} 52$ & 0.980308 & 2.263627 & 1.726103 \\
$\mathbf{5 3}$ & $\mathrm{H} 53$ & 2.677335 & 1.810894 & 1.950122 \\
$\mathbf{5 4}$ & $\mathrm{H} 54$ & 1.787350 & 1.280537 & 0.512569 \\
$\mathbf{5 5}$ & $\mathrm{H} 55$ & 5.766744 & -0.206342 & 6.565983 \\
$\mathbf{5 6}$ & $\mathrm{H} 56$ & 4.185988 & 0.519905 & 6.396233 \\
$\mathbf{5 7}$ & $\mathrm{H} 57$ & 4.323766 & -1.243209 & 6.595540 \\
$\mathbf{5 8}$ & $\mathrm{H} 58$ & 6.819341 & -1.484411 & 4.779332 \\
$\mathbf{5 9}$ & $\mathrm{H} 59$ & 5.472918 & -2.626047 & 4.584195 \\
$\mathbf{6 0}$ & $\mathrm{H} 60$ & 6.091068 & -1.682006 & 3.205451 \\
$\mathbf{6 1}$ & $\mathrm{H} 61$ & 4.696305 & 1.348551 & 4.145894 \\
\hline
\end{tabular}




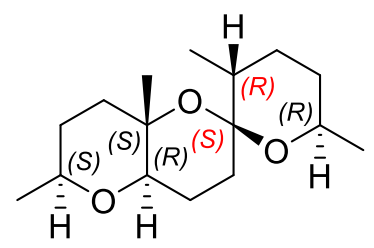

Table S16. Conformer 1c-A/5c-A. PCM- $\mathrm{CHCl}_{3}$ energy and Boltzmann Populations 1160.10035892074 hartrees, $32.38 \%$.

\begin{tabular}{rrrrr}
\hline \multicolumn{1}{r}{ Atom number } & \multicolumn{3}{r}{ Coordinates } & \\
\hline & & $\mathbf{X}$ & $\mathbf{Y}$ & $\mathbf{Z}$ \\
$\mathbf{1}$ & $\mathrm{O} 1$ & 2.269392 & -1.799543 & -3.604991 \\
$\mathbf{2}$ & $\mathrm{C} 2$ & 1.222254 & -2.643488 & -3.129672 \\
$\mathbf{3}$ & $\mathrm{C} 3$ & 1.554844 & -3.188521 & -1.731890 \\
$\mathbf{4}$ & $\mathrm{C} 4$ & 2.817978 & -4.057795 & -1.908433 \\
$\mathbf{5}$ & $\mathrm{C} 5$ & 3.947196 & -3.307201 & -2.626546 \\
$\mathbf{6}$ & $\mathrm{C} 6$ & 3.464060 & -2.569622 & -3.893516 \\
$\mathbf{7}$ & $\mathrm{C} 7$ & -0.121972 & -1.929405 & -3.128012 \\
$\mathbf{8}$ & $\mathrm{C} 8$ & -1.183364 & -2.921720 & -2.651637 \\
$\mathbf{9}$ & $\mathrm{C} 9$ & -0.841640 & -3.522450 & -1.265053 \\
$\mathbf{1 0}$ & $\mathrm{O} 10$ & 0.505595 & -4.050344 & -1.267339 \\
$\mathbf{1 1}$ & $\mathrm{O} 11$ & -0.950004 & -2.532064 & -0.231965 \\
$\mathbf{1 2}$ & $\mathrm{C} 12$ & -2.278834 & -2.072119 & 0.085438 \\
$\mathbf{1 3}$ & $\mathrm{C} 13$ & -3.176880 & -3.240579 & 0.492162 \\
$\mathbf{1 4}$ & $\mathrm{C} 14$ & -3.170274 & -4.321666 & -0.578500 \\
$\mathbf{1 5}$ & $\mathrm{C} 15$ & -1.732063 & -4.740831 & -0.884737 \\
$\mathbf{1 6}$ & $\mathrm{C} 16$ & 4.517828 & -1.583902 & -4.478467 \\
$\mathbf{1 7}$ & $\mathrm{C} 17$ & 3.999821 & -0.935418 & -5.769732 \\
$\mathbf{1 8}$ & $\mathrm{O} 18$ & 4.735650 & -0.524229 & -3.533753 \\
$\mathbf{1 9}$ & $\mathrm{C} 19$ & 5.867136 & -2.251816 & -4.746770 \\
$\mathbf{2 0}$ & $\mathrm{C} 20$ & 1.866427 & -2.091344 & -0.695625 \\
$\mathbf{2 1}$ & $\mathrm{C} 21$ & -1.715210 & -5.881289 & -1.908098 \\
$\mathbf{2 2}$ & $\mathrm{C} 22$ & -2.151265 & -0.966502 & 1.177443 \\
$\mathbf{2 3}$ & $\mathrm{C} 23$ & -3.503302 & -0.348716 & 1.539062 \\
$\mathbf{2 4}$ & $\mathrm{C} 24$ & -1.433302 & -1.443989 & 2.444796 \\
$\mathbf{2 5}$ & $\mathrm{O} 25$ & -1.353026 & 0.096347 & 0.623338 \\
$\mathbf{2 6}$ & $\mathrm{H} 26$ & 1.114259 & -3.483271 & -3.833198 \\
$\mathbf{2 7}$ & $\mathrm{H} 27$ & 2.561321 & -4.956023 & -2.486884 \\
$\mathbf{2 8}$ & $\mathrm{H} 28$ & 3.169039 & -4.430881 & -0.938071 \\
$\mathbf{2 9}$ & $\mathrm{H} 29$ & 4.736602 & -4.022189 & -2.884869 \\
$\mathbf{3 0}$ & $\mathrm{H} 30$ & 4.394984 & -2.592801 & -1.926855 \\
$\mathbf{3 1}$ & $\mathrm{H} 31$ & 3.214317 & -3.311839 & -4.664008 \\
$\mathbf{3 2}$ & $\mathrm{H} 32$ & -0.358283 & -1.580768 & -4.140513 \\
$\mathbf{3 3}$ & $\mathrm{H} 33$ & -0.103563 & -1.031279 & -2.501618 \\
$\mathbf{3 4}$ & $\mathrm{H} 34$ & -1.228898 & -3.718904 & -3.402452 \\
$\mathbf{3 5}$ & $\mathrm{H} 35$ & -2.158967 & -2.428670 & -2.661295 \\
$\mathbf{3 6}$ & $\mathrm{H} 36$ & -2.687571 & -1.589688 & -0.809761 \\
$\mathbf{3 7}$ & $\mathrm{H} 37$ & -4.205606 & -2.905965 & 0.660055 \\
$\mathbf{3 8}$ & $\mathrm{H} 38$ & -2.818862 & -3.687377 & 1.426733 \\
$\mathbf{3 9}$ & $\mathrm{H} 39$ & -3.666328 & -3.952776 & -1.484261 \\
$\mathbf{4 0}$ & $\mathrm{H} 40$ & -3.750688 & -5.183234 & -0.227405 \\
$\mathbf{4 1}$ & $\mathrm{H} 41$ & -1.315485 & -5.160593 & 0.043697 \\
$\mathbf{4 2}$ & $\mathrm{H} 42$ & 3.080998 & -0.365771 & -5.592014 \\
$\mathbf{4 3}$ & $\mathrm{H} 43$ & 4.728698 & -0.217400 & -6.163330
\end{tabular}




\begin{tabular}{llrrr}
$\mathbf{4 4}$ & $\mathrm{H} 44$ & 3.800774 & -1.685158 & -6.542304 \\
$\mathbf{4 5}$ & $\mathrm{H} 45$ & 3.840806 & -0.241937 & -3.257351 \\
$\mathbf{4 6}$ & $\mathrm{H} 46$ & 6.567693 & -1.542495 & -5.202970 \\
$\mathbf{4 7}$ & $\mathrm{H} 47$ & 6.343328 & -2.581820 & -3.817446 \\
$\mathbf{4 8}$ & $\mathrm{H} 48$ & 5.763529 & -3.111855 & -5.415996 \\
$\mathbf{4 9}$ & $\mathrm{H} 49$ & 1.071472 & -1.349139 & -0.624416 \\
$\mathbf{5 0}$ & $\mathrm{H} 50$ & 1.980408 & -2.528812 & 0.302694 \\
$\mathbf{5 1}$ & $\mathrm{H} 51$ & 2.774907 & -1.529847 & -0.929783 \\
$\mathbf{5 2}$ & $\mathrm{H} 52$ & -0.692503 & -6.173490 & -2.165724 \\
$\mathbf{5 3}$ & $\mathrm{H} 53$ & -2.216238 & -6.765187 & -1.497715 \\
$\mathbf{5 4}$ & $\mathrm{H} 54$ & -2.240228 & -5.610895 & -2.829412 \\
$\mathbf{5 5}$ & $\mathrm{H} 55$ & -3.369638 & 0.529666 & 2.181397 \\
$\mathbf{5 6}$ & $\mathrm{H} 56$ & -4.022076 & 0.003917 & 0.640468 \\
$\mathbf{5 7}$ & $\mathrm{H} 57$ & -4.153619 & -1.055671 & 2.062841 \\
$\mathbf{5 8}$ & $\mathrm{H} 58$ & -0.439554 & -1.843362 & 2.214339 \\
$\mathbf{5 9}$ & $\mathrm{H} 59$ & -2.002952 & -2.212154 & 2.975739 \\
$\mathbf{6 0}$ & $\mathrm{H} 60$ & -1.270419 & -0.606205 & 3.133168 \\
$\mathbf{6 1}$ & $\mathrm{H} 61$ & -0.551564 & -0.339282 & 0.276808 \\
\hline
\end{tabular}


Table S17. Conformer 1c-B/5c-B. PCM- $\mathrm{CHCl}_{3}$ energy and Boltzmann Populations 1160.10072622252 hartrees, $18.04 \%$.

\begin{tabular}{|c|c|c|c|c|}
\hline \multicolumn{2}{|c|}{ Atom number } & \multicolumn{3}{|c|}{ Coordinates } \\
\hline & & $x$ & $\mathbf{Y}$ & $\mathbf{Z}$ \\
\hline 1 & 01 & 2.374533 & -1.738324 & -3.536263 \\
\hline 2 & $\mathrm{C} 2$ & 1.307973 & -2.571367 & -3.083628 \\
\hline 3 & $\mathrm{C} 3$ & 1.589069 & -3.105259 & -1.669742 \\
\hline 4 & $\mathrm{C} 4$ & 2.850326 & -3.988088 & -1.792291 \\
\hline 5 & $\mathrm{C} 5$ & 4.014285 & -3.251147 & -2.467813 \\
\hline 6 & $\mathrm{C} 6$ & 3.581270 & -2.514876 & -3.748929 \\
\hline 7 & $\mathrm{C} 7$ & -0.030661 & -1.848626 & -3.135052 \\
\hline 8 & $\mathrm{C} 8$ & -1.114301 & -2.829381 & -2.686711 \\
\hline 9 & $\mathrm{C} 9$ & -0.825511 & -3.419084 & -1.283719 \\
\hline 10 & 010 & 0.518155 & -3.953059 & -1.230145 \\
\hline 11 & 011 & -0.971718 & -2.421652 & -0.261537 \\
\hline 12 & $\mathrm{C} 12$ & -2.307951 & -1.936442 & -0.015558 \\
\hline 13 & C13 & -3.223749 & -3.103478 & 0.368192 \\
\hline 14 & C14 & -3.178554 & -4.202723 & -0.682059 \\
\hline 15 & C15 & -1.732515 & -4.630897 & -0.925790 \\
\hline 16 & C16 & 4.653592 & -1.556205 & -4.341018 \\
\hline 17 & C17 & 5.927699 & -2.297409 & -4.745965 \\
\hline 18 & 018 & 4.098065 & -0.987534 & -5.543083 \\
\hline 19 & C19 & 4.993797 & -0.380578 & -3.418585 \\
\hline 20 & $\mathrm{C} 20$ & 1.872196 & -1.999768 & -0.635697 \\
\hline 21 & $\mathrm{C} 21$ & -1.679313 & -5.783007 & -1.934792 \\
\hline 22 & $\mathrm{C} 22$ & -2.209044 & -0.852789 & 1.104165 \\
\hline 23 & $\mathrm{C} 23$ & -1.388308 & 0.354047 & 0.631867 \\
\hline 24 & $\mathrm{C} 24$ & -3.577395 & -0.366489 & 1.588056 \\
\hline 25 & $\mathrm{O} 25$ & -1.523226 & -1.414805 & 2.233852 \\
\hline 26 & $\mathrm{H} 26$ & 1.217879 & -3.418018 & -3.781387 \\
\hline 27 & $\mathrm{H} 27$ & 2.608057 & -4.886660 & -2.376338 \\
\hline 28 & $\mathrm{H} 28$ & 3.158790 & -4.359622 & -0.806959 \\
\hline 29 & $\mathrm{H} 29$ & 4.809298 & -3.970007 & -2.695808 \\
\hline 30 & $\mathrm{H} 30$ & 4.437008 & -2.544261 & -1.747446 \\
\hline 31 & H31 & 3.339005 & -3.258852 & -4.521082 \\
\hline 32 & H32 & -0.229613 & -1.508063 & -4.158297 \\
\hline 33 & $\mathrm{H} 33$ & -0.027818 & -0.944701 & -2.516753 \\
\hline 34 & H34 & -1.139016 & -3.633304 & -3.431263 \\
\hline 35 & H35 & -2.085664 & -2.330268 & -2.735224 \\
\hline 36 & H36 & -2.692934 & -1.452078 & -0.919664 \\
\hline 37 & $\mathrm{H} 37$ & -4.259470 & -2.767197 & 0.482568 \\
\hline 38 & H38 & -2.906534 & -3.528221 & 1.328700 \\
\hline 39 & H39 & -3.638352 & -3.847941 & -1.612287 \\
\hline 40 & $\mathrm{H} 40$ & -3.775190 & -5.056545 & -0.339292 \\
\hline 41 & $\mathrm{H} 41$ & -1.354673 & -5.041214 & 0.023232 \\
\hline 42 & $\mathrm{H} 42$ & 6.461061 & -2.701476 & -3.880207 \\
\hline 43 & $\mathrm{H} 43$ & 5.696671 & -3.122115 & -5.429640 \\
\hline 44 & $\mathrm{H} 44$ & 6.609577 & -1.631262 & -5.287298 \\
\hline 45 & $\mathrm{H} 45$ & 3.195728 & -0.702113 & -5.294183 \\
\hline 46 & $\mathrm{H} 46$ & 5.651617 & 0.331717 & -3.930635 \\
\hline 47 & $\mathrm{H} 47$ & 4.095493 & 0.181861 & -3.141497 \\
\hline 48 & $\mathrm{H} 48$ & 5.496573 & -0.705824 & -2.503308 \\
\hline 49 & $\mathrm{H} 49$ & 1.955505 & -2.427077 & 0.369977 \\
\hline 50 & $\mathrm{H} 50$ & 1.074507 & -1.259038 & -0.596917 \\
\hline 51 & H51 & 2.787049 & -1.440135 & -0.848378 \\
\hline
\end{tabular}




\begin{tabular}{rrrrr}
$\mathbf{5 2}$ & $\mathrm{H} 52$ & -0.648322 & -6.081814 & -2.14804 \\
$\mathbf{5 3}$ & $\mathrm{H} 53$ & -2.199844 & -6.660381 & -1.534786 \\
$\mathbf{5 4}$ & $\mathrm{H} 54$ & -2.166015 & -5.521484 & -2.879374 \\
$\mathbf{5 5}$ & $\mathrm{H} 55$ & -0.357200 & 0.074333 & 0.402722 \\
$\mathbf{5 6}$ & $\mathrm{H} 56$ & -1.831073 & 0.818276 & -0.255211 \\
$\mathbf{5 7}$ & $\mathrm{H} 57$ & -1.321747 & 1.111869 & 1.421350 \\
$\mathbf{5 8}$ & $\mathrm{H} 58$ & -4.126814 & -1.160150 & 2.105155 \\
$\mathbf{5 9}$ & $\mathrm{H} 59$ & -4.187122 & 0.006694 & 0.759070 \\
$\mathbf{6 0}$ & $\mathrm{H} 60$ & -3.466045 & 0.439322 & 2.323244 \\
$\mathbf{6 1}$ & $\mathrm{H} 61$ & -0.768156 & -1.908374 & 1.853389 \\
\hline
\end{tabular}


Table S18. Conformer 1c-C/5c-C. PCM- $\mathrm{CHCl}_{3}$ energy and Boltzmann Populations 1160.09605300637 hartrees, $23.92 \%$.

\begin{tabular}{|c|c|c|c|c|}
\hline \multicolumn{2}{|c|}{ Atom number } & \multicolumn{3}{|c|}{ Coordinates } \\
\hline & & $x$ & $\mathbf{Y}$ & $\mathbf{Z}$ \\
\hline 1 & 01 & 2.435019 & -1.739605 & -3.549363 \\
\hline 2 & $\mathrm{C} 2$ & 1.355107 & -2.574542 & -3.132896 \\
\hline 3 & C3 & 1.661844 & -3.237707 & -1.780236 \\
\hline 4 & $\mathrm{C} 4$ & 2.887170 & -4.146454 & -2.021874 \\
\hline 5 & C5 & 4.054086 & -3.391475 & -2.671830 \\
\hline 6 & $\mathrm{C} 6$ & 3.606804 & -2.532736 & -3.869029 \\
\hline 7 & $\mathrm{C} 7$ & 0.040524 & -1.809151 & -3.077923 \\
\hline 8 & $\mathrm{C} 8$ & -1.060796 & -2.790253 & -2.674610 \\
\hline 9 & $\mathrm{C} 9$ & -0.746378 & -3.500083 & -1.333860 \\
\hline 10 & 010 & 0.575857 & -4.087636 & -1.381587 \\
\hline 11 & 011 & -0.806586 & -2.579983 & -0.235366 \\
\hline 12 & C12 & -2.104315 & -2.078443 & 0.111740 \\
\hline 13 & C13 & -3.052622 & -3.232136 & 0.457453 \\
\hline 14 & C14 & -3.107082 & -4.237631 & -0.683649 \\
\hline 15 & C15 & -1.691896 & -4.699853 & -1.034876 \\
\hline 16 & C16 & 4.692577 & -1.560485 & -4.412394 \\
\hline 17 & C17 & 5.927182 & -2.303257 & -4.923006 \\
\hline 18 & O18 & 4.119781 & -0.871534 & -5.541187 \\
\hline 19 & C19 & 5.101619 & -0.481377 & -3.403930 \\
\hline 20 & $\mathrm{C} 20$ & 2.017123 & -2.235178 & -0.665182 \\
\hline 21 & $\mathrm{C} 21$ & -1.735671 & -5.761791 & -2.138562 \\
\hline 22 & $\mathrm{C} 22$ & -1.977480 & -1.035145 & 1.264678 \\
\hline 23 & $\mathrm{C} 23$ & -1.310712 & -1.612767 & 2.519163 \\
\hline 24 & $\mathrm{C} 24$ & -1.185452 & 0.191142 & 0.790176 \\
\hline 25 & $\mathrm{O} 25$ & -3.291003 & -0.578779 & 1.622390 \\
\hline 26 & $\mathrm{H} 26$ & 1.214535 & -3.354656 & -3.896776 \\
\hline 27 & $\mathrm{H} 27$ & 2.595246 & -4.982832 & -2.671883 \\
\hline 28 & $\mathrm{H} 28$ & 3.213637 & -4.611709 & -1.083236 \\
\hline 29 & $\mathrm{H} 29$ & 4.815626 & -4.113040 & -2.988360 \\
\hline 30 & $\mathrm{H} 30$ & 4.524404 & -2.764196 & -1.908566 \\
\hline 31 & H31 & 3.314916 & -3.198378 & -4.693743 \\
\hline 32 & H32 & -0.179553 & -1.378169 & -4.061979 \\
\hline 33 & $\mathrm{H} 33$ & 0.094049 & -0.960738 & -2.387431 \\
\hline 34 & H34 & -1.135067 & -3.530044 & -3.479954 \\
\hline 35 & H35 & -2.016201 & -2.259661 & -2.650070 \\
\hline 36 & H36 & -2.527903 & -1.545863 & -0.748003 \\
\hline 37 & H37 & -4.061607 & -2.861599 & 0.667915 \\
\hline 38 & H38 & -2.701267 & -3.758259 & 1.352349 \\
\hline 39 & H39 & -3.593736 & -3.785983 & -1.556402 \\
\hline 40 & $\mathrm{H} 40$ & -3.723197 & -5.093829 & -0.384075 \\
\hline 41 & $\mathrm{H} 41$ & -1.289649 & -5.205424 & -0.143612 \\
\hline 42 & $\mathrm{H} 42$ & 5.646788 & -3.057261 & -5.667176 \\
\hline 43 & $\mathrm{H} 43$ & 6.614407 & -1.614168 & -5.427613 \\
\hline 44 & $\mathrm{H} 44$ & 6.473064 & -2.798183 & -4.114091 \\
\hline 45 & $\mathrm{H} 45$ & 3.235163 & -0.582394 & -5.238414 \\
\hline 46 & $\mathrm{H} 46$ & 5.767075 & 0.252022 & -3.874652 \\
\hline 47 & $\mathrm{H} 47$ & 4.232216 & 0.082578 & -3.048671 \\
\hline 48 & $\mathrm{H} 48$ & 5.621389 & -0.901102 & -2.537926 \\
\hline 49 & $\mathrm{H} 49$ & 1.251114 & -1.470835 & -0.534545 \\
\hline 50 & $\mathrm{H} 50$ & 2.115374 & -2.751123 & 0.296632 \\
\hline 51 & H51 & 2.946008 & -1.692373 & -0.860012 \\
\hline
\end{tabular}




\begin{tabular}{rrrrr}
$\mathbf{5 2}$ & $\mathrm{H} 52$ & -2.275315 & -6.649003 & -1.788813 \\
$\mathbf{5 3}$ & $\mathrm{H} 53$ & -2.253281 & -5.400259 & -3.032403 \\
$\mathbf{5 4}$ & $\mathrm{H} 54$ & -0.729566 & -6.081643 & -2.426887 \\
$\mathbf{5 5}$ & $\mathrm{H} 55$ & -0.315247 & -2.014370 & 2.305380 \\
$\mathbf{5 6}$ & $\mathrm{H} 56$ & -1.206947 & -0.841795 & 3.291930 \\
$\mathbf{5 7}$ & $\mathrm{H} 57$ & -1.920827 & -2.402810 & 2.968438 \\
$\mathbf{5 8}$ & $\mathrm{H} 58$ & -1.179207 & 0.973580 & 1.558053 \\
$\mathbf{5 9}$ & $\mathrm{H} 59$ & -1.652080 & 0.635871 & -0.096150 \\
$\mathbf{6 0}$ & $\mathrm{H} 60$ & -0.148603 & -0.056310 & 0.549986 \\
$\mathbf{6 1}$ & $\mathrm{H} 61$ & -3.193579 & 0.059826 & 2.351133 \\
\hline
\end{tabular}


Table S19. Conformer 1c-D/5c-D. PCM- $\mathrm{CHCl}_{3}$ energy and Boltzmann Populations 1160.09564920643 hartrees, $24.33 \%$.

\begin{tabular}{|c|c|c|c|c|}
\hline \multicolumn{2}{|c|}{ Atom number } & \multicolumn{3}{|c|}{ Coordinates } \\
\hline & & $x$ & $\mathbf{Y}$ & $\mathbf{Z}$ \\
\hline 1 & 01 & 2.169904 & -1.650918 & -3.598589 \\
\hline 2 & $\mathrm{C} 2$ & 1.159732 & -2.526013 & -3.100422 \\
\hline 3 & C3 & 1.498212 & -3.004947 & -1.679314 \\
\hline 4 & $\mathrm{C} 4$ & 2.804550 & -3.819030 & -1.805166 \\
\hline 5 & C5 & 3.909251 & -3.035424 & -2.526368 \\
\hline 6 & $\mathrm{C} 6$ & 3.404278 & -2.364070 & -3.822406 \\
\hline 7 & $\mathrm{C} 7$ & -0.216809 & -1.877598 & -3.140933 \\
\hline 8 & $\mathrm{C} 8$ & -1.236191 & -2.903018 & -2.644554 \\
\hline 9 & $\mathrm{C} 9$ & -0.887257 & -3.440581 & -1.234178 \\
\hline 10 & 010 & 0.484589 & -3.898296 & -1.195041 \\
\hline 11 & 011 & -1.069724 & -2.427976 & -0.232786 \\
\hline 12 & C12 & -2.425886 & -2.011384 & 0.028609 \\
\hline 13 & C13 & -3.268025 & -3.217406 & 0.458033 \\
\hline 14 & C14 & -3.181129 & -4.338211 & -0.566508 \\
\hline 15 & C15 & -1.718223 & -4.691490 & -0.828854 \\
\hline 16 & C16 & 4.448399 & -1.412326 & -4.477345 \\
\hline 17 & C17 & 4.763872 & -0.188366 & -3.608496 \\
\hline 18 & 018 & 5.667167 & -2.137721 & -4.692626 \\
\hline 19 & C19 & 3.940747 & -0.938733 & -5.847394 \\
\hline 20 & $\mathrm{C} 20$ & 1.738556 & -1.857465 & -0.680608 \\
\hline 21 & $\mathrm{C} 21$ & -1.619346 & -5.864113 & -1.810420 \\
\hline 22 & $\mathrm{C} 22$ & -2.366155 & -0.896810 & 1.120364 \\
\hline 23 & $\mathrm{C} 23$ & -1.620809 & 0.340591 & 0.604174 \\
\hline 24 & $\mathrm{C} 24$ & -3.750186 & -0.473585 & 1.618750 \\
\hline 25 & $\mathrm{O} 25$ & -1.630690 & -1.392959 & 2.249700 \\
\hline 26 & $\mathrm{H} 26$ & 1.100158 & -3.395572 & -3.772938 \\
\hline 27 & $\mathrm{H} 27$ & 2.602141 & -4.746121 & -2.358968 \\
\hline 28 & $\mathrm{H} 28$ & 3.154046 & -4.143855 & -0.817069 \\
\hline 29 & $\mathrm{H} 29$ & 4.743834 & -3.709661 & -2.751036 \\
\hline 30 & $\mathrm{H} 30$ & 4.300324 & -2.282983 & -1.835170 \\
\hline 31 & H31 & 3.201531 & -3.170175 & -4.542647 \\
\hline 32 & H32 & -0.454702 & -1.575390 & -4.167909 \\
\hline 33 & $\mathrm{H} 33$ & -0.250498 & -0.958783 & -2.545974 \\
\hline 34 & $\mathrm{H} 34$ & -1.232911 & -3.725882 & -3.368513 \\
\hline 35 & H35 & -2.233916 & -2.458088 & -2.684082 \\
\hline 36 & H36 & -2.854230 & -1.571200 & -0.878539 \\
\hline 37 & H37 & -4.318557 & -2.936108 & 0.585115 \\
\hline 38 & H38 & -2.909775 & -3.600066 & 1.421813 \\
\hline 39 & H39 & -3.677335 & -4.032722 & -1.495503 \\
\hline 40 & $\mathrm{H} 40$ & -3.722627 & -5.214981 & -0.191659 \\
\hline 41 & $\mathrm{H} 41$ & -1.300253 & -5.055983 & 0.122060 \\
\hline 42 & $\mathrm{H} 42$ & 5.261071 & -0.475156 & -2.676571 \\
\hline 43 & $\mathrm{H} 43$ & 5.463542 & 0.480896 & -4.123252 \\
\hline 44 & $\mathrm{H} 44$ & 3.863474 & 0.384486 & -3.365690 \\
\hline 45 & $\mathrm{H} 45$ & 6.303697 & -1.528296 & -5.107111 \\
\hline 46 & $\mathrm{H} 46$ & 4.695135 & -0.325502 & -6.353841 \\
\hline 47 & $\mathrm{H} 47$ & 3.748385 & -1.793378 & -6.505995 \\
\hline 48 & $\mathrm{H} 48$ & 3.021303 & -0.350719 & -5.762758 \\
\hline 49 & $\mathrm{H} 49$ & 0.899862 & -1.163526 & -0.641743 \\
\hline 50 & $\mathrm{H} 50$ & 1.868724 & -2.252210 & 0.333365 \\
\hline 51 & H51 & 2.613997 & -1.251357 & -0.928754 \\
\hline
\end{tabular}




\begin{tabular}{rrrrr}
$\mathbf{5 2}$ & $\mathrm{H} 52$ & -0.577406 & -6.110187 & -2.037328 \\
$\mathbf{5 3}$ & $\mathrm{H} 53$ & -2.081842 & -6.758734 & -1.378587 \\
$\mathbf{5 4}$ & $\mathrm{H} 54$ & -2.137931 & -5.654430 & -2.750947 \\
$\mathbf{5 5}$ & $\mathrm{H} 55$ & -1.583009 & 1.120705 & 1.373592 \\
$\mathbf{5 6}$ & $\mathrm{H} 56$ & -0.579755 & 0.111772 & 0.363773 \\
$\mathbf{5 7}$ & $\mathrm{H} 57$ & -2.102885 & 0.757433 & -0.285930 \\
$\mathbf{5 8}$ & $\mathrm{H} 58$ & -4.246280 & -1.283056 & 2.164479 \\
$\mathbf{5 9}$ & $\mathrm{H} 59$ & -3.669908 & 0.354627 & 2.332824 \\
$\mathbf{6 0}$ & $\mathrm{H} 60$ & -4.393972 & -0.154081 & 0.793092 \\
$\mathbf{6 1}$ & $\mathrm{H} 61$ & -0.856239 & -1.853281 & 1.866450 \\
\hline
\end{tabular}




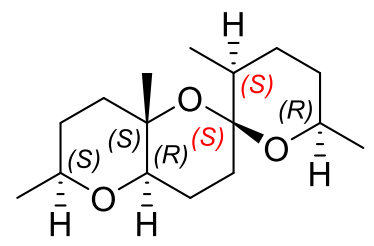

Table S20. Conformer 1d-A/5d-A. PCM- $\mathrm{CHCl}_{3}$ energy and Boltzmann Populations 1160.09866574623 hartrees, $23.10 \%$.

\begin{tabular}{|c|c|c|c|c|}
\hline \multicolumn{2}{|c|}{ Atom number } & \multicolumn{3}{|c|}{ Coordinates } \\
\hline & & $x$ & $\mathbf{Y}$ & $\mathbf{Z}$ \\
\hline 1 & 01 & -5.312822 & -1.664226 & -3.029541 \\
\hline 2 & $\mathrm{C} 2$ & -4.352866 & -2.687407 & -3.287227 \\
\hline 3 & C3 & -4.993114 & -3.856296 & -4.050895 \\
\hline 4 & $\mathrm{C} 4$ & -6.068034 & -4.439195 & -3.109761 \\
\hline 5 & C5 & -7.031671 & -3.365102 & -2.589087 \\
\hline 6 & C6 & -6.300634 & -2.110428 & -2.066164 \\
\hline 7 & $\mathrm{C} 7$ & -3.146269 & -2.148293 & -4.040925 \\
\hline 8 & $\mathrm{C} 8$ & -2.158791 & -3.295517 & -4.248395 \\
\hline 9 & C9 & -2.800212 & -4.518252 & -4.956680 \\
\hline 10 & 010 & -4.023242 & -4.887176 & -4.281806 \\
\hline 11 & 011 & -3.070462 & -4.261131 & -6.340067 \\
\hline 12 & C12 & -1.928396 & -4.030789 & -7.191046 \\
\hline 13 & C13 & -0.954695 & -5.215803 & -7.123040 \\
\hline 14 & C14 & -0.611543 & -5.583701 & -5.686100 \\
\hline 15 & C15 & -1.876652 & -5.765391 & -4.843867 \\
\hline 16 & C16 & -7.250651 & -0.908084 & -1.789898 \\
\hline 17 & C17 & -6.470717 & 0.281828 & -1.213634 \\
\hline 18 & 018 & -7.821854 & -0.473392 & -3.033954 \\
\hline 19 & C19 & -8.402347 & -1.268358 & -0.850473 \\
\hline 20 & $\mathrm{C} 20$ & -5.676372 & -3.436656 & -5.365398 \\
\hline 21 & $\mathrm{C} 21$ & -2.600474 & -7.064431 & -5.246101 \\
\hline 22 & $\mathrm{C} 22$ & -2.467562 & -3.783446 & -8.636169 \\
\hline 23 & $\mathrm{C} 23$ & -3.352253 & -2.531760 & -8.691697 \\
\hline 24 & $\mathrm{C} 24$ & -1.349293 & -3.652722 & -9.673348 \\
\hline 25 & $\mathrm{O} 25$ & -3.289570 & -4.896624 & -9.020893 \\
\hline 26 & $\mathrm{H} 26$ & -3.967852 & -3.047741 & -2.321010 \\
\hline 27 & $\mathrm{H} 27$ & -5.576732 & -4.923513 & -2.254616 \\
\hline 28 & $\mathrm{H} 28$ & -6.627743 & -5.238833 & -3.611061 \\
\hline 29 & $\mathrm{H} 29$ & -7.648410 & -3.800600 & -1.794753 \\
\hline 30 & H30 & -7.718315 & -3.087625 & -3.396519 \\
\hline 31 & H31 & -5.778330 & -2.364975 & -1.133793 \\
\hline 32 & $\mathrm{H} 32$ & -2.675314 & -1.345478 & -3.460957 \\
\hline 33 & H33 & -3.434294 & -1.693607 & -4.994760 \\
\hline 34 & H34 & -1.804443 & -3.606590 & -3.256291 \\
\hline 35 & H35 & -1.285951 & -2.910875 & -4.782077 \\
\hline 36 & H36 & -1.415095 & -3.119270 & -6.865814 \\
\hline 37 & $\mathrm{H} 37$ & -0.027485 & -4.981797 & -7.657594 \\
\hline 38 & H38 & -1.392772 & -6.091682 & -7.615666 \\
\hline 39 & H39 & 0.014486 & -4.795978 & -5.250372 \\
\hline 40 & $\mathrm{H} 40$ & -0.004197 & -6.496555 & -5.672627 \\
\hline 41 & $\mathrm{H} 41$ & -1.580964 & -5.898116 & -3.794435 \\
\hline 42 & $\mathrm{H} 42$ & -5.697263 & 0.628579 & -1.907809 \\
\hline 43 & $\mathrm{H} 43$ & -7.134647 & 1.138492 & -1.049196 \\
\hline 44 & $\mathrm{H} 44$ & -5.994691 & 0.025187 & -0.261734 \\
\hline 45 & $\mathrm{H} 45$ & -7.068950 & -0.425810 & -3.656609 \\
\hline
\end{tabular}




\begin{tabular}{llrrr}
$\mathbf{4 6}$ & $\mathrm{H} 46$ & -9.021183 & -0.388294 & -0.639391 \\
$\mathbf{4 7}$ & $\mathrm{H} 47$ & -9.076466 & -2.001981 & -1.305046 \\
$\mathbf{4 8}$ & $\mathrm{H} 48$ & -8.035103 & -1.666976 & 0.100593 \\
$\mathbf{4 9}$ & $\mathrm{H} 49$ & -5.001866 & -2.886266 & -6.020739 \\
$\mathbf{5 0}$ & $\mathrm{H} 50$ & -6.010310 & -4.318771 & -5.923254 \\
$\mathbf{5 1}$ & $\mathrm{H} 51$ & -6.538301 & -2.782593 & -5.208292 \\
$\mathbf{5 2}$ & $\mathrm{H} 52$ & -3.395164 & -7.306410 & -4.532458 \\
$\mathbf{5 3}$ & $\mathrm{H} 53$ & -3.056069 & -6.992052 & -6.238570 \\
$\mathbf{5 4}$ & $\mathrm{H} 54$ & -1.905154 & -7.910757 & -5.251205 \\
$\mathbf{5 5}$ & $\mathrm{H} 55$ & -3.707917 & -2.352161 & -9.713132 \\
$\mathbf{5 6}$ & $\mathrm{H} 56$ & -4.252461 & -2.649852 & -8.083296 \\
$\mathbf{5 7}$ & $\mathrm{H} 57$ & -2.811691 & -1.641856 & -8.353844 \\
$\mathbf{5 8}$ & $\mathrm{H} 58$ & -0.802482 & -4.593443 & -9.796607 \\
$\mathbf{5 9}$ & $\mathrm{H} 59$ & -1.761401 & -3.421530 & -10.662754 \\
$\mathbf{6 0}$ & $\mathrm{H} 60$ & -0.641298 & -2.862900 & -9.402781 \\
$\mathbf{6 1}$ & $\mathrm{H} 61$ & -3.851285 & -5.080858 & -8.240729 \\
\hline
\end{tabular}


Table S21. Conformer 1d-B/5d-B. PCM- $\mathrm{CHCl}_{3}$ energy and Boltzmann Populations 1160.09895700992 hartrees, $17.37 \%$.

\begin{tabular}{|c|c|c|c|c|}
\hline \multicolumn{2}{|c|}{ Atom number } & \multicolumn{3}{|c|}{ Coordinates } \\
\hline & & $x$ & $\mathbf{Y}$ & Z \\
\hline 1 & 01 & -5.448123 & -1.674230 & -3.163589 \\
\hline 2 & $\mathrm{C} 2$ & -4.445885 & -2.666449 & -3.381556 \\
\hline 3 & C3 & -5.029834 & -3.889371 & -4.105897 \\
\hline 4 & C4 & -6.083427 & -4.488902 & -3.149532 \\
\hline 5 & C5 & -7.104818 & -3.445149 & -2.679673 \\
\hline 6 & C6 & -6.437702 & -2.139341 & -2.210499 \\
\hline 7 & $\mathrm{C} 7$ & -3.257798 & -2.103120 & -4.147022 \\
\hline 8 & $\mathrm{C} 8$ & -2.220647 & -3.213354 & -4.307727 \\
\hline 9 & $\mathrm{C9}$ & -2.803858 & -4.485896 & -4.977121 \\
\hline 10 & 010 & -4.014437 & -4.884127 & -4.296511 \\
\hline 11 & 011 & -3.075641 & -4.287654 & -6.369866 \\
\hline 12 & C12 & -1.938676 & -4.037250 & -7.222138 \\
\hline 13 & C13 & -0.916580 & -5.177128 & -7.109394 \\
\hline 14 & C14 & -0.566895 & -5.480699 & -5.659057 \\
\hline 15 & C15 & -1.828236 & -5.687469 & -4.816875 \\
\hline 16 & C16 & -7.422819 & -0.963772 & -1.951255 \\
\hline 17 & C17 & -8.429693 & -1.288799 & -0.847941 \\
\hline 18 & 018 & -6.638985 & 0.153723 & -1.488988 \\
\hline 19 & C19 & -8.152637 & -0.492698 & -3.213910 \\
\hline 20 & $\mathrm{C} 20$ & -5.722583 & -3.546213 & -5.437490 \\
\hline 21 & $\mathrm{C} 21$ & -2.493078 & -7.029587 & -5.177184 \\
\hline 22 & $\mathrm{C} 22$ & -2.478547 & -3.861005 & -8.677351 \\
\hline 23 & $\mathrm{C} 23$ & -3.412051 & -2.648303 & -8.779481 \\
\hline 24 & $\mathrm{C} 24$ & -1.360446 & -3.720800 & -9.713467 \\
\hline 25 & $\mathrm{O} 25$ & -3.253206 & -5.018654 & -9.027471 \\
\hline 26 & $\mathrm{H} 26$ & -4.052438 & -2.975825 & -2.401199 \\
\hline 27 & $\mathrm{H} 27$ & -5.576449 & -4.914010 & -2.272332 \\
\hline 28 & $\mathrm{H} 28$ & -6.597415 & -5.334816 & -3.623137 \\
\hline 29 & $\mathrm{H} 29$ & -7.708182 & -3.872930 & -1.871158 \\
\hline 30 & $\mathrm{H} 30$ & -7.794773 & -3.244518 & -3.504899 \\
\hline 31 & $\mathrm{H} 31$ & -5.897931 & -2.333185 & -1.272721 \\
\hline 32 & $\mathrm{H} 32$ & -2.825809 & -1.260704 & -3.593483 \\
\hline 33 & $\mathrm{H} 33$ & -3.558249 & -1.695482 & -5.118139 \\
\hline 34 & H34 & -1.861347 & -3.474494 & -3.303098 \\
\hline 35 & $\mathrm{H} 35$ & -1.360769 & -2.810359 & -4.848876 \\
\hline 36 & $\mathrm{H} 36$ & -1.466248 & -3.094590 & -6.924943 \\
\hline 37 & $\mathrm{H} 37$ & 0.003197 & -4.922554 & -7.647396 \\
\hline 38 & $\mathrm{H} 38$ & -1.314641 & -6.086921 & -7.573675 \\
\hline 39 & H39 & 0.022319 & -4.652490 & -5.247775 \\
\hline 40 & $\mathrm{H} 40$ & 0.078698 & -6.365724 & -5.611377 \\
\hline 41 & $\mathrm{H} 41$ & -1.533525 & -5.771101 & -3.762108 \\
\hline 42 & $\mathrm{H} 42$ & -9.017995 & -0.401949 & -0.584989 \\
\hline 43 & $\mathrm{H} 43$ & -9.122421 & -2.082279 & -1.144194 \\
\hline 44 & $\mathrm{H} 44$ & -7.915116 & -1.598912 & 0.068544 \\
\hline 45 & $\mathrm{H} 45$ & -5.884109 & 0.207051 & -2.109464 \\
\hline 46 & $\mathrm{H} 46$ & -8.733267 & 0.414002 & -3.006460 \\
\hline 47 & $\mathrm{H} 47$ & -7.445642 & -0.224871 & -4.006694 \\
\hline 48 & $\mathrm{H} 48$ & -8.838836 & -1.251112 & -3.601520 \\
\hline 49 & $\mathrm{H} 49$ & -6.611379 & -2.922780 & -5.308048 \\
\hline 50 & $\mathrm{H} 50$ & -5.067622 & -2.992499 & -6.109614 \\
\hline
\end{tabular}




\begin{tabular}{lllll}
$\mathbf{5 1}$ & H51 & -6.016707 & -4.460976 & -5.964339 \\
$\mathbf{5 2}$ & H52 & -3.280957 & -7.281021 & -4.459257 \\
$\mathbf{5 3}$ & H53 & -2.945277 & -7.011110 & -6.173667 \\
$\mathbf{5 4}$ & H54 & -1.762006 & -7.844796 & -5.149792 \\
$\mathbf{5 5}$ & H55 & -2.908977 & -1.726412 & -8.470734 \\
$\mathbf{5 6}$ & H56 & -3.769428 & -2.518363 & -9.807807 \\
$\mathbf{5 7}$ & H57 & -4.309843 & -2.781024 & -8.170551 \\
$\mathbf{5 8}$ & H58 & -0.776087 & -4.642629 & -9.802408 \\
$\mathbf{5 9}$ & H59 & -1.775786 & -3.539657 & -10.711906 \\
$\mathbf{6 0}$ & H60 & -0.685890 & -2.894727 & -9.466757 \\
$\mathbf{6 1}$ & H61 & -3.810020 & -5.199826 & -8.243048 \\
\hline
\end{tabular}


Table S22. Conformer 1d-C/5d-C. PCM- $\mathrm{CHCl}_{3}$ energy and Boltzmann Populations 1160.09383378936 hartrees, $32.78 \%$.

\begin{tabular}{|c|c|c|c|c|}
\hline \multicolumn{2}{|c|}{ Atom number } & \multicolumn{3}{|c|}{ Coordinates } \\
\hline & & $x$ & $\mathbf{Y}$ & $\mathbf{Z}$ \\
\hline 1 & 01 & -5.355525 & -1.640370 & -3.018368 \\
\hline 2 & $\mathrm{C} 2$ & -4.377110 & -2.653516 & -3.244873 \\
\hline 3 & $\mathrm{C} 3$ & -5.014999 & -3.894684 & -3.887105 \\
\hline 4 & $\mathrm{C} 4$ & -6.025419 & -4.433659 & -2.852624 \\
\hline 5 & C5 & -6.997169 & -3.350262 & -2.367245 \\
\hline 6 & C6 & -6.282768 & -2.038939 & -1.977075 \\
\hline 7 & $\mathrm{C} 7$ & -3.221728 & -2.140980 & -4.091599 \\
\hline 8 & $\mathrm{C} 8$ & -2.211049 & -3.275044 & -4.259289 \\
\hline 9 & C9 & -2.849832 & -4.559099 & -4.852087 \\
\hline 10 & O10 & -4.023366 & -4.912294 & -4.085121 \\
\hline 11 & O11 & -3.205156 & -4.395235 & -6.228919 \\
\hline 12 & C12 & -2.124405 & -4.210351 & -7.154114 \\
\hline 13 & C13 & -1.120854 & -5.370241 & -7.080775 \\
\hline 14 & C14 & -0.675812 & -5.621310 & -5.645616 \\
\hline 15 & C15 & -1.881790 & -5.770618 & -4.711814 \\
\hline 16 & C16 & -7.256317 & -0.845902 & -1.746732 \\
\hline 17 & C17 & -6.488514 & 0.405412 & -1.298473 \\
\hline 18 & 018 & -7.895932 & -0.523013 & -2.991604 \\
\hline 19 & C19 & -8.352880 & -1.165187 & -0.729809 \\
\hline 20 & $\mathrm{C} 20$ & -5.774332 & -3.596523 & -5.194036 \\
\hline 21 & $\mathrm{C} 21$ & -2.590933 & -7.113323 & -4.971659 \\
\hline 22 & $\mathrm{C} 22$ & -2.692430 & -3.997648 & -8.592422 \\
\hline 23 & $\mathrm{C} 23$ & -3.567274 & -5.165656 & -9.064952 \\
\hline 24 & $\mathrm{C} 24$ & -3.508306 & -2.699041 & -8.658664 \\
\hline 25 & $\mathrm{O} 25$ & -1.595318 & -3.856910 & -9.507712 \\
\hline 26 & $\mathrm{H} 26$ & -3.938106 & -2.928742 & -2.273656 \\
\hline 27 & $\mathrm{H} 27$ & -5.479302 & -4.837089 & -1.988780 \\
\hline 28 & $\mathrm{H} 28$ & -6.582464 & -5.284789 & -3.264107 \\
\hline 29 & $\mathrm{H} 29$ & -7.561474 & -3.740693 & -1.512741 \\
\hline 30 & $\mathrm{H} 30$ & -7.729867 & -3.155056 & -3.158114 \\
\hline 31 & $\mathrm{H} 31$ & -5.709916 & -2.206152 & -1.054678 \\
\hline 32 & H32 & -2.749377 & -1.284857 & -3.595059 \\
\hline 33 & $\mathrm{H} 33$ & -3.565845 & -1.767378 & -5.061947 \\
\hline 34 & H34 & -1.807441 & -3.506459 & -3.264250 \\
\hline 35 & H35 & -1.371978 & -2.908124 & -4.855825 \\
\hline 36 & H36 & -1.585096 & -3.291940 & -6.893855 \\
\hline 37 & $\mathrm{H} 37$ & -0.243181 & -5.160447 & -7.702431 \\
\hline 38 & H38 & -1.569656 & -6.291048 & -7.466870 \\
\hline 39 & H39 & -0.047382 & -4.787961 & -5.309661 \\
\hline 40 & $\mathrm{H} 40$ & -0.042999 & -6.515828 & -5.605048 \\
\hline 41 & $\mathrm{H} 41$ & -1.516887 & -5.818643 & -3.676972 \\
\hline 42 & $\mathrm{H} 42$ & -5.757722 & 0.719201 & -2.052020 \\
\hline 43 & $\mathrm{H} 43$ & -7.170575 & 1.253658 & -1.167958 \\
\hline 44 & $\mathrm{H} 44$ & -5.962941 & 0.235367 & -0.353228 \\
\hline 45 & $\mathrm{H} 45$ & -7.173564 & -0.504665 & -3.650999 \\
\hline 46 & $\mathrm{H} 46$ & -8.988504 & -0.288940 & -0.556100 \\
\hline 47 & $\mathrm{H} 47$ & -9.023769 & -1.949424 & -1.096075 \\
\hline 48 & $\mathrm{H} 48$ & -7.931123 & -1.480142 & 0.229969 \\
\hline 49 & $\mathrm{H} 49$ & -5.152586 & -3.080285 & -5.925688 \\
\hline 50 & H50 & -6.107847 & -4.528481 & -5.664388 \\
\hline
\end{tabular}




\begin{tabular}{rrrrr}
$\mathbf{5 1}$ & $\mathrm{H} 51$ & -6.647862 & -2.956591 & -5.042618 \\
$\mathbf{5 2}$ & $\mathrm{H} 52$ & -3.114225 & -7.123629 & -5.932872 \\
$\mathbf{5 3}$ & $\mathrm{H} 53$ & -1.872249 & -7.939887 & -4.965638 \\
$\mathbf{5 4}$ & $\mathrm{H} 54$ & -3.328253 & -7.323788 & -4.189663 \\
$\mathbf{5 5}$ & $\mathrm{H} 55$ & -3.979881 & -4.961633 & -10.060192 \\
$\mathbf{5 6}$ & $\mathrm{H} 56$ & -2.984838 & -6.086448 & -9.169101 \\
$\mathbf{5 7}$ & $\mathrm{H} 57$ & -4.403187 & -5.352625 & -8.383575 \\
$\mathbf{5 8}$ & $\mathrm{H} 58$ & -2.904471 & -1.843390 & -8.335793 \\
$\mathbf{5 9}$ & $\mathrm{H} 59$ & -3.818085 & -2.484132 & -9.688112 \\
$\mathbf{6 0}$ & $\mathrm{H} 60$ & -4.406263 & -2.745888 & -8.037777 \\
$\mathbf{6 1}$ & $\mathrm{H} 61$ & -1.967950 & -3.739963 & -10.399821 \\
\hline
\end{tabular}


Table S23. Conformer 1d-D/5d-D. PCM- $\mathrm{CHCl}_{3}$ energy and Boltzmann Populations 1160.09408876724 hartrees, $24.33 \%$.

\begin{tabular}{|c|c|c|c|c|}
\hline \multicolumn{2}{|c|}{ Atom number } & \multicolumn{3}{|c|}{ Coordinates } \\
\hline & & $x$ & $\mathbf{Y}$ & $\mathbf{Z}$ \\
\hline 1 & 01 & -5.499278 & -1.662425 & -3.149121 \\
\hline 2 & $\mathrm{C} 2$ & -4.469779 & -2.632740 & -3.337314 \\
\hline 3 & C3 & -5.039185 & -3.933078 & -3.926033 \\
\hline 4 & $\mathrm{C} 4$ & -6.016428 & -4.487516 & -2.866683 \\
\hline 5 & C5 & -7.054709 & -3.446096 & -2.429921 \\
\hline 6 & C6 & -6.419755 & -2.080667 & -2.109056 \\
\hline 7 & $\mathrm{C} 7$ & -3.342905 & -2.094158 & -4.206216 \\
\hline 8 & $\mathrm{C} 8$ & -2.272567 & -3.178077 & -4.329156 \\
\hline 9 & $\mathrm{C} 9$ & -2.841221 & -4.516708 & -4.869722 \\
\hline 10 & 010 & -3.993612 & -4.902149 & -4.086528 \\
\hline 11 & 011 & -3.205535 & -4.425901 & -6.250949 \\
\hline 12 & C12 & -2.136570 & -4.219349 & -7.185239 \\
\hline 13 & C13 & -1.072882 & -5.320723 & -7.069838 \\
\hline 14 & C14 & -0.614014 & -5.491615 & -5.627254 \\
\hline 15 & C15 & -1.809597 & -5.668272 & -4.684923 \\
\hline 16 & C16 & -7.438854 & -0.924752 & -1.897337 \\
\hline 17 & C17 & -8.375097 & -1.191119 & -0.718672 \\
\hline 18 & 018 & -6.680229 & 0.256829 & -1.572950 \\
\hline 19 & C19 & -8.249645 & -0.591518 & -3.154491 \\
\hline 20 & C20 & -5.815019 & -3.730021 & -5.241498 \\
\hline 21 & $\mathrm{C} 21$ & -2.446262 & -7.056051 & -4.890192 \\
\hline 22 & $\mathrm{C} 22$ & -2.715807 & -4.092490 & -8.629165 \\
\hline 23 & $\mathrm{C} 23$ & -3.530944 & -5.320888 & -9.052752 \\
\hline 24 & $\mathrm{C} 24$ & -3.596274 & -2.840289 & -8.744091 \\
\hline 25 & $\mathrm{O} 25$ & -1.628155 & -3.932726 & -9.552576 \\
\hline 26 & $\mathrm{H} 26$ & -4.016787 & -2.843975 & -2.356561 \\
\hline 27 & $\mathrm{H} 27$ & -5.447776 & -4.817374 & -1.986324 \\
\hline 28 & $\mathrm{H} 28$ & -6.518477 & -5.389198 & -3.239470 \\
\hline 29 & $\mathrm{H} 29$ & -7.596745 & -3.825235 & -1.556292 \\
\hline 30 & $\mathrm{H} 30$ & -7.794568 & -3.343034 & -3.229438 \\
\hline 31 & H31 & -5.826115 & -2.172673 & -1.188464 \\
\hline 32 & H32 & -2.917444 & -1.194231 & -3.746071 \\
\hline 33 & $\mathrm{H} 33$ & -3.706304 & -1.779782 & -5.190466 \\
\hline 34 & $\mathrm{H} 34$ & -1.858248 & -3.347724 & -3.326122 \\
\hline 35 & H35 & -1.453664 & -2.790873 & -4.940715 \\
\hline 36 & H36 & -1.646673 & -3.264314 & -6.961699 \\
\hline 37 & H37 & -0.208005 & -5.089034 & -7.701550 \\
\hline 38 & H38 & -1.472498 & -6.278323 & -7.418684 \\
\hline 39 & H39 & -0.030667 & -4.613515 & -5.325743 \\
\hline 40 & $\mathrm{H} 40$ & 0.065614 & -6.348916 & -5.553669 \\
\hline 41 & $\mathrm{H} 41$ & -1.441874 & -5.656187 & -3.650038 \\
\hline 42 & $\mathrm{H} 42$ & -7.803021 & -1.401875 & 0.191954 \\
\hline 43 & $\mathrm{H} 43$ & -8.986139 & -0.307581 & -0.500174 \\
\hline 44 & $\mathrm{H} 44$ & -9.047973 & -2.032488 & -0.910047 \\
\hline 45 & $\mathrm{H} 45$ & -5.959386 & 0.283834 & -2.234312 \\
\hline 46 & $\mathrm{H} 46$ & -8.856560 & 0.307329 & -2.993212 \\
\hline 47 & $\mathrm{H} 47$ & -7.594769 & -0.366561 & -4.003389 \\
\hline 48 & $\mathrm{H} 48$ & -8.922099 & -1.405486 & -3.440095 \\
\hline 49 & $\mathrm{H} 49$ & -5.222419 & -3.212433 & -5.996005 \\
\hline 50 & $\mathrm{H} 50$ & -6.099356 & -4.696983 & -5.671708 \\
\hline 51 & H51 & -6.720779 & -3.131070 & -5.113887 \\
\hline
\end{tabular}




\begin{tabular}{rrrrr}
$\mathbf{5 2}$ & $\mathrm{H} 52$ & -3.170098 & -7.275084 & -4.098025 \\
$\mathbf{5 3}$ & $\mathrm{H} 53$ & -2.969695 & -7.131403 & -5.848413 \\
$\mathbf{5 4}$ & $\mathrm{H} 54$ & -1.684446 & -7.842233 & -4.854867 \\
$\mathbf{5 5}$ & $\mathrm{H} 55$ & -3.953192 & -5.177943 & -10.054577 \\
$\mathbf{5 6}$ & $\mathrm{H} 56$ & -2.903066 & -6.214789 & -9.120895 \\
$\mathbf{5 7}$ & $\mathrm{H} 57$ & -4.356533 & -5.521984 & -8.362856 \\
$\mathbf{5 8}$ & $\mathrm{H} 58$ & -3.035474 & -1.943037 & -8.458687 \\
$\mathbf{5 9}$ & $\mathrm{H} 59$ & -3.918989 & -2.683047 & -9.779957 \\
$\mathbf{6 0}$ & $\mathrm{H} 60$ & -4.489167 & -2.906924 & -8.117730 \\
$\mathbf{6 1}$ & $\mathrm{H} 61$ & -2.007023 & -3.870353 & -10.447519 \\
\hline
\end{tabular}


Table S24. J-DP4 results for 1a-1d (Isomers 1-4).

\begin{tabular}{|c|c|c|c|c|c|}
\hline Atom/J & Exp & 1a $(14 R-15 R)$ & 1b (14R-15S) & 1c $(14 S-15 R)$ & 1d (14S-15S) \\
\hline $\mathrm{C}$ & 86.00 & 106.86 & 106.76 & 106.19 & 105.98 \\
\hline $\mathrm{C}$ & 23.30 & 164.93 & 164.94 & 165.17 & 165.32 \\
\hline C & 38.70 & 151.48 & 151.62 & 152.33 & 152.34 \\
\hline $\mathrm{C}$ & 72.00 & 117.44 & 117.47 & 116.47 & 116.59 \\
\hline $\mathrm{C}$ & 75.60 & 114.91 & 115.08 & 110.08 & 110.05 \\
\hline $\mathrm{C}$ & 20.30 & 169.06 & 169.04 & 168.48 & 167.98 \\
\hline $\mathrm{C}$ & 27.90 & 161.45 & 160.64 & 166.09 & 159.04 \\
\hline C & 99.10 & 89.09 & 89.36 & 87.12 & 88.80 \\
\hline $\mathrm{C}$ & 38.80 & 150.08 & 151.18 & 148.84 & 150.83 \\
\hline $\mathrm{C}$ & 27.40 & 162.18 & 163.39 & 161.84 & 162.99 \\
\hline C & 27.10 & 163.68 & 169.26 & 164.08 & 169.18 \\
\hline $\mathrm{C}$ & 74.60 & 114.41 & 113.79 & 110.53 & 111.37 \\
\hline C & 19.70 & 170.74 & 170.91 & 170.77 & 170.91 \\
\hline $\mathrm{C}$ & 17.10 & 173.00 & 175.35 & 173.43 & 176.46 \\
\hline $\mathrm{H}$ & 3.06 & 28.29 & 28.30 & 28.26 & 28.29 \\
\hline $\mathrm{H}$ & 1.73 & 30.18 & 30.18 & 30.19 & 30.21 \\
\hline $\mathrm{H}$ & 1.43 & 29.85 & 29.87 & 29.76 & 29.77 \\
\hline $\mathrm{H}$ & 1.59 & 29.91 & 29.91 & 30.18 & 30.20 \\
\hline $\mathrm{H}$ & 1.78 & 29.86 & 29.88 & 29.96 & 29.93 \\
\hline $\mathrm{H}$ & 3.96 & 27.49 & 27.55 & 28.41 & 28.39 \\
\hline $\mathrm{H}$ & 1.87 & 29.64 & 29.70 & 30.19 & 30.19 \\
\hline $\mathrm{H}$ & 1.35 & 30.18 & 30.17 & 29.75 & 29.74 \\
\hline $\mathrm{H}$ & 1.55 & 30.09 & 29.86 & 30.13 & 30.34 \\
\hline $\mathrm{H}$ & 2.17 & 29.40 & 29.76 & 29.47 & 29.07 \\
\hline $\mathrm{H}$ & 1.35 & 30.25 & 30.17 & 30.15 & 30.14 \\
\hline $\mathrm{H}$ & 1.56 & 29.99 & 29.45 & 30.39 & 29.92 \\
\hline $\mathrm{H}$ & 1.38 & 30.40 & 30.46 & 30.17 & 30.21 \\
\hline $\mathrm{H}$ & 1.53 & 30.19 & 30.48 & 30.26 & 30.52 \\
\hline $\mathrm{H}$ & 1.18 & 30.25 & 30.08 & 30.15 & 29.84 \\
\hline $\mathrm{H}$ & 3.77 & 27.73 & 27.68 & 28.10 & 28.05 \\
\hline $\mathrm{H}$ & 1.12 & 30.46 & 30.48 & 30.25 & 30.17 \\
\hline $\mathrm{H}$ & 0.84 & 30.85 & 30.75 & 30.83 & 30.52 \\
\hline${ }^{3} J_{\mathrm{H} 7-\mathrm{H} 8 \mathrm{a}}$ & 2.40 & 2.50 & 2.49 & 2.81 & 2.75 \\
\hline${ }^{3} J_{H 7-H 8 b}$ & 11.40 & 10.51 & 10.53 & 10.66 & 10.65 \\
\hline${ }^{3} J_{\mathrm{H} 8 \mathrm{a}-\mathrm{H} 9 \mathrm{a}}$ & 4.30 & 4.47 & 4.47 & 4.69 & 4.72 \\
\hline${ }^{3} J_{\text {H8a-H9b }}$ & 3.00 & 2.38 & 2.38 & 2.17 & 2.16 \\
\hline${ }^{3} J_{\mathrm{H} 8 \mathrm{~b}-\mathrm{H} 9 \mathrm{a}}$ & 13.50 & 12.67 & 12.68 & 12.54 & 12.57 \\
\hline${ }^{3} J_{H 8 b-H 9 b}$ & 2.60 & 4.31 & 4.29 & 4.66 & 4.65 \\
\hline${ }^{3} J_{\mathrm{H} 11-\mathrm{H} 12 \mathrm{a}}$ & 8.10 & 7.32 & 7.21 & 3.36 & 3.38 \\
\hline${ }^{3} J_{H 11-H 12 b}$ & 9.80 & 9.38 & 9.64 & 11.21 & 11.21 \\
\hline${ }^{3} J_{\mathrm{H} 12 \mathrm{a}-\mathrm{H} 13 \mathrm{a}}$ & 10.50 & 10.01 & 7.50 & 4.11 & 4.12 \\
\hline${ }^{3} J_{H 12 a-H 13 b}$ & 8.00 & 7.03 & 9.53 & 2.87 & 2.88 \\
\hline${ }^{3} J_{H 12 b-H 13 a}$ & 1.00 & 1.03 & 8.69 & 12.18 & 12.35 \\
\hline
\end{tabular}




$\begin{array}{lccccc}{ }^{3} J_{\mathrm{H} 12 \mathrm{~b}-\mathrm{H} 13 \mathrm{~b}} & 11.20 & 10.37 & 2.91 & 3.53 & 3.58 \\ { }^{3} \mathrm{~J}_{\mathrm{H} 18-\mathrm{H} 17 \mathrm{a}} & 2.30 & 2.12 & 2.40 & 2.31 & 2.97 \\ { }^{3} \mathrm{~J}_{\mathrm{H} 18-\mathrm{H} 17 \mathrm{~b}} & 12.50 & 10.71 & 10.89 & 10.60 & 10.69\end{array}$

\begin{tabular}{|c|c|c|c|c|c|}
\hline & $\mathrm{H}$ & 100.00 & 0.00 & 0.00 & 0.00 \\
\cline { 2 - 6 } & $\mathrm{C}$ & 98.96 & 0.66 & 0.35 & 0.02 \\
\cline { 2 - 6 } DP4 (\%) & $\mathrm{H}+\mathrm{C}$ & 100.00 & 0.00 & 0.00 & 0.00 \\
\cline { 2 - 6 } & $\mathrm{J}$ & 100.00 & 0.00 & 0.00 & 0.00 \\
\cline { 2 - 6 } & all data & $\mathbf{1 0 0 . 0 0}$ & $\mathbf{0 . 0 0}$ & $\mathbf{0 . 0 0}$ & $\mathbf{0 . 0 0}$ \\
\hline
\end{tabular}


Table S25. J-DP4 results for 5a-5d (Isomers 1-4).

\begin{tabular}{|c|c|c|c|c|c|}
\hline Atom/J & Exp & 5a $(14 R-15 R)$ & 5b $(14 R-15 S)$ & 5c $(14 S-15 R)$ & 5d (14S-15S) \\
\hline $\mathrm{C}$ & 83.20 & 106.86 & 106.76 & 106.19 & 105.98 \\
\hline C & 24.50 & 164.93 & 164.94 & 165.17 & 165.32 \\
\hline C & 38.70 & 151.48 & 151.62 & 152.33 & 152.34 \\
\hline C & 71.80 & 117.44 & 117.47 & 116.47 & 116.59 \\
\hline C & 75.50 & 114.91 & 115.08 & 110.08 & 110.05 \\
\hline C & 20.20 & 169.06 & 169.04 & 168.48 & 167.98 \\
\hline $\mathrm{C}$ & 27.80 & 161.45 & 160.64 & 166.09 & 159.04 \\
\hline C & 99.00 & 89.09 & 89.36 & 87.12 & 88.80 \\
\hline C & 38.60 & 150.08 & 151.18 & 148.84 & 150.83 \\
\hline C & 27.20 & 162.18 & 163.39 & 161.84 & 162.99 \\
\hline $\mathrm{C}$ & 27.00 & 163.68 & 169.26 & 164.08 & 169.18 \\
\hline C & 74.50 & 114.41 & 113.79 & 110.53 & 111.37 \\
\hline C & 19.50 & 170.74 & 170.91 & 170.77 & 170.91 \\
\hline C & 16.90 & 173.00 & 175.35 & 173.43 & 176.46 \\
\hline $\mathrm{H}$ & 3.30 & 28.29 & 28.30 & 28.26 & 28.29 \\
\hline $\mathrm{H}$ & 1.69 & 30.18 & 30.18 & 30.19 & 30.21 \\
\hline $\mathrm{H}$ & 1.51 & 29.85 & 29.87 & 29.76 & 29.77 \\
\hline $\mathrm{H}$ & 1.65 & 29.91 & 29.91 & 30.18 & 30.20 \\
\hline $\mathrm{H}$ & 1.83 & 29.86 & 29.88 & 29.96 & 29.93 \\
\hline $\mathrm{H}$ & 4.03 & 27.49 & 27.55 & 28.41 & 28.39 \\
\hline $\mathrm{H}$ & 1.93 & 29.64 & 29.70 & 30.19 & 30.19 \\
\hline $\mathrm{H}$ & 1.42 & 30.18 & 30.17 & 29.75 & 29.74 \\
\hline $\mathrm{H}$ & 1.58 & 30.09 & 29.86 & 30.13 & 30.34 \\
\hline $\mathrm{H}$ & 2.19 & 29.40 & 29.76 & 29.47 & 29.07 \\
\hline $\mathrm{H}$ & 1.38 & 30.25 & 30.17 & 30.15 & 30.14 \\
\hline $\mathrm{H}$ & 1.57 & 29.99 & 29.45 & 30.39 & 29.92 \\
\hline $\mathrm{H}$ & 1.42 & 30.40 & 30.46 & 30.17 & 30.21 \\
\hline $\mathrm{H}$ & 1.55 & 30.19 & 30.48 & 30.26 & 30.52 \\
\hline $\mathrm{H}$ & 1.20 & 30.25 & 30.08 & 30.15 & 29.84 \\
\hline $\mathrm{H}$ & 3.80 & 27.73 & 27.68 & 28.10 & 28.05 \\
\hline $\mathrm{H}$ & 1.17 & 30.46 & 30.48 & 30.25 & 30.17 \\
\hline $\mathrm{H}$ & 0.87 & 30.85 & 30.75 & 30.83 & 30.52 \\
\hline${ }^{3} J_{\mathrm{H} 7-\mathrm{H} 8 \mathrm{a}}$ & 2.60 & 2.50 & 2.49 & 2.81 & 2.75 \\
\hline${ }^{3} J_{\mathrm{H} 7-\mathrm{H} 8 \mathrm{~b}}$ & 11.80 & 10.51 & 10.53 & 10.66 & 10.65 \\
\hline${ }^{3} J_{\mathrm{H} 8 \mathrm{a}-\mathrm{H} 9 \mathrm{a}}$ & 4.50 & 4.47 & 4.47 & 4.69 & 4.72 \\
\hline${ }^{3} J_{\mathrm{H} 8 \mathrm{a}-\mathrm{H} 9 \mathrm{~b}}$ & 2.90 & 2.38 & 2.38 & 2.17 & 2.16 \\
\hline${ }^{3} J_{\mathrm{H} 8 \mathrm{~b}-\mathrm{H} 9 \mathrm{a}}$ & 13.50 & 12.67 & 12.68 & 12.54 & 12.57 \\
\hline${ }^{3} J_{\text {H8b-H9b }}$ & 2.90 & 4.31 & 4.29 & 4.66 & 4.65 \\
\hline${ }^{3} J_{H 11-H 12 a}$ & 8.10 & 7.32 & 7.21 & 3.36 & 3.38 \\
\hline${ }^{3} J_{H 11-H 12 b}$ & 10.00 & 9.38 & 9.64 & 11.21 & 11.21 \\
\hline${ }^{3} J_{\mathrm{H} 12 \mathrm{a}-\mathrm{H} 13 \mathrm{a}}$ & 10.40 & 10.01 & 7.50 & 4.11 & 4.12 \\
\hline${ }^{3} J_{H 12 a-H 13 b}$ & 7.50 & 7.03 & 9.53 & 2.87 & 2.88 \\
\hline${ }^{3} J_{\mathrm{H} 12 \mathrm{~b}-\mathrm{H} 13 \mathrm{a}}$ & 1.30 & 1.03 & 8.69 & 12.18 & 12.35 \\
\hline${ }^{3} J_{H 12 b-H 13 b}$ & 11.10 & 10.37 & 2.91 & 3.53 & 3.58 \\
\hline${ }^{3} J_{\mathrm{H} 15-\mathrm{H} 16 \mathrm{a}}$ & 12.50 & 11.33 & 4.24 & 11.95 & 3.92 \\
\hline
\end{tabular}




$\begin{array}{lccccc}3 J_{\mathrm{H} 15-\mathrm{H} 16 \mathrm{~b}} & 3.80 & 3.52 & 2.08 & 3.42 & 3.31 \\ { }^{3} J_{\mathrm{H} 16 \mathrm{a}-\mathrm{H} 17 \mathrm{a}} & 4.20 & 3.71 & 4.05 & 3.56 & 4.65 \\ { }^{3} J_{\mathrm{H} 16 \mathrm{a}-\mathrm{H} 17 \mathrm{~b}} & 13.00 & 12.43 & 12.94 & 12.48 & 11.95 \\ { }^{3} J_{\mathrm{H} 16 \mathrm{~b}-\mathrm{H} 17 \mathrm{a}} & 2.90 & 2.68 & 2.57 & 2.68 & 2.97 \\ { }^{3} J_{\mathrm{H} 16 \mathrm{~b}-\mathrm{H} 17 \mathrm{~b}} & 4.40 & 3.90 & 3.97 & 4.02 & 4.84 \\ { }^{3} J_{\mathrm{H} 18-\mathrm{H} 17 \mathrm{a}} & 2.30 & 2.12 & 2.40 & 2.31 & 2.97 \\ { }^{3} J_{\mathrm{H} 18-\mathrm{H} 17 \mathrm{~b}} & 12.50 & 10.71 & 10.89 & 10.60 & 10.69\end{array}$

\begin{tabular}{|c|c|c|c|c|c|}
\hline & $\mathrm{H}$ & 100.00 & 0.00 & 0.00 & 0.00 \\
\cline { 2 - 6 } DP4 (\%) & $\mathrm{C}$ & 98.79 & 0.74 & 0.45 & 0.02 \\
\cline { 2 - 6 } & $\mathrm{H}+\mathrm{C}$ & 100.00 & 0.00 & 0.00 & 0.00 \\
\cline { 2 - 6 } & $\mathrm{J}$ & 100.00 & 0.00 & 0.00 & 0.00 \\
\cline { 2 - 6 } & all data & $\mathbf{1 0 0 . 0 0}$ & $\mathbf{0 . 0 0}$ & $\mathbf{0 . 0 0}$ & $\mathbf{0 . 0 0}$ \\
\hline
\end{tabular}


Table S26. Scaled ${ }^{13} \mathrm{C}$ average chemical shifts for $\mathbf{2 a - 2} \mathbf{d} / \mathbf{3 a - 3} \mathbf{d}$ on B3LYP-D3 in PCM-CHCl 3 .

\begin{tabular}{|c|c|c|c|c|}
\hline C & $\mathbf{2 a} / 3 \mathbf{a}(14 R-15 R)$ & 2b/3b (14R-15S) & 2c/3c $(14 S-15 R)$ & $\mathbf{2 d} / \mathbf{3 d}(14 S-15 S)$ \\
\hline 7 & 82,5537907 & 82,1838891 & 81,3508066 & 81,7045147 \\
\hline 8 & 24,2537122 & 23,9028182 & 23,4398952 & 23,0610274 \\
\hline 9 & 37,4584160 & 37,0448910 & 35,9103475 & 35,6103902 \\
\hline 10 & 73,1919117 & 72,9008556 & 71,8524946 & 73,6933277 \\
\hline 11 & 73,5330028 & 73,5513178 & 76,5858649 & 77,2555980 \\
\hline 12 & 19,2344090 & 19,4920716 & 20,4168904 & 20,0396983 \\
\hline 13 & 23,2902863 & 23,9753742 & 23,0866273 & 25,1203813 \\
\hline 14 & 101,9605939 & 101,7398041 & 101,2467896 & 99,3955450 \\
\hline 15 & 72,1965495 & 71,8014036 & 71,2607126 & 70,3829578 \\
\hline 16 & 28,1363151 & 27,4688435 & 28,4850613 & 28,8473347 \\
\hline 17 & 23,1331804 & 21,1416935 & 22,2205777 & 20,1843941 \\
\hline 18 & 75,5736831 & 74,4557712 & 76,0586572 & 76,7474868 \\
\hline 27 & 17,9914831 & 18,0325313 & 17,6757105 & 17,6320577 \\
\hline 28 & 53,2926661 & 58,1087354 & 56,2095645 & 56,1252862 \\
\hline
\end{tabular}

Table S27. Scaled ${ }^{1} \mathrm{H}$ average chemical shifts for $\mathbf{2 a - 2 d / 3 a - 3 d}$ on B3LYP-D3 in PCM$\mathrm{CHCl}_{3}$.

\begin{tabular}{ccccc}
\hline $\mathbf{H}$ & $\mathbf{2 a} / \mathbf{3 a}(14 R-15 R)$ & $\mathbf{2 b} / \mathbf{3 b}(14 R-15 S)$ & $\mathbf{2 c} / \mathbf{3 c}(14 S-15 R)$ & $\mathbf{2 d} / \mathbf{3 d}(14 S-15 S)$ \\
\hline $\mathbf{7}$ & 3.3060752 & 3.2764358 & 3.3525464 & 3.7267500 \\
$\mathbf{8 a}$ & 1.7865999 & 1.7680556 & 1.8381808 & 1.9144785 \\
$\mathbf{8 b}$ & 1.5107448 & 1.4725334 & 1.4306757 & 1.4569163 \\
$\mathbf{9 a}$ & 1.7835108 & 1.7402810 & 1.6694055 & 1.7099697 \\
$\mathbf{9 b}$ & 1.7363288 & 1.7223694 & 1.4624911 & 1.6258767 \\
$\mathbf{1 1}$ & 3.9270499 & 3.9944390 & 3.2705657 & 3.6935298 \\
$\mathbf{1 2 a}$ & 1.4767939 & 1.4496518 & 1.8265686 & 2.0284532 \\
$\mathbf{1 2 b}$ & 1.8709048 & 1.9436329 & 1.4631519 & 1.4020754 \\
$\mathbf{1 3 a}$ & 2.1967052 & 1.8648547 & 2.2247175 & 2.3811529 \\
$\mathbf{1 3 b}$ & 1.4795821 & 2.0496578 & 2.1349425 & 1.4262972 \\
$\mathbf{1 6 a}$ & 1.9774396 & 1.7086814 & 1.7662602 & 1.6237268 \\
$\mathbf{1 6 b}$ & 1.5968416 & 1.7047466 & 1.3898893 & 1.2067652 \\
$\mathbf{1 7 a}$ & 1.5129324 & 1.6464327 & 1.5798815 & 1.9823512 \\
$\mathbf{1 7 b}$ & 1.4169147 & 1.3387146 & 1.2949362 & 1.0596866 \\
$\mathbf{1 8}$ & 3.8466050 & 3.8066413 & 3.6201071 & 3.8361886 \\
$\mathbf{2 7}$ & 1.2143268 & 1.1747687 & 1.3207331 & 1.3727083 \\
$\mathbf{2 8 a}$ & 3.4977224 & 3.5731959 & 4.2259595 & 3.6163727 \\
$\mathbf{2 8 b}$ & 3.7629222 & 3.6649074 & 4.0289873 & 3.8367008 \\
\hline
\end{tabular}




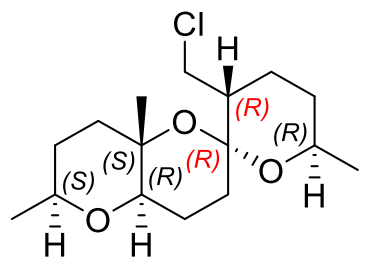

Table S28. Conformer 2a-A/3a-A. $\mathrm{PCM}-\mathrm{CHCl}_{3}$ energy and Boltzmann Populations 1694.8125931 hartrees, $35.55 \%$.

\begin{tabular}{|c|c|c|c|c|}
\hline \multicolumn{2}{|c|}{ Atom number } & \multicolumn{3}{|c|}{ Coordinates } \\
\hline & & $\mathbf{X}$ & $\mathbf{Y}$ & $\mathbf{Z}$ \\
\hline 1 & 0 & 2.83730 & -0.48820 & 0.81870 \\
\hline 2 & $\mathrm{C}$ & 1.42870 & -0.39390 & 0.64480 \\
\hline 3 & $\mathrm{C}$ & 1.01460 & -1.04950 & -0.68470 \\
\hline 4 & $\mathrm{C}$ & 1.71540 & -0.27030 & -1.80770 \\
\hline 5 & $\mathrm{C}$ & 3.23700 & -0.20380 & -1.57810 \\
\hline 6 & $\mathrm{C}$ & 3.58420 & 0.26600 & -0.15770 \\
\hline 7 & $\mathrm{C}$ & 0.72130 & -1.02240 & 1.84150 \\
\hline 8 & $\mathrm{C}$ & -0.74880 & -1.38700 & 1.50550 \\
\hline 9 & C & -1.27670 & -0.68940 & 0.24480 \\
\hline 10 & $\mathrm{O}$ & -0.41810 & -0.88140 & -0.87820 \\
\hline 11 & $\mathrm{O}$ & -1.39920 & 0.69010 & 0.58080 \\
\hline 12 & C & -1.79200 & 1.57680 & -0.49410 \\
\hline 13 & $\mathrm{C}$ & -3.16480 & 1.16310 & -1.02230 \\
\hline 14 & $\mathrm{C}$ & -3.14830 & -0.32280 & -1.41400 \\
\hline 15 & $\mathrm{C}$ & -2.66390 & -1.23610 & -0.26740 \\
\hline 16 & C & 5.07030 & 0.06790 & 0.24050 \\
\hline 17 & $\mathrm{C}$ & 5.33080 & 0.68040 & 1.62730 \\
\hline 18 & $\mathrm{O}$ & 5.34920 & -1.33780 & 0.27060 \\
\hline 19 & C & 6.02520 & 0.66740 & -0.79320 \\
\hline 20 & C & 1.34210 & -2.54690 & -0.75340 \\
\hline 21 & C & -3.64170 & -1.30200 & 0.91240 \\
\hline 22 & C & -1.64200 & 3.00790 & 0.07570 \\
\hline 23 & $\mathrm{C}$ & -1.76480 & 4.04770 & -1.03930 \\
\hline 24 & C & -2.62580 & 3.30280 & 1.21870 \\
\hline 25 & 0 & -0.29700 & 3.13020 & 0.56650 \\
\hline 26 & $\mathrm{Cl}$ & -5.35660 & -1.66740 & 0.43620 \\
\hline 27 & $\mathrm{O}$ & -2.49580 & -2.57080 & -0.72210 \\
\hline 28 & $\mathrm{H}$ & 1.15390 & 0.66830 & 0.57410 \\
\hline 29 & $\mathrm{H}$ & 1.49220 & -0.72430 & -2.78080 \\
\hline 30 & $\mathrm{H}$ & 1.29830 & 0.74470 & -1.82280 \\
\hline 31 & $\mathrm{H}$ & 3.69980 & -1.18300 & -1.74150 \\
\hline 32 & $\mathrm{H}$ & 3.67860 & 0.48490 & -2.30700 \\
\hline 33 & $\mathrm{H}$ & 3.31800 & 1.33060 & -0.04850 \\
\hline 34 & $\mathrm{H}$ & 1.26930 & -1.92270 & 2.13920 \\
\hline 35 & $\mathrm{H}$ & 0.76860 & -0.33210 & 2.68910 \\
\hline 36 & $\mathrm{H}$ & -0.85790 & -2.46310 & 1.34590 \\
\hline 37 & $\mathrm{H}$ & -1.39610 & -1.11950 & 2.34430 \\
\hline 38 & $\mathrm{H}$ & -1.05180 & 1.47570 & -1.29740 \\
\hline 39 & $\mathrm{H}$ & -3.93470 & 1.34480 & -0.26320 \\
\hline 40 & $\mathrm{H}$ & -3.42810 & 1.77140 & -1.89580 \\
\hline 41 & $\mathrm{H}$ & -4.13820 & -0.65730 & -1.73390 \\
\hline 42 & $\mathrm{H}$ & -2.46670 & -0.46770 & -2.25980 \\
\hline 43 & $\mathrm{H}$ & 5.19890 & 1.76910 & 1.61310 \\
\hline 44 & $\mathrm{H}$ & 4.64260 & 0.26590 & 2.36990 \\
\hline
\end{tabular}




\begin{tabular}{llrrr}
$\mathbf{4 5}$ & $\mathrm{H}$ & 6.35680 & 0.45880 & 1.93710 \\
$\mathbf{4 6}$ & $\mathrm{H}$ & 4.66450 & -1.72420 & 0.84300 \\
$\mathbf{4 7}$ & $\mathrm{H}$ & 5.82920 & 1.73550 & -0.93980 \\
$\mathbf{4 8}$ & $\mathrm{H}$ & 7.05650 & 0.55250 & -0.44490 \\
$\mathbf{4 9}$ & $\mathrm{H}$ & 5.93870 & 0.15590 & -1.75550 \\
$\mathbf{5 0}$ & $\mathrm{H}$ & 2.40780 & -2.72660 & -0.59090 \\
$\mathbf{5 1}$ & $\mathrm{H}$ & 0.78560 & -3.12060 & -0.00750 \\
$\mathbf{5 2}$ & $\mathrm{H}$ & 1.07640 & -2.93560 & -1.74290 \\
$\mathbf{5 3}$ & $\mathrm{H}$ & -3.35330 & -2.10890 & 1.58400 \\
$\mathbf{5 4}$ & $\mathrm{H}$ & -3.66620 & -0.35760 & 1.45470 \\
$\mathbf{5 5}$ & $\mathrm{H}$ & -1.05720 & 3.83410 & -1.84750 \\
$\mathbf{5 6}$ & $\mathrm{H}$ & -2.77730 & 4.07600 & -1.45410 \\
$\mathbf{5 7}$ & $\mathrm{H}$ & -1.52970 & 5.03840 & -0.63780 \\
$\mathbf{5 8}$ & $\mathrm{H}$ & -3.66500 & 3.33120 & 0.87320 \\
$\mathbf{5 9}$ & $\mathrm{H}$ & -2.38410 & 4.27580 & 1.65730 \\
$\mathbf{6 0}$ & $\mathrm{H}$ & -2.54820 & 2.54210 & 2.00300 \\
$\mathbf{6 1}$ & $\mathrm{H}$ & -0.18480 & 2.39300 & 1.18970 \\
$\mathbf{6 2}$ & $\mathrm{H}$ & -1.75060 & -2.53220 & -1.34640 \\
\hline
\end{tabular}


Table S29. Conformer 2a-B/3a-B. $\mathrm{PCM}-\mathrm{CHCl}_{3}$ energy and Boltzmann Populations 1694.8119361 hartrees, $17.73 \%$.

\begin{tabular}{|c|c|c|c|c|}
\hline \multicolumn{2}{|c|}{ Atom number } & \multicolumn{3}{|c|}{ Coordinates } \\
\hline & & $x$ & $\mathbf{Y}$ & $\mathbf{Z}$ \\
\hline 1 & $\mathrm{O}$ & -2.89900 & -0.38830 & -0.81630 \\
\hline 2 & $\mathrm{C}$ & -1.48920 & -0.31400 & -0.63700 \\
\hline 3 & $\mathrm{C}$ & -1.08140 & -1.02270 & 0.66650 \\
\hline 4 & $\mathrm{C}$ & -1.78220 & -0.28710 & 1.81850 \\
\hline 5 & $\mathrm{C}$ & -3.30250 & -0.20330 & 1.58930 \\
\hline 6 & $\mathrm{C}$ & -3.64450 & 0.32570 & 0.18880 \\
\hline 7 & $\mathrm{C}$ & -0.78770 & -0.90010 & -1.85720 \\
\hline 8 & $\mathrm{C}$ & 0.67500 & -1.29810 & -1.53210 \\
\hline 9 & C & 1.21930 & -0.66680 & -0.24270 \\
\hline 10 & $\mathrm{O}$ & 0.34880 & -0.86300 & 0.87210 \\
\hline 11 & $\mathrm{O}$ & 1.38840 & 0.71910 & -0.52340 \\
\hline 12 & C & 1.86050 & 1.56680 & 0.55290 \\
\hline 13 & $\mathrm{C}$ & 3.18260 & 1.03340 & 1.10730 \\
\hline 14 & $\mathrm{C}$ & 3.08570 & -0.46150 & 1.43800 \\
\hline 15 & $\mathrm{C}$ & 2.57780 & -1.29610 & 0.24680 \\
\hline 16 & $\mathrm{C}$ & -5.13080 & 0.15080 & -0.22090 \\
\hline 17 & C & -5.38470 & 0.82320 & -1.58100 \\
\hline 18 & 0 & -5.41490 & -1.25070 & -0.31130 \\
\hline 19 & C & -6.08600 & 0.70940 & 0.83520 \\
\hline 20 & $\mathrm{C}$ & -1.41580 & -2.52050 & 0.67630 \\
\hline 21 & $\mathrm{C}$ & 3.56690 & -1.34230 & -0.92460 \\
\hline 22 & $\mathrm{C}$ & 1.95020 & 2.98280 & -0.07720 \\
\hline 23 & $\mathrm{C}$ & 0.55550 & 3.47840 & -0.49660 \\
\hline 24 & $\mathrm{C}$ & 2.59790 & 3.98400 & 0.88180 \\
\hline 25 & 0 & 2.80500 & 2.92570 & -1.22530 \\
\hline 26 & $\mathrm{Cl}$ & 5.24820 & -1.83860 & -0.45080 \\
\hline 27 & 0 & 2.34890 & -2.64470 & 0.62930 \\
\hline 28 & $\mathrm{H}$ & -1.20050 & 0.74160 & -0.53080 \\
\hline 29 & $\mathrm{H}$ & -1.56260 & -0.78010 & 2.77330 \\
\hline 30 & $\mathrm{H}$ & -1.35780 & 0.72380 & 1.87670 \\
\hline 31 & $\mathrm{H}$ & -3.76950 & -1.18660 & 1.71090 \\
\hline 32 & $\mathrm{H}$ & -3.74350 & 0.45570 & 2.34550 \\
\hline 33 & $\mathrm{H}$ & -3.37540 & 1.39390 & 0.12500 \\
\hline 34 & $\mathrm{H}$ & -1.34590 & -1.78020 & -2.19420 \\
\hline 35 & $\mathrm{H}$ & -0.82330 & -0.17240 & -2.67330 \\
\hline 36 & $\mathrm{H}$ & 0.76750 & -2.38140 & -1.41940 \\
\hline 37 & $\mathrm{H}$ & 1.32890 & -1.00600 & -2.35760 \\
\hline 38 & $\mathrm{H}$ & 1.10110 & 1.57120 & 1.34630 \\
\hline 39 & $\mathrm{H}$ & 3.97650 & 1.22160 & 0.37540 \\
\hline 40 & $\mathrm{H}$ & 3.44740 & 1.58820 & 2.01440 \\
\hline 41 & $\mathrm{H}$ & 4.05390 & -0.85540 & 1.75750 \\
\hline 42 & $\mathrm{H}$ & 2.38420 & -0.60940 & 2.26710 \\
\hline 43 & $\mathrm{H}$ & -5.24990 & 1.91000 & -1.51970 \\
\hline 44 & $\mathrm{H}$ & -4.69520 & 0.43910 & -2.33840 \\
\hline 45 & $\mathrm{H}$ & -6.41040 & 0.61850 & -1.90320 \\
\hline 46 & $\mathrm{H}$ & -4.73330 & -1.61480 & -0.90190 \\
\hline 47 & $\mathrm{H}$ & -5.88740 & 1.77000 & 1.02680 \\
\hline 48 & $\mathrm{H}$ & -7.11690 & 0.61250 & 0.48030 \\
\hline 49 & $\mathrm{H}$ & -6.00310 & 0.15800 & 1.77550 \\
\hline 50 & $\mathrm{H}$ & -2.48230 & -2.68860 & 0.50660 \\
\hline 51 & $\mathrm{H}$ & -0.86170 & -3.06690 & -0.09150 \\
\hline
\end{tabular}




\begin{tabular}{rrrrr}
$\mathbf{5 2}$ & $\mathrm{H}$ & -1.15240 & -2.94890 & 1.64990 \\
$\mathbf{5 3}$ & $\mathrm{H}$ & 3.24430 & -2.08500 & -1.65250 \\
$\mathbf{5 4}$ & $\mathrm{H}$ & 3.65590 & -0.36700 & -1.40170 \\
$\mathbf{5 5}$ & $\mathrm{H}$ & -0.11210 & 3.57430 & 0.36850 \\
$\mathbf{5 6}$ & $\mathrm{H}$ & 0.64480 & 4.45880 & -0.97490 \\
$\mathbf{5 7}$ & $\mathrm{H}$ & 0.09330 & 2.78840 & -1.20900 \\
$\mathbf{5 8}$ & $\mathrm{H}$ & 2.04600 & 4.04030 & 1.82700 \\
$\mathbf{5 9}$ & $\mathrm{H}$ & 3.63540 & 3.71310 & 1.09330 \\
$\mathbf{6 0}$ & $\mathrm{H}$ & 2.59770 & 4.97820 & 0.42420 \\
$\mathbf{6 1}$ & $\mathrm{H}$ & 2.44840 & 2.20980 & -1.77800 \\
$\mathbf{6 2}$ & $\mathrm{H}$ & 1.62090 & -2.60490 & 1.27290 \\
\hline
\end{tabular}




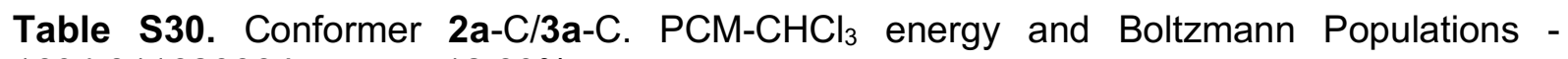
1694.81162066 hartrees, $12.69 \%$.

\begin{tabular}{|c|c|c|c|c|}
\hline \multicolumn{2}{|c|}{ Atom number } & \multicolumn{3}{|c|}{ Coordinates } \\
\hline & & $x$ & $\mathbf{Y}$ & $\mathbf{Z}$ \\
\hline 1 & 0 & -2.89960 & -0.48310 & -0.80340 \\
\hline 2 & $\mathrm{C}$ & -1.48900 & -0.38030 & -0.63490 \\
\hline 3 & C & -1.06450 & -1.07550 & 0.67200 \\
\hline 4 & $\mathrm{C}$ & -1.77870 & -0.35510 & 1.82770 \\
\hline 5 & $\mathrm{C}$ & -3.30210 & -0.30310 & 1.60000 \\
\hline 6 & $\mathrm{C}$ & -3.63110 & 0.24330 & 0.20450 \\
\hline 7 & $\mathrm{C}$ & -0.77920 & -0.96010 & -1.85260 \\
\hline 8 & $\mathrm{C}$ & 0.69450 & -1.31880 & -1.52930 \\
\hline 9 & $\mathrm{C}$ & 1.22660 & -0.67290 & -0.24150 \\
\hline 10 & 0 & 0.36100 & -0.88270 & 0.87580 \\
\hline 11 & 0 & 1.36840 & 0.71480 & -0.52520 \\
\hline 12 & $\mathrm{C}$ & 1.82400 & 1.57390 & 0.54960 \\
\hline 13 & $\mathrm{C}$ & 3.15570 & 1.06670 & 1.10610 \\
\hline 14 & $\mathrm{C}$ & 3.08820 & -0.42930 & 1.43900 \\
\hline 15 & $\mathrm{C}$ & 2.59710 & -1.27530 & 0.24890 \\
\hline 16 & $\mathrm{C}$ & -5.11300 & 0.20330 & -0.23990 \\
\hline 17 & $\mathrm{C}$ & -5.97060 & 1.12270 & 0.63160 \\
\hline 18 & 0 & -5.16710 & 0.75990 & -1.55910 \\
\hline 19 & C & -5.68290 & -1.22460 & -0.26800 \\
\hline 20 & $\mathrm{C}$ & -1.36230 & -2.58110 & 0.68410 \\
\hline 21 & $\mathrm{C}$ & 3.58710 & -1.30340 & -0.92220 \\
\hline 22 & C & 1.88870 & 2.98980 & -0.08350 \\
\hline 23 & $\mathrm{C}$ & 0.48630 & 3.45860 & -0.50730 \\
\hline 24 & $\mathrm{C}$ & 2.51560 & 4.00440 & 0.87540 \\
\hline 25 & $\mathrm{O}$ & 2.74770 & 2.94600 & -1.22910 \\
\hline 26 & $\mathrm{Cl}$ & 5.27690 & -1.76860 & -0.44740 \\
\hline 27 & 0 & 2.39410 & -2.62770 & 0.63310 \\
\hline 28 & $\mathrm{H}$ & -1.21920 & 0.68090 & -0.53440 \\
\hline 29 & $\mathrm{H}$ & -1.54520 & -0.84460 & 2.78080 \\
\hline 30 & $\mathrm{H}$ & -1.37850 & 0.66530 & 1.88550 \\
\hline 31 & $\mathrm{H}$ & -3.73210 & -1.30360 & 1.72030 \\
\hline 32 & $\mathrm{H}$ & -3.76360 & 0.33530 & 2.36220 \\
\hline 33 & $\mathrm{H}$ & -3.31310 & 1.29810 & 0.15110 \\
\hline 34 & $\mathrm{H}$ & -1.31710 & -1.85610 & -2.17990 \\
\hline 35 & $\mathrm{H}$ & -0.83440 & -0.23990 & -2.67440 \\
\hline 36 & $\mathrm{H}$ & 0.81560 & -2.39930 & -1.41700 \\
\hline 37 & $\mathrm{H}$ & 1.33910 & -1.00970 & -2.35590 \\
\hline 38 & $\mathrm{H}$ & 1.06380 & 1.56620 & 1.34230 \\
\hline 39 & $\mathrm{H}$ & 3.94630 & 1.26990 & 0.37470 \\
\hline 40 & $\mathrm{H}$ & 3.40860 & 1.62760 & 2.01290 \\
\hline 41 & $\mathrm{H}$ & 4.06400 & -0.80360 & 1.75910 \\
\hline 42 & $\mathrm{H}$ & 2.38980 & -0.58970 & 2.26830 \\
\hline 43 & $\mathrm{H}$ & -6.05810 & 0.74540 & 1.65550 \\
\hline 44 & $\mathrm{H}$ & -5.55070 & 2.13390 & 0.65960 \\
\hline 45 & $\mathrm{H}$ & -6.97550 & 1.18820 & 0.20290 \\
\hline 46 & $\mathrm{H}$ & -4.45100 & 0.32360 & -2.05230 \\
\hline 47 & $\mathrm{H}$ & -5.76250 & -1.66120 & 0.73390 \\
\hline 48 & $\mathrm{H}$ & -6.68390 & -1.19800 & -0.70940 \\
\hline 49 & $\mathrm{H}$ & -5.05370 & -1.87910 & -0.87960 \\
\hline 50 & $\mathrm{H}$ & -2.42050 & -2.77720 & 0.49430 \\
\hline 51 & $\mathrm{H}$ & -0.78180 & -3.11590 & -0.07190 \\
\hline
\end{tabular}




\begin{tabular}{rrrrr}
$\mathbf{5 2}$ & $\mathrm{H}$ & -1.10390 & -2.99940 & 1.66360 \\
$\mathbf{5 3}$ & $\mathrm{H}$ & 3.27850 & -2.05180 & -1.65040 \\
$\mathbf{5 4}$ & $\mathrm{H}$ & 3.65810 & -0.32650 & -1.39910 \\
$\mathbf{5 5}$ & $\mathrm{H}$ & -0.18550 & 3.54390 & 0.35570 \\
$\mathbf{5 6}$ & $\mathrm{H}$ & 0.55830 & 4.43970 & -0.98720 \\
$\mathbf{5 7}$ & $\mathrm{H}$ & 0.03840 & 2.75910 & -1.21960 \\
$\mathbf{5 8}$ & $\mathrm{H}$ & 1.96100 & 4.05120 & 1.81950 \\
$\mathbf{5 9}$ & $\mathrm{H}$ & 3.55770 & 3.75380 & 1.08890 \\
$\mathbf{6 0}$ & $\mathrm{H}$ & 2.49690 & 4.99790 & 0.41660 \\
$\mathbf{6 1}$ & $\mathrm{H}$ & 2.40220 & 2.22700 & -1.78480 \\
$\mathbf{6 2}$ & $\mathrm{H}$ & 1.66830 & -2.60040 & 1.27990 \\
\hline
\end{tabular}


Table S31. Conformer 2a-D/3a-D. $\mathrm{PCM}-\mathrm{CHCl}_{3}$ energy and Boltzmann Populations 1694.8121798 hartrees, $22.95 \%$.

\begin{tabular}{|c|c|c|c|c|}
\hline \multicolumn{2}{|c|}{ Atom number } & \multicolumn{3}{|c|}{ Coordinates } \\
\hline & & $x$ & $\mathbf{Y}$ & $\mathbf{Z}$ \\
\hline 1 & $\mathrm{O}$ & -2.83550 & -0.58220 & -0.80210 \\
\hline 2 & $\mathrm{C}$ & -1.42650 & -0.46100 & -0.63920 \\
\hline 3 & C & -0.99610 & -1.10450 & 0.69240 \\
\hline 4 & $\mathrm{C}$ & -1.71230 & -0.34350 & 1.82080 \\
\hline 5 & C & -3.23680 & -0.31020 & 1.59260 \\
\hline 6 & $\mathrm{C}$ & -3.56930 & 0.18220 & 0.17800 \\
\hline 7 & $\mathrm{C}$ & -0.71100 & -1.08250 & -1.83390 \\
\hline 8 & $\mathrm{C}$ & 0.76990 & -1.40520 & -1.50160 \\
\hline 9 & C & 1.28420 & -0.69400 & -0.24220 \\
\hline 10 & $\mathrm{O}$ & 0.43170 & -0.90170 & 0.88370 \\
\hline 11 & $\mathrm{O}$ & 1.37710 & 0.68680 & -0.57960 \\
\hline 12 & C & 1.75650 & 1.58250 & 0.49320 \\
\hline 13 & $\mathrm{C}$ & 3.13860 & 1.19620 & 1.01780 \\
\hline 14 & $\mathrm{C}$ & 3.15160 & -0.28880 & 1.41240 \\
\hline 15 & $\mathrm{C}$ & 2.68310 & -1.21260 & 0.26800 \\
\hline 16 & $\mathrm{C}$ & -5.05100 & 0.12080 & -0.26370 \\
\hline 17 & C & -5.90910 & 1.07480 & 0.56920 \\
\hline 18 & 0 & -5.10700 & 0.62150 & -1.60520 \\
\hline 19 & $\mathrm{C}$ & -5.61970 & -1.30740 & -0.23320 \\
\hline 20 & $\mathrm{C}$ & -1.28510 & -2.61000 & 0.76110 \\
\hline 21 & $\mathrm{C}$ & 3.65950 & -1.25970 & -0.91400 \\
\hline 22 & $\mathrm{C}$ & 1.57750 & 3.01000 & -0.07690 \\
\hline 23 & $\mathrm{C}$ & 1.69090 & 4.05270 & 1.03650 \\
\hline 24 & $\mathrm{C}$ & 2.54790 & 3.32100 & -1.22710 \\
\hline 25 & 0 & 0.22710 & 3.10970 & -0.55650 \\
\hline 26 & $\mathrm{Cl}$ & 5.38230 & -1.59000 & -0.44130 \\
\hline 27 & 0 & 2.54260 & -2.55010 & 0.72390 \\
\hline 28 & $\mathrm{H}$ & -1.16950 & 0.60590 & -0.57320 \\
\hline 29 & $\mathrm{H}$ & -1.47550 & -0.79570 & 2.79130 \\
\hline 30 & $\mathrm{H}$ & -1.32090 & 0.68130 & 1.83780 \\
\hline 31 & $\mathrm{H}$ & -3.66180 & -1.30770 & 1.75100 \\
\hline 32 & $\mathrm{H}$ & -3.70050 & 0.35470 & 2.33030 \\
\hline 33 & $\mathrm{H}$ & -3.25310 & 1.23410 & 0.08340 \\
\hline 34 & $\mathrm{H}$ & -1.23740 & -1.99910 & -2.12030 \\
\hline 35 & $\mathrm{H}$ & -0.77930 & -0.40060 & -2.68720 \\
\hline 36 & $\mathrm{H}$ & 0.90960 & -2.47800 & -1.34370 \\
\hline 37 & $\mathrm{H}$ & 1.40720 & -1.11900 & -2.34180 \\
\hline 38 & $\mathrm{H}$ & 1.02090 & 1.46820 & 1.29900 \\
\hline 39 & $\mathrm{H}$ & 3.90270 & 1.39090 & 0.25590 \\
\hline 40 & $\mathrm{H}$ & 3.39280 & 1.81120 & 1.88920 \\
\hline 41 & $\mathrm{H}$ & 4.14830 & -0.60380 & 1.73110 \\
\hline 42 & $\mathrm{H}$ & 2.47440 & -0.44520 & 2.25970 \\
\hline 43 & $\mathrm{H}$ & -5.99030 & 0.74390 & 1.60960 \\
\hline 44 & $\mathrm{H}$ & -5.49300 & 2.08780 & 0.54960 \\
\hline 45 & $\mathrm{H}$ & -6.91630 & 1.11670 & 0.14270 \\
\hline 46 & $\mathrm{H}$ & -4.38620 & 0.17080 & -2.07830 \\
\hline 47 & $\mathrm{H}$ & -5.70100 & -1.70260 & 0.78570 \\
\hline 48 & $\mathrm{H}$ & -6.62010 & -1.29940 & -0.67670 \\
\hline 49 & $\mathrm{H}$ & -4.98970 & -1.98640 & -0.81670 \\
\hline 50 & $\mathrm{H}$ & -2.34160 & -2.81950 & 0.57610 \\
\hline 51 & $\mathrm{H}$ & -0.69900 & -3.16960 & 0.02750 \\
\hline
\end{tabular}




\begin{tabular}{rrrrr}
$\mathbf{5 2}$ & $\mathrm{H}$ & -1.02690 & -2.98930 & 1.75630 \\
$\mathbf{5 3}$ & $\mathrm{H}$ & 3.38570 & -2.07270 & -1.58450 \\
$\mathbf{5 4}$ & $\mathrm{H}$ & 3.66340 & -0.31540 & -1.45690 \\
$\mathbf{5 5}$ & $\mathrm{H}$ & 2.70580 & 4.09910 & 1.44350 \\
$\mathbf{5 6}$ & $\mathrm{H}$ & 1.43530 & 5.03860 & 0.63590 \\
$\mathbf{5 7}$ & $\mathrm{H}$ & 0.99320 & 3.82780 & 1.85020 \\
$\mathbf{5 8}$ & $\mathrm{H}$ & 3.58890 & 3.36750 & -0.88910 \\
$\mathbf{5 9}$ & $\mathrm{H}$ & 2.47780 & 2.55860 & -2.01050 \\
$\mathbf{6 0}$ & $\mathrm{H}$ & 2.28620 & 4.28940 & -1.66430 \\
$\mathbf{6 1}$ & $\mathrm{H}$ & 0.12380 & 2.37870 & -1.18830 \\
$\mathbf{6 2}$ & $\mathrm{H}$ & 1.80100 & -2.52530 & 1.35310 \\
\hline
\end{tabular}




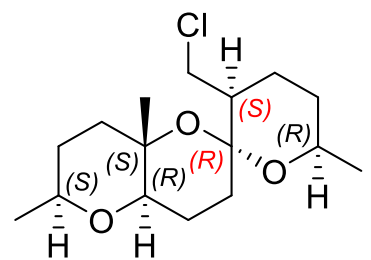

Table S32. Conformer 2b-A/3b-A. PCM-CHCl 3 energy and Boltzmann Populations 1694.81068 hartrees, $9.30 \%$.

\begin{tabular}{|c|c|c|c|c|}
\hline \multicolumn{2}{|c|}{ Atom number } & \multicolumn{3}{|c|}{ Coordinates } \\
\hline & & $\mathbf{X}$ & $\mathbf{Y}$ & $\mathbf{Z}$ \\
\hline 1 & 0 & 2.8072 & -0.3853 & 0.7993 \\
\hline 2 & $\mathrm{C}$ & 1.3971 & -0.2236 & 0.6727 \\
\hline 3 & $\mathrm{C}$ & 0.876 & -1.072 & -0.5027 \\
\hline 4 & C & 1.5604 & -0.5466 & -1.7742 \\
\hline 5 & $\mathrm{C}$ & 3.0933 & -0.5325 & -1.6258 \\
\hline 6 & $\mathrm{C}$ & 3.5418 & 0.1433 & -0.3211 \\
\hline 7 & $\mathrm{C}$ & 0.7167 & -0.5695 & 1.9957 \\
\hline 8 & $\mathrm{C}$ & -0.7763 & -0.9312 & 1.7882 \\
\hline 9 & $\mathrm{C}$ & -1.3265 & -0.4287 & 0.4444 \\
\hline 10 & 0 & -0.5519 & -0.8565 & -0.6694 \\
\hline 11 & 0 & -1.3117 & 0.9901 & 0.5661 \\
\hline 12 & $\mathrm{C}$ & -1.8006 & 1.7831 & -0.5384 \\
\hline 13 & $\mathrm{C}$ & -3.228 & 1.3641 & -0.8857 \\
\hline 14 & $\mathrm{C}$ & -3.3187 & -0.1492 & -1.0921 \\
\hline 15 & $\mathrm{C}$ & -2.7838 & -0.9184 & 0.1313 \\
\hline 16 & $\mathrm{C}$ & 5.034 & -0.0812 & 0.0397 \\
\hline 17 & $\mathrm{C}$ & 5.4031 & 0.7378 & 1.2885 \\
\hline 18 & $\mathrm{O}$ & 5.2326 & -1.4768 & 0.2985 \\
\hline 19 & $\mathrm{C}$ & 5.9643 & 0.2718 & -1.1218 \\
\hline 20 & $\mathrm{C}$ & 1.1281 & -2.5758 & -0.3213 \\
\hline 21 & $\mathrm{C}$ & -2.7862 & -2.4357 & -0.1259 \\
\hline 22 & $\mathrm{C}$ & -1.6716 & 3.2509 & -0.0502 \\
\hline 23 & $\mathrm{C}$ & -0.1997 & 3.6075 & 0.2224 \\
\hline 24 & $\mathrm{C}$ & -2.2682 & 4.2317 & -1.0631 \\
\hline 25 & 0 & -2.4325 & 3.4066 & 1.152 \\
\hline 26 & $\mathrm{Cl}$ & -4.4895 & -3.0816 & -0.2987 \\
\hline 27 & 0 & -3.5348 & -0.6124 & 1.3018 \\
\hline 28 & $\mathrm{H}$ & 1.1856 & 0.8256 & 0.4282 \\
\hline 29 & $\mathrm{H}$ & 1.2633 & -1.1497 & -2.6407 \\
\hline 30 & $\mathrm{H}$ & 1.193 & 0.472 & -1.9545 \\
\hline 31 & $\mathrm{H}$ & 3.4944 & -1.5513 & -1.6425 \\
\hline 32 & $\mathrm{H}$ & 3.5307 & -0.0029 & -2.4796 \\
\hline 33 & $\mathrm{H}$ & 3.3446 & 1.2272 & -0.3831 \\
\hline 34 & $\mathrm{H}$ & 1.2432 & -1.4148 & 2.4521 \\
\hline 35 & $\mathrm{H}$ & 0.822 & 0.2757 & 2.6822 \\
\hline 36 & $\mathrm{H}$ & -0.903 & -2.0155 & 1.833 \\
\hline 37 & $\mathrm{H}$ & -1.4013 & -0.5131 & 2.578 \\
\hline 38 & $\mathrm{H}$ & -1.1407 & 1.6271 & -1.4035 \\
\hline 39 & $\mathrm{H}$ & -3.8884 & 1.6756 & -0.0707 \\
\hline 40 & $\mathrm{H}$ & -3.5543 & 1.8769 & -1.7969 \\
\hline 41 & $\mathrm{H}$ & -4.3578 & -0.447 & -1.2738 \\
\hline 42 & $\mathrm{H}$ & -2.7396 & -0.4473 & -1.9741 \\
\hline 43 & $\mathrm{H}$ & 4.7336 & 0.4994 & 2.1201 \\
\hline
\end{tabular}




\begin{tabular}{llrrr}
$\mathbf{4 4}$ & $\mathrm{H}$ & 6.4304 & 0.506 & 1.5864 \\
$\mathbf{4 5}$ & $\mathrm{H}$ & 5.332 & 1.8149 & 1.0941 \\
$\mathbf{4 6}$ & $\mathrm{H}$ & 4.5566 & -1.7149 & 0.956 \\
$\mathbf{4 7}$ & $\mathrm{H}$ & 5.7967 & -0.3879 & -1.9773 \\
$\mathbf{4 8}$ & $\mathrm{H}$ & 5.8215 & 1.3104 & -1.4413 \\
$\mathbf{4 9}$ & $\mathrm{H}$ & 7.005 & 0.1507 & -0.8049 \\
$\mathbf{5 0}$ & $\mathrm{H}$ & 0.6101 & -2.9746 & 0.555 \\
$\mathbf{5 1}$ & $\mathrm{H}$ & 0.765 & -3.1165 & -1.2019 \\
$\mathbf{5 2}$ & $\mathrm{H}$ & 2.1938 & -2.7859 & -0.2017 \\
$\mathbf{5 3}$ & $\mathrm{H}$ & -2.263 & -2.6904 & -1.0448 \\
$\mathbf{5 4}$ & $\mathrm{H}$ & -2.3631 & -2.9879 & 0.7117 \\
$\mathbf{5 5}$ & $\mathrm{H}$ & 0.2248 & 2.9509 & 0.9871 \\
$\mathbf{5 6}$ & $\mathrm{H}$ & 0.4106 & 3.5193 & -0.6854 \\
$\mathbf{5 7}$ & $\mathrm{H}$ & -0.1359 & 4.6389 & 0.5831 \\
$\mathbf{5 8}$ & $\mathrm{H}$ & -3.3426 & 4.0709 & -1.1826 \\
$\mathbf{5 9}$ & $\mathrm{H}$ & -1.783 & 4.1357 & -2.0413 \\
$\mathbf{6 0}$ & $\mathrm{H}$ & -2.1215 & 5.2562 & -0.7065 \\
$\mathbf{6 1}$ & $\mathrm{H}$ & -2.211 & 2.6358 & 1.7035 \\
$\mathbf{6 2}$ & $\mathrm{H}$ & -4.431 & -0.9635 & 1.1645 \\
\hline
\end{tabular}


Table S33. Conformer 2b-B/3b-B. $\mathrm{PCM}-\mathrm{CHCl}_{3}$ energy and Boltzmann Populations 1694.81128 hartrees, $17.07 \%$.

\begin{tabular}{|c|c|c|c|c|}
\hline \multicolumn{2}{|c|}{ Atom number } & \multicolumn{3}{|c|}{ Coordinates } \\
\hline & & X & $\mathbf{Y}$ & Z \\
\hline 1 & O & 2.7562 & -0.4799 & 0.8075 \\
\hline 2 & C & 1.3502 & -0.2847 & 0.6913 \\
\hline 3 & $\mathrm{C}$ & 0.8083 & -1.0833 & -0.5097 \\
\hline 4 & $\mathrm{C}$ & 1.4969 & -0.5253 & -1.7649 \\
\hline 5 & $\mathrm{C}$ & 3.0307 & -0.5471 & -1.6228 \\
\hline 6 & $\mathrm{C}$ & 3.4964 & 0.0757 & -0.298 \\
\hline 7 & $\mathrm{C}$ & 0.6658 & -0.6636 & 2.0042 \\
\hline 8 & $\mathrm{C}$ & -0.837 & -0.9848 & 1.793 \\
\hline 9 & $\mathrm{C}$ & -1.3738 & -0.42 & 0.4699 \\
\hline 10 & $\mathrm{O}$ & -0.6183 & -0.8409 & -0.6594 \\
\hline 11 & $\mathrm{O}$ & -1.3135 & 0.9918 & 0.6399 \\
\hline 12 & $\mathrm{C}$ & -1.7442 & 1.8055 & -0.4705 \\
\hline 13 & $\mathrm{C}$ & -3.2091 & 1.5024 & -0.7716 \\
\hline 14 & $\mathrm{C}$ & -3.3779 & 0.002 & -1.0371 \\
\hline 15 & $\mathrm{C}$ & -2.8511 & -0.8436 & 0.1426 \\
\hline 16 & $\mathrm{C}$ & 4.9853 & -0.1871 & 0.0488 \\
\hline 17 & $\mathrm{C}$ & 5.3733 & 0.5802 & 1.3244 \\
\hline 18 & $\mathrm{O}$ & 5.1617 & -1.5945 & 0.2578 \\
\hline 19 & $\mathrm{C}$ & 5.9171 & 0.1916 & -1.1033 \\
\hline 20 & $\mathrm{C}$ & 1.0336 & -2.5965 & -0.3801 \\
\hline 21 & $\mathrm{C}$ & -2.9 & -2.3455 & -0.19 \\
\hline 22 & $\mathrm{C}$ & -1.3896 & 3.2613 & -0.0785 \\
\hline 23 & $\mathrm{C}$ & -1.5151 & 4.1923 & -1.2852 \\
\hline 24 & $\mathrm{C}$ & -2.2223 & 3.7725 & 1.1077 \\
\hline 25 & 0 & 0.0025 & 3.2823 & 0.2767 \\
\hline 26 & $\mathrm{Cl}$ & -4.6232 & -2.9351 & -0.3728 \\
\hline 27 & 0 & -3.5873 & -0.574 & 1.331 \\
\hline 28 & $\mathrm{H}$ & 1.1642 & 0.7757 & 0.4758 \\
\hline 29 & $\mathrm{H}$ & 1.1848 & -1.0915 & -2.651 \\
\hline 30 & $\mathrm{H}$ & 1.1518 & 0.5068 & -1.9055 \\
\hline 31 & $\mathrm{H}$ & 3.4126 & -1.572 & -1.6768 \\
\hline 32 & $\mathrm{H}$ & 3.4742 & 0.0041 & -2.4595 \\
\hline 33 & $\mathrm{H}$ & 3.3166 & 1.1635 & -0.3211 \\
\hline 34 & $\mathrm{H}$ & 1.1764 & -1.5362 & 2.426 \\
\hline 35 & $\mathrm{H}$ & 0.793 & 0.1513 & 2.7232 \\
\hline 36 & $\mathrm{H}$ & -0.9894 & -2.0667 & 1.7991 \\
\hline 37 & $\mathrm{H}$ & -1.4488 & -0.5774 & 2.5984 \\
\hline 38 & $\mathrm{H}$ & -1.1286 & 1.547 & -1.3425 \\
\hline 39 & $\mathrm{H}$ & -3.8286 & 1.7905 & 0.0835 \\
\hline 40 & $\mathrm{H}$ & -3.547 & 2.0752 & -1.6431 \\
\hline 41 & $\mathrm{H}$ & -4.4333 & -0.2423 & -1.203 \\
\hline 42 & $\mathrm{H}$ & -2.8343 & -0.2819 & -1.946 \\
\hline 43 & $\mathrm{H}$ & 5.3175 & 1.6646 & 1.17 \\
\hline 44 & $\mathrm{H}$ & 4.7047 & 0.3215 & 2.1507 \\
\hline 45 & $\mathrm{H}$ & 6.3985 & 0.322 & 1.6079 \\
\hline 46 & $\mathrm{H}$ & 4.4815 & -1.8446 & 0.9064 \\
\hline 47 & $\mathrm{H}$ & 5.7901 & 1.243 & -1.3848 \\
\hline 48 & $\mathrm{H}$ & 6.9568 & 0.042 & -0.7954 \\
\hline 49 & $\mathrm{H}$ & 5.735 & -0.4345 & -1.9809 \\
\hline 50 & $\mathrm{H}$ & 0.6532 & -3.1005 & -1.2751 \\
\hline
\end{tabular}




\begin{tabular}{rrrrr}
$\mathbf{5 1}$ & $\mathrm{H}$ & 2.096 & -2.8309 & -0.2773 \\
$\mathbf{5 2}$ & $\mathrm{H}$ & 0.5151 & -3.0147 & 0.487 \\
$\mathbf{5 3}$ & $\mathrm{H}$ & -2.3946 & -2.5684 & -1.1267 \\
$\mathbf{5 4}$ & $\mathrm{H}$ & -2.482 & -2.9495 & 0.614 \\
$\mathbf{5 5}$ & $\mathrm{H}$ & -2.5523 & 4.2742 & -1.6252 \\
$\mathbf{5 6}$ & $\mathrm{H}$ & -1.1634 & 5.1919 & -1.0107 \\
$\mathbf{5 7}$ & $\mathrm{H}$ & -0.8982 & 3.8328 & -2.1157 \\
$\mathbf{5 8}$ & $\mathrm{H}$ & -3.2772 & 3.9048 & 0.8438 \\
$\mathbf{5 9}$ & $\mathrm{H}$ & -2.1695 & 3.0719 & 1.9478 \\
$\mathbf{6 0}$ & $\mathrm{H}$ & -1.8261 & 4.7395 & 1.433 \\
$\mathbf{6 1}$ & $\mathrm{H}$ & 0.0962 & 2.6114 & 0.9735 \\
$\mathbf{6 2}$ & $\mathrm{H}$ & -4.4868 & -0.9152 & 1.1894 \\
\hline
\end{tabular}




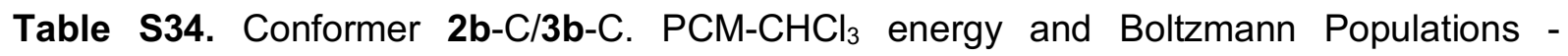
1694.81034 hartrees, $6.49 \%$.

\begin{tabular}{|c|c|c|c|c|}
\hline \multicolumn{2}{|c|}{ Atom number } & \multicolumn{3}{|c|}{ Coordinates } \\
\hline & & $x$ & $\mathbf{Y}$ & $\mathbf{Z}$ \\
\hline 1 & 0 & 2.7987 & -0.4959 & 0.7944 \\
\hline 2 & C & 1.3915 & -0.2984 & 0.6753 \\
\hline 3 & $\mathrm{C}$ & 0.8496 & -1.129 & -0.505 \\
\hline 4 & $\mathrm{C}$ & 1.55 & -0.625 & -1.779 \\
\hline 5 & $\mathrm{C}$ & 3.0839 & -0.6538 & -1.6272 \\
\hline 6 & $\mathrm{C}$ & 3.5227 & 0.0404 & -0.331 \\
\hline 7 & $\mathrm{C}$ & 0.7026 & -0.6354 & 1.9956 \\
\hline 8 & $\mathrm{C}$ & -0.8004 & -0.9531 & 1.7846 \\
\hline 9 & $\mathrm{C}$ & -1.3351 & -0.4288 & 0.4425 \\
\hline 10 & 0 & -0.5703 & -0.8707 & -0.6738 \\
\hline 11 & $\mathrm{O}$ & -1.2828 & 0.9878 & 0.5716 \\
\hline 12 & $\mathrm{C}$ & -1.7487 & 1.7994 & -0.5296 \\
\hline 13 & $\mathrm{C}$ & -3.1862 & 1.4201 & -0.8811 \\
\hline 14 & $\mathrm{C}$ & -3.3174 & -0.0893 & -1.0947 \\
\hline 15 & $\mathrm{C}$ & -2.8043 & -0.8786 & 0.1254 \\
\hline 16 & $\mathrm{C}$ & 5.02 & -0.0491 & 0.0498 \\
\hline 17 & $\mathrm{C}$ & 5.8901 & 0.6925 & -0.9668 \\
\hline 18 & 0 & 5.1771 & 0.661 & 1.2844 \\
\hline 19 & $\mathrm{C}$ & 5.4974 & -1.4993 & 0.2342 \\
\hline 20 & $\mathrm{C}$ & 1.0546 & -2.6401 & -0.3245 \\
\hline 21 & $\mathrm{C}$ & -2.8465 & -2.3942 & -0.1393 \\
\hline 22 & $\mathrm{C}$ & -1.5817 & 3.261 & -0.0341 \\
\hline 23 & $\mathrm{C}$ & -0.1016 & 3.5774 & 0.2433 \\
\hline 24 & $\mathrm{C}$ & -2.1497 & 4.2616 & -1.0441 \\
\hline 25 & 0 & -2.3418 & 3.4315 & 1.1666 \\
\hline 26 & $\mathrm{Cl}$ & -4.5656 & -2.9934 & -0.3203 \\
\hline 27 & 0 & -3.548 & -0.5589 & 1.2967 \\
\hline 28 & $\mathrm{H}$ & 1.2036 & 0.7564 & 0.4359 \\
\hline 29 & $\mathrm{H}$ & 1.235 & -1.2215 & -2.6436 \\
\hline 30 & $\mathrm{H}$ & 1.2149 & 0.404 & -1.9616 \\
\hline 31 & $\mathrm{H}$ & 3.4407 & -1.6894 & -1.6345 \\
\hline 32 & $\mathrm{H}$ & 3.5452 & -0.1523 & -2.4859 \\
\hline 33 & $\mathrm{H}$ & 3.2788 & 1.1136 & -0.4023 \\
\hline 34 & $\mathrm{H}$ & 1.205 & -1.4984 & 2.4458 \\
\hline 35 & $\mathrm{H}$ & 0.8301 & 0.2022 & 2.6877 \\
\hline 36 & $\mathrm{H}$ & -0.9582 & -2.0333 & 1.8254 \\
\hline 37 & $\mathrm{H}$ & -1.4141 & -0.5199 & 2.575 \\
\hline 38 & $\mathrm{H}$ & -1.0913 & 1.6305 & -1.3942 \\
\hline 39 & $\mathrm{H}$ & -3.8392 & 1.7458 & -0.0658 \\
\hline 40 & $\mathrm{H}$ & -3.4973 & 1.9453 & -1.7905 \\
\hline 41 & $\mathrm{H}$ & -4.3639 & -0.3579 & -1.2786 \\
\hline 42 & $\mathrm{H}$ & -2.7458 & -0.3986 & -1.9778 \\
\hline 43 & $\mathrm{H}$ & 5.907 & 0.1835 & -1.9359 \\
\hline 44 & $\mathrm{H}$ & 5.5321 & 1.7177 & -1.1099 \\
\hline 45 & $\mathrm{H}$ & 6.9162 & 0.7451 & -0.5892 \\
\hline 46 & $\mathrm{H}$ & 4.4513 & 0.3501 & 1.853 \\
\hline 47 & $\mathrm{H}$ & 5.4957 & -2.0602 & -0.7072 \\
\hline 48 & $\mathrm{H}$ & 6.5197 & -1.4893 & 0.6244 \\
\hline 49 & $\mathrm{H}$ & 4.8597 & -2.027 & 0.9506 \\
\hline 50 & $\mathrm{H}$ & 0.6867 & -3.1686 & -1.2107 \\
\hline
\end{tabular}




\begin{tabular}{rrrrr}
$\mathbf{5 1}$ & $\mathrm{H}$ & 2.1112 & -2.8828 & -0.1879 \\
$\mathbf{5 2}$ & $\mathrm{H}$ & 0.5133 & -3.0241 & 0.544 \\
$\mathbf{5 3}$ & $\mathrm{H}$ & -2.3273 & -2.6581 & -1.0578 \\
$\mathbf{5 4}$ & $\mathrm{H}$ & -2.4409 & -2.9613 & 0.697 \\
$\mathbf{5 5}$ & $\mathrm{H}$ & 0.5084 & 3.4773 & -0.6633 \\
$\mathbf{5 6}$ & $\mathrm{H}$ & -0.0112 & 4.6051 & 0.6087 \\
$\mathbf{5 7}$ & $\mathrm{H}$ & 0.3042 & 2.9069 & 1.006 \\
$\mathbf{5 8}$ & $\mathrm{H}$ & -1.6644 & 4.1575 & -2.0213 \\
$\mathbf{5 9}$ & $\mathrm{H}$ & -3.2277 & 4.1296 & -1.1672 \\
$\mathbf{6 0}$ & $\mathrm{H}$ & -1.9773 & 5.2802 & -0.6824 \\
$\mathbf{6 1}$ & $\mathrm{H}$ & -2.1381 & 2.6559 & 1.718 \\
$\mathbf{6 2}$ & $\mathrm{H}$ & -4.4538 & -0.8833 & 1.1559 \\
\hline
\end{tabular}




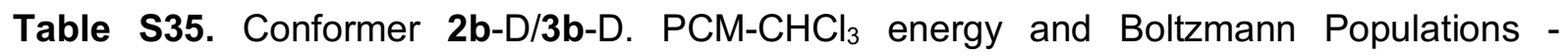
1694.81083 hartrees, $10.91 \%$.

\begin{tabular}{|c|c|c|c|c|}
\hline \multicolumn{2}{|c|}{ Atom number } & \multicolumn{3}{|c|}{ Coordinates } \\
\hline & & $x$ & $\mathrm{Y}$ & Z \\
\hline 1 & $\mathrm{O}$ & 2.7446 & -0.5924 & 0.7988 \\
\hline 2 & C & 1.3425 & -0.3594 & 0.6922 \\
\hline 3 & C & 0.7799 & -1.1357 & -0.5155 \\
\hline 4 & $\mathrm{C}$ & 1.4834 & -0.5932 & -1.7718 \\
\hline 5 & $\mathrm{C}$ & 3.0177 & -0.6568 & -1.6288 \\
\hline 6 & $\mathrm{C}$ & 3.4753 & -0.0236 & -0.3081 \\
\hline 7 & $\mathrm{C}$ & 0.6497 & -0.732 & 2.0018 \\
\hline 8 & $\mathrm{C}$ & -0.8621 & -1.007 & 1.788 \\
\hline 9 & $\mathrm{C}$ & -1.383 & -0.4187 & 0.4685 \\
\hline 10 & $\mathrm{O}$ & -0.6386 & -0.8523 & -0.6647 \\
\hline 11 & $\mathrm{O}$ & -1.2847 & 0.9893 & 0.6464 \\
\hline 12 & $\mathrm{C}$ & -1.6936 & 1.82 & -0.4598 \\
\hline 13 & $\mathrm{C}$ & -3.1668 & 1.5586 & -0.7601 \\
\hline 14 & $\mathrm{C}$ & -3.377 & 0.065 & -1.034 \\
\hline 15 & $\mathrm{C}$ & -2.8712 & -0.8018 & 0.1393 \\
\hline 16 & $\mathrm{C}$ & 4.9717 & -0.1585 & 0.0622 \\
\hline 17 & $\mathrm{C}$ & 5.8515 & 0.6121 & -0.9241 \\
\hline 18 & 0 & 5.1474 & 0.4911 & 1.3273 \\
\hline 19 & $\mathrm{C}$ & 5.4232 & -1.624 & 0.1794 \\
\hline 20 & $\mathrm{C}$ & 0.96 & -2.6554 & -0.3921 \\
\hline 21 & $\mathrm{C}$ & -2.9596 & -2.2999 & -0.2022 \\
\hline 22 & $\mathrm{C}$ & -1.298 & 3.2637 & -0.0639 \\
\hline 23 & $\mathrm{C}$ & -1.4071 & 4.203 & -1.266 \\
\hline 24 & $\mathrm{C}$ & -2.1082 & 3.7921 & 1.1302 \\
\hline 25 & 0 & 0.0968 & 3.2471 & 0.2797 \\
\hline 26 & $\mathrm{Cl}$ & -4.6971 & -2.8417 & -0.3938 \\
\hline 27 & 0 & -3.5992 & -0.5207 & 1.3301 \\
\hline 28 & $\mathrm{H}$ & 1.1815 & 0.7067 & 0.485 \\
\hline 29 & $\mathrm{H}$ & 1.1544 & -1.1493 & -2.658 \\
\hline 30 & $\mathrm{H}$ & 1.1691 & 0.4487 & -1.9099 \\
\hline 31 & $\mathrm{H}$ & 3.3562 & -1.6974 & -1.6812 \\
\hline 32 & $\mathrm{H}$ & 3.4834 & -0.1279 & -2.4684 \\
\hline 33 & $\mathrm{H}$ & 3.2512 & 1.0555 & -0.3339 \\
\hline 34 & $\mathrm{H}$ & 1.1351 & -1.6229 & 2.4144 \\
\hline 35 & $\mathrm{H}$ & 0.7996 & 0.0727 & 2.7281 \\
\hline 36 & $\mathrm{H}$ & -1.0464 & -2.0839 & 1.788 \\
\hline 37 & $\mathrm{H}$ & -1.4618 & -0.5864 & 2.5959 \\
\hline 38 & $\mathrm{H}$ & -1.0866 & 1.548 & -1.3336 \\
\hline 39 & $\mathrm{H}$ & -3.7766 & 1.8589 & 0.0977 \\
\hline 40 & $\mathrm{H}$ & -3.4905 & 2.145 & -1.6279 \\
\hline 41 & $\mathrm{H}$ & -4.4389 & -0.1492 & -1.1991 \\
\hline 42 & $\mathrm{H}$ & -2.8436 & -0.2282 & -1.9461 \\
\hline 43 & $\mathrm{H}$ & 5.8492 & 0.1508 & -1.917 \\
\hline 44 & $\mathrm{H}$ & 5.5154 & 1.6506 & -1.0138 \\
\hline 45 & $\mathrm{H}$ & 6.8814 & 0.6229 & -0.5535 \\
\hline 46 & $\mathrm{H}$ & 4.4139 & 0.1734 & 1.882 \\
\hline 47 & $\mathrm{H}$ & 5.4104 & -2.142 & -0.7863 \\
\hline 48 & $\mathrm{H}$ & 6.4461 & -1.6498 & 0.5675 \\
\hline 49 & $\mathrm{H}$ & 4.7773 & -2.1721 & 0.8728 \\
\hline 50 & $\mathrm{H}$ & 0.5745 & -3.1443 & -1.2934 \\
\hline
\end{tabular}




\begin{tabular}{rrrrr}
$\mathbf{5 1}$ & $\mathrm{H}$ & 2.0133 & -2.9213 & -0.2754 \\
$\mathbf{5 2}$ & $\mathrm{H}$ & 0.42 & -3.0615 & 0.4674 \\
$\mathbf{5 3}$ & $\mathrm{H}$ & -2.4568 & -2.5306 & -1.1384 \\
$\mathbf{5 4}$ & $\mathrm{H}$ & -2.5607 & -2.9194 & 0.5998 \\
$\mathbf{5 5}$ & $\mathrm{H}$ & -2.4443 & 4.3167 & -1.5963 \\
$\mathbf{5 6}$ & $\mathrm{H}$ & -1.0236 & 5.1905 & -0.9904 \\
$\mathbf{5 7}$ & $\mathrm{H}$ & -0.8082 & 3.8295 & -2.1034 \\
$\mathbf{5 8}$ & $\mathrm{H}$ & -3.1612 & 3.9526 & 0.8746 \\
$\mathbf{5 9}$ & $\mathrm{H}$ & -2.0676 & 3.0875 & 1.9676 \\
$\mathbf{6 0}$ & $\mathrm{H}$ & -1.6847 & 4.7474 & 1.4555 \\
$\mathbf{6 1}$ & $\mathrm{H}$ & 0.1789 & 2.583 & 0.9842 \\
$\mathbf{6 2}$ & $\mathrm{H}$ & -4.5083 & -0.8344 & 1.1861 \\
\hline
\end{tabular}


Table S36. Conformer 2b-E/3b-E. PCM-CHCl ${ }_{3}$ energy and Boltzmann Populations 1694.81036 hartrees, $6.63 \%$.

\begin{tabular}{|c|c|c|c|c|}
\hline \multicolumn{2}{|c|}{ Atom number } & \multicolumn{3}{|c|}{ Coordinates } \\
\hline & & $x$ & $Y$ & Z \\
\hline 1 & 0 & 2.8122 & -0.3952 & 0.7986 \\
\hline 2 & C & 1.4018 & -0.2362 & 0.6837 \\
\hline 3 & $\mathrm{C}$ & 0.872 & -1.0727 & -0.4961 \\
\hline 4 & $\mathrm{C}$ & 1.5459 & -0.5339 & -1.7674 \\
\hline 5 & $\mathrm{C}$ & 3.0799 & -0.521 & -1.6306 \\
\hline 6 & $\mathrm{C}$ & 3.5384 & 0.143 & -0.3233 \\
\hline 7 & $\mathrm{C}$ & 0.7313 & -0.5955 & 2.0084 \\
\hline 8 & $\mathrm{C}$ & -0.7562 & -0.9748 & 1.8017 \\
\hline 9 & $\mathrm{C}$ & -1.3262 & -0.4578 & 0.4736 \\
\hline 10 & 0 & -0.5583 & -0.8509 & -0.6478 \\
\hline 11 & $\mathrm{O}$ & -1.3445 & 0.9743 & 0.6182 \\
\hline 12 & $\mathrm{C}$ & -1.7632 & 1.7518 & -0.5299 \\
\hline 13 & $\mathrm{C}$ & -3.1675 & 1.3296 & -0.9653 \\
\hline 14 & $\mathrm{C}$ & -3.2938 & -0.1945 & -1.0855 \\
\hline 15 & $\mathrm{C}$ & -2.8065 & -0.9089 & 0.1902 \\
\hline 16 & $\mathrm{C}$ & 5.0335 & -0.0846 & 0.0232 \\
\hline 17 & $\mathrm{C}$ & 5.4123 & 0.7189 & 1.2791 \\
\hline 18 & 0 & 5.2362 & -1.4829 & 0.2622 \\
\hline 19 & $\mathrm{C}$ & 5.9534 & 0.2845 & -1.1416 \\
\hline 20 & $\mathrm{C}$ & 1.1248 & -2.5785 & -0.3366 \\
\hline 21 & $\mathrm{C}$ & -2.8819 & -2.4323 & 0.0694 \\
\hline 22 & $\mathrm{C}$ & -1.6584 & 3.229 & -0.0632 \\
\hline 23 & $\mathrm{C}$ & -0.1974 & 3.6065 & 0.2315 \\
\hline 24 & $\mathrm{C}$ & -2.2513 & 4.1873 & -1.0984 \\
\hline 25 & 0 & -2.4456 & 3.395 & 1.1242 \\
\hline 26 & $\mathrm{Cl}$ & -4.5224 & -3.0541 & -0.4063 \\
\hline 27 & 0 & -3.6014 & -0.5518 & 1.3183 \\
\hline 28 & $\mathrm{H}$ & 1.1861 & 0.8154 & 0.4503 \\
\hline 29 & $\mathrm{H}$ & 1.2418 & -1.1279 & -2.6377 \\
\hline 30 & $\mathrm{H}$ & 1.1786 & 0.4869 & -1.9352 \\
\hline 31 & $\mathrm{H}$ & 3.4813 & -1.5393 & -1.6602 \\
\hline 32 & $\mathrm{H}$ & 3.5102 & 0.0168 & -2.4827 \\
\hline 33 & $\mathrm{H}$ & 3.3408 & 1.2275 & -0.3738 \\
\hline 34 & $\mathrm{H}$ & 1.2689 & -1.4368 & 2.4591 \\
\hline 35 & $\mathrm{H}$ & 0.8292 & 0.2476 & 2.6988 \\
\hline 36 & $\mathrm{H}$ & -0.8653 & -2.0617 & 1.8161 \\
\hline 37 & $\mathrm{H}$ & -1.3817 & -0.5942 & 2.6108 \\
\hline 38 & $\mathrm{H}$ & -1.0516 & 1.5761 & -1.3472 \\
\hline 39 & $\mathrm{H}$ & -3.8937 & 1.7191 & -0.2414 \\
\hline 40 & $\mathrm{H}$ & -3.4085 & 1.7901 & -1.9294 \\
\hline 41 & $\mathrm{H}$ & -4.337 & -0.4706 & -1.2592 \\
\hline 42 & $\mathrm{H}$ & -2.7063 & -0.5545 & -1.9375 \\
\hline 43 & $\mathrm{H}$ & 4.7517 & 0.468 & 2.1142 \\
\hline 44 & $\mathrm{H}$ & 6.443 & 0.4858 & 1.5641 \\
\hline 45 & $\mathrm{H}$ & 5.337 & 1.7983 & 1.0996 \\
\hline 46 & $\mathrm{H}$ & 4.5703 & -1.7307 & 0.9264 \\
\hline 47 & $\mathrm{H}$ & 5.8063 & 1.3267 & -1.447 \\
\hline 48 & $\mathrm{H}$ & 6.9969 & 0.1612 & -0.8348 \\
\hline 49 & $\mathrm{H}$ & 5.7798 & -0.365 & -2.0036 \\
\hline 50 & $\mathrm{H}$ & 2.1921 & -2.7895 & -0.2324 \\
\hline
\end{tabular}




\begin{tabular}{rrrrr}
$\mathbf{5 1}$ & $\mathrm{H}$ & 0.6175 & -2.991 & 0.5393 \\
$\mathbf{5 2}$ & $\mathrm{H}$ & 0.7522 & -3.1068 & -1.2207 \\
$\mathbf{5 3}$ & $\mathrm{H}$ & -2.1824 & -2.787 & -0.6856 \\
$\mathbf{5 4}$ & $\mathrm{H}$ & -2.6689 & -2.9002 & 1.0298 \\
$\mathbf{5 5}$ & $\mathrm{H}$ & 0.4254 & 3.5272 & -0.6678 \\
$\mathbf{5 6}$ & $\mathrm{H}$ & -0.1515 & 4.6383 & 0.594 \\
$\mathbf{5 7}$ & $\mathrm{H}$ & 0.229 & 2.9528 & 0.9989 \\
$\mathbf{5 8}$ & $\mathrm{H}$ & -2.1068 & 5.2195 & -0.7648 \\
$\mathbf{5 9}$ & $\mathrm{H}$ & -3.3248 & 4.0219 & -1.2201 \\
$\mathbf{6 0}$ & $\mathrm{H}$ & -1.7624 & 4.068 & -2.0719 \\
$\mathbf{6 1}$ & $\mathrm{H}$ & -2.0826 & 2.7621 & 1.7664 \\
$\mathbf{6 2}$ & $\mathrm{H}$ & -3.4658 & 0.3988 & 1.4646 \\
\hline
\end{tabular}


Table S37. Conformer 2b-F/3b-F. $\mathrm{PCM}_{-} \mathrm{CHCl}_{3}$ energy and Boltzmann Populations 1694.81146 hartrees, $21.44 \%$.

\begin{tabular}{|c|c|c|c|c|}
\hline \multicolumn{2}{|c|}{ Atom number } & \multicolumn{3}{|c|}{ Coordinates } \\
\hline & & $x$ & $\mathbf{Y}$ & $\mathbf{Z}$ \\
\hline 1 & 0 & 2.7551 & -0.5037 & 0.8064 \\
\hline 2 & C & 1.3491 & -0.3096 & 0.7008 \\
\hline 3 & $\mathrm{C}$ & 0.7995 & -1.0918 & -0.5069 \\
\hline 4 & $\mathrm{C}$ & 1.48 & -0.5186 & -1.7593 \\
\hline 5 & $\mathrm{C}$ & 3.0146 & -0.5441 & -1.6263 \\
\hline 6 & $\mathrm{C}$ & 3.4883 & 0.0648 & -0.298 \\
\hline 7 & $\mathrm{C}$ & 0.6721 & -0.7067 & 2.0125 \\
\hline 8 & $\mathrm{C}$ & -0.8258 & -1.0429 & 1.7972 \\
\hline 9 & $\mathrm{C}$ & -1.3792 & -0.4562 & 0.4917 \\
\hline 10 & 0 & -0.6294 & -0.8388 & -0.6422 \\
\hline 11 & $\mathrm{O}$ & -1.3518 & 0.9726 & 0.6937 \\
\hline 12 & $\mathrm{C}$ & -1.6915 & 1.7765 & -0.4629 \\
\hline 13 & $\mathrm{C}$ & -3.1333 & 1.4811 & -0.8731 \\
\hline 14 & $\mathrm{C}$ & -3.3415 & -0.0311 & -1.0518 \\
\hline 15 & $\mathrm{C}$ & -2.8792 & -0.8244 & 0.1906 \\
\hline 16 & $\mathrm{C}$ & 4.9798 & -0.1998 & 0.0354 \\
\hline 17 & $\mathrm{C}$ & 5.3755 & 0.5502 & 1.3188 \\
\hline 18 & 0 & 5.1614 & -1.6094 & 0.2223 \\
\hline 19 & $\mathrm{C}$ & 5.9021 & 0.1978 & -1.1182 \\
\hline 20 & $\mathrm{C}$ & 1.0199 & -2.6072 & -0.4036 \\
\hline 21 & $\mathrm{C}$ & -3.0263 & -2.3351 & 0.0009 \\
\hline 22 & $\mathrm{C}$ & -1.3414 & 3.2344 & -0.0784 \\
\hline 23 & $\mathrm{C}$ & -1.409 & 4.1447 & -1.3055 \\
\hline 24 & $\mathrm{C}$ & -2.2144 & 3.7801 & 1.063 \\
\hline 25 & 0 & 0.0347 & 3.2494 & 0.3342 \\
\hline 26 & $\mathrm{Cl}$ & -4.7034 & -2.8586 & -0.4657 \\
\hline 27 & 0 & -3.653 & -0.4751 & 1.3361 \\
\hline 28 & $\mathrm{H}$ & 1.16 & 0.7539 & 0.5004 \\
\hline 29 & $\mathrm{H}$ & 1.1613 & -1.0727 & -2.6505 \\
\hline 30 & $\mathrm{H}$ & 1.1381 & 0.5165 & -1.8851 \\
\hline 31 & $\mathrm{H}$ & 3.3954 & -1.5685 & -1.6933 \\
\hline 32 & $\mathrm{H}$ & 3.4533 & 0.0155 & -2.4599 \\
\hline 33 & $\mathrm{H}$ & 3.307 & 1.1526 & -0.3077 \\
\hline 34 & $\mathrm{H}$ & 1.1915 & -1.5778 & 2.426 \\
\hline 35 & $\mathrm{H}$ & 0.7911 & 0.1032 & 2.7387 \\
\hline 36 & $\mathrm{H}$ & -0.9624 & -2.1265 & 1.7665 \\
\hline 37 & $\mathrm{H}$ & -1.4372 & -0.6795 & 2.6247 \\
\hline 38 & $\mathrm{H}$ & -1.017 & 1.4898 & -1.2779 \\
\hline 39 & $\mathrm{H}$ & -3.8276 & 1.8603 & -0.1131 \\
\hline 40 & $\mathrm{H}$ & -3.3749 & 1.9996 & -1.808 \\
\hline 41 & $\mathrm{H}$ & -4.399 & -0.2475 & -1.222 \\
\hline 42 & $\mathrm{H}$ & -2.7822 & -0.383 & -1.9259 \\
\hline 43 & $\mathrm{H}$ & 4.7146 & 0.2777 & 2.1468 \\
\hline 44 & $\mathrm{H}$ & 6.4037 & 0.2911 & 1.5902 \\
\hline 45 & $\mathrm{H}$ & 5.3155 & 1.6365 & 1.1806 \\
\hline 46 & $\mathrm{H}$ & 4.4915 & -1.871 & 0.877 \\
\hline 47 & $\mathrm{H}$ & 5.7693 & 1.2524 & -1.3847 \\
\hline 48 & $\mathrm{H}$ & 6.9445 & 0.0479 & -0.8196 \\
\hline 49 & $\mathrm{H}$ & 5.7161 & -0.4172 & -2.0027 \\
\hline 50 & $\mathrm{H}$ & 2.0828 & -2.8452 & -0.3144 \\
\hline
\end{tabular}




\begin{tabular}{rrrrr}
$\mathbf{5 1}$ & $\mathrm{H}$ & 0.5085 & -3.0402 & 0.46 \\
$\mathbf{5 2}$ & $\mathrm{H}$ & 0.6307 & -3.0945 & -1.3039 \\
$\mathbf{5 3}$ & $\mathrm{H}$ & -2.3591 & -2.6842 & -0.7855 \\
$\mathbf{5 4}$ & $\mathrm{H}$ & -2.8144 & -2.8567 & 0.9335 \\
$\mathbf{5 5}$ & $\mathrm{H}$ & -2.4333 & 4.2433 & -1.678 \\
$\mathbf{5 6}$ & $\mathrm{H}$ & -1.0423 & 5.1407 & -1.0383 \\
$\mathbf{5 7}$ & $\mathrm{H}$ & -0.7761 & 3.7558 & -2.1102 \\
$\mathbf{5 8}$ & $\mathrm{H}$ & -1.8394 & 4.7638 & 1.3615 \\
$\mathbf{5 9}$ & $\mathrm{H}$ & -2.1722 & 3.1192 & 1.936 \\
$\mathbf{6 0}$ & $\mathrm{H}$ & -3.2634 & 3.8895 & 0.7672 \\
$\mathbf{6 1}$ & $\mathrm{H}$ & 0.0967 & 2.5912 & 1.0466 \\
$\mathbf{6 2}$ & $\mathrm{H}$ & -3.3862 & 0.4275 & 1.5775 \\
\hline
\end{tabular}


Table S38. Conformer 2b-G/3b-G. PCM- $\mathrm{CHCl}_{3}$ energy and Boltzmann Populations 1694.81003 hartrees, $4.69 \%$.

\begin{tabular}{|c|c|c|c|c|}
\hline \multicolumn{2}{|c|}{ Atom number } & \multicolumn{3}{|c|}{ Coordinates } \\
\hline & & $x$ & $\mathbf{Y}$ & $\mathbf{Z}$ \\
\hline 1 & 0 & 2.8028 & -0.5113 & 0.7921 \\
\hline 2 & C & 1.3952 & -0.3164 & 0.6857 \\
\hline 3 & $\mathrm{C}$ & 0.8443 & -1.1327 & -0.5002 \\
\hline 4 & $\mathrm{C}$ & 1.5344 & -0.6132 & -1.7735 \\
\hline 5 & $\mathrm{C}$ & 3.0696 & -0.6432 & -1.6334 \\
\hline 6 & $\mathrm{C}$ & 3.5175 & 0.0379 & -0.3336 \\
\hline 7 & $\mathrm{C}$ & 0.7161 & -0.6689 & 2.0072 \\
\hline 8 & $\mathrm{C}$ & -0.7827 & -1.0007 & 1.7967 \\
\hline 9 & $\mathrm{C}$ & -1.3358 & -0.4589 & 0.4709 \\
\hline 10 & $\mathrm{O}$ & -0.5779 & -0.8671 & -0.6532 \\
\hline 11 & $\mathrm{O}$ & -1.3132 & 0.9715 & 0.6238 \\
\hline 12 & $\mathrm{C}$ & -1.7076 & 1.7675 & -0.5207 \\
\hline 13 & $\mathrm{C}$ & -3.1225 & 1.388 & -0.9609 \\
\hline 14 & $\mathrm{C}$ & -3.293 & -0.1312 & -1.0891 \\
\hline 15 & $\mathrm{C}$ & -2.828 & -0.8658 & 0.1836 \\
\hline 16 & $\mathrm{C}$ & 5.0179 & -0.0526 & 0.0348 \\
\hline 17 & $\mathrm{C}$ & 5.8783 & 0.7011 & -0.9812 \\
\hline 18 & 0 & 5.1833 & 0.6458 & 1.2749 \\
\hline 19 & $\mathrm{C}$ & 5.4993 & -1.5036 & 0.2004 \\
\hline 20 & $\mathrm{C}$ & 1.0491 & -2.6463 & -0.3441 \\
\hline 21 & $\mathrm{C}$ & -2.9478 & -2.3858 & 0.055 \\
\hline 22 & $\mathrm{C}$ & -1.5624 & 3.2388 & -0.0459 \\
\hline 23 & $\mathrm{C}$ & -0.0927 & 3.5731 & 0.2566 \\
\hline 24 & $\mathrm{C}$ & -2.1237 & 4.2181 & -1.0791 \\
\hline 25 & 0 & -2.3503 & 3.4213 & 1.1386 \\
\hline 26 & $\mathrm{Cl}$ & -4.6046 & -2.9575 & -0.4263 \\
\hline 27 & 0 & -3.6133 & -0.4912 & 1.3126 \\
\hline 28 & $\mathrm{H}$ & 1.2033 & 0.7413 & 0.4594 \\
\hline 29 & $\mathrm{H}$ & 1.2127 & -1.1994 & -2.6425 \\
\hline 30 & $\mathrm{H}$ & 1.1992 & 0.4181 & -1.9422 \\
\hline 31 & $\mathrm{H}$ & 3.4269 & -1.6783 & -1.6546 \\
\hline 32 & $\mathrm{H}$ & 3.5239 & -0.1322 & -2.4902 \\
\hline 33 & $\mathrm{H}$ & 3.2713 & 1.1115 & -0.3913 \\
\hline 34 & $\mathrm{H}$ & 1.2281 & -1.5304 & 2.4492 \\
\hline 35 & $\mathrm{H}$ & 0.8382 & 0.1648 & 2.7052 \\
\hline 36 & $\mathrm{H}$ & -0.9252 & -2.0837 & 1.8059 \\
\hline 37 & $\mathrm{H}$ & -1.3969 & -0.6049 & 2.607 \\
\hline 38 & $\mathrm{H}$ & -0.9993 & 1.5763 & -1.3374 \\
\hline 39 & $\mathrm{H}$ & -3.8383 & 1.7948 & -0.236 \\
\hline 40 & $\mathrm{H}$ & -3.3488 & 1.8598 & -1.9231 \\
\hline 41 & $\mathrm{H}$ & -4.3437 & -0.3758 & -1.2649 \\
\hline 42 & $\mathrm{H}$ & -2.7155 & -0.5037 & -1.9424 \\
\hline 43 & $\mathrm{H}$ & 5.887 & 0.2029 & -1.956 \\
\hline 44 & $\mathrm{H}$ & 5.5183 & 1.7276 & -1.1098 \\
\hline 45 & $\mathrm{H}$ & 6.9077 & 0.7505 & -0.6123 \\
\hline 46 & $\mathrm{H}$ & 4.4675 & 0.3223 & 1.849 \\
\hline 47 & $\mathrm{H}$ & 5.4908 & -2.0546 & -0.7467 \\
\hline 48 & $\mathrm{H}$ & 6.5247 & -1.4958 & 0.5822 \\
\hline 49 & $\mathrm{H}$ & 4.8685 & -2.0401 & 0.9163 \\
\hline 50 & $\mathrm{H}$ & 0.5162 & -3.0456 & 0.5224 \\
\hline
\end{tabular}




\begin{tabular}{rrrrr}
$\mathbf{5 1}$ & $\mathrm{H}$ & 0.6731 & -3.1601 & -1.2354 \\
$\mathbf{5 2}$ & $\mathrm{H}$ & 2.1071 & -2.8905 & -0.2217 \\
$\mathbf{5 3}$ & $\mathrm{H}$ & -2.2575 & -2.7566 & -0.7008 \\
$\mathbf{5 4}$ & $\mathrm{H}$ & -2.7501 & -2.8644 & 1.0134 \\
$\mathbf{5 5}$ & $\mathrm{H}$ & 0.5317 & 3.4799 & -0.6402 \\
$\mathbf{5 6}$ & $\mathrm{H}$ & -0.0186 & 4.6016 & 0.6237 \\
$\mathbf{5 7}$ & $\mathrm{H}$ & 0.3118 & 2.9045 & 1.0229 \\
$\mathbf{5 8}$ & $\mathrm{H}$ & -1.634 & 4.0896 & -2.051 \\
$\mathbf{5 9}$ & $\mathrm{H}$ & -3.2009 & 4.0837 & -1.2063 \\
$\mathbf{6 0}$ & $\mathrm{H}$ & -1.9515 & 5.2442 & -0.7399 \\
$\mathbf{6 1}$ & $\mathrm{H}$ & -2.002 & 2.783 & 1.7834 \\
$\mathbf{6 2}$ & $\mathrm{H}$ & -3.4495 & 0.4542 & 1.4644 \\
\hline
\end{tabular}


Table S39. Conformer 2b-H/3b-H. PCM- $\mathrm{CHCl}_{3}$ energy and Boltzmann Populations 1694.81103 hartrees, $13.57 \%$.

\begin{tabular}{|c|c|c|c|c|}
\hline \multicolumn{2}{|c|}{ Atom number } & \multicolumn{3}{|c|}{ Coordinates } \\
\hline & & $\mathbf{X}$ & $\mathbf{Y}$ & $\mathbf{Z}$ \\
\hline 1 & 0 & 2.7436 & -0.6149 & 0.7961 \\
\hline 2 & $\mathrm{C}$ & 1.3413 & -0.3842 & 0.7001 \\
\hline 3 & $\mathrm{C}$ & 0.7716 & -1.1447 & -0.5138 \\
\hline 4 & C & 1.4675 & -0.5878 & -1.7677 \\
\hline 5 & $\mathrm{C}$ & 3.0026 & -0.6543 & -1.6334 \\
\hline 6 & C & 3.467 & -0.033 & -0.3095 \\
\hline 7 & $\mathrm{C}$ & 0.6558 & -0.775 & 2.0084 \\
\hline 8 & C & -0.8516 & -1.065 & 1.7911 \\
\hline 9 & $\mathrm{C}$ & -1.3886 & -0.4549 & 0.489 \\
\hline 10 & 0 & -0.6488 & -0.8503 & -0.6485 \\
\hline 11 & 0 & -1.3223 & 0.9705 & 0.6988 \\
\hline 12 & C & -1.6438 & 1.7898 & -0.4527 \\
\hline 13 & C & -3.0942 & 1.5357 & -0.8593 \\
\hline 14 & $\mathrm{C}$ & -3.3419 & 0.0309 & -1.0486 \\
\hline 15 & $\mathrm{C}$ & -2.898 & -0.7832 & 0.1867 \\
\hline 16 & $\mathrm{C}$ & 4.9659 & -0.1681 & 0.0499 \\
\hline 17 & $\mathrm{C}$ & 5.8372 & 0.6124 & -0.9362 \\
\hline 18 & $\mathrm{O}$ & 5.1489 & 0.4719 & 1.3188 \\
\hline 19 & $\mathrm{C}$ & 5.421 & -1.6335 & 0.152 \\
\hline 20 & $\mathrm{C}$ & 0.9466 & -2.6664 & -0.4159 \\
\hline 21 & $\mathrm{C}$ & -3.0833 & -2.288 & -0.0145 \\
\hline 22 & $\mathrm{C}$ & -1.2526 & 3.2357 & -0.0639 \\
\hline 23 & $\mathrm{C}$ & -1.3102 & 4.1543 & -1.2855 \\
\hline 24 & $\mathrm{C}$ & -2.099 & 3.797 & 1.0897 \\
\hline 25 & 0 & 0.128 & 3.2144 & 0.3322 \\
\hline 26 & $\mathrm{Cl}$ & -4.7735 & -2.7653 & -0.4835 \\
\hline 27 & 0 & -3.6614 & -0.4239 & 1.336 \\
\hline 28 & $\mathrm{H}$ & 1.1764 & 0.6846 & 0.5074 \\
\hline 29 & $\mathrm{H}$ & 1.1326 & -1.1326 & -2.6584 \\
\hline 30 & $\mathrm{H}$ & 1.156 & 0.4567 & -1.8921 \\
\hline 31 & $\mathrm{H}$ & 3.3405 & -1.6944 & -1.6973 \\
\hline 32 & $\mathrm{H}$ & 3.4636 & -0.1179 & -2.4706 \\
\hline 33 & $\mathrm{H}$ & 3.2402 & 1.0456 & -0.3228 \\
\hline 34 & $\mathrm{H}$ & 1.1502 & -1.6647 & 2.4128 \\
\hline 35 & $\mathrm{H}$ & 0.7977 & 0.0249 & 2.7418 \\
\hline 36 & $\mathrm{H}$ & -1.0204 & -2.1439 & 1.755 \\
\hline 37 & $\mathrm{H}$ & -1.4518 & -0.688 & 2.6206 \\
\hline 38 & $\mathrm{H}$ & -0.9804 & 1.4887 & -1.2716 \\
\hline 39 & $\mathrm{H}$ & -3.7757 & 1.9275 & -0.0941 \\
\hline 40 & $\mathrm{H}$ & -3.3255 & 2.067 & -1.7896 \\
\hline 41 & $\mathrm{H}$ & -4.4049 & -0.1572 & -1.2185 \\
\hline 42 & $\mathrm{H}$ & -2.7935 & -0.3288 & -1.9265 \\
\hline 43 & $\mathrm{H}$ & 5.4987 & 1.651 & -1.0148 \\
\hline 44 & $\mathrm{H}$ & 5.8287 & 0.1594 & -1.9328 \\
\hline 45 & $\mathrm{H}$ & 6.8697 & 0.622 & -0.5728 \\
\hline 46 & $\mathrm{H}$ & 4.4248 & 0.1427 & 1.8789 \\
\hline 47 & $\mathrm{H}$ & 4.7817 & -2.1887 & 0.8458 \\
\hline 48 & $\mathrm{H}$ & 5.4019 & -2.144 & -0.8177 \\
\hline 49 & $\mathrm{H}$ & 6.4469 & -1.6605 & 0.5319 \\
\hline 50 & $\mathrm{H}$ & 0.5545 & -3.1385 & -1.3231 \\
\hline
\end{tabular}




\begin{tabular}{rrrrr}
$\mathbf{5 1}$ & $\mathrm{H}$ & 2.0003 & -2.9358 & -0.311 \\
$\mathbf{5 2}$ & $\mathrm{H}$ & 0.4116 & -3.0874 & 0.439 \\
$\mathbf{5 3}$ & $\mathrm{H}$ & -2.4258 & -2.6477 & -0.8044 \\
$\mathbf{5 4}$ & $\mathrm{H}$ & -2.8841 & -2.8223 & 0.9137 \\
$\mathbf{5 5}$ & $\mathrm{H}$ & -2.3357 & 4.2832 & -1.6449 \\
$\mathbf{5 6}$ & $\mathrm{H}$ & -0.9126 & 5.138 & -1.0174 \\
$\mathbf{5 7}$ & $\mathrm{H}$ & -0.6978 & 3.7529 & -2.0999 \\
$\mathbf{5 8}$ & $\mathrm{H}$ & -3.1486 & 3.9326 & 0.8065 \\
$\mathbf{5 9}$ & $\mathrm{H}$ & -2.0627 & 3.1312 & 1.9593 \\
$\mathbf{6 0}$ & $\mathrm{H}$ & -1.6974 & 4.7702 & 1.388 \\
$\mathbf{6 1}$ & $\mathrm{H}$ & 0.1817 & 2.5647 & 1.0526 \\
$\mathbf{6 2}$ & $\mathrm{H}$ & -3.3754 & 0.4716 & 1.5821 \\
\hline
\end{tabular}




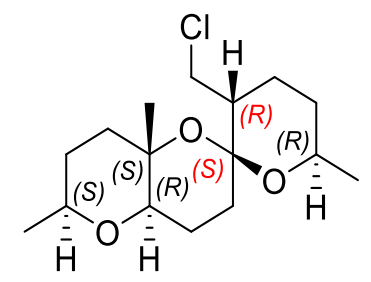

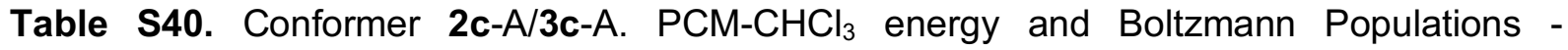
1694.808387 hartrees, $28.31 \%$.

\begin{tabular}{|c|c|c|c|c|}
\hline \multicolumn{2}{|c|}{ Atom number } & \multicolumn{3}{|c|}{ Coordinates } \\
\hline & & $x$ & $\mathbf{Y}$ & $\mathbf{Z}$ \\
\hline 1 & 0 & -3.1114 & 0.3613 & 0.7233 \\
\hline 2 & $\mathrm{C}$ & -1.7998 & -0.1779 & 0.8195 \\
\hline 3 & $\mathrm{C}$ & -1.1709 & -0.2878 & -0.5804 \\
\hline 4 & $\mathrm{C}$ & -2.0209 & -1.301 & -1.36 \\
\hline 5 & $\mathrm{C}$ & -3.5062 & -0.8879 & -1.3585 \\
\hline 6 & $\mathrm{C}$ & -4.024 & -0.5254 & 0.0444 \\
\hline 7 & $\mathrm{C}$ & -0.9207 & 0.6254 & 1.761 \\
\hline 8 & $\mathrm{C}$ & 0.4281 & -0.1053 & 1.8655 \\
\hline 9 & $\mathrm{C}$ & 1.07 & -0.401 & 0.4909 \\
\hline 10 & $\mathrm{O}$ & 0.1429 & -0.8853 & -0.4484 \\
\hline 11 & $\mathrm{O}$ & 1.653 & 0.7889 & -0.0838 \\
\hline 12 & $\mathrm{C}$ & 2.7977 & 1.3938 & 0.5536 \\
\hline 13 & C & 3.918 & 0.3676 & 0.7403 \\
\hline 14 & C & 3.39 & -0.9339 & 1.3519 \\
\hline 15 & $\mathrm{C}$ & 2.1831 & -1.5043 & 0.5839 \\
\hline 16 & $\mathrm{C}$ & -5.3816 & 0.2254 & 0.0477 \\
\hline 17 & $\mathrm{C}$ & -5.8721 & 0.425 & 1.4921 \\
\hline 18 & 0 & -5.1954 & 1.4999 & -0.581 \\
\hline 19 & $\mathrm{C}$ & -6.4431 & -0.5042 & -0.7773 \\
\hline 20 & $\mathrm{C}$ & -1.0922 & 1.0513 & -1.3255 \\
\hline 21 & $\mathrm{C}$ & 2.5404 & -1.9494 & -0.8474 \\
\hline 22 & $\mathrm{C}$ & 3.181 & 2.5801 & -0.3771 \\
\hline 23 & $\mathrm{C}$ & 2.0791 & 3.6532 & -0.3747 \\
\hline 24 & $\mathrm{C}$ & 4.5175 & 3.2069 & 0.0277 \\
\hline 25 & 0 & 3.3633 & 2.0746 & -1.7037 \\
\hline 26 & $\mathrm{Cl}$ & 3.802 & -3.2746 & -0.8399 \\
\hline 27 & 0 & 1.6183 & -2.5997 & 1.2958 \\
\hline 28 & $\mathrm{H}$ & -1.8707 & -1.2036 & 1.221 \\
\hline 29 & $\mathrm{H}$ & -1.6535 & -1.3978 & -2.3888 \\
\hline 30 & $\mathrm{H}$ & -1.897 & -2.2805 & -0.8808 \\
\hline 31 & $\mathrm{H}$ & -3.6665 & -0.0284 & -2.0174 \\
\hline 32 & $\mathrm{H}$ & -4.1071 & -1.7119 & -1.7594 \\
\hline 33 & $\mathrm{H}$ & -4.1177 & -1.4445 & 0.648 \\
\hline 34 & $\mathrm{H}$ & -0.7962 & 1.6515 & 1.3983 \\
\hline 35 & $\mathrm{H}$ & -1.3891 & 0.6857 & 2.75 \\
\hline 36 & $\mathrm{H}$ & 1.1219 & 0.4699 & 2.4845 \\
\hline 37 & $\mathrm{H}$ & 0.2812 & -1.0664 & 2.3658 \\
\hline 38 & $\mathrm{H}$ & 2.4993 & 1.7993 & 1.5325 \\
\hline 39 & $\mathrm{H}$ & 4.3855 & 0.176 & -0.2318 \\
\hline 40 & $\mathrm{H}$ & 4.6938 & 0.7874 & 1.3904 \\
\hline 41 & $\mathrm{H}$ & 4.1832 & -1.6889 & 1.3797 \\
\hline 42 & $\mathrm{H}$ & 3.0773 & -0.7723 & 2.3901 \\
\hline 43 & $\mathrm{H}$ & -6.7842 & 1.0301 & 1.4872 \\
\hline 44 & $\mathrm{H}$ & -6.095 & -0.5349 & 1.9737 \\
\hline
\end{tabular}




\begin{tabular}{llrrr}
$\mathbf{4 5}$ & $\mathrm{H}$ & -5.1142 & 0.9369 & 2.092 \\
$\mathbf{4 6}$ & $\mathrm{H}$ & -4.4375 & 1.9037 & -0.1241 \\
$\mathbf{4 7}$ & $\mathrm{H}$ & -6.1637 & -0.5465 & -1.8332 \\
$\mathbf{4 8}$ & $\mathrm{H}$ & -6.5968 & -1.5251 & -0.4097 \\
$\mathbf{4 9}$ & $\mathrm{H}$ & -7.3938 & 0.0334 & -0.7038 \\
$\mathbf{5 0}$ & $\mathrm{H}$ & -0.627 & 0.8925 & -2.3039 \\
$\mathbf{5 1}$ & $\mathrm{H}$ & -0.4908 & 1.7806 & -0.7836 \\
$\mathbf{5 2}$ & $\mathrm{H}$ & -2.0888 & 1.4724 & -1.479 \\
$\mathbf{5 3}$ & $\mathrm{H}$ & 1.6661 & -2.3739 & -1.3314 \\
$\mathbf{5 4}$ & $\mathrm{H}$ & 2.9465 & -1.1386 & -1.4494 \\
$\mathbf{5 5}$ & $\mathrm{H}$ & 2.3513 & 4.4554 & -1.0677 \\
$\mathbf{5 6}$ & $\mathrm{H}$ & 1.1211 & 3.2352 & -0.6953 \\
$\mathbf{5 7}$ & $\mathrm{H}$ & 1.9454 & 4.0886 & 0.6232 \\
$\mathbf{5 8}$ & $\mathrm{H}$ & 5.3403 & 2.4976 & -0.091 \\
$\mathbf{5 9}$ & $\mathrm{H}$ & 4.7233 & 4.0682 & -0.6154 \\
$\mathbf{6 0}$ & $\mathrm{H}$ & 4.4941 & 3.5533 & 1.0673 \\
$\mathbf{6 1}$ & $\mathrm{H}$ & 2.5567 & 1.5651 & -1.8953 \\
$\mathbf{6 2}$ & $\mathrm{H}$ & 2.2636 & -3.3256 & 1.2521 \\
\hline
\end{tabular}


Table S41. Conformer 2c-B/3c-B. $\mathrm{PCM}-\mathrm{CHCl}_{3}$ energy and Boltzmann Populations 1694.807499 hartrees, $11.06 \%$.

\begin{tabular}{|c|c|c|c|c|}
\hline \multicolumn{2}{|c|}{ Atom number } & \multicolumn{2}{|c|}{ Coordinates } & \multirow[b]{2}{*}{$\mathbf{Z}$} \\
\hline & & X & $\mathbf{Y}$ & \\
\hline 1 & 0 & -3.0831 & 0.3769 & 0.7023 \\
\hline 2 & C & -1.7769 & -0.1714 & 0.8164 \\
\hline 3 & C & -1.1557 & -0.3425 & -0.5807 \\
\hline 4 & $\mathrm{C}$ & -2.0179 & -1.378 & -1.3174 \\
\hline 5 & $\mathrm{C}$ & -3.5031 & -0.9629 & -1.3165 \\
\hline 6 & $\mathrm{C}$ & -4.0065 & -0.5348 & 0.0733 \\
\hline 7 & $\mathrm{C}$ & -0.8849 & 0.655 & 1.726 \\
\hline 8 & $\mathrm{C}$ & 0.4581 & -0.0841 & 1.8447 \\
\hline 9 & C & 1.09 & -0.4191 & 0.4745 \\
\hline 10 & $\mathrm{O}$ & 0.1521 & -0.9481 & -0.4303 \\
\hline 11 & $\mathrm{O}$ & 1.6586 & 0.7454 & -0.1599 \\
\hline 12 & $\mathrm{C}$ & 2.7796 & 1.3905 & 0.4762 \\
\hline 13 & $\mathrm{C}$ & 3.935 & 0.4012 & 0.6388 \\
\hline 14 & $\mathrm{C}$ & 3.4405 & -0.8886 & 1.3069 \\
\hline 15 & $\mathrm{C}$ & 2.2195 & -1.5052 & 0.5942 \\
\hline 16 & $\mathrm{C}$ & -5.3609 & 0.2214 & 0.0541 \\
\hline 17 & $\mathrm{C}$ & -5.834 & 0.4953 & 1.492 \\
\hline 18 & $\mathrm{O}$ & -5.1773 & 1.4618 & -0.6401 \\
\hline 19 & $\mathrm{C}$ & -6.4342 & -0.5445 & -0.7211 \\
\hline 20 & $\mathrm{C}$ & -1.0721 & 0.9653 & -1.3808 \\
\hline 21 & $\mathrm{C}$ & 2.5422 & -2.015 & -0.823 \\
\hline 22 & $\mathrm{C}$ & 3.0659 & 2.6616 & -0.3642 \\
\hline 23 & $\mathrm{C}$ & 4.1153 & 3.5378 & 0.3232 \\
\hline 24 & $\mathrm{C}$ & 3.4757 & 2.3403 & -1.8106 \\
\hline 25 & $\mathrm{O}$ & 1.8667 & 3.4461 & -0.3755 \\
\hline 26 & $\mathrm{Cl}$ & 3.8402 & -3.3052 & -0.7949 \\
\hline 27 & 0 & 1.6899 & -2.573 & 1.3723 \\
\hline 28 & $\mathrm{H}$ & -1.8574 & -1.1804 & 1.2566 \\
\hline 29 & $\mathrm{H}$ & -1.661 & -1.5121 & -2.3458 \\
\hline 30 & $\mathrm{H}$ & -1.8908 & -2.3401 & -0.8053 \\
\hline 31 & $\mathrm{H}$ & -3.6714 & -0.1346 & -2.0122 \\
\hline 32 & $\mathrm{H}$ & -4.1082 & -1.8041 & -1.6731 \\
\hline 33 & $\mathrm{H}$ & -4.0991 & -1.4248 & 0.719 \\
\hline 34 & $\mathrm{H}$ & -0.751 & 1.6693 & 1.3348 \\
\hline 35 & $\mathrm{H}$ & -1.3466 & 0.7495 & 2.7153 \\
\hline 36 & $\mathrm{H}$ & 1.1564 & 0.4985 & 2.4508 \\
\hline 37 & $\mathrm{H}$ & 0.3059 & -1.033 & 2.3661 \\
\hline 38 & $\mathrm{H}$ & 2.4689 & 1.7501 & 1.4679 \\
\hline 39 & $\mathrm{H}$ & 4.3751 & 0.1714 & -0.3383 \\
\hline 40 & $\mathrm{H}$ & 4.7281 & 0.8516 & 1.2467 \\
\hline 41 & $\mathrm{H}$ & 4.2439 & -1.6324 & 1.3413 \\
\hline 42 & $\mathrm{H}$ & 3.1523 & -0.6915 & 2.3458 \\
\hline 43 & $\mathrm{H}$ & -5.0686 & 1.036 & 2.0561 \\
\hline 44 & $\mathrm{H}$ & -6.7455 & 1.1008 & 1.4669 \\
\hline 45 & $\mathrm{H}$ & -6.052 & -0.4384 & 2.0247 \\
\hline 46 & $\mathrm{H}$ & -4.4152 & 1.8874 & -0.2107 \\
\hline 47 & $\mathrm{H}$ & -6.5893 & -1.5437 & -0.2985 \\
\hline 48 & $\mathrm{H}$ & -7.3813 & 0.0016 & -0.6667 \\
\hline 49 & $\mathrm{H}$ & -6.1658 & -0.6441 & -1.7761 \\
\hline 50 & $\mathrm{H}$ & -0.5707 & 1.7545 & -0.8195 \\
\hline
\end{tabular}




\begin{tabular}{rrrrr}
$\mathbf{5 1}$ & $\mathrm{H}$ & -0.5052 & 0.7877 & -2.2996 \\
$\mathbf{5 2}$ & $\mathrm{H}$ & -2.0679 & 1.3275 & -1.6463 \\
$\mathbf{5 3}$ & $\mathrm{H}$ & 1.6639 & -2.4868 & -1.2529 \\
$\mathbf{5 4}$ & $\mathrm{H}$ & 2.9011 & -1.2258 & -1.4807 \\
$\mathbf{5 5}$ & $\mathrm{H}$ & 5.1001 & 3.0604 & 0.3296 \\
$\mathbf{5 6}$ & $\mathrm{H}$ & 4.1967 & 4.4877 & -0.2141 \\
$\mathbf{5 7}$ & $\mathrm{H}$ & 3.8214 & 3.7592 & 1.3549 \\
$\mathbf{5 8}$ & $\mathrm{H}$ & 2.7295 & 1.6984 & -2.2903 \\
$\mathbf{5 9}$ & $\mathrm{H}$ & 3.5436 & 3.2743 & -2.3768 \\
$\mathbf{6 0}$ & $\mathrm{H}$ & 4.4471 & 1.8366 & -1.8666 \\
$\mathbf{6 1}$ & $\mathrm{H}$ & 1.1754 & 2.8603 & -0.7254 \\
$\mathbf{6 2}$ & $\mathrm{H}$ & 2.3457 & -3.2904 & 1.3491 \\
\hline
\end{tabular}


Table S42. Conformer 2c-C/3c-C. $\mathrm{PCM}^{-} \mathrm{CHCl}_{3}$ energy and Boltzmann Populations 1694.807950 hartrees, $17.82 \%$.

\begin{tabular}{|c|c|c|c|c|}
\hline \multicolumn{2}{|c|}{ Atom number } & \multicolumn{2}{|c|}{ Coordinates } & \multirow[b]{2}{*}{$\mathbf{Z}$} \\
\hline & & X & $Y$ & \\
\hline 1 & 0 & -3.1117 & 0.4527 & 0.6681 \\
\hline 2 & C & -1.8057 & -0.1009 & 0.7739 \\
\hline 3 & C & -1.1633 & -0.2005 & -0.6221 \\
\hline 4 & C & -2.0247 & -1.1766 & -1.4397 \\
\hline 5 & $\mathrm{C}$ & -3.5038 & -0.7361 & -1.4419 \\
\hline 6 & $\mathrm{C}$ & -4.0097 & -0.4425 & -0.022 \\
\hline 7 & $\mathrm{C}$ & -0.9233 & 0.6772 & 1.7328 \\
\hline 8 & $\mathrm{C}$ & 0.4104 & -0.0811 & 1.8439 \\
\hline 9 & $\mathrm{C}$ & 1.0602 & -0.3864 & 0.4747 \\
\hline 10 & $\mathrm{O}$ & 0.1318 & -0.835 & -0.4817 \\
\hline 11 & $\mathrm{O}$ & 1.688 & 0.7878 & -0.0839 \\
\hline 12 & $\mathrm{C}$ & 2.8428 & 1.3524 & 0.5727 \\
\hline 13 & C & 3.9276 & 0.2904 & 0.767 \\
\hline 14 & $\mathrm{C}$ & 3.3507 & -0.9975 & 1.3627 \\
\hline 15 & $\mathrm{C}$ & 2.137 & -1.5251 & 0.5744 \\
\hline 16 & $\mathrm{C}$ & -5.4167 & 0.188 & 0.1079 \\
\hline 17 & $\mathrm{C}$ & -6.4962 & -0.7741 & -0.3918 \\
\hline 18 & $\mathrm{O}$ & -5.6687 & 0.3598 & 1.5082 \\
\hline 19 & $\mathrm{C}$ & -5.5215 & 1.5516 & -0.5959 \\
\hline 20 & $\mathrm{C}$ & -1.0362 & 1.1492 & -1.3403 \\
\hline 21 & $\mathrm{C}$ & 2.5003 & -1.971 & -0.8552 \\
\hline 22 & $\mathrm{C}$ & 3.2751 & 2.5323 & -0.3447 \\
\hline 23 & $\mathrm{C}$ & 2.2064 & 3.6383 & -0.3503 \\
\hline 24 & $\mathrm{C}$ & 4.6242 & 3.1152 & 0.0833 \\
\hline 25 & $\mathrm{O}$ & 3.4607 & 2.0296 & -1.6719 \\
\hline 26 & $\mathrm{Cl}$ & 3.719 & -3.3356 & -0.8401 \\
\hline 27 & 0 & 1.5287 & -2.607 & 1.2701 \\
\hline 28 & $\mathrm{H}$ & -1.8912 & -1.1315 & 1.1597 \\
\hline 29 & $\mathrm{H}$ & -1.6451 & -1.2521 & -2.4657 \\
\hline 30 & $\mathrm{H}$ & -1.9282 & -2.171 & -0.9862 \\
\hline 31 & $\mathrm{H}$ & -3.6282 & 0.1517 & -2.0709 \\
\hline 32 & $\mathrm{H}$ & -4.1177 & -1.5277 & -1.887 \\
\hline 33 & $\mathrm{H}$ & -4.0332 & -1.3866 & 0.5477 \\
\hline 34 & $\mathrm{H}$ & -0.7769 & 1.705 & 1.3844 \\
\hline 35 & $\mathrm{H}$ & -1.3997 & 0.7337 & 2.7183 \\
\hline 36 & $\mathrm{H}$ & 1.1103 & 0.4778 & 2.4709 \\
\hline 37 & $\mathrm{H}$ & 0.2393 & -1.0405 & 2.3395 \\
\hline 38 & $\mathrm{H}$ & 2.5442 & 1.7605 & 1.5503 \\
\hline 39 & $\mathrm{H}$ & 4.402 & 0.0905 & -0.2001 \\
\hline 40 & $\mathrm{H}$ & 4.7072 & 0.6816 & 1.43 \\
\hline 41 & $\mathrm{H}$ & 4.1194 & -1.7772 & 1.3964 \\
\hline 42 & $\mathrm{H}$ & 3.0289 & -0.8327 & 2.3975 \\
\hline 43 & $\mathrm{H}$ & -6.4392 & -0.9199 & -1.4753 \\
\hline 44 & $\mathrm{H}$ & -6.4082 & -1.7474 & 0.1029 \\
\hline 45 & $\mathrm{H}$ & -7.4819 & -0.3631 & -0.1519 \\
\hline 46 & $\mathrm{H}$ & -4.8595 & 0.7642 & 1.8661 \\
\hline 47 & $\mathrm{H}$ & -5.4373 & 1.4659 & -1.6853 \\
\hline 48 & $\mathrm{H}$ & -6.4932 & 1.9975 & -0.3626 \\
\hline 49 & $\mathrm{H}$ & -4.7371 & 2.2288 & -0.2428 \\
\hline 50 & $\mathrm{H}$ & -2.0174 & 1.6038 & -1.4958 \\
\hline
\end{tabular}




\begin{tabular}{rrrrr}
$\mathbf{5 1}$ & $\mathrm{H}$ & -0.4209 & 1.8504 & -0.7779 \\
$\mathbf{5 2}$ & $\mathrm{H}$ & -0.5628 & 0.9948 & -2.3156 \\
$\mathbf{5 3}$ & $\mathrm{H}$ & 1.62 & -2.3642 & -1.3547 \\
$\mathbf{5 4}$ & $\mathrm{H}$ & 2.9405 & -1.1692 & -1.4453 \\
$\mathbf{5 5}$ & $\mathrm{H}$ & 2.0724 & 4.0718 & 0.6483 \\
$\mathbf{5 6}$ & $\mathrm{H}$ & 2.5125 & 4.4358 & -1.0346 \\
$\mathbf{5 7}$ & $\mathrm{H}$ & 1.2406 & 3.2516 & -0.6863 \\
$\mathbf{5 8}$ & $\mathrm{H}$ & 4.5953 & 3.4583 & 1.1238 \\
$\mathbf{5 9}$ & $\mathrm{H}$ & 5.4261 & 2.3806 & -0.0253 \\
$\mathbf{6 0}$ & $\mathrm{H}$ & 4.8673 & 3.972 & -0.5528 \\
$\mathbf{6 1}$ & $\mathrm{H}$ & 2.6424 & 1.5446 & -1.8772 \\
$\mathbf{6 2}$ & $\mathrm{H}$ & 2.153 & -3.3515 & 1.2323 \\
\hline
\end{tabular}




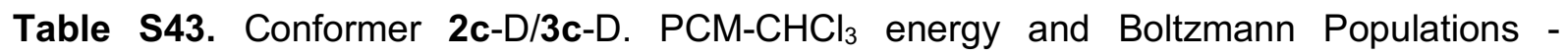
1694.807078 hartrees, $7.08 \%$.

\begin{tabular}{|c|c|c|c|c|}
\hline \multicolumn{2}{|c|}{ Atom number } & \multicolumn{2}{|c|}{ Coordinates } & \multirow[b]{2}{*}{$\mathbf{Z}$} \\
\hline & & X & $\mathbf{Y}$ & \\
\hline 1 & 0 & -3.0861 & 0.4579 & 0.651 \\
\hline 2 & C & -1.7847 & -0.1036 & 0.7715 \\
\hline 3 & C & -1.1489 & -0.2543 & -0.6228 \\
\hline 4 & $\mathrm{C}$ & -2.0212 & -1.2481 & -1.4074 \\
\hline 5 & $\mathrm{C}$ & -3.4994 & -0.804 & -1.4121 \\
\hline 6 & $\mathrm{C}$ & -3.9942 & -0.4555 & -0.0006 \\
\hline 7 & $\mathrm{C}$ & -0.8912 & 0.6944 & 1.7042 \\
\hline 8 & $\mathrm{C}$ & 0.4401 & -0.0666 & 1.825 \\
\hline 9 & C & 1.0798 & -0.4065 & 0.4588 \\
\hline 10 & $\mathrm{O}$ & 0.1393 & -0.8979 & -0.4654 \\
\hline 11 & $\mathrm{O}$ & 1.6926 & 0.7444 & -0.1577 \\
\hline 12 & $\mathrm{C}$ & 2.825 & 1.349 & 0.4978 \\
\hline 13 & C & 3.9464 & 0.3228 & 0.6656 \\
\hline 14 & $\mathrm{C}$ & 3.4043 & -0.9562 & 1.3171 \\
\hline 15 & $\mathrm{C}$ & 2.1734 & -1.529 & 0.5841 \\
\hline 16 & $\mathrm{C}$ & -5.3962 & 0.1888 & 0.1139 \\
\hline 17 & $\mathrm{C}$ & -6.4848 & -0.7835 & -0.3447 \\
\hline 18 & $\mathrm{O}$ & -5.6392 & 0.4126 & 1.5083 \\
\hline 19 & $\mathrm{C}$ & -5.4951 & 1.5268 & -0.6381 \\
\hline 20 & $\mathrm{C}$ & -1.015 & 1.0708 & -1.3861 \\
\hline 21 & $\mathrm{C}$ & 2.4988 & -2.0375 & -0.833 \\
\hline 22 & $\mathrm{C}$ & 3.1629 & 2.6165 & -0.329 \\
\hline 23 & $\mathrm{C}$ & 4.2269 & 3.4564 & 0.381 \\
\hline 24 & $\mathrm{C}$ & 3.586 & 2.2924 & -1.771 \\
\hline 25 & $\mathrm{O}$ & 1.9886 & 3.4374 & -0.3543 \\
\hline 26 & $\mathrm{Cl}$ & 3.7544 & -3.3689 & -0.7983 \\
\hline 27 & 0 & 1.6003 & -2.5857 & 1.3459 \\
\hline 28 & $\mathrm{H}$ & -1.8783 & -1.1209 & 1.1895 \\
\hline 29 & $\mathrm{H}$ & -1.6493 & -1.3544 & -2.4335 \\
\hline 30 & $\mathrm{H}$ & -1.9243 & -2.2292 & -0.9261 \\
\hline 31 & $\mathrm{H}$ & -3.6282 & 0.0596 & -2.0732 \\
\hline 32 & $\mathrm{H}$ & -4.1184 & -1.6102 & -1.8225 \\
\hline 33 & $\mathrm{H}$ & -4.0208 & -1.378 & 0.6036 \\
\hline 34 & $\mathrm{H}$ & -0.7407 & 1.714 & 1.3341 \\
\hline 35 & $\mathrm{H}$ & -1.3605 & 0.7763 & 2.6914 \\
\hline 36 & $\mathrm{H}$ & 1.143 & 0.5013 & 2.4395 \\
\hline 37 & $\mathrm{H}$ & 0.2684 & -1.0156 & 2.3399 \\
\hline 38 & $\mathrm{H}$ & 2.513 & 1.7102 & 1.4884 \\
\hline 39 & $\mathrm{H}$ & 4.3908 & 0.0873 & -0.3081 \\
\hline 40 & $\mathrm{H}$ & 4.7457 & 0.7431 & 1.2866 \\
\hline 41 & $\mathrm{H}$ & 4.1835 & -1.725 & 1.355 \\
\hline 42 & $\mathrm{H}$ & 3.1093 & -0.7585 & 2.3541 \\
\hline 43 & $\mathrm{H}$ & -6.4002 & -1.7388 & 0.1843 \\
\hline 44 & $\mathrm{H}$ & -7.4664 & -0.3575 & -0.1142 \\
\hline 45 & $\mathrm{H}$ & -6.4352 & -0.9686 & -1.4226 \\
\hline 46 & $\mathrm{H}$ & -4.8268 & 0.8275 & 1.8464 \\
\hline 47 & $\mathrm{H}$ & -5.4176 & 1.4015 & -1.7243 \\
\hline 48 & $\mathrm{H}$ & -6.4621 & 1.9885 & -0.416 \\
\hline 49 & $\mathrm{H}$ & -4.7036 & 2.2101 & -0.314 \\
\hline 50 & $\mathrm{H}$ & -0.4924 & 1.8267 & -0.7996 \\
\hline
\end{tabular}




\begin{tabular}{rrrrr}
$\mathbf{5 1}$ & $\mathrm{H}$ & -0.4467 & 0.8992 & -2.3053 \\
$\mathbf{5 2}$ & $\mathrm{H}$ & -1.9955 & 1.4752 & -1.6467 \\
$\mathbf{5 3}$ & $\mathrm{H}$ & 1.612 & -2.4777 & -1.2786 \\
$\mathbf{5 4}$ & $\mathrm{H}$ & 2.8916 & -1.2552 & -1.4794 \\
$\mathbf{5 5}$ & $\mathrm{H}$ & 5.1975 & 2.9511 & 0.3988 \\
$\mathbf{5 6}$ & $\mathrm{H}$ & 4.3438 & 4.4076 & -0.1476 \\
$\mathbf{5 7}$ & $\mathrm{H}$ & 3.9237 & 3.6787 & 1.4099 \\
$\mathbf{5 8}$ & $\mathrm{H}$ & 2.8285 & 1.6764 & -2.2669 \\
$\mathbf{5 9}$ & $\mathrm{H}$ & 3.6911 & 3.2276 & -2.3295 \\
$\mathbf{6 0}$ & $\mathrm{H}$ & 4.5425 & 1.7598 & -1.8155 \\
$\mathbf{6 1}$ & $\mathrm{H}$ & 1.2855 & 2.876 & -0.7203 \\
$\mathbf{6 2}$ & $\mathrm{H}$ & 2.2329 & -3.3238 & 1.3245 \\
\hline
\end{tabular}


Table S44. Conformer 2c-E/3c-E. PCM-CHCl 3 energy and Boltzmann Populations 1694.806944 hartrees, $6.14 \%$.

\begin{tabular}{|c|c|c|c|c|}
\hline \multicolumn{2}{|c|}{ Atom number } & \multicolumn{2}{|c|}{ Coordinates } & \multirow[b]{2}{*}{$\mathbf{Z}$} \\
\hline & & X & $\mathbf{Y}$ & \\
\hline 1 & 0 & 2.9286 & -0.6521 & 0.7176 \\
\hline 2 & C & 1.6568 & -0.0394 & 0.8785 \\
\hline 3 & C & 1.0443 & 0.2833 & -0.4959 \\
\hline 4 & $\mathrm{C}$ & 1.9692 & 1.3171 & -1.1535 \\
\hline 5 & $\mathrm{C}$ & 3.4209 & 0.8003 & -1.2045 \\
\hline 6 & $\mathrm{C}$ & 3.904 & 0.2445 & 0.1462 \\
\hline 7 & $\mathrm{C}$ & 0.7185 & -0.8851 & 1.7208 \\
\hline 8 & $\mathrm{C}$ & -0.5784 & -0.0824 & 1.9141 \\
\hline 9 & $\mathrm{C}$ & -1.1904 & 0.4191 & 0.5863 \\
\hline 10 & $\mathrm{O}$ & -0.2248 & 0.953 & -0.2839 \\
\hline 11 & $\mathrm{O}$ & -1.8534 & -0.6533 & -0.1218 \\
\hline 12 & $\mathrm{C}$ & -3.0301 & -1.2411 & 0.4702 \\
\hline 13 & $\mathrm{C}$ & -4.0708 & -0.1636 & 0.7918 \\
\hline 14 & $\mathrm{C}$ & -3.4469 & 1.0219 & 1.5373 \\
\hline 15 & $\mathrm{C}$ & -2.2171 & 1.5849 & 0.8041 \\
\hline 16 & $\mathrm{C}$ & 5.2088 & -0.5906 & 0.0636 \\
\hline 17 & $\mathrm{C}$ & 5.6745 & -0.9841 & 1.4761 \\
\hline 18 & $\mathrm{O}$ & 4.9432 & -1.7705 & -0.7052 \\
\hline 19 & $\mathrm{C}$ & 6.3218 & 0.1561 & -0.6734 \\
\hline 20 & $\mathrm{C}$ & 0.871 & -0.9458 & -1.3971 \\
\hline 21 & $\mathrm{C}$ & -2.692 & 2.174 & -0.555 \\
\hline 22 & $\mathrm{C}$ & -3.5119 & -2.2938 & -0.5687 \\
\hline 23 & $\mathrm{C}$ & -2.4862 & -3.4321 & -0.7017 \\
\hline 24 & $\mathrm{C}$ & -4.881 & -2.8686 & -0.1971 \\
\hline 25 & $\mathrm{O}$ & -3.6835 & -1.6429 & -1.8316 \\
\hline 26 & $\mathrm{Cl}$ & -1.6656 & 3.5344 & -1.1961 \\
\hline 27 & 0 & -1.6797 & 2.5898 & 1.6424 \\
\hline 28 & $\mathrm{H}$ & 1.7972 & 0.9234 & 1.4013 \\
\hline 29 & $\mathrm{H}$ & 1.6137 & 1.5632 & -2.1606 \\
\hline 30 & $\mathrm{H}$ & 1.916 & 2.241 & -0.5633 \\
\hline 31 & $\mathrm{H}$ & 3.5224 & 0.0117 & -1.9572 \\
\hline 32 & $\mathrm{H}$ & 4.0802 & 1.6197 & -1.5122 \\
\hline 33 & $\mathrm{H}$ & 4.0533 & 1.0794 & 0.852 \\
\hline 34 & $\mathrm{H}$ & 0.523 & -1.8486 & 1.2378 \\
\hline 35 & $\mathrm{H}$ & 1.1766 & -1.0967 & 2.6937 \\
\hline 36 & $\mathrm{H}$ & -1.3111 & -0.6792 & 2.464 \\
\hline 37 & $\mathrm{H}$ & -0.3751 & 0.8018 & 2.5256 \\
\hline 38 & $\mathrm{H}$ & -2.7496 & -1.7693 & 1.3942 \\
\hline 39 & $\mathrm{H}$ & -4.5292 & 0.1657 & -0.1479 \\
\hline 40 & $\mathrm{H}$ & -4.87 & -0.5976 & 1.4025 \\
\hline 41 & $\mathrm{H}$ & -4.1744 & 1.8309 & 1.6678 \\
\hline 42 & $\mathrm{H}$ & -3.1358 & 0.7235 & 2.5454 \\
\hline 43 & $\mathrm{H}$ & 4.88 & -1.5088 & 2.0146 \\
\hline 44 & $\mathrm{H}$ & 6.5443 & -1.6443 & 1.4022 \\
\hline 45 & $\mathrm{H}$ & 5.9567 & -0.1015 & 2.063 \\
\hline 46 & $\mathrm{H}$ & 4.1548 & -2.1699 & -0.2989 \\
\hline 47 & $\mathrm{H}$ & 7.2356 & -0.4463 & -0.6593 \\
\hline 48 & $\mathrm{H}$ & 6.0543 & 0.3339 & -1.7183 \\
\hline 49 & $\mathrm{H}$ & 6.5371 & 1.1177 & -0.1938 \\
\hline 50 & $\mathrm{H}$ & 1.8353 & -1.4161 & -1.6036 \\
\hline
\end{tabular}




\begin{tabular}{rrrrr}
$\mathbf{5 1}$ & $\mathrm{H}$ & 0.2145 & -1.69 & -0.9472 \\
$\mathbf{5 2}$ & $\mathrm{H}$ & 0.4249 & -0.6341 & -2.3473 \\
$\mathbf{5 3}$ & $\mathrm{H}$ & -2.7154 & 1.4118 & -1.3311 \\
$\mathbf{5 4}$ & $\mathrm{H}$ & -3.6839 & 2.6125 & -0.439 \\
$\mathbf{5 5}$ & $\mathrm{H}$ & -2.3703 & -3.9787 & 0.2421 \\
$\mathbf{5 6}$ & $\mathrm{H}$ & -2.8212 & -4.136 & -1.47 \\
$\mathbf{5 7}$ & $\mathrm{H}$ & -1.5057 & -3.0467 & -0.9945 \\
$\mathbf{5 8}$ & $\mathrm{H}$ & -4.8624 & -3.3228 & 0.8001 \\
$\mathbf{5 9}$ & $\mathrm{H}$ & -5.6548 & -2.0971 & -0.2205 \\
$\mathbf{6 0}$ & $\mathrm{H}$ & -5.158 & -3.6418 & -0.9205 \\
$\mathbf{6 1}$ & $\mathrm{H}$ & -2.8408 & -1.1877 & -2.0021 \\
$\mathbf{6 2}$ & $\mathrm{H}$ & -0.9987 & 3.0522 & 1.1225 \\
\hline
\end{tabular}




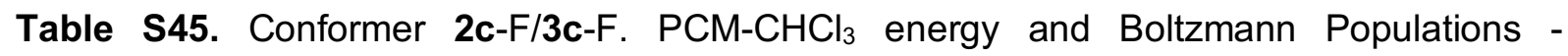
1694.806519 hartrees, $3.92 \%$.

\begin{tabular}{|c|c|c|c|c|}
\hline \multicolumn{2}{|c|}{ Atom number } & \multicolumn{3}{|c|}{ Coordinates } \\
\hline & & X & $\mathbf{Y}$ & Z \\
\hline 1 & O & 2.9207 & -0.7201 & 0.661 \\
\hline 2 & C & 1.6573 & -0.0918 & 0.8369 \\
\hline 3 & $\mathrm{C}$ & 1.0327 & 0.2269 & -0.5344 \\
\hline 4 & $\mathrm{C}$ & 1.966 & 1.2347 & -1.2243 \\
\hline 5 & $\mathrm{C}$ & 3.4086 & 0.6904 & -1.288 \\
\hline 6 & $\mathrm{C}$ & 3.8864 & 0.1836 & 0.0812 \\
\hline 7 & $\mathrm{C}$ & 0.7158 & -0.9156 & 1.6968 \\
\hline 8 & $\mathrm{C}$ & -0.5652 & -0.0886 & 1.8984 \\
\hline 9 & $\mathrm{C}$ & -1.1838 & 0.4164 & 0.5749 \\
\hline 10 & $\mathrm{O}$ & -0.2187 & 0.9248 & -0.312 \\
\hline 11 & $\mathrm{O}$ & -1.877 & -0.6465 & -0.1181 \\
\hline 12 & $\mathrm{C}$ & -3.0592 & -1.2062 & 0.4905 \\
\hline 13 & $\mathrm{C}$ & -4.0738 & -0.1047 & 0.8143 \\
\hline 14 & $\mathrm{C}$ & -3.4172 & 1.0708 & 1.5469 \\
\hline 15 & $\mathrm{C}$ & -2.1836 & 1.6046 & 0.798 \\
\hline 16 & $\mathrm{C}$ & 5.2404 & -0.5649 & 0.1225 \\
\hline 17 & $\mathrm{C}$ & 6.3935 & 0.3673 & -0.2546 \\
\hline 18 & $\mathrm{O}$ & 5.471 & -0.9324 & 1.4886 \\
\hline 19 & $\mathrm{C}$ & 5.2431 & -1.8311 & -0.7495 \\
\hline 20 & $\mathrm{C}$ & 0.8197 & -1.0102 & -1.4158 \\
\hline 21 & $\mathrm{C}$ & -2.6603 & 2.1973 & -0.559 \\
\hline 22 & $\mathrm{C}$ & -3.5734 & -2.2564 & -0.535 \\
\hline 23 & $\mathrm{C}$ & -2.5734 & -3.4172 & -0.6693 \\
\hline 24 & $\mathrm{C}$ & -4.9503 & -2.7998 & -0.1451 \\
\hline 25 & 0 & -3.744 & -1.6119 & -1.8013 \\
\hline 26 & $\mathrm{Cl}$ & -1.6137 & 3.5347 & -1.2159 \\
\hline 27 & 0 & -1.617 & 2.6018 & 1.6261 \\
\hline 28 & $\mathrm{H}$ & 1.8158 & 0.8742 & 1.3483 \\
\hline 29 & $\mathrm{H}$ & 1.597 & 1.4704 & -2.2289 \\
\hline 30 & $\mathrm{H}$ & 1.9412 & 2.1685 & -0.6484 \\
\hline 31 & $\mathrm{H}$ & 3.4684 & -0.1174 & -2.0252 \\
\hline 32 & $\mathrm{H}$ & 4.0808 & 1.4842 & -1.6338 \\
\hline 33 & $\mathrm{H}$ & 3.9806 & 1.0445 & 0.7643 \\
\hline 34 & $\mathrm{H}$ & 0.4997 & -1.8805 & 1.226 \\
\hline 35 & $\mathrm{H}$ & 1.1806 & -1.1247 & 2.6672 \\
\hline 36 & $\mathrm{H}$ & -1.3022 & -0.6689 & 2.4599 \\
\hline 37 & $\mathrm{H}$ & -0.3391 & 0.7952 & 2.5023 \\
\hline 38 & $\mathrm{H}$ & -2.7799 & -1.7329 & 1.4157 \\
\hline 39 & $\mathrm{H}$ & -4.5333 & 0.2285 & -0.1234 \\
\hline 40 & $\mathrm{H}$ & -4.8766 & -0.5175 & 1.4349 \\
\hline 41 & $\mathrm{H}$ & -4.1259 & 1.8958 & 1.6807 \\
\hline 42 & $\mathrm{H}$ & -3.1018 & 0.7709 & 2.5532 \\
\hline 43 & $\mathrm{H}$ & 6.3747 & 1.2758 & 0.357 \\
\hline 44 & $\mathrm{H}$ & 7.3434 & -0.1435 & -0.0676 \\
\hline 45 & $\mathrm{H}$ & 6.3578 & 0.65 & -1.3116 \\
\hline 46 & $\mathrm{H}$ & 4.6309 & -1.315 & 1.7957 \\
\hline 47 & $\mathrm{H}$ & 4.405 & -2.4834 & -0.4843 \\
\hline 48 & $\mathrm{H}$ & 5.1754 & -1.6004 & -1.8187 \\
\hline 49 & $\mathrm{H}$ & 6.1747 & -2.3787 & -0.5767 \\
\hline 50 & $\mathrm{H}$ & 1.7691 & -1.5108 & -1.619 \\
\hline
\end{tabular}




\begin{tabular}{rrrrr}
$\mathbf{5 1}$ & $\mathrm{H}$ & 0.1477 & -1.7294 & -0.9492 \\
$\mathbf{5 2}$ & $\mathrm{H}$ & 0.3741 & -0.7016 & -2.3673 \\
$\mathbf{5 3}$ & $\mathrm{H}$ & -2.706 & 1.4328 & -1.3318 \\
$\mathbf{5 4}$ & $\mathrm{H}$ & -3.6423 & 2.6557 & -0.4353 \\
$\mathbf{5 5}$ & $\mathrm{H}$ & -2.9308 & -4.1193 & -1.4291 \\
$\mathbf{5 6}$ & $\mathrm{H}$ & -1.5875 & -3.0555 & -0.9741 \\
$\mathbf{5 7}$ & $\mathrm{H}$ & -2.4599 & -3.9596 & 0.2773 \\
$\mathbf{5 8}$ & $\mathrm{H}$ & -4.9313 & -3.2448 & 0.8563 \\
$\mathbf{5 9}$ & $\mathrm{H}$ & -5.7087 & -2.0132 & -0.1686 \\
$\mathbf{6 0}$ & $\mathrm{H}$ & -5.2497 & -3.5741 & -0.8582 \\
$\mathbf{6 1}$ & $\mathrm{H}$ & -2.8944 & -1.1739 & -1.9825 \\
$\mathbf{6 2}$ & $\mathrm{H}$ & -0.9333 & 3.0497 & 1.0971 \\
\hline
\end{tabular}


Table S46. Conformer 2c-G/3c-G. PCM-CHCl 3 energy and Boltzmann Populations 1694.806626 hartrees, $4.38 \%$.

\begin{tabular}{|c|c|c|c|c|}
\hline \multicolumn{2}{|c|}{ Atom number } & \multicolumn{3}{|c|}{ Coordinates } \\
\hline & & X & $\mathbf{Y}$ & Z \\
\hline 1 & 0 & -3.0716 & 0.358 & 0.691 \\
\hline 2 & C & -1.7751 & -0.2063 & 0.8347 \\
\hline 3 & $\mathrm{C}$ & -1.1515 & -0.4579 & -0.5504 \\
\hline 4 & $\mathrm{C}$ & -2.0291 & -1.5165 & -1.2323 \\
\hline 5 & $\mathrm{C}$ & -3.5027 & -1.0638 & -1.2699 \\
\hline 6 & $\mathrm{C}$ & -4.0087 & -0.5601 & 0.0937 \\
\hline 7 & $\mathrm{C}$ & -0.8715 & 0.6435 & 1.711 \\
\hline 8 & $\mathrm{C}$ & 0.4555 & -0.1167 & 1.8707 \\
\hline 9 & $\mathrm{C}$ & 1.094 & -0.4691 & 0.5088 \\
\hline 10 & $\mathrm{O}$ & 0.1499 & -1.0777 & -0.3536 \\
\hline 11 & $\mathrm{O}$ & 1.5977 & 0.6908 & -0.1598 \\
\hline 12 & $\mathrm{C}$ & 2.6717 & 1.3961 & 0.4749 \\
\hline 13 & $\mathrm{C}$ & 3.8735 & 0.4627 & 0.6766 \\
\hline 14 & $\mathrm{C}$ & 3.4389 & -0.8484 & 1.352 \\
\hline 15 & $\mathrm{C}$ & 2.2618 & -1.5129 & 0.6248 \\
\hline 16 & $\mathrm{C}$ & -5.3445 & 0.2264 & 0.0265 \\
\hline 17 & $\mathrm{C}$ & -5.8265 & 0.5707 & 1.4464 \\
\hline 18 & $\mathrm{O}$ & -5.1228 & 1.4323 & -0.7152 \\
\hline 19 & $\mathrm{C}$ & -6.4276 & -0.5451 & -0.7292 \\
\hline 20 & $\mathrm{C}$ & -1.0401 & 0.8047 & -1.4177 \\
\hline 21 & $\mathrm{C}$ & 2.6211 & -1.9705 & -0.806 \\
\hline 22 & $\mathrm{C}$ & 2.9274 & 2.6652 & -0.369 \\
\hline 23 & $\mathrm{C}$ & 4.1017 & 3.4697 & 0.2106 \\
\hline 24 & $\mathrm{C}$ & 3.1488 & 2.3573 & -1.8556 \\
\hline 25 & 0 & 1.7167 & 3.4272 & -0.205 \\
\hline 26 & $\mathrm{Cl}$ & 4.0913 & -3.0382 & -0.8766 \\
\hline 27 & 0 & 1.8654 & -2.6263 & 1.4136 \\
\hline 28 & $\mathrm{H}$ & -1.8797 & -1.1918 & 1.3234 \\
\hline 29 & $\mathrm{H}$ & -1.667 & -1.7199 & -2.2472 \\
\hline 30 & $\mathrm{H}$ & -1.9362 & -2.4519 & -0.6643 \\
\hline 31 & $\mathrm{H}$ & -3.6375 & -0.2635 & -2.0044 \\
\hline 32 & $\mathrm{H}$ & -4.1265 & -1.904 & -1.5956 \\
\hline 33 & $\mathrm{H}$ & -4.1289 & -1.4178 & 0.7777 \\
\hline 34 & $\mathrm{H}$ & -0.7054 & 1.6307 & 1.267 \\
\hline 35 & $\mathrm{H}$ & -1.3385 & 0.7962 & 2.6908 \\
\hline 36 & $\mathrm{H}$ & 1.1501 & 0.4634 & 2.4824 \\
\hline 37 & $\mathrm{H}$ & 0.2881 & -1.0569 & 2.4049 \\
\hline 38 & $\mathrm{H}$ & 2.3374 & 1.764 & 1.4558 \\
\hline 39 & $\mathrm{H}$ & 4.347 & 0.2435 & -0.288 \\
\hline 40 & $\mathrm{H}$ & 4.6296 & 0.9557 & 1.2973 \\
\hline 41 & $\mathrm{H}$ & 4.2715 & -1.5556 & 1.3933 \\
\hline 42 & $\mathrm{H}$ & 3.1347 & -0.6618 & 2.388 \\
\hline 43 & $\mathrm{H}$ & -5.054 & 1.1154 & 1.9968 \\
\hline 44 & $\mathrm{H}$ & -6.7222 & 1.1968 & 1.3853 \\
\hline 45 & $\mathrm{H}$ & -6.0732 & -0.3348 & 2.0143 \\
\hline 46 & $\mathrm{H}$ & -4.3496 & 1.8516 & -0.2993 \\
\hline 47 & $\mathrm{H}$ & -7.3621 & 0.0245 & -0.7077 \\
\hline 48 & $\mathrm{H}$ & -6.1506 & -0.6936 & -1.7762 \\
\hline 49 & $\mathrm{H}$ & -6.6107 & -1.5225 & -0.2685 \\
\hline 50 & $\mathrm{H}$ & -0.6109 & 0.5325 & -2.3883 \\
\hline
\end{tabular}




\begin{tabular}{rrrrr}
$\mathbf{5 1}$ & $\mathrm{H}$ & -2.0284 & 1.2393 & -1.5877 \\
$\mathbf{5 2}$ & $\mathrm{H}$ & -0.3949 & 1.5603 & -0.9704 \\
$\mathbf{5 3}$ & $\mathrm{H}$ & 1.8048 & -2.5522 & -1.2294 \\
$\mathbf{5 4}$ & $\mathrm{H}$ & 2.8151 & -1.1209 & -1.458 \\
$\mathbf{5 5}$ & $\mathrm{H}$ & 5.0606 & 2.9567 & 0.0831 \\
$\mathbf{5 6}$ & $\mathrm{H}$ & 4.1744 & 4.4359 & -0.3035 \\
$\mathbf{5 7}$ & $\mathrm{H}$ & 3.9468 & 3.6696 & 1.2765 \\
$\mathbf{5 8}$ & $\mathrm{H}$ & 3.2701 & 3.2933 & -2.4165 \\
$\mathbf{5 9}$ & $\mathrm{H}$ & 4.0548 & 1.7637 & -2.0192 \\
$\mathbf{6 0}$ & $\mathrm{H}$ & 2.2934 & 1.8139 & -2.2619 \\
$\mathbf{6 1}$ & $\mathrm{H}$ & 1.7744 & 4.1929 & -0.7982 \\
$\mathbf{6 2}$ & $\mathrm{H}$ & 1.0856 & -3.0108 & 0.9782 \\
\hline
\end{tabular}




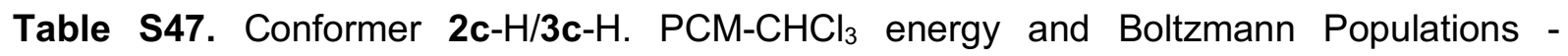
1694.806744 hartrees, $4.97 \%$.

\begin{tabular}{|c|c|c|c|c|}
\hline \multicolumn{2}{|c|}{ Atom number } & \multicolumn{2}{|c|}{ Coordinates } & \multirow[b]{2}{*}{$\mathbf{Z}$} \\
\hline & & X & $\mathbf{Y}$ & \\
\hline 1 & 0 & -3.1269 & 0.4709 & 0.6706 \\
\hline 2 & C & -1.8254 & -0.0874 & 0.7792 \\
\hline 3 & C & -1.1804 & -0.2227 & -0.613 \\
\hline 4 & C & -2.0504 & -1.2041 & -1.4141 \\
\hline 5 & $\mathrm{C}$ & -3.5207 & -0.7404 & -1.4282 \\
\hline 6 & $\mathrm{C}$ & -4.0231 & -0.4188 & -0.0117 \\
\hline 7 & $\mathrm{C}$ & -0.9383 & 0.702 & 1.7248 \\
\hline 8 & $\mathrm{C}$ & 0.397 & -0.0513 & 1.85 \\
\hline 9 & C & 1.0524 & -0.3784 & 0.4886 \\
\hline 10 & $\mathrm{O}$ & 0.112 & -0.8665 & -0.4498 \\
\hline 11 & $\mathrm{O}$ & 1.6785 & 0.7823 & -0.0838 \\
\hline 12 & $\mathrm{C}$ & 2.8318 & 1.3326 & 0.5949 \\
\hline 13 & C & 3.8899 & 0.2556 & 0.87 \\
\hline 14 & $\mathrm{C}$ & 3.2877 & -1.032 & 1.4474 \\
\hline 15 & $\mathrm{C}$ & 2.1175 & -1.5292 & 0.5934 \\
\hline 16 & $\mathrm{C}$ & -5.448 & 0.1906 & 0.0607 \\
\hline 17 & $\mathrm{C}$ & -5.554 & 1.5274 & -0.6756 \\
\hline 18 & $\mathrm{O}$ & -6.3461 & -0.7012 & -0.6208 \\
\hline 19 & $\mathrm{C}$ & -5.8763 & 0.3459 & 1.5258 \\
\hline 20 & $\mathrm{C}$ & -1.0318 & 1.1102 & -1.3564 \\
\hline 21 & $\mathrm{C}$ & 2.5507 & -1.913 & -0.8398 \\
\hline 22 & $\mathrm{C}$ & 3.3252 & 2.4714 & -0.3431 \\
\hline 23 & $\mathrm{C}$ & 2.2809 & 3.5975 & -0.4255 \\
\hline 24 & $\mathrm{C}$ & 4.6692 & 3.0402 & 0.118 \\
\hline 25 & $\mathrm{O}$ & 3.5506 & 1.9226 & -1.646 \\
\hline 26 & $\mathrm{Cl}$ & 3.8549 & -3.1763 & -0.8721 \\
\hline 27 & 0 & 1.5567 & -2.6528 & 1.2559 \\
\hline 28 & $\mathrm{H}$ & -1.9166 & -1.1116 & 1.1858 \\
\hline 29 & $\mathrm{H}$ & -1.6677 & -1.3081 & -2.4364 \\
\hline 30 & $\mathrm{H}$ & -1.9725 & -2.1918 & -0.9399 \\
\hline 31 & $\mathrm{H}$ & -3.6241 & 0.1438 & -2.0648 \\
\hline 32 & $\mathrm{H}$ & -4.1611 & -1.5135 & -1.8635 \\
\hline 33 & $\mathrm{H}$ & -4.0518 & -1.3677 & 0.5594 \\
\hline 34 & $\mathrm{H}$ & -0.7909 & 1.7235 & 1.3583 \\
\hline 35 & $\mathrm{H}$ & -1.4164 & 0.7775 & 2.708 \\
\hline 36 & $\mathrm{H}$ & 1.092 & 0.5205 & 2.4699 \\
\hline 37 & $\mathrm{H}$ & 0.2325 & -1.0033 & 2.3639 \\
\hline 38 & $\mathrm{H}$ & 2.5076 & 1.7825 & 1.5453 \\
\hline 39 & $\mathrm{H}$ & 4.4218 & 0.0378 & -0.0622 \\
\hline 40 & $\mathrm{H}$ & 4.6305 & 0.6555 & 1.5713 \\
\hline 41 & $\mathrm{H}$ & 4.0448 & -1.8191 & 1.504 \\
\hline 42 & $\mathrm{H}$ & 2.9244 & -0.8726 & 2.4689 \\
\hline 43 & $\mathrm{H}$ & -5.4011 & 1.3961 & -1.7507 \\
\hline 44 & $\mathrm{H}$ & -6.5531 & 1.9503 & -0.5315 \\
\hline 45 & $\mathrm{H}$ & -4.8107 & 2.2334 & -0.2948 \\
\hline 46 & $\mathrm{H}$ & -6.4528 & -1.4904 & -0.0652 \\
\hline 47 & $\mathrm{H}$ & -5.8323 & -0.6203 & 2.0479 \\
\hline 48 & $\mathrm{H}$ & -5.2193 & 1.0389 & 2.0578 \\
\hline 49 & $\mathrm{H}$ & -6.9053 & 0.7162 & 1.5762 \\
\hline 50 & $\mathrm{H}$ & -0.5647 & 0.9305 & -2.3305 \\
\hline
\end{tabular}




\begin{tabular}{rrrrr}
$\mathbf{5 1}$ & $\mathrm{H}$ & -2.0065 & 1.5769 & -1.5162 \\
$\mathbf{5 2}$ & $\mathrm{H}$ & -0.403 & 1.8106 & -0.8082 \\
$\mathbf{5 3}$ & $\mathrm{H}$ & 1.7088 & -2.3311 & -1.3881 \\
$\mathbf{5 4}$ & $\mathrm{H}$ & 2.9331 & -1.0537 & -1.388 \\
$\mathbf{5 5}$ & $\mathrm{H}$ & 2.1195 & 4.0665 & 0.5527 \\
$\mathbf{5 6}$ & $\mathrm{H}$ & 2.6286 & 4.3653 & -1.1237 \\
$\mathbf{5 7}$ & $\mathrm{H}$ & 1.3197 & 3.2185 & -0.7837 \\
$\mathbf{5 8}$ & $\mathrm{H}$ & 5.4579 & 2.2854 & 0.0674 \\
$\mathbf{5 9}$ & $\mathrm{H}$ & 4.9561 & 3.8687 & -0.537 \\
$\mathbf{6 0}$ & $\mathrm{H}$ & 4.6068 & 3.42 & 1.1442 \\
$\mathbf{6 1}$ & $\mathrm{H}$ & 2.7214 & 1.4716 & -1.8812 \\
$\mathbf{6 2}$ & $\mathrm{H}$ & 0.787 & -2.9275 & 0.7287 \\
\hline
\end{tabular}




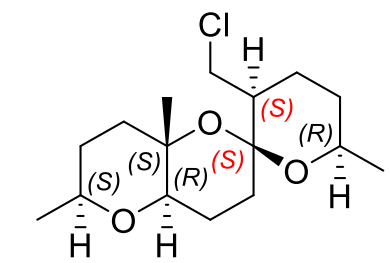

Table S48. Conformer 2d-A/3d-A. $\mathrm{PCM}-\mathrm{CHCl}_{3}$ energy and Boltzmann Populations 1694.806402 hartrees, $12.46 \%$.

\begin{tabular}{|c|c|c|c|c|}
\hline \multicolumn{2}{|c|}{ Atom number } & \multicolumn{2}{|c|}{ Coordinates } & \multirow[b]{2}{*}{$\mathbf{Z}$} \\
\hline & & $x$ & $\mathbf{Y}$ & \\
\hline 1 & 0 & 2.7338 & -0.5913 & 0.7723 \\
\hline 2 & $\mathrm{C}$ & 1.4991 & 0.109 & 0.7205 \\
\hline 3 & $\mathrm{C}$ & 0.9453 & 0.0834 & -0.7158 \\
\hline 4 & $\mathrm{C}$ & 1.9393 & 0.877 & -1.577 \\
\hline 5 & $\mathrm{C}$ & 3.3689 & 0.3167 & -1.425 \\
\hline 6 & $\mathrm{C}$ & 3.7761 & 0.0888 & 0.0415 \\
\hline 7 & $\mathrm{C}$ & 0.4913 & -0.4226 & 1.7268 \\
\hline 8 & $\mathrm{C}$ & -0.7394 & 0.497 & 1.6542 \\
\hline 9 & $\mathrm{C}$ & -1.3075 & 0.6005 & 0.2227 \\
\hline 10 & 0 & -0.2999 & 0.8311 & -0.7398 \\
\hline 11 & 0 & -1.926 & -0.6417 & -0.1511 \\
\hline 12 & $\mathrm{C}$ & -3.1956 & -0.9885 & 0.4317 \\
\hline 13 & $\mathrm{C}$ & -4.2168 & 0.0964 & 0.11 \\
\hline 14 & $\mathrm{C}$ & -3.6994 & 1.4463 & 0.6098 \\
\hline 15 & $\mathrm{C}$ & -2.3214 & 1.7901 & 0.0004 \\
\hline 16 & $\mathrm{C}$ & 5.0334 & -0.8025 & 0.221 \\
\hline 17 & $\mathrm{C}$ & 5.4292 & -0.8648 & 1.7063 \\
\hline 18 & 0 & 4.7268 & -2.1194 & -0.2542 \\
\hline 19 & $\mathrm{C}$ & 6.21 & -0.3093 & -0.6226 \\
\hline 20 & $\mathrm{C}$ & 0.7512 & -1.3305 & -1.2796 \\
\hline 21 & $\mathrm{C}$ & -1.9176 & 3.1411 & 0.6296 \\
\hline 22 & $\mathrm{C}$ & -3.5163 & -2.4185 & -0.0718 \\
\hline 23 & $\mathrm{C}$ & -4.75 & -2.9775 & 0.6398 \\
\hline 24 & $\mathrm{C}$ & -3.6798 & -2.4821 & -1.5982 \\
\hline 25 & 0 & -2.4334 & -3.2678 & 0.331 \\
\hline 26 & $\mathrm{Cl}$ & -0.3547 & 3.8713 & 0.0571 \\
\hline 27 & 0 & -2.528 & 1.957 & -1.3895 \\
\hline 28 & $\mathrm{H}$ & 1.6885 & 1.1667 & 0.9714 \\
\hline 29 & $\mathrm{H}$ & 1.6354 & 0.8557 & -2.6307 \\
\hline 30 & $\mathrm{H}$ & 1.9018 & 1.9238 & -1.2529 \\
\hline 31 & $\mathrm{H}$ & 3.4696 & -0.6327 & -1.9605 \\
\hline 32 & $\mathrm{H}$ & 4.0747 & 1.0165 & -1.8862 \\
\hline 33 & $\mathrm{H}$ & 3.9505 & 1.0634 & 0.528 \\
\hline 34 & $\mathrm{H}$ & 0.2208 & -1.462 & 1.5144 \\
\hline 35 & $\mathrm{H}$ & 0.9216 & -0.3972 & 2.734 \\
\hline 36 & $\mathrm{H}$ & -1.5208 & 0.1488 & 2.3368 \\
\hline 37 & $\mathrm{H}$ & -0.4397 & 1.4936 & 1.9925 \\
\hline 38 & $\mathrm{H}$ & -3.0797 & -1.0701 & 1.5242 \\
\hline 39 & $\mathrm{H}$ & -4.3747 & 0.1526 & -0.9706 \\
\hline 40 & $\mathrm{H}$ & -5.1772 & -0.1309 & 0.5863 \\
\hline 41 & $\mathrm{H}$ & -4.3959 & 2.2461 & 0.3337 \\
\hline 42 & $\mathrm{H}$ & -3.631 & 1.4403 & 1.7059 \\
\hline 43 & $\mathrm{H}$ & 5.7386 & 0.1195 & 2.0783 \\
\hline 44 & $\mathrm{H}$ & 4.5895 & -1.209 & 2.317 \\
\hline
\end{tabular}




\begin{tabular}{llrrr}
$\mathbf{4 5}$ & $\mathrm{H}$ & 6.264 & -1.5612 & 1.8323 \\
$\mathbf{4 6}$ & $\mathrm{H}$ & 3.9111 & -2.3773 & 0.2087 \\
$\mathbf{4 7}$ & $\mathrm{H}$ & 6.466 & 0.7272 & -0.3755 \\
$\mathbf{4 8}$ & $\mathrm{H}$ & 7.0861 & -0.9354 & -0.4262 \\
$\mathbf{4 9}$ & $\mathrm{H}$ & 5.9856 & -0.3735 & -1.6906 \\
$\mathbf{5 0}$ & $\mathrm{H}$ & 0.1377 & -1.9529 & -0.6287 \\
$\mathbf{5 1}$ & $\mathrm{H}$ & 0.2508 & -1.2643 & -2.2504 \\
$\mathbf{5 2}$ & $\mathrm{H}$ & 1.7126 & -1.8315 & -1.4132 \\
$\mathbf{5 3}$ & $\mathrm{H}$ & -2.6852 & 3.8661 & 0.3559 \\
$\mathbf{5 4}$ & $\mathrm{H}$ & -1.8498 & 3.0888 & 1.7171 \\
$\mathbf{5 5}$ & $\mathrm{H}$ & -4.6317 & -2.9192 & 1.7273 \\
$\mathbf{5 6}$ & $\mathrm{H}$ & -5.6606 & -2.4429 & 0.3527 \\
$\mathbf{5 7}$ & $\mathrm{H}$ & -4.8711 & -4.0316 & 0.3713 \\
$\mathbf{5 8}$ & $\mathrm{H}$ & -4.5707 & -1.944 & -1.9401 \\
$\mathbf{5 9}$ & $\mathrm{H}$ & -2.8092 & -2.0473 & -2.0992 \\
$\mathbf{6 0}$ & $\mathrm{H}$ & -3.7728 & -3.529 & -1.9034 \\
$\mathbf{6 1}$ & $\mathrm{H}$ & -1.6304 & -2.8481 & -0.0183 \\
$\mathbf{6 2}$ & $\mathrm{H}$ & -1.6616 & 1.7946 & -1.805 \\
\hline
\end{tabular}




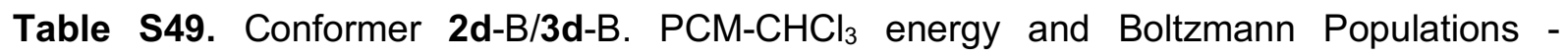
1694.805943 hartrees, $7.66 \%$.

\begin{tabular}{|c|c|c|c|c|}
\hline \multicolumn{2}{|c|}{ Atom number } & \multicolumn{3}{|c|}{ Coordinates } \\
\hline & & $\mathrm{X}$ & $\mathbf{Y}$ & Z \\
\hline 1 & 0 & -2.7234 & -0.6838 & -0.7253 \\
\hline 2 & C & -1.4985 & 0.0362 & -0.6865 \\
\hline 3 & C & -0.9277 & 0.0095 & 0.7443 \\
\hline 4 & $\mathrm{C}$ & -1.9311 & 0.762 & 1.6346 \\
\hline 5 & $\mathrm{C}$ & -3.3488 & 0.1653 & 1.4921 \\
\hline 6 & $\mathrm{C}$ & -3.7553 & 0.0005 & 0.0196 \\
\hline 7 & $\mathrm{C}$ & -0.4904 & -0.4662 & -1.707 \\
\hline 8 & $\mathrm{C}$ & 0.7183 & 0.4834 & -1.6417 \\
\hline 9 & $\mathrm{C}$ & 1.2983 & 0.5979 & -0.2156 \\
\hline 10 & $\mathrm{O}$ & 0.2942 & 0.7944 & 0.7593 \\
\hline 11 & $\mathrm{O}$ & 1.9578 & -0.6265 & 0.1466 \\
\hline 12 & $\mathrm{C}$ & 3.233 & -0.9318 & -0.4476 \\
\hline 13 & $\mathrm{C}$ & 4.2228 & 0.1824 & -0.129 \\
\hline 14 & $\mathrm{C}$ & 3.6606 & 1.5169 & -0.6211 \\
\hline 15 & $\mathrm{C}$ & 2.278 & 1.8174 & 0.0009 \\
\hline 16 & $\mathrm{C}$ & -5.0648 & -0.7705 & -0.2706 \\
\hline 17 & $\mathrm{C}$ & -6.278 & -0.0158 & 0.2761 \\
\hline 18 & $\mathrm{O}$ & -5.2294 & -0.7872 & -1.6943 \\
\hline 19 & $\mathrm{C}$ & -5.0288 & -2.2165 & 0.2511 \\
\hline 20 & $\mathrm{C}$ & -0.6792 & -1.4033 & 1.289 \\
\hline 21 & $\mathrm{C}$ & 1.8289 & 3.1569 & -0.6215 \\
\hline 22 & $\mathrm{C}$ & 3.6004 & -2.3534 & 0.0473 \\
\hline 23 & $\mathrm{C}$ & 4.8459 & -2.8717 & -0.6747 \\
\hline 24 & $\mathrm{C}$ & 3.7763 & -2.4183 & 1.5724 \\
\hline 25 & 0 & 2.5414 & -3.2339 & -0.3518 \\
\hline 26 & $\mathrm{Cl}$ & 0.2502 & 3.8388 & -0.0329 \\
\hline 27 & 0 & 2.4921 & 1.9872 & 1.3895 \\
\hline 28 & $\mathrm{H}$ & -1.7082 & 1.093 & -0.9248 \\
\hline 29 & $\mathrm{H}$ & -1.6091 & 0.7316 & 2.6825 \\
\hline 30 & $\mathrm{H}$ & -1.9303 & 1.8144 & 1.3272 \\
\hline 31 & $\mathrm{H}$ & -3.3997 & -0.8018 & 2.0034 \\
\hline 32 & $\mathrm{H}$ & -4.0683 & 0.8237 & 1.9923 \\
\hline 33 & $\mathrm{H}$ & -3.8724 & 1.0013 & -0.4283 \\
\hline 34 & $\mathrm{H}$ & -0.1937 & -1.5006 & -1.5066 \\
\hline 35 & $\mathrm{H}$ & -0.9313 & -0.4424 & -2.7098 \\
\hline 36 & $\mathrm{H}$ & 1.5018 & 0.1574 & -2.3329 \\
\hline 37 & $\mathrm{H}$ & 0.3902 & 1.4731 & -1.9737 \\
\hline 38 & $\mathrm{H}$ & 3.1111 & -1.0121 & -1.5396 \\
\hline 39 & $\mathrm{H}$ & 4.3855 & 0.2402 & 0.9508 \\
\hline 40 & $\mathrm{H}$ & 5.1867 & -0.0144 & -0.6119 \\
\hline 41 & $\mathrm{H}$ & 4.3346 & 2.3371 & -0.3491 \\
\hline 42 & $\mathrm{H}$ & 3.5828 & 1.511 & -1.7166 \\
\hline 43 & $\mathrm{H}$ & -6.2898 & 1.0163 & -0.0903 \\
\hline 44 & $\mathrm{H}$ & -7.1922 & -0.5066 & -0.0722 \\
\hline 45 & $\mathrm{H}$ & -6.2901 & -0.0042 & 1.3707 \\
\hline 46 & $\mathrm{H}$ & -4.3613 & -1.0458 & -2.0494 \\
\hline 47 & $\mathrm{H}$ & -5.0176 & -2.2645 & 1.3459 \\
\hline 48 & $\mathrm{H}$ & -5.9193 & -2.7436 & -0.1046 \\
\hline 49 & $\mathrm{H}$ & -4.1442 & -2.7403 & -0.1251 \\
\hline 50 & $\mathrm{H}$ & -0.055 & -1.9973 & 0.6227 \\
\hline
\end{tabular}




\begin{tabular}{rrrrr}
$\mathbf{5 1}$ & $\mathrm{H}$ & -0.1675 & -1.3308 & 2.2535 \\
$\mathbf{5 2}$ & $\mathrm{H}$ & -1.6203 & -1.9397 & 1.4283 \\
$\mathbf{5 3}$ & $\mathrm{H}$ & 2.5772 & 3.9037 & -0.3532 \\
$\mathbf{5 4}$ & $\mathrm{H}$ & 1.7527 & 3.1047 & -1.7084 \\
$\mathbf{5 5}$ & $\mathrm{H}$ & 4.7184 & -2.8135 & -1.7612 \\
$\mathbf{5 6}$ & $\mathrm{H}$ & 5.7413 & -2.3099 & -0.3918 \\
$\mathbf{5 7}$ & $\mathrm{H}$ & 5.0015 & -3.9223 & -0.4107 \\
$\mathbf{5 8}$ & $\mathrm{H}$ & 3.9026 & -3.4632 & 1.8724 \\
$\mathbf{5 9}$ & $\mathrm{H}$ & 4.6531 & -1.8553 & 1.9105 \\
$\mathbf{6 0}$ & $\mathrm{H}$ & 2.8966 & -2.0118 & 2.0812 \\
$\mathbf{6 1}$ & $\mathrm{H}$ & 1.7278 & -2.8382 & 0.0006 \\
$\mathbf{6 2}$ & $\mathrm{H}$ & 1.634 & 1.8017 & 1.8122 \\
\hline
\end{tabular}


Table S50. Conformer 2d-C/3d-C. $\mathrm{PCM}_{-} \mathrm{CHCl}_{3}$ energy and Boltzmann Populations 1694.806929 hartrees, $21.78 \%$.

\begin{tabular}{|c|c|c|c|c|}
\hline \multicolumn{2}{|c|}{ Atom number } & \multicolumn{3}{|c|}{ Coordinates } \\
\hline & & 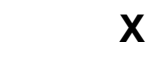 & $\mathbf{Y}$ & Z \\
\hline 1 & $\mathrm{O}$ & 2.7555 & -0.5663 & 0.7891 \\
\hline 2 & C & 1.515 & 0.1232 & 0.7283 \\
\hline 3 & C & 0.9441 & 0.0503 & -0.7 \\
\hline 4 & $\mathrm{C}$ & 1.9238 & 0.8207 & -1.5967 \\
\hline 5 & $\mathrm{C}$ & 3.3538 & 0.2608 & -1.4502 \\
\hline 6 & $\mathrm{C}$ & 3.7843 & 0.0887 & 0.017 \\
\hline 7 & $\mathrm{C}$ & 0.5239 & -0.3909 & 1.7594 \\
\hline 8 & $\mathrm{C}$ & -0.7171 & 0.5143 & 1.6789 \\
\hline 9 & $\mathrm{C}$ & -1.2999 & 0.5917 & 0.2519 \\
\hline 10 & $\mathrm{O}$ & -0.3065 & 0.7907 & -0.731 \\
\hline 11 & 0 & -1.9303 & -0.6601 & -0.078 \\
\hline 12 & $\mathrm{C}$ & -3.2057 & -0.9989 & 0.5014 \\
\hline 13 & $\mathrm{C}$ & -4.2143 & 0.1065 & 0.2071 \\
\hline 14 & $\mathrm{C}$ & -3.6712 & 1.4596 & 0.6637 \\
\hline 15 & $\mathrm{C}$ & -2.3075 & 1.7809 & 0.0164 \\
\hline 16 & $\mathrm{C}$ & 5.0467 & -0.7919 & 0.2115 \\
\hline 17 & $\mathrm{C}$ & 5.466 & -0.7948 & 1.6917 \\
\hline 18 & $\mathrm{O}$ & 4.7364 & -2.1273 & -0.2064 \\
\hline 19 & $\mathrm{C}$ & 6.209 & -0.3298 & -0.6688 \\
\hline 20 & $\mathrm{C}$ & 0.7447 & -1.3809 & -1.2139 \\
\hline 21 & $\mathrm{C}$ & -1.8854 & 3.1464 & 0.6004 \\
\hline 22 & $\mathrm{C}$ & -3.5678 & -2.378 & -0.1183 \\
\hline 23 & $\mathrm{C}$ & -2.5817 & -3.4615 & 0.3529 \\
\hline 24 & $\mathrm{C}$ & -4.9983 & -2.7945 & 0.2356 \\
\hline 25 & 0 & -3.5252 & -2.2641 & -1.5422 \\
\hline 26 & $\mathrm{Cl}$ & -0.3279 & 3.8522 & -0.0169 \\
\hline 27 & 0 & -2.5402 & 1.91 & -1.3734 \\
\hline 28 & $\mathrm{H}$ & 1.6984 & 1.1891 & 0.9466 \\
\hline 29 & $\mathrm{H}$ & 1.6039 & 0.7713 & -2.6446 \\
\hline 30 & $\mathrm{H}$ & 1.894 & 1.8763 & -1.3007 \\
\hline 31 & $\mathrm{H}$ & 3.4403 & -0.7089 & -1.951 \\
\hline 32 & $\mathrm{H}$ & 4.054 & 0.9397 & -1.9497 \\
\hline 33 & $\mathrm{H}$ & 3.9628 & 1.0818 & 0.4633 \\
\hline 34 & $\mathrm{H}$ & 0.2662 & -1.4382 & 1.5727 \\
\hline 35 & $\mathrm{H}$ & 0.965 & -0.3353 & 2.7609 \\
\hline 36 & $\mathrm{H}$ & -1.4918 & 0.1702 & 2.3718 \\
\hline 37 & $\mathrm{H}$ & -0.4243 & 1.5185 & 2.0004 \\
\hline 38 & $\mathrm{H}$ & -3.0946 & -1.1238 & 1.591 \\
\hline 39 & $\mathrm{H}$ & -4.4126 & 0.1257 & -0.868 \\
\hline 40 & $\mathrm{H}$ & -5.1574 & -0.099 & 0.7247 \\
\hline 41 & $\mathrm{H}$ & -4.3701 & 2.258 & 0.3891 \\
\hline 42 & $\mathrm{H}$ & -3.5747 & 1.4782 & 1.7578 \\
\hline 43 & $\mathrm{H}$ & 4.6359 & -1.1127 & 2.3293 \\
\hline 44 & $\mathrm{H}$ & 6.3024 & -1.4867 & 1.8323 \\
\hline 45 & $\mathrm{H}$ & 5.7822 & 0.2034 & 2.0185 \\
\hline 46 & $\mathrm{H}$ & 3.9258 & -2.3663 & 0.2752 \\
\hline 47 & $\mathrm{H}$ & 6.4655 & 0.7162 & -0.4667 \\
\hline 48 & $\mathrm{H}$ & 7.0899 & -0.9455 & -0.4612 \\
\hline 49 & $\mathrm{H}$ & 5.9687 & -0.4368 & -1.7299 \\
\hline 50 & $\mathrm{H}$ & 1.698 & -1.9127 & -1.2573 \\
\hline
\end{tabular}




\begin{tabular}{rrrrr}
$\mathbf{5 1}$ & $\mathrm{H}$ & 0.0609 & -1.9482 & -0.5842 \\
$\mathbf{5 2}$ & $\mathrm{H}$ & 0.3206 & -1.3447 & -2.2229 \\
$\mathbf{5 3}$ & $\mathrm{H}$ & -2.6537 & 3.8673 & 0.3182 \\
$\mathbf{5 4}$ & $\mathrm{H}$ & -1.8005 & 3.125 & 1.6877 \\
$\mathbf{5 5}$ & $\mathrm{H}$ & -2.8245 & -4.4094 & -0.1372 \\
$\mathbf{5 6}$ & $\mathrm{H}$ & -1.5523 & -3.1996 & 0.0952 \\
$\mathbf{5 7}$ & $\mathrm{H}$ & -2.6365 & -3.6093 & 1.4387 \\
$\mathbf{5 8}$ & $\mathrm{H}$ & -5.7287 & -2.1108 & -0.2039 \\
$\mathbf{5 9}$ & $\mathrm{H}$ & -5.1936 & -3.794 & -0.1654 \\
$\mathbf{6 0}$ & $\mathrm{H}$ & -5.1467 & -2.8263 & 1.3213 \\
$\mathbf{6 1}$ & $\mathrm{H}$ & -2.7147 & -1.7623 & -1.7398 \\
$\mathbf{6 2}$ & $\mathrm{H}$ & -1.6757 & 1.7655 & -1.7988 \\
\hline
\end{tabular}




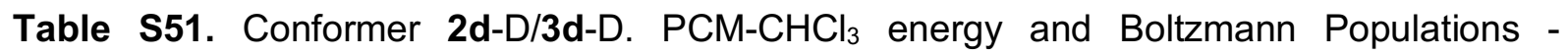
1694.806502 hartrees, $13.86 \%$.

\begin{tabular}{|c|c|c|c|c|}
\hline \multicolumn{2}{|c|}{ Atom number } & \multicolumn{3}{|c|}{ Coordinates } \\
\hline & & 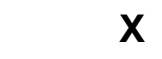 & $\mathbf{Y}$ & Z \\
\hline 1 & $\mathrm{O}$ & 2.7451 & -0.6632 & 0.7452 \\
\hline 2 & C & 1.5144 & 0.0469 & 0.6989 \\
\hline 3 & C & 0.9268 & -0.0242 & -0.724 \\
\hline 4 & $\mathrm{C}$ & 1.9155 & 0.7073 & -1.6464 \\
\hline 5 & $\mathrm{C}$ & 3.3333 & 0.111 & -1.509 \\
\hline 6 & $\mathrm{C}$ & 3.7632 & -0.0018 & -0.0384 \\
\hline 7 & $\mathrm{C}$ & 0.5221 & -0.4411 & 1.7411 \\
\hline 8 & $\mathrm{C}$ & -0.697 & 0.4947 & 1.6685 \\
\hline 9 & $\mathrm{C}$ & -1.2909 & 0.5869 & 0.2465 \\
\hline 10 & $\mathrm{O}$ & -0.3014 & 0.7524 & -0.7474 \\
\hline 11 & 0 & -1.9643 & -0.6445 & -0.0745 \\
\hline 12 & $\mathrm{C}$ & -3.2448 & -0.9417 & 0.5167 \\
\hline 13 & $\mathrm{C}$ & -4.2198 & 0.1959 & 0.2317 \\
\hline 14 & $\mathrm{C}$ & -3.6281 & 1.5311 & 0.6811 \\
\hline 15 & $\mathrm{C}$ & -2.2625 & 1.8071 & 0.0181 \\
\hline 16 & $\mathrm{C}$ & 5.0798 & -0.7582 & 0.2581 \\
\hline 17 & $\mathrm{C}$ & 6.2817 & -0.017 & -0.3306 \\
\hline 18 & $\mathrm{O}$ & 5.265 & -0.7279 & 1.6791 \\
\hline 19 & $\mathrm{C}$ & 5.0435 & -2.2204 & -0.216 \\
\hline 20 & $\mathrm{C}$ & 0.6762 & -1.4541 & -1.2184 \\
\hline 21 & $\mathrm{C}$ & -1.7912 & 3.1604 & 0.5926 \\
\hline 22 & $\mathrm{C}$ & -3.6564 & -2.3083 & -0.0998 \\
\hline 23 & $\mathrm{C}$ & -2.7023 & -3.4237 & 0.363 \\
\hline 24 & $\mathrm{C}$ & -5.0965 & -2.6786 & 0.2662 \\
\hline 25 & 0 & -3.6222 & -2.1958 & -1.524 \\
\hline 26 & $\mathrm{Cl}$ & -0.2205 & 3.8159 & -0.0463 \\
\hline 27 & 0 & -2.5065 & 1.9391 & -1.3695 \\
\hline 28 & $\mathrm{H}$ & 1.7179 & 1.111 & 0.9074 \\
\hline 29 & $\mathrm{H}$ & 1.5779 & 0.6515 & -2.6882 \\
\hline 30 & $\mathrm{H}$ & 1.9214 & 1.7671 & -1.3651 \\
\hline 31 & $\mathrm{H}$ & 3.3698 & -0.8744 & -1.9855 \\
\hline 32 & $\mathrm{H}$ & 4.0465 & 0.7479 & -2.0448 \\
\hline 33 & $\mathrm{H}$ & 3.8834 & 1.0142 & 0.373 \\
\hline 34 & $\mathrm{H}$ & 0.2383 & -1.4829 & 1.5624 \\
\hline 35 & $\mathrm{H}$ & 0.9726 & -0.3905 & 2.7388 \\
\hline 36 & $\mathrm{H}$ & -1.4744 & 0.1712 & 2.3683 \\
\hline 37 & $\mathrm{H}$ & -0.376 & 1.4913 & 1.9865 \\
\hline 38 & $\mathrm{H}$ & -3.1276 & -1.07 & 1.6052 \\
\hline 39 & $\mathrm{H}$ & -4.4281 & 0.2209 & -0.8414 \\
\hline 40 & $\mathrm{H}$ & -5.1638 & 0.0213 & 0.7589 \\
\hline 41 & $\mathrm{H}$ & -4.3038 & 2.3516 & 0.414 \\
\hline 42 & $\mathrm{H}$ & -3.518 & 1.5469 & 1.774 \\
\hline 43 & $\mathrm{H}$ & 7.2031 & -0.4909 & 0.0225 \\
\hline 44 & $\mathrm{H}$ & 6.2795 & -0.0439 & -1.425 \\
\hline 45 & $\mathrm{H}$ & 6.293 & 1.0274 & -0.0007 \\
\hline 46 & $\mathrm{H}$ & 4.4023 & -0.9752 & 2.0548 \\
\hline 47 & $\mathrm{H}$ & 5.0135 & -2.3035 & -1.3083 \\
\hline 48 & $\mathrm{H}$ & 5.9434 & -2.7309 & 0.1406 \\
\hline 49 & $\mathrm{H}$ & 4.169 & -2.737 & 0.1924 \\
\hline 50 & $\mathrm{H}$ & -0.0305 & -1.9852 & -0.5831 \\
\hline
\end{tabular}




\begin{tabular}{rrrrr}
$\mathbf{5 1}$ & $\mathrm{H}$ & 0.2575 & -1.4177 & -2.2298 \\
$\mathbf{5 2}$ & $\mathrm{H}$ & 1.608 & -2.0232 & -1.2464 \\
$\mathbf{5 3}$ & $\mathrm{H}$ & -2.5403 & 3.9039 & 0.3178 \\
$\mathbf{5 4}$ & $\mathrm{H}$ & -1.6934 & 3.1397 & 1.6789 \\
$\mathbf{5 5}$ & $\mathrm{H}$ & -2.9801 & -4.3627 & -0.1258 \\
$\mathbf{5 6}$ & $\mathrm{H}$ & -1.6669 & -3.1959 & 0.097 \\
$\mathbf{5 7}$ & $\mathrm{H}$ & -2.753 & -3.5708 & 1.449 \\
$\mathbf{5 8}$ & $\mathrm{H}$ & -5.8081 & -1.9717 & -0.1673 \\
$\mathbf{5 9}$ & $\mathrm{H}$ & -5.3272 & -3.6712 & -0.1333 \\
$\mathbf{6 0}$ & $\mathrm{H}$ & -5.2366 & -2.706 & 1.3531 \\
$\mathbf{6 1}$ & $\mathrm{H}$ & -2.7981 & -1.7196 & -1.7286 \\
$\mathbf{6 2}$ & $\mathrm{H}$ & -1.6505 & 1.7725 & -1.8037 \\
\hline
\end{tabular}


Table S52. Conformer 2d-E/3d-E. PCM-CHCl 3 energy and Boltzmann Populations 1694.806597 hartrees, $15.33 \%$.

\begin{tabular}{|c|c|c|c|c|}
\hline \multicolumn{2}{|c|}{ Atom number } & \multicolumn{3}{|c|}{ Coordinates } \\
\hline & & X & $\mathbf{Y}$ & Z \\
\hline 1 & 0 & 2.9428 & -0.2289 & 0.7814 \\
\hline 2 & C & 1.6337 & 0.3212 & 0.7385 \\
\hline 3 & $\mathrm{C}$ & 1.0908 & 0.2773 & -0.7028 \\
\hline 4 & $\mathrm{C}$ & 1.9892 & 1.2082 & -1.5287 \\
\hline 5 & $\mathrm{C}$ & 3.4701 & 0.7994 & -1.3933 \\
\hline 6 & $\mathrm{C}$ & 3.9025 & 0.577 & 0.0673 \\
\hline 7 & $\mathrm{C}$ & 0.6921 & -0.3556 & 1.722 \\
\hline 8 & $\mathrm{C}$ & -0.6418 & 0.4095 & 1.6636 \\
\hline 9 & $\mathrm{C}$ & -1.2107 & 0.4443 & 0.2283 \\
\hline 10 & $\mathrm{O}$ & -0.2312 & 0.8901 & -0.6987 \\
\hline 11 & $\mathrm{O}$ & -1.5928 & -0.861 & -0.1863 \\
\hline 12 & $\mathrm{C}$ & -2.7345 & -1.4567 & 0.4399 \\
\hline 13 & $\mathrm{C}$ & -3.9671 & -0.5785 & 0.2137 \\
\hline 14 & $\mathrm{C}$ & -3.6835 & 0.8605 & 0.6658 \\
\hline 15 & $\mathrm{C}$ & -2.425 & 1.4218 & -0.0137 \\
\hline 16 & $\mathrm{C}$ & 5.2476 & -0.1807 & 0.2227 \\
\hline 17 & $\mathrm{C}$ & 5.6529 & -0.239 & 1.7056 \\
\hline 18 & $\mathrm{O}$ & 5.079 & -1.5098 & -0.2859 \\
\hline 19 & $\mathrm{C}$ & 6.3637 & 0.4537 & -0.6086 \\
\hline 20 & $\mathrm{C}$ & 1.0586 & -1.1329 & -1.3092 \\
\hline 21 & $\mathrm{C}$ & -2.0719 & 2.8376 & 0.4836 \\
\hline 22 & $\mathrm{C}$ & -2.8199 & -2.9043 & -0.0941 \\
\hline 23 & $\mathrm{C}$ & -4.0207 & -3.6363 & 0.5252 \\
\hline 24 & $\mathrm{C}$ & -2.8647 & -2.9665 & -1.6261 \\
\hline 25 & 0 & -1.6067 & -3.515 & 0.3866 \\
\hline 26 & $\mathrm{Cl}$ & -3.3462 & 4.0659 & 0.0811 \\
\hline 27 & 0 & -2.6874 & 1.4479 & -1.4065 \\
\hline 28 & $\mathrm{H}$ & 1.6991 & 1.3868 & 1.0239 \\
\hline 29 & $\mathrm{H}$ & 1.6879 & 1.197 & -2.5832 \\
\hline 30 & $\mathrm{H}$ & 1.8438 & 2.2337 & -1.1643 \\
\hline 31 & $\mathrm{H}$ & 3.6646 & -0.1203 & -1.9536 \\
\hline 32 & $\mathrm{H}$ & 4.0983 & 1.5807 & -1.8358 \\
\hline 33 & $\mathrm{H}$ & 3.9745 & 1.5523 & 0.5785 \\
\hline 34 & $\mathrm{H}$ & 0.5445 & -1.411 & 1.4726 \\
\hline 35 & $\mathrm{H}$ & 1.1124 & -0.3067 & 2.7328 \\
\hline 36 & $\mathrm{H}$ & -1.3703 & -0.0345 & 2.3478 \\
\hline 37 & $\mathrm{H}$ & -0.4717 & 1.4349 & 2.0085 \\
\hline 38 & $\mathrm{H}$ & -2.5502 & -1.5516 & 1.5214 \\
\hline 39 & $\mathrm{H}$ & -4.2242 & -0.5672 & -0.8495 \\
\hline 40 & $\mathrm{H}$ & -4.8241 & -0.9759 & 0.7686 \\
\hline 41 & $\mathrm{H}$ & -4.5264 & 1.51 & 0.4117 \\
\hline 42 & $\mathrm{H}$ & -3.563 & 0.9038 & 1.7568 \\
\hline 43 & $\mathrm{H}$ & 6.555 & -0.8494 & 1.8131 \\
\hline 44 & $\mathrm{H}$ & 5.8615 & 0.7623 & 2.102 \\
\hline 45 & $\mathrm{H}$ & 4.8551 & -0.6823 & 2.3084 \\
\hline 46 & $\mathrm{H}$ & 4.2897 & -1.8597 & 0.1625 \\
\hline 47 & $\mathrm{H}$ & 6.5123 & 1.5045 & -0.3352 \\
\hline 48 & $\mathrm{H}$ & 7.3007 & -0.0831 & -0.4294 \\
\hline 49 & $\mathrm{H}$ & 6.1436 & 0.394 & -1.6777 \\
\hline 50 & $\mathrm{H}$ & 2.0695 & -1.5436 & -1.3674 \\
\hline
\end{tabular}




\begin{tabular}{rrrrr}
$\mathbf{5 1}$ & $\mathrm{H}$ & 0.4325 & -1.8179 & -0.7388 \\
$\mathbf{5 2}$ & $\mathrm{H}$ & 0.6514 & -1.0743 & -2.3245 \\
$\mathbf{5 3}$ & $\mathrm{H}$ & -1.9602 & 2.8758 & 1.5677 \\
$\mathbf{5 4}$ & $\mathrm{H}$ & -1.1515 & 3.181 & 0.0101 \\
$\mathbf{5 5}$ & $\mathrm{H}$ & -3.9937 & -3.5718 & 1.6185 \\
$\mathbf{5 6}$ & $\mathrm{H}$ & -4.9771 & -3.238 & 0.1716 \\
$\mathbf{5 7}$ & $\mathrm{H}$ & -3.9854 & -4.698 & 0.2515 \\
$\mathbf{5 8}$ & $\mathrm{H}$ & -2.0048 & -2.4471 & -2.0532 \\
$\mathbf{5 9}$ & $\mathrm{H}$ & -2.8484 & -4.0129 & -1.958 \\
$\mathbf{6 0}$ & $\mathrm{H}$ & -3.7787 & -2.5128 & -2.0232 \\
$\mathbf{6 1}$ & $\mathrm{H}$ & -1.5634 & -4.4069 & 0.0067 \\
$\mathbf{6 2}$ & $\mathrm{H}$ & -1.8185 & 1.3829 & -1.8421 \\
\hline
\end{tabular}


Table S53. Conformer 2d-F/3d-F. $\mathrm{PCM}^{-} \mathrm{CHCl}_{3}$ energy and Boltzmann Populations 1694.806092 hartrees, $8.98 \%$.

\begin{tabular}{|c|c|c|c|c|}
\hline \multicolumn{2}{|c|}{ Atom number } & \multicolumn{2}{|c|}{ Coordinates } & \multirow[b]{2}{*}{$\mathbf{Z}$} \\
\hline & & X & $\mathbf{Y}$ & \\
\hline 1 & 0 & -2.9414 & -0.3372 & -0.7285 \\
\hline 2 & C & -1.6415 & 0.238 & -0.7005 \\
\hline 3 & C & -1.0836 & 0.1971 & 0.7364 \\
\hline 4 & C & -1.9989 & 1.0914 & 1.5878 \\
\hline 5 & $\mathrm{C}$ & -3.4713 & 0.6447 & 1.4611 \\
\hline 6 & $\mathrm{C}$ & -3.8927 & 0.4742 & -0.007 \\
\hline 7 & $\mathrm{C}$ & -0.6925 & -0.4101 & -1.6955 \\
\hline 8 & $\mathrm{C}$ & 0.6197 & 0.3932 & -1.6469 \\
\hline 9 & C & 1.2022 & 0.4413 & -0.2172 \\
\hline 10 & $\mathrm{O}$ & 0.2181 & 0.8511 & 0.7228 \\
\hline 11 & $\mathrm{O}$ & 1.6302 & -0.8527 & 0.1873 \\
\hline 12 & $\mathrm{C}$ & 2.7844 & -1.4073 & -0.4538 \\
\hline 13 & C & 3.9899 & -0.4924 & -0.2296 \\
\hline 14 & $\mathrm{C}$ & 3.6574 & 0.9388 & -0.6736 \\
\hline 15 & $\mathrm{C}$ & 2.3863 & 1.4572 & 0.0173 \\
\hline 16 & C & -5.2768 & -0.1643 & -0.273 \\
\hline 17 & $\mathrm{C}$ & -6.4026 & 0.7268 & 0.2549 \\
\hline 18 & $\mathrm{O}$ & -5.4489 & -0.203 & -1.6956 \\
\hline 19 & $\mathrm{C}$ & -5.3886 & -1.5913 & 0.2884 \\
\hline 20 & $\mathrm{C}$ & -0.998 & -1.217 & 1.3283 \\
\hline 21 & $\mathrm{C}$ & 1.9831 & 2.8619 & -0.4729 \\
\hline 22 & $\mathrm{C}$ & 2.9187 & -2.8562 & 0.0657 \\
\hline 23 & $\mathrm{C}$ & 4.1347 & -3.5458 & -0.5727 \\
\hline 24 & $\mathrm{C}$ & 2.9812 & -2.9309 & 1.5964 \\
\hline 25 & $\mathrm{O}$ & 1.7195 & -3.4986 & -0.4085 \\
\hline 26 & $\mathrm{Cl}$ & 3.2181 & 4.1307 & -0.0739 \\
\hline 27 & 0 & 2.6598 & 1.4881 & 1.4079 \\
\hline 28 & $\mathrm{H}$ & -1.7314 & 1.3041 & -0.9772 \\
\hline 29 & $\mathrm{H}$ & -1.6832 & 1.0725 & 2.6377 \\
\hline 30 & $\mathrm{H}$ & -1.889 & 2.1258 & 1.2369 \\
\hline 31 & $\mathrm{H}$ & -3.62 & -0.2943 & 2.0041 \\
\hline 32 & $\mathrm{H}$ & -4.1186 & 1.3908 & 1.9362 \\
\hline 33 & $\mathrm{H}$ & -3.9068 & 1.4671 & -0.4875 \\
\hline 34 & $\mathrm{H}$ & -0.5137 & -1.4621 & -1.4533 \\
\hline 35 & $\mathrm{H}$ & -1.1227 & -0.3672 & -2.7027 \\
\hline 36 & $\mathrm{H}$ & 1.354 & -0.0283 & -2.339 \\
\hline 37 & $\mathrm{H}$ & 0.4163 & 1.4136 & -1.9886 \\
\hline 38 & $\mathrm{H}$ & 2.5941 & -1.4981 & -1.5345 \\
\hline 39 & $\mathrm{H}$ & 4.2521 & -0.4777 & 0.8323 \\
\hline 40 & $\mathrm{H}$ & 4.8562 & -0.8598 & -0.7908 \\
\hline 41 & $\mathrm{H}$ & 4.4808 & 1.614 & -0.4229 \\
\hline 42 & $\mathrm{H}$ & 3.5274 & 0.9823 & -1.7636 \\
\hline 43 & $\mathrm{H}$ & -6.3074 & 1.7456 & -0.1362 \\
\hline 44 & $\mathrm{H}$ & -7.3643 & 0.3265 & -0.0812 \\
\hline 45 & $\mathrm{H}$ & -6.4102 & 0.7658 & 1.3489 \\
\hline 46 & $\mathrm{H}$ & -4.6147 & -0.5626 & -2.0444 \\
\hline 47 & $\mathrm{H}$ & -5.3719 & -1.6109 & 1.384 \\
\hline 48 & $\mathrm{H}$ & -6.3335 & -2.0302 & -0.0463 \\
\hline 49 & $\mathrm{H}$ & -4.5679 & -2.2153 & -0.0793 \\
\hline 50 & $\mathrm{H}$ & -0.358 & -1.8765 & 0.7438 \\
\hline
\end{tabular}




\begin{tabular}{rrrrr}
$\mathbf{5 1}$ & $\mathrm{H}$ & -0.5797 & -1.1545 & 2.3389 \\
$\mathbf{5 2}$ & $\mathrm{H}$ & -1.9936 & -1.6624 & 1.3921 \\
$\mathbf{5 3}$ & $\mathrm{H}$ & 1.8632 & 2.8999 & -1.5561 \\
$\mathbf{5 4}$ & $\mathrm{H}$ & 1.0548 & 3.1729 & 0.0075 \\
$\mathbf{5 5}$ & $\mathrm{H}$ & 4.0945 & -3.4715 & -1.6649 \\
$\mathbf{5 6}$ & $\mathrm{H}$ & 5.0824 & -3.1224 & -0.2247 \\
$\mathbf{5 7}$ & $\mathrm{H}$ & 4.1341 & -4.6107 & -0.3094 \\
$\mathbf{5 8}$ & $\mathrm{H}$ & 2.9993 & -3.9802 & 1.9189 \\
$\mathbf{5 9}$ & $\mathrm{H}$ & 3.8854 & -2.4536 & 1.9883 \\
$\mathbf{6 0}$ & $\mathrm{H}$ & 2.1107 & -2.4412 & 2.0369 \\
$\mathbf{6 1}$ & $\mathrm{H}$ & 1.7101 & -4.3971 & -0.0421 \\
$\mathbf{6 2}$ & $\mathrm{H}$ & 1.7972 & 1.3973 & 1.851 \\
\hline
\end{tabular}


Table S54. J-DP4 results for $\mathbf{2 a - 2 d}$ (Isomers 1-4).

\begin{tabular}{|c|c|c|c|c|c|}
\hline Atom/J & Exp & 2a $(14 R-15 R)$ & 2b (14R-15S) & 2c $(14 S-15 R)$ & 2d (14S-15S) \\
\hline $\mathrm{C}$ & 85.8 & 106.636 & 106.601 & 106.148 & 105.913 \\
\hline C & 23.1 & 164.825 & 164.971 & 165.341 & 165.285 \\
\hline C & 38.2 & 151.646 & 151.809 & 152.594 & 152.580 \\
\hline C & 73.5 & 115.980 & 115.898 & 115.857 & 114.024 \\
\hline $\mathrm{C}$ & 75 & 115.639 & 115.247 & 111.019 & 110.417 \\
\hline C & 19.7 & 169.835 & 169.389 & 168.430 & 168.344 \\
\hline C & 23.6 & 165.787 & 164.899 & 165.702 & 163.200 \\
\hline C & 100 & 87.266 & 87.015 & 85.812 & 88.002 \\
\hline C & 71.5 & 116.973 & 116.999 & 116.462 & 117.375 \\
\hline C & 28 & 160.950 & 161.400 & 160.184 & 159.427 \\
\hline C & 21.8 & 165.944 & 167.737 & 166.587 & 168.197 \\
\hline C & 74.2 & 113.602 & 114.341 & 111.557 & 110.932 \\
\hline C & 19.7 & 171.076 & 170.851 & 171.232 & 170.781 \\
\hline $\mathrm{H}$ & 3.05 & 28.296 & 28.312 & 28.290 & 28.248 \\
\hline $\mathrm{H}$ & 1.45 & 29.853 & 29.872 & 29.784 & 29.757 \\
\hline $\mathrm{H}$ & 1.74 & 30.135 & 30.177 & 30.186 & 30.138 \\
\hline $\mathrm{H}$ & 1.78 & 29.856 & 29.900 & 29.951 & 29.927 \\
\hline $\mathrm{H}$ & 1.61 & 29.904 & 29.919 & 30.155 & 29.997 \\
\hline $\mathrm{H}$ & 3.91 & 27.660 & 27.570 & 28.371 & 28.276 \\
\hline $\mathrm{H}$ & 1.4 & 30.170 & 30.201 & 29.796 & 29.662 \\
\hline $\mathrm{H}$ & 1.88 & 29.766 & 29.690 & 30.154 & 30.183 \\
\hline $\mathrm{H}$ & 2.04 & 29.433 & 29.772 & 29.403 & 29.368 \\
\hline $\mathrm{H}$ & 1.95 & 30.167 & 29.581 & 29.491 & 30.163 \\
\hline $\mathrm{H}$ & 1.91 & 29.657 & 29.933 & 29.855 & 29.999 \\
\hline $\mathrm{H}$ & 1.85 & 30.047 & 29.937 & 30.226 & 30.346 \\
\hline $\mathrm{H}$ & 1.5 & 30.133 & 29.997 & 30.039 & 29.700 \\
\hline $\mathrm{H}$ & 1.47 & 30.231 & 30.316 & 30.320 & 30.468 \\
\hline $\mathrm{H}$ & 3.83 & 27.743 & 27.764 & 28.026 & 28.157 \\
\hline $\mathrm{H}$ & 1.14 & 30.439 & 30.485 & 30.295 & 30.208 \\
\hline${ }^{3} J_{\mathrm{H} 7-\mathrm{H} 8 \mathrm{a}}$ & 11.60 & 10.548 & 10.561 & 10.639 & 10.676 \\
\hline${ }^{3} J_{\mathrm{H} 7-\mathrm{H} 8 \mathrm{~b}}$ & 2.50 & 2.481 & 2.512 & 2.746 & 2.794 \\
\hline${ }^{3} J_{\text {н8а-н9а }}$ & 4.50 & 4.256 & 4.346 & 4.623 & 4.705 \\
\hline${ }^{3} J_{\mathrm{H} 8 \mathrm{a}-\mathrm{H}}$ & 12.30 & 12.710 & 12.629 & 12.603 & 12.482 \\
\hline${ }^{3} \mathrm{~J}_{\mathrm{Hzb}-\mathrm{H} 9 \mathrm{a}}$ & 2.70 & 2.428 & 2.356 & 2.189 & 2.149 \\
\hline${ }^{3} J_{\mathrm{H} 8 \mathrm{~b}-\mathrm{H} 9 \mathrm{a}}$ & 4.30 & 4.457 & 4.523 & 4.709 & 4.767 \\
\hline${ }^{3} J_{\mathrm{H} 11-\mathrm{H} 12 \mathrm{a}}$ & 9.80 & 9.799 & 9.338 & 11.213 & 11.232 \\
\hline${ }^{3} J_{H 11-H 12 b}$ & 8.10 & 7.114 & 7.375 & 3.437 & 3.295 \\
\hline${ }^{3} J_{\mathrm{H} 12 \mathrm{a}-\mathrm{H} 13 \mathrm{a}}$ & 11.10 & 10.740 & 10.203 & 3.726 & 3.320 \\
\hline${ }^{3} J_{\mathrm{H} 12 \mathrm{a}-\mathrm{H} 13 \mathrm{~b}}$ & 1.30 & 1.152 & 0.913 & 12.700 & 11.898 \\
\hline${ }^{3} J_{\mathrm{H} 12 \mathrm{~b}-\mathrm{H} 13 \mathrm{a}}$ & 7.70 & 6.828 & 7.218 & 2.755 & 3.053 \\
\hline${ }^{3} J_{H 12 b-H 13 b}$ & 10.50 & 10.224 & 9.988 & 4.474 & 3.917 \\
\hline
\end{tabular}




\begin{tabular}{|c|c|c|c|c|c|}
\hline \multirow{4}{*}{ DP4 (\%) } & $\mathrm{H}$ & 17.88 & 82.12 & 0.00 & 0.00 \\
\cline { 2 - 6 } & $\mathrm{C}$ & 24.79 & 70.20 & 3.57 & 1.43 \\
\cline { 2 - 6 } & $\mathrm{H}+\mathrm{C}$ & 7.14 & 92.86 & 0.00 & 0.00 \\
\cline { 2 - 6 } & $\mathrm{J}$ & 25.60 & 74.40 & 0.00 & 0.00 \\
\cline { 2 - 6 } & all data & 2.58 & 97.42 & 0.00 & 0.00 \\
\hline
\end{tabular}


Table S55. DP4 results for $\mathbf{3 a - 3 d}$ (Isomers 1-4).

\begin{tabular}{cccccc}
\hline Atom/J & Exp & 3a $(14 R-15 R)$ & 3b (14R-15S) & 3c $(14 S-15 R)$ & 3d (14S-15S) \\
\hline C & 86.1 & 106.636 & 106.601 & 106.148 & 105.913 \\
C & 23.4 & 164.825 & 164.971 & 165.341 & 165.285 \\
C & 38.5 & 151.646 & 151.809 & 152.594 & 152.580 \\
C & 74 & 115.980 & 115.898 & 115.857 & 114.024 \\
C & 75 & 115.639 & 115.247 & 111.019 & 110.417 \\
C & 19.4 & 169.835 & 169.389 & 168.430 & 168.344 \\
C & 23.2 & 165.787 & 164.899 & 165.702 & 163.200 \\
C & 99.9 & 87.266 & 87.015 & 85.812 & 88.002 \\
C & 72.3 & 116.973 & 116.999 & 116.462 & 117.375 \\
C & 28.4 & 160.950 & 161.400 & 160.184 & 159.427 \\
C & 24.8 & 165.944 & 167.737 & 166.587 & 168.197 \\
C & 74.9 & 113.602 & 114.341 & 111.557 & 110.932 \\
C & 19.7 & 171.076 & 170.851 & 171.232 & 170.781 \\
H & 3.05 & 28.296 & 28.312 & 28.290 & 28.248 \\
H & 1.78 & 29.853 & 29.872 & 29.784 & 29.757 \\
H & 1.46 & 30.135 & 30.177 & 30.186 & 30.138 \\
H & 1.84 & 29.856 & 29.900 & 29.951 & 29.927 \\
H & 1.65 & 29.904 & 29.919 & 30.155 & 29.997 \\
H & 3.87 & 27.660 & 27.570 & 28.371 & 28.276 \\
H & 1.43 & 30.170 & 30.201 & 29.796 & 29.662 \\
H & 1.83 & 29.766 & 29.690 & 30.154 & 30.183 \\
H & 2.19 & 29.433 & 29.772 & 29.403 & 29.368 \\
H & 1.62 & 30.167 & 29.581 & 29.491 & 30.163 \\
H & 2.17 & 29.657 & 29.933 & 29.855 & 29.999 \\
H & 1.8 & 30.047 & 29.937 & 30.226 & 30.346 \\
H & 1.56 & 30.133 & 29.997 & 30.039 & 29.700 \\
H & 1.24 & 30.231 & 30.316 & 30.320 & 30.468 \\
H & 3.85 & 27.743 & 27.764 & 28.026 & 28.157 \\
H & 1.2 & 30.439 & 30.485 & 30.295 & 30.208 \\
& & & & &
\end{tabular}

\begin{tabular}{|c|c|c|c|c|c|}
\hline & $\mathrm{H}$ & 99.92 & 0.08 & 0.00 & 0.00 \\
\cline { 2 - 6 } & $\mathrm{C}$ & 70.63 & 27.02 & 2.07 & 0.28 \\
\cline { 2 - 6 } DP4 (\%) & $\mathrm{H}+\mathrm{C}$ & 99.97 & 0.03 & 0.00 & 0.00 \\
\cline { 2 - 6 } & $\mathrm{J}$ & - & - & - & - \\
\cline { 2 - 6 } & all data & $\mathbf{1 0 0 . 0 0}$ & $\mathbf{0 . 0 0}$ & $\mathbf{0 . 0 0}$ & $\mathbf{0 . 0 0}$ \\
\hline
\end{tabular}


Table S56. Scaled ${ }^{13} \mathrm{C}$ average chemical shifts for $4 a-4 d$ on B3LYP-D3 in PCM-CHCl 3 .

\begin{tabular}{ccccc}
\hline $\mathbf{C}$ & 4a $(14 R-15 R)$ & 4b $(14 R-15 S)$ & 4c $(14 S-15 R)$ & 4d $(14 S-15 S)$ \\
\hline $\mathbf{7}$ & 82.5537907 & 82.1838891 & 81.3508066 & 81.7045147 \\
$\mathbf{8}$ & 24.2537122 & 23.9028182 & 23.4398952 & 23.0610274 \\
$\mathbf{9}$ & 37.4584160 & 37.0448910 & 35.9103475 & 35.6103902 \\
$\mathbf{1 0}$ & 73.1919117 & 72.9008556 & 71.8524946 & 73.6933277 \\
$\mathbf{1 1}$ & 73.5330028 & 73.5513178 & 76.5858649 & 77.2555980 \\
$\mathbf{1 2}$ & 19.2344090 & 19.4920716 & 20.4168904 & 20.0396983 \\
$\mathbf{1 3}$ & 23.2902863 & 23.9753742 & 23.0866273 & 25.1203813 \\
$\mathbf{1 4}$ & 101.9605939 & 101.7398041 & 101.2467896 & 99.3955450 \\
$\mathbf{1 5}$ & 72.1965495 & 71.8014036 & 71.2607126 & 70.3829578 \\
$\mathbf{1 6}$ & 28.1363151 & 27.4688435 & 28.4850613 & 28.8473347 \\
$\mathbf{1 7}$ & 23.1331804 & 21.1416935 & 22.2205777 & 20.1843941 \\
$\mathbf{1 8}$ & 75.5736831 & 74.4557712 & 76.0586572 & 76.7474868 \\
$\mathbf{2 7}$ & 17.9914831 & 18.0325313 & 17.6757105 & 17.6320577 \\
$\mathbf{2 8}$ & 53.2926661 & 58.1087354 & 56.2095645 & 56.1252862 \\
\hline
\end{tabular}

Table S57. Scaled ${ }^{1} \mathrm{H}$ average chemical shifts for $4 a-4 d$ on B3LYP-D3 in PCM-CHCl 3 .

\begin{tabular}{ccccc}
\hline $\mathbf{H}$ & $\mathbf{4 a}(14 R-15 R)$ & $\mathbf{4 b}(14 R-15 S)$ & $\mathbf{4 c}(14 S-15 R)$ & 4d $(14 S-15 S)$ \\
\hline $\mathbf{7}$ & 3.3060752 & 3.2764358 & 3.3525464 & 3.7267500 \\
$\mathbf{8 a}$ & 1.7865999 & 1.7680556 & 1.8381808 & 1.9144785 \\
$\mathbf{8 b}$ & 1.5107448 & 1.4725334 & 1.4306757 & 1.4569163 \\
$\mathbf{9 a}$ & 1.7835108 & 1.7402810 & 1.6694055 & 1.7099697 \\
$\mathbf{9 b}$ & 1.7363288 & 1.7223694 & 1.4624911 & 1.6258767 \\
$\mathbf{1 1}$ & 3.9270499 & 3.9944390 & 3.2705657 & 3.6935298 \\
$\mathbf{1 2 a}$ & 1.4767939 & 1.4496518 & 1.8265686 & 2.0284532 \\
$\mathbf{1 2 b}$ & 1.8709048 & 1.9436329 & 1.4631519 & 1.4020754 \\
$\mathbf{1 3 a}$ & 2.1967052 & 1.8648547 & 2.2247175 & 2.3811529 \\
$\mathbf{1 3 b}$ & 1.4795821 & 2.0496578 & 2.1349425 & 1.4262972 \\
$\mathbf{1 6 a}$ & 1.9774396 & 1.7086814 & 1.7662602 & 1.6237268 \\
$\mathbf{1 6 b}$ & 1.5968416 & 1.7047466 & 1.3898893 & 1.2067652 \\
$\mathbf{1 7 a}$ & 1.5129324 & 1.6464327 & 1.5798815 & 1.9823512 \\
$\mathbf{1 7 b}$ & 1.4169147 & 1.3387146 & 1.2949362 & 1.0596866 \\
$\mathbf{1 8}$ & 3.8466050 & 3.8066413 & 3.6201071 & 3.8361886 \\
$\mathbf{2 7}$ & 1.2143268 & 1.1747687 & 1.3207331 & 1.3727083 \\
$\mathbf{2 8 a}$ & 3.4977224 & 3.5731959 & 4.2259595 & 3.6163727 \\
$\mathbf{2 8 b}$ & 3.7629222 & 3.6649074 & 4.0289873 & 3.8367008 \\
\hline
\end{tabular}




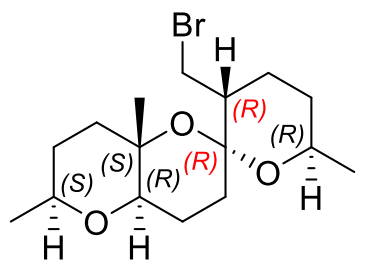

Table S58. Conformer 4a-A. PCM-CHCl ${ }_{3}$ energy and Boltzmann Populations -3806.322917 hartrees, $16.17 \%$.

\begin{tabular}{|c|c|c|c|c|}
\hline \multicolumn{2}{|c|}{ Atom number } & \multicolumn{3}{|c|}{ Coordinates } \\
\hline & & $x$ & $\mathrm{Y}$ & Z \\
\hline 1 & $\mathrm{O}$ & -3.2771 & -0.5283 & -0.8233 \\
\hline 2 & C & -1.8795 & -0.3436 & -0.6285 \\
\hline 3 & C & -1.4348 & -1.0015 & 0.6894 \\
\hline 4 & $\mathrm{C}$ & -2.2065 & -0.31 & 1.8234 \\
\hline 5 & $\mathrm{C}$ & -3.7256 & -0.348 & 1.5742 \\
\hline 6 & $\mathrm{C}$ & -4.087 & 0.1396 & 0.1634 \\
\hline 7 & $\mathrm{C}$ & -1.1184 & -0.8902 & -1.8312 \\
\hline 8 & $\mathrm{C}$ & 0.3685 & -1.159 & -1.4834 \\
\hline 9 & $\mathrm{C}$ & 0.8398 & -0.4676 & -0.1962 \\
\hline 10 & 0 & -0.0244 & -0.726 & 0.9104 \\
\hline 11 & 0 & 0.8938 & 0.9245 & -0.4934 \\
\hline 12 & $\mathrm{C}$ & 1.2845 & 1.8256 & 0.5727 \\
\hline 13 & $\mathrm{C}$ & 2.6357 & 1.4082 & 1.1563 \\
\hline 14 & $\mathrm{C}$ & 2.6526 & -0.0838 & 1.5083 \\
\hline 15 & $\mathrm{C}$ & 2.2427 & -0.9695 & 0.3173 \\
\hline 16 & $\mathrm{C}$ & -5.5507 & -0.1484 & -0.2621 \\
\hline 17 & $\mathrm{C}$ & -5.8361 & 0.4861 & -1.6341 \\
\hline 18 & $\mathrm{O}$ & -5.7309 & -1.568 & -0.3371 \\
\hline 19 & $\mathrm{C}$ & -6.5571 & 0.3525 & 0.7752 \\
\hline 20 & $\mathrm{C}$ & -1.6489 & -2.521 & 0.7142 \\
\hline 21 & $\mathrm{C}$ & 3.2435 & -0.9326 & -0.8398 \\
\hline 22 & $\mathrm{C}$ & 1.2744 & 3.2312 & -0.0859 \\
\hline 23 & $\mathrm{C}$ & -0.147 & 3.6069 & -0.5388 \\
\hline 24 & $\mathrm{C}$ & 1.8244 & 4.2994 & 0.8616 \\
\hline 25 & 0 & 2.1513 & 3.2171 & -1.2187 \\
\hline 26 & $\mathrm{Br}$ & 5.1077 & -1.3001 & -0.289 \\
\hline 27 & 0 & 2.1342 & -2.331 & 0.7072 \\
\hline 28 & $\mathrm{H}$ & -1.6739 & 0.7324 & -0.534 \\
\hline 29 & $\mathrm{H}$ & -1.9615 & -0.7732 & 2.7868 \\
\hline 30 & $\mathrm{H}$ & -1.8641 & 0.7319 & 1.8735 \\
\hline 31 & $\mathrm{H}$ & -4.1162 & -1.3633 & 1.6991 \\
\hline 32 & $\mathrm{H}$ & -4.2271 & 0.2816 & 2.3177 \\
\hline 33 & $\mathrm{H}$ & -3.8961 & 1.224 & 0.0908 \\
\hline 34 & $\mathrm{H}$ & -1.5976 & -1.8191 & -2.1587 \\
\hline 35 & $\mathrm{H}$ & -1.2045 & -0.1818 & -2.6604 \\
\hline 36 & $\mathrm{H}$ & 0.5512 & -2.2288 & -1.353 \\
\hline 37 & $\mathrm{H}$ & 1.0068 & -0.8243 & -2.3049 \\
\hline 38 & $\mathrm{H}$ & 0.5154 & 1.7861 & 1.3558 \\
\hline 39 & $\mathrm{H}$ & 3.4269 & 1.6441 & 0.4354 \\
\hline 40 & $\mathrm{H}$ & 2.8403 & 1.9959 & 2.0583 \\
\hline 41 & $\mathrm{H}$ & 3.6438 & -0.3943 & 1.8485 \\
\hline 42 & $\mathrm{H}$ & 1.9489 & -0.2788 & 2.3254 \\
\hline 43 & $\mathrm{H}$ & -5.7796 & 1.5805 & -1.5871 \\
\hline
\end{tabular}




\begin{tabular}{llrrr}
$\mathbf{4 4}$ & $\mathrm{H}$ & -5.1128 & 0.1417 & -2.3791 \\
$\mathbf{4 5}$ & $\mathrm{H}$ & -6.841 & 0.2048 & -1.9641 \\
$\mathbf{4 6}$ & $\mathrm{H}$ & -5.0157 & -1.8892 & -0.9127 \\
$\mathbf{4 7}$ & $\mathrm{H}$ & -6.435 & 1.4261 & 0.9582 \\
$\mathbf{4 8}$ & $\mathrm{H}$ & -7.5738 & 0.1809 & 0.408 \\
$\mathbf{4 9}$ & $\mathrm{H}$ & -6.4489 & -0.1829 & 1.7222 \\
$\mathbf{5 0}$ & $\mathrm{H}$ & -2.6962 & -2.7767 & 0.5336 \\
$\mathbf{5 1}$ & $\mathrm{H}$ & -1.0432 & -3.0306 & -0.0399 \\
$\mathbf{5 2}$ & $\mathrm{H}$ & -1.3651 & -2.9159 & 1.6962 \\
$\mathbf{5 3}$ & $\mathrm{H}$ & 3.0201 & -1.7138 & -1.5626 \\
$\mathbf{5 4}$ & $\mathrm{H}$ & 3.2683 & 0.0411 & -1.3241 \\
$\mathbf{5 5}$ & $\mathrm{H}$ & -0.8354 & 3.6693 & 0.3131 \\
$\mathbf{5 6}$ & $\mathrm{H}$ & -0.126 & 4.5807 & -1.0381 \\
$\mathbf{5 7}$ & $\mathrm{H}$ & -0.541 & 2.8671 & -1.2421 \\
$\mathbf{5 8}$ & $\mathrm{H}$ & 1.2537 & 4.3297 & 1.7967 \\
$\mathbf{5 9}$ & $\mathrm{H}$ & 2.8764 & 4.1164 & 1.0944 \\
$\mathbf{6 0}$ & $\mathrm{H}$ & 1.753 & 5.2815 & 0.3836 \\
$\mathbf{6 1}$ & $\mathrm{H}$ & 1.861 & 2.4639 & -1.7606 \\
$\mathbf{6 2}$ & $\mathrm{H}$ & 1.3982 & -2.3548 & 1.3425 \\
\hline
\end{tabular}


Table S59. Conformer 4a-B. PCM-CHCl ${ }_{3}$ energy and Boltzmann Populations -3806.323604 hartrees, $33.49 \%$.

\begin{tabular}{|c|c|c|c|c|}
\hline \multicolumn{2}{|c|}{ Atom number } & \multicolumn{3}{|c|}{ Coordinates } \\
\hline & & $\mathbf{x}$ & $\mathbf{Y}$ & Z \\
\hline 1 & O & -3.222 & -0.6134 & -0.8246 \\
\hline 2 & $\mathrm{C}$ & -1.8261 & -0.4176 & -0.6346 \\
\hline 3 & $\mathrm{C}$ & -1.3852 & -1.0192 & 0.7116 \\
\hline 4 & $\mathrm{C}$ & -2.1563 & -0.2747 & 1.8118 \\
\hline 5 & $\mathrm{C}$ & -3.6755 & -0.3214 & 1.5612 \\
\hline 6 & $\mathrm{C}$ & -4.0336 & 0.1019 & 0.1287 \\
\hline 7 & $\mathrm{C}$ & -1.0602 & -1.0173 & -1.8097 \\
\hline 8 & $\mathrm{C}$ & 0.429 & -1.2605 & -1.4497 \\
\hline 9 & $\mathrm{C}$ & 0.8853 & -0.506 & -0.194 \\
\hline 10 & $\mathrm{O}$ & 0.0291 & -0.7452 & 0.9209 \\
\hline 11 & $\mathrm{O}$ & 0.9121 & 0.8741 & -0.5494 \\
\hline 12 & $\mathrm{C}$ & 1.2329 & 1.8037 & 0.5137 \\
\hline 13 & C & 2.6225 & 1.4929 & 1.0684 \\
\hline 14 & $\mathrm{C}$ & 2.6987 & 0.017 & 1.4854 \\
\hline 15 & $\mathrm{C}$ & 2.3045 & -0.9395 & 0.3405 \\
\hline 16 & $\mathrm{C}$ & -5.4964 & -0.2045 & -0.2854 \\
\hline 17 & $\mathrm{C}$ & -5.7781 & 0.3605 & -1.6882 \\
\hline 18 & 0 & -5.6798 & -1.6262 & -0.2899 \\
\hline 19 & $\mathrm{C}$ & -6.5039 & 0.3501 & 0.7232 \\
\hline 20 & $\mathrm{C}$ & -1.6042 & -2.5353 & 0.8012 \\
\hline 21 & $\mathrm{C}$ & 3.2922 & -0.935 & -0.8285 \\
\hline 22 & $\mathrm{C}$ & 0.9979 & 3.2114 & -0.0852 \\
\hline 23 & $\mathrm{C}$ & 1.0322 & 4.2767 & 1.0118 \\
\hline 24 & $\mathrm{C}$ & 1.9812 & 3.5504 & -1.2164 \\
\hline 25 & 0 & -0.343 & 3.2353 & -0.6006 \\
\hline 26 & $\mathrm{Br}$ & 5.1815 & -1.1653 & -0.2863 \\
\hline 27 & 0 & 2.2403 & -2.2806 & 0.8031 \\
\hline 28 & $\mathrm{H}$ & -1.6261 & 0.6622 & -0.5805 \\
\hline 29 & $\mathrm{H}$ & -1.9149 & -0.6954 & 2.7955 \\
\hline 30 & $\mathrm{H}$ & -1.8146 & 0.7681 & 1.8146 \\
\hline 31 & $\mathrm{H}$ & -4.0694 & -1.3287 & 1.7327 \\
\hline 32 & $\mathrm{H}$ & -4.1758 & 0.3441 & 2.2735 \\
\hline 33 & $\mathrm{H}$ & -3.8404 & 1.1808 & 0.0058 \\
\hline 34 & $\mathrm{H}$ & -1.535 & -1.9632 & -2.0913 \\
\hline 35 & $\mathrm{H}$ & -1.15 & -0.3519 & -2.6737 \\
\hline 36 & $\mathrm{H}$ & 0.6195 & -2.322 & -1.2701 \\
\hline 37 & $\mathrm{H}$ & 1.0655 & -0.9572 & -2.2847 \\
\hline 38 & $\mathrm{H}$ & 0.4906 & 1.6679 & 1.3101 \\
\hline 39 & $\mathrm{H}$ & 3.3936 & 1.7077 & 0.319 \\
\hline 40 & $\mathrm{H}$ & 2.8298 & 2.1341 & 1.9333 \\
\hline 41 & $\mathrm{H}$ & 3.7034 & -0.2455 & 1.8256 \\
\hline 42 & $\mathrm{H}$ & 2.0115 & -0.1634 & 2.3195 \\
\hline 43 & $\mathrm{H}$ & -5.7181 & 1.4556 & -1.6968 \\
\hline 44 & $\mathrm{H}$ & -5.055 & -0.0236 & -2.4137 \\
\hline 45 & $\mathrm{H}$ & -6.7835 & 0.0658 & -2.0051 \\
\hline 46 & $\mathrm{H}$ & -4.9639 & -1.977 & -0.8469 \\
\hline 47 & $\mathrm{H}$ & -6.3782 & 1.4309 & 0.8534 \\
\hline 48 & $\mathrm{H}$ & -7.5203 & 0.1638 & 0.3623 \\
\hline 49 & $\mathrm{H}$ & -6.3996 & -0.1385 & 1.6956 \\
\hline 50 & $\mathrm{H}$ & -2.6513 & -2.7958 & 0.6265 \\
\hline
\end{tabular}




\begin{tabular}{rrrrr}
$\mathbf{5 1}$ & $\mathrm{H}$ & -0.9957 & -3.0785 & 0.0731 \\
$\mathbf{5 2}$ & $\mathrm{H}$ & -1.3264 & -2.8879 & 1.8009 \\
$\mathbf{5 3}$ & $\mathrm{H}$ & 3.0997 & -1.7775 & -1.4886 \\
$\mathbf{5 4}$ & $\mathrm{H}$ & 3.2586 & -0.0002 & -1.3834 \\
$\mathbf{5 5}$ & $\mathrm{H}$ & 2.032 & 4.3767 & 1.446 \\
$\mathbf{5 6}$ & $\mathrm{H}$ & 0.7437 & 5.2432 & 0.5867 \\
$\mathbf{5 7}$ & $\mathrm{H}$ & 0.3227 & 4.0334 & 1.8099 \\
$\mathbf{5 8}$ & $\mathrm{H}$ & 3.0095 & 3.6533 & -0.853 \\
$\mathbf{5 9}$ & $\mathrm{H}$ & 1.9683 & 2.7727 & -1.9876 \\
$\mathbf{6 0}$ & $\mathrm{H}$ & 1.6841 & 4.4974 & -1.6773 \\
$\mathbf{6 1}$ & $\mathrm{H}$ & -0.3957 & 2.4788 & -1.2084 \\
$\mathbf{6 2}$ & $\mathrm{H}$ & 1.4932 & -2.2984 & 1.4259 \\
\hline
\end{tabular}


Table S60. Conformer 4a-C. PCM- $\mathrm{CHCl}_{3}$ energy and Boltzmann Populations -3806.322622 hartrees, $11.84 \%$.

\begin{tabular}{|c|c|c|c|c|}
\hline \multicolumn{2}{|c|}{ Atom number } & \multicolumn{3}{|c|}{ Coordinates } \\
\hline & & $x$ & $\mathbf{Y}$ & $\mathbf{Z}$ \\
\hline 1 & $\mathrm{O}$ & -3.2718 & -0.6252 & -0.8087 \\
\hline 2 & $\mathrm{C}$ & -1.8747 & -0.4188 & -0.6242 \\
\hline 3 & C & -1.4163 & -1.0678 & 0.695 \\
\hline 4 & $\mathrm{C}$ & -2.2011 & -0.3985 & 1.8361 \\
\hline 5 & $\mathrm{C}$ & -3.7207 & -0.4635 & 1.5855 \\
\hline 6 & $\mathrm{C}$ & -4.0649 & 0.0566 & 0.1839 \\
\hline 7 & $\mathrm{C}$ & -1.1074 & -0.9582 & -1.8255 \\
\hline 8 & $\mathrm{C}$ & 0.387 & -1.1881 & -1.4803 \\
\hline 9 & C & 0.8447 & -0.4858 & -0.1936 \\
\hline 10 & $\mathrm{O}$ & -0.013 & -0.7606 & 0.9152 \\
\hline 11 & $\mathrm{O}$ & 0.87 & 0.9065 & -0.4911 \\
\hline 12 & C & 1.2445 & 1.8162 & 0.5739 \\
\hline 13 & C & 2.6035 & 1.4252 & 1.1577 \\
\hline 14 & C & 2.6503 & -0.0662 & 1.5096 \\
\hline 15 & $\mathrm{C}$ & 2.2581 & -0.9591 & 0.3183 \\
\hline 16 & $\mathrm{C}$ & -5.5337 & -0.0778 & -0.2836 \\
\hline 17 & $\mathrm{C}$ & -6.46 & 0.7928 & 0.5674 \\
\hline 18 & 0 & -5.6 & 0.4663 & -1.6075 \\
\hline 19 & $\mathrm{C}$ & -6.0152 & -1.5378 & -0.3106 \\
\hline 20 & $\mathrm{C}$ & -1.5945 & -2.5921 & 0.7161 \\
\hline 21 & $\mathrm{C}$ & 3.2566 & -0.8995 & -0.8398 \\
\hline 22 & $\mathrm{C}$ & 1.2082 & 3.2206 & -0.0864 \\
\hline 23 & $\mathrm{C}$ & -0.2189 & 3.5681 & -0.5435 \\
\hline 24 & $\mathrm{C}$ & 1.7344 & 4.3002 & 0.8617 \\
\hline 25 & 0 & 2.0887 & 3.2226 & -1.2164 \\
\hline 26 & $\mathrm{Br}$ & 5.1287 & -1.2278 & -0.2918 \\
\hline 27 & 0 & 2.1787 & -2.3231 & 0.7067 \\
\hline 28 & $\mathrm{H}$ & -1.6836 & 0.66 & -0.5328 \\
\hline 29 & $\mathrm{H}$ & -1.945 & -0.8645 & 2.7951 \\
\hline 30 & $\mathrm{H}$ & -1.8818 & 0.65 & 1.8938 \\
\hline 31 & $\mathrm{H}$ & -4.076 & -1.4936 & 1.6982 \\
\hline 32 & $\mathrm{H}$ & -4.2408 & 0.1378 & 2.3402 \\
\hline 33 & $\mathrm{H}$ & -3.8141 & 1.1294 & 0.1308 \\
\hline 34 & $\mathrm{H}$ & -1.566 & -1.9007 & -2.1431 \\
\hline 35 & $\mathrm{H}$ & -1.2123 & -0.2583 & -2.66 \\
\hline 36 & $\mathrm{H}$ & 0.5973 & -2.253 & -1.3518 \\
\hline 37 & $\mathrm{H}$ & 1.0149 & -0.8359 & -2.3025 \\
\hline 38 & $\mathrm{H}$ & 0.4763 & 1.7632 & 1.3573 \\
\hline 39 & $\mathrm{H}$ & 3.39 & 1.6765 & 0.437 \\
\hline 40 & $\mathrm{H}$ & 2.7964 & 2.0169 & 2.0597 \\
\hline 41 & $\mathrm{H}$ & 3.6476 & -0.3568 & 1.8495 \\
\hline 42 & $\mathrm{H}$ & 1.9507 & -0.2754 & 2.3268 \\
\hline 43 & $\mathrm{H}$ & -6.5362 & 0.4209 & 1.5942 \\
\hline 44 & $\mathrm{H}$ & -6.1058 & 1.829 & 0.5897 \\
\hline 45 & $\mathrm{H}$ & -7.4615 & 0.7897 & 0.1259 \\
\hline 46 & $\mathrm{H}$ & -4.849 & 0.0733 & -2.0851 \\
\hline 47 & $\mathrm{H}$ & -6.0886 & -1.9706 & 0.6935 \\
\hline 48 & $\mathrm{H}$ & -7.0068 & -1.5751 & -0.7718 \\
\hline 49 & $\mathrm{H}$ & -5.3355 & -2.1579 & -0.9038 \\
\hline 50 & $\mathrm{H}$ & -2.6302 & -2.8727 & 0.5084 \\
\hline 51 & $\mathrm{H}$ & -0.9594 & -3.086 & -0.0238 \\
\hline
\end{tabular}




\begin{tabular}{rrrrr}
$\mathbf{5 2}$ & $\mathrm{H}$ & -1.3233 & -2.9811 & 1.7041 \\
$\mathbf{5 3}$ & $\mathrm{H}$ & 3.0491 & -1.684 & -1.5638 \\
$\mathbf{5 4}$ & $\mathrm{H}$ & 3.2597 & 0.0753 & -1.3226 \\
$\mathbf{5 5}$ & $\mathrm{H}$ & -0.9112 & 3.6172 & 0.306 \\
$\mathbf{5 6}$ & $\mathrm{H}$ & -0.2158 & 4.542 & -1.0428 \\
$\mathbf{5 7}$ & $\mathrm{H}$ & -0.5969 & 2.821 & -1.248 \\
$\mathbf{5 8}$ & $\mathrm{H}$ & 1.1613 & 4.3192 & 1.7957 \\
$\mathbf{5 9}$ & $\mathrm{H}$ & 2.7895 & 4.1392 & 1.0967 \\
$\mathbf{6 0}$ & $\mathrm{H}$ & 1.6435 & 5.2804 & 0.3832 \\
$\mathbf{6 1}$ & $\mathrm{H}$ & 1.8099 & 2.4684 & -1.7629 \\
$\mathbf{6 2}$ & $\mathrm{H}$ & 1.4485 & -2.3625 & 1.3478 \\
\hline
\end{tabular}


Table S61. Conformer 4a-D. PCM- $\mathrm{CHCl}_{3}$ energy and Boltzmann Populations -3806.32317 hartrees, $21.14 \%$.

\begin{tabular}{|c|c|c|c|c|}
\hline \multicolumn{2}{|c|}{ Atom number } & \multicolumn{3}{|c|}{ Coordinates } \\
\hline & & $x$ & $\mathbf{Y}$ & $\mathbf{Z}$ \\
\hline 1 & $\mathrm{O}$ & -3.2167 & -0.7025 & -0.8068 \\
\hline 2 & $\mathrm{C}$ & -1.8215 & -0.4859 & -0.6263 \\
\hline 3 & C & -1.3667 & -1.0846 & 0.7182 \\
\hline 4 & $\mathrm{C}$ & -2.1533 & -0.3691 & 1.8296 \\
\hline 5 & $\mathrm{C}$ & -3.6728 & -0.4449 & 1.5775 \\
\hline 6 & $\mathrm{C}$ & -4.0131 & 0.0203 & 0.1558 \\
\hline 7 & $\mathrm{C}$ & -1.0491 & -1.0725 & -1.8028 \\
\hline 8 & $\mathrm{C}$ & 0.4465 & -1.2815 & -1.4468 \\
\hline 9 & C & 0.8899 & -0.52 & -0.1902 \\
\hline 10 & $\mathrm{O}$ & 0.0399 & -0.7763 & 0.9272 \\
\hline 11 & $\mathrm{O}$ & 0.8893 & 0.8601 & -0.5435 \\
\hline 12 & C & 1.1982 & 1.7952 & 0.5187 \\
\hline 13 & $\mathrm{C}$ & 2.5944 & 1.508 & 1.0696 \\
\hline 14 & C & 2.6974 & 0.0335 & 1.4857 \\
\hline 15 & $\mathrm{C}$ & 2.3179 & -0.9284 & 0.3405 \\
\hline 16 & $\mathrm{C}$ & -5.4801 & -0.1336 & -0.3112 \\
\hline 17 & C & -6.4091 & 0.7725 & 0.4988 \\
\hline 18 & 0 & -5.5417 & 0.3541 & -1.6573 \\
\hline 19 & $\mathrm{C}$ & -5.9624 & -1.5933 & -0.2803 \\
\hline 20 & $\mathrm{C}$ & -1.5474 & -2.6066 & 0.797 \\
\hline 21 & $\mathrm{C}$ & 3.3026 & -0.903 & -0.8309 \\
\hline 22 & $\mathrm{C}$ & 0.9382 & 3.1988 & -0.0794 \\
\hline 23 & $\mathrm{C}$ & 0.9682 & 4.2657 & 1.0164 \\
\hline 24 & $\mathrm{C}$ & 1.9064 & 3.5502 & -1.2198 \\
\hline 25 & 0 & -0.4075 & 3.2047 & -0.5812 \\
\hline 26 & $\mathrm{Br}$ & 5.1971 & -1.0993 & -0.2939 \\
\hline 27 & 0 & 2.2798 & -2.2713 & 0.8006 \\
\hline 28 & $\mathrm{H}$ & -1.6362 & 0.5963 & -0.5699 \\
\hline 29 & $\mathrm{H}$ & -1.9003 & -0.7969 & 2.8071 \\
\hline 30 & $\mathrm{H}$ & -1.8368 & 0.6812 & 1.8446 \\
\hline 31 & $\mathrm{H}$ & -4.0297 & -1.4695 & 1.7298 \\
\hline 32 & $\mathrm{H}$ & -4.1938 & 0.1866 & 2.3062 \\
\hline 33 & $\mathrm{H}$ & -3.7609 & 1.0892 & 0.0604 \\
\hline 34 & $\mathrm{H}$ & -1.5044 & -2.0292 & -2.0799 \\
\hline 35 & $\mathrm{H}$ & -1.1553 & -0.4107 & -2.6679 \\
\hline 36 & $\mathrm{H}$ & 0.6622 & -2.3388 & -1.2715 \\
\hline 37 & $\mathrm{H}$ & 1.0737 & -0.9606 & -2.282 \\
\hline 38 & $\mathrm{H}$ & 0.4606 & 1.6475 & 1.3173 \\
\hline 39 & $\mathrm{H}$ & 3.3599 & 1.7365 & 0.3185 \\
\hline 40 & $\mathrm{H}$ & 2.7924 & 2.1524 & 1.9342 \\
\hline 41 & $\mathrm{H}$ & 3.7071 & -0.2117 & 1.824 \\
\hline 42 & $\mathrm{H}$ & 2.0147 & -0.1593 & 2.3209 \\
\hline 43 & $\mathrm{H}$ & -6.4874 & 0.4461 & 1.5409 \\
\hline 44 & $\mathrm{H}$ & -6.0555 & 1.8088 & 0.4763 \\
\hline 45 & $\mathrm{H}$ & -7.4097 & 0.7489 & 0.0556 \\
\hline 46 & $\mathrm{H}$ & -4.7855 & -0.0539 & -2.1134 \\
\hline 47 & $\mathrm{H}$ & -5.2812 & -2.2371 & -0.8458 \\
\hline 48 & $\mathrm{H}$ & -6.0398 & -1.9855 & 0.7401 \\
\hline 49 & $\mathrm{H}$ & -6.9524 & -1.6488 & -0.7431 \\
\hline 50 & $\mathrm{H}$ & -2.5829 & -2.8933 & 0.5971 \\
\hline 51 & $\mathrm{H}$ & -0.9106 & -3.1293 & 0.0785 \\
\hline
\end{tabular}




\begin{tabular}{rrrrr}
$\mathbf{5 2}$ & $\mathrm{H}$ & -1.2796 & -2.9578 & 1.8 \\
$\mathbf{5 3}$ & $\mathrm{H}$ & 3.1244 & -1.7474 & -1.4927 \\
$\mathbf{5 4}$ & $\mathrm{H}$ & 3.2498 & 0.0323 & -1.3834 \\
$\mathbf{5 5}$ & $\mathrm{H}$ & 1.9706 & 4.3802 & 1.441 \\
$\mathbf{5 6}$ & $\mathrm{H}$ & 0.6619 & 5.2273 & 0.5929 \\
$\mathbf{5 7}$ & $\mathrm{H}$ & 0.2696 & 4.0135 & 1.8214 \\
$\mathbf{5 8}$ & $\mathrm{H}$ & 2.9367 & 3.6665 & -0.8662 \\
$\mathbf{5 9}$ & $\mathrm{H}$ & 1.8962 & 2.7724 & -1.9908 \\
$\mathbf{6 0}$ & $\mathrm{H}$ & 1.5927 & 4.4932 & -1.6778 \\
$\mathbf{6 1}$ & $\mathrm{H}$ & -0.4548 & 2.4553 & -1.198 \\
$\mathbf{6 2}$ & $\mathrm{H}$ & 1.538 & -2.3032 & 1.4291 \\
\hline
\end{tabular}




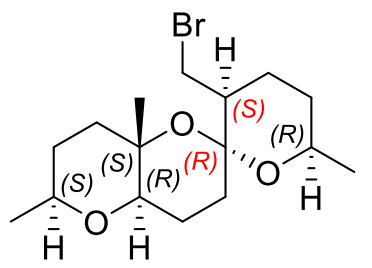

Table S62. Conformer 4b-A. PCM-CHCl ${ }_{3}$ energy and Boltzmann Populations -3806.322662 hartrees, $15.35 \%$.

\begin{tabular}{|c|c|c|c|c|}
\hline \multicolumn{2}{|c|}{ Atom number } & \multicolumn{3}{|c|}{ Coordinates } \\
\hline & & $x$ & $\mathbf{Y}$ & $\mathbf{Z}$ \\
\hline 1 & 0 & 3.1259 & -0.6022 & 0.797 \\
\hline 2 & $\mathrm{C}$ & 1.7561 & -0.2295 & 0.6758 \\
\hline 3 & $\mathrm{C}$ & 1.098 & -1.0166 & -0.4733 \\
\hline 4 & $\mathrm{C}$ & 1.8406 & -0.6343 & -1.7626 \\
\hline 5 & $\mathrm{C}$ & 3.3585 & -0.8569 & -1.6257 \\
\hline 6 & $\mathrm{C}$ & 3.9217 & -0.2257 & -0.3433 \\
\hline 7 & $\mathrm{C}$ & 1.046 & -0.4363 & 2.0123 \\
\hline 8 & $\mathrm{C}$ & -0.4862 & -0.5777 & 1.826 \\
\hline 9 & $\mathrm{C}$ & -0.971 & -0.0268 & 0.4763 \\
\hline 10 & $\mathrm{O}$ & -0.2818 & -0.5892 & -0.6342 \\
\hline 11 & $\mathrm{O}$ & -0.7458 & 1.3757 & 0.5662 \\
\hline 12 & $\mathrm{C}$ & -1.1254 & 2.2137 & -0.5487 \\
\hline 13 & $\mathrm{C}$ & -2.5994 & 1.9952 & -0.8873 \\
\hline 14 & $\mathrm{C}$ & -2.9087 & 0.507 & -1.0583 \\
\hline 15 & $\mathrm{C}$ & -2.4887 & -0.3006 & 0.1855 \\
\hline 16 & $\mathrm{C}$ & 5.3634 & -0.6745 & 0.0143 \\
\hline 17 & $\mathrm{C}$ & 5.8731 & 0.1114 & 1.2346 \\
\hline 18 & 0 & 5.3396 & -2.0755 & 0.3154 \\
\hline 19 & $\mathrm{C}$ & 6.3249 & -0.5097 & -1.1638 \\
\hline 20 & $\mathrm{C}$ & 1.1182 & -2.5363 & -0.2545 \\
\hline 21 & $\mathrm{C}$ & -2.7199 & -1.8033 & -0.0274 \\
\hline 22 & $\mathrm{C}$ & -0.7905 & 3.6548 & -0.0787 \\
\hline 23 & $\mathrm{C}$ & 0.7171 & 3.804 & 0.1917 \\
\hline 24 & $\mathrm{C}$ & -1.2437 & 4.6978 & -1.1036 \\
\hline 25 & 0 & -1.5208 & 3.9299 & 1.121 \\
\hline 26 & $\mathrm{Br}$ & -4.6672 & -2.2048 & -0.1857 \\
\hline 27 & 0 & -3.1766 & 0.1469 & 1.3492 \\
\hline 28 & $\mathrm{H}$ & 1.7053 & 0.8339 & 0.4067 \\
\hline 29 & $\mathrm{H}$ & 1.443 & -1.203 & -2.6119 \\
\hline 30 & $\mathrm{H}$ & 1.6362 & 0.4252 & -1.9644 \\
\hline 31 & $\mathrm{H}$ & 3.5958 & -1.9259 & -1.6157 \\
\hline 32 & $\mathrm{H}$ & 3.864 & -0.4252 & -2.4969 \\
\hline 33 & $\mathrm{H}$ & 3.8973 & 0.8736 & -0.4339 \\
\hline 34 & $\mathrm{H}$ & 1.447 & -1.3385 & 2.4868 \\
\hline 35 & $\mathrm{H}$ & 1.2832 & 0.401 & 2.6751 \\
\hline 36 & $\mathrm{H}$ & -0.7725 & -1.6301 & 1.8948 \\
\hline 37 & $\mathrm{H}$ & -1.0329 & -0.0552 & 2.6117 \\
\hline 38 & $\mathrm{H}$ & -0.4967 & 1.9559 & -1.4128 \\
\hline 39 & $\mathrm{H}$ & -3.2069 & 2.4167 & -0.0809 \\
\hline 40 & $\mathrm{H}$ & -2.8504 & 2.5283 & -1.8108 \\
\hline 41 & $\mathrm{H}$ & -3.9793 & 0.3549 & -1.2343 \\
\hline 42 & $\mathrm{H}$ & -2.3779 & 0.1072 & -1.9306 \\
\hline 43 & $\mathrm{H}$ & 5.183 & 0.0099 & 2.0773 \\
\hline
\end{tabular}




\begin{tabular}{llrrr}
$\mathbf{4 4}$ & $\mathrm{H}$ & 6.8524 & -0.2747 & 1.5341 \\
$\mathbf{4 5}$ & $\mathrm{H}$ & 5.9757 & 1.1794 & 1.0069 \\
$\mathbf{4 6}$ & $\mathrm{H}$ & 4.6385 & -2.1826 & 0.981 \\
$\mathbf{4 7}$ & $\mathrm{H}$ & 6.3455 & 0.5281 & -1.5153 \\
$\mathbf{4 8}$ & $\mathrm{H}$ & 7.3364 & -0.7852 & -0.8491 \\
$\mathbf{4 9}$ & $\mathrm{H}$ & 6.0449 & -1.1601 & -1.9966 \\
$\mathbf{5 0}$ & $\mathrm{H}$ & 0.6588 & -3.0358 & -1.1143 \\
$\mathbf{5 1}$ & $\mathrm{H}$ & 2.1404 & -2.9077 & -0.1457 \\
$\mathbf{5 2}$ & $\mathrm{H}$ & 0.562 & -2.8275 & 0.6407 \\
$\mathbf{5 3}$ & $\mathrm{H}$ & -2.2662 & -2.1705 & -0.9438 \\
$\mathbf{5 4}$ & $\mathrm{H}$ & -2.4036 & -2.3986 & 0.8262 \\
$\mathbf{5 5}$ & $\mathrm{H}$ & 1.3092 & 3.6272 & -0.7151 \\
$\mathbf{5 6}$ & $\mathrm{H}$ & 0.9237 & 4.8187 & 0.5466 \\
$\mathbf{5 7}$ & $\mathrm{H}$ & 1.0461 & 3.0989 & 0.9601 \\
$\mathbf{5 8}$ & $\mathrm{H}$ & -0.7752 & 4.5253 & -2.0793 \\
$\mathbf{5 9}$ & $\mathrm{H}$ & -2.3299 & 4.6868 & -1.2244 \\
$\mathbf{6 0}$ & $\mathrm{H}$ & -0.9566 & 5.6957 & -0.7572 \\
$\mathbf{6 1}$ & $\mathrm{H}$ & -1.415 & 3.1391 & 1.6784 \\
$\mathbf{6 2}$ & $\mathrm{H}$ & -4.1028 & -0.138 & 1.2525 \\
\hline
\end{tabular}


Table S63. Conformer 4b-B. PCM- $\mathrm{CHCl}_{3}$ energy and Boltzmann Populations -3806.323268 hartrees, $29.17 \%$.

\begin{tabular}{|c|c|c|c|c|}
\hline \multicolumn{2}{|c|}{ Atom number } & \multicolumn{3}{|c|}{ Coordinates } \\
\hline & & $x$ & $\mathbf{Y}$ & $\mathbf{Z}$ \\
\hline 1 & $\mathrm{O}$ & 3.0745 & -0.692 & 0.8065 \\
\hline 2 & $\mathrm{C}$ & 1.7116 & -0.2924 & 0.7014 \\
\hline 3 & $\mathrm{C}$ & 1.0391 & -1.0291 & -0.4729 \\
\hline 4 & $\mathrm{C}$ & 1.7832 & -0.6081 & -1.7494 \\
\hline 5 & $\mathrm{C}$ & 3.2984 & -0.8565 & -1.625 \\
\hline 6 & $\mathrm{C}$ & 3.8737 & -0.2819 & -0.3216 \\
\hline 7 & $\mathrm{C}$ & 0.9988 & -0.5383 & 2.0308 \\
\hline 8 & C & -0.5382 & -0.6365 & 1.8459 \\
\hline 9 & C & -1.0063 & -0.0289 & 0.516 \\
\hline 10 & 0 & -0.3381 & -0.5829 & -0.6112 \\
\hline 11 & 0 & -0.7398 & 1.3622 & 0.6489 \\
\hline 12 & $\mathrm{C}$ & -1.062 & 2.2059 & -0.4764 \\
\hline 13 & $\mathrm{C}$ & -2.5569 & 2.1038 & -0.7685 \\
\hline 14 & $\mathrm{C}$ & -2.9378 & 0.6366 & -0.9922 \\
\hline 15 & $\mathrm{C}$ & -2.5348 & -0.2389 & 0.2141 \\
\hline 16 & $\mathrm{C}$ & 5.3095 & -0.7642 & 0.0151 \\
\hline 17 & $\mathrm{C}$ & 5.8328 & -0.0315 & 1.2623 \\
\hline 18 & 0 & 5.2668 & -2.1754 & 0.264 \\
\hline 19 & $\mathrm{C}$ & 6.2703 & -0.5689 & -1.1588 \\
\hline 20 & $\mathrm{C}$ & 1.0394 & -2.5555 & -0.3074 \\
\hline 21 & $\mathrm{C}$ & -2.8099 & -1.7231 & -0.065 \\
\hline 22 & $\mathrm{C}$ & -0.5079 & 3.6058 & -0.1128 \\
\hline 23 & $\mathrm{C}$ & -0.5125 & 4.5243 & -1.3356 \\
\hline 24 & $\mathrm{C}$ & -1.2535 & 4.2467 & 1.068 \\
\hline 25 & 0 & 0.8767 & 3.4411 & 0.2349 \\
\hline 26 & $\mathrm{Br}$ & -4.7688 & -2.062 & -0.2393 \\
\hline 27 & 0 & -3.2118 & 0.1764 & 1.3957 \\
\hline 28 & $\mathrm{H}$ & 1.6817 & 0.7792 & 0.4637 \\
\hline 29 & $\mathrm{H}$ & 1.3765 & -1.1406 & -2.6178 \\
\hline 30 & $\mathrm{H}$ & 1.5942 & 0.4609 & -1.9102 \\
\hline 31 & $\mathrm{H}$ & 3.5215 & -1.9282 & -1.6571 \\
\hline 32 & $\mathrm{H}$ & 3.807 & -0.3977 & -2.4803 \\
\hline 33 & $\mathrm{H}$ & 3.863 & 0.8198 & -0.3697 \\
\hline 34 & $\mathrm{H}$ & 1.381 & -1.469 & 2.464 \\
\hline 35 & $\mathrm{H}$ & 1.2577 & 0.2622 & 2.7303 \\
\hline 36 & $\mathrm{H}$ & -0.8501 & -1.6834 & 1.8802 \\
\hline 37 & $\mathrm{H}$ & -1.0695 & -0.1244 & 2.6488 \\
\hline 38 & $\mathrm{H}$ & -0.4925 & 1.8483 & -1.3447 \\
\hline 39 & $\mathrm{H}$ & -3.1268 & 2.499 & 0.078 \\
\hline 40 & $\mathrm{H}$ & -2.8126 & 2.6954 & -1.6554 \\
\hline 41 & $\mathrm{H}$ & -4.0167 & 0.5355 & -1.1532 \\
\hline 42 & $\mathrm{H}$ & -2.4388 & 0.2514 & -1.8895 \\
\hline 43 & $\mathrm{H}$ & 6.8079 & -0.4411 & 1.544 \\
\hline 44 & $\mathrm{H}$ & 5.9481 & 1.0429 & 1.0744 \\
\hline 45 & $\mathrm{H}$ & 5.1436 & -0.156 & 2.1027 \\
\hline 46 & $\mathrm{H}$ & 4.5636 & -2.2972 & 0.925 \\
\hline 47 & $\mathrm{H}$ & 7.2781 & -0.8733 & -0.8588 \\
\hline 48 & $\mathrm{H}$ & 5.9769 & -1.1813 & -2.0156 \\
\hline 49 & $\mathrm{H}$ & 6.3066 & 0.4814 & -1.4691 \\
\hline 50 & $\mathrm{H}$ & 0.484 & -2.8694 & 0.5808 \\
\hline
\end{tabular}




\begin{tabular}{rrrrr}
$\mathbf{5 1}$ & $\mathrm{H}$ & 0.567 & -3.0175 & -1.1811 \\
$\mathbf{5 2}$ & $\mathrm{H}$ & 2.0566 & -2.9449 & -0.2186 \\
$\mathbf{5 3}$ & $\mathrm{H}$ & -2.3663 & -2.0637 & -0.9964 \\
$\mathbf{5 4}$ & $\mathrm{H}$ & -2.5119 & -2.3635 & 0.7623 \\
$\mathbf{5 5}$ & $\mathrm{H}$ & 0.0385 & 4.0669 & -2.1643 \\
$\mathbf{5 6}$ & $\mathrm{H}$ & -1.5312 & 4.7471 & -1.6684 \\
$\mathbf{5 7}$ & $\mathrm{H}$ & -0.0197 & 5.4682 & -1.0822 \\
$\mathbf{5 8}$ & $\mathrm{H}$ & -1.2956 & 3.5576 & 1.918 \\
$\mathbf{5 9}$ & $\mathrm{H}$ & -0.7225 & 5.1525 & 1.3767 \\
$\mathbf{6 0}$ & $\mathrm{H}$ & -2.2805 & 4.5229 & 0.8055 \\
$\mathbf{6 1}$ & $\mathrm{H}$ & 0.8832 & 2.7744 & 0.9418 \\
$\mathbf{6 2}$ & $\mathrm{H}$ & -4.1411 & -0.0967 & 1.2928 \\
\hline
\end{tabular}


Table S64. Conformer 4b-C. PCM- $\mathrm{CHCl}_{3}$ energy and Boltzmann Populations -3806.322286 hartrees, $10.31 \%$.

\begin{tabular}{|c|c|c|c|c|}
\hline \multicolumn{2}{|c|}{ Atom number } & \multicolumn{2}{|c|}{ Coordinates } & \multirow[b]{2}{*}{$\mathbf{Z}$} \\
\hline & & X & $\mathbf{Y}$ & \\
\hline 1 & 0 & 3.1079 & -0.7141 & 0.7949 \\
\hline 2 & $\mathrm{C}$ & 1.7451 & -0.3102 & 0.6819 \\
\hline 3 & C & 1.0693 & -1.0791 & -0.4708 \\
\hline 4 & $\mathrm{C}$ & 1.8231 & -0.7223 & -1.7635 \\
\hline 5 & $\mathrm{C}$ & 3.3364 & -0.9817 & -1.6224 \\
\hline 6 & $\mathrm{C}$ & 3.8905 & -0.3245 & -0.3517 \\
\hline 7 & $\mathrm{C}$ & 1.0297 & -0.5088 & 2.0161 \\
\hline 8 & $\mathrm{C}$ & -0.5063 & -0.6032 & 1.8271 \\
\hline 9 & $\mathrm{C}$ & -0.974 & -0.0365 & 0.4774 \\
\hline 10 & 0 & -0.2964 & -0.612 & -0.635 \\
\hline 11 & 0 & -0.7142 & 1.359 & 0.5724 \\
\hline 12 & $\mathrm{C}$ & -1.0714 & 2.2108 & -0.5397 \\
\hline 13 & $\mathrm{C}$ & -2.5495 & 2.0292 & -0.882 \\
\hline 14 & $\mathrm{C}$ & -2.8948 & 0.5498 & -1.0592 \\
\hline 15 & $\mathrm{C}$ & -2.4974 & -0.2724 & 0.1824 \\
\hline 16 & $\mathrm{C}$ & 5.3619 & -0.6291 & 0.019 \\
\hline 17 & $\mathrm{C}$ & 6.32 & -0.0572 & -1.0279 \\
\hline 18 & 0 & 5.6419 & 0.0832 & 1.2302 \\
\hline 19 & $\mathrm{C}$ & 5.6169 & -2.1291 & 0.2426 \\
\hline 20 & $\mathrm{C}$ & 1.0437 & -2.5985 & -0.2494 \\
\hline 21 & $\mathrm{C}$ & -2.7651 & -1.7682 & -0.0364 \\
\hline 22 & $\mathrm{C}$ & -0.7034 & 3.6415 & -0.0627 \\
\hline 23 & $\mathrm{C}$ & 0.8053 & 3.7521 & 0.2193 \\
\hline 24 & $\mathrm{C}$ & -1.1229 & 4.6983 & -1.0879 \\
\hline 25 & 0 & -1.4359 & 3.9318 & 1.1321 \\
\hline 26 & $\mathrm{Br}$ & -4.7211 & -2.1212 & -0.2023 \\
\hline 27 & 0 & -3.1765 & 0.1873 & 1.3465 \\
\hline 28 & $\mathrm{H}$ & 1.7157 & 0.7551 & 0.4171 \\
\hline 29 & $\mathrm{H}$ & 1.4101 & -1.2855 & -2.609 \\
\hline 30 & $\mathrm{H}$ & 1.6485 & 0.3411 & -1.9711 \\
\hline 31 & $\mathrm{H}$ & 3.5318 & -2.0592 & -1.598 \\
\hline 32 & $\mathrm{H}$ & 3.8585 & -0.5831 & -2.4999 \\
\hline 33 & $\mathrm{H}$ & 3.8103 & 0.771 & -0.453 \\
\hline 34 & $\mathrm{H}$ & 1.404 & -1.4266 & 2.4823 \\
\hline 35 & $\mathrm{H}$ & 1.2898 & 0.3159 & 2.6862 \\
\hline 36 & $\mathrm{H}$ & -0.8246 & -1.6462 & 1.8962 \\
\hline 37 & $\mathrm{H}$ & -1.0377 & -0.0643 & 2.612 \\
\hline 38 & $\mathrm{H}$ & -0.4472 & 1.9416 & -1.4036 \\
\hline 39 & $\mathrm{H}$ & -3.1481 & 2.4624 & -0.0751 \\
\hline 40 & $\mathrm{H}$ & -2.7856 & 2.5721 & -1.8038 \\
\hline 41 & $\mathrm{H}$ & -3.9685 & 0.4245 & -1.2375 \\
\hline 42 & $\mathrm{H}$ & -2.3727 & 0.1399 & -1.9321 \\
\hline 43 & $\mathrm{H}$ & 6.2404 & -0.586 & -1.9832 \\
\hline 44 & $\mathrm{H}$ & 6.1231 & 1.0075 & -1.1934 \\
\hline 45 & $\mathrm{H}$ & 7.348 & -0.1564 & -0.6652 \\
\hline 46 & $\mathrm{H}$ & 4.8856 & -0.0982 & 1.8148 \\
\hline 47 & $\mathrm{H}$ & 5.513 & -2.7113 & -0.6802 \\
\hline 48 & $\mathrm{H}$ & 6.6358 & -2.264 & 0.6185 \\
\hline 49 & $\mathrm{H}$ & 4.9198 & -2.5321 & 0.9842 \\
\hline 50 & $\mathrm{H}$ & 0.4603 & -2.8713 & 0.6338 \\
\hline
\end{tabular}




\begin{tabular}{rrrrr}
$\mathbf{5 1}$ & $\mathrm{H}$ & 0.589 & -3.0881 & -1.1175 \\
$\mathbf{5 2}$ & $\mathrm{H}$ & 2.0522 & -2.9967 & -0.114 \\
$\mathbf{5 3}$ & $\mathrm{H}$ & -2.3178 & -2.1438 & -0.9526 \\
$\mathbf{5 4}$ & $\mathrm{H}$ & -2.467 & -2.3741 & 0.8163 \\
$\mathbf{5 5}$ & $\mathrm{H}$ & 1.4001 & 3.5616 & -0.6829 \\
$\mathbf{5 6}$ & $\mathrm{H}$ & 1.0348 & 4.7606 & 0.5773 \\
$\mathbf{5 7}$ & $\mathrm{H}$ & 1.1109 & 3.0381 & 0.9891 \\
$\mathbf{5 8}$ & $\mathrm{H}$ & -0.6523 & 4.5162 & -2.0609 \\
$\mathbf{5 9}$ & $\mathrm{H}$ & -2.2081 & 4.7158 & -1.2161 \\
$\mathbf{6 0}$ & $\mathrm{H}$ & -0.8122 & 5.6876 & -0.737 \\
$\mathbf{6 1}$ & $\mathrm{H}$ & -1.3484 & 3.1403 & 1.6917 \\
$\mathbf{6 2}$ & $\mathrm{H}$ & -4.1097 & -0.0719 & 1.2459 \\
\hline
\end{tabular}


Table S65. Conformer 4b-D. PCM-CHCl 3 energy and Boltzmann Populations -3806.322782 hartrees, $17.43 \%$.

\begin{tabular}{|c|c|c|c|c|}
\hline \multicolumn{2}{|c|}{ Atom number } & \multicolumn{3}{|c|}{ Coordinates } \\
\hline & & $\mathbf{x}$ & $\mathbf{Y}$ & $\mathbf{Z}$ \\
\hline 1 & $\mathrm{O}$ & 3.0557 & -0.7974 & 0.8012 \\
\hline 2 & C & 1.7002 & -0.3674 & 0.7048 \\
\hline 3 & C & 1.012 & -1.0845 & -0.474 \\
\hline 4 & $\mathrm{C}$ & 1.7672 & -0.6846 & -1.7532 \\
\hline 5 & $\mathrm{C}$ & 3.2772 & -0.97 & -1.626 \\
\hline 6 & $\mathrm{C}$ & 3.8434 & -0.3728 & -0.331 \\
\hline 7 & C & 0.9817 & -0.6066 & 2.0315 \\
\hline 8 & $\mathrm{C}$ & -0.5576 & -0.6604 & 1.8447 \\
\hline 9 & $\mathrm{C}$ & -1.0094 & -0.0373 & 0.5156 \\
\hline 10 & 0 & -0.3518 & -0.6013 & -0.6142 \\
\hline 11 & 0 & -0.7115 & 1.3462 & 0.6541 \\
\hline 12 & $\mathrm{C}$ & -1.0164 & 2.2025 & -0.4669 \\
\hline 13 & $\mathrm{C}$ & -2.5132 & 2.1345 & -0.7582 \\
\hline 14 & C & -2.9257 & 0.6774 & -0.9902 \\
\hline 15 & $\mathrm{C}$ & -2.5418 & -0.213 & 0.2113 \\
\hline 16 & $\mathrm{C}$ & 5.31 & -0.717 & 0.0235 \\
\hline 17 & $\mathrm{C}$ & 6.2753 & -0.1148 & -0.9995 \\
\hline 18 & 0 & 5.6037 & -0.064 & 1.2647 \\
\hline 19 & $\mathrm{C}$ & 5.5418 & -2.2291 & 0.1814 \\
\hline 20 & $\mathrm{C}$ & 0.9687 & -2.6104 & -0.3089 \\
\hline 21 & $\mathrm{C}$ & -2.8485 & -1.6895 & -0.0765 \\
\hline 22 & $\mathrm{C}$ & -0.4316 & 3.5884 & -0.0977 \\
\hline 23 & $\mathrm{C}$ & -0.4268 & 4.515 & -1.3145 \\
\hline 24 & $\mathrm{C}$ & -1.156 & 4.2359 & 1.0926 \\
\hline 25 & 0 & 0.9518 & 3.3942 & 0.2376 \\
\hline 26 & $\mathrm{Br}$ & -4.814 & -1.9862 & -0.2529 \\
\hline 27 & 0 & -3.2104 & 0.2097 & 1.395 \\
\hline 28 & $\mathrm{H}$ & 1.691 & 0.7057 & 0.4725 \\
\hline 29 & $\mathrm{H}$ & 1.3458 & -1.2085 & -2.6196 \\
\hline 30 & $\mathrm{H}$ & 1.6079 & 0.3887 & -1.9161 \\
\hline 31 & $\mathrm{H}$ & 3.4581 & -2.0502 & -1.6466 \\
\hline 32 & $\mathrm{H}$ & 3.8028 & -0.542 & -2.4873 \\
\hline 33 & $\mathrm{H}$ & 3.7795 & 0.7265 & -0.3869 \\
\hline 34 & $\mathrm{H}$ & 1.3381 & -1.5513 & 2.456 \\
\hline 35 & $\mathrm{H}$ & 1.261 & 0.181 & 2.7381 \\
\hline 36 & $\mathrm{H}$ & -0.8992 & -1.6979 & 1.8789 \\
\hline 37 & $\mathrm{H}$ & -1.0747 & -0.134 & 2.6475 \\
\hline 38 & $\mathrm{H}$ & -0.4554 & 1.8371 & -1.3375 \\
\hline 39 & $\mathrm{H}$ & -3.0739 & 2.5371 & 0.091 \\
\hline 40 & $\mathrm{H}$ & -2.7562 & 2.737 & -1.6412 \\
\hline 41 & $\mathrm{H}$ & -4.0064 & 0.6001 & -1.1517 \\
\hline 42 & $\mathrm{H}$ & -2.4347 & 0.2866 & -1.8895 \\
\hline 43 & $\mathrm{H}$ & 7.3023 & -0.245 & -0.6435 \\
\hline 44 & $\mathrm{H}$ & 6.1865 & -0.6005 & -1.9767 \\
\hline 45 & $\mathrm{H}$ & 6.0937 & 0.9587 & -1.1184 \\
\hline 46 & $\mathrm{H}$ & 4.8428 & -0.2546 & 1.8403 \\
\hline 47 & $\mathrm{H}$ & 6.5593 & -2.3959 & 0.5481 \\
\hline 48 & $\mathrm{H}$ & 4.8403 & -2.6526 & 0.9074 \\
\hline 49 & $\mathrm{H}$ & 5.4265 & -2.7698 & -0.765 \\
\hline 50 & $\mathrm{H}$ & 0.3871 & -2.9077 & 0.568 \\
\hline
\end{tabular}




\begin{tabular}{rrrrr}
$\mathbf{5 1}$ & $\mathrm{H}$ & 0.5012 & -3.061 & -1.1911 \\
$\mathbf{5 2}$ & $\mathrm{H}$ & 1.9725 & -3.0268 & -0.1956 \\
$\mathbf{5 3}$ & $\mathrm{H}$ & -2.4124 & -2.0341 & -1.0099 \\
$\mathbf{5 4}$ & $\mathrm{H}$ & -2.5646 & -2.3411 & 0.747 \\
$\mathbf{5 5}$ & $\mathrm{H}$ & 0.1081 & 4.0519 & -2.1506 \\
$\mathbf{5 6}$ & $\mathrm{H}$ & -1.4431 & 4.7616 & -1.6377 \\
$\mathbf{5 7}$ & $\mathrm{H}$ & 0.088 & 5.4464 & -1.0587 \\
$\mathbf{5 8}$ & $\mathrm{H}$ & -1.2042 & 3.5427 & 1.9389 \\
$\mathbf{5 9}$ & $\mathrm{H}$ & -0.6061 & 5.13 & 1.4022 \\
$\mathbf{6 0}$ & $\mathrm{H}$ & -2.1798 & 4.5323 & 0.8399 \\
$\mathbf{6 1}$ & $\mathrm{H}$ & 0.9519 & 2.7335 & 0.9499 \\
$\mathbf{6 2}$ & $\mathrm{H}$ & -4.1454 & -0.0419 & 1.29 \\
\hline
\end{tabular}


Table S66. Conformer 4b-E. PCM-CHCl 3 energy and Boltzmann Populations -3806.322446 hartrees, $12.20 \%$.

\begin{tabular}{|c|c|c|c|c|}
\hline \multicolumn{2}{|c|}{ Atom number } & \multicolumn{3}{|c|}{ Coordinates } \\
\hline & & $\mathbf{x}$ & $\mathbf{Y}$ & Z \\
\hline 1 & 0 & -2.7713 & -0.6508 & -0.7653 \\
\hline 2 & $\mathrm{C}$ & -1.4228 & -0.2283 & -0.5905 \\
\hline 3 & $\mathrm{C}$ & -0.8659 & -0.7724 & 0.7393 \\
\hline 4 & $\mathrm{C}$ & -1.7326 & -0.1794 & 1.861 \\
\hline 5 & $\mathrm{C}$ & -3.2271 & -0.4732 & 1.6299 \\
\hline 6 & $\mathrm{C}$ & -3.6735 & -0.0935 & 0.2106 \\
\hline 7 & $\mathrm{C}$ & -0.5906 & -0.6642 & -1.7946 \\
\hline 8 & $\mathrm{C}$ & 0.9204 & -0.7305 & -1.4523 \\
\hline 9 & $\mathrm{C}$ & 1.2799 & 0.0667 & -0.1906 \\
\hline 10 & $\mathrm{O}$ & 0.4857 & -0.2767 & 0.9386 \\
\hline 11 & $\mathrm{O}$ & 1.0641 & 1.4238 & -0.5777 \\
\hline 12 & $\mathrm{C}$ & 1.2771 & 2.4364 & 0.4235 \\
\hline 13 & C & 2.7352 & 2.3895 & 0.8678 \\
\hline 14 & $\mathrm{C}$ & 3.0735 & 0.9786 & 1.3641 \\
\hline 15 & $\mathrm{C}$ & 2.7718 & -0.1007 & 0.2967 \\
\hline 16 & $\mathrm{C}$ & -5.0717 & -0.6322 & -0.1913 \\
\hline 17 & $\mathrm{C}$ & -5.4684 & -0.0857 & -1.5736 \\
\hline 18 & 0 & -5.011 & -2.0636 & -0.2348 \\
\hline 19 & $\mathrm{C}$ & -6.141 & -0.2835 & 0.8452 \\
\hline 20 & $\mathrm{C}$ & -0.8418 & -2.3067 & 0.8072 \\
\hline 21 & $\mathrm{C}$ & 3.0535 & -1.4471 & 0.9853 \\
\hline 22 & $\mathrm{C}$ & 0.7729 & 3.7578 & -0.2084 \\
\hline 23 & $\mathrm{C}$ & 0.6639 & 4.8573 & 0.849 \\
\hline 24 & $\mathrm{C}$ & 1.6366 & 4.2109 & -1.3957 \\
\hline 25 & 0 & -0.5709 & 3.5276 & -0.6612 \\
\hline 26 & $\mathrm{Br}$ & 3.3339 & -2.9743 & -0.2689 \\
\hline 27 & 0 & 3.6696 & 0.1338 & -0.7767 \\
\hline 28 & $\mathrm{H}$ & -1.4086 & 0.8675 & -0.5133 \\
\hline 29 & $\mathrm{H}$ & -1.4096 & -0.5682 & 2.8344 \\
\hline 30 & $\mathrm{H}$ & -1.5657 & 0.9053 & 1.8766 \\
\hline 31 & $\mathrm{H}$ & -3.4449 & -1.5349 & 1.7882 \\
\hline 32 & $\mathrm{H}$ & -3.8197 & 0.0882 & 2.3611 \\
\hline 33 & $\mathrm{H}$ & -3.6631 & 1.0042 & 0.1045 \\
\hline 34 & $\mathrm{H}$ & -0.937 & -1.6518 & -2.1178 \\
\hline 35 & $\mathrm{H}$ & -0.7764 & 0.0215 & -2.6269 \\
\hline 36 & $\mathrm{H}$ & 1.23 & -1.7659 & -1.3131 \\
\hline 37 & $\mathrm{H}$ & 1.5206 & -0.3257 & -2.2703 \\
\hline 38 & $\mathrm{H}$ & 0.6235 & 2.2172 & 1.2787 \\
\hline 39 & $\mathrm{H}$ & 3.3899 & 2.6412 & 0.0281 \\
\hline 40 & $\mathrm{H}$ & 2.9174 & 3.1143 & 1.6702 \\
\hline 41 & $\mathrm{H}$ & 4.1379 & 0.9097 & 1.6142 \\
\hline 42 & $\mathrm{H}$ & 2.4966 & 0.7536 & 2.2687 \\
\hline 43 & $\mathrm{H}$ & -4.6988 & -0.315 & -2.3165 \\
\hline 44 & $\mathrm{H}$ & -6.4104 & -0.544 & -1.8905 \\
\hline 45 & $\mathrm{H}$ & -5.6019 & 1.0026 & -1.5485 \\
\hline 46 & $\mathrm{H}$ & -4.2478 & -2.27 & -0.8015 \\
\hline 47 & $\mathrm{H}$ & -6.1983 & 0.7991 & 1.0051 \\
\hline 48 & $\mathrm{H}$ & -7.1174 & -0.6302 & 0.4922 \\
\hline 49 & $\mathrm{H}$ & -5.9389 & -0.7725 & 1.8019 \\
\hline 50 & $\mathrm{H}$ & -0.121 & -2.736 & 0.1062 \\
\hline
\end{tabular}




\begin{tabular}{rrrrr}
$\mathbf{5 1}$ & $\mathrm{H}$ & -0.5535 & -2.6252 & 1.8153 \\
$\mathbf{5 2}$ & $\mathrm{H}$ & -1.8257 & -2.7277 & 0.5842 \\
$\mathbf{5 3}$ & $\mathrm{H}$ & 3.9955 & -1.4031 & 1.5301 \\
$\mathbf{5 4}$ & $\mathrm{H}$ & 2.2386 & -1.7476 & 1.6377 \\
$\mathbf{5 5}$ & $\mathrm{H}$ & 0.0299 & 4.5313 & 1.6805 \\
$\mathbf{5 6}$ & $\mathrm{H}$ & 1.6462 & 5.1377 & 1.2422 \\
$\mathbf{5 7}$ & $\mathrm{H}$ & 0.2061 & 5.746 & 0.4031 \\
$\mathbf{5 8}$ & $\mathrm{H}$ & 2.6362 & 4.5292 & -1.08 \\
$\mathbf{5 9}$ & $\mathrm{H}$ & 1.7535 & 3.3985 & -2.1205 \\
$\mathbf{6 0}$ & $\mathrm{H}$ & 1.1482 & 5.0551 & -1.8922 \\
$\mathbf{6 1}$ & $\mathrm{H}$ & -0.5124 & 2.7463 & -1.2363 \\
$\mathbf{6 2}$ & $\mathrm{H}$ & 3.7675 & -0.7032 & -1.2645 \\
\hline
\end{tabular}


Table S67. Conformer 4b-F. PCM-CHCl 3 energy and Boltzmann Populations -3806.321970 hartrees, $7.37 \%$.

\begin{tabular}{|c|c|c|c|c|}
\hline \multicolumn{2}{|c|}{ Atom number } & \multicolumn{3}{|c|}{ Coordinates } \\
\hline & & $\mathbf{x}$ & $\mathbf{Y}$ & $\mathbf{Z}$ \\
\hline 1 & 0 & -2.7474 & -0.7793 & -0.7497 \\
\hline 2 & $\mathrm{C}$ & -1.4118 & -0.3089 & -0.5882 \\
\hline 3 & $\mathrm{C}$ & -0.8331 & -0.8285 & 0.7437 \\
\hline 4 & $\mathrm{C}$ & -1.7171 & -0.267 & 1.8706 \\
\hline 5 & $\mathrm{C}$ & -3.2019 & -0.6157 & 1.643 \\
\hline 6 & $\mathrm{C}$ & -3.6465 & -0.2194 & 0.2294 \\
\hline 7 & $\mathrm{C}$ & -0.5682 & -0.7222 & -1.7915 \\
\hline 8 & $\mathrm{C}$ & 0.9454 & -0.7291 & -1.4527 \\
\hline 9 & $\mathrm{C}$ & 1.28 & 0.0821 & -0.1925 \\
\hline 10 & $\mathrm{O}$ & 0.4989 & -0.2834 & 0.9399 \\
\hline 11 & $\mathrm{O}$ & 1.0192 & 1.4303 & -0.581 \\
\hline 12 & $\mathrm{C}$ & 1.2013 & 2.45 & 0.4195 \\
\hline 13 & $\mathrm{C}$ & 2.6616 & 2.4515 & 0.8594 \\
\hline 14 & $\mathrm{C}$ & 3.0475 & 1.0534 & 1.3566 \\
\hline 15 & $\mathrm{C}$ & 2.7774 & -0.0374 & 0.2926 \\
\hline 16 & $\mathrm{C}$ & -5.0673 & -0.646 & -0.2115 \\
\hline 17 & $\mathrm{C}$ & -6.1353 & 0.0795 & 0.6092 \\
\hline 18 & 0 & -5.2394 & -0.1917 & -1.5595 \\
\hline 19 & $\mathrm{C}$ & -5.2699 & -2.1697 & -0.1606 \\
\hline 20 & $\mathrm{C}$ & -0.7501 & -2.3611 & 0.8115 \\
\hline 21 & $\mathrm{C}$ & 3.1031 & -1.3721 & 0.9849 \\
\hline 22 & $\mathrm{C}$ & 0.6515 & 3.7543 & -0.2088 \\
\hline 23 & $\mathrm{C}$ & 0.5188 & 4.8508 & 0.849 \\
\hline 24 & $\mathrm{C}$ & 1.4903 & 4.2328 & -1.4039 \\
\hline 25 & 0 & -0.6889 & 3.4838 & -0.6481 \\
\hline 26 & $\mathrm{Br}$ & 3.4289 & -2.8945 & -0.2637 \\
\hline 27 & 0 & 3.6654 & 0.222 & -0.7832 \\
\hline 28 & $\mathrm{H}$ & -1.4337 & 0.7871 & -0.516 \\
\hline 29 & $\mathrm{H}$ & -1.3747 & -0.6443 & 2.8418 \\
\hline 30 & $\mathrm{H}$ & -1.593 & 0.8232 & 1.8858 \\
\hline 31 & $\mathrm{H}$ & -3.3616 & -1.6886 & 1.7963 \\
\hline 32 & $\mathrm{H}$ & -3.8175 & -0.0914 & 2.3831 \\
\hline 33 & $\mathrm{H}$ & -3.5996 & 0.8781 & 0.1351 \\
\hline 34 & $\mathrm{H}$ & -0.8782 & -1.7242 & -2.107 \\
\hline 35 & $\mathrm{H}$ & -0.7805 & -0.0486 & -2.6275 \\
\hline 36 & $\mathrm{H}$ & 1.2948 & -1.7521 & -1.3158 \\
\hline 37 & $\mathrm{H}$ & 1.5273 & -0.3012 & -2.2721 \\
\hline 38 & $\mathrm{H}$ & 0.5579 & 2.2091 & 1.2765 \\
\hline 39 & $\mathrm{H}$ & 3.305 & 2.7238 & 0.0174 \\
\hline 40 & $\mathrm{H}$ & 2.8224 & 3.1828 & 1.6605 \\
\hline 41 & $\mathrm{H}$ & 4.1145 & 1.0196 & 1.603 \\
\hline 42 & $\mathrm{H}$ & 2.4817 & 0.8116 & 2.264 \\
\hline 43 & $\mathrm{H}$ & -5.9725 & 1.1624 & 0.5869 \\
\hline 44 & $\mathrm{H}$ & -7.1197 & -0.1216 & 0.1745 \\
\hline 45 & $\mathrm{H}$ & -6.1448 & -0.2572 & 1.6508 \\
\hline 46 & $\mathrm{H}$ & -4.4202 & -0.4432 & -2.0205 \\
\hline 47 & $\mathrm{H}$ & -5.2525 & -2.5572 & 0.8644 \\
\hline 48 & $\mathrm{H}$ & -6.241 & -2.4136 & -0.6021 \\
\hline 49 & $\mathrm{H}$ & -4.4915 & -2.6815 & -0.7355 \\
\hline 50 & $\mathrm{H}$ & 0.0041 & -2.7576 & 0.1269 \\
\hline
\end{tabular}




\begin{tabular}{rrrrr}
$\mathbf{5 1}$ & $\mathrm{H}$ & -0.472 & -2.6712 & 1.8252 \\
$\mathbf{5 2}$ & $\mathrm{H}$ & -1.7095 & -2.8207 & 0.5609 \\
$\mathbf{5 3}$ & $\mathrm{H}$ & 4.0446 & -1.2959 & 1.5269 \\
$\mathbf{5 4}$ & $\mathrm{H}$ & 2.3001 & -1.6957 & 1.6408 \\
$\mathbf{5 5}$ & $\mathrm{H}$ & -0.0949 & 4.5055 & 1.688 \\
$\mathbf{5 6}$ & $\mathrm{H}$ & 1.4958 & 5.1635 & 1.2307 \\
$\mathbf{5 7}$ & $\mathrm{H}$ & 0.027 & 5.7233 & 0.4076 \\
$\mathbf{5 8}$ & $\mathrm{H}$ & 2.4835 & 4.5789 & -1.0975 \\
$\mathbf{5 9}$ & $\mathrm{H}$ & 1.6234 & 3.4249 & -2.131 \\
$\mathbf{6 0}$ & $\mathrm{H}$ & 0.9735 & 5.0633 & -1.8948 \\
$\mathbf{6 1}$ & $\mathrm{H}$ & -0.6134 & 2.7127 & -1.2346 \\
$\mathbf{6 2}$ & $\mathrm{H}$ & 3.7895 & -0.6134 & -1.2681 \\
\hline
\end{tabular}




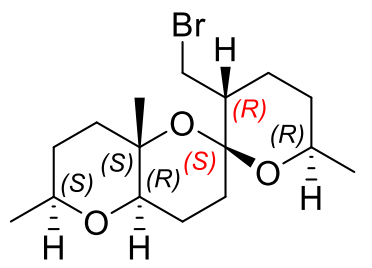

Table S68. Conformer 4c-A. PCM- $\mathrm{CHCl}_{3}$ energy and Boltzmann Populations 3806.321079 hartrees, $36.14 \%$.

\begin{tabular}{|c|c|c|c|c|}
\hline \multicolumn{2}{|c|}{ Atom number } & \multicolumn{3}{|c|}{ Coordinates } \\
\hline & & $X$ & $\mathbf{Y}$ & $\mathbf{Z}$ \\
\hline 1 & $\mathrm{O}$ & -3.5165 & 0.2796 & 0.6737 \\
\hline 2 & $\mathrm{C}$ & -2.161 & -0.1067 & 0.8477 \\
\hline 3 & $\mathrm{C}$ & -1.482 & -0.2813 & -0.5225 \\
\hline 4 & C & -2.199 & -1.447 & -1.2173 \\
\hline 5 & C & -3.7183 & -1.1937 & -1.2863 \\
\hline 6 & C & -4.3144 & -0.7556 & 0.0629 \\
\hline 7 & C & -1.3997 & 0.8693 & 1.7263 \\
\hline 8 & C & 0.0201 & 0.3118 & 1.9212 \\
\hline 9 & C & 0.7338 & -0.0249 & 0.5929 \\
\hline 10 & $\mathrm{O}$ & -0.1146 & -0.7186 & -0.3028 \\
\hline 11 & 0 & 1.1919 & 1.1652 & -0.0704 \\
\hline 12 & C & 2.2498 & 1.9279 & 0.5573 \\
\hline 13 & C & 3.4456 & 1.0394 & 0.928 \\
\hline 14 & C & 3.022 & -0.2671 & 1.6101 \\
\hline 15 & C & 1.954 & -0.9956 & 0.7908 \\
\hline 16 & C & -5.7388 & -0.1483 & -0.0384 \\
\hline 17 & C & -6.2911 & 0.1408 & 1.368 \\
\hline 18 & 0 & -5.6578 & 1.0705 & -0.7878 \\
\hline 19 & C & -6.6975 & -1.0577 & -0.8087 \\
\hline 20 & C & -1.5177 & 0.981 & -1.3931 \\
\hline 21 & C & 2.458 & -1.4168 & -0.604 \\
\hline 22 & C & 2.592 & 3.0376 & -0.4773 \\
\hline 23 & C & 1.4026 & 3.9957 & -0.6588 \\
\hline 24 & C & 3.8378 & 3.8261 & -0.0666 \\
\hline 25 & 0 & 2.9081 & 2.4138 & -1.7265 \\
\hline 26 & $\mathrm{Br}$ & 4.0854 & -2.5375 & -0.52 \\
\hline 27 & 0 & 1.5554 & -2.1373 & 1.5353 \\
\hline 28 & $\mathrm{H}$ & -2.1389 & -1.0925 & 1.346 \\
\hline 29 & $\mathrm{H}$ & -1.792 & -1.6041 & -2.2233 \\
\hline 30 & $\mathrm{H}$ & -1.9953 & -2.3602 & -0.6423 \\
\hline 31 & $\mathrm{H}$ & -3.9451 & -0.4231 & -2.0298 \\
\hline 32 & $\mathrm{H}$ & -4.2187 & -2.1108 & -1.6169 \\
\hline 33 & $\mathrm{H}$ & -4.3368 & -1.6182 & 0.7506 \\
\hline 34 & $\mathrm{H}$ & -1.3796 & 1.8661 & 1.2726 \\
\hline 35 & $\mathrm{H}$ & -1.8995 & 0.9657 & 2.6968 \\
\hline 36 & $\mathrm{H}$ & 0.6218 & 1.0167 & 2.5005 \\
\hline 37 & $\mathrm{H}$ & -0.0255 & -0.6124 & 2.5057 \\
\hline 38 & $\mathrm{H}$ & 1.8534 & 2.4091 & 1.4641 \\
\hline 39 & $\mathrm{H}$ & 4.0192 & 0.8175 & 0.0218 \\
\hline 40 & $\mathrm{H}$ & 4.1104 & 1.5984 & 1.5957 \\
\hline 41 & $\mathrm{H}$ & 3.8818 & -0.9306 & 1.7356 \\
\hline 42 & $\mathrm{H}$ & 2.6197 & -0.0777 & 2.6113 \\
\hline 43 & $\mathrm{H}$ & -5.6063 & 0.783 & 1.9295 \\
\hline
\end{tabular}




\begin{tabular}{llrrr}
$\mathbf{4 4}$ & $\mathrm{H}$ & -7.2577 & 0.6471 & 1.2833 \\
$\mathbf{4 5}$ & $\mathrm{H}$ & -6.4329 & -0.7848 & 1.9391 \\
$\mathbf{4 6}$ & $\mathrm{H}$ & -4.9671 & 1.5971 & -0.35 \\
$\mathbf{4 7}$ & $\mathrm{H}$ & -7.6965 & -0.6105 & -0.8146 \\
$\mathbf{4 8}$ & $\mathrm{H}$ & -6.3798 & -1.179 & -1.8476 \\
$\mathbf{4 9}$ & $\mathrm{H}$ & -6.7666 & -2.0462 & -0.3409 \\
$\mathbf{5 0}$ & $\mathrm{H}$ & -1.0183 & 1.8206 & -0.9108 \\
$\mathbf{5 1}$ & $\mathrm{H}$ & -1.0019 & 0.7811 & -2.3379 \\
$\mathbf{5 2}$ & $\mathrm{H}$ & -2.5474 & 1.2721 & -1.6135 \\
$\mathbf{5 3}$ & $\mathrm{H}$ & 1.7171 & -2.0259 & -1.1156 \\
$\mathbf{5 4}$ & $\mathrm{H}$ & 2.7165 & -0.5651 & -1.228 \\
$\mathbf{5 5}$ & $\mathrm{H}$ & 0.5073 & 3.4566 & -0.9813 \\
$\mathbf{5 6}$ & $\mathrm{H}$ & 1.1667 & 4.5215 & 0.2743 \\
$\mathbf{5 7}$ & $\mathrm{H}$ & 1.6483 & 4.7401 & -1.4225 \\
$\mathbf{5 8}$ & $\mathrm{H}$ & 4.01 & 4.6323 & -0.7864 \\
$\mathbf{5 9}$ & $\mathrm{H}$ & 3.7126 & 4.2731 & 0.9261 \\
$\mathbf{6 0}$ & $\mathrm{H}$ & 4.7259 & 3.1894 & -0.0591 \\
$\mathbf{6 1}$ & $\mathrm{H}$ & 2.1512 & 1.8352 & -1.9233 \\
$\mathbf{6 2}$ & $\mathrm{H}$ & 0.8448 & -2.5643 & 1.027 \\
\hline
\end{tabular}


Table S69. Conformer 4c-B. PCM-CHCl ${ }_{3}$ energy and Boltzmann Populations -3806.320139 hartrees, $13.36 \%$.

\begin{tabular}{|c|c|c|c|c|}
\hline \multicolumn{2}{|c|}{ Atom number } & \multicolumn{3}{|c|}{ Coordinates } \\
\hline & & $x$ & $\mathbf{Y}$ & $\mathbf{Z}$ \\
\hline 1 & $\mathrm{O}$ & -3.5071 & 0.2868 & 0.6556 \\
\hline 2 & $\mathrm{C}$ & -2.154 & -0.1023 & 0.8405 \\
\hline 3 & $\mathrm{C}$ & -1.4736 & -0.3125 & -0.5238 \\
\hline 4 & $\mathrm{C}$ & -2.1986 & -1.4849 & -1.1991 \\
\hline 5 & $\mathrm{C}$ & -3.7173 & -1.2286 & -1.2695 \\
\hline 6 & $\mathrm{C}$ & -4.3085 & -0.761 & 0.0719 \\
\hline 7 & $\mathrm{C}$ & -1.3883 & 0.8893 & 1.6977 \\
\hline 8 & C & 0.0314 & 0.3346 & 1.901 \\
\hline 9 & $\mathrm{C}$ & 0.7419 & -0.0413 & 0.5806 \\
\hline 10 & 0 & -0.1128 & -0.7611 & -0.288 \\
\hline 11 & 0 & 1.2094 & 1.1162 & -0.1303 \\
\hline 12 & $\mathrm{C}$ & 2.2501 & 1.9001 & 0.4965 \\
\hline 13 & $\mathrm{C}$ & 3.464 & 1.0304 & 0.8477 \\
\hline 14 & $\mathrm{C}$ & 3.0448 & -0.2493 & 1.584 \\
\hline 15 & $\mathrm{C}$ & 1.9631 & -1.0076 & 0.8067 \\
\hline 16 & $\mathrm{C}$ & -5.7336 & -0.1568 & -0.0367 \\
\hline 17 & $\mathrm{C}$ & -6.2781 & 0.1668 & 1.3651 \\
\hline 18 & 0 & -5.6581 & 1.0424 & -0.8173 \\
\hline 19 & $\mathrm{C}$ & -6.6956 & -1.0862 & -0.7786 \\
\hline 20 & $\mathrm{C}$ & -1.4958 & 0.9325 & -1.4204 \\
\hline 21 & $\mathrm{C}$ & 2.4379 & -1.4899 & -0.5783 \\
\hline 22 & $\mathrm{C}$ & 2.5127 & 3.0897 & -0.4633 \\
\hline 23 & $\mathrm{C}$ & 3.4518 & 4.1069 & 0.1884 \\
\hline 24 & $\mathrm{C}$ & 3.0455 & 2.6449 & -1.8346 \\
\hline 25 & 0 & 1.2697 & 3.7814 & -0.6399 \\
\hline 26 & $\mathrm{Br}$ & 4.1263 & -2.5199 & -0.5063 \\
\hline 27 & 0 & 1.5778 & -2.1176 & 1.6046 \\
\hline 28 & $\mathrm{H}$ & -2.1374 & -1.077 & 1.3607 \\
\hline 29 & $\mathrm{H}$ & -1.7939 & -1.6602 & -2.2031 \\
\hline 30 & $\mathrm{H}$ & -1.9967 & -2.3891 & -0.6096 \\
\hline 31 & $\mathrm{H}$ & -3.9452 & -0.4729 & -2.0279 \\
\hline 32 & $\mathrm{H}$ & -4.2207 & -2.151 & -1.58 \\
\hline 33 & $\mathrm{H}$ & -4.328 & -1.6083 & 0.7785 \\
\hline 34 & $\mathrm{H}$ & -1.3677 & 1.8788 & 1.2285 \\
\hline 35 & $\mathrm{H}$ & -1.8851 & 1.0044 & 2.6676 \\
\hline 36 & $\mathrm{H}$ & 0.6332 & 1.0522 & 2.4632 \\
\hline 37 & $\mathrm{H}$ & -0.0142 & -0.5746 & 2.5084 \\
\hline 38 & $\mathrm{H}$ & 1.8457 & 2.3431 & 1.4178 \\
\hline 39 & $\mathrm{H}$ & 4.0163 & 0.7576 & -0.0585 \\
\hline 40 & $\mathrm{H}$ & 4.1516 & 1.6084 & 1.4751 \\
\hline 41 & $\mathrm{H}$ & 3.9027 & -0.9116 & 1.7268 \\
\hline 42 & $\mathrm{H}$ & 2.6535 & -0.0176 & 2.5806 \\
\hline 43 & $\mathrm{H}$ & -5.5918 & 0.8249 & 1.9059 \\
\hline 44 & $\mathrm{H}$ & -7.2466 & 0.6683 & 1.2733 \\
\hline 45 & $\mathrm{H}$ & -6.4138 & -0.7443 & 1.9604 \\
\hline 46 & $\mathrm{H}$ & -4.9676 & 1.5826 & -0.396 \\
\hline 47 & $\mathrm{H}$ & -6.7618 & -2.0625 & -0.2852 \\
\hline 48 & $\mathrm{H}$ & -7.6949 & -0.6397 & -0.7906 \\
\hline 49 & $\mathrm{H}$ & -6.3834 & -1.234 & -1.8157 \\
\hline 50 & $\mathrm{H}$ & -2.5185 & 1.1871 & -1.7078 \\
\hline
\end{tabular}




\begin{tabular}{rrrrr}
$\mathbf{5 1}$ & $\mathrm{H}$ & -1.0613 & 1.7996 & -0.9223 \\
$\mathbf{5 2}$ & $\mathrm{H}$ & -0.9148 & 0.7339 & -2.3262 \\
$\mathbf{5 3}$ & $\mathrm{H}$ & 1.7113 & -2.1671 & -1.0201 \\
$\mathbf{5 4}$ & $\mathrm{H}$ & 2.6197 & -0.6669 & -1.2642 \\
$\mathbf{5 5}$ & $\mathrm{H}$ & 4.4632 & 3.7063 & 0.3067 \\
$\mathbf{5 6}$ & $\mathrm{H}$ & 3.5063 & 4.9994 & -0.4426 \\
$\mathbf{5 7}$ & $\mathrm{H}$ & 3.0737 & 4.4118 & 1.1703 \\
$\mathbf{5 8}$ & $\mathrm{H}$ & 3.1106 & 3.5182 & -2.491 \\
$\mathbf{5 9}$ & $\mathrm{H}$ & 4.0409 & 2.1926 & -1.7685 \\
$\mathbf{6 0}$ & $\mathrm{H}$ & 2.3691 & 1.9175 & -2.2957 \\
$\mathbf{6 1}$ & $\mathrm{H}$ & 0.6424 & 3.1136 & -0.9624 \\
$\mathbf{6 2}$ & $\mathrm{H}$ & 0.8521 & -2.5595 & 1.1314 \\
\hline
\end{tabular}


Table S70. Conformer 4c-C. PCM- $\mathrm{CHCl}_{3}$ energy and Boltzmann Populations -3806.320567 hartrees, $21.02 \%$.

\begin{tabular}{|c|c|c|c|c|}
\hline \multicolumn{2}{|c|}{ Atom number } & \multicolumn{3}{|c|}{ Coordinates } \\
\hline & & $x$ & $\mathbf{Y}$ & $\mathbf{Z}$ \\
\hline 1 & 0 & -3.5195 & 0.3565 & 0.6154 \\
\hline 2 & $\mathrm{C}$ & -2.1681 & -0.0428 & 0.7992 \\
\hline 3 & $\mathrm{C}$ & -1.4771 & -0.199 & -0.5685 \\
\hline 4 & $\mathrm{C}$ & -2.2067 & -1.331 & -1.309 \\
\hline 5 & $\mathrm{C}$ & -3.7227 & -1.0507 & -1.3847 \\
\hline 6 & $\mathrm{C}$ & -4.3022 & -0.6826 & -0.0106 \\
\hline 7 & $\mathrm{C}$ & -1.4034 & 0.9091 & 1.7004 \\
\hline 8 & $\mathrm{C}$ & 0.0074 & 0.3295 & 1.9 \\
\hline 9 & $\mathrm{C}$ & 0.7293 & -0.0106 & 0.5765 \\
\hline 10 & 0 & -0.1235 & -0.6725 & -0.3397 \\
\hline 11 & 0 & 1.2264 & 1.1743 & -0.0682 \\
\hline 12 & $\mathrm{C}$ & 2.2967 & 1.9029 & 0.5785 \\
\hline 13 & $\mathrm{C}$ & 3.4646 & 0.9805 & 0.9521 \\
\hline 14 & $\mathrm{C}$ & 2.999 & -0.3199 & 1.6185 \\
\hline 15 & $\mathrm{C}$ & 1.9225 & -1.014 & 0.7803 \\
\hline 16 & $\mathrm{C}$ & -5.767 & -0.1839 & 0.0258 \\
\hline 17 & C & -6.7329 & -1.286 & -0.4126 \\
\hline 18 & 0 & -6.0816 & 0.0821 & 1.3983 \\
\hline 19 & $\mathrm{C}$ & -5.9756 & 1.0972 & -0.7992 \\
\hline 20 & $\mathrm{C}$ & -1.4699 & 1.0864 & -1.4053 \\
\hline 21 & $\mathrm{C}$ & 2.4325 & -1.4358 & -0.6122 \\
\hline 22 & $\mathrm{C}$ & 2.6768 & 3.0141 & -0.4415 \\
\hline 23 & $\mathrm{C}$ & 1.5159 & 4.0069 & -0.6203 \\
\hline 24 & $\mathrm{C}$ & 3.9411 & 3.7635 & -0.0143 \\
\hline 25 & 0 & 2.9843 & 2.3951 & -1.6951 \\
\hline 26 & $\mathrm{Br}$ & 4.0339 & -2.5925 & -0.5198 \\
\hline 27 & 0 & 1.4874 & -2.1516 & 1.5103 \\
\hline 28 & $\mathrm{H}$ & -2.1585 & -1.0383 & 1.2785 \\
\hline 29 & $\mathrm{H}$ & -1.789 & -1.4624 & -2.3141 \\
\hline 30 & $\mathrm{H}$ & -2.0279 & -2.2656 & -0.7612 \\
\hline 31 & $\mathrm{H}$ & -3.916 & -0.2424 & -2.0975 \\
\hline 32 & $\mathrm{H}$ & -4.2371 & -1.9395 & -1.7674 \\
\hline 33 & $\mathrm{H}$ & -4.2547 & -1.5688 & 0.6445 \\
\hline 34 & $\mathrm{H}$ & -1.368 & 1.9133 & 1.2648 \\
\hline 35 & $\mathrm{H}$ & -1.9095 & 0.9945 & 2.6688 \\
\hline 36 & $\mathrm{H}$ & 0.6147 & 1.0223 & 2.488 \\
\hline 37 & $\mathrm{H}$ & -0.0566 & -0.5971 & 2.4787 \\
\hline 38 & $\mathrm{H}$ & 1.9034 & 2.3844 & 1.4865 \\
\hline 39 & $\mathrm{H}$ & 4.0413 & 0.7507 & 0.0498 \\
\hline 40 & $\mathrm{H}$ & 4.1373 & 1.5159 & 1.6309 \\
\hline 41 & $\mathrm{H}$ & 3.8396 & -1.0068 & 1.7483 \\
\hline 42 & $\mathrm{H}$ & 2.5899 & -0.1284 & 2.6165 \\
\hline 43 & $\mathrm{H}$ & -6.5678 & -2.2007 & 0.1671 \\
\hline 44 & $\mathrm{H}$ & -7.7603 & -0.9549 & -0.2317 \\
\hline 45 & $\mathrm{H}$ & -6.629 & -1.5166 & -1.4777 \\
\hline 46 & $\mathrm{H}$ & -5.3336 & 0.6033 & 1.7376 \\
\hline 47 & $\mathrm{H}$ & -6.9922 & 1.4655 & -0.631 \\
\hline 48 & $\mathrm{H}$ & -5.2707 & 1.8748 & -0.4882 \\
\hline 49 & $\mathrm{H}$ & -5.8473 & 0.9267 & -1.8742 \\
\hline 50 & $\mathrm{H}$ & -0.9484 & 1.897 & -0.8981 \\
\hline
\end{tabular}




\begin{tabular}{rrrrr}
$\mathbf{5 1}$ & $\mathrm{H}$ & -0.9549 & 0.8968 & -2.3528 \\
$\mathbf{5 2}$ & $\mathrm{H}$ & -2.4889 & 1.4166 & -1.6197 \\
$\mathbf{5 3}$ & $\mathrm{H}$ & 1.684 & -2.0247 & -1.1366 \\
$\mathbf{5 4}$ & $\mathrm{H}$ & 2.7162 & -0.5851 & -1.2265 \\
$\mathbf{5 5}$ & $\mathrm{H}$ & 0.6083 & 3.497 & -0.9555 \\
$\mathbf{5 6}$ & $\mathrm{H}$ & 1.2879 & 4.5284 & 0.3172 \\
$\mathbf{5 7}$ & $\mathrm{H}$ & 1.7879 & 4.7529 & -1.3735 \\
$\mathbf{5 8}$ & $\mathrm{H}$ & 4.1422 & 4.5697 & -0.7265 \\
$\mathbf{5 9}$ & $\mathrm{H}$ & 3.8206 & 4.2065 & 0.9808 \\
$\mathbf{6 0}$ & $\mathrm{H}$ & 4.8104 & 3.1014 & -0.0046 \\
$\mathbf{6 1}$ & $\mathrm{H}$ & 2.215 & 1.8363 & -1.9009 \\
$\mathbf{6 2}$ & $\mathrm{H}$ & 0.7753 & -2.5601 & 0.9891 \\
\hline
\end{tabular}


Table S71. Conformer 4c-D. PCM-CHCl 3 energy and Boltzmann Populations -3806.319669 hartrees, $8.12 \%$.

\begin{tabular}{|c|c|c|c|c|}
\hline \multicolumn{2}{|c|}{ Atom number } & \multicolumn{3}{|c|}{ Coordinates } \\
\hline & & $\mathbf{x}$ & $\mathbf{Y}$ & Z \\
\hline 1 & 0 & -3.5108 & 0.3602 & 0.5986 \\
\hline 2 & $\mathrm{C}$ & -2.1615 & -0.0411 & 0.7912 \\
\hline 3 & $\mathrm{C}$ & -1.4697 & -0.2299 & -0.5718 \\
\hline 4 & $\mathrm{C}$ & -2.2068 & -1.3683 & -1.2952 \\
\hline 5 & $\mathrm{C}$ & -3.7224 & -1.0863 & -1.3712 \\
\hline 6 & $\mathrm{C}$ & -4.2971 & -0.6903 & -0.003 \\
\hline 7 & $\mathrm{C}$ & -1.3927 & 0.9258 & 1.6728 \\
\hline 8 & $\mathrm{C}$ & 0.0186 & 0.3501 & 1.8794 \\
\hline 9 & $\mathrm{C}$ & 0.7366 & -0.0274 & 0.5629 \\
\hline 10 & $\mathrm{O}$ & -0.1232 & -0.715 & -0.3274 \\
\hline 11 & $\mathrm{O}$ & 1.2429 & 1.1255 & -0.1287 \\
\hline 12 & $\mathrm{C}$ & 2.295 & 1.8764 & 0.5187 \\
\hline 13 & C & 3.4814 & 0.9732 & 0.877 \\
\hline 14 & $\mathrm{C}$ & 3.0201 & -0.303 & 1.5945 \\
\hline 15 & $\mathrm{C}$ & 1.9305 & -1.0265 & 0.7951 \\
\hline 16 & $\mathrm{C}$ & -5.7613 & -0.1898 & 0.0286 \\
\hline 17 & C & -6.7292 & -1.2994 & -0.3863 \\
\hline 18 & 0 & -6.0714 & 0.1015 & 1.3968 \\
\hline 19 & $\mathrm{C}$ & -5.9711 & 1.0759 & -0.8194 \\
\hline 20 & $\mathrm{C}$ & -1.4486 & 1.0391 & -1.4342 \\
\hline 21 & $\mathrm{C}$ & 2.4116 & -1.5071 & -0.5883 \\
\hline 22 & $\mathrm{C}$ & 2.5998 & 3.0673 & -0.4267 \\
\hline 23 & $\mathrm{C}$ & 3.5548 & 4.0552 & 0.2466 \\
\hline 24 & $\mathrm{C}$ & 3.1407 & 2.6216 & -1.7947 \\
\hline 25 & 0 & 1.3767 & 3.7908 & -0.6142 \\
\hline 26 & $\mathrm{Br}$ & 4.0746 & -2.5765 & -0.5052 \\
\hline 27 & 0 & 1.5087 & -2.1344 & 1.5771 \\
\hline 28 & $\mathrm{H}$ & -2.1559 & -1.0266 & 1.2907 \\
\hline 29 & $\mathrm{H}$ & -1.7919 & -1.5158 & -2.2994 \\
\hline 30 & $\mathrm{H}$ & -2.0288 & -2.2952 & -0.7344 \\
\hline 31 & $\mathrm{H}$ & -3.918 & -0.292 & -2.099 \\
\hline 32 & $\mathrm{H}$ & -4.2394 & -1.9816 & -1.7349 \\
\hline 33 & $\mathrm{H}$ & -4.2483 & -1.5633 & 0.6696 \\
\hline 34 & $\mathrm{H}$ & -1.3579 & 1.9239 & 1.2235 \\
\hline 35 & $\mathrm{H}$ & -1.8955 & 1.0275 & 2.6413 \\
\hline 36 & $\mathrm{H}$ & 0.6261 & 1.0554 & 2.4511 \\
\hline 37 & $\mathrm{H}$ & -0.0443 & -0.5626 & 2.4799 \\
\hline 38 & $\mathrm{H}$ & 1.8901 & 2.3212 & 1.439 \\
\hline 39 & $\mathrm{H}$ & 4.039 & 0.6947 & -0.0242 \\
\hline 40 & $\mathrm{H}$ & 4.175 & 1.5275 & 1.5192 \\
\hline 41 & $\mathrm{H}$ & 3.8589 & -0.9881 & 1.7418 \\
\hline 42 & $\mathrm{H}$ & 2.6211 & -0.0718 & 2.5882 \\
\hline 43 & $\mathrm{H}$ & -6.5626 & -2.2033 & 0.2096 \\
\hline 44 & $\mathrm{H}$ & -7.7558 & -0.9644 & -0.2082 \\
\hline 45 & $\mathrm{H}$ & -6.6288 & -1.5497 & -1.4473 \\
\hline 46 & $\mathrm{H}$ & -5.324 & 0.6316 & 1.7234 \\
\hline 47 & $\mathrm{H}$ & -6.9865 & 1.4489 & -0.6544 \\
\hline 48 & $\mathrm{H}$ & -5.2638 & 1.858 & -0.5258 \\
\hline 49 & $\mathrm{H}$ & -5.8471 & 0.8849 & -1.8915 \\
\hline 50 & $\mathrm{H}$ & -2.4602 & 1.3265 & -1.7294 \\
\hline
\end{tabular}




\begin{tabular}{rrrrr}
$\mathbf{5 1}$ & $\mathrm{H}$ & -1.004 & 1.8827 & -0.9059 \\
$\mathbf{5 2}$ & $\mathrm{H}$ & -0.857 & 0.8514 & -2.3354 \\
$\mathbf{5 3}$ & $\mathrm{H}$ & 1.675 & -2.1632 & -1.0452 \\
$\mathbf{5 4}$ & $\mathrm{H}$ & 2.6208 & -0.6824 & -1.2642 \\
$\mathbf{5 5}$ & $\mathrm{H}$ & 3.1712 & 4.3608 & 1.2261 \\
$\mathbf{5 6}$ & $\mathrm{H}$ & 4.5541 & 3.6281 & 0.3748 \\
$\mathbf{5 7}$ & $\mathrm{H}$ & 3.6401 & 4.9515 & -0.3754 \\
$\mathbf{5 8}$ & $\mathrm{H}$ & 3.233 & 3.4985 & -2.4428 \\
$\mathbf{5 9}$ & $\mathrm{H}$ & 4.1252 & 2.1476 & -1.7194 \\
$\mathbf{6 0}$ & $\mathrm{H}$ & 2.455 & 1.9125 & -2.2704 \\
$\mathbf{6 1}$ & $\mathrm{H}$ & 0.7376 & 3.1408 & -0.9497 \\
$\mathbf{6 2}$ & $\mathrm{H}$ & 0.7818 & -2.5581 & 1.0893 \\
\hline
\end{tabular}


Table S72. Conformer 4c-E. PCM-CHCl 3 energy and Boltzmann Populations -3806.319029 hartrees, $4.12 \%$.

\begin{tabular}{|c|c|c|c|c|}
\hline \multicolumn{2}{|c|}{ Atom number } & \multicolumn{3}{|c|}{ Coordinates } \\
\hline & & $\mathbf{x}$ & $\mathbf{Y}$ & Z \\
\hline 1 & 0 & 3.0785 & -0.8721 & 0.6745 \\
\hline 2 & $\mathrm{C}$ & 1.7745 & -0.3576 & 0.9059 \\
\hline 3 & $\mathrm{C}$ & 1.1253 & 0.0664 & -0.4229 \\
\hline 4 & $\mathrm{C}$ & 1.9797 & 1.2121 & -0.9842 \\
\hline 5 & $\mathrm{C}$ & 3.4574 & 0.7885 & -1.0985 \\
\hline 6 & $\mathrm{C}$ & 3.9902 & 0.1344 & 0.1876 \\
\hline 7 & $\mathrm{C}$ & 0.8981 & -1.342 & 1.6592 \\
\hline 8 & $\mathrm{C}$ & -0.4384 & -0.6395 & 1.9518 \\
\hline 9 & $\mathrm{C}$ & -1.0941 & -0.0149 & 0.7 \\
\hline 10 & $\mathrm{O}$ & -0.1722 & 0.6405 & -0.1309 \\
\hline 11 & $\mathrm{O}$ & -1.7367 & -1.0225 & -0.1157 \\
\hline 12 & $\mathrm{C}$ & -2.8961 & -1.7032 & 0.4035 \\
\hline 13 & $\mathrm{C}$ & -3.965 & -0.6926 & 0.829 \\
\hline 14 & $\mathrm{C}$ & -3.3707 & 0.408 & 1.7131 \\
\hline 15 & $\mathrm{C}$ & -2.1506 & 1.0894 & 1.0681 \\
\hline 16 & $\mathrm{C}$ & 5.3427 & -0.606 & 0.0168 \\
\hline 17 & $\mathrm{C}$ & 5.85 & -1.1011 & 1.3822 \\
\hline 18 & 0 & 5.1397 & -1.723 & -0.8582 \\
\hline 19 & $\mathrm{C}$ & 6.3978 & 0.2743 & -0.6552 \\
\hline 20 & $\mathrm{C}$ & 1.0033 & -1.0761 & -1.4395 \\
\hline 21 & $\mathrm{C}$ & -2.622 & 1.841 & -0.2019 \\
\hline 22 & $\mathrm{C}$ & -3.3431 & -2.6542 & -0.7431 \\
\hline 23 & $\mathrm{C}$ & -2.285 & -3.7437 & -0.9858 \\
\hline 24 & $\mathrm{C}$ & -4.6978 & -3.3007 & -0.4443 \\
\hline 25 & 0 & -3.5255 & -1.8802 & -1.933 \\
\hline 26 & $\mathrm{Br}$ & -1.4789 & 3.3879 & -0.7038 \\
\hline 27 & 0 & -1.648 & 1.9853 & 2.0423 \\
\hline 28 & $\mathrm{H}$ & 1.8631 & 0.5536 & 1.5237 \\
\hline 29 & $\mathrm{H}$ & 1.5944 & 1.5341 & -1.9584 \\
\hline 30 & $\mathrm{H}$ & 1.8804 & 2.0694 & -0.3065 \\
\hline 31 & $\mathrm{H}$ & 3.5944 & 0.0843 & -1.9255 \\
\hline 32 & $\mathrm{H}$ & 4.0647 & 1.6711 & -1.3292 \\
\hline 33 & $\mathrm{H}$ & 4.0964 & 0.9046 & 0.9707 \\
\hline 34 & $\mathrm{H}$ & 0.7516 & -2.2571 & 1.0753 \\
\hline 35 & $\mathrm{H}$ & 1.3814 & -1.6307 & 2.5994 \\
\hline 36 & $\mathrm{H}$ & -1.1323 & -1.3365 & 2.4304 \\
\hline 37 & $\mathrm{H}$ & -0.2721 & 0.1751 & 2.6628 \\
\hline 38 & $\mathrm{H}$ & -2.605 & -2.3178 & 1.2693 \\
\hline 39 & $\mathrm{H}$ & -4.4181 & -0.2672 & -0.0739 \\
\hline 40 & $\mathrm{H}$ & -4.7619 & -1.2077 & 1.3765 \\
\hline 41 & $\mathrm{H}$ & -4.1166 & 1.1774 & 1.9415 \\
\hline 42 & $\mathrm{H}$ & -3.0512 & -0.0058 & 2.6771 \\
\hline 43 & $\mathrm{H}$ & 5.0943 & -1.7196 & 1.8751 \\
\hline 44 & $\mathrm{H}$ & 6.7553 & -1.6993 & 1.2396 \\
\hline 45 & $\mathrm{H}$ & 6.0889 & -0.2624 & 2.0474 \\
\hline 46 & $\mathrm{H}$ & 4.3825 & -2.2065 & -0.4852 \\
\hline 47 & $\mathrm{H}$ & 7.3481 & -0.2669 & -0.7022 \\
\hline 48 & $\mathrm{H}$ & 6.1064 & 0.5288 & -1.6776 \\
\hline 49 & $\mathrm{H}$ & 6.5575 & 1.2004 & -0.0913 \\
\hline 50 & $\mathrm{H}$ & 0.3907 & -1.8938 & -1.0597 \\
\hline
\end{tabular}




\begin{tabular}{rrrrr}
$\mathbf{5 1}$ & $\mathrm{H}$ & 0.5305 & -0.6973 & -2.3515 \\
$\mathbf{5 2}$ & $\mathrm{H}$ & 1.9876 & -1.4746 & -1.6978 \\
$\mathbf{5 3}$ & $\mathrm{H}$ & -2.637 & 1.1978 & -1.0777 \\
$\mathbf{5 4}$ & $\mathrm{H}$ & -3.6004 & 2.2917 & -0.0401 \\
$\mathbf{5 5}$ & $\mathrm{H}$ & -1.3153 & -3.3022 & -1.2314 \\
$\mathbf{5 6}$ & $\mathrm{H}$ & -2.1566 & -4.3811 & -0.1025 \\
$\mathbf{5 7}$ & $\mathrm{H}$ & -2.5967 & -4.3741 & -1.8245 \\
$\mathbf{5 8}$ & $\mathrm{H}$ & -4.9479 & -4.0047 & -1.2441 \\
$\mathbf{5 9}$ & $\mathrm{H}$ & -4.6739 & -3.8521 & 0.5025 \\
$\mathbf{6 0}$ & $\mathrm{H}$ & -5.4925 & -2.5517 & -0.3985 \\
$\mathbf{6 1}$ & $\mathrm{H}$ & -2.698 & -1.3802 & -2.0426 \\
$\mathbf{6 2}$ & $\mathrm{H}$ & -1.0541 & 2.6045 & 1.5791 \\
\hline
\end{tabular}




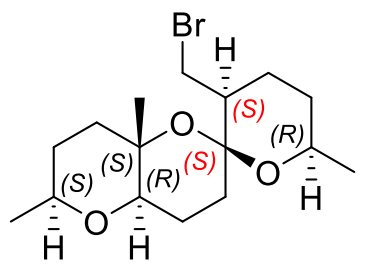

Table S73. Conformer 4d-A. PCM-CHCl 3 energy and Boltzmann Populations -3806.319568 hartrees, $5.27 \%$.

\begin{tabular}{|c|c|c|c|c|}
\hline \multicolumn{2}{|c|}{ Atom number } & \multicolumn{3}{|c|}{ Coordinates } \\
\hline & & $x$ & $\mathbf{Y}$ & $\mathbf{Z}$ \\
\hline 1 & $\mathrm{O}$ & -3.2752 & 0.087 & 0.7743 \\
\hline 2 & $\mathrm{C}$ & -1.8969 & -0.249 & 0.7373 \\
\hline 3 & $\mathrm{C}$ & -1.3649 & -0.1107 & -0.701 \\
\hline 4 & $\mathrm{C}$ & -2.0973 & -1.1707 & -1.536 \\
\hline 5 & $\mathrm{C}$ & -3.6264 & -1.0193 & -1.3972 \\
\hline 6 & $\mathrm{C}$ & -4.0904 & -0.8685 & 0.0632 \\
\hline 7 & $\mathrm{C}$ & -1.0775 & 0.5611 & 1.7289 \\
\hline 8 & $\mathrm{C}$ & 0.3641 & 0.0264 & 1.6696 \\
\hline 9 & $\mathrm{C}$ & 0.9413 & 0.0718 & 0.2383 \\
\hline 10 & $\mathrm{O}$ & 0.0386 & -0.4888 & -0.7039 \\
\hline 11 & $\mathrm{O}$ & 1.1713 & 1.4242 & -0.161 \\
\hline 12 & $\mathrm{C}$ & 2.2676 & 2.1338 & 0.4594 \\
\hline 13 & $\mathrm{C}$ & 3.5785 & 1.3779 & 0.2509 \\
\hline 14 & $\mathrm{C}$ & 3.4415 & -0.0765 & 0.7122 \\
\hline 15 & $\mathrm{C}$ & 2.2632 & -0.7614 & 0.0073 \\
\hline 16 & $\mathrm{C}$ & -5.5422 & -0.3443 & 0.2168 \\
\hline 17 & $\mathrm{C}$ & -5.9489 & -0.3405 & 1.7004 \\
\hline 18 & 0 & -5.6001 & 0.9885 & -0.3064 \\
\hline 19 & $\mathrm{C}$ & -6.5378 & -1.1657 & -0.6039 \\
\hline 20 & $\mathrm{C}$ & -1.5655 & 1.2867 & -1.3027 \\
\hline 21 & $\mathrm{C}$ & 2.0625 & -2.2126 & 0.4803 \\
\hline 22 & $\mathrm{C}$ & 2.2026 & 3.5755 & -0.1056 \\
\hline 23 & $\mathrm{C}$ & 3.1795 & 4.4907 & 0.6356 \\
\hline 24 & $\mathrm{C}$ & 2.4394 & 3.6251 & -1.6231 \\
\hline 25 & 0 & 0.9003 & 4.0972 & 0.1942 \\
\hline 26 & $\mathrm{Br}$ & 3.5991 & -3.3695 & 0.0263 \\
\hline 27 & 0 & 2.5479 & -0.7401 & -1.3817 \\
\hline 28 & $\mathrm{H}$ & -1.7913 & -1.3128 & 1.0164 \\
\hline 29 & $\mathrm{H}$ & -1.8038 & -1.101 & -2.5904 \\
\hline 30 & $\mathrm{H}$ & -1.7801 & -2.1591 & -1.179 \\
\hline 31 & $\mathrm{H}$ & -3.9776 & -0.1489 & -1.9602 \\
\hline 32 & $\mathrm{H}$ & -4.1117 & -1.8975 & -1.8375 \\
\hline 33 & $\mathrm{H}$ & -4.0003 & -1.8402 & 0.5782 \\
\hline 34 & $\mathrm{H}$ & -1.1098 & 1.6302 & 1.4959 \\
\hline 35 & $\mathrm{H}$ & -1.4854 & 0.4353 & 2.7379 \\
\hline 36 & $\mathrm{H}$ & 1.0103 & 0.5881 & 2.3495 \\
\hline 37 & $\mathrm{H}$ & 0.3622 & -1.0095 & 2.0241 \\
\hline 38 & $\mathrm{H}$ & 2.0633 & 2.2219 & 1.5375 \\
\hline 39 & $\mathrm{H}$ & 3.8484 & 1.3838 & -0.8083 \\
\hline 40 & $\mathrm{H}$ & 4.3814 & 1.8727 & 0.8091 \\
\hline 41 & $\mathrm{H}$ & 4.3495 & -0.6415 & 0.4812 \\
\hline 42 & $\mathrm{H}$ & 3.2987 & -0.1276 & 1.8002 \\
\hline 43 & $\mathrm{H}$ & -6.9417 & 0.1079 & 1.8048 \\
\hline
\end{tabular}




\begin{tabular}{llrrr}
$\mathbf{4 4}$ & $\mathrm{H}$ & -5.9833 & -1.3587 & 2.1068 \\
$\mathbf{4 5}$ & $\mathrm{H}$ & -5.2378 & 0.238 & 2.2972 \\
$\mathbf{4 6}$ & $\mathrm{H}$ & -4.8973 & 1.4794 & 0.1531 \\
$\mathbf{4 7}$ & $\mathrm{H}$ & -6.5078 & -2.2233 & -0.3187 \\
$\mathbf{4 8}$ & $\mathrm{H}$ & -7.5507 & -0.7912 & -0.4262 \\
$\mathbf{4 9}$ & $\mathrm{H}$ & -6.3336 & -1.0817 & -1.6745 \\
$\mathbf{5 0}$ & $\mathrm{H}$ & -2.6254 & 1.4909 & -1.4691 \\
$\mathbf{5 1}$ & $\mathrm{H}$ & -1.1724 & 2.0706 & -0.6559 \\
$\mathbf{5 2}$ & $\mathrm{H}$ & -1.0414 & 1.3437 & -2.2613 \\
$\mathbf{5 3}$ & $\mathrm{H}$ & 1.9626 & -2.2996 & 1.5614 \\
$\mathbf{5 4}$ & $\mathrm{H}$ & 1.2039 & -2.6658 & -0.0129 \\
$\mathbf{5 5}$ & $\mathrm{H}$ & 3.0128 & 5.5242 & 0.3166 \\
$\mathbf{5 6}$ & $\mathrm{H}$ & 3.0139 & 4.4419 & 1.7174 \\
$\mathbf{5 7}$ & $\mathrm{H}$ & 4.2207 & 4.2289 & 0.4242 \\
$\mathbf{5 8}$ & $\mathrm{H}$ & 2.2442 & 4.6407 & -1.9809 \\
$\mathbf{5 9}$ & $\mathrm{H}$ & 3.4678 & 3.3583 & -1.8881 \\
$\mathbf{6 0}$ & $\mathrm{H}$ & 1.7682 & 2.9341 & -2.1429 \\
$\mathbf{6 1}$ & $\mathrm{H}$ & 0.272 & 3.4722 & -0.2022 \\
$\mathbf{6 2}$ & $\mathrm{H}$ & 1.6869 & -0.7935 & -1.8333 \\
\hline
\end{tabular}


Table S74. Conformer 4d-B. PCM-CHCl 3 energy and Boltzmann Populations -3806.320124 hartrees, $9.50 \%$.

\begin{tabular}{|c|c|c|c|c|}
\hline \multicolumn{2}{|c|}{ Atom number } & \multicolumn{3}{|c|}{ Coordinates } \\
\hline & & $\mathbf{x}$ & $\mathbf{Y}$ & $\mathbf{Z}$ \\
\hline 1 & O & -3.292 & 0.107 & 0.7844 \\
\hline 2 & $\mathrm{C}$ & -1.9137 & -0.2285 & 0.7423 \\
\hline 3 & $\mathrm{C}$ & -1.3748 & -0.0544 & -0.6898 \\
\hline 4 & $\mathrm{C}$ & -2.1078 & -1.0896 & -1.5541 \\
\hline 5 & $\mathrm{C}$ & -3.6359 & -0.9254 & -1.4235 \\
\hline 6 & $\mathrm{C}$ & -4.1071 & -0.8216 & 0.0382 \\
\hline 7 & $\mathrm{C}$ & -1.0991 & 0.5608 & 1.7539 \\
\hline 8 & $\mathrm{C}$ & 0.3419 & 0.024 & 1.6946 \\
\hline 9 & $\mathrm{C}$ & 0.9306 & 0.081 & 0.2685 \\
\hline 10 & 0 & 0.0281 & -0.4358 & -0.6968 \\
\hline 11 & 0 & 1.1955 & 1.4418 & -0.0916 \\
\hline 12 & $\mathrm{C}$ & 2.3115 & 2.1225 & 0.5323 \\
\hline 13 & $\mathrm{C}$ & 3.599 & 1.3143 & 0.3673 \\
\hline 14 & $\mathrm{C}$ & 3.4097 & -0.1467 & 0.7796 \\
\hline 15 & $\mathrm{C}$ & 2.2317 & -0.7789 & 0.0322 \\
\hline 16 & C & -5.5577 & -0.2975 & 0.2032 \\
\hline 17 & C & -5.9723 & -0.3457 & 1.6838 \\
\hline 18 & 0 & -5.6078 & 1.0538 & -0.2713 \\
\hline 19 & $\mathrm{C}$ & -6.5515 & -1.0847 & -0.6523 \\
\hline 20 & $\mathrm{C}$ & -1.562 & 1.3597 & -1.2527 \\
\hline 21 & $\mathrm{C}$ & 1.9861 & -2.239 & 0.4543 \\
\hline 22 & $\mathrm{C}$ & 2.3495 & 3.5178 & -0.1519 \\
\hline 23 & $\mathrm{C}$ & 1.078 & 4.3208 & 0.1744 \\
\hline 24 & $\mathrm{C}$ & 3.5861 & 4.3153 & 0.2727 \\
\hline 25 & $\mathrm{O}$ & 2.459 & 3.3329 & -1.5648 \\
\hline 26 & $\mathrm{Br}$ & 3.5027 & -3.4185 & -0.0075 \\
\hline 27 & 0 & 2.54 & -0.7186 & -1.3507 \\
\hline 28 & $\mathrm{H}$ & -1.8087 & -1.2986 & 0.9967 \\
\hline 29 & $\mathrm{H}$ & -1.8045 & -0.9984 & -2.6039 \\
\hline 30 & $\mathrm{H}$ & -1.8042 & -2.0894 & -1.217 \\
\hline 31 & $\mathrm{H}$ & -3.9713 & -0.0308 & -1.9575 \\
\hline 32 & $\mathrm{H}$ & -4.1278 & -1.7818 & -1.898 \\
\hline 33 & $\mathrm{H}$ & -4.023 & -1.8103 & 0.521 \\
\hline 34 & $\mathrm{H}$ & -1.1316 & 1.6328 & 1.5351 \\
\hline 35 & $\mathrm{H}$ & -1.5131 & 0.4182 & 2.7582 \\
\hline 36 & $\mathrm{H}$ & 0.9877 & 0.5779 & 2.3816 \\
\hline 37 & $\mathrm{H}$ & 0.3355 & -1.0144 & 2.0417 \\
\hline 38 & $\mathrm{H}$ & 2.0925 & 2.2658 & 1.6022 \\
\hline 39 & $\mathrm{H}$ & 3.9081 & 1.3616 & -0.6802 \\
\hline 40 & $\mathrm{H}$ & 4.3908 & 1.7657 & 0.9747 \\
\hline 41 & $\mathrm{H}$ & 4.3086 & -0.7272 & 0.552 \\
\hline 42 & $\mathrm{H}$ & 3.2408 & -0.2296 & 1.8621 \\
\hline 43 & $\mathrm{H}$ & -6.9638 & 0.1032 & 1.7995 \\
\hline 44 & $\mathrm{H}$ & -6.0134 & -1.3777 & 2.0529 \\
\hline 45 & $\mathrm{H}$ & -5.2619 & 0.2076 & 2.3048 \\
\hline 46 & $\mathrm{H}$ & -4.9043 & 1.5239 & 0.2083 \\
\hline 47 & $\mathrm{H}$ & -6.3424 & -0.9598 & -1.718 \\
\hline 48 & $\mathrm{H}$ & -6.5244 & -2.1527 & -0.4081 \\
\hline 49 & $\mathrm{H}$ & -7.5647 & -0.7156 & -0.4646 \\
\hline 50 & $\mathrm{H}$ & -1.0606 & 2.1089 & -0.642 \\
\hline
\end{tabular}




\begin{tabular}{rrrrr}
$\mathbf{5 1}$ & $\mathrm{H}$ & -1.135 & 1.4061 & -2.2599 \\
$\mathbf{5 2}$ & $\mathrm{H}$ & -2.6222 & 1.6159 & -1.3138 \\
$\mathbf{5 3}$ & $\mathrm{H}$ & 1.8633 & -2.3573 & 1.5301 \\
$\mathbf{5 4}$ & $\mathrm{H}$ & 1.1261 & -2.6557 & -0.068 \\
$\mathbf{5 5}$ & $\mathrm{H}$ & 0.9879 & 4.5106 & 1.2513 \\
$\mathbf{5 6}$ & $\mathrm{H}$ & 0.1808 & 3.7917 & -0.1567 \\
$\mathbf{5 7}$ & $\mathrm{H}$ & 1.1149 & 5.2839 & -0.3442 \\
$\mathbf{5 8}$ & $\mathrm{H}$ & 3.5553 & 5.3041 & -0.1959 \\
$\mathbf{5 9}$ & $\mathrm{H}$ & 3.6182 & 4.4526 & 1.3597 \\
$\mathbf{6 0}$ & $\mathrm{H}$ & 4.5051 & 3.8211 & -0.0513 \\
$\mathbf{6 1}$ & $\mathrm{H}$ & 1.8412 & 2.6134 & -1.7839 \\
$\mathbf{6 2}$ & $\mathrm{H}$ & 1.689 & -0.7914 & -1.8182 \\
\hline
\end{tabular}


Table S75. Conformer 4d-C. PCM-CHCl 3 energy and Boltzmann Populations -3806.319635 hartrees, $5.66 \%$.

\begin{tabular}{|c|c|c|c|c|}
\hline \multicolumn{2}{|c|}{ Atom number } & \multicolumn{3}{|c|}{ Coordinates } \\
\hline & & $\mathbf{x}$ & $\mathbf{Y}$ & $\mathbf{Z}$ \\
\hline 1 & O & -3.2965 & 0.2274 & 0.7355 \\
\hline 2 & $\mathrm{C}$ & -1.9239 & -0.1361 & 0.7069 \\
\hline 3 & $\mathrm{C}$ & -1.369 & 0.0361 & -0.7207 \\
\hline 4 & $\mathrm{C}$ & -2.1272 & -0.9614 & -1.6109 \\
\hline 5 & $\mathrm{C}$ & -3.6523 & -0.7543 & -1.4873 \\
\hline 6 & $\mathrm{C}$ & -4.104 & -0.7027 & -0.0197 \\
\hline 7 & $\mathrm{C}$ & -1.0968 & 0.625 & 1.7293 \\
\hline 8 & $\mathrm{C}$ & 0.3272 & 0.0429 & 1.6798 \\
\hline 9 & $\mathrm{C}$ & 0.9306 & 0.0871 & 0.2592 \\
\hline 10 & 0 & 0.02 & -0.3935 & -0.7184 \\
\hline 11 & 0 & 1.2455 & 1.4398 & -0.0905 \\
\hline 12 & $\mathrm{C}$ & 2.3776 & 2.0791 & 0.5481 \\
\hline 13 & C & 3.6389 & 1.2298 & 0.3892 \\
\hline 14 & $\mathrm{C}$ & 3.3967 & -0.2265 & 0.7906 \\
\hline 15 & $\mathrm{C}$ & 2.2044 & -0.8148 & 0.0295 \\
\hline 16 & C & -5.5736 & -0.3028 & 0.2529 \\
\hline 17 & C & -6.5395 & -1.3432 & -0.3172 \\
\hline 18 & 0 & -5.7586 & -0.3422 & 1.6734 \\
\hline 19 & $\mathrm{C}$ & -5.9092 & 1.1065 & -0.2633 \\
\hline 20 & $\mathrm{C}$ & -1.4983 & 1.4628 & -1.2673 \\
\hline 21 & $\mathrm{C}$ & 1.907 & -2.2681 & 0.4419 \\
\hline 22 & $\mathrm{C}$ & 2.4683 & 3.4763 & -0.127 \\
\hline 23 & $\mathrm{C}$ & 1.2237 & 4.322 & 0.1962 \\
\hline 24 & $\mathrm{C}$ & 3.7289 & 4.2283 & 0.3105 \\
\hline 25 & $\mathrm{O}$ & 2.5804 & 3.296 & -1.5403 \\
\hline 26 & $\mathrm{Br}$ & 3.3874 & -3.495 & -0.0137 \\
\hline 27 & 0 & 2.5271 & -0.7573 & -1.3501 \\
\hline 28 & $\mathrm{H}$ & -1.8441 & -1.2103 & 0.9526 \\
\hline 29 & $\mathrm{H}$ & -1.8106 & -0.8597 & -2.6557 \\
\hline 30 & $\mathrm{H}$ & -1.8608 & -1.9767 & -1.2901 \\
\hline 31 & $\mathrm{H}$ & -3.9448 & 0.1686 & -1.9987 \\
\hline 32 & $\mathrm{H}$ & -4.1711 & -1.5753 & -1.9952 \\
\hline 33 & $\mathrm{H}$ & -3.9611 & -1.6995 & 0.4306 \\
\hline 34 & $\mathrm{H}$ & -1.0949 & 1.6986 & 1.5164 \\
\hline 35 & $\mathrm{H}$ & -1.5228 & 0.4893 & 2.7297 \\
\hline 36 & $\mathrm{H}$ & 0.9844 & 0.5733 & 2.3745 \\
\hline 37 & $\mathrm{H}$ & 0.2849 & -0.9961 & 2.0219 \\
\hline 38 & $\mathrm{H}$ & 2.1535 & 2.2232 & 1.6168 \\
\hline 39 & $\mathrm{H}$ & 3.9588 & 1.2731 & -0.6551 \\
\hline 40 & $\mathrm{H}$ & 4.4397 & 1.6505 & 1.0067 \\
\hline 41 & $\mathrm{H}$ & 4.2773 & -0.8356 & 0.5667 \\
\hline 42 & $\mathrm{H}$ & 3.2162 & -0.3101 & 1.8711 \\
\hline 43 & $\mathrm{H}$ & -7.5542 & -1.1134 & 0.0227 \\
\hline 44 & $\mathrm{H}$ & -6.5361 & -1.3434 & -1.4119 \\
\hline 45 & $\mathrm{H}$ & -6.285 & -2.347 & 0.0395 \\
\hline 46 & $\mathrm{H}$ & -5.0021 & 0.143 & 2.0457 \\
\hline 47 & $\mathrm{H}$ & -5.8754 & 1.1686 & -1.357 \\
\hline 48 & $\mathrm{H}$ & -6.9194 & 1.3725 & 0.0626 \\
\hline 49 & $\mathrm{H}$ & -5.2101 & 1.8436 & 0.1445 \\
\hline 50 & $\mathrm{H}$ & -2.5459 & 1.7691 & -1.3111 \\
\hline
\end{tabular}




\begin{tabular}{rrrrr}
$\mathbf{5 1}$ & $\mathrm{H}$ & -0.9566 & 2.1807 & -0.6541 \\
$\mathbf{5 2}$ & $\mathrm{H}$ & -1.0799 & 1.5014 & -2.2786 \\
$\mathbf{5 3}$ & $\mathrm{H}$ & 1.7719 & -2.3871 & 1.516 \\
$\mathbf{5 4}$ & $\mathrm{H}$ & 1.0375 & -2.6534 & -0.0887 \\
$\mathbf{5 5}$ & $\mathrm{H}$ & 1.2962 & 5.2842 & -0.3203 \\
$\mathbf{5 6}$ & $\mathrm{H}$ & 0.3094 & 3.8256 & -0.1391 \\
$\mathbf{5 7}$ & $\mathrm{H}$ & 1.1361 & 4.5129 & 1.2731 \\
$\mathbf{5 8}$ & $\mathrm{H}$ & 3.7335 & 5.2215 & -0.1495 \\
$\mathbf{5 9}$ & $\mathrm{H}$ & 3.76 & 4.3555 & 1.3989 \\
$\mathbf{6 0}$ & $\mathrm{H}$ & 4.6326 & 3.7066 & -0.0133 \\
$\mathbf{6 1}$ & $\mathrm{H}$ & 1.9436 & 2.5957 & -1.7667 \\
$\mathbf{6 2}$ & $\mathrm{H}$ & 1.6791 & -0.8039 & -1.826 \\
\hline
\end{tabular}


Table S76. Conformer 4d-D. PCM-CHCl 3 energy and Boltzmann Populations -3806.320655 hartrees, $16.66 \%$.

\begin{tabular}{|c|c|c|c|c|}
\hline \multicolumn{2}{|c|}{ Atom number } & \multicolumn{3}{|c|}{ Coordinates } \\
\hline & & $x$ & $Y$ & Z \\
\hline 1 & $\mathrm{O}$ & 3.025 & 0.1559 & -0.7943 \\
\hline 2 & C & 1.7056 & -0.2795 & -0.4991 \\
\hline 3 & C & 1.282 & 0.2517 & 0.8821 \\
\hline 4 & C & 2.2039 & -0.4238 & 1.9072 \\
\hline 5 & C & 3.686 & -0.1827 & 1.5546 \\
\hline 6 & C & 4.0085 & -0.4471 & 0.0721 \\
\hline 7 & C & 0.7112 & 0.1008 & -1.5835 \\
\hline 8 & C & -0.6392 & -0.5292 & -1.1997 \\
\hline 9 & $\mathrm{C}$ & -1.1012 & -0.0928 & 0.2035 \\
\hline 10 & $\mathrm{O}$ & -0.0604 & -0.2303 & 1.1626 \\
\hline 11 & 0 & -1.4752 & 1.2909 & 0.1975 \\
\hline 12 & $\mathrm{C}$ & -2.7073 & 1.6442 & -0.4716 \\
\hline 13 & $\mathrm{C}$ & -3.8809 & 0.8913 & 0.1509 \\
\hline 14 & $\mathrm{C}$ & -3.6062 & -0.6167 & 0.1444 \\
\hline 15 & $\mathrm{C}$ & -2.2763 & -0.9231 & 0.8413 \\
\hline 16 & $\mathrm{C}$ & 5.365 & 0.1448 & -0.3938 \\
\hline 17 & $\mathrm{C}$ & 5.653 & -0.2725 & -1.8459 \\
\hline 18 & 0 & 5.2899 & 1.5731 & -0.3042 \\
\hline 19 & $\mathrm{C}$ & 6.5178 & -0.2771 & 0.5187 \\
\hline 20 & $\mathrm{C}$ & 1.3586 & 1.7787 & 1.0085 \\
\hline 21 & $\mathrm{C}$ & -1.9907 & -2.433 & 0.9718 \\
\hline 22 & $\mathrm{C}$ & -2.7735 & 3.1914 & -0.4331 \\
\hline 23 & $\mathrm{C}$ & -3.9344 & 3.7049 & -1.2875 \\
\hline 24 & $\mathrm{C}$ & -2.853 & 3.7426 & 0.9991 \\
\hline 25 & $\mathrm{O}$ & -1.5886 & 3.6867 & -1.0724 \\
\hline 26 & $\mathrm{Br}$ & -2.2812 & -3.5604 & -0.645 \\
\hline 27 & 0 & -2.4129 & -0.4906 & 2.1979 \\
\hline 28 & $\mathrm{H}$ & 1.7104 & -1.3819 & -0.4314 \\
\hline 29 & $\mathrm{H}$ & 1.9907 & -0.0559 & 2.9183 \\
\hline 30 & $\mathrm{H}$ & 1.9824 & -1.4988 & 1.9031 \\
\hline 31 & $\mathrm{H}$ & 3.9712 & 0.8476 & 1.7898 \\
\hline 32 & $\mathrm{H}$ & 4.3102 & -0.8336 & 2.177 \\
\hline 33 & $\mathrm{H}$ & 4.0045 & -1.5343 & -0.1154 \\
\hline 34 & $\mathrm{H}$ & 0.6264 & 1.1876 & -1.6858 \\
\hline 35 & $\mathrm{H}$ & 1.047 & -0.2924 & -2.5492 \\
\hline 36 & $\mathrm{H}$ & -1.4052 & -0.2727 & -1.9365 \\
\hline 37 & $\mathrm{H}$ & -0.5466 & -1.6175 & -1.2248 \\
\hline 38 & $\mathrm{H}$ & -2.6216 & 1.371 & -1.5342 \\
\hline 39 & $\mathrm{H}$ & -4.0278 & 1.2208 & 1.1831 \\
\hline 40 & $\mathrm{H}$ & -4.7982 & 1.1045 & -0.4093 \\
\hline 41 & $\mathrm{H}$ & -4.4012 & -1.1532 & 0.6756 \\
\hline 42 & $\mathrm{H}$ & -3.5869 & -1.0094 & -0.8778 \\
\hline 43 & $\mathrm{H}$ & 6.5685 & 0.2176 & -2.1915 \\
\hline 44 & $\mathrm{H}$ & 5.787 & -1.3579 & -1.9293 \\
\hline 45 & $\mathrm{H}$ & 4.8292 & 0.018 & -2.5043 \\
\hline 46 & $\mathrm{H}$ & 4.4965 & 1.8235 & -0.8081 \\
\hline 47 & $\mathrm{H}$ & 6.6032 & -1.3685 & 0.5686 \\
\hline 48 & $\mathrm{H}$ & 7.4575 & 0.123 & 0.1254 \\
\hline 49 & $\mathrm{H}$ & 6.3854 & 0.1143 & 1.5308 \\
\hline 50 & $\mathrm{H}$ & 2.394 & 2.1213 & 0.9521 \\
\hline
\end{tabular}




\begin{tabular}{llrrr}
$\mathbf{5 1}$ & $\mathrm{H}$ & 0.7956 & 2.2831 & 0.224 \\
$\mathbf{5 2}$ & $\mathrm{H}$ & 0.9394 & 2.0825 & 1.9726 \\
$\mathbf{5 3}$ & $\mathrm{H}$ & -0.9623 & -2.618 & 1.2783 \\
$\mathbf{5 4}$ & $\mathrm{H}$ & -2.6797 & -2.8416 & 1.7079 \\
$\mathbf{5 5}$ & $\mathrm{H}$ & -3.8682 & 4.7947 & -1.3626 \\
$\mathbf{5 6}$ & $\mathrm{H}$ & -3.8826 & 3.2941 & -2.3016 \\
$\mathbf{5 7}$ & $\mathrm{H}$ & -4.9041 & 3.4482 & -0.8502 \\
$\mathbf{5 8}$ & $\mathrm{H}$ & -2.7669 & 4.8331 & 0.9673 \\
$\mathbf{5 9}$ & $\mathrm{H}$ & -3.7998 & 3.4856 & 1.4862 \\
$\mathbf{6 0}$ & $\mathrm{H}$ & -2.0386 & 3.3442 & 1.6123 \\
$\mathbf{6 1}$ & $\mathrm{H}$ & -0.8481 & 3.2588 & -0.6126 \\
$\mathbf{6 2}$ & $\mathrm{H}$ & -1.5141 & -0.2484 & 2.4873 \\
\hline
\end{tabular}


Table S77. Conformer 4d-E. PCM-CHCl 3 energy and Boltzmann Populations -3806.320082 hartrees, $9.09 \%$.

\begin{tabular}{|c|c|c|c|c|}
\hline \multicolumn{2}{|c|}{ Atom number } & \multicolumn{3}{|c|}{ Coordinates } \\
\hline & & $x$ & $\mathbf{Y}$ & $\mathbf{Z}$ \\
\hline 1 & $\mathrm{O}$ & -3.0237 & -0.2863 & -0.7706 \\
\hline 2 & $\mathrm{C}$ & -1.7126 & 0.1834 & -0.4857 \\
\hline 3 & $\mathrm{C}$ & -1.2648 & -0.3471 & 0.8894 \\
\hline 4 & $\mathrm{C}$ & -2.2063 & 0.2774 & 1.9318 \\
\hline 5 & $\mathrm{C}$ & -3.682 & -0.0107 & 1.5816 \\
\hline 6 & $\mathrm{C}$ & -4.0002 & 0.3085 & 0.1117 \\
\hline 7 & $\mathrm{C}$ & -0.712 & -0.1584 & -1.5769 \\
\hline 8 & $\mathrm{C}$ & 0.6174 & 0.5165 & -1.1951 \\
\hline 9 & $\mathrm{C}$ & 1.1007 & 0.0927 & 0.2052 \\
\hline 10 & 0 & 0.0584 & 0.1849 & 1.1691 \\
\hline 11 & 0 & 1.529 & -1.2751 & 0.1929 \\
\hline 12 & $\mathrm{C}$ & 2.7724 & -1.5774 & -0.4807 \\
\hline 13 & $\mathrm{C}$ & 3.9175 & -0.78 & 0.1395 \\
\hline 14 & $\mathrm{C}$ & 3.5825 & 0.7156 & 0.1389 \\
\hline 15 & $\mathrm{C}$ & 2.2443 & 0.9661 & 0.8425 \\
\hline 16 & $\mathrm{C}$ & -5.3808 & -0.1462 & -0.4196 \\
\hline 17 & $\mathrm{C}$ & -6.5132 & 0.5936 & 0.2954 \\
\hline 18 & 0 & -5.4552 & 0.2657 & -1.7898 \\
\hline 19 & $\mathrm{C}$ & -5.5738 & -1.6697 & -0.3367 \\
\hline 20 & $\mathrm{C}$ & -1.2765 & -1.8775 & 0.9985 \\
\hline 21 & $\mathrm{C}$ & 1.9001 & 2.4633 & 0.9799 \\
\hline 22 & $\mathrm{C}$ & 2.901 & -3.1209 & -0.4462 \\
\hline 23 & $\mathrm{C}$ & 4.081 & -3.5848 & -1.3029 \\
\hline 24 & $\mathrm{C}$ & 3.0042 & -3.6727 & 0.9843 \\
\hline 25 & 0 & 1.7368 & -3.6624 & -1.086 \\
\hline 26 & $\mathrm{Br}$ & 2.1449 & 3.6067 & -0.6327 \\
\hline 27 & 0 & 2.4034 & 0.5332 & 2.1964 \\
\hline 28 & $\mathrm{H}$ & -1.747 & 1.2844 & -0.4075 \\
\hline 29 & $\mathrm{H}$ & -1.9721 & -0.0989 & 2.9349 \\
\hline 30 & $\mathrm{H}$ & -2.028 & 1.3601 & 1.9439 \\
\hline 31 & $\mathrm{H}$ & -3.914 & -1.0596 & 1.7929 \\
\hline 32 & $\mathrm{H}$ & -4.3306 & 0.5909 & 2.2285 \\
\hline 33 & $\mathrm{H}$ & -3.9505 & 1.4006 & -0.034 \\
\hline 34 & $\mathrm{H}$ & -0.5909 & -1.2408 & -1.6867 \\
\hline 35 & $\mathrm{H}$ & -1.0643 & 0.2298 & -2.539 \\
\hline 36 & $\mathrm{H}$ & 1.3892 & 0.289 & -1.9352 \\
\hline 37 & $\mathrm{H}$ & 0.487 & 1.601 & -1.2165 \\
\hline 38 & $\mathrm{H}$ & 2.6722 & -1.3057 & -1.5424 \\
\hline 39 & $\mathrm{H}$ & 4.0828 & -1.1067 & 1.1697 \\
\hline 40 & $\mathrm{H}$ & 4.8396 & -0.9548 & -0.426 \\
\hline 41 & $\mathrm{H}$ & 4.3575 & 1.2821 & 0.6685 \\
\hline 42 & $\mathrm{H}$ & 3.5436 & 1.1101 & -0.8819 \\
\hline 43 & $\mathrm{H}$ & -7.4602 & 0.3675 & -0.2046 \\
\hline 44 & $\mathrm{H}$ & -6.5978 & 0.291 & 1.344 \\
\hline 45 & $\mathrm{H}$ & -6.3589 & 1.677 & 0.2492 \\
\hline 46 & $\mathrm{H}$ & -4.6135 & -0.0138 & -2.1896 \\
\hline 47 & $\mathrm{H}$ & -5.6274 & -2.0273 & 0.6979 \\
\hline 48 & $\mathrm{H}$ & -6.509 & -1.9364 & -0.8383 \\
\hline 49 & $\mathrm{H}$ & -4.7522 & -2.1905 & -0.8385 \\
\hline 50 & $\mathrm{H}$ & -0.835 & -2.1739 & 1.9549 \\
\hline
\end{tabular}




\begin{tabular}{rrrrr}
$\mathbf{5 1}$ & $\mathrm{H}$ & -2.2961 & -2.2641 & 0.9452 \\
$\mathbf{5 2}$ & $\mathrm{H}$ & -0.7019 & -2.3495 & 0.2026 \\
$\mathbf{5 3}$ & $\mathrm{H}$ & 0.8658 & 2.6079 & 1.2884 \\
$\mathbf{5 4}$ & $\mathrm{H}$ & 2.5739 & 2.8949 & 1.7168 \\
$\mathbf{5 5}$ & $\mathrm{H}$ & 4.0582 & -4.6762 & -1.3812 \\
$\mathbf{5 6}$ & $\mathrm{H}$ & 4.0121 & -3.1736 & -2.3158 \\
$\mathbf{5 7}$ & $\mathrm{H}$ & 5.0402 & -3.2911 & -0.8655 \\
$\mathbf{5 8}$ & $\mathrm{H}$ & 2.9638 & -4.7658 & 0.9489 \\
$\mathbf{5 9}$ & $\mathrm{H}$ & 3.9396 & -3.3779 & 1.4718 \\
$\mathbf{6 0}$ & $\mathrm{H}$ & 2.1741 & -3.3108 & 1.5992 \\
$\mathbf{6 1}$ & $\mathrm{H}$ & 0.9796 & -3.2675 & -0.6241 \\
$\mathbf{6 2}$ & $\mathrm{H}$ & 1.5161 & 0.2555 & 2.4888 \\
\hline
\end{tabular}


Table S78. Conformer 4d-F. PCM-CHCl 3 energy and Boltzmann Populations -3806.321204 hartrees, $29.81 \%$.

\begin{tabular}{|c|c|c|c|c|}
\hline \multicolumn{2}{|c|}{ Atom number } & \multicolumn{3}{|c|}{ Coordinates } \\
\hline & & $\mathbf{x}$ & $\mathbf{Y}$ & $\mathbf{Z}$ \\
\hline 1 & $\mathrm{O}$ & -3.0386 & -0.1622 & -0.7977 \\
\hline 2 & C & -1.7174 & 0.267 & -0.5014 \\
\hline 3 & C & -1.2744 & -0.3055 & 0.8575 \\
\hline 4 & $\mathrm{C}$ & -2.1919 & 0.3269 & 1.9127 \\
\hline 5 & $\mathrm{C}$ & -3.6732 & 0.0705 & 1.5695 \\
\hline 6 & $\mathrm{C}$ & -4.0166 & 0.3959 & 0.1047 \\
\hline 7 & C & -0.7363 & -0.0826 & -1.6075 \\
\hline 8 & $\mathrm{C}$ & 0.618 & 0.5412 & -1.227 \\
\hline 9 & $\mathrm{C}$ & 1.0991 & 0.09 & 0.165 \\
\hline 10 & 0 & 0.0674 & 0.1811 & 1.138 \\
\hline 11 & 0 & 1.5049 & -1.2866 & 0.1195 \\
\hline 12 & $\mathrm{C}$ & 2.7443 & -1.6167 & -0.5511 \\
\hline 13 & $\mathrm{C}$ & 3.9023 & -0.811 & 0.0376 \\
\hline 14 & $\mathrm{C}$ & 3.5791 & 0.6849 & 0.0759 \\
\hline 15 & $\mathrm{C}$ & 2.258 & 0.9353 & 0.8067 \\
\hline 16 & $\mathrm{C}$ & -5.3732 & -0.1867 & -0.3724 \\
\hline 17 & $\mathrm{C}$ & -5.6857 & 0.3018 & -1.7972 \\
\hline 18 & 0 & -5.281 & -1.6167 & -0.3578 \\
\hline 19 & $\mathrm{C}$ & -6.5177 & 0.1741 & 0.576 \\
\hline 20 & $\mathrm{C}$ & -1.3264 & -1.836 & 0.9334 \\
\hline 21 & $\mathrm{C}$ & 1.9334 & 2.4329 & 0.9845 \\
\hline 22 & $\mathrm{C}$ & 2.887 & -3.1543 & -0.3774 \\
\hline 23 & $\mathrm{C}$ & 1.7583 & -3.8934 & -1.1177 \\
\hline 24 & $\mathrm{C}$ & 4.2452 & -3.6524 & -0.8802 \\
\hline 25 & 0 & 2.8391 & -3.4648 & 1.0175 \\
\hline 26 & $\mathrm{Br}$ & 2.1707 & 3.6153 & -0.6014 \\
\hline 27 & 0 & 2.4289 & 0.4676 & 2.1474 \\
\hline 28 & $\mathrm{H}$ & -1.7233 & 1.3668 & -0.4002 \\
\hline 29 & $\mathrm{H}$ & -1.9598 & -0.0658 & 2.91 \\
\hline 30 & $\mathrm{H}$ & -1.9899 & 1.4058 & 1.9364 \\
\hline 31 & $\mathrm{H}$ & -3.9335 & -0.9756 & 1.7591 \\
\hline 32 & $\mathrm{H}$ & -4.3027 & 0.6793 & 2.2282 \\
\hline 33 & $\mathrm{H}$ & -4.0231 & 1.4902 & -0.0353 \\
\hline 34 & $\mathrm{H}$ & -0.6563 & -1.1671 & -1.7339 \\
\hline 35 & $\mathrm{H}$ & -1.085 & 0.334 & -2.5589 \\
\hline 36 & $\mathrm{H}$ & 1.3783 & 0.2939 & -1.9732 \\
\hline 37 & $\mathrm{H}$ & 0.5243 & 1.6296 & -1.2389 \\
\hline 38 & $\mathrm{H}$ & 2.6435 & -1.3958 & -1.6252 \\
\hline 39 & $\mathrm{H}$ & 4.1 & -1.1742 & 1.0497 \\
\hline 40 & $\mathrm{H}$ & 4.8032 & -0.9744 & -0.5635 \\
\hline 41 & $\mathrm{H}$ & 4.371 & 1.2313 & 0.6018 \\
\hline 42 & $\mathrm{H}$ & 3.5247 & 1.1045 & -0.9346 \\
\hline 43 & $\mathrm{H}$ & -4.8653 & 0.0605 & -2.4794 \\
\hline 44 & $\mathrm{H}$ & -6.5972 & -0.1847 & -2.1579 \\
\hline 45 & $\mathrm{H}$ & -5.8384 & 1.3876 & -1.8213 \\
\hline 46 & $\mathrm{H}$ & -4.487 & -1.8299 & -0.8776 \\
\hline 47 & $\mathrm{H}$ & -6.3686 & -0.2712 & 1.5632 \\
\hline 48 & $\mathrm{H}$ & -6.6116 & 1.2603 & 0.6871 \\
\hline 49 & $\mathrm{H}$ & -7.4592 & -0.2122 & 0.1731 \\
\hline 50 & $\mathrm{H}$ & -2.3453 & -2.198 & 0.7781 \\
\hline
\end{tabular}




\begin{tabular}{rrrrr}
$\mathbf{5 1}$ & $\mathrm{H}$ & -0.678 & -2.3007 & 0.1924 \\
$\mathbf{5 2}$ & $\mathrm{H}$ & -0.9906 & -2.1599 & 1.9242 \\
$\mathbf{5 3}$ & $\mathrm{H}$ & 0.9051 & 2.5827 & 1.3098 \\
$\mathbf{5 4}$ & $\mathrm{H}$ & 2.6225 & 2.8379 & 1.7225 \\
$\mathbf{5 5}$ & $\mathrm{H}$ & 0.776 & -3.5801 & -0.7549 \\
$\mathbf{5 6}$ & $\mathrm{H}$ & 1.8568 & -4.9698 & -0.946 \\
$\mathbf{5 7}$ & $\mathrm{H}$ & 1.7996 & -3.7099 & -2.1986 \\
$\mathbf{5 8}$ & $\mathrm{H}$ & 4.2868 & -4.7424 & -0.7881 \\
$\mathbf{5 9}$ & $\mathrm{H}$ & 4.3999 & -3.3921 & -1.9337 \\
$\mathbf{6 0}$ & $\mathrm{H}$ & 5.0632 & -3.2364 & -0.287 \\
$\mathbf{6 1}$ & $\mathrm{H}$ & 2.1109 & -2.93 & 1.3791 \\
$\mathbf{6 2}$ & $\mathrm{H}$ & 1.5354 & 0.2314 & 2.4571 \\
\hline
\end{tabular}


Table S79. Conformer 4d-G. PCM-CHCl 3 energy and Boltzmann Populations -3806.320636 hartrees, $16.33 \%$.

\begin{tabular}{|c|c|c|c|c|}
\hline \multicolumn{2}{|c|}{ Atom number } & \multicolumn{3}{|c|}{ Coordinates } \\
\hline & & $\mathbf{x}$ & $\mathbf{Y}$ & $\mathbf{Z}$ \\
\hline 1 & 0 & -3.0341 & -0.3084 & -0.7771 \\
\hline 2 & $\mathrm{C}$ & -1.7222 & 0.1585 & -0.4919 \\
\hline 3 & $\mathrm{C}$ & -1.2532 & -0.4087 & 0.8619 \\
\hline 4 & $\mathrm{C}$ & -2.191 & 0.1717 & 1.9321 \\
\hline 5 & $\mathrm{C}$ & -3.6644 & -0.1351 & 1.5898 \\
\hline 6 & $\mathrm{C}$ & -4.0039 & 0.2436 & 0.1397 \\
\hline 7 & $\mathrm{C}$ & -0.7352 & -0.1526 & -1.6036 \\
\hline 8 & $\mathrm{C}$ & 0.5959 & 0.5212 & -1.2259 \\
\hline 9 & $\mathrm{C}$ & 1.0995 & 0.089 & 0.1647 \\
\hline 10 & $\mathrm{O}$ & 0.0677 & 0.1314 & 1.142 \\
\hline 11 & $\mathrm{O}$ & 1.5666 & -1.268 & 0.1153 \\
\hline 12 & $\mathrm{C}$ & 2.8173 & -1.5414 & -0.5607 \\
\hline 13 & $\mathrm{C}$ & 3.9403 & -0.6823 & 0.0209 \\
\hline 14 & $\mathrm{C}$ & 3.5481 & 0.7967 & 0.065 \\
\hline 15 & $\mathrm{C}$ & 2.2214 & 0.9837 & 0.8046 \\
\hline 16 & $\mathrm{C}$ & -5.3881 & -0.1957 & -0.3947 \\
\hline 17 & C & -6.5155 & 0.5086 & 0.3628 \\
\hline 18 & 0 & -5.4799 & 0.2719 & -1.746 \\
\hline 19 & $\mathrm{C}$ & -5.5739 & -1.7221 & -0.3728 \\
\hline 20 & $\mathrm{C}$ & -1.2367 & -1.9406 & 0.9222 \\
\hline 21 & $\mathrm{C}$ & 1.8304 & 2.4642 & 0.9918 \\
\hline 22 & $\mathrm{C}$ & 3.0321 & -3.0706 & -0.3849 \\
\hline 23 & $\mathrm{C}$ & 1.9359 & -3.8634 & -1.1186 \\
\hline 24 & $\mathrm{C}$ & 4.4096 & -3.5061 & -0.8932 \\
\hline 25 & 0 & 3.0049 & -3.38 & 1.0108 \\
\hline 26 & $\mathrm{Br}$ & 2.009 & 3.6643 & -0.588 \\
\hline 27 & 0 & 2.4212 & 0.5171 & 2.1418 \\
\hline 28 & $\mathrm{H}$ & -1.7598 & 1.2568 & -0.3825 \\
\hline 29 & $\mathrm{H}$ & -1.9369 & -0.2269 & 2.9215 \\
\hline 30 & $\mathrm{H}$ & -2.0347 & 1.2574 & 1.971 \\
\hline 31 & $\mathrm{H}$ & -3.8691 & -1.198 & 1.7535 \\
\hline 32 & $\mathrm{H}$ & -4.318 & 0.4217 & 2.271 \\
\hline 33 & $\mathrm{H}$ & -3.9616 & 1.3413 & 0.04 \\
\hline 34 & $\mathrm{H}$ & -0.6159 & -1.2327 & -1.7353 \\
\hline 35 & $\mathrm{H}$ & -1.1019 & 0.2553 & -2.5522 \\
\hline 36 & $\mathrm{H}$ & 1.3631 & 0.3028 & -1.9742 \\
\hline 37 & $\mathrm{H}$ & 0.4614 & 1.6053 & -1.2375 \\
\hline 38 & $\mathrm{H}$ & 2.701 & -1.3281 & -1.6347 \\
\hline 39 & $\mathrm{H}$ & 4.1631 & -1.0373 & 1.0306 \\
\hline 40 & $\mathrm{H}$ & 4.8427 & -0.802 & -0.5879 \\
\hline 41 & $\mathrm{H}$ & 4.3171 & 1.3779 & 0.5876 \\
\hline 42 & $\mathrm{H}$ & 3.4683 & 1.2162 & -0.9437 \\
\hline 43 & $\mathrm{H}$ & -7.4671 & 0.2995 & -0.136 \\
\hline 44 & $\mathrm{H}$ & -6.5873 & 0.162 & 1.3986 \\
\hline 45 & $\mathrm{H}$ & -6.366 & 1.5937 & 0.3605 \\
\hline 46 & $\mathrm{H}$ & -4.6405 & 0.0149 & -2.1652 \\
\hline 47 & $\mathrm{H}$ & -5.6086 & -2.1234 & 0.6465 \\
\hline 48 & $\mathrm{H}$ & -6.5162 & -1.9719 & -0.8699 \\
\hline 49 & $\mathrm{H}$ & -4.7588 & -2.2173 & -0.91 \\
\hline 50 & $\mathrm{H}$ & -0.5606 & -2.3667 & 0.183 \\
\hline
\end{tabular}




\begin{tabular}{rrrrr}
$\mathbf{5 1}$ & $\mathrm{H}$ & -0.8952 & -2.2594 & 1.9128 \\
$\mathbf{5 2}$ & $\mathrm{H}$ & -2.2356 & -2.3475 & 0.7499 \\
$\mathbf{5 3}$ & $\mathrm{H}$ & 0.7979 & 2.5666 & 1.3219 \\
$\mathbf{5 4}$ & $\mathrm{H}$ & 2.5038 & 2.8951 & 1.7296 \\
$\mathbf{5 5}$ & $\mathrm{H}$ & 2.0879 & -4.9338 & -0.9482 \\
$\mathbf{5 6}$ & $\mathrm{H}$ & 0.9418 & -3.5987 & -0.7496 \\
$\mathbf{5 7}$ & $\mathrm{H}$ & 1.9614 & -3.6778 & -2.1996 \\
$\mathbf{5 8}$ & $\mathrm{H}$ & 4.5465 & -3.2435 & -1.9485 \\
$\mathbf{5 9}$ & $\mathrm{H}$ & 5.21 & -3.0497 & -0.3059 \\
$\mathbf{6 0}$ & $\mathrm{H}$ & 4.5027 & -4.5926 & -0.797 \\
$\mathbf{6 1}$ & $\mathrm{H}$ & 2.2566 & -2.8756 & 1.375 \\
$\mathbf{6 2}$ & $\mathrm{H}$ & 1.5409 & 0.2415 & 2.4564 \\
\hline
\end{tabular}


Table S80. DP4 results for $\mathbf{4 a - 4 d ~ ( I s o m e r s ~ 1 - 4 ) . ~}$

\begin{tabular}{|c|c|c|c|c|c|}
\hline Atom & Exp & 4a $(14 R-15 R)$ & 4b (14R-15S) & 4c $(14 S-15 R)$ & 4d (14S-15S) \\
\hline $\mathrm{C}$ & 86.1 & 106.636 & 106.601 & 106.148 & 105.913 \\
\hline C & 23.4 & 164.825 & 164.971 & 165.341 & 165.285 \\
\hline C & 38.5 & 151.646 & 151.809 & 152.594 & 152.580 \\
\hline C & 74 & 115.980 & 115.898 & 115.857 & 114.024 \\
\hline C & 75 & 115.639 & 115.247 & 111.019 & 110.417 \\
\hline C & 19.4 & 169.835 & 169.389 & 168.430 & 168.344 \\
\hline C & 23.2 & 165.787 & 164.899 & 165.702 & 163.200 \\
\hline C & 99.9 & 87.266 & 87.015 & 85.812 & 88.002 \\
\hline $\mathrm{C}$ & 71.6 & 116.973 & 116.999 & 116.462 & 117.375 \\
\hline $\mathrm{C}$ & 29.9 & 160.950 & 161.400 & 160.184 & 159.427 \\
\hline C & 24.8 & 165.944 & 167.737 & 166.587 & 168.197 \\
\hline C & 74.9 & 113.602 & 114.341 & 111.557 & 110.932 \\
\hline C & 19.7 & 171.076 & 170.851 & 171.232 & 170.781 \\
\hline $\mathrm{H}$ & 3.05 & 28.296 & 28.312 & 28.290 & 28.248 \\
\hline $\mathrm{H}$ & 1.78 & 29.853 & 29.872 & 29.784 & 29.757 \\
\hline $\mathrm{H}$ & 1.46 & 30.135 & 30.177 & 30.186 & 30.138 \\
\hline $\mathrm{H}$ & 1.84 & 29.856 & 29.900 & 29.951 & 29.927 \\
\hline $\mathrm{H}$ & 1.65 & 29.904 & 29.919 & 30.155 & 29.997 \\
\hline $\mathrm{H}$ & 3.87 & 27.660 & 27.570 & 28.371 & 28.276 \\
\hline $\mathrm{H}$ & 1.43 & 30.170 & 30.201 & 29.796 & 29.662 \\
\hline $\mathrm{H}$ & 1.83 & 29.766 & 29.690 & 30.154 & 30.183 \\
\hline $\mathrm{H}$ & 2.19 & 29.433 & 29.772 & 29.403 & 29.368 \\
\hline $\mathrm{H}$ & 1.62 & 30.167 & 29.581 & 29.491 & 30.163 \\
\hline $\mathrm{H}$ & 2.17 & 29.657 & 29.933 & 29.855 & 29.999 \\
\hline $\mathrm{H}$ & 1.8 & 30.047 & 29.937 & 30.226 & 30.346 \\
\hline $\mathrm{H}$ & 1.56 & 30.133 & 29.997 & 30.039 & 29.700 \\
\hline $\mathrm{H}$ & 1.24 & 30.231 & 30.316 & 30.320 & 30.468 \\
\hline $\mathrm{H}$ & 3.85 & 27.743 & 27.764 & 28.026 & 28.157 \\
\hline $\mathrm{H}$ & 1.2 & 30.439 & 30.485 & 30.295 & 30.208 \\
\hline \multirow{5}{*}{ DP4 (\%) } & $\mathrm{H}$ & 99.92 & 0.08 & 0.00 & 0.00 \\
\hline & $\mathrm{C}$ & 72.83 & 21.23 & 4.73 & 1.21 \\
\hline & $\mathrm{H}+\mathrm{C}$ & 99.98 & 0.02 & 0.00 & 0.00 \\
\hline & $J$ & - & - & - & - \\
\hline & all data & 99.98 & 0.02 & 0.00 & 0.00 \\
\hline
\end{tabular}


Biogenetic calculations were performed at $P C M($ water)-M06-2X//6-31+G(d,p) and PCM(water)-B3LYP-D3//6-31+G(d,p) with total energies(au) provided. Lowest conformer for each stationary point is reported.

\section{Computational details}

All computations were carried out at the B3LYP-D3/6-31+G(d,p) and M06-2X/6-31+G(d,p) level of theories, including an implicit solvent environment (PCM-water) (A.D. Becke, J.Chem.Phys. 1993, 98, 5648-5652; C. Lee, W. Yang, R.G. Parr, Phys. Rev. B 1988, 37, 785789; S.H. Vosko, L. Wilk, M. Nusair, Can. J. Phys. 1980, 58, 1200-1211; P.J. Stephens, F.J. Devlin, C.F. Chabalowski, M.J. Frisch, J.Phys.Chem. 1994, 98, 11623-11627) using GAUSSIANO9 (Gaussian 09, Revision D.01, M. J. Frisch, G. W. Trucks, H. B. Schlegel, G. E. Scuseria, M. A. Robb, J. R. Cheeseman, G. Scalmani, V. Barone, B. Mennucci, G. A. Petersson, H. Nakatsuji, M. Caricato, X. Li, H. P. Hratchian, A. F. Izmaylov, J. Bloino, G. Zheng, J. L. Sonnenberg, M. Hada, M. Ehara, K. Toyota, R. Fukuda, J. Hasegawa, M. Ishida, T. Nakajima, Y. Honda, O. Kitao, H. Nakai, T. Vreven, J. A. Montgomery, Jr., J. E. Peralta, F. Ogliaro, M. Bearpark, J. J. Heyd, E. Brothers, K. N. Kudin, V. N. Staroverov, T. Keith, R. Kobayashi, J. Normand, K. Raghavachari, A. Rendell, J. C. Burant, S. S. Iyengar, J. Tomasi, M. Cossi, N. Rega, J. M. Millam, M. Klene, J. E. Knox, J. B. Cross, V. Bakken, C. Adamo, J. Jaramillo, R. Gomperts, R. E. Stratmann, O. Yazyev, A. J. Austin, R. Cammi, C. Pomelli, J. W. Ochterski, R. L. Martin, K. Morokuma, V. G. Zakrzewski, G. A. Voth, P. Salvador, J. J. Dannenberg, S. Dapprich, A. D. Daniels, O. Farkas, J. B. Foresman, J. V. Ortiz, J. Cioslowski, and D. J. Fox, Gaussian, Inc., Wallingford CT, 2013.).

Structures are confirmed to be minima by frequency analysis. Transition state structures were also confirmed to have relevant vibrational mode by frequency analysis. For each structure, electronic energy (EE), EE + zero-point energy, free energy ( $E E+$ Thermal Free Energy Correction), and the lowest frequency are shown. 
Table S81. Cartesian coordinates structures in Path A.

\section{Protonated 14-Ketodehydrothyrsiferol}

$E E=-927.538816$

EE + Zero-point Energy $=-927.099201$

$\mathrm{EE}+$ Thermal Free Energy Correction $=-927.149562$

Number of imaginary freq. $=0$

Lowest freq. $=17.54$

M06-2X/6-31+G(d,p), scrf $=(p c m$, Solvent $=$ Water $)$

11

C

$-1.70701000$

1.78880200

0.17349000

C

$-2.14605900$

$-0.53629200$

0.44826100

C

$-3.38683600$

$-0.51666700$

$-0.46365000$

C

$-3.98028700$

0.89718300

$-0.40518800$

C

$-2.93331700$

1.96797300

$-0.71619900$

C

$-0.57329700$

2.73079000

$-0.17512600$

C

$-3.06176400$

$-0.93686700$

$-1.89527900$

C

$-1.40596500-1.86148400$

0.48881600

$-0.18936000$

$-1.76318300$

1.42300300

0.88999300

$-0.91245000$

0.83173200

1.56399400

0.19746400

1.51776200

2.56896200

1.00992500

0.73812200

1.28870500

0.44804400

2.80842600

4.24312600

0.23678300

$-1.01354800$

5.56989000

$-0.42809000$

$-1.31980200$

3.90485400

0.27338100

0.46727700

$-2.00159600$

1.93186900

1.22702500

$-4.82841300$

0.96848600

$-1.09675600$

$-4.37039400$

1.05096400

0.60931700

$-2.61780200$

1.91236500

$-1.76569200$

$-3.35430700$

2.96573800

$-0.55443600$

$-2.49510500$

$-0.29267600$

1.46757300

$-0.92297500$

3.76615900

$-0.14459300$

0.24918000

2.62502100

0.53666300

$-0.20262200$

2.51726700

$-1.18306100$

$-2.81061600$

$-1.99974800$

$-1.93303700$

$-3.92969700$

$-0.76602600$

$-2.54102700$

$-2.21958800$

$-0.36447200$

$-2.29378800$

$-5.12046500$

$-1.43350400$

$-0.35862000$

$-1.07411500$

$-2.15015600$

$-0.51223400$

$-2.07973500$

$-2.63681600$

0.85968200

0.25631500

$-2.75677300$

1.55126000

$-0.49716600$

$-1.40516200$

2.40342800

2.11557100

1.31602700

$-0.21353700$

2.75841400

1.93105200

1.29248900

0.58568700

$-0.13010600$

3.39463100

1.79302400

1.26230600

3.31968700

4.21318700

1.25214100

$-1.42457400$

5.73595500

$-0.48134500$

$-2.39879100$

6.38501500

0.14851700

$-0.87447200$

5.58895700

$-1.44155000$

$-0.90879900$

3.03681300

$-0.28191100$

$-2.55906000$

2.06220700

$-0.82404200$

$-0.85752400$

3.87313100

$-0.75900000$

0.83993900

4.72033200

0.76765800

1.00051900

$-1.21072000$

0.45126200

0.03741400

$-4.28850700$

$-1.44926000$

0.13275900 


\section{Protonated 14-Ketodehydrothyrsiferol}

$E E=-928.022203$

EE + Zero-point Energy $=-927.585395$

$\mathrm{EE}+$ Thermal Free Energy Correction $=-927.635791$

Number of imaginary freq. $=0$

Lowest freq. $=16.55$

B3LYP(D3)/6-31+G(d,p), scrf $=(p c m$, Solvent=Water $)$

11

C

C

$-1.69716000$

$-2.16064500$

1.77324800

0.37729600

$-3.44914700$

$-0.58720900$

0.35637700

C

$-4.01858600$

$-0.42834300$

$-0.48850300$

C

$-2.97196800$

0.97549700

$-0.20454100$

C

$-0.56464800$

2.07804000

$-0.41368800$

C

$-3.20757900$

2.74472500

0.09240400

C

$-1.43134100$

$-0.65822400$

$-1.98409000$

C

$-0.19246200$

$-1.91191300$

0.18370800

0.89644700

$-1.98095100$

1.10237200

1.57881600

$-1.05119600$

0.66182500

C

2.58910900

$-0.07866300$

1.51759800

1.31029600

0.86761500

0.90078400

C

4.31291600

$-0.05280100$

2.84086100

C

5.66947900

0.40126000

$-0.93317200$

C

3.95543900

$-0.16844700$

$-1.31598900$

C

$-1.93055800$

0.21504500

0.53664300

$\mathrm{H}$

$-4.89662300$

1.77947100

1.45487400

$-4.36165700$

1.14939200

$-0.83819300$

$\mathrm{H}$

$-2.71529900$

0.98806900

0.83769600

$-3.37306000$

2.17126700

$-1.47575800$

$-2.46006400$

3.04520000

$-0.09067000$

$-0.89656800$

$-0.48396800$

1.41294100

$\mathrm{H}$

0.29443300

3.77056800

0.27935600

$-0.25027600$

2.54125800

0.73528200

$-2.95777300$

2.66632100

$-0.95371400$

$-4.11180900$

$-1.70449700$

$-2.17662200$

$-2.39117000$

$-0.41383800$

$-2.55199500$

$-5.20191400$

$-0.03171900$

$-2.35305400$

$-1.12260800$

$-1.33530400$

$-0.41976100$

$-2.10796100$

$-2.05019900$

$-0.85489000$

0.23856200

$-2.72803200$

0.44549000

$-0.48497200$

$-2.98838500$

1.04752400

2.14937100

$-1.80228300$

2.13498400

2.74957100

1.32339500

0.00676000

0.60455300

1.68695200

1.60409900

1.82132700

$-0.71726200$

3.32256900

4.25676900

0.65544300

3.48524800

5.84654600

1.46540200

$-1.19249400$

6.46223300

$-0.05645500$

$-2.39004000$

5.72425100

0.36403700

$-0.78105500$

3.16907400

$-1.23129600$

$-1.05975300$

2.06336400

0.05942600

$-2.57641900$

$\mathrm{H}$

3.95573200

$-0.72438200$

$-0.96746300$

$\mathrm{H}$

4.74972700

$-0.85868300$

0.76146700

O

$-1.22700700$

0.65508000

1.14546400

0.44970900

0.03685000 


$\begin{array}{lrrr}\mathrm{O} & -4.34818900 & -1.43106400 & 0.02631000 \\ \mathrm{O} & 1.24704100 & -1.22815600 & -0.55487700 \\ \mathrm{O} & 3.26103200 & -0.29312400 & -1.67968100\end{array}$

\section{TSS to INT1A(S)}

$E=-927.525903$

$E E+$ Zero-point Energy $=-927.083661$

$\mathrm{EE}+$ Thermal Free Energy Correction $=-927.130539$

Number of imaginary freq. $=1$

Lowest freq. $=-139.10$

M06-2X/6-31+G(d,p), scrf $=(p c m$, Solvent $=$ Water $)$

11

C

$-1.19401100$

0.13670600

0.70431100

C

$-1.77545200$

$-0.57181900$

$-0.53357300$

C

$-3.61738400$

0.90952200

$-0.68566100$

C

$-3.08235700$

1.77363900

0.45619400

C

$-1.56498600$

1.61733800

0.60256500

$\mathrm{H}$

$-1.36672600$

$-0.05535900$

$-1.42247600$

$\mathrm{H}$

$-3.20948300$

1.28769000

$-1.63819500$

$-3.33277100$

2.82101700

0.25986900

$\mathrm{H}$

$-3.58704900$

1.48766800

1.38684000

$-1.07195900$

2.04205700

$-0.28351300$

$-5.13137600$

0.88938500

$-0.74563100$

$-5.51665600$

1.90252600

$-0.88780700$

$-5.47505400$

0.26551400

$-1.57440800$

$-5.53817300$

0.48985700

0.18860800

$-1.42191400$

$-2.04838900$

$-0.63463000$

$-1.68864300$

$-2.58168100$

0.28277600

$-2.01479300$

$-2.47986400$

$-1.44519000$

2.49143400

$-0.36652200$

$-1.35551100$

2.81151000

$-0.89788200$

$-2.25822900$

1.51280400

0.06517300

$-1.58203400$

3.47255700

0.77610700

$-1.07096800$

3.52558500

1.40791200

$-1.96468300$

4.48133700

0.38913700

$-0.89419100$

3.07584100

1.64645900

0.11345500

3.08021000

1.04575400

1.03439200

4.00243100

2.83832000

0.27850700

3.98810100

3.45752900

$-0.62363200$

3.70479000

3.45190300

1.13410600

5.02608500

2.49548700

0.45175800

1.72225800

2.08363300

$-0.12722900$

1.52400100

2.83076200

0.45439700

$-3.18425600$

$-0.44836600$

$-0.53807000$

0.23877900

$-0.01643200$

0.65668900

2.36548300

$-1.37130200$

$-0.22463600$

3.41586800

$-1.89649500$

0.42085000

4.42743200

$-1.58561800$

0.18020800

3.32549100

$-2.68193500$

1.16502400

$-1.19635100$

2.15696000

1.48208400

$-1.67428400$

$-0.47731900$

2.01539300

$-1.37227800$

0.16072700

2.85097000

$-2.76194900$

$-0.57569300$

2.02061000

$-1.23830400$

$-1.46636500$

2.16711200

$1.02874200-1.90945700$

0.09223600

$0.79726600-2.49906600$

1.21974000

1.44807900

$-2.26932600$

1.90662600 


$\begin{array}{rrrr}\mathrm{C} & 0.05540500 & -2.25795200 & -0.98431200 \\ \mathrm{H} & 0.24762000 & -3.32316900 & -1.17421000 \\ \mathrm{H} & 0.31652700 & -1.72248400 & -1.89835600 \\ \mathrm{H} & 0.68224800 & 0.79422200 & 0.30096100\end{array}$

TSS to INT1A(S)

$E E=-928.009151$

$\mathrm{EE}+$ Zero-point Energy $=-927.570220$

$\mathrm{EE}+$ Thermal Free Energy Correction $=-927.616849$

Number of imaginary freq. $=1$

Lowest freq. $=-148.81$

B3LYP(D3)/6-31+G(d,p), scrf=(pcm, Solvent=Water $)$

11

C

C

C

C

C

$\mathrm{H}$

$\mathrm{H}$

$\mathrm{H}$

$\mathrm{H}$

$\mathrm{H}$

C

$\mathrm{H}$

$\mathrm{H}$

$\mathrm{H}$

C

$\mathrm{H}$

$\mathrm{H}$

C

$\mathrm{H}$

$\mathrm{H}$

$\mathrm{C}$

$\mathrm{H}$

$\mathrm{H}$

C

$\mathrm{H}$

C

$\mathrm{H}$

$\mathrm{H}$

$\mathrm{H}$

$\mathrm{O}$

$\mathrm{H}$

O

O

C

C

$\mathrm{H}$

$\mathrm{H}$

$\mathrm{H}$

C

$\mathrm{H}$

$\mathrm{H}$

$\mathrm{H}$

C

$-1.19571900$

0.08077300

0.68985200

$-3.61278700$

$-0.59099500$

$-0.56571700$

$-3.03303300$

0.94789000

$-0.68030100$

$-1.51402100$

1.78694000

0.46611600

$-1.38396300$

1.58046600

0.61141200

$-3.21048800$

$-0.06057900$

$-1.44200300$

$-3.24136600$

1.32430200

2.84506500

$-1.63566700$

$-3.54302000$

1.52168300

0.27556000

$-1.00829100$

2.00586000

1.39889800

$-5.13150300$

0.96607600

$-0.26441000$

$-5.49221900$

1.99123800

$-0.72206300$

$-5.50060700$

0.36026200

$-0.85084600$

$-5.54305400$

0.56756900

$-1.55476000$

$-1.44411200$

$-2.06666300$

0.21130800

$-1.75340500$

$-2.62834200$

$-0.70050800$

$-1.99306600$

$-2.47483500$

0.18485900

2.53707200

$-0.36999300$

$-1.55360000$

2.91092100

1.57054900

$-0.91738700$

$-1.37469500$

3.49365500

0.04208600

$-2.24675300$

3.52776800

0.80043800

$-1.67106600$

4.51152300

1.44048200

$-1.08029900$

3.09821200

0.43298300

$-1.96933600$

3.17773800

1.64718600

$-0.91313600$

3.94321500

1.04570900

0.12671300

3.83987600

2.90560000

1.04170200

3.64832300

3.53730300

0.26448100

4.99732100

3.48353000

$-0.62372900$

1.68826200

2.63482200

1.14689600

1.46356600

1.99402400

0.37978300

$-3.20936000$

2.71066700

$-0.04677500$

0.26549800

$-0.43587700$

0.56572000

2.36167300

$-0.10645900$

$-0.55358600$

3.40001400

$-1.35289900$

0.62558500

4.41952400

$-1.92605000$

$-0.22512600$

3.28712900

$-1.67868200$

0.40218600

$-1.13700800$

$-2.68212000$

0.12540400

$-1.66462400$

2.09627800

1.17192200

1.50070800

$-1.26406500$

$-0.53806400$

2.00559100

$-2.75439700$

0.03905300

2.84367800

$-1.32507500$

$-0.52935600$

2.05873800

0.97936000

$-1.56842100$

2.10558800

0.77461500

$-1.78717300$

0.13107800

$\mathrm{O}$

$-2.46050000$

1.25326300 


$\begin{array}{lrrr}\mathrm{H} & 1.38470300 & -2.18566400 & 1.95873500 \\ \mathrm{C} & 0.05746400 & -2.24258400 & -0.97452800 \\ \mathrm{H} & 0.29766600 & -3.30406400 & -1.11117300 \\ \mathrm{H} & 0.32867000 & -1.73573800 & -1.90175000 \\ \mathrm{H} & 0.75493900 & 0.71111800 & 0.29568500\end{array}$

\section{TSS to INT1A(R)}

$E E=-927.522663$

EE + Zero-point Energy $=-927.080788$

$\mathrm{EE}+$ Thermal Free Energy Correction= -927.127159

Number of imaginary freq. $=1$

Lowest freq. $=-168.99$

M06-2X/6-31+G(d,p), scrf $=(p c m$, Solvent=Water $)$

11

C

1.25551000

$-0.53745000$

0.16010900

C

2.07596400

0.56140400

$-0.53600000$

C

$4.10300000-0.62264700$

$-0.29703200$

C

$\begin{array}{lll}3.37880800 & -1.85291700 & 0.25328400\end{array}$

C

$\begin{array}{lll}1.90419800 & -1.87998300 & -0.17205200\end{array}$

$\begin{array}{llll}2.13333900 & 0.28830800 & -1.60440100\end{array}$

$\mathrm{H}$

4.15910800

$-0.70446800$

$-1.39499500$

$\mathrm{H}$

3.88349300

$-2.75327500$

$-0.11040700$

$3.45984400-1.85716500 \quad 1.34657400$

$\mathrm{H}$

$1.82942700-2.04055800$

$-1.25429800$

$5.49444000-0.45963200$

0.28007300

6.10147600

$-1.33957900$

0.05140400

5.98397900

0.42167600

$-0.14139000$

5.43845700

$-0.34624300$

1.36723700

1.45117700

1.94466400

$-0.43814700$

1.30995500

2.23358100

0.60798600

2.14952400

2.66057200

$-0.87979100$

$-2.33565800$

0.34069100

1.41459800

$-1.76455500$

$-0.59296200$

1.46878700

$-2.40715600$

0.69915300

2.44341800

$-3.75815700$

0.04168500

0.88186600

$-4.07641900$

0.81000900

0.16561600

$-4.47112400$

0.07796500

1.70975700

$-3.86849500$

$-1.32867300$

0.23288800

$-3.65548300$

$-2.10576400$

0.97718200

$-5.21841200$

$-1.57106400$

$-0.41491200$

$-5.41285100$

$-0.81479300$

$-1.18150300$

$-5.26184600$

$-2.56244500$

$-0.87521200$

$-6.00712900$

$-1.51513300$

0.34007500

$-2.81003800$

$-1.37449900$

$-0.75269100$

$-2.92172300$

$-2.13243200$

$-1.34376800$

3.37706500

0.57754100

$-0.03571300$

$-0.45617100$

0.00831700

$-1.54894600$

1.39659200

$-0.50784400$

$-1.39147800$

2.61825100

0.66339600

$\begin{array}{ll}-1.83417200 & 2.85438300\end{array}$

1.18643600

$-0.85085000$

3.41647700

2.14934100

$1.37221300-2.69666700$

0.69166500

1.10778800

$-0.33119500$

0.32804900

0.60734200

$-1.19300000$

1.66154800

C

2.09490600

$-0.23079100$

2.11429500

$\mathrm{H}$

0.53891800

0.56913200

2.11636900

$-0.99849000$

1.17126200

1.89699900

C

$-0.71575000$ 


$\begin{array}{lrrr}\mathrm{O} & -1.80065800 & 0.78004500 & -1.66689600 \\ \mathrm{H} & -2.43484400 & 0.06094800 & -1.37070900 \\ \mathrm{H} & -0.64710400 & -1.17989600 & -0.27246200 \\ \mathrm{C} & 0.14097500 & 2.01326800 & -1.23366500 \\ \mathrm{H} & -0.22610900 & 3.04317200 & -1.28867600 \\ \mathrm{H} & 0.31080600 & 1.68893900 & -2.26406800\end{array}$

\section{TSS to INT1A(R)}

$E E=-928.002688$

EE + Zero-point Energy $=-927.500889$

$\mathrm{EE}+$ Thermal Free Energy Correction $=-927.609163$

Number of imaginary freq. $=1$

Lowest freq. $=-95.41$

B3LYP(D3)/6-31+G(d,p), scrf $=(p c m$, Solvent=Water $)$

11

C

1.18630800

$-0.47658200$

0.16798600

C

2.06242000

0.59562900

$-0.51507600$

C

$4.03953000-0.70294700$

$-0.28456100$

C

$\begin{array}{lll}3.24120800 & -1.90865500 & 0.23369000\end{array}$

C

$1.75997500-1.85079500$

2.11348600

0.33088700

$-0.19070800$

$\mathrm{H}$

4.10483200

$-0.76445500$

$-1.58418300$

$\mathrm{H}$

$3.69454200-2.82680400$

$-1.38312600$

$\mathrm{H}$

$\begin{array}{llll}3.31959800 & -1.94911600 & 1.32586100\end{array}$

$\mathrm{H}$

$1.67430900-1.99087400$

$-1.27429600$

$5.43543400-0.61821300$

0.30906000

6.00327600

$-1.52178700$

0.06684100

5.97106100

0.24734400

$-0.09140400$

5.37983700

$-0.52379700$

1.39868100

1.48879500

2.00427200

$-0.41423600$

1.35963400

2.30333200

0.62940400

2.20461200

2.69821800

$-0.86358600$

$-2.34782400$

0.41583500

1.42241200

$-1.71613600$

$-0.46480900$

1.56972900

$-2.51041100$

0.82188200

2.42276200

$-3.71515600$

$-0.03841400$

0.84019800

$-4.08235400 \quad 0.67615900$

0.09370300

$-4.45822900$

$-0.04730900$

1.64289700

$-3.68355600-1.43913500$

0.23770300

$-3.40439600$

$-2.16545700$

1.01133200

$-4.99648400$

$-1.84294400$

$-0.41595400$

$-5.26273500$

$-1.13944800$

$-1.21153900$

$-4.93141800$

$-284996500$

$-0.84119500$

$-5.79699800-1.84550500$

0.33020500

$-2.60290400$

$-1.42376900$

$-0.74909400$

$-2.65081800$

$-2.20853500$

$-1.31560800$

3.36749300

0.53950200

0.04013000

$-0.11449700$

$-0.32510900$

$-0.53537000$

$-1.57967500$

1.48288200

0.65729000

$-1.47156400$

2.72000400

1.16453400

$\begin{array}{ll}-1.94970700 & 2.96570100\end{array}$

2.10755000

$-0.932745003 .52445700$

0.67979000

$1.18802800-2.64745300$

0.29703200

$1.02738500-0.28430900$

1.67219200

$0.47399700-1.11891600$

2.11138400

$2.01560600-0.25131100$

2.13289300

$\mathrm{H}$

0.51795100

0.64679300

1.91695600 


$\begin{array}{lrrr}\mathrm{C} & -0.96477200 & 1.22268800 & -0.70235800 \\ \mathrm{O} & -1.79574700 & 0.92357500 & -1.70700000 \\ \mathrm{H} & -2.42637000 & 0.20681500 & -1.45923200 \\ \mathrm{H} & -0.79063000 & -1.02354300 & -0.36440600 \\ \mathrm{C} & 0.17037200 & 2.09888100 & -1.20151700 \\ \mathrm{H} & -0.19568400 & 3.12857000 & -1.22633300 \\ \mathrm{H} & 0.33873100 & 1.80616000 & -2.24147200\end{array}$

\section{INT1A(S)}

$E E=-927.539024$

$\mathrm{EE}+$ Zero-point Energy $=-927.098537$

$\mathrm{EE}+$ Thermal Free Energy Correction $=-927.145210$

Number of imaginary freq. $=0$

Lowest freq. $=35.99$

M06-2X/6-31+G(d,p), scrf $=(\mathrm{pcm}$, Solvent=Water $)$

11

C

C

C

C

C

$\mathrm{H}$

$\mathrm{H}$

$\mathrm{H}$

$\mathrm{H}$

$\mathrm{H}$

$$
-1.14065100
$$

0.16074400

0.52786900

$-1.90582400$

$-3.66131200$

$-0.70843300$

$-0.48227200$

$-2.91098600$

0.83981000

$-0.70586400$

$-1.39639300$

1.87958800

0.12898500

$-1.60453200$

1.62137100

0.16119300

$-3.38436100$

$-0.36716500-1.48915900$

$-3.09892600$

$0.96407600-1.76560300$

$-3.31623500$

$2.87294300-0.28948900$

$-0.96315800$

1.87849100

1.14733900

C

$-5.16438300$

1.81539300

$-0.82814000$

$\mathrm{H}$

$-5.50547000$

0.94597000

$-0.54921600$

$\mathrm{H}$

$-5.66805900$

1.93917300

$-0.85288800$

$\mathrm{H}$

$-5.44477800$

0.19908400

$-1.16760300$

C

$-1.54896300$

0.78500300

0.49641500

$-1.78028100$

$-2.17549400$

$-0.36032400$

$\mathrm{H}$

$\mathrm{H}$

$-2.13605100$

$-2.55515300$

0.63961800

2.44702800

$-2.75094500$

$-1.08110300$

C

$\mathrm{H}$

2.82326200

$-0.38094800-1.49994200$

$\mathrm{H}$

1.49013900

$-0.93583700$

$-2.36423700$

C

3.44169300

$0.05031400-1.80834500$

3.38555800

0.75310800

$-1.18633400$

$\mathrm{H}$

4.46356900

1.50250200

$-1.98329400$

$\mathrm{H}$

3.24111500

0.35917500

$-1.18338000$

C

3.46659300

1.43193400

0.15827500

4.04905000

0.73606000

0.97282100

C

3.76701800

2.70457700

0.30354300

$\mathrm{H}$

$\mathrm{H}$

3.90555900

3.42518700

$-0.46902200$

5.11106800

3.15763900

1.28835500

1.81428300

2.47160200

0.19346800

$\mathrm{O}$

1.67929100

1.76583500

0.27387400

$-3.28773800$

2.35496500

1.03381900

$\mathrm{O}$

0.29051200

$-0.49054600$

$-0.31693600$

2.24051100

$-0.10071100$

0.27237900

C

3.22407300

$-1.34048300$

$-0.34130700$

C

4.22198500

$-2.09725800$

0.14715400

3.06638900

$-2.04578500$

$-0.27938200$

$\mathrm{H}$

$-0.92144000$

$-2.79659000$

0.96155900

$\mathrm{H}$

$-1.46759100$

2.29677900

0.88078500

C

$-0.85167200$

$-0.12800600$

1.98644400

$\mathrm{H}$

0.50863800

2.62850800

$\mathrm{H}$

$-2.51802900$

0.10069600

2.17345400 


$\begin{array}{lrrr}\mathrm{H} & -1.28557700 & -1.16806200 & 2.25303100 \\ \mathrm{C} & 0.83627100 & -1.48266600 & 0.22746100 \\ \mathrm{O} & 0.79522100 & -2.02630300 & 1.49321800 \\ \mathrm{H} & 1.43616800 & -1.57964200 & 2.06658700 \\ \mathrm{C} & -0.05923400 & -2.32396700 & -0.68017700 \\ \mathrm{H} & 0.27691800 & -3.35775300 & -0.57020600 \\ \mathrm{H} & 0.12467100 & -2.02395100 & -1.71731700 \\ \mathrm{H} & 1.01572800 & 0.77787700 & 0.32020600\end{array}$

INT1A(S)

$E E=-928.013304$

$E E+$ Zero-point Energy $=-927.575118$

$\mathrm{EE}+$ Thermal Free Energy Correction $=-927.622868$

Number of imaginary freq. $=0$

Lowest freq. $=16.51$

B3LYP(D3)/6-31+G(d,p), scrf=(pcm, Solvent=Water $)$

11

$\begin{array}{llll}\text { C } & -1.16115900 & 0.20493800 & 0.50622600\end{array}$

C $\quad-1.93629700 \quad-0.73042800 \quad-0.44372400$

$\begin{array}{llll}\text { C } & -3.72537800 & 0.80305700 & -0.71200700\end{array}$

$\begin{array}{llll}\text { C } & -2.95784200 & 1.89352900 & 0.04983500\end{array}$

C $\quad-1.43499600 \quad 1.64560000 \quad 0.06286000$

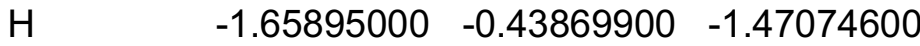

$\mathrm{H} \quad-3.47658400 \quad 0.87313700 \quad-1.78354800$

$\mathrm{H} \quad-3.16065700 \quad 2.86276200 \quad-0.41717400$

$\mathrm{H} \quad-3.33816400 \quad 1.94797900 \quad 1.07581200$

$\mathrm{H} \quad-1.02404500 \quad 1.78787500 \quad-0.94296600$

C $\quad-5.23014300 \quad 0.90075200 \quad-0.52796900$

$\mathrm{H} \quad-5.59041300 \quad 1.87080000 \quad-0.88404500$

$\mathrm{H} \quad-5.73780100 \quad 0.11281000 \quad-1.09182600$

H $\quad-5.49201700 \quad 0.79972200 \quad 0.53053900$

C $\quad-1.56169800 \quad-2.19191200 \quad-0.26295000$

$\mathrm{H} \quad-1.77052700 \quad-2.53077100 \quad 0.75557200$

$\mathrm{H} \quad-2.16140400-2.80172800 \quad-0.94480400$

C $\quad 2.45789000 \quad-0.39538100 \quad-1.52748700$

$\mathrm{H} \quad 2.85383400 \quad-0.94391000 \quad-2.38751300$

$\mathrm{H} \quad 1.50001300 \quad 0.01964900 \quad-1.84760100$

C $\quad 3.44026300 \quad 0.75973700 \quad-1.20906800$

$\mathrm{H} \quad 3.35094700 \quad 1.52006100 \quad-1.99293000$

$\begin{array}{lll}4.46753300 & 0.38096800 & -1.24457300\end{array}$

C

$0.38096800-1.24457300$

$3.28480700 \quad 1.41932600 \quad 0.15729500$

$\begin{array}{lll}3.51343800 & 0.69738300 & 0.94802900\end{array}$

$\begin{array}{lll}4.14124400 & 2.66476500 & 0.31663600\end{array}$

$3.87952400 \quad 3.41264700 \quad-0.43808500$

$\begin{array}{lll}4.01879600 & 3.10484400 & 1.31161300\end{array}$

$\begin{array}{lll}5.19596700 & 2.40024800 & 0.19632100\end{array}$

$\begin{array}{lll}1.86181400 & 1.78937900 & 0.32096200\end{array}$

$\begin{array}{lll}1.76013600 & 2.32682200 & 1.12308200\end{array}$

$-3.32655800 \quad-0.51524000 \quad-0.26076200$

$\begin{array}{lll}0.28354500 & -0.07372500 & 0.21001300\end{array}$

$\begin{array}{llll}2.25149300 & -1.36240700 & -0.37138700\end{array}$

$3.23182300 \quad-2.14784900 \quad 0.08869500$

$\begin{array}{lll}4.21963700 & -2.11653700 & -0.36132500\end{array}$

$3.08293300 \quad-2.84817800 \quad 0.90347700$

$\begin{array}{lll}-0.94584000 & 2.36160500 & 0.73074700\end{array}$

$\begin{array}{lrrr}\mathrm{C} & -1.42988400 & -0.00842900 & 1.99205400 \\ \mathrm{H} & -0.80266900 & 0.66867400 & 2.57890400\end{array}$ 


$\begin{array}{rrrr}\mathrm{H} & -2.47640200 & 0.21441700 & 2.20450400 \\ \mathrm{H} & -1.22392100 & -1.02995400 & 2.30445100 \\ \mathrm{C} & 0.85823400 & -1.48691300 & 0.23562900 \\ \mathrm{O} & 0.83850700 & -1.95933900 & 1.53231700 \\ \mathrm{H} & 1.50483700 & -1.50045100 & 2.06760300 \\ \mathrm{C} & -0.07408400 & -2.35822400 & -0.61122300 \\ \mathrm{H} & 0.25649000 & -3.38941900 & -0.46666100 \\ \mathrm{H} & 0.08759600 & -2.10624400 & -1.66366800 \\ \mathrm{H} & 0.98362000 & 0.75392300 & 0.32607800\end{array}$

INT1A(R)

$E E=-927.528802$

EE + Zero-point Energy $=-927.086403$

$\mathrm{EE}+$ Thermal Free Energy Correction $=-927.132594$

Number of imaginary freq. $=0$

Lowest freq. $=47.25$

M06-2X/6-31+G(d,p), scrf $=(p c m$, Solvent=Water $)$

11

C

C

1.03329700

$-0.33503400$

0.15158100

2.01045000

0.66071700

$-0.49274000$

C

$3.83850300-0.80628800$

$-0.26973900$

C

$2.93045300-1.94160100$

0.21134400

C

1.47288900

$-1.74269700$

$-0.23336000$

$\mathrm{H}$

$2.05475900 \quad 0.42202300 \quad-1.56937500$

$\mathrm{H}$

$\begin{array}{llll}3.91195700 & -0.84927100 & -1.36853700\end{array}$

$3.30620900-2.88850000-0.18739000$

$\begin{array}{lll}2.98671400 & -2.00697600 & 1.30414000\end{array}$

$\begin{array}{lll}1.39513200 & -1.84810400 & -1.32200200\end{array}$

$\begin{array}{lll}5.22154500 & -0.87130000 & 0.34468300\end{array}$

$\begin{array}{llll}5.70006300 & -1.82219200 & 0.09607700\end{array}$

$\begin{array}{lll}5.84656400 & -0.05703200 & -0.02963100\end{array}$

$\begin{array}{lll}5.15146100 & -0.79004500 & 1.43385500\end{array}$

$\begin{array}{lll}1.52967300 & 2.09143700 & -0.34326500\end{array}$

$\begin{array}{lll}1.41780300 & 2.36083800 & 0.71249600\end{array}$

$\begin{array}{lll}2.26914100 & 2.76756200 & -0.77996000\end{array}$

$\begin{array}{lll}-2.35722900 & 0.49304600 & 1.38584000\end{array}$

$\begin{array}{lll}-1.64601900 & -0.28276300 & 1.68876400\end{array}$

$\begin{array}{lll}-2.70937100 & 0.94201900 & 2.31629800\end{array}$

$\begin{array}{lll}-3.55559200 & -0.20093200 & 0.69093200\end{array}$

$\begin{array}{lll}-3.95537600 & 0.38506000 & -0.14714100\end{array}$

$\begin{array}{lll}-4.37945400 & -0.26289200 & 1.40760000\end{array}$

$\begin{array}{lll}-3.27597800 & -1.62866000 & 0.24780900\end{array}$

$\begin{array}{lll}-2.94324200 & -2.21623100 & 1.11104500\end{array}$

$-4.46810500 \quad-2.28449900 \quad-0.42070900$

$-4.77630700 \quad-1.70870700 \quad-1.29805700$

$-4.23346300-3.30655300-0.73067900$

$\begin{array}{lll}-5.30321700 & -2.33039400 & 0.28243600\end{array}$

$\begin{array}{lll}-2.16864000 & -1.58215700 & -0.69867800\end{array}$

$\begin{array}{lll}-2.00886600 & -2.45513600 & -1.08840700\end{array}$

$\begin{array}{lll}3.28458100 & 0.47394400 & 0.07496700\end{array}$

$\begin{array}{lll}-0.25786100 & -0.09045900 & -0.53696900\end{array}$

$\begin{array}{lll}-1.62015900 & 1.59511200 & 0.64320800\end{array}$

$\begin{array}{lll}-1.62119800 & 2.84194600 & 1.12107800\end{array}$

$\begin{array}{lll}-2.17377100 & 3.07902900 & 2.02474300\end{array}$

$\begin{array}{lll}-1.08911000 & 3.66052000 & 0.65084800\end{array}$

$\begin{array}{lll}0.82386200 & -2.49356300 & 0.23016900\end{array}$

$\begin{array}{lll}0.91236600 & -0.16073400 & 1.65815500\end{array}$ 


$\begin{array}{rrrr}\mathrm{H} & 0.37040000 & -0.99964500 & 2.10230200 \\ \mathrm{H} & 1.91740800 & -0.14051400 & 2.08416900 \\ \mathrm{H} & 0.42005800 & 0.77309200 & 1.93338800 \\ \mathrm{C} & -0.89379200 & 1.28261700 & -0.66168700 \\ \mathrm{O} & -1.75614300 & 1.15294800 & -1.73165100 \\ \mathrm{H} & -2.59032200 & 0.73787600 & -1.47268400 \\ \mathrm{H} & -1.02751700 & -0.84384300 & -0.51943100 \\ \mathrm{C} & 0.21495000 & 2.22535200 & -1.11207200 \\ \mathrm{H} & -0.17095400 & 3.24485100 & -1.08670600 \\ \mathrm{H} & 0.38751900 & 1.98438500 & -2.16603700\end{array}$

\begin{tabular}{|c|c|c|c|}
\hline $\begin{array}{l}\text { EE } \\
\text { EE } \\
\text { NE } \\
\text { Lov } \\
\text { B3l } \\
11\end{array}$ & $\begin{array}{l}3897 \\
\text { int Energy = } \\
\text { l Free Energ, } \\
\text { laginary freq. } \\
=40.53 \\
-31+G(\mathrm{~d}, \mathrm{p}), \mathrm{s}\end{array}$ & $\begin{array}{l}-927.564921 \\
\text { Correction= }-\mathrm{c} \\
=0\end{array}$ & 927.611690 \\
\hline C & 1.06432900 & -0.35886000 & 0.16664900 \\
\hline C & 2.02552000 & 0.65599300 & -0.48400400 \\
\hline C & 3.88906300 & -0.80001900 & -0.27327500 \\
\hline C & 2.98885700 & -1.95308800 & 0.19812200 \\
\hline C & 1.51855300 & -1.76351100 & -0.23118100 \\
\hline $\mathrm{H}$ & 2.06822400 & 0.41548600 & -1.55889900 \\
\hline $\mathrm{H}$ & 3.96497600 & -0.83294900 & -1.37221200 \\
\hline $\mathrm{H}$ & 3.36818100 & -2.89180900 & -0.21816900 \\
\hline $\mathrm{H}$ & 3.05376900 & -2.03694800 & 1.28859500 \\
\hline $\mathrm{H}$ & 1.42899500 & -1.86502300 & -1.31863200 \\
\hline C & 5.27819500 & -0.84660900 & 0.33950300 \\
\hline $\mathrm{H}$ & 5.77452600 & -1.78569800 & 0.07638100 \\
\hline $\mathrm{H}$ & 5.88776800 & -0.01614900 & -0.02808100 \\
\hline $\mathrm{H}$ & 5.21519800 & -0.78021800 & 1.43073100 \\
\hline C & 1.53315900 & 2.08886100 & -0.34542300 \\
\hline $\mathrm{H}$ & 1.41870900 & 2.36947400 & 0.70558100 \\
\hline $\mathrm{H}$ & 2.27338700 & 2.76313300 & -0.78497300 \\
\hline C & -2.39762500 & 0.51154300 & 1.37697500 \\
\hline $\mathrm{H}$ & -1.69724400 & -0.26811200 & 1.68811700 \\
\hline $\mathrm{H}$ & -2.75609700 & 0.96140900 & 2.30469100 \\
\hline C & -3.59950100 & -0.17528200 & 0.66688900 \\
\hline $\mathrm{H}$ & -3.96206200 & 0.40310200 & -0.19274200 \\
\hline $\mathrm{H}$ & -4.44334000 & -0.19920200 & 1.36269200 \\
\hline C & -3.34432400 & -1.62163800 & 0.25279300 \\
\hline $\mathrm{H}$ & -3.03071500 & -2.19842100 & 1.13133800 \\
\hline C & -4.54600100 & -2.27579400 & -0.41190700 \\
\hline $\mathrm{H}$ & -4.84661800 & -1.71331900 & -1.30142200 \\
\hline $\mathrm{H}$ & -4.32004600 & -3.30582500 & -0.70577600 \\
\hline $\mathrm{H}$ & -5.38656600 & -2.30344600 & 0.28779800 \\
\hline 0 & -2.21703100 & -1.61057600 & -0.68737700 \\
\hline $\mathrm{H}$ & -2.05703000 & -2.50151000 & -1.03445500 \\
\hline 0 & 3.31375100 & 0.48348300 & 0.08203600 \\
\hline $\mathrm{O}$ & -0.24269300 & -0.11070500 & -0.53815500 \\
\hline C & -1.63713300 & 1.60682500 & 0.64147400 \\
\hline C & -1.61355400 & 2.85235800 & 1.13327600 \\
\hline $\mathrm{H}$ & -2.16209500 & 3.09526300 & 2.03783800 \\
\hline & -1.06559400 & 3.66628200 & 0.67467800 \\
\hline
\end{tabular}




$\begin{array}{lrrr}\mathrm{H} & 0.88299900 & -2.52516800 & 0.23223700 \\ \mathrm{C} & 0.91990400 & -0.19100800 & 1.67398900 \\ \mathrm{H} & 0.33829500 & -1.01084900 & 2.10154600 \\ \mathrm{H} & 1.91449700 & -0.20907900 & 2.12206400 \\ \mathrm{H} & 0.45533600 & 0.75696100 & 1.94387900 \\ \mathrm{C} & -0.91303300 & 1.30359600 & -0.66872500 \\ \mathrm{O} & -1.76321900 & 1.15552000 & -1.74440600 \\ \mathrm{H} & -2.58211800 & 0.70150700 & -1.49620700 \\ \mathrm{H} & -0.98442300 & -0.84415600 & -0.49676100 \\ \mathrm{C} & 0.21548400 & 2.22866700 & -1.11978400 \\ \mathrm{H} & -0.15527500 & 3.25409700 & -1.09557900 \\ \mathrm{H} & 0.38653000 & 1.98718600 & -2.17326600\end{array}$

\section{INT2A}

$E E=-851.117142$

EE + Zero-point Energy $=-850.702830$

$\mathrm{EE}+$ Thermal Free Energy Correction $=-850.751899$

Number of imaginary freq. $=0$

Lowest freq. $=21.67$

M06-2X/6-31+G(d,p), scrf $=(p c m$, Solvent=Water $)$

11

$\begin{array}{lrrr}\mathrm{C} & -3.59758700 & 1.10868800 & -0.63362600 \\ \mathrm{C} & -3.12893700 & 1.61802900 & 0.72909300 \\ \mathrm{C} & -1.64445900 & 1.31138800 & 0.96749100 \\ \mathrm{C} & -1.41086300 & -0.17052300 & 0.71573600 \\ \mathrm{C} & -1.88391600 & -0.51249600 & -0.70290000 \\ \mathrm{H} & -3.29104800 & 2.69852200 & 0.78263100 \\ \mathrm{H} & -3.07111200 & 1.66397500 & -1.42640900 \\ \mathrm{H} & -1.02922900 & 1.88843300 & 0.26647300 \\ \mathrm{H} & -1.34239200 & 1.57899300 & 1.98431300 \\ \mathrm{H} & -1.35224600 & 0.15954500 & -1.40036800 \\ \mathrm{H} & -3.74378500 & 1.15914300 & 1.51244300 \\ \mathrm{C} & -5.09546600 & 1.23242900 & -0.82051800 \\ \mathrm{H} & -5.39700600 & 2.27996900 & -0.73953300 \\ \mathrm{H} & -5.39281900 & 0.85827800 & -1.80289400 \\ \mathrm{H} & -5.61904000 & 0.65831800 & -0.05003400 \\ \mathrm{C} & -1.55246700 & -1.94749400 & -1.03946300 \\ \mathrm{H} & -2.10802100 & -2.62144800 & -0.38303100 \\ \mathrm{H} & -1.84849600 & -2.16936900 & -2.06636100 \\ \mathrm{C} & -0.03869600 & -2.15569400 & -0.89569800 \\ \mathrm{H} & 0.46101300 & -1.97768000 & -1.85571100 \\ \mathrm{H} & 0.19682800 & -3.18953500 & -0.62429200 \\ \mathrm{O} & 0.06015100 & -0.36772300 & 0.75663000 \\ \mathrm{O} & -3.26795800 & -0.28245300 & -0.78936800 \\ \mathrm{C} & -1.98605600 & -1.06564600 & 1.80262400 \\ \mathrm{H} & -1.71148500 & -0.68138800 & 2.78765300 \\ \mathrm{H} & -3.07445000 & -1.08206400 & 1.71606500 \\ \mathrm{H} & -1.62055700 & -2.09140900 & 1.71478200 \\ \mathrm{C} & 0.66567300 & -1.26231800 & 0.07938100 \\ \mathrm{C} & 2.10417700 & -1.31134600 & 0.32155100 \\ \mathrm{C} & 2.70522000 & -0.33052300 & 1.30023800 \\ \mathrm{C} & 2.82418500 & 1.09561300 & 0.73997100 \\ \mathrm{H} & 3.70117200 & -0.69410100 & 1.56062700 \\ \mathrm{H} & 2.10820100 & -0.31046300 & 2.21650400 \\ \mathrm{H} & 3.33067800 & 1.71440400 & 1.49046100 \\ \mathrm{H} & 1.83211100 & 1.53134100 & 0.57798900 \\ \mathrm{O} & 4.84304800 & 0.49046100 & -0.34226400 \\ & & & \end{array}$




$\begin{array}{lrrr}\mathrm{C} & 3.60574600 & 1.16855400 & -0.56508000 \\ \mathrm{H} & 3.04545500 & 0.63674100 & -1.34921700 \\ \mathrm{C} & 2.84431900 & -2.21311500 & -0.34927700 \\ \mathrm{H} & 2.42841000 & -2.91204300 & -1.06643500 \\ \mathrm{H} & 3.91642000 & -2.25537300 & -0.18361400 \\ \mathrm{C} & 3.83466500 & 2.60669800 & -1.00708400 \\ \mathrm{H} & 2.87899600 & 3.11339300 & -1.17172100 \\ \mathrm{H} & 4.39368300 & 3.15188300 & -0.24003400 \\ \mathrm{H} & 4.40151500 & 2.64088200 & -1.94253200 \\ \mathrm{H} & 5.35204400 & 0.49531000 & -1.16237100\end{array}$

\section{INT2A}

$E E=-851.567476$

$\mathrm{EE}+$ Zero-point Energy $=-851.155526$

$\mathrm{EE}+$ Thermal Free Energy Correction $=-851.202353$

Number of imaginary freq. $=0$

Lowest freq. $=37.05$

B3LYP(D3)/6-31+G(d,p), scrf=(pcm, Solvent=Water $)$ 11

$\begin{array}{lrrr}\text { C } & 1.18630800 & -0.47658200 & 0.16798600 \\ \mathrm{C} & 2.06242000 & 0.59562900 & -0.51507600 \\ \mathrm{C} & 4.03953000 & -0.70294700 & -0.28456100 \\ \mathrm{C} & 3.24120800 & -1.90865500 & 0.23369000 \\ \mathrm{C} & 1.75997500 & -1.85079500 & -0.19070800 \\ \mathrm{H} & 2.11348600 & 0.33088700 & -1.58418300 \\ \mathrm{H} & 4.10483200 & -0.76445500 & -1.38312600 \\ \mathrm{H} & 3.69454200 & -2.82680400 & -0.15391100 \\ \mathrm{H} & 3.31959800 & -1.94911600 & 1.32586100 \\ \mathrm{H} & 1.67430900 & -1.99087400 & -1.27429600 \\ \mathrm{C} & 5.43543400 & -0.61821300 & 0.30906000 \\ \mathrm{H} & 6.00327600 & -1.52178700 & 0.06684100 \\ \mathrm{H} & 5.97106100 & 0.24734400 & -0.09140400 \\ \mathrm{H} & 5.37983700 & -0.52379700 & 1.39868100 \\ \mathrm{C} & 1.48879500 & 2.00427200 & -0.41423600 \\ \mathrm{H} & 1.35963400 & 2.30333200 & 0.62940400 \\ \mathrm{H} & 2.20461200 & 2.69821800 & -0.86358600 \\ \mathrm{C} & -2.34782400 & 0.41583500 & 1.42241200 \\ \mathrm{H} & -1.71613600 & -0.46480900 & 1.56972900 \\ \mathrm{H} & -2.51041100 & 0.82188200 & 2.42276200 \\ \mathrm{C} & -3.71515600 & -0.03841400 & 0.84019800 \\ \mathrm{H} & -4.08235400 & 0.67615900 & 0.09370300 \\ \mathrm{H} & -4.45822900 & -0.04730900 & 1.64289700 \\ \mathrm{C} & -3.68355600 & -1.43913500 & 0.23770300 \\ \mathrm{H} & -3.40439600 & -2.16545700 & 1.01133200 \\ \mathrm{C} & -4.99648400 & -1.84294400 & -0.41595400 \\ \mathrm{H} & -5.26273500 & -1.13944800 & -1.21153900 \\ \mathrm{H} & -4.93141800 & -2.84996500 & -0.84119500 \\ \mathrm{H} & -5.79699800 & -1.84550500 & 0.33020500 \\ \mathrm{O} & -2.60290400 & -1.42376900 & -0.74909400 \\ \mathrm{H} & -2.65081800 & -2.20853500 & -1.31560800 \\ \mathrm{O} & 3.36749300 & 0.53950200 & 0.04013000\end{array}$

\section{TSS to INT3A(R)+}

$E E=-851.111968$

$E E+$ Zero-point Energy $=-850.696228$

EE + Thermal Free Energy Correction $=-850.740045$

Number of imaginary freq. $=1$ 
Lowest freq. $=-86.24$

M06-2X/6-31+G(d,p), scrf=(pcm, Solvent=Water $)$

11

C

C

3.91099600

0.15238500

0.31779800

$\begin{array}{llll}3.59838600 & 0.45295700 & -1.15000300\end{array}$

$\begin{array}{lll}2.17012300 & 0.98165600 & -1.34692100\end{array}$

C

1.18096900

$0.08200300-0.61266100$

C

1.62905000

$-0.01965000$

0.84984100

4.31494500

1.19291500

3.93817100

1.09966500

$-1.51922400$

2.08141000

1.99199600

0.88018400

1.91715000

1.03495100

$-0.92995400$

1.73486500

1.01205200

$-2.41073400$

3.75373300

$-0.45901400$

1.23053700

5.21962300

$-0.58966700$

$-1.73813800$

6.04370500

$-0.00008600$

0.49404500

5.41636800

$-0.77625000$

0.08363100

5.18133000

$-1.54846000$

1.55243500

0.59046100

$-0.72592400$

$-0.03229500$

0.43653900

$-1.75597500$

1.69430300

0.92123000

$-0.77164300$

1.35908100

$-0.69462600$

0.10065000

2.73450400

$-0.56166600$

1.03254300

1.61503900

$-1.54051200$

$-0.41274900$

2.18028300

$-0.10613900$

0.78398900

2.07382600

2.88033900

$-0.66239100$

$-0.66244900$

1.02759900

$-1.26450900$

0.90153300

0.49705400

$-1.12553900$

$-1.31303000$

2.00819800

$-1.69019200$

$-2.25853400$

$\mathrm{H}$

0.50926900

$-2.01279900$

$-1.52827300$

$-1.06282900$

0.57079900

$-0.70576200$

$-2.16531000$

1.58496600

0.21949700

$-3.48725700$

1.19926000

0.13511700

$-4.12052100$

0.00885800

0.74943400

$-4.15799300$

2.05962600

0.01959000

$-3.36051400$

0.95496000

0.69964900

$-4.31888800$

0.27446300

1.81145900

$-5.07518100$

$-0.25001700$

$-1.02512600$

$-1.93678100$

$-0.80505300$

0.48666500

$-3.24078700$

$-1.22458300$

$-0.49174300$

$-3.06988400$

$-1.55400600$

0.05421800

$-1.92259300$

2.76172000

1.08506000

$-0.95711700$

2.99279500

$-0.44294500$

$\mathrm{H}$

$-2.69517400$

3.52351400

$-0.87914200$

$-3.75017600$

$-2.37084700$

$-0.47637600$

$-4.71796000$

$-2.69421300$

$-0.79392300$

$-3.87729700$

$-2.05104300$

$-0.40266700$

$-3.07048500$

$-3.22643400$

$-1.83120100$

$-1.34929200-1.57385800$

$-0.75832600$

$\mathrm{H}$

$-0.59669100$

\section{TSS to INT3A(S)+}

$E E=-851.104500$

EE + Zero-point Energy $=-850.687848$

$\mathrm{EE}+$ Thermal Free Energy Correction $=-850.731461$

Number of imaginary freq. $=1$

Lowest freq. $=-66.77$

M06-2X/6-31+G(d,p), scrf $=(p c m$, Solvent=Water $)$ 


$\begin{array}{lrrr}11 & & & \\ \mathrm{C} & 3.58417300 & -0.79890300 & -0.23895600 \\ \mathrm{C} & 3.10828600 & -1.06220400 & 1.19048500 \\ \mathrm{C} & 1.57758000 & -1.06650100 & 1.27901500 \\ \mathrm{C} & 1.03254400 & 0.21010100 & 0.64927200 \\ \mathrm{C} & 1.56454300 & 0.29786400 & -0.78542400 \\ \mathrm{H} & 3.50305700 & -2.02638400 & 1.52519000 \\ \mathrm{H} & 3.27765700 & -1.64329300 & -0.87825100 \\ \mathrm{H} & 1.17318300 & -1.92350900 & 0.72543000 \\ \mathrm{H} & 1.24083900 & -1.14928200 & 2.31714300 \\ \mathrm{H} & 1.27063900 & -0.63047800 & -1.31085100 \\ \mathrm{H} & 3.52637900 & -0.29372600 & 1.85180700 \\ \mathrm{C} & 5.08326000 & -0.59939200 & -0.33075100 \\ \mathrm{H} & 5.60356800 & -1.48812800 & 0.03570900 \\ \mathrm{H} & 5.38498500 & -0.41960700 & -1.36535400 \\ \mathrm{H} & 5.38538600 & 0.25781100 & 0.27876300 \\ \mathrm{C} & 0.96141800 & 1.48745300 & -1.49413100 \\ \mathrm{H} & 1.23995400 & 2.40888900 & -0.97670500 \\ \mathrm{H} & 1.34561900 & 1.55415500 & -2.51445000 \\ \mathrm{C} & -0.56272300 & 1.31263400 & -1.54803600 \\ \mathrm{H} & -0.81905500 & 0.71687100 & -2.42832500 \\ \mathrm{H} & -1.04837600 & 2.27172400 & -1.71247100 \\ \mathrm{O} & -0.41745800 & 0.07288400 & 0.62462600 \\ \mathrm{O} & 2.96984800 & 0.38717200 & -0.76604000 \\ \mathrm{C} & 1.34546700 & 1.44915700 & 1.48363800 \\ \mathrm{H} & 1.10664000 & 1.25329100 & 2.53229100 \\ \mathrm{H} & 2.40234200 & 1.71195100 & 1.40294300 \\ \mathrm{H} & 0.75054500 & 2.30518000 & 1.15485500 \\ \mathrm{C} & -1.20471600 & 0.63245700 & -0.32669500 \\ \mathrm{C} & -2.38512900 & 1.29843900 & 0.34772300 \\ \mathrm{C} & -3.13936000 & 0.40882200 & 1.30710200 \\ \mathrm{C} & -3.69880900 & -0.82681000 & 0.59552100 \\ \mathrm{H} & -3.95211800 & 0.97931500 & 1.76053800 \\ \mathrm{H} & -2.46571200 & 0.09008500 & 2.11063600 \\ \mathrm{H} & -4.44528800 & -0.53267600 & -0.15098600 \\ \mathrm{H} & -4.18662500 & -1.49021700 & 1.31524400 \\ \mathrm{O} & -1.93589000 & -0.65728500 & -1.01368700 \\ \mathrm{C} & -2.60712800 & -1.62125300 & -0.08853100 \\ \mathrm{H} & -1.83590900 & -1.93715800 & 0.61947400 \\ \mathrm{C} & -2.68185900 & 2.58598500 & 0.17225200 \\ \mathrm{H} & -2.13904700 & 3.24957400 & -0.48960000 \\ \mathrm{H} & -3.49892900 & 3.02647800 & 0.73498100 \\ \mathrm{H} & -3.10364200 & -2.77089000 & -0.93531400 \\ \mathrm{H} & -3.60221100 & -3.48699600 & -0.27774000 \\ & -3.82131100 & -2.41986000 & -1.68058000 \\ & -2.28300700 & -3.29199300 & -1.43515700 \\ -1.28862600 & -1.13594300 & -1.56674200\end{array}$

TSS to INT3A(S)+

$E E=-851.546258$

$\mathrm{EE}+$ Zero-point Energy $=-851.133528$

$\mathrm{EE}+$ Thermal Free Energy Correction $=-851.178033$

Number of imaginary freq. $=1$

Lowest freq. $=-177.58$

B3LYP(D3)/6-31+G(d,p), scrf=(pcm, Solvent=Water $)$ 11

C

$3.73923500-0.74122500-0.30276400$ 


\begin{tabular}{lrrr}
$\mathrm{C}$ & 3.18427900 & -1.29806200 & 1.01707300 \\
$\mathrm{C}$ & 1.64516900 & -1.37644500 & 1.01420300 \\
$\mathrm{C}$ & 1.07333500 & -0.02917800 & 0.58010800 \\
$\mathrm{C}$ & 1.67242200 & 0.32102900 & -0.79441700 \\
$\mathrm{H}$ & 3.60291600 & -2.29618400 & 1.18309300 \\
$\mathrm{H}$ & 3.53788900 & -1.46685100 & -1.10798800 \\
$\mathrm{H}$ & 1.30701200 & -2.13997900 & 0.30381400 \\
$\mathrm{H}$ & 1.26650900 & -1.65120700 & 2.00374100 \\
$\mathrm{H}$ & 1.47994100 & -0.53441600 & -1.46465900 \\
$\mathrm{H}$ & 3.52756700 & -0.66580100 & 1.84384100 \\
$\mathrm{C}$ & 5.22721300 & -0.44116900 & -0.24235900 \\
$\mathrm{H}$ & 5.78544400 & -1.35227600 & -0.00619500 \\
$\mathrm{H}$ & 5.58146200 & -0.05521000 & -1.20263000 \\
$\mathrm{H}$ & 5.43381200 & 0.30382100 & 0.53331800 \\
$\mathrm{C}$ & 1.00349600 & 1.54678700 & -1.38801100 \\
$\mathrm{H}$ & 1.13841400 & 2.42111500 & -0.74588500 \\
$\mathrm{H}$ & 1.44156600 & 1.78177000 & -2.36157700 \\
$\mathrm{C}$ & -0.48420500 & 1.20061100 & -1.58455300 \\
$\mathrm{H}$ & -0.54194900 & 0.46019800 & -2.38791700 \\
$\mathrm{H}$ & -1.06410500 & 2.05635300 & -1.92572300 \\
$\mathrm{O}$ & -0.39249200 & -0.21956600 & 0.43585500 \\
$\mathrm{O}$ & 3.07376000 & 0.49695000 & -0.66034600 \\
$\mathrm{C}$ & 1.26518200 & 1.05971400 & 1.63664100 \\
$\mathrm{H}$ & 0.90559400 & 0.69936300 & 2.60391100 \\
$\mathrm{H}$ & 2.32122100 & 1.32025600 & 1.72351800 \\
$\mathrm{H}$ & 0.71027600 & 1.96591600 & 1.38607900 \\
$\mathrm{C}$ & -1.13442800 & 0.59595800 & -0.34169600 \\
$\mathrm{C}$ & -2.15114400 & 1.36511100 & 0.44909900 \\
$\mathrm{C}$ & -2.95696700 & 0.54892900 & 1.43413700 \\
$\mathrm{C}$ & -3.81740900 & -0.50335800 & 0.71094600 \\
$\mathrm{H}$ & -3.59866700 & 1.21385300 & 2.01675300 \\
& & & \\
\hline
\end{tabular}

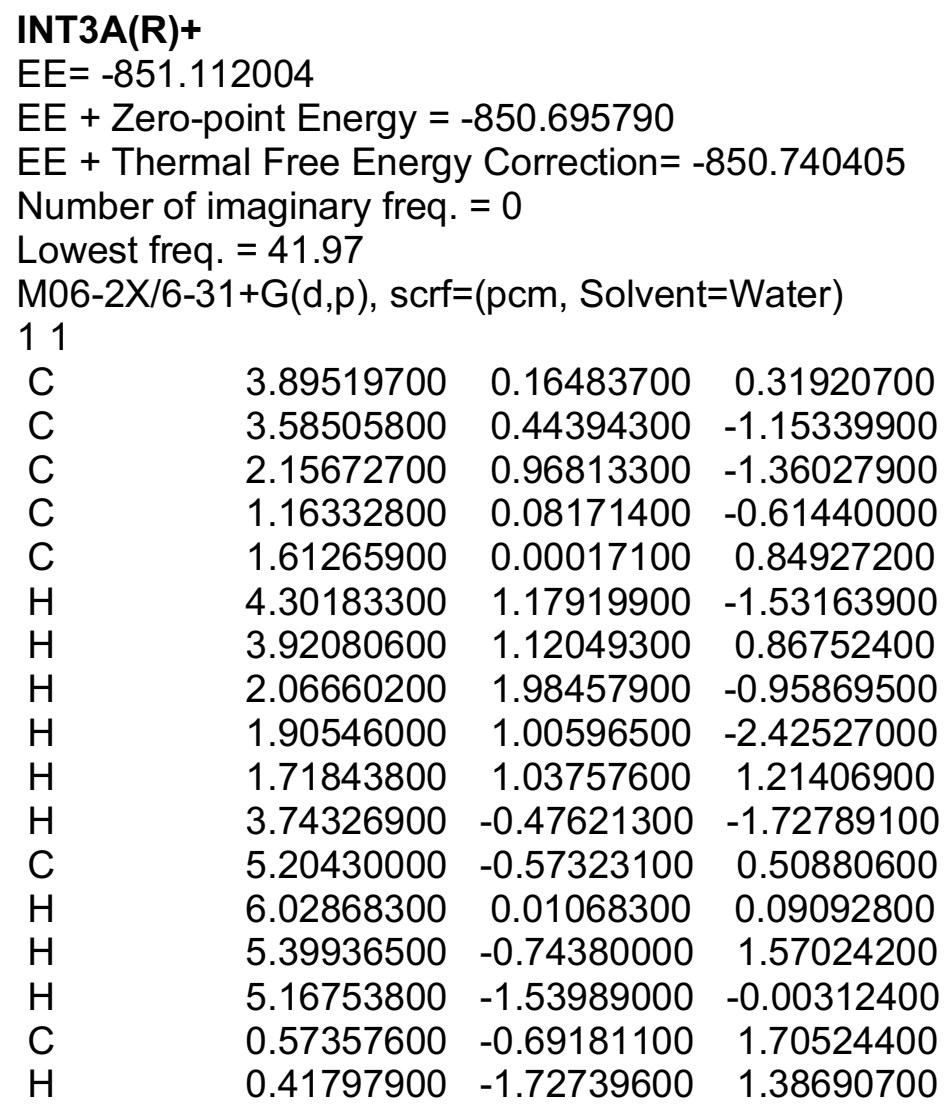




$\begin{array}{lrrr}\text { H } & 0.90474300 & -0.72227900 & 2.74601400 \\ \mathrm{C} & -0.70977400 & 0.13633900 & 1.61321600 \\ \mathrm{H} & -0.56311200 & 1.08906500 & 2.13674100 \\ \mathrm{H} & -1.54795900 & -0.35714000 & 2.10726300 \\ \mathrm{O} & -0.11726700 & 0.78618500 & -0.67496000 \\ \mathrm{O} & 2.86438500 & -0.64185600 & 0.91286800 \\ \mathrm{C} & 1.02201900 & -1.27801400 & -1.29580700 \\ \mathrm{H} & 0.49552900 & -1.15727300 & -2.24628000 \\ \mathrm{H} & 2.00580000 & -1.70130500 & -1.50106800 \\ \mathrm{H} & 0.51100100 & -2.02739900 & -0.68159400 \\ \mathrm{C} & -1.09877100 & 0.54213100 & 0.19749900 \\ \mathrm{C} & -2.19061600 & 1.57708300 & 0.11649700 \\ \mathrm{C} & -3.51957300 & 1.18744900 & 0.71316300 \\ \mathrm{C} & -4.11341000 & -0.03377100 & 0.00164100 \\ \mathrm{H} & -4.20466300 & 2.03345300 & 0.62772000 \\ \mathrm{H} & -3.40974900 & 0.97121600 & 1.78335700 \\ \mathrm{H} & -4.31015200 & 0.20307000 & -1.04999100 \\ \mathrm{H} & -5.06256700 & -0.31617400 & 0.46606500 \\ \mathrm{O} & -1.88925700 & -0.77382300 & -0.46915600 \\ \mathrm{C} & -3.19598300 & -1.23620400 & 0.07024600 \\ \mathrm{H} & -3.01805700 & -1.54378500 & 1.10533200 \\ \mathrm{C} & -1.93549600 & 2.76032600 & -0.44012200 \\ \mathrm{H} & -0.96397500 & 2.99104600 & -0.86267100 \\ \mathrm{H} & -2.70396100 & 3.52636100 & -0.47156100 \\ \mathrm{C} & -3.63507700 & -2.41056000 & -0.77596500 \\ \mathrm{H} & -4.59690600 & -2.76693400 & -0.39941600 \\ \mathrm{H} & -3.75458700 & -2.10831000 & -1.81914000 \\ \mathrm{H} & -2.92368400 & -3.23836900 & -0.71357700 \\ \mathrm{H} & -1.27552400 & -1.52169700 & -0.58918000 \\ & & & \end{array}$

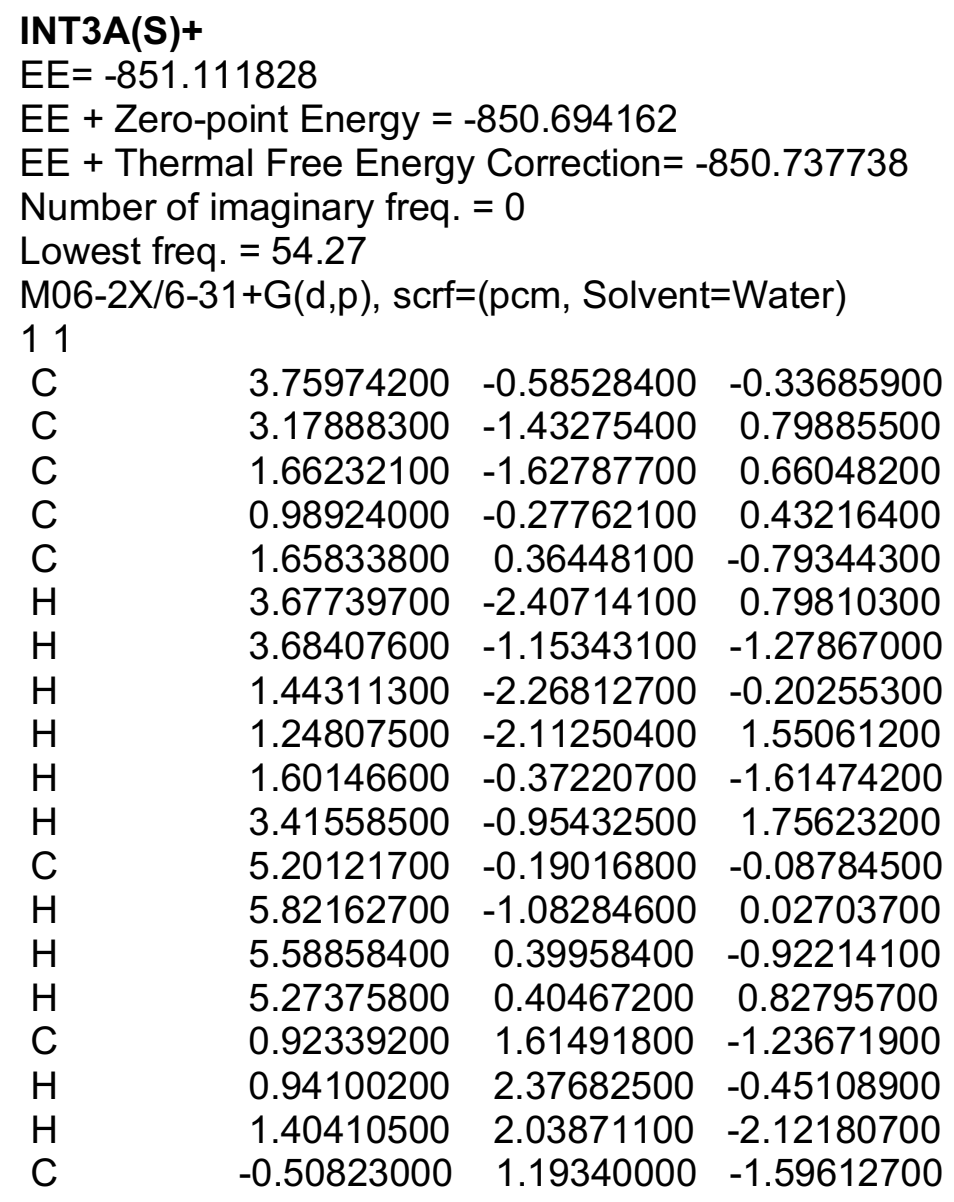




$\begin{array}{rrrr}H & -0.44438900 & 0.50379400 & -2.44642000 \\ \mathrm{H} & -1.12901200 & 2.03373200 & -1.90797600 \\ \mathrm{O} & -0.39340100 & -0.57083900 & 0.08832100 \\ \mathrm{O} & 3.01304200 & 0.62938400 & -0.50869900 \\ \mathrm{C} & 1.05653300 & 0.59181500 & 1.68494800 \\ \mathrm{H} & 0.59184000 & 0.05950400 & 2.52000200 \\ \mathrm{H} & 2.09738200 & 0.79906400 & 1.93828600 \\ \mathrm{H} & 0.54906900 & 1.54861500 & 1.56073200 \\ \mathrm{C} & -1.17368700 & 0.45290600 & -0.44283900 \\ \mathrm{C} & -1.88148500 & 1.27740800 & 0.61614800 \\ \mathrm{C} & -2.64125700 & 0.45102600 & 1.62096900 \\ \mathrm{C} & -3.71041400 & -0.37798300 & 0.89730800 \\ \mathrm{H} & -3.10944100 & 1.10594200 & 2.35803600 \\ \mathrm{H} & -1.95517400 & -0.22028700 & 2.14998700 \\ \mathrm{H} & -4.45761400 & 0.28108300 & 0.44174600 \\ \mathrm{H} & -4.22700400 & -1.03803200 & 1.60005000 \\ \mathrm{O} & -2.31475200 & -0.32931600 & -1.07805800 \\ \mathrm{C} & -3.10752900 & -1.26036600 & -0.17251000 \\ \mathrm{H} & -2.36207800 & -1.94494200 & 0.23637800 \\ \mathrm{C} & -1.86182700 & 2.60933800 & 0.61671700 \\ \mathrm{H} & -1.33121300 & 3.19584400 & -0.12529200 \\ \mathrm{H} & -2.37722700 & 3.15905000 & 1.39816900 \\ \mathrm{C} & -4.10162200 & -1.95702600 & -1.06802500 \\ \mathrm{H} & -4.68952600 & -2.63791500 & -0.44780600 \\ \mathrm{H} & -4.77663300 & -1.23691800 & -1.53571200 \\ \mathrm{H} & -3.60525200 & -2.55346700 & -1.83782800 \\ \mathrm{H} & -1.97332400 & -0.83538300 & -1.84314800 \\ & & & \end{array}$

\section{INT3A(S)+}

$E E=-851.548629$

$\mathrm{EE}+$ Zero-point Energy $=-851.134715$

$\mathrm{EE}+$ Thermal Free Energy Correction $=-851.178557$

Number of imaginary freq. $=0$

Lowest freq. $=48.86$

B3LYP(D3)/6-31+G(d,p), scrf=(pcm, Solvent=Water $)$

11

$\begin{array}{lrrr}\mathrm{C} & 3.78440600 & -0.61329500 & -0.33190000 \\ \mathrm{C} & 3.19594100 & -1.42941600 & 0.83037700 \\ \mathrm{C} & 1.66910000 & -1.60614500 & 0.71275500 \\ \mathrm{C} & 1.00925600 & -0.24889000 & 0.45675000 \\ \mathrm{C} & 1.67206400 & 0.35635700 & -0.79640200 \\ \mathrm{H} & 3.67902300 & -2.41226600 & 0.84796600 \\ \mathrm{H} & 3.69846300 & -1.20358500 & -1.25918700 \\ \mathrm{H} & 1.42997800 & -2.26692700 & -0.12893700 \\ \mathrm{H} & 1.26026500 & -2.06365800 & 1.61962800 \\ \mathrm{H} & 1.59860700 & -0.40104900 & -1.59548700 \\ \mathrm{H} & 3.44723300 & -0.93531500 & 1.77578800 \\ \mathrm{C} & 5.23557100 & -0.22340500 & -0.10655700 \\ \mathrm{H} & 5.85143400 & -1.11939200 & 0.01855300 \\ \mathrm{H} & 5.61846700 & 0.34534000 & -0.95901700 \\ \mathrm{H} & 5.32908100 & 0.39072900 & 0.79543600 \\ \mathrm{C} & 0.94271700 & 1.60295800 & -1.27235100 \\ \mathrm{H} & 0.97228900 & 2.38941200 & -0.51316300 \\ \mathrm{H} & 1.42049100 & 1.99631500 & -2.17370100 \\ \mathrm{C} & -0.50325000 & 1.19058900 & -1.60980700 \\ \mathrm{H} & -0.45820900 & 0.49044400 & -2.45191100 \\ \mathrm{H} & -1.11232900 & 2.03541700 & -1.93164000\end{array}$




\begin{tabular}{lrrr}
$\mathrm{O}$ & -0.39522900 & -0.53940200 & 0.12457400 \\
$\mathrm{O}$ & 3.04262700 & 0.61557900 & -0.53294700 \\
$\mathrm{C}$ & 1.07828700 & 0.65218600 & 1.69187700 \\
$\mathrm{H}$ & 0.61207000 & 0.14302900 & 2.53979500 \\
$\mathrm{H}$ & 2.11866200 & 0.86727900 & 1.93927600 \\
$\mathrm{H}$ & 0.56890600 & 1.60358200 & 1.54207700 \\
$\mathrm{C}$ & -1.17264500 & 0.47329700 & -0.43753800 \\
$\mathrm{C}$ & -1.91551300 & 1.29859200 & 0.59603200 \\
$\mathrm{C}$ & -2.68332900 & 0.47332100 & 1.60115000 \\
$\mathrm{C}$ & -3.74487500 & -0.38412400 & 0.88728100 \\
$\mathrm{H}$ & -3.16534200 & 1.12874600 & 2.32980100 \\
$\mathrm{H}$ & -1.99332700 & -0.18179900 & 2.14458300 \\
$\mathrm{H}$ & -4.49866700 & 0.25793300 & 0.41875000 \\
$\mathrm{H}$ & -4.25808400 & -1.03179000 & 1.60530100 \\
$\mathrm{O}$ & -2.32825400 & -0.35374100 & -1.09266200 \\
$\mathrm{C}$ & -3.13529900 & -1.28956700 & -0.16349300 \\
$\mathrm{H}$ & -2.38435500 & -1.95833500 & 0.25987700 \\
$\mathrm{C}$ & -1.89575900 & 2.63448400 & 0.59534900 \\
$\mathrm{H}$ & -1.35311100 & 3.22138700 & -0.13641300 \\
$\mathrm{H}$ & -2.42110000 & 3.18873700 & 1.36699200 \\
$\mathrm{C}$ & -4.11396300 & -2.01314100 & -1.05855400 \\
$\mathrm{H}$ & -4.69659800 & -2.69588700 & -0.43283700 \\
$\mathrm{H}$ & -4.79813200 & -1.31205200 & -1.54325700 \\
$\mathrm{H}$ & -3.60308600 & -2.61382100 & -1.81707200 \\
$\mathrm{H}$ & -1.95203200 & -0.88478500 & -1.82274400 \\
& & & \\
\hline & & & \\
\hline
\end{tabular}


Table S82. Cartesian coordinates structures in Path B.

\begin{tabular}{|c|c|c|c|}
\hline $\begin{array}{l}\text { Pro } \\
\text { EE } \\
\text { EE } \\
\text { EE } \\
\text { Nur } \\
\text { Lov } \\
\text { MO } \\
11\end{array}$ & $\begin{array}{l}14-K e t o d e h y \\
8816 \\
\text { oint Energy = } \\
\text { al Free Energs } \\
\text { naginary freq. } \\
=17.54 \\
1+G(d, p) \text {, scrf }\end{array}$ & $\begin{array}{l}\text { drothyrsiferol } \\
-927.099201 \\
\text { Correction= - } \\
=0\end{array}$ & 927.149562 \\
\hline C & -1.70701000 & 1.78880200 & 0.17349000 \\
\hline C & -2.14605900 & -0.53629200 & 0.44826100 \\
\hline C & -3.38683600 & -0.51666700 & -0.46365000 \\
\hline C & -3.98028700 & 0.89718300 & -0.40518800 \\
\hline C & -2.93331700 & 1.96797300 & -0.71619900 \\
\hline C & -0.57329700 & 2.73079000 & -0.17512600 \\
\hline C & -3.06176400 & -0.93686700 & -1.89527900 \\
\hline C & -1.40596500 & -1.86148400 & 0.48881600 \\
\hline C & -0.18936000 & -1.76318300 & 1.42300300 \\
\hline C & 0.88999300 & -0.91245000 & 0.83173200 \\
\hline C & 1.56399400 & 0.19746400 & 1.51776200 \\
\hline C & 2.56896200 & 1.00992500 & 0.73812200 \\
\hline C & 1.28870500 & 0.44804400 & 2.80842600 \\
\hline C & 4.24312600 & 0.23678300 & -1.01354800 \\
\hline C & 5.56989000 & -0.42809000 & -1.31980200 \\
\hline C & 3.90485400 & 0.27338100 & 0.46727700 \\
\hline $\mathrm{H}$ & -2.00159600 & 1.93186900 & 1.22702500 \\
\hline $\mathrm{H}$ & -4.82841300 & 0.96848600 & -1.09675600 \\
\hline $\mathrm{H}$ & -4.37039400 & 1.05096400 & 0.60931700 \\
\hline $\mathrm{H}$ & -2.61780200 & 1.91236500 & -1.76569200 \\
\hline $\mathrm{H}$ & -3.35430700 & 2.96573800 & -0.55443600 \\
\hline $\mathrm{H}$ & -2.49510500 & -0.29267600 & 1.46757300 \\
\hline $\mathrm{H}$ & -0.92297500 & 3.76615900 & -0.14459300 \\
\hline $\mathrm{H}$ & 0.24918000 & 2.62502100 & 0.53666300 \\
\hline $\mathrm{H}$ & -0.20262200 & 2.51726700 & -1.18306100 \\
\hline $\mathrm{H}$ & -2.81061600 & -1.99974800 & -1.93303700 \\
\hline $\mathrm{H}$ & -3.92969700 & -0.76602600 & -2.54102700 \\
\hline $\mathrm{H}$ & -2.21958800 & -0.36447200 & -2.29378800 \\
\hline $\mathrm{H}$ & -5.12046500 & -1.43350400 & -0.35862000 \\
\hline $\mathrm{H}$ & -1.07411500 & -2.15015600 & -0.51223400 \\
\hline $\mathrm{H}$ & -2.07973500 & -2.63681600 & 0.85968200 \\
\hline $\mathrm{H}$ & 0.25631500 & -2.75677300 & 1.55126000 \\
\hline $\mathrm{H}$ & -0.49716600 & -1.40516200 & 2.40342800 \\
\hline $\mathrm{H}$ & 2.11557100 & 1.31602700 & -0.21353700 \\
\hline $\mathrm{H}$ & 2.75841400 & 1.93105200 & 1.29248900 \\
\hline $\mathrm{H}$ & 0.58568700 & -0.13010600 & 3.39463100 \\
\hline $\mathrm{H}$ & 1.79302400 & 1.26230600 & 3.31968700 \\
\hline $\mathrm{H}$ & 4.21318700 & 1.25214100 & -1.42457400 \\
\hline $\mathrm{H}$ & 5.73595500 & -0.48134500 & -2.39879100 \\
\hline $\mathrm{H}$ & 6.38501500 & 0.14851700 & -0.87447200 \\
\hline $\mathrm{H}$ & 5.58895700 & -1.44155000 & -0.90879900 \\
\hline $\mathrm{H}$ & 3.03681300 & -0.28191100 & -2.55906000 \\
\hline $\mathrm{H}$ & 2.06220700 & -0.82404200 & -0.85752400 \\
\hline $\mathrm{H}$ & 3.87313100 & -0.75900000 & 0.83993900 \\
\hline $\mathrm{H}$ & 4.72033200 & 0.76765800 & 1.00051900 \\
\hline $\mathrm{O}$ & -1.21072000 & 0.45126200 & 0.03741400 \\
\hline $\mathrm{O}$ & -4.28850700 & -1.44926000 & 0.13275900 \\
\hline
\end{tabular}




\section{Protonated 14-Ketodehydrothyrsiferol}

$E E=-928.022203$

EE + Zero-point Energy $=-927.585395$

EE + Thermal Free Energy Correction $=-927.635791$

Number of imaginary freq. $=0$

Lowest freq. $=16.55$

B3LYP(D3)/6-31+G(d,p), scrf= $(p c m$, Solvent=Water $)$

11

C

$-1.69716000$

1.77324800

0.37729600

C

$-2.16064500$

$-0.58720900$

0.35637700

C

$-3.44914700$

$-0.42834300$

$-0.48850300$

C

$-4.01858600$

0.97549700

$-0.20454100$

C

$-2.97196800$

2.07804000

$-0.41368800$

C

$-0.56464800$

2.74472500

0.09240400

$-3.20757900 \quad-0.65822400 \quad-1.98409000$

C

$-1.43134100-1.91191300$

0.18370800

$-0.19246200-1.98095100$

1.10237200

C

0.89644700

$-1.05119600$

0.66182500

C

1.57881600

$-0.07866300$

1.51759800

2.58910900

0.86761500

0.90078400

C

1.31029600

$-0.05280100$

2.84086100

C

4.31291600

0.40126000

$-0.93317200$

C

5.66947900

$-0.16844700$

$-1.31598900$

C

3.95543900

0.21504500

0.53664300

$-1.93055800$

1.77947100

1.45487400

$-4.89662300$

1.14939200

$-0.83819300$

$-4.36165700$

0.98806900

0.83769600

$-2.71529900$

2.17126700

$-1.47575800$

$-3.37306000$

3.04520000

$-0.09067000$

$-2.46006400$

$-0.48396800$

1.41294100

$-0.89656800$

3.77056800

0.27935600

0.29443300

2.54125800

0.73528200

$-0.25027600$

2.66632100

$-0.95371400$

$-2.95777300$

$-1.70449700$

$-2.17662200$

$-4.11180900$

$-0.41383800$

$-2.55199500$

$-2.39117000$

$-0.03171900$

$-2.35305400$

$-5.20191400$

$-1.33530400$

$-0.41976100$

$-1.12260800$

$-2.05019900$

$-0.85489000$

$-2.10796100$

$-2.72803200$

0.44549000

0.23856200

$-2.98838500$

1.04752400

$-0.48497200$

$-1.80228300$

2.13498400

2.14937100

1.32339500

0.00676000

2.74957100

1.68695200

1.60409900

0.60455300

$-0.71726200$

3.32256900

1.82132700

0.65544300

3.48524800

4.25676900

1.46540200

$-1.19249400$

5.84654600

$-0.05645500$

$-2.39004000$

6.46223300

0.36403700

$-0.78105500$

5.72425100

$-1.23129600$

$-1.05975300$

3.16907400

0.05942600

$-2.57641900$

2.06336400

$-0.72438200$

$-0.96746300$

3.95573200

$-0.85868300$

0.76146700

$\mathrm{H}$

4.74972700

0.65508000

1.14546400 


$\begin{array}{lrrr}\mathrm{O} & -1.22700700 & 0.44970900 & 0.03685000 \\ \mathrm{O} & -4.34818900 & -1.43106400 & 0.02631000 \\ \mathrm{O} & 1.24704100 & -1.22815600 & -0.55487700 \\ \mathrm{O} & 3.26103200 & -0.29312400 & -1.67968100\end{array}$

\section{TSS to INT1B(R)}

$E E=-927.523879$

EE + Zero-point Energy $=-927.082144$

$\mathrm{EE}+$ Thermal Free Energy Correction $=-927.129308$

Number of imaginary freq. $=1$

Lowest freq. $=-154.86$

M06-2X/6-31+G(d,p), scrf $=(p c m$, Solvent=Water $)$

11

C

$-1.81360400$

$-0.24233500$

$-0.49396700$

C

$-4.54834400$

0.37594500

0.17387000

$\mathrm{H}$

$-1.92272500$

0.04512100

$-1.55416500$

C

$-4.98509200$

$-2.08305700$

$-0.26253600$

$-2.77143900$

$-1.23967700$

$-0.18295200$

C

$-3.52338600$

$1.49529200-0.01812600$

$-4.66993900$

0.14368700

1.23880000

$-5.52528900$

0.69294600

$-0.20420600$

$-6.01767300$

$-1.87361200$

$-0.55397200$

$-4.63845600$

$-2.96241300$

$-0.81070400$

$-4.96347500$

$-2.30485300$

0.80896400

$-4.10577000$

$-0.88570500$

$-0.56251300$

$-2.11858700$

1.01308400

0.34859700

$-3.78524500$

2.37062200

0.58794800

$-3.51051700$

1.80923800

$-1.06954500$

$-4.10359800$

$-0.68403900$

$-1.64686700$

$-0.43660000$

$-0.87710500$

$-0.29134300$

$-1.96643000$

0.77843500

1.84803500

$-1.15657600$

2.00057100

$-0.08639400$

0.34231700

0.44265000

$-1.86119900$

1.25457400

$-1.02708900-1.64377200$

0.72206500

$-0.24964000$

$-1.09675300$

$-0.91833400$

0.63580700

2.12309700

$-2.34713900$

1.64199500

2.40193000

$-2.52490000$

$-0.10773200$

2.15799300

$-1.43268400$

2.88240300

0.20560600

$-0.19086200$

$-0.93229300$

0.77206100

$-0.54398200$

$-1.91139800$

$-0.62730700$

1.74188100

0.57447200

$-0.34253500$

3.10328400

0.70216600

$-0.94281900$

1.31297300

1.62138200

0.31445700

3.82906300

$-0.56786000$

$-1.34234300$

3.59977000

1.92648700

$-1.13234200$

0.30247100

1.73845600

0.24885800

3.54088100

$-0.84872800$

$-2.36185700$

4.89664800

$-0.33561700$

$-1.37861100$

3.57644200

$-1.76717900$

$-0.41046700$

3.03874500

2.81191300

$-0.85564800$

4.57869000

2.06070300

$-1.58256700$

3.37428100

$-1.36453400$

1.03978600

$2.69775400-2.33651200$

$-0.72849400$

$4.42325200-2.45647000$

$-0.45879800$

$\begin{array}{lll}3.11906100 & -2.24428300 & 1.63622700\end{array}$

$\begin{array}{lll}4.53602500 & -0.61772600 & 1.66824500\end{array}$ 


$\begin{array}{rrrr}\mathrm{O} & 2.15828300 & -0.53658200 & 1.04772100 \\ \mathrm{H} & 5.41301600 & -1.27015100 & 1.67149500 \\ \mathrm{H} & 4.77871900 & 0.29234500 & 1.11508900 \\ \mathrm{H} & 4.31351300 & -0.35274700 & 2.70584700 \\ \mathrm{H} & 2.13927100 & 0.05007000 & 1.82440800\end{array}$

\section{TSS to INT1B(R)}

$E E=-928.007705$

EE + Zero-point Energy $=-927.555411$

EE + Thermal Free Energy Correction $=-927.615519$

Number of imaginary freq. $=1$

Lowest freq. $=-261.28$

$B 3 L Y P(D 3) / 6-31+G(d, p)$, scrf $=(p c m$, Solvent=Water $)$

11

C

1.79928200

$0.79665800 \quad 0.69889900$

$\begin{array}{lll}1.60588500 & -0.35623000 & -0.31552600\end{array}$

C

3.95161100

$-0.83409700$

$-0.39564400$

C

$4.25067400 \quad 0.18642700$

$3.00928800 \quad 0.44771600$

0.70835600

C

$1.52159400-1.28805200$

1.57662600

$\mathrm{H}$

3.74826600

$-1.81328600$

0.26909900

5.06862900

$-0.18959000$

0.06924900

$\mathrm{H}$

4.59751700

1.11954900

1.33211600

2.76388600

$-0.45205000$

0.24960700

$\mathrm{H}$

5.07829300

$-0.97057500$

2.15467800

C

$5.99632100-1.29123900$

$-1.40609000$

$\mathrm{H}$

4.82300000

$-1.71031200$

$-0.90410500$

$\mathrm{H}$

5.26797500

$-0.00996300$

$-2.17043600$

0.37839800

$-0.22465600$

$-1.89660700$

C

0.03525500

0.81012700

$-1.23250300$

0.71921000

$-0.48219700$

$-1.29658100$

$-3.94901100$

$-0.89605800$

$-2.23916600$

C

$\mathrm{H}$

$\mathrm{H}$

$-3.54300200$

$-1.08847700$

$-0.85165600$

$-4.92831600$

$-1.38031300$

$-1.85195700$

$-4.13888600$

0.62495700

$-0.82026900$

$-4.66357100$

0.82007200

$-0.66772700$

$\mathrm{H}$

$-4.76496600$

1.00704600

0.27481200

$-2.83204000$

1.40981400

$-1.48017800$

$-2.28401900$

1.24525000

$-0.66403000$

$-2.99725100$

2.89891800

$-1.59968600$

$-3.51816100$

3.07091800

$-0.40797400$

$-2.02539800$

3.40186000

0.53853200

$-3.58296300$

3.34253100

$-0.37777200$

$-2.04943400$

0.82151300

$-1.21873600$

$-1.18688800$

1.25778600

0.42771200

2.76865200

$-0.45067100$

0.58490200

0.57486700

0.76520500

$-1.13570100$

$-3.07265200$

$-1.53007600$

1.49385400

$-3.50384000-2.47527300$

0.21195400

C

$-4.51907400$

$-2.85371400$

1.05936400

$-2.85697000-2.90392200$

0.99373300

3.20081900

1.25623800

1.81671600

1.92950500

2.17473000

2.29162200

2.14699400

2.92668800

0.05053900

C

2.73837300

0.81531400

$\mathrm{H}$

1.00618600

2.18128500

$-0.68273700$

$\mathrm{H}$

$-1.65676200$

2.46633900

$-0.45540600$

C

$-1.07049400$

0.30622400 


$\begin{array}{lrrr}\mathrm{O} & -1.10357100 & -1.23824700 & 1.48490900 \\ \mathrm{H} & -0.25188200 & -0.73295200 & 1.56974800 \\ \mathrm{H} & 0.69349000 & 1.25866300 & 2.32016700 \\ \mathrm{C} & -0.81456700 & -1.15841600 & -0.95554800 \\ \mathrm{H} & -1.48697700 & -1.08932000 & -1.80986900 \\ \mathrm{H} & -0.46698500 & -2.20143000 & -0.93456800\end{array}$

\section{TSS to INT1B(S)}

$E E=-927.520776$

EE + Zero-point Energy $=-927.078722$

$\mathrm{EE}+$ Thermal Free Energy Correction $=-927.125310$

Number of imaginary freq. $=1$

Lowest freq. $=-190.88$

$M 06-2 X / 6-31+G(d, p), s c r f=(p c m$, Solvent=Water $)$

11

C

1.80003900

$-0.55901100$

0.25327400

C

$4.25671200 \quad 0.93850300$

0.28299900

$\mathrm{H}$

$\begin{array}{lll}1.84905100 & -0.80102200 & 1.32974200\end{array}$

C

$5.34268900-1.23705900$

$2.98249300-1.02123600$

$-0.43481200$

$\mathrm{O}$

$2.97160800 \quad 1.54429800$

$-0.37540100$

C

$4.42464600 \quad 1.28773800$

0.85130000

$\mathrm{H}$

$5.11814200 \quad 1.25488400$

$-0.74317000$

$\mathrm{H}$

$6.28411400-0.95322100$

0.87978200

$\mathrm{H}$

$5.25143300-2.32573600$

0.04318600

$\mathrm{H}$

5.36972000

$-0.91089400$

$-0.40846800$

$\mathrm{H}$

4.17697200

$-0.58570800$

$-1.47926800$

C

1.73941800

0.98222900

0.28189900

C

2.98571500

2.63734600

0.13719100

2.88768000

1.30040100

0.75999000

$\mathrm{H}$

$4.13265500-0.94058000$

1.91843700

C

0.62309600

$-1.30869700$

1.32551400

1.65835700

1.46205600

$-0.37108200$

C

$\mathrm{O}$

0.54787600

1.38835200

$-1.31050500$

$-0.51964800$

$-0.66404300$

0.84191400

$-0.55328700-2.40023000$

1.38722200

$\mathrm{H}$

$-0.56543500$

$-1.42012500$

1.09842700

0.68636400

1.22152700

0.60216600

$\mathrm{H}$

1.78720200

2.54861100

$-1.74864800$

2.43740300

0.99852200

$-1.34726400$

$\mathrm{H}$

0.63453800

2.31654100

$-1.92039800$

$\mathrm{H}$

0.31232900

$-0.82577600$

1.10150700

0.97567900

$-2.30740900$

$-1.30145300$

$-1.89805900$

$-1.32300900$

$-0.63831200$

$-3.14845400$

$-1.05969500$

$-0.05631400$

$-1.95531500$

$-1.85177400$

0.67896100

$-4.32594600$

$-0.51140400$

$-1.23809100$

C

$-3.18930700$

$-1.33376200$

$-0.10468300$

$-2.85791200$

$-1.90689900$

1.98678000

$-4.85665400$

$-1.32803400$

$-1.59653500$

$-5.03236800$

$-0.11225800$

$-0.61056800$

$-3.96070100$

0.57618900

0.62753400

$-2.33874200$

$-1.73519600$

$-1.13598500$

$-4.10405400$

$-1.17324900$

2.52706400

$-2.83298600$

1.50133500

2.54927800

C

$-3.66612800$

0.13269700

$-0.69304300$

$\mathrm{H}$

$-4.85079000$

1.17583800

$-2.09379400$

$\mathrm{H}$ 


$\begin{array}{lrrr}\mathrm{H} & -2.65829300 & 2.23580900 & -1.48800800 \\ \mathrm{C} & -3.09288300 & 2.22852400 & 0.61751200 \\ \mathrm{O} & -1.65065700 & 0.67821700 & -0.61643700 \\ \mathrm{H} & -3.99614600 & 2.83837400 & 0.52667100 \\ \mathrm{H} & -3.22454300 & 1.52907300 & 1.44691500 \\ \mathrm{H} & -2.25977900 & 2.89603500 & 0.85671700 \\ \mathrm{H} & -0.94424000 & 1.09468500 & -0.06861500\end{array}$

TSS to INT1B(S)

$E E=-928.002948$

$E E+$ Zero-point Energy $=-927.564049$

$\mathrm{EE}+$ Thermal Free Energy Correction $=-927.610781$

Number of imaginary freq. $=1$

Lowest freq. $=-167.57$

B3LYP(D3)/6-31+G(d,p), scrf $=(p c m$, Solvent=Water $)$

11

C

1.79673900

$-0.58300500$

0.24970700

C

$\begin{array}{ll}4.23643200 & 0.97759500\end{array}$

0.30346900

$\mathrm{H}$

$\begin{array}{lll}1.83506300 & -0.79634100 & 1.33081800\end{array}$

C

$5.38814100-1.19349900$

$3.01105200-1.03091200$

$-0.37076600$

O

$2.91789800 \quad 1.56745700$

$-0.35358100$

C

4.42870500

1.31899700

0.82515900

$\mathrm{H}$

$5.07132000 \quad 1.32444300$

$-0.72039700$

$\mathrm{H}$

$6.31705000-0.88551500$

0.92200300

$\mathrm{H}$

$5.31822700-2.28450400$

0.11922800

$\mathrm{H}$

5.43007300

$-0.88408900$

$-0.32977000$

$\mathrm{H}$

4.19563300

$-0.55356100$

$-1.42055500$

C

1.70879100

0.96012700

0.32078700

$\mathrm{H}$

2.91241000

2.65809200

0.09686600

$2.81245800 \quad 1.35010600$

0.70848900

$\mathrm{H}$

$4.14544600-0.89742300$

1.89559400

$\mathrm{H}$

0.65270100

$-1.39592000$

1.36774800

1.62053700

1.42166600

$-0.36872100$

C

$\mathrm{O}$

0.48658400

1.35620500

$-1.35968500$

$-0.50457300$

$-0.82009600$

0.79620700

$-0.67944300-2.50255700$

1.41244200

$\mathrm{H}$

$-0.59023100$

$-1.48542800$

0.95187700

0.68577900

1.09766200

0.55339400

1.66088200

2.51468400

$-1.82232800$

$\mathrm{H}$

2.45020000

1.01824000

$-1.40540700$

0.53884100

2.29896400

$-1.94383700$

$\mathrm{H}$

0.38377400

$-0.99572300$

1.01509900

1.04208000

$-2.40068300$

$-1.34810500$

$-1.90521100$

$-1.21525900$

$-0.54692300$

$-3.16691000$

$-1.05713100$

$-0.12355700$

$-1.97988500$

$-1.77490200$

0.65171500

$-4.35240300$

$-0.44055800$

$-1.32480500$

C

$-3.22470700$

$-1.45241500$

$-0.07786400$

$-2.88260900$

$-1.79844800$

1.92991800

$-4.91724500$

$-1.21785400$

$-1.68056500$

$-5.03303900$

$-0.04832900$

$-0.60815000$

$-3.96860900$

0.67569100

0.68171000

$-2.38596300$

$-1.90029500$

$-1.07684500$

$-4.14611400$

$-1.34511300$

2.44972500

$\mathrm{H}$

$-2.77677600$

1.51463600

2.49394400

$-3.73210400$

0.26062000

0.62520600

$\mathrm{H}$

$-2.06240400$ 


$\begin{array}{lrrr}\mathrm{H} & -4.82490300 & 1.33893300 & -1.22570300 \\ \mathrm{H} & -2.52649200 & 2.23327200 & -1.41245300 \\ \mathrm{C} & -2.96529900 & 2.24256400 & 0.70055400 \\ \mathrm{O} & -1.62639700 & 0.59719600 & -0.57509900 \\ \mathrm{H} & -3.82674600 & 2.91258100 & 0.62065600 \\ \mathrm{H} & -3.13912900 & 1.54711600 & 1.52439900 \\ \mathrm{H} & -2.08954400 & 2.85286700 & 0.93879300 \\ \mathrm{H} & -0.87559000 & 0.97193900 & -0.02125700\end{array}$

\section{INT1B(R)}

$E E=-927.524317$

EE + Zero-point Energy $=-927.081470$

$\mathrm{EE}+$ Thermal Free Energy Correction $=-927.128693$

Number of imaginary freq. $=0$

Lowest freq. $=44.24$

M06-2X/6-31+G(d,p), scrf $=(p c m$, Solvent=Water $)$

11

C $\quad-1.81192900-0.26498400 \quad-0.48095100$

$\begin{array}{llll}\text { C } & -4.55063600 & 0.39005200 & 0.13475400\end{array}$

$\mathrm{H} \quad-1.90654200 \quad-0.01334900 \quad-1.55172600$

C $\quad-4.99709600 \quad-2.07964800 \quad-0.22135100$

O $\quad-2.77974200-1.24614800 \quad-0.14725500$

$\begin{array}{llll}\text { C } & -3.51646100 & 1.49557600 & -0.08349400\end{array}$

$\mathrm{H} \quad \begin{array}{llll}\mathrm{H} & -4.68595600 & 0.19464700 & 1.20551400\end{array}$

$\begin{array}{llll}\mathrm{H} & -5.52145600 & 0.70019300 & -0.26478800\end{array}$

$\mathrm{H} \quad-6.02579800 \quad-1.87436100 \quad-0.52953700$

$\mathrm{H} \quad-4.65080300 \quad-2.97945400 \quad-0.73576400$

$\mathrm{H} \quad-4.98695800 \quad-2.26478700 \quad 0.85733400$

C $\quad-4.10777100-0.89814400 \quad-0.55315200$

$\begin{array}{llll}\text { C } & -2.11733400 & 1.02105500 & 0.31369500\end{array}$

$\mathrm{H} \quad-3.77980000 \quad 2.39274700 \quad 0.48937500$

$\mathrm{H} \quad-3.49138100 \quad 1.77404100 \quad-1.14484600$

$\mathrm{H} \quad-4.09302100-0.73397400 \quad-1.64387200$

C $\quad-0.44164200 \quad-0.90159600 \quad-0.24240700$

$\begin{array}{llll}\text { C } & -1.98363400 & 0.83380700 & 1.82198500\end{array}$

$\begin{array}{llll}\mathrm{O} & -1.14639800 & 1.98742900 & -0.14143400\end{array}$

$\mathrm{H} \quad 0.37012800 \quad 0.44853400 \quad-1.77129200$

$\mathrm{H} \quad 1.24161200 \quad-1.05294000 \quad-1.60332100$

C $\quad 0.72991500 \quad-0.27761900-1.03133200$

$\mathrm{H} \quad-0.93852800 \quad 0.69727200 \quad 2.11141800$

$\mathrm{H} \quad-2.36629500 \quad 1.71651200 \quad 2.34387500$

$\mathrm{H} \quad-2.54783000-0.04017800 \quad 2.15603800$

$\mathrm{H} \quad-1.42520100 \quad 2.87771700 \quad 0.11919200$

$\mathrm{H} \quad-0.22304100-0.94345400 \quad 0.82845300$

$\mathrm{H} \quad-0.54443100-1.94104400 \quad-0.56407800$

$\begin{array}{llll}\text { C } & 1.77572700 & 0.48636900 & -0.23176700\end{array}$

C $\quad 3.08720000 \quad 0.68311700 \quad-0.94430400$

$\begin{array}{llll}\text { O } & 1.33548900 & 1.57075500 & 0.41989200\end{array}$

C $\quad 3.84065100 \quad-0.56489300-1.36151700$

C $\quad 3.50645700 \quad 1.92186300 \quad-1.20049300$

$\begin{array}{llll}\mathrm{H} & 0.35026200 & 1.71459600 & 0.28192800\end{array}$

H $\quad 3.54554500 \quad-0.85444700 \quad-2.37625100$

$\mathrm{H} \quad 4.90376000 \quad-0.31447700 \quad-1.40715700$

C $\quad 3.61140500 \quad-1.75939400 \quad-0.41904400$

$\mathrm{H} \quad 2.92597300 \quad 2.78607100 \quad-0.89808500$

$\mathrm{H} \quad 4.44091700 \quad 2.08938100 \quad-1.72792900$

C $\quad 3.40553100-1.33235300 \quad 1.02063900$ 


$\begin{array}{lrrr}\mathrm{H} & 2.74521400 & -2.35161500 & -0.72817500 \\ \mathrm{H} & 4.47178900 & -2.43257100 & -0.44636100 \\ \mathrm{H} & 3.12981000 & -2.18820600 & 1.63890500 \\ \mathrm{C} & 4.54668300 & -0.55482700 & 1.64293900 \\ \mathrm{O} & 2.16203100 & -0.50287100 & 1.01340400 \\ \mathrm{H} & 5.42711600 & -1.20238000 & 1.66501400 \\ \mathrm{H} & 4.78847800 & 0.34400300 & 1.07170400 \\ \mathrm{H} & 4.31533800 & -0.27275300 & 2.67353400 \\ \mathrm{H} & 2.09767300 & 0.06067300 & 1.80883900\end{array}$

\section{INT1B(R)}

$E E=-928.010277$

EE + Zero-point Energy $=-927.570985$

$\mathrm{EE}+$ Thermal Free Energy Correction $=-927.617500$

Number of imaginary freq. $=0$

Lowest freq. $=41.14$

B3LYP(D3)/6-31+G(d,p), scrf=(pcm, Solvent=Water $)$

11

C

C

1.72051300

0.95488000

0.44971300

1.52736700

$-0.40392600$

$-0.27179000$

C

3.86053100

$-0.94284800$

$-0.11441900$

C

4.15286000

0.31163700

0.71444200

2.88854100

0.80504300

1.43510900

$\mathrm{H}$

$1.39018400-1.16811600$

0.51330600

$\mathrm{H}$

3.60183300

$-1.77045100$

0.56686500

$\mathrm{H}$

4.93313600

0.08483800

1.44858100

$\mathrm{H}$

4.54731600

1.09340300

0.05519800

$\mathrm{H}$

2.59382100

0.08241600

2.20597500

$5.01776000-1.35661200-1.00779300$

C

5.90364000

1.56720400

$-0.40084100$

$\mathrm{H}$

4.76326300

$-225596100$

$-1.57604100$

5.26139000

$-0.55369300$

$-1.71152200$

0.35301100

$-0.46845400$

$-1.26529600$

0.02625300

0.53498800

$-1.54587900$

0.75151700

$-0.90981000$

$-2.18294300$

$-4.01713200$

$-0.69822600$

$-0.81153800$

$-3.65648700$

$-0.91986000$

$-1.82481900$

$-5.04119400$

$-1.07489700$

$-0.75514800$

$-4.00773900$

0.82527500

$-0.60253600$

$-4.47667500$

1.07996700

0.35447100

$-4.57780400$

1.31759700

$-1.39648600$

$-2.59766900$

1.38830800

$-0.62562600$

$-2.10786200$

1.18130400

$-1.58160400$

$-2.49195600$

2.85516200

$-0.25839500$

$-2.91777500$

3.03524800

0.73245700

$-1.45728500$

3.20698300

$-0.27623800$

$-3.05679500$

3.43558700

$-0.99333600$

$-1.84059900$

0.62309600

0.41816900

$-0.90067500$

0.98397400

0.65794200

2.72218300

$-0.72067300$

$-0.98029300$

0.48352000

1.14004800

1.21428200

$-3.16820600$

$-1.41400200$

0.21612100

$-3.61102200$

$-2.42375600$

0.97104900

$-4.63026300$

$-2.78286100$

0.86609800

$-2.97615900$

$-2.91716200$

1.69776700

3.07828400

1.76050200

1.93741500

C

1.91143400

2.13516300

$-0.50110600$ 


$\begin{array}{lrrr}\mathrm{H} & 2.11420200 & 3.04461900 & 0.07249200 \\ \mathrm{H} & 2.75406200 & 1.95250600 & -1.17084500 \\ \mathrm{H} & 1.02333900 & 2.31061000 & -1.11127600 \\ \mathrm{C} & -1.73369500 & -0.94368200 & 0.33784700 \\ \mathrm{O} & -1.21758700 & -1.28765800 & 1.55902100 \\ \mathrm{H} & -0.26071600 & -1.13919600 & 1.59658600 \\ \mathrm{H} & 0.57735700 & 1.87209600 & 1.84379400 \\ \mathrm{C} & -0.86575500 & -1.32826700 & -0.87820700 \\ \mathrm{H} & -1.51531300 & -1.36924500 & -1.75508400 \\ \mathrm{H} & -0.55778400 & -2.36085800 & -0.68177000\end{array}$

\section{INT1B(S)}

$E E=-927.532259$

EE + Zero-point Energy $=-927.089707$

$E E+$ Thermal Free Energy Correction $=-927.135793$

Number of imaginary freq. $=0$

Lowest freq. $=44.91$

M06-2X/6-31+G(d,p), scrf $=(p c m$, Solvent $=$ Water $)$

11

C

C

$\mathrm{H}$

C

$\mathrm{O}$

1.78022900

$4.14054400 \quad 1.02128200$

0.25082600

$\begin{array}{lll}1.81483100 & -0.83703300 & 1.33308200\end{array}$

C

$5.37080100-1.09704600$

$-0.34576400$

$3.00057600-1.02322900-0.34255300$

$\begin{array}{lll}2.80706100 & 1.56059600 & 0.83043500\end{array}$

$\mathrm{H}$

4.31637700

1.36342800

$-0.72021300$

$\mathrm{H}$

4.96222100

1.40102100

0.92163800

$\mathrm{H}$

6.28245800

$-0.74825000$

0.14648100

$\mathrm{H}$

5.34424800

$-2.18841000$

$-0.29984400$

5.40129900

$-0.78882300$

$-1.39543300$

C

4.15296700

$-0.50506200$

0.33395400

1.64144000

0.90488400

2.75705500

2.64950500

0.09206500

2.70857200

1.33165100

0.71390100

4.10531800

$-0.84444400$

1.89913000

$\mathrm{H}$

0.66893200

$-1.46834600$

1.38195000

C

1.54819600

1.35541400

$-0.37368700$

0.40343800

1.25436600

$-1.36084100$

$\mathrm{O}$

$-0.46878800$

$-1.05353400$

0.78290500

$-0.84399700$

$-2.59718100$

1.44755100

$-0.61556700$

$-1.55072300$

0.69999000

0.60885900

1.03801800

0.48463300

$\mathrm{H}$

1.60559600

2.44638800

$-1.82053100$

2.37078400

0.93115300

$-1.41837000$

0.41546200

2.19014700

$-1.94054100$

$\mathrm{H}$

0.47515400

$-1.10505000$

1.03866300

1.08414800

$-2.47155200$

$-1.39061300$

$\mathrm{H}$

C

$-1.87040600$

$-0.99157000$

$-0.48898000$

$-3.13723200$

$-0.99872000$

$-0.16837800$

$-2.17119700$

$-1.57586500$

0.65689400

O

$-4.32723200$

$-0.37305000$

$-1.37864600$

$-3.18735400$

$-1.50045800$

$-0.04241100$

C

$-1.37032100$

$-1.71037200$

1.88897700

$-4.88972000$

$-1.15119600$

$-1.90570800$

$\mathrm{H}$

$-4.99364400$

0.04644000

$-0.56819200$

$\mathrm{H}$

$-3.90356100$

0.70184000

0.71618100

$-2.33417300$

$-1.95965400$

2.37534400 


$\begin{array}{lrrr}\mathrm{H} & -4.11578000 & -1.46842500 & 2.45167000 \\ \mathrm{C} & -2.66304600 & 1.45327000 & -0.60823800 \\ \mathrm{H} & -3.71010100 & 0.25176000 & -2.03336500 \\ \mathrm{H} & -4.69977300 & 1.43904100 & -1.19003800 \\ \mathrm{H} & -2.30082300 & 2.10678600 & -1.40430800 \\ \mathrm{C} & -2.83069500 & 2.22049700 & 0.69020700 \\ \mathrm{O} & -1.55710300 & 0.46279500 & -0.45610200 \\ \mathrm{H} & -3.64428400 & 2.93964600 & 0.56424800 \\ \mathrm{H} & -3.07657600 & 1.55604500 & 1.52201900 \\ \mathrm{H} & -1.93057800 & 2.78638600 & 0.94135500 \\ \mathrm{H} & -0.72999500 & 0.84883300 & 0.11626500\end{array}$

\section{INT1B(S)}

$E E=-928.004963$

$\mathrm{EE}+$ Zero-point Energy= -927.566021

$E E+$ Thermal Free Energy Correction $=-927.613128$

Number of imaginary freq. $=0$

Lowest freq. $=25.74$

B3LYP(D3)/6-31+G(d,p), scrf=(pcm, Solvent=Water $)$ 11

\begin{tabular}{lrrr}
$\mathrm{C}$ & 1.79260800 & -0.60327300 & 0.27734300 \\
$\mathrm{C}$ & 4.19426200 & 1.02319900 & 0.26240400 \\
$\mathrm{H}$ & 1.82270000 & -0.76470700 & 1.36753700 \\
$\mathrm{C}$ & 5.40380800 & -1.14908600 & -0.29545200 \\
$\mathrm{O}$ & 3.02355400 & -1.04535500 & -0.29546000 \\
$\mathrm{C}$ & 2.85970300 & 1.60238900 & 0.75409400 \\
$\mathrm{H}$ & 4.37830100 & 1.31748600 & -0.77737900 \\
$\mathrm{H}$ & 5.01726700 & 1.42462800 & 0.86317800 \\
$\mathrm{H}$ & 6.32246200 & -0.79187100 & 0.18018500 \\
$\mathrm{H}$ & 5.36188900 & -2.23782700 & -0.19750200 \\
$\mathrm{H}$ & 5.44155400 & -0.89392600 & -1.35981100 \\
$\mathrm{C}$ & 4.19261000 & -0.50532800 & 0.35889300 \\
$\mathrm{C}$ & 1.67438500 & 0.92597700 & 0.05230500 \\
$\mathrm{H}$ & 2.82385000 & 2.68521700 & 0.58489100 \\
$\mathrm{H}$ & 2.75767500 & 1.43206700 & 1.83306200 \\
$\mathrm{H}$ & 4.14596700 & -0.79648800 & 1.42170100 \\
$\mathrm{C}$ & 0.68340500 & -1.48032300 & -0.31489900 \\
$\mathrm{C}$ & 1.55920500 & 1.32264500 & -1.41903600 \\
$\mathrm{O}$ & 0.44077200 & 1.32193000 & 0.75385500 \\
$\mathrm{H}$ & -0.51072400 & -0.99404400 & 1.46959800 \\
$\mathrm{H}$ & -0.84703700 & -2.56580300 & 0.76958900 \\
$\mathrm{C}$ & -0.62271400 & -1.52609900 & 0.52237500 \\
$\mathrm{H}$ & 0.62994000 & 0.95688800 & -1.86081500 \\
$\mathrm{H}$ & 1.57846800 & 2.41247400 & -1.51632900 \\
$\mathrm{H}$ & 2.39327300 & 0.90745900 & -1.98815100 \\
$\mathrm{H}$ & 0.45887200 & 2.27687700 & 0.92528100 \\
$\mathrm{H}$ & 0.47959000 & -1.19654900 & -1.34954100 \\
$\mathrm{H}$ & 1.10032500 & -2.48920100 & -0.35898700 \\
$\mathrm{C}$ & -1.87028700 & -1.01233800 & -0.18411000 \\
$\mathrm{C}$ & -3.18023100 & -1.05505400 & 0.57466300 \\
$\mathrm{O}$ & -1.93558900 & -1.59143300 & -1.43316300 \\
$\mathrm{C}$ & -4.35625800 & -0.38082000 & -0.11939900 \\
$\mathrm{C}$ & -3.29226600 & -1.64060200 & 1.77003700 \\
$\mathrm{H}$ & -2.83222400 & -1.57767700 & -1.79612300 \\
$\mathrm{H}$ & -4.91946900 & -1.11341700 & -0.71024400 \\
$\mathrm{H}$ & -5.04536100 & -0.02503600 & 0.65048600 \\
$\mathrm{C}$ & -3.92672800 & 0.78572100 & -1.03674900 \\
& & & \\
\hline & & &
\end{tabular}




$\begin{array}{lrrr}\mathrm{H} & -2.46718500 & -2.13105400 & 2.27218000 \\ \mathrm{H} & -4.24708100 & -1.64518200 & 2.28749300 \\ \mathrm{C} & -2.70780800 & 1.52038900 & -0.50062900 \\ \mathrm{H} & -3.70294800 & 0.43916700 & -2.05035500 \\ \mathrm{H} & -4.74146200 & 1.50781600 & -1.13484000 \\ \mathrm{H} & -2.34034900 & 2.23824100 & -1.23673700 \\ \mathrm{C} & -2.89495600 & 2.18227900 & 0.85583200 \\ \mathrm{O} & -1.59082700 & 0.51420200 & -0.43732400 \\ \mathrm{H} & -3.69455400 & 2.92405400 & 0.76978100 \\ \mathrm{H} & -3.16996700 & 1.45936900 & 1.62629000 \\ \mathrm{H} & -1.99017300 & 2.70624300 & 1.17327500 \\ \mathrm{H} & -0.75930200 & 0.88200400 & 0.09299600\end{array}$

\section{INT2B}

$E E=-851.123241$

EE + Zero-point Energy $=-850.708486$

$\mathrm{EE}+$ Thermal Free Energy Correction $=-850.755912$

Number of imaginary freq. $=0$

Lowest freq. $=13.50$

M06-2X/6-31+G(d,p), scrf $=(p c m$, Solvent=Water $)$

11

C $\quad 3.78049200 \quad-0.55264700 \quad-0.53545100$

C $\quad 3.62637800 \quad-1.08283700 \quad 0.88619500$

C $\quad 2.16553100 \quad-1.44137800 \quad 1.15672600$

C $\quad 1.23073200 \quad-0.27086500 \quad 0.83079500$

C $\quad 1.50200100 \quad 0.15724800 \quad-0.62639900$

$\mathrm{H} \quad 4.26407700 \quad-1.96321500 \quad 1.01713400$

$\mathrm{H} \quad 3.53282900 \quad-1.35911500 \quad-1.24661100$

$\mathrm{H} \quad 1.87155700 \quad-2.29012300 \quad 0.52571700$

$\mathrm{H} \quad 2.02215900-1.74300200 \quad 2.20136400$

$\mathrm{H} \quad \begin{array}{llll}\mathrm{H} & 1.29648400 & -0.72009100 & -1.26562800\end{array}$

$\mathrm{H} \quad 3.97560800 \quad-0.31795700 \quad 1.59104100$

C $\quad 5.17033100 \quad-0.02151300-0.82378200$

$\mathrm{H} \quad 5.91156600 \quad-0.81343900-0.68751100$

$\mathrm{H} \quad 5.23722000 \quad 0.34708900 \quad-1.85039700$

$\mathrm{H} \quad 5.40772700 \quad 0.79897800 \quad-0.13930100$

C $\quad 0.69530700 \quad 1.34981000 \quad-1.13970200$

$\begin{array}{llll}\mathrm{H} & 0.63338500 & 2.12999200 & -0.37725700\end{array}$

$\mathrm{H} \quad 1.26787900 \quad 1.76889000 \quad-1.97167200$

C $\quad-0.69408600 \quad 1.01930200-1.71814300$

$\mathrm{H} \quad-0.60548300 \quad 0.25113900-2.48932000$

$\mathrm{H} \quad-1.09403100 \quad 1.91970900-2.19768000$

$\begin{array}{llll}\mathrm{H} & -0.12626500 & -0.73279900 & 0.84626400\end{array}$

$\begin{array}{llll}0 & 2.86604100 & 0.52449300 & -0.76119600\end{array}$

C $\quad 1.39796700 \quad 0.87939000 \quad 1.82153000$

$\begin{array}{llll}\mathrm{H} & 1.39133700 & 0.49307400 & 2.84617800\end{array}$

$\begin{array}{llll}\mathrm{H} & 2.34228800 & 1.40520000 & 1.65780800\end{array}$

$\mathrm{H} \quad 0.57711300 \quad 1.59533900 \quad 1.72550400$

C $\quad-1.72466200 \quad 0.54770100 \quad-0.74471400$

$\begin{array}{llll}\text { C } & -2.23102300 & 1.34294600 & 0.37123500\end{array}$

C $\quad-3.09111900 \quad 0.63186600 \quad 1.38062500$

C $\quad-4.00172000 \quad-0.35306100 \quad 0.64940500$

$\mathrm{H} \quad-3.67153100 \quad 1.36142500 \quad 1.94806300$

$\mathrm{H} \quad-2.45105700 \quad 0.09251300 \quad 2.08879600$

$\mathrm{H} \quad-4.69325600 \quad 0.18262200 \quad-0.01112600$

$\mathrm{H} \quad-4.59845900-0.93357800 \quad 1.35769200$

$\begin{array}{llll}\mathrm{H} & -2.19665600 & -0.60176900 & -1.01296300\end{array}$ 


$\begin{array}{lrrr}\mathrm{C} & -3.17438800 & -1.32528700 & -0.16261900 \\ \mathrm{H} & -2.52790500 & -1.92839300 & 0.48267000 \\ \mathrm{C} & -1.98930200 & 2.66333000 & 0.38356000 \\ \mathrm{H} & -1.40037800 & 3.16417300 & -0.37689700 \\ \mathrm{H} & -2.40760400 & 3.28561000 & 1.16887800 \\ \mathrm{C} & -3.96449500 & -2.19606100 & -1.11125600 \\ \mathrm{H} & -4.63455600 & -2.83078100 & -0.52653600 \\ \mathrm{H} & -4.56430500 & -1.58089600 & -1.78672800 \\ \mathrm{H} & -3.30079100 & -2.83579900 & -1.69574200 \\ \mathrm{H} & -0.31448700 & -1.08641200 & 1.72669300\end{array}$

\section{INT2B}

$\mathrm{EE}=-851.570566$

EE + Zero-point Energy $=-851.158571$

$\mathrm{EE}+$ Thermal Free Energy Correction $=-851.205808$

Number of imaginary freq. $=0$

Lowest freq. $=21.18$

B3LYP(D3)/6-31+G(d,p), scrf=(pcm, Solvent=Water $)$

11

C

C

3.91293900

$-0.32436500-0.04836600$

C

3.65668200

$0.46991100 \quad 1.23606700$

C

2.27765600

$0.13037700 \quad 1.82163100$

1.16398300

0.26075300

0.76628400

C

1.56308300

$-0.60961900$

$-0.44995700$

$\mathrm{H}$

$4.44220800 \quad 0.24124800$

1.96500800

$3.97873800-1.39675200$

0.20251600

$\mathrm{H}$

2.27414900

$-0.90595200$

2.18242000

$\mathrm{H}$

2.04987200

0.77522100

2.67923600

1.68140800

$-1.64115900$

$-0.07658000$

$\mathrm{H}$

3.72556600

1.54109500

1.01225000

5.17373900

0.10727300

$-0.77972200$

6.04785300

$-0.02583200$

$-0.13460400$

$\mathrm{H}$

5.31707600

$-0.48742600$

$-1.68679400$

$\mathrm{H}$

5.10740700

$1.16396100-1.06039600$

C

0.58528100

$-0.64052300-1.62921200$

$\mathrm{H}$

0.34011700

$0.36885000-1.96729100$

1.11622600

$-1.12805800$

$-2.45153600$

$-0.69281600$

$-1.46248600$

$-1.37192800$

$-0.43940200$

$-2.39369300$

$-0.86885700$

$-1.13499400$

$-1.73839300$

$-2.34038900$

$-0.05011100$

$-0.34757000$

1.26443000

2.81406800

$-0.15549400$

$-0.96875000$

0.90193100

1.72040400

0.39073800

0.66917200

2.30214700

1.28920200

1.77679300

2.16367100

$-0.08990300$

0.05867400

1.79728300

$-0.29495300$

$-1.81010200$

$-0.76916600$

$-0.65450200$

$-2.72747900$

$-1.40887300$

0.27971100

$-3.83508600$

$-0.56980600$

0.88966600

$-3.49739900$

0.92013200

0.83719300

$-4.00533700$

$-0.89367300$

1.91940400

$-4.76418200$

$-0.75316100$

0.33646300

$-2.66294300$

1.15988400

1.50522600

$-4.35528300$

1.52147800

1.14979100

$-1.99076000$

0.44251700

$-1.04305600$

$-3.13079500$

1.30992800

$-0.57926700$

H

$-3.93444000$

1.06133000

$-1.27884900$ 


$\begin{array}{lrrr}\mathrm{C} & -2.59437900 & -2.72149100 & 0.56310200 \\ \mathrm{H} & -1.82636500 & -3.35469900 & 0.13828400 \\ \mathrm{H} & -3.28951100 & -3.19953900 & 1.24668200 \\ \mathrm{C} & -2.66826800 & 2.73721500 & -0.77292700 \\ \mathrm{H} & -3.52020800 & 3.40117400 & -0.60072200 \\ \mathrm{H} & -1.87831300 & 2.99753500 & -0.06447100 \\ \mathrm{H} & -2.30777300 & 2.89495100 & -1.79245000 \\ \mathrm{H} & -0.32589000 & 0.11491100 & 2.06925600\end{array}$

\section{TSS to INT3B(R)+}

$E E=-851.108336$

EE + Zero-point Energy $=-850.692035$

$\mathrm{EE}+$ Thermal Free Energy Correction $=-850.735431$

Number of imaginary freq. $=1$

Lowest freq. $=-61.72$

M06-2X/6-31+G(d,p), scrf=(pcm, Solvent=Water)

11

C $\quad-3.85166800 \quad-0.27080500 \quad 0.06777300$

$\begin{array}{llll}\text { C } & -3.44970800 & 0.06840400 & -1.36871200\end{array}$

C $\quad-2.00712400 \quad-0.36185200-1.68813800$

C $\quad-1.08650200 \quad 0.14940300 \quad-0.58525600$

C $\quad-1.60675700 \quad-0.38332800 \quad 0.75869300$

$\mathrm{H} \quad-4.13366800 \quad-0.43515200 \quad-2.05821800$

$\mathrm{H} \quad-3.90732700 \quad-1.36576900 \quad 0.17650700$

$\mathrm{H} \quad-1.94230000 \quad-1.45547800 \quad-1.72079500$

$\begin{array}{llll}\mathrm{H} & -1.70146600 & 0.02975200 & -2.66420900\end{array}$

H $\quad-1.73244700 \quad-1.47435200 \quad 0.64524700$

$\mathrm{H} \quad-3.56903100 \quad 1.14564600 \quad-1.52880800$

$\begin{array}{llll}\text { C } & -5.17274700 & 0.35558400 & 0.46369500\end{array}$

$\begin{array}{llll}\mathrm{H} & -5.96686800 & 0.00962700 & -0.20308400\end{array}$

$\begin{array}{llll}\mathrm{H} & -5.43377200 & 0.08353900 & 1.48908900\end{array}$

$\begin{array}{llll}\mathrm{H} & -5.10773400 & 1.44569100 & 0.39290600\end{array}$

C $\quad-0.63959200 \quad-0.14165700 \quad 1.90104300$

$\begin{array}{llll}\mathrm{H} & -0.42617400 & 0.92374700 & 2.02587000\end{array}$

$\mathrm{H} \quad-1.09008700 \quad-0.50381100 \quad 2.82858600$

C $\quad 0.63655000 \quad-0.93740100 \quad 1.61768200$

$\mathrm{H} \quad 0.40965600 \quad-2.00547100 \quad 1.55198000$

$\mathrm{H} \quad 1.37370000 \quad-0.80503000 \quad 2.41576600$

O $\quad 0.22774900 \quad-0.57600100-0.76166500$

$\begin{array}{llll}0 & -2.86292900 & 0.20313200 & 0.99754000\end{array}$

C $\quad-0.87632100 \quad 1.65184500 \quad-0.64908600$

$\mathrm{H} \quad-0.39892700 \quad 1.92148300 \quad-1.59604300$

$\mathrm{H} \quad-1.85312000 \quad 2.13649200 \quad-0.60882900$

$\mathrm{H} \quad-0.26567800 \quad 2.02800800 \quad 0.16862700$

$\begin{array}{llll}\text { C } & 1.34569900 & -0.50935700 & 0.33876800\end{array}$

C $\quad 2.39073600-1.49422100 \quad-0.15875600$

C $\quad 3.82315700 \quad-1.05023200-0.03470800$

$\begin{array}{llll}C & 3.94799000 & 0.39109500 & -0.52107700\end{array}$

$\mathrm{H} \quad 4.46035100 \quad-1.73010500-0.60441000$

$\mathrm{H} \quad 4.14168200 \quad-1.10340000 \quad 1.01528600$

$\mathrm{H} \quad 3.64287400 \quad 0.47178000 \quad-1.57216600$

$\mathrm{H} \quad 4.98145300 \quad 0.74102000 \quad-0.44308900$

$\begin{array}{llll}\mathrm{O} & 1.70110300 & 0.80681100 & 0.38377400\end{array}$

$\begin{array}{llll}\text { C } & 3.07712900 & 1.28051800 & 0.34095900\end{array}$

$\begin{array}{llll}\mathrm{H} & 3.44207700 & 1.24676400 & 1.37580500\end{array}$

C $\quad 2.01803500-2.67173900-0.66236500$

$\mathrm{H} \quad 0.97554300 \quad-2.96563700 \quad-0.74342900$ 


$\begin{array}{lrrr}\mathrm{H} & 2.76485500 & -3.37748500 & -1.01205300 \\ \mathrm{C} & 2.99316000 & 2.71499200 & -0.13541100 \\ \mathrm{H} & 3.98739500 & 3.16837500 & -0.10679900 \\ \mathrm{H} & 2.61650900 & 2.75112800 & -1.16185000 \\ \mathrm{H} & 2.32716400 & 3.29534700 & 0.50766300 \\ \mathrm{H} & 0.62574000 & -0.39574300 & -1.63735900\end{array}$

\begin{tabular}{|c|c|c|c|}
\hline \multicolumn{4}{|c|}{$\begin{array}{l}\text { EE }+ \text { Zero-point Energy }=-851.134708 \\
E E+\text { Thermal Free Energy Correction }=-851.178044 \\
\text { Number of imaginary freq. }=1 \\
\text { Lowest freq. }=-74.04 \\
\text { B3LYP(D3)/6-31+G(d,p), scrf=(pcm, Solvent=Water }) \\
11\end{array}$} \\
\hline $\mathrm{C}$ & -3.89879700 & -0.25771300 & 0.15081300 \\
\hline C & -3.53243500 & -0.09445700 & -1.33151700 \\
\hline C & -2.08901100 & -0.55508000 & -1.63333000 \\
\hline C & -1.14357500 & 0.10195600 & -0.62866200 \\
\hline C & -1.61276000 & -0.26901200 & 0.79204100 \\
\hline $\mathrm{H}$ & -4.22922500 & -0.68124800 & -1.93838500 \\
\hline $\mathrm{H}$ & -3.94463000 & -1.33181800 & 0.39222700 \\
\hline $\mathrm{H}$ & -2.01427000 & -1.64276700 & -1.52708900 \\
\hline $\mathrm{H}$ & -1.81318600 & -0.29411300 & -2.66039500 \\
\hline $\mathrm{H}$ & -1.72529000 & -1.36556800 & 0.81785300 \\
\hline $\mathrm{H}$ & -3.66092000 & 0.95439600 & -1.62026600 \\
\hline C & -5.21548100 & 0.40824600 & 0.51147100 \\
\hline $\mathrm{H}$ & -6.02962800 & -0.02564800 & -0.07696800 \\
\hline $\mathrm{H}$ & -5.43908000 & 0.26618000 & 1.57261000 \\
\hline $\mathrm{H}$ & -5.16914600 & 1.48212700 & 0.30221700 \\
\hline C & -0.61372000 & 0.11899600 & 1.87079400 \\
\hline $\mathrm{H}$ & -0.40314100 & 1.19144600 & 1.85429900 \\
\hline $\mathrm{H}$ & -1.03885700 & -0.12124600 & 2.84902400 \\
\hline C & 0.66682600 & -0.70529800 & 1.66500200 \\
\hline $\mathrm{H}$ & 0.44835500 & -1.77257100 & 1.75678500 \\
\hline $\mathrm{H}$ & 1.41452600 & -0.46087200 & 2.42595600 \\
\hline $\mathrm{O}$ & 0.19390800 & -0.64929500 & -0.75416300 \\
\hline $\mathrm{O}$ & -2.87944500 & 0.33278600 & 0.99823600 \\
\hline C & -0.93251800 & 1.58630300 & -0.88310400 \\
\hline $\mathrm{H}$ & -0.54218600 & 1.74646300 & -1.89212000 \\
\hline $\mathrm{H}$ & -1.89815500 & 2.08815400 & -0.80633800 \\
\hline $\mathrm{H}$ & -0.24801100 & 2.03748800 & -0.16960400 \\
\hline C & 1.35784000 & -0.46440100 & 0.32461300 \\
\hline C & 2.37829700 & -1.52407200 & -0.06431900 \\
\hline C & 3.82742800 & -1.12043400 & 0.07444700 \\
\hline C & 4.04916500 & 0.29589500 & -0.46622700 \\
\hline $\mathrm{H}$ & 4.45696500 & -1.84997900 & -0.44146700 \\
\hline $\mathrm{H}$ & 4.11328000 & -1.14445400 & 1.13561800 \\
\hline $\mathrm{H}$ & 3.83720000 & 0.33573000 & -1.54174200 \\
\hline $\mathrm{H}$ & 5.08842300 & 0.60541700 & -0.31647800 \\
\hline $\mathrm{O}$ & 1.73723000 & 0.84405000 & 0.15243700 \\
\hline C & 3.13981400 & 1.26583000 & 0.26780200 \\
\hline $\mathrm{H}$ & 3.38046300 & 1.25970700 & 1.33937300 \\
\hline C & 1.98485800 & -2.73664600 & -0.47032100 \\
\hline $\mathrm{H}$ & 0.93878200 & -3.01270200 & -0.55065800 \\
\hline $\mathrm{H}$ & 2.71866900 & -3.49055400 & -0.73788200 \\
\hline C & 3.16976600 & 2.68627900 & -0.26421300 \\
\hline
\end{tabular}




$\begin{array}{rrrr}\mathrm{H} & 4.17592300 & 3.09894300 & -0.14525800 \\ \mathrm{H} & 2.90565100 & 2.70171100 & -1.32615200 \\ \mathrm{H} & 2.46638900 & 3.31850200 & 0.28450600 \\ \mathrm{H} & 0.58337000 & -0.53140200 & -1.64348000\end{array}$

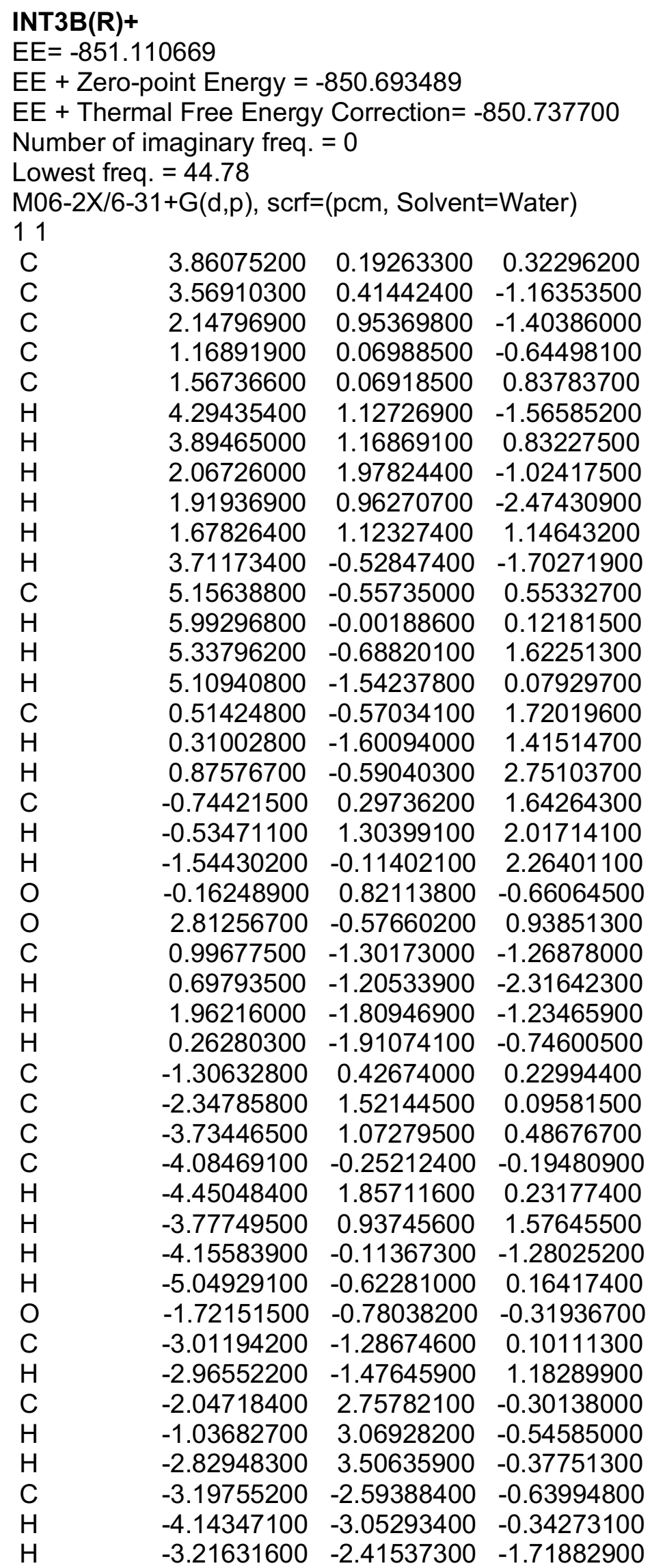


$\begin{array}{llll}\mathrm{H} & -2.38424900 & -3.28548600 & -0.40798900\end{array}$

$\begin{array}{lllll}\mathrm{H} & -0.49889600 & 0.92808900 & -1.57553800\end{array}$

\section{INT3B(R)+}

$E E=-851.548768$

$E E+$ Zero-point Energy $=-851.134885$

$\mathrm{EE}+$ Thermal Free Energy Correction $=-851.179063$

Number of imaginary freq. $=0$

Lowest freq. $=52.10$

B3LYP(D3)/6-31+G(d,p), scrf=(pcm, Solvent=Water $)$

11

C

C

C

$\begin{array}{lll}-3.89766400 & -0.20790100 & 0.31610900\end{array}$

$\begin{array}{lll}-3.60260200 & -0.38689400 & -1.18089700\end{array}$

$-2.17042600-0.90631700-1.44104300$

$\begin{array}{llll}\text { C } & -1.19582400 & -0.03195800 & -0.66011400 \\ \text { C } & -1.58230200 & -0.08095800 & 0.82985600\end{array}$

$\mathrm{H} \quad-4.32090500-1.09570300-1.60438700$

$\mathrm{H} \quad-3.92700900-1.19806700 \quad 0.79802200$

$\mathrm{H} \quad-2.07727200-1.94031300 \quad-1.09226200$

$\mathrm{H} \quad-1.94650500 \quad-0.88518300 \quad-2.51211000$

$\mathrm{H} \quad-1.68297300 \quad-1.14401500 \quad 1.10412600$

H $\quad-3.75327800 \quad 0.56859300 \quad-1.69482600$

$\begin{array}{llll}\text { C } & -5.19874400 & 0.53089800 & 0.57704300\end{array}$

$\mathrm{H} \quad-6.03682400 \quad-0.01849600 \quad 0.13769600$

$\mathrm{H} \quad-5.37363900 \quad 0.63108900 \quad 1.65195400$

$\mathrm{H} \quad-5.16514700 \quad 1.53024200 \quad 0.13088800$

$\begin{array}{llll}\text { C } & -0.52894700 & 0.53607900 & 1.73450100\end{array}$

$\begin{array}{llll}\mathrm{H} & -0.32681100 & 1.57513000 & 1.46203700\end{array}$

$\begin{array}{llll}\mathrm{H} & -0.89497800 & 0.52698100 & 2.76462900\end{array}$

C $\quad 0.73970000-0.32453900 \quad 1.64345100$

$\mathrm{H} \quad 0.53628300 \quad-1.33759700 \quad 2.00199000$

$\mathrm{H} \quad \begin{array}{llll}\mathrm{H} & 1.53100000 & 0.08385800 & 2.27735800\end{array}$

$\begin{array}{llll}\mathrm{O} & 0.16547800 & -0.79491400 & -0.69472600\end{array}$

$\begin{array}{llll}0 & -2.84226400 & 0.55446600 & 0.95913800\end{array}$

C $\quad-1.01170600 \quad 1.35476200 \quad-1.25055900$

$\begin{array}{llll}\mathrm{H} & -0.74496200 & 1.28586800 & -2.30894800\end{array}$

$\mathrm{H} \quad-1.96369400 \quad 1.88227700 \quad-1.17057400$

$\mathrm{H} \quad-0.24945400 \quad 1.92960500-0.73080000$

C $\quad 1.32595300 \quad-0.42642600 \quad 0.23287100$

C $\quad 2.35641700 \quad-1.53420600 \quad 0.08049700$

C $\quad 3.75471400 \quad-1.10572200 \quad 0.46559500$

C $\quad 4.12460200 \quad 0.22903200 \quad-0.19797900$

$\mathrm{H} \quad 4.46332900-1.89301800 \quad 0.19599200$

$\mathrm{H} \quad 3.80814000 \quad-0.98855600 \quad 1.55751500$

$\mathrm{H} \quad 4.18997400 \quad 0.10568600 \quad-1.28564800$

$\begin{array}{llll}\mathrm{H} & 5.09888400 & 0.57597200 & 0.16136200\end{array}$

$\begin{array}{llll}\mathrm{O} & 1.75319600 & 0.79563200 & -0.28558800\end{array}$

C $\quad 3.07196100 \quad 1.28182200 \quad 0.12280100$

$\begin{array}{llll}\mathrm{H} & 3.04038800 & 1.45771900 & 1.20684700\end{array}$

C $\quad 2.03914400 \quad-2.76816700-0.32427600$

$\mathrm{H} \quad 1.02454300 \quad-3.06549700 \quad-0.56649600$

$\mathrm{H} \quad 2.81045100 \quad-3.52685400 \quad-0.41477100$

$\begin{array}{llll}\text { C } & 3.25971300 & 2.60039400 & -0.60451700\end{array}$

$\mathrm{H} \quad 4.21573600 \quad 3.04539300 \quad-0.31386600$

$\mathrm{H} \quad 3.26081200 \quad 2.44185100 \quad-1.68744000$

$\mathrm{H} \quad 2.45757500 \quad 3.29831700 \quad-0.34954800$

H $\quad 0.51109800 \quad-0.84615300 \quad-1.60936900$ 


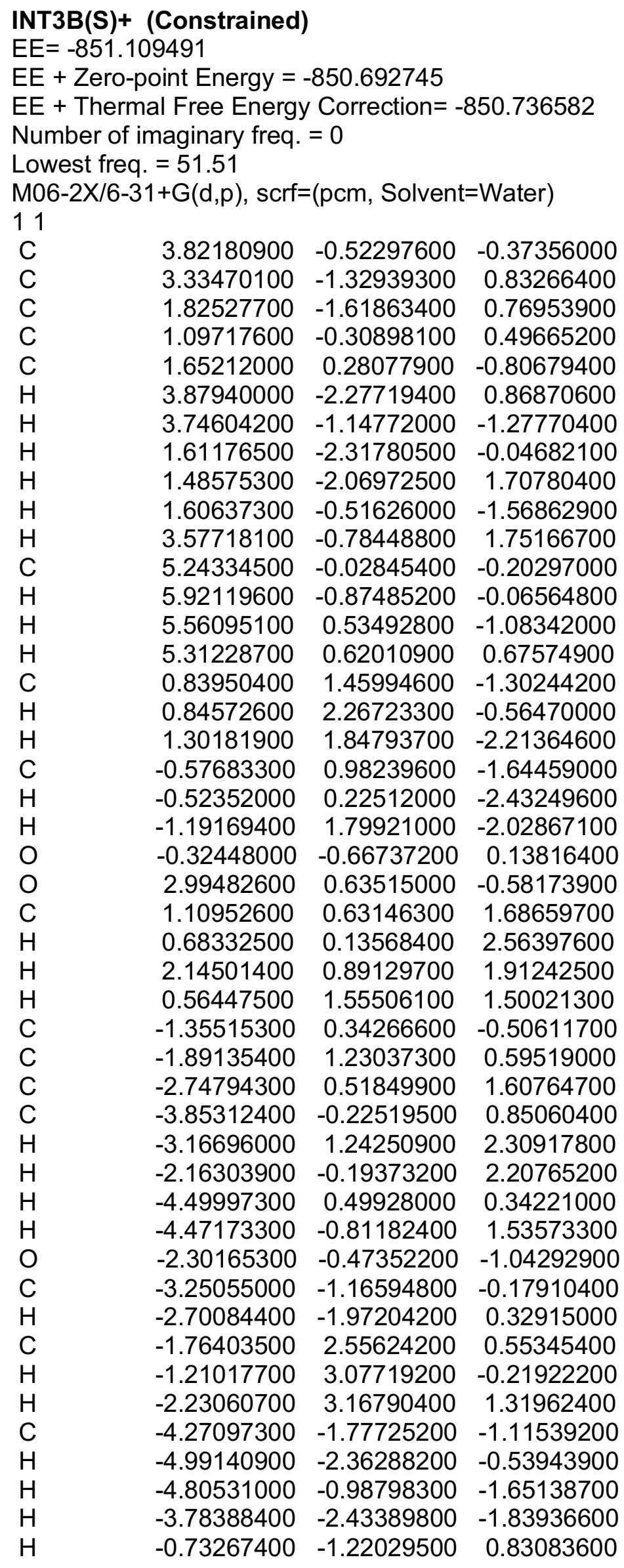

B $2228 \mathrm{~F}$ 


\begin{tabular}{|c|c|c|c|}
\hline $\begin{array}{l}\text { INT } \\
\text { EE } \\
\text { EE } \\
\text { EE } \\
\text { NuI } \\
\text { Lov } \\
\text { B3l } \\
11\end{array}$ & $\begin{array}{l}\text { (Constrainec } \\
48360 \\
\text { ooint Energy = } \\
\text { lal Free Energ } \\
\text { maginary freq. } \\
=45.57 \\
6-31+G(d, p), s\end{array}$ & $\begin{array}{l}-851.134988 \\
\text { Correction }=- \\
=0\end{array}$ & 851.179272 \\
\hline C & -3.84063800 & -0.58918300 & 0.34205900 \\
\hline C & -3.36133000 & -1.25018400 & -0.95911800 \\
\hline C & -1.83832500 & -1.51048700 & -0.95895300 \\
\hline C & -1.12920100 & -0.21871500 & -0.56272000 \\
\hline C & -1.65865800 & 0.22194300 & 0.81350900 \\
\hline $\mathrm{H}$ & -3.88577000 & -2.20178600 & -1.09043900 \\
\hline $\mathrm{H}$ & -3.72988500 & -1.30642000 & 1.17076100 \\
\hline $\mathrm{H}$ & -1.59127000 & -2.28806900 & -0.22800200 \\
\hline $\mathrm{H}$ & -1.51187300 & -1.85302500 & -1.94621800 \\
\hline $\mathrm{H}$ & -1.57611700 & -0.64776500 & 1.48546000 \\
\hline $\mathrm{H}$ & -3.63306200 & -0.61411100 & -1.80849600 \\
\hline C & -5.27942900 & -0.10860800 & 0.26597500 \\
\hline $\mathrm{H}$ & -5.94606300 & -0.95110600 & 0.05847200 \\
\hline $\mathrm{H}$ & -5.58118200 & 0.34879000 & 1.21245500 \\
\hline $\mathrm{H}$ & -5.39302800 & 0.63025500 & -0.53410700 \\
\hline C & -0.85140100 & 1.36003100 & 1.41456700 \\
\hline $\mathrm{H}$ & -0.87391900 & 2.23683000 & 0.76306800 \\
\hline $\mathrm{H}$ & -1.30780400 & 1.65146400 & 2.36442100 \\
\hline C & 0.57936800 & 0.87000800 & 1.69727300 \\
\hline $\mathrm{H}$ & 0.54078300 & 0.05560100 & 2.42625000 \\
\hline $\mathrm{H}$ & 1.18613000 & 1.66124900 & 2.14141600 \\
\hline $\mathrm{O}$ & 0.33140700 & -0.60129900 & -0.25179800 \\
\hline $\mathrm{O}$ & -3.02406900 & 0.56827000 & 0.66020900 \\
\hline C & -1.15811400 & 0.84449900 & -1.64729400 \\
\hline $\mathrm{H}$ & -0.79938300 & 0.43514300 & -2.59596000 \\
\hline $\mathrm{H}$ & -2.19083400 & 1.16961000 & -1.78273300 \\
\hline $\mathrm{H}$ & -0.56131100 & 1.71732300 & -1.39251500 \\
\hline C & 1.36742000 & 0.31910800 & 0.50786600 \\
\hline C & 1.95923700 & 1.28794200 & -0.49930500 \\
\hline C & 2.87058600 & 0.65287700 & -1.52287400 \\
\hline C & 3.92842400 & -0.19288500 & -0.79607600 \\
\hline $\mathrm{H}$ & 3.33932500 & 1.42918800 & -2.13212900 \\
\hline $\mathrm{H}$ & 2.30775700 & 0.01471600 & -2.21974400 \\
\hline $\mathrm{H}$ & 4.57141000 & 0.45759000 & -0.19190200 \\
\hline $\mathrm{H}$ & 4.56301800 & -0.72186300 & -1.51417200 \\
\hline $\mathrm{O}$ & 2.28135900 & -0.57010900 & 1.00225400 \\
\hline C & 3.25569400 & -1.21692400 & 0.10696700 \\
\hline $\mathrm{H}$ & 2.69758200 & -1.94159500 & -0.50288900 \\
\hline C & 1.80046400 & 2.61045600 & -0.38980000 \\
\hline $\mathrm{H}$ & 1.19345500 & 3.07968400 & 0.37486600 \\
\hline $\mathrm{H}$ & 2.29472600 & 3.27720000 & -1.08957400 \\
\hline C & 4.20628000 & -1.96360700 & 1.02366400 \\
\hline $\mathrm{H}$ & 4.93233300 & -2.51860700 & 0.42270700 \\
\hline $\mathrm{H}$ & 4.74575200 & -1.26002500 & 1.66515300 \\
\hline $\mathrm{H}$ & 3.65988200 & -2.67125800 & 1.65276800 \\
\hline $\mathrm{H}$ & 0.73749700 & -1.06153100 & -1.00867400 \\
\hline
\end{tabular}


Table S83. Cartesian coordinates of structures in proposed mechanism in forming yucatecone.

\begin{tabular}{|c|c|c|c|}
\hline $\begin{array}{l}\text { Pro } \\
\text { EE } \\
\text { EE } \\
\text { EE } \\
\text { NuI } \\
\text { Lov } \\
\text { M0 } \\
11\end{array}$ & $\begin{array}{l}\text { _aurokanol } \\
0100 \\
\text { int Energy = } \\
\text { l Free Energy } \\
\text { laginary freq. } \\
=48.65 \\
+G(d, p), \text { scrf }\end{array}$ & $\begin{array}{l}-851.906373 \\
\text { Correction }= \\
=0\end{array}$ & 851.951335 \\
\hline $\mathrm{C}$ & 3.43579500 & 0.85751500 & -0.11477700 \\
\hline C & 1.40739800 & 0.05311600 & 0.79313300 \\
\hline C & 0.97585000 & -0.61350400 & -0.51897300 \\
\hline C & 1.55532300 & 0.19802700 & -1.67329300 \\
\hline C & 3.07287200 & 0.33750500 & -1.50563400 \\
\hline $\mathrm{H}$ & 3.05310500 & 1.88581900 & -0.00618800 \\
\hline $\mathrm{H}$ & 1.30419200 & -0.27440700 & -2.62829000 \\
\hline $\mathrm{H}$ & 1.08984100 & 1.19191100 & -1.66058000 \\
\hline $\mathrm{H}$ & 3.56644300 & -0.62883100 & -1.66416800 \\
\hline $\mathrm{H}$ & 3.46636200 & 1.02821600 & -2.25771900 \\
\hline $\mathrm{H}$ & 1.06927500 & 1.10510000 & 0.74203400 \\
\hline C & 4.92615000 & 0.82999000 & 0.15487800 \\
\hline $\mathrm{H}$ & 5.45164000 & 1.44594000 & -0.57957800 \\
\hline $\mathrm{H}$ & 5.14288600 & 1.21597200 & 1.15389300 \\
\hline $\mathrm{H}$ & 5.30209000 & -0.19522200 & 0.08319500 \\
\hline C & 0.80766900 & -0.64277800 & 2.00593000 \\
\hline $\mathrm{H}$ & 1.43331600 & -1.50804600 & 2.24138200 \\
\hline $\mathrm{H}$ & 0.84881700 & 0.02458200 & 2.86954100 \\
\hline C & -0.63873700 & -1.11749100 & 1.73565900 \\
\hline $\mathrm{H}$ & -0.66411200 & -2.20546800 & 1.64570800 \\
\hline $\mathrm{H}$ & -1.31059300 & -0.86591200 & 2.55904300 \\
\hline $\mathrm{O}$ & 2.81128500 & 0.05430100 & 0.89912000 \\
\hline O & -0.47521300 & -0.51190600 & -0.63779000 \\
\hline C & -1.25964600 & -0.58618000 & 0.45616200 \\
\hline C & -2.63175400 & -1.15080500 & 0.09193500 \\
\hline C & -2.18630100 & 1.79868400 & -0.23073500 \\
\hline C & -3.29341800 & -0.32045000 & -1.01623200 \\
\hline $\mathrm{H}$ & -3.24617000 & -1.09602300 & 0.99881300 \\
\hline C & -3.49275000 & 1.14107500 & -0.61947600 \\
\hline $\mathrm{H}$ & -1.45582200 & 1.74457900 & -1.04337500 \\
\hline $\mathrm{H}$ & -4.26289600 & -0.77137500 & -1.24542800 \\
\hline $\mathrm{H}$ & -2.68345000 & -0.38411800 & -1.92505000 \\
\hline $\mathrm{H}$ & -4.18955300 & 1.21923000 & 0.22392500 \\
\hline $\mathrm{H}$ & -3.91543600 & 1.70977000 & -1.45308500 \\
\hline $\mathrm{O}$ & -1.64082800 & 0.94219000 & 0.86355500 \\
\hline C & -2.31324300 & 3.19990900 & 0.31951100 \\
\hline $\mathrm{H}$ & -1.34143300 & 3.60836100 & 0.61077300 \\
\hline $\mathrm{H}$ & -2.72201300 & 3.84049800 & -0.46563300 \\
\hline $\mathrm{H}$ & -2.99094200 & 3.22004100 & 1.17652200 \\
\hline C & -2.48943000 & -2.61184700 & -0.34425700 \\
\hline $\mathrm{H}$ & -1.81033800 & -2.69174300 & -1.19855200 \\
\hline $\mathrm{H}$ & -2.12279100 & -3.25658500 & 0.45778200 \\
\hline $\mathrm{H}$ & -3.46847900 & -2.98927400 & -0.64915400 \\
\hline C & 1.36105000 & -2.08539600 & -0.60321800 \\
\hline $\mathrm{H}$ & 0.86073300 & -2.68615300 & 0.15987000 \\
\hline $\mathrm{H}$ & 1.08215600 & -2.47766700 & -1.58498600 \\
\hline $\mathrm{H}$ & 2.43824600 & -2.20171300 & -0.46822000 \\
\hline
\end{tabular}




\begin{tabular}{|c|c|c|c|}
\hline $\begin{array}{l}\text { Prc } \\
\text { EE } \\
\text { EE } \\
\text { EE } \\
\text { Nul } \\
\text { Lov } \\
\text { B3L } \\
11\end{array}$ & $\begin{array}{l}\text { Laurokanol } \\
91445 \\
\text { oint Energy = } \\
\text { lal Free Energ } \\
\text { maginary freq } \\
=-177.98 \\
6-31+G(d, p)\end{array}$ & $\begin{array}{l}-852.354825 \\
\text { Correction= } \\
=1\end{array}$ & 852.399646 \\
\hline C & 3.45741200 & 0.87682400 & -0.16051400 \\
\hline C & 1.40635400 & 0.11346500 & 0.77729300 \\
\hline C & 1.01229600 & -0.65989500 & -0.49559700 \\
\hline C & 1.59111000 & 0.08342200 & -1.69947300 \\
\hline C & 3.11035400 & 0.25341100 & -1.51826100 \\
\hline $\mathrm{H}$ & 3.07046300 & 1.90854400 & -0.12825400 \\
\hline $\mathrm{H}$ & 1.36349100 & -0.45993500 & -2.62225200 \\
\hline $\mathrm{H}$ & 1.11184300 & 1.06721600 & -1.76575800 \\
\hline $\mathrm{H}$ & 3.61474700 & -0.71686600 & -1.59786800 \\
\hline $\mathrm{H}$ & 3.50753000 & 0.88832600 & -2.31695100 \\
\hline $\mathrm{H}$ & 1.05667000 & 1.14981300 & 0.63361900 \\
\hline C & 4.94718000 & 0.87753500 & 0.13595300 \\
\hline $\mathrm{H}$ & 5.48173700 & 1.45105300 & -0.62743100 \\
\hline $\mathrm{H}$ & 5.14474700 & 1.32940500 & 1.11235500 \\
\hline $\mathrm{H}$ & 5.33581500 & -0.14623700 & 0.13784000 \\
\hline $\mathrm{C}$ & 0.77475100 & -0.47982300 & 2.03645600 \\
\hline $\mathrm{H}$ & 1.44047200 & -1.24826900 & 2.43583900 \\
\hline $\mathrm{H}$ & 0.69401600 & 0.29297100 & 2.80562000 \\
\hline C & -0.60847500 & -1.10903500 & 1.73488200 \\
\hline $\mathrm{H}$ & -0.50309600 & -2.19124200 & 1.61872800 \\
\hline $\mathrm{H}$ & -1.31492300 & -0.95070600 & 2.55107800 \\
\hline $\mathrm{O}$ & 2.81647600 & 0.13747600 & 0.91030600 \\
\hline $\mathrm{O}$ & -0.46308100 & -0.56568200 & -0.63644000 \\
\hline C & -1.24564200 & -0.63650000 & 0.44381900 \\
\hline C & -2.63914800 & -1.16352900 & 0.10212600 \\
\hline C & -2.22005500 & 1.81819300 & -0.26268900 \\
\hline C & -3.29763900 & -0.34445500 & -1.02491700 \\
\hline $\mathrm{H}$ & -3.24064000 & -1.06608700 & 1.01245300 \\
\hline C & -3.51383300 & 1.12593200 & -0.65099400 \\
\hline $\mathrm{H}$ & -1.48380900 & 1.75919600 & -1.06968500 \\
\hline $\mathrm{H}$ & -4.26348000 & -0.80355300 & -1.25596300 \\
\hline $\mathrm{H}$ & -2.68342300 & -0.41737100 & -1.92962400 \\
\hline $\mathrm{H}$ & -4.22025600 & 1.20675800 & 0.18414500 \\
\hline $\mathrm{H}$ & -3.94200000 & 1.67258100 & -1.49745000 \\
\hline $\mathrm{O}$ & -1.66420000 & 0.99276500 & 0.86166300 \\
\hline C & -2.37679300 & 3.23779200 & 0.24456200 \\
\hline $\mathrm{H}$ & -1.41575500 & 3.66846100 & 0.54276800 \\
\hline $\mathrm{H}$ & -2.78028800 & 3.85292000 & -0.56507800 \\
\hline $\mathrm{H}$ & -3.06822400 & 3.27555300 & 1.09093100 \\
\hline C & -2.54770700 & -2.65041400 & -0.28553600 \\
\hline $\mathrm{H}$ & -1.93031000 & -2.77977300 & -1.17936900 \\
\hline $\mathrm{H}$ & -2.13652100 & -3.26737400 & 0.51758100 \\
\hline $\mathrm{H}$ & -3.55162000 & -3.02245600 & -0.50751800 \\
\hline C & 1.41261200 & -2.14934200 & -0.46548000 \\
\hline $\mathrm{H}$ & 1.91446900 & -2.42091900 & 0.46546100 \\
\hline $\mathrm{H}$ & 0.52829600 & -2.77908900 & -0.58023700 \\
\hline $\mathrm{H}$ & 2.09132300 & -2.38980500 & -1.28609500 \\
\hline
\end{tabular}


B $2435 \mathrm{~F}$

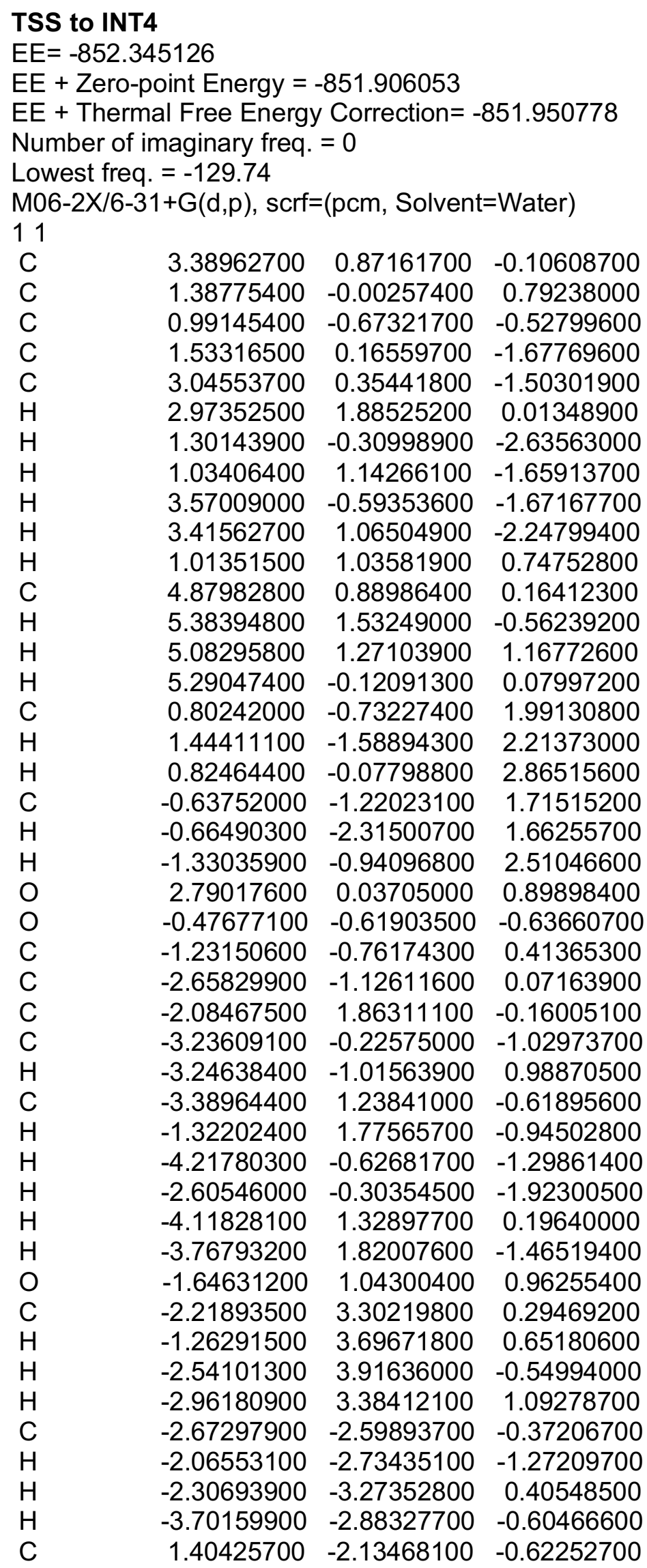




$\begin{array}{rrrr}\mathrm{H} & 0.92236300 & -2.75030300 & 0.14065600 \\ \mathrm{H} & 1.13611500 & -2.52928400 & -1.60575800 \\ \mathrm{H} & 2.48418600 & -2.22001100 & -0.48777200 \\ \mathrm{H} & -0.92721800 & 1.48357700 & 1.44414800\end{array}$

\section{INT4}

$E E=-852.339437$

EE + Zero-point Energy $=-851.901951$

$E E+$ Thermal Free Energy Correction $=-851.951418$

Number of imaginary freq. $=0$

Lowest freq. $=12.85$

M06-2X/6-31+G(d,p), scrf $=(p c m$, Solvent=Water $)$

11

C

1.02495500

$-0.32796100$

0.13073100

C

1.92213300

0.88583000

$-0.14968500$

C

3.83836800

$-0.46353600$

$-0.49661700$

C

$3.03416400-1.75104900-0.32703000$

C

$\begin{array}{lll}1.55413100 & -1.52531000 & -0.65466200\end{array}$

$\mathrm{H}$

$\mathrm{H}$

$\mathrm{H}$

$\mathrm{H}$

$\mathrm{H}$

$\begin{array}{lll}1.91243900 & 1.04419900 & -1.24172600\end{array}$

$3.82267200-0.16688200-1.55812700$

$3.44166200-2.52083700-0.98931900$

$3.15222700 \quad-2.11216000 \quad 0.70162100$

$1.44107900-1.30720800-1.72326700$

$\mathrm{H}$

$0.96233600-2.41925200$

$-0.43044600$

$-0.73777700$

1.41862200

$-0.58217900$

1.43733200

2.14397100

0.55720000

1.77103600

2.10424100

1.59840300

1.92005000

3.00792500

0.09462700

$-0.10180600$

2.27704900

0.50112700

$-0.54786700$

2.03262100

1.46971500

$-0.37634200$

3.31253500

0.28553800

5.26977500

$-0.59774500$

$-0.01893900$

5.78080600

$-1.38434100$

$-0.58025500$

5.81078800

0.34098800

$-0.16001800$

5.28837300

$-0.85956100$

1.04348200

0.83839800

$-0.63236300$

1.60924100

0.39269900

0.20427400

2.15081300

0.20091900

$-1.51303800$

1.73477300

1.80985600

$-0.84390200$

2.06118100

3.23930700

0.59684700

0.26193300

$-0.28290200$

0.01382700

$-0.46456200$

$-2.26498200$

1.35255400

$-0.56951500$

$-2.54148700$

0.70055800

$-1.41024400$

$-2.82258200$

0.74604100

0.74207600

$-2.01614700$

0.38679100

1.39312400

$-3.33966800$

1.52315000

1.31142300

$-3.79242200$

$-0.40570100$

0.50324800

$-4.34124600$

$-0.66933900$

1.41569900

$-4.56650200$

$-0.13670800$

$-0.22960600$

$-3.15259400$

$-1.67126200$

0.01100300

$-2.86537800$

2.73026200

$-0.85788400$

$-2.46270500$

3.15647900

$-1.78141900$

$-3.94949100$

2.64189300

$-0.96881300$

$-2.66968500$

3.42583000

$-0.03665500$

$-3.99088300$

$-2.90202300$

$-0.08003500$

$-5.00316000$

$-2.66009700$

$-0.40927800$

$\mathrm{H}$

$-3.52656100$

$-3.63434300$

$-0.73926400$ 


$\begin{array}{lrrr}H & -4.06588300 & -3.32459500 & 0.92868700 \\ \mathrm{O} & -1.95314100 & -1.73551100 & -0.28960000 \\ \mathrm{H} & -1.07405700 & -0.73357600 & -0.32953500 \\ \mathrm{H} & -0.39724100 & 1.73876900 & -1.57175300\end{array}$

\section{INT4}

$E E=-852.793828$

EE + Zero-point Energy $=-852.359298$

$\mathrm{EE}+$ Thermal Free Energy Correction $=-852.409579$

Number of imaginary freq. $=0$

Lowest freq. $=7.08$

B3LYP(D3)/6-31+G(d,p), scrf=(pcm, Solvent=Water $)$

11

C

$-4.42901400$

$-0.31754800$

$-0.69452200$

$-2.19373300$

$-0.85811700$

$-0.09614200$

C

$-1.89234400$

0.58560400

0.33819700

$\begin{array}{lll}-2.67363400 & 1.53720400 & -0.56005000\end{array}$

C

$-4.17119100$

$1.17963700-0.46527500$

$\mathrm{H}$

$-4.19087700$

$-0.56759700-1.74088700$

$\mathrm{H}$

$-2.50068300$

$2.57634000-0.26585000$

$\begin{array}{lll}-2.32389400 & 1.41517600 & -1.59132300\end{array}$

$\mathrm{H}$

$-4.56288700$

$1.46410600 \quad 0.51766200$

$-4.72888000$

$1.75461900-1.21125700$

$-1.99779200$

$-0.92418600$

$-1.18016700$

$\mathrm{H}$

$-5.85643600$

$-0.72883300$

$-0.37914600$

$-6.55482200$

$-0.17293500$

$-1.01198300$

$-5.99734300$

$-1.79798400$

$-0.56135100$

$-6.09172700$

$-0.51630900$

0.66894700

$-1.28901700-1.84531100$

0.61561000

$-1.44834100-1.80984400$

1.69595400

$-1.50679500-2.86252800$

0.28287000

$0.16346200-1.49264700$

0.25839700

C

$0.89011100-1.93586800$

0.94601500

$0.41351900-1.91207200$

$-0.72820100$

$-3.56230000$

$-1.12203800$

0.14988500

$-0.42497600$

0.85845000

0.07032400

0.47757100

$-0.03818300$

0.11972800

$-2.06616800$

0.86765000

1.82587300

$-1.37418100$

0.27969300

2.43212400

$-1.89034200$

1.92694800

2.02753000

$-3.08419400$

0.61368500

2.12535800

1.99133900

1.97096600

$-0.09403600$

1.41926800

2.46906300

0.69214100

3.03527200

2.26742300

0.00934700

1.62051500

2.31925700

$-1.06312400$

1.88570200

0.44741100

0.02150700

2.33543300

0.13036500

0.97616300

2.66268900

$-0.31138300$

$-1.10055500$

2.53141700

$-1.39093200$

$-0.98036000$

2.21506700

$-0.03635800$

$-2.06269200$

4.17201600

$-0.02645300$

$-1.15205500$

4.37252200

1.04478700

$-1.26520100$

$\begin{array}{lll}4.98132000 & -0.55885000 & 0.03128900\end{array}$

C

$4.70201800-1.60952000$

0.20730200

$\mathrm{H}$

4.55517500

$-0.50948000$

$-2.05822700$ 


$\begin{array}{rrrr}\mathrm{O} & 4.61263800 & 0.21934500 & 1.19211000 \\ \mathrm{H} & 5.06901800 & -0.14268900 & 1.96428400 \\ \mathrm{C} & 6.48637300 & -0.47212500 & -0.21701300 \\ \mathrm{H} & 7.04331700 & -0.82357300 & 0.65848700 \\ \mathrm{H} & 6.77404200 & -1.09334100 & -1.07190600 \\ \mathrm{H} & 6.77956200 & 0.56357100 & -0.42104500\end{array}$

TSS to Yucatecone Epimer ${ }^{+}$

$E E=-852.31395$

EE + Zero-point Energy $=-851.878543$

$\mathrm{EE}+$ Thermal Free Energy Correction $=-851.923220$

Number of imaginary freq. $=1$

Lowest freq. $=-590.48$

M06-2X/6-31+G(d,p), scrf $=(\mathrm{pcm}$, Solvent=Water $)$

11

C

1.14951500

$-1.01529300$

$-0.06711700$

C

1.28725300

0.37195500

0.57657300

C

3.38139000

0.79544600

$-0.43670600$

C

3.32784700

$-0.51989500-1.21268300$

C

1.88262200

$-0.99020400-1.40688700$

$\mathrm{H}$

0.87279200

$1.09554100-0.14798500$

$\mathrm{H}$

2.90402800

$1.58573300-1.04014100$

3.80730200

$-0.38033600-2.18674000$

$\mathrm{H}$

3.90931700

$-1.27571700$

$-0.67086800$

1.35216300

$-0.29339100$

$-2.06896000$

1.84665600

$-1.98101900$

$-1.87150700$

$-1.21450700$

$-0.65589400$

0.38158700

0.53079800

0.46365800

1.89310400

1.14638200

0.01293800

2.67694500

0.39231500

1.51474200

2.15826700

$-0.82261700$

$-0.27171000$

1.80040500

$-0.79223000$

$-1.21245300$

2.36096900

$-1.62607300$

0.32094300

2.24196400

4.79300300

1.20667400

$-0.07155000$

5.39333700

1.33034700

$-0.97687400$

4.78962200

2.15148700

0.47739100

5.25837100

0.43759100

0.55280900

1.63906700

$-2.14413400$

0.83375100

1.03753500

$-2.22782600$

1.74131800

1.58186100

$-3.09311700$

0.29399700

2.67561400

$-1.96784000$

1.12969400

2.64519100

0.68158100

0.78934800

$-0.25182500$

$-1.24744900$

$-0.37571400$

$-2.57028400$

$-1.34183300$

0.22066400

$-2.65052200$

$-2.04115900$

1.06336400

$-3.70220400$

$-0.31295700$

0.36691000

$-4.66209100$

$-0.80613800$

0.19936200

$-3.72882800$

0.10313400

1.37927800

$-3.53253700$

0.82382300

$-0.65009800$

$-3.54663600$

0.44655000

$-1.67445200$

$-4.34348200$

1.55313000

$-0.54457000$

$-2.25888300$

1.58123200

$-0.41994300$

$-2.67290800$

$-2.13505700$

$-1.08351900$

$-1.96061200$

$-2.96106800$

$-1.09333300$

$-3.68212200$

$-2.54320900$

$-1.18118500$

$-2.46610000$

$-1.51289300$

$-1.95934500$

C

$-1.48731500$

2.15418200

$-1.56876000$ 


$\begin{array}{lrrr}\mathrm{H} & -2.11437800 & 2.93574800 & -2.01515500 \\ \mathrm{H} & -0.54565100 & 2.60466400 & -1.24633700 \\ \mathrm{H} & -1.29505400 & 1.39137700 & -2.32456100 \\ \mathrm{O} & -2.26298700 & 2.24193200 & 0.73128900 \\ \mathrm{H} & -1.55012500 & 2.89823800 & 0.78837200 \\ \mathrm{H} & -1.42785200 & 0.44553100 & -0.15549800\end{array}$

TSS to Yucatecone Epimer ${ }^{+}$

$E E=-852.771012$

EE + Zero-point Energy $=-852.338077$

$\mathrm{EE}+$ Thermal Free Energy Correction $=-852.382702$

Number of imaginary freq. $=1$

Lowest freq. $=-404.86$

B3LYP(D3)/6-31+G(d,p), scrf=(pcm, Solvent=Water $)$

11

C

1.16120100

$-1.01105500$

$-0.09272200$

$\begin{array}{lll}1.30811800 & 0.35155800 & 0.61261200\end{array}$

$\begin{array}{lll}3.40999500 & 0.81502900 & -0.41659000\end{array}$

$3.32859000-0.46434000-1.25842500$

$\begin{array}{lll}1.87330000 & -0.92465400 & -1.44655800\end{array}$

$\begin{array}{llll}0.88711700 & 1.10665600 & -0.07182500\end{array}$

$\begin{array}{lll}2.93622500 & 1.63997500 & -0.97450800\end{array}$

$3.78756500-0.27835200-2.23546200$

$3.91789600 \quad-1.24985600-0.77088900$

$1.33218100 \quad-0.20046300-2.06747200$

$1.82935500-1.89235500-1.95692400$

$\begin{array}{lll}-1.22001600 & -0.65490900 & 0.38920000\end{array}$

$\begin{array}{lll}0.56178300 & 0.39490400 & 1.94000400\end{array}$

$\begin{array}{lll}1.16888400 & -0.10605800 & 2.69888500\end{array}$

$\begin{array}{lll}0.45020200 & 1.43544400 & 2.25508800\end{array}$

$\begin{array}{lll}-0.81646900 & -0.30335800 & 1.82117500\end{array}$

$\begin{array}{lll}-0.81370000 & -1.25199800 & 2.36849200\end{array}$

$\begin{array}{lll}-1.60465700 & 0.30537700 & 2.26750500\end{array}$

$\begin{array}{lll}4.83226400 & 1.19815600 & -0.04437500\end{array}$

$\begin{array}{llll}5.42570200 & 1.36443400 & -0.94874000\end{array}$

$\begin{array}{lll}4.84127400 & 2.11572000 & 0.55126000\end{array}$

$\begin{array}{llll}5.30223100 & 0.39781800 & 0.53703000\end{array}$

$\begin{array}{lll}1.64951700 & -2.19249800 & 0.74766600\end{array}$

$\begin{array}{lll}1.05743500 & -2.31504600 & 1.65631200\end{array}$

$\begin{array}{lll}1.57506200 & -3.11368000 & 0.16346100\end{array}$

$\begin{array}{lll}2.69088900 & -2.04250800 & 1.03980000\end{array}$

$\begin{array}{lll}2.68005200 & 0.65081100 & 0.82337800\end{array}$

$\begin{array}{lll}-0.25946800 & -1.23148000 & -0.39225100\end{array}$

$\begin{array}{lll}-2.58252600 & -1.34824800 & 0.22374800\end{array}$

$\begin{array}{lll}-2.66971500 & -2.03767200 & 1.07372600\end{array}$

$\begin{array}{lll}-3.72421900 & -0.31794600 & 0.35698400\end{array}$

$\begin{array}{lll}-4.68132800 & -0.81330500 & 0.17714700\end{array}$

$\begin{array}{lll}-3.76320300 & 0.09045300 & 1.37164300\end{array}$

$\begin{array}{lll}-3.55632600 & 0.83681300 & -0.65146600\end{array}$

$\begin{array}{lll}-3.56197500 & 0.46557700 & -1.67779000\end{array}$

$\begin{array}{lll}-4.38239000 & 1.54957200 & -0.54536000\end{array}$

$\begin{array}{llll}-2.28995700 & 1.61092100 & -0.41567700\end{array}$

$-2.68227500 \quad-2.16908200-1.07030700$

$\begin{array}{lll}-1.94894200 & -2.97754300 & -1.07602600\end{array}$

$\begin{array}{lll}-3.68231400 & -2.60575900 & -1.14690300\end{array}$

$-2.50417400-1.55863300-1.96030900$

$\begin{array}{llll}-1.49659600 & 2.17068300 & -1.55917400\end{array}$ 


$\begin{array}{lrrr}\mathrm{H} & -2.10208000 & 2.96453300 & -2.01590600 \\ \mathrm{H} & -0.54960100 & 2.60481200 & -1.22858400 \\ \mathrm{H} & -1.30688500 & 1.40584100 & -2.31362800 \\ \mathrm{O} & -2.31267300 & 2.28861300 & 0.73480400 \\ \mathrm{H} & -1.58528800 & 2.92971300 & 0.80262600 \\ \mathrm{H} & -1.44736900 & 0.45190300 & -0.11666700\end{array}$

\begin{tabular}{|c|c|c|c|}
\hline \multicolumn{4}{|c|}{$\begin{array}{l}\text { Yucatecone Epimer }{ }^{+} \\
E E=-852.351329 \\
E E+\text { Zero-point Energy }=-851.918330 \\
E E+\text { Thermal Free Energy Correction }=-851.961546 \\
\text { Number of imaginary freq. }=0 \\
\text { Lowest freq. }=46.07 \\
\text { M06-2X/6-31+G(d,p), scrf }=(p c m, \text { Solvent=Water }) \\
11\end{array}$} \\
\hline C & 1.02495500 & -0.32796100 & 0.13073100 \\
\hline C & 1.92213300 & 0.88583000 & -0.14968500 \\
\hline C & 3.83836800 & -0.46353600 & -0.49661700 \\
\hline C & 3.03416400 & -1.75104900 & -0.32703000 \\
\hline C & 1.55413100 & -1.52531000 & -0.65466200 \\
\hline $\mathrm{H}$ & 1.91243900 & 1.04419900 & -1.24172600 \\
\hline $\mathrm{H}$ & 3.82267200 & -0.16688200 & -1.55812700 \\
\hline $\mathrm{H}$ & 3.44166200 & -2.52083700 & -0.98931900 \\
\hline $\mathrm{H}$ & 3.15222700 & -2.11216000 & 0.70162100 \\
\hline $\mathrm{H}$ & 1.44107900 & -1.30720800 & -1.72326700 \\
\hline $\mathrm{H}$ & 0.96233600 & -2.41925200 & -0.43044600 \\
\hline C & -0.73777700 & 1.41862200 & -0.58217900 \\
\hline C & 1.43733200 & 2.14397100 & 0.55720000 \\
\hline $\mathrm{H}$ & 1.77103600 & 2.10424100 & 1.59840300 \\
\hline $\mathrm{H}$ & 1.92005000 & 3.00792500 & 0.09462700 \\
\hline C & -0.10180600 & 2.27704900 & 0.50112700 \\
\hline $\mathrm{H}$ & -0.54786700 & 2.03262100 & 1.46971500 \\
\hline $\mathrm{H}$ & -0.37634200 & 3.31253500 & 0.28553800 \\
\hline C & 5.26977500 & -0.59774500 & -0.01893900 \\
\hline $\mathrm{H}$ & 5.78080600 & -1.38434100 & -0.58025500 \\
\hline $\mathrm{H}$ & 5.81078800 & 0.34098800 & -0.16001800 \\
\hline $\mathrm{H}$ & 5.28837300 & -0.85956100 & 1.04348200 \\
\hline C & 0.83839800 & -0.63236300 & 1.60924100 \\
\hline $\mathrm{H}$ & 0.39269900 & 0.20427400 & 2.15081300 \\
\hline $\mathrm{H}$ & 0.20091900 & -1.51303800 & 1.73477300 \\
\hline $\mathrm{H}$ & 1.80985600 & -0.84390200 & 2.06118100 \\
\hline $\mathrm{O}$ & 3.23930700 & 0.59684700 & 0.26193300 \\
\hline $\mathrm{O}$ & -0.28290200 & 0.01382700 & -0.46456200 \\
\hline C & -2.26498200 & 1.35255400 & -0.56951500 \\
\hline $\mathrm{H}$ & -2.54148700 & 0.70055800 & -1.41024400 \\
\hline C & -2.82258200 & 0.74604100 & 0.74207600 \\
\hline $\mathrm{H}$ & -2.01614700 & 0.38679100 & 1.39312400 \\
\hline $\mathrm{H}$ & -3.33966800 & 1.52315000 & 1.31142300 \\
\hline C & -3.79242200 & -0.40570100 & 0.50324800 \\
\hline $\mathrm{H}$ & -4.34124600 & -0.66933900 & 1.41569900 \\
\hline $\mathrm{H}$ & -4.56650200 & -0.13670800 & -0.22960600 \\
\hline C & -3.15259400 & -1.67126200 & 0.01100300 \\
\hline C & -2.86537800 & 2.73026200 & -0.85788400 \\
\hline $\mathrm{H}$ & -2.46270500 & 3.15647900 & -1.78141900 \\
\hline $\mathrm{H}$ & -3.94949100 & 2.64189300 & -0.96881300 \\
\hline $\mathrm{H}$ & -2.66968500 & 3.42583000 & -0.03665500 \\
\hline C & -3.99088300 & -2.90202300 & -0.08003500 \\
\hline
\end{tabular}




$\begin{array}{lrrr}\mathrm{H} & -5.00316000 & -2.66009700 & -0.40927800 \\ \mathrm{H} & -3.52656100 & -3.63434300 & -0.73926400 \\ \mathrm{H} & -4.06588300 & -3.32459500 & 0.92868700 \\ \mathrm{O} & -1.95314100 & -1.73551100 & -0.28960000 \\ \mathrm{H} & -1.07405700 & -0.73357600 & -0.32953500 \\ \mathrm{H} & -0.39724100 & 1.73876900 & -1.57175300\end{array}$

\begin{tabular}{|c|c|c|c|}
\hline $\begin{array}{l}\text { Yuc } \\
\text { EE: } \\
\text { EE } \\
\text { EE } \\
\text { Nur } \\
\text { Lov } \\
\text { B3L } \\
11\end{array}$ & $\begin{array}{l}\text { Epimer } \\
\text { O4125 } \\
\text { oint Energy = } \\
\text { lal Free Energy } \\
\text { maginary freq. } \\
=37.05 \\
6-31+G(d, p), s\end{array}$ & $\begin{array}{l}-852.370299 \\
\text { / Correction= - } \\
=0\end{array}$ & 852.417760 \\
\hline C & 1.04841100 & -0.34402400 & 0.12256900 \\
\hline C & 1.93502600 & 0.89080400 & -0.12742400 \\
\hline C & 3.88164100 & -0.44702800 & -0.49719900 \\
\hline C & 3.07348900 & -1.74484900 & -0.38035300 \\
\hline C & 1.58692300 & -1.51431100 & -0.70457300 \\
\hline $\mathrm{H}$ & 1.92937700 & 1.06883700 & -1.21491700 \\
\hline $\mathrm{H}$ & 3.88427800 & -0.11854100 & -1.54934300 \\
\hline $\mathrm{H}$ & 3.48511000 & -2.48974200 & -1.06918400 \\
\hline $\mathrm{H}$ & 3.18606900 & -2.14630400 & 0.63334300 \\
\hline $\mathrm{H}$ & 1.47247700 & -1.26305000 & -1.76510300 \\
\hline $\mathrm{H}$ & 1.00097500 & -2.41897200 & -0.51288200 \\
\hline C & -0.75050900 & 1.44427100 & -0.56230800 \\
\hline C & 1.43859700 & 2.14317900 & 0.59166000 \\
\hline $\mathrm{H}$ & 1.77627800 & 2.10601300 & 1.63129400 \\
\hline $\mathrm{H}$ & 1.91124100 & 3.01372400 & 0.12954600 \\
\hline C & -0.10729200 & 2.26656400 & 0.54790800 \\
\hline $\mathrm{H}$ & -0.54114000 & 1.98150400 & 1.50929100 \\
\hline $\mathrm{H}$ & -0.39199800 & 3.30782100 & 0.37771300 \\
\hline C & 5.31000700 & -0.58599700 & 0.00130900 \\
\hline $\mathrm{H}$ & 5.83749700 & -1.34929900 & -0.57889200 \\
\hline $\mathrm{H}$ & 5.84687500 & 0.36154400 & -0.10063400 \\
\hline $\mathrm{H}$ & 5.31856200 & -0.88303900 & 1.05533000 \\
\hline C & 0.84722500 & -0.69596400 & 1.59187400 \\
\hline $\mathrm{H}$ & 0.38799300 & 0.11717300 & 2.15466900 \\
\hline $\mathrm{H}$ & 0.21909200 & -1.58599800 & 1.68607400 \\
\hline $\mathrm{H}$ & 1.81718000 & -0.90918200 & 2.04476400 \\
\hline $\mathrm{O}$ & 3.26147400 & 0.60043700 & 0.28791800 \\
\hline $\mathrm{O}$ & -0.27898800 & 0.01524300 & -0.48148800 \\
\hline C & -2.28268400 & 1.37468800 & -0.57515100 \\
\hline $\mathrm{H}$ & -2.53980400 & 0.72325100 & -1.42089900 \\
\hline C & -2.88000300 & 0.76527200 & 0.72651900 \\
\hline $\mathrm{H}$ & -2.09232100 & 0.42685800 & 1.40770400 \\
\hline $\mathrm{H}$ & -3.42729000 & 1.53897000 & 1.27161400 \\
\hline C & -3.83140900 & -0.40722200 & 0.47602100 \\
\hline $\mathrm{H}$ & -4.40425100 & -0.65078800 & 1.38027100 \\
\hline $\mathrm{H}$ & -4.59139500 & -0.15446400 & -0.27742100 \\
\hline C & -3.17978000 & -1.68742100 & 0.02291700 \\
\hline C & -2.87782300 & 2.75747200 & -0.88709800 \\
\hline $\mathrm{H}$ & -2.46189700 & 3.17487800 & -1.80986200 \\
\hline $\mathrm{H}$ & -3.96096400 & 2.67060800 & -1.01354600 \\
\hline & -2.69310000 & 3.46405800 & -0.07216500 \\
\hline
\end{tabular}




$\begin{array}{lrrr}\mathrm{C} & -4.02643300 & -2.91922000 & -0.05769000 \\ \mathrm{H} & -5.00908300 & -2.69100200 & -0.47973200 \\ \mathrm{H} & -3.52477400 & -3.69522500 & -0.63666500 \\ \mathrm{H} & -4.19249400 & -3.28392800 & 0.96390700 \\ \mathrm{O} & -1.97163400 & -1.76064800 & -0.25743700 \\ \mathrm{H} & -1.04527000 & -0.71052200 & -0.32919500 \\ \mathrm{H} & -0.39635200 & 1.77727400 & -1.54090200\end{array}$

TSS to Yucatecone *

$E E=-852.316115$

$E E+$ Thermal Energy Correction $=-851.880456$

$E E+$ Thermal Free Energy Correction $=-851.925023$

Number of imaginary freq. $=1$

Lowest freq. $=-619.80$

M06-2X/6-31+G(d,p), scrf $=(p c m$, Solvent=Water $)$

11

$\begin{array}{llll}\text { C } & 1.06240800 & -0.33281200 & -0.37719700\end{array}$

C $\quad 1.66318900 \quad 0.82455500 \quad 0.43620000$

$\begin{array}{llll}\text { C } & 3.87211200 & 0.31471800 & -0.21948500\end{array}$

C $\quad 3.38836200 \quad-0.77844000-1.17379700$

C $\quad 1.93575500 \quad-0.54837400-1.60784700$

$\mathrm{H} \quad 1.73082700 \quad 1.69923100 \quad-0.23529900$

$\mathrm{H} \quad 3.90898300 \quad 1.27287600 \quad-0.76392300$

$\mathrm{H} \quad 4.03894500 \quad-0.79439700 \quad-2.05364300$

$\mathrm{H} \quad 3.49031700 \quad-1.75260400 \quad-0.68061400$

$\mathrm{H} \quad \begin{array}{llll}\mathrm{H} & 1.87125300 & 0.34996500 & -2.23385700\end{array}$

$\mathrm{H} \quad \begin{array}{llll}1.56043900 & -1.39297600 & -2.19475900\end{array}$

C $\quad-1.11728800 \quad 0.74095300-0.10392300$

$\begin{array}{llll}\text { C } & 0.76503000 & 1.17541400 & 1.60188600\end{array}$

$\begin{array}{llll}\mathrm{H} & 0.65482900 & 0.32361300 & 2.27985300\end{array}$

$\mathrm{H} \quad 1.19813200 \quad 1.99856100 \quad 2.17606300$

$\begin{array}{llll}\text { C } & -0.57474300 & 1.61933300 & 1.01888200\end{array}$

$\mathrm{H} \quad-1.33862500 \quad 1.73952300 \quad 1.78980900$

$\mathrm{H} \quad-0.44205300 \quad 2.60607600 \quad 0.55460700$

$\begin{array}{llll}\text { C } & 5.22953800 & 0.00928100 & 0.38001400\end{array}$

$\mathrm{H} \quad 5.97560400 \quad-0.09813100-0.41170600$

$\mathrm{H} \quad 5.54398000 \quad 0.81354600 \quad 1.04956400$

$\mathrm{H} \quad 5.18729700 \quad-0.92556400 \quad 0.94758900$

C $\quad 0.92935400 \quad-1.60661300 \quad 0.45024100$

H $\quad 0.16258300 \quad-1.50847800 \quad 1.22445300$

$\mathrm{H} \quad 0.69277400 \quad-2.45198900-0.20044800$

$\mathrm{H} \quad 1.87153300 \quad-1.81380100 \quad 0.96154800$

$\begin{array}{llll}\mathrm{O} & 2.95622000 & 0.47460700 & 0.87420100\end{array}$

$\begin{array}{llll}0 & -0.24334200 & 0.07551000 & -0.88387000\end{array}$

$\begin{array}{llll}\text { C } & -2.24298000 & 1.32830600 & -0.95397600\end{array}$

$\mathrm{H} \quad-1.72520900 \quad 1.81856000 \quad-1.78766600$

C $\quad-3.10818600 \quad 0.19520200-1.52684200$

$\mathrm{H} \quad-3.90261000 \quad 0.61943000 \quad-2.14492200$

$\mathrm{H} \quad-2.50407500 \quad-0.45898500 \quad-2.16220400$

C $\quad-3.71814500 \quad-0.61126000-0.37641600$

$\mathrm{H} \quad-4.36260600 \quad 0.00990500 \quad 0.24835600$

$\mathrm{H} \quad-4.31972800-1.44086600 \quad-0.76294500$

C $\quad-2.64220000 \quad-1.23242900 \quad 0.47138900$

$\begin{array}{llll}C & -3.08753700 & 2.37098100 & -0.21570400\end{array}$

$\mathrm{H} \quad-2.51324900 \quad 3.27079200 \quad 0.01217400$

$\mathrm{H} \quad-3.92639700 \quad 2.66382600-0.85218900$

$\mathrm{H} \quad-3.50111100 \quad 1.99299300 \quad 0.72398900$ 


$\begin{array}{lrrr}\mathrm{C} & -2.87608200 & -1.46845000 & 1.93815900 \\ \mathrm{H} & -3.63333900 & -2.25541400 & 2.02859900 \\ \mathrm{H} & -1.96441000 & -1.79779400 & 2.44313800 \\ \mathrm{H} & -3.25117900 & -0.56462100 & 2.41920500 \\ \mathrm{O} & -1.98008200 & -2.17409200 & -0.20794900 \\ \mathrm{H} & -1.46165900 & -2.75004600 & 0.37468400 \\ \mathrm{H} & -1.77513500 & -0.18363600 & 0.54199000\end{array}$

\section{TSS to Yucatecone ${ }^{+}$}

$E E=-852.771403$

EE + Zero-point Energy $=-852.339178$

EE + Thermal Free Energy Correction $=-852.384325$

Number of imaginary freq. $=1$

Lowest freq. $=-629.56$

B3LYP(D3)/6-31+G(d,p), scrf $=(p c m$, Solvent=Water $)$

11

C

C

C

C

C

$\mathrm{H}$

$\mathrm{H}$

$\mathrm{H}$

$\mathrm{H}$

$\mathrm{H}$

$\mathrm{H}$

C

C

$\mathrm{H}$

$\mathrm{H}$

C

$\mathrm{H}$

$\mathrm{H}$

C

$\mathrm{H}$

$\mathrm{H}$

$\mathrm{H}$

C

$\mathrm{H}$

$\mathrm{H}$

$\mathrm{H}$

$\mathrm{O}$

O

C

$\mathrm{H}$

C

$\mathrm{H}$

$\mathrm{H}$

C

$\mathrm{H}$

$\mathrm{H}$

C

C

1.08994300

$1.66926700 \quad 0.76427800$

3.90972200

0.33683500

.42755800

$\begin{array}{lll}0.33683500 & -0.15848900\end{array}$

$1.98364100 \quad-0.38913200-1.66808500$

$\begin{array}{llll}1.75019400 & 1.69544900 & -0.07770100\end{array}$

$\begin{array}{lll}3.95533800 & 1.35137700 & -0.58774200\end{array}$

$\begin{array}{lll}4.10021900 & -0.54036400 & -2.11811700\end{array}$

$\begin{array}{llll}3.55983500 & -1.66750600 & -0.87821600\end{array}$

$\begin{array}{lll}1.90750500 & 0.56635500 & -2.20035500\end{array}$

1.63052200

$-1.17406700$

$-2.34474100$

$-1.12040700$

0.74025900

$-0.08313900$

0.74274000

1.02220700

1.68205300

0.60335900

0.11801300

2.28170600

1.17203500

1.78474800

2.33818000

$-0.58353800$

1.53452500

1.10990900

$-1.35548400$

1.60642900

1.87865900

$-0.42945300$

2.55223100

0.72812600

$5.26049400-0.03023000$

6.02452100

$-0.03835500$

0.43242500

$5.55538900 \quad 0.69340400$

$-0.35108300$

$5.21914700-1.02555700$

1.19800600

0.94146400

$-1.67548500$

0.88751400

0.21110400

$-1.64419800$

0.25123200

0.64174500

$-2.42692200$

1.06176700

1.89303600

$-1.97482200$

$-0.48227400$

2.96411100

0.37291500

0.69237300

$-0.22635500$

0.14180300

0.93863100

$-2.24758100$

1.37600200

$-0.91162400$

$-1.72908100$

1.86486300

$-0.91384500$

$-3.14796300$

0.26914500

$-1.74739200$

$-3.94628800$

0.72304300

$-1.50510600$

$-2.56502200$

$-0.37183600$

$-2.09733700$

$-3.75803800$

$-0.57835200$

$-2.17312500$

$-4.37962500$

0.03075000

$-0.37534900$

$-4.38759000$

$-1.37188400$

0.28314200

$-2.68879900$

$-1.26183700$

$-0.79289300$

$-3.06188500$

2.43897000

0.43937600

$-2.45232800$

3.30757400

$-0.15930700$

$-3.87584100$

2.78619200

0.09949100

$-3.50818100$

2.05825100

$-0.80198300$

0.76362600 


$\begin{array}{lrrr}\mathrm{C} & -2.88504700 & -1.50162600 & 1.91375900 \\ \mathrm{H} & -3.66249200 & -2.26741700 & 2.02495500 \\ \mathrm{H} & -1.96929300 & -1.86150100 & 2.39026400 \\ \mathrm{H} & -3.21946300 & -0.59197400 & 2.41404700 \\ \mathrm{O} & -2.07671300 & -2.22392100 & -0.27150600 \\ \mathrm{H} & -1.55381800 & -2.81779800 & 0.29030800 \\ \mathrm{H} & -1.76658600 & -0.22761000 & 0.48090400\end{array}$

\begin{tabular}{|c|c|c|c|}
\hline $\begin{array}{l}\text { Yu } \\
\text { EE } \\
\text { EE } \\
\text { EE } \\
\text { Nur } \\
\text { Lov } \\
\text { M0 } \\
11\end{array}$ & $\begin{array}{l}5^{+} \\
55696 \\
\text { oint Energy }= \\
\text { lal Free Energy } \\
\text { maginary freq. } \\
=46.07 \\
1+G(d, p), \text { scrf }\end{array}$ & $\begin{array}{l}-851.918330 \\
\text { y Correction= } \\
=0\end{array}$ & 851.964725 \\
\hline C & 1.00916600 & -0.29902200 & 0.03805900 \\
\hline C & 1.88571200 & 0.96079400 & 0.01913200 \\
\hline C & 3.83189700 & -0.27304300 & -0.50575500 \\
\hline C & 3.03495800 & -1.57039700 & -0.64737700 \\
\hline C & 1.56258200 & -1.29129900 & -0.97739100 \\
\hline $\mathrm{H}$ & 1.90981600 & 1.32839100 & -1.02175100 \\
\hline $\mathrm{H}$ & 3.86399700 & 0.23613300 & -1.48299500 \\
\hline $\mathrm{H}$ & 3.47902300 & -2.17930000 & -1.44049100 \\
\hline $\mathrm{H}$ & 3.11445000 & -2.14231600 & 0.28478400 \\
\hline $\mathrm{H}$ & 1.48115600 & -0.84678100 & -1.97628900 \\
\hline $\mathrm{H}$ & 0.97842400 & -2.21763800 & -0.96908800 \\
\hline C & -1.02664400 & 1.23967900 & 0.19229800 \\
\hline C & 1.31101500 & 2.04259500 & 0.90730700 \\
\hline $\mathrm{H}$ & 1.26681300 & 1.69907400 & 1.94586600 \\
\hline $\mathrm{H}$ & 1.96169000 & 2.92067400 & 0.87918700 \\
\hline C & -0.07450500 & 2.41844300 & 0.37666600 \\
\hline $\mathrm{H}$ & -0.55424300 & 3.12472100 & 1.05820300 \\
\hline $\mathrm{H}$ & 0.03597100 & 2.92258200 & -0.59121600 \\
\hline C & 5.24004500 & -0.50549800 & 0.00235000 \\
\hline $\mathrm{H}$ & 5.78553600 & -1.15712000 & -0.68529000 \\
\hline $\mathrm{H}$ & 5.77843900 & 0.44145100 & 0.08788000 \\
\hline $\mathrm{H}$ & 5.20941700 & -0.98406200 & 0.98615600 \\
\hline C & 0.85129300 & -0.90799100 & 1.42640500 \\
\hline $\mathrm{H}$ & 0.23117200 & -0.29693900 & 2.08472000 \\
\hline $\mathrm{H}$ & 0.41215400 & -1.90625900 & 1.36089800 \\
\hline $\mathrm{H}$ & 1.83772600 & -0.98851200 & 1.88799400 \\
\hline 0 & 3.19006200 & 0.61253500 & 0.42391300 \\
\hline 0 & -0.31921400 & 0.12646600 & -0.48970900 \\
\hline C & -2.22959400 & 1.59976700 & -0.68579600 \\
\hline $\mathrm{H}$ & -1.82251500 & 2.07746000 & -1.58587600 \\
\hline C & -3.06716200 & 0.40816200 & -1.18589400 \\
\hline $\mathrm{H}$ & -3.87986400 & 0.83712900 & -1.77786200 \\
\hline $\mathrm{H}$ & -2.48234200 & -0.19469500 & -1.88927200 \\
\hline C & -3.69162000 & -0.51071300 & -0.12602300 \\
\hline $\mathrm{H}$ & -3.85067500 & 0.00048000 & 0.83518100 \\
\hline $\mathrm{H}$ & -4.68980600 & -0.84180900 & -0.42993300 \\
\hline C & -2.89993900 & -1.74622300 & 0.18755100 \\
\hline C & -3.10513300 & 2.62287800 & 0.04824800 \\
\hline $\mathrm{H}$ & -2.57553700 & 3.56281300 & 0.21656600 \\
\hline $\mathrm{H}$ & -3.99270100 & 2.84553100 & -0.54921800 \\
\hline & -3.43866200 & 2.24792200 & 1.02191800 \\
\hline
\end{tabular}




$\begin{array}{lrrr}\mathrm{C} & -3.59670400 & -2.90453900 & 0.81571300 \\ \mathrm{H} & -4.19235300 & -3.39812300 & 0.03908700 \\ \mathrm{H} & -2.87745800 & -3.61144800 & 1.22629600 \\ \mathrm{H} & -4.29206500 & -2.55801300 & 1.58409800 \\ \mathrm{O} & -1.69334600 & -1.82924800 & -0.07774300 \\ \mathrm{H} & -0.97595800 & -0.74686600 & -0.42778500 \\ \mathrm{H} & -1.35382100 & 0.84594300 & 1.16358700\end{array}$

\begin{tabular}{|c|c|c|c|}
\hline $\begin{array}{l}\text { Yu } \\
\text { EE } \\
\text { EE } \\
\text { EE } \\
\text { Nu } \\
\text { Lov } \\
\text { B3l } \\
11\end{array}$ & $\begin{array}{l}9125 \\
\text { oint Energy = } \\
\text { lal Free Energ } \\
\text { maginary freq } \\
=38.50 \\
6-31+G(d, p),\end{array}$ & $\begin{array}{l}-852.374321 \\
\text { y Correction= } \\
=0\end{array}$ & 852.420810 \\
\hline $\mathrm{C}$ & 1.03010800 & -0.30798700 & 0.05586700 \\
\hline C & 1.90331100 & 0.95918500 & 0.01427600 \\
\hline C & 3.86647000 & -0.28725000 & -0.50514900 \\
\hline C & 3.06178900 & -1.58700400 & -0.64251200 \\
\hline C & 1.57824700 & -1.31258400 & -0.95665000 \\
\hline $\mathrm{H}$ & 1.92491900 & 1.30553600 & -1.03250700 \\
\hline $\mathrm{H}$ & 3.90312300 & 0.21604900 & -1.48505600 \\
\hline $\mathrm{H}$ & 3.49603900 & -2.19428100 & -1.44320500 \\
\hline $\mathrm{H}$ & 3.15170600 & -2.16428900 & 0.28453600 \\
\hline $\mathrm{H}$ & 1.48230900 & -0.88139400 & -1.95953300 \\
\hline $\mathrm{H}$ & 0.99946800 & -2.24123600 & -0.93326100 \\
\hline C & -1.03192800 & 1.27437800 & 0.18913800 \\
\hline C & 1.32978500 & 2.06903000 & 0.87560000 \\
\hline $\mathrm{H}$ & 1.28285700 & 1.75847100 & 1.92389000 \\
\hline $\mathrm{H}$ & 1.98696700 & 2.94194300 & 0.82398500 \\
\hline C & -0.05787200 & 2.44526900 & 0.33422100 \\
\hline $\mathrm{H}$ & -0.52424200 & 3.17794300 & 0.99696000 \\
\hline $\mathrm{H}$ & 0.05631300 & 2.92231600 & -0.64618300 \\
\hline C & 5.27788000 & -0.51432100 & 0.00878200 \\
\hline $\mathrm{H}$ & 5.82610500 & -1.16909900 & -0.67548500 \\
\hline $\mathrm{H}$ & 5.81577300 & 0.43479600 & 0.08781900 \\
\hline $\mathrm{H}$ & 5.25229700 & -0.98689500 & 0.99635000 \\
\hline C & 0.85107100 & -0.90255800 & 1.44987200 \\
\hline $\mathrm{H}$ & 0.28740600 & -0.24899100 & 2.11656400 \\
\hline $\mathrm{H}$ & 0.34013800 & -1.86588100 & 1.39403800 \\
\hline $\mathrm{H}$ & 1.83640000 & -1.05224800 & 1.89443200 \\
\hline 0 & 3.21813900 & 0.61606400 & 0.42453600 \\
\hline $\mathrm{O}$ & -0.31915600 & 0.12912100 & -0.48974700 \\
\hline C & -2.25699200 & 1.62007000 & -0.67493700 \\
\hline $\mathrm{H}$ & -1.87064100 & 2.10209200 & -1.58133200 \\
\hline C & -3.10113400 & 0.42117800 & -1.17056900 \\
\hline $\mathrm{H}$ & -3.92336200 & 0.85163800 & -1.74900800 \\
\hline $\mathrm{H}$ & -2.52263100 & -0.17020800 & -1.88785800 \\
\hline C & -3.71325900 & -0.51813000 & -0.11180900 \\
\hline $\mathrm{H}$ & -3.85299400 & -0.01953900 & 0.85844700 \\
\hline $\mathrm{H}$ & -4.72206100 & -0.82957000 & -0.40323800 \\
\hline C & -2.92839600 & -1.77025800 & 0.17388400 \\
\hline C & -3.13357400 & 2.64327000 & 0.07379800 \\
\hline $\mathrm{H}$ & -2.59888000 & 3.57613700 & 0.26658500 \\
\hline $\mathrm{H}$ & -4.01302700 & 2.88556800 & -0.52950300 \\
\hline
\end{tabular}




$\begin{array}{lrrr}\mathrm{H} & -3.48265100 & 2.25315300 & 1.03620400 \\ \mathrm{C} & -3.63531700 & -2.92865700 & 0.80107900 \\ \mathrm{H} & -4.28100900 & -3.38816700 & 0.04202300 \\ \mathrm{H} & -2.92150100 & -3.66770200 & 1.16560800 \\ \mathrm{H} & -4.28962800 & -2.58674500 & 1.60890600 \\ \mathrm{O} & -1.72209600 & -1.86422100 & -0.10848400 \\ \mathrm{H} & -0.95973200 & -0.72153800 & -0.43870000 \\ \mathrm{H} & -1.33569900 & 0.89265300 & 1.16873600\end{array}$

